


\section{ADVANCES IN \\ FLIGHT CONTROL SYSTEMS}

Edited by Agneta Balint 
Advances in Flight Control Systems

http://dx.doi.org/10.5772/587

Edited by Agneta Balint

\section{Contributors}

Ron Barrett, Qi-Ping Chu, Lars Sonneveldt, Jan Albert Mulder, Gianfranco Morani, Federico Corraro, Adolfo Sollazzo, Yuta Kobayashi, Masaki Takahashi, Yue Bao, Syuhei Saito, Yutaro Koya, Atsushi Fujimori, Maria Agneta Balint, Stefan Balint, Thomas Lombaerts, Jan Albert (Bob) Mulder, Olaf Stroosma, Bateman Francois, Francois Bateman, François Bateman, Nhan Nguyen, Thomas Jones, lain Peddle, S. Hossein Sadati, M. Sabzeh Parvar, M.B. Menhaj, Hammad Ahmad, Trevor Young, Daniel Toal, Edin Omerdic, Ben M. Chen, Ali Karimoddini, Guowei Cai, Hai Lin, Tong H. Lee

\section{(c) The Editor(s) and the Author(s) 2011}

The moral rights of the and the author(s) have been asserted.

All rights to the book as a whole are reserved by INTECH. The book as a whole (compilation) cannot be reproduced, distributed or used for commercial or non-commercial purposes without INTECH's written permission.

Enquiries concerning the use of the book should be directed to INTECH rights and permissions department (permissions@intechopen.com).

Violations are liable to prosecution under the governing Copyright Law.

\section{(c)) BY}

Individual chapters of this publication are distributed under the terms of the Creative Commons Attribution 3.0 Unported License which permits commercial use, distribution and reproduction of the individual chapters, provided the original author(s) and source publication are appropriately acknowledged. If so indicated, certain images may not be included under the Creative Commons license. In such cases users will need to obtain permission from the license holder to reproduce the material. More details and guidelines concerning content reuse and adaptation can be foundat http://www.intechopen.com/copyright-policy.html.

\section{Notice}

Statements and opinions expressed in the chapters are these of the individual contributors and not necessarily those of the editors or publisher. No responsibility is accepted for the accuracy of information contained in the published chapters. The publisher assumes no responsibility for any damage or injury to persons or property arising out of the use of any materials, instructions, methods or ideas contained in the book.

First published in Croatia, 2011 by INTECH d.o.o.

eBook (PDF) Published by IN TECH d.o.o.

Place and year of publication of eBook (PDF): Rijeka, 2019.

IntechOpen is the global imprint of IN TECH d.o.o.

Printed in Croatia

Legal deposit, Croatia: National and University Library in Zagreb

Additional hard and PDF copies can be obtained from orders@intechopen.com

Advances in Flight Control Systems

Edited by Agneta Balint

p. $\mathrm{cm}$.

ISBN 978-953-307-218-0

eBook (PDF) ISBN 978-953-51-6001-4 


\section{We are IntechOpen, \\ the world's leading publisher of Open Access books}

Built by scientists, for scientists

\section{$4,000+$ \\ Open access books available \\ $116,000+$ \\ International authors and editors

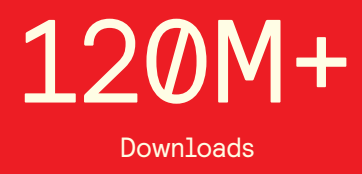

Our authors are among the

151

Countries delivered to

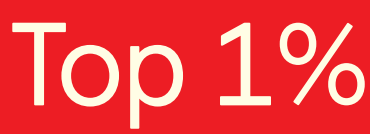

most cited scientists

Contributors from top 500 universities

$12.2 \%$

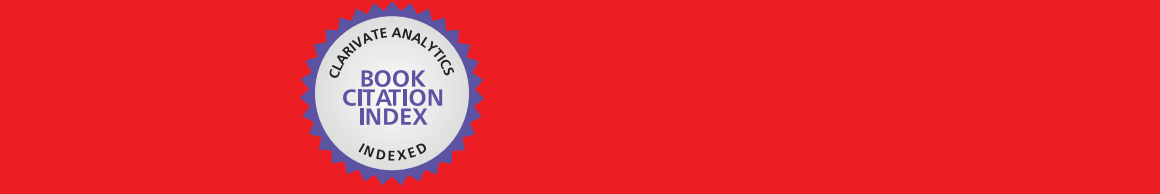

WEB OF SCIENCE ${ }^{\mathrm{M}}$

Selection of our books indexed in the Book Citation Index in Web of Science ${ }^{\mathrm{TM}}$ Core Collection (BKCI)

\section{Interested in publishing with us? \\ Contact book.department@intechopen.com}





\section{Meet the editor}

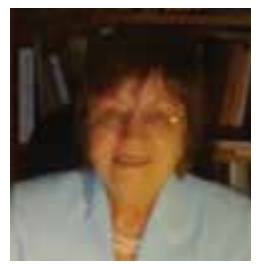

Professor Agneta M. Balint, $\mathrm{PhD}$ is a professor in the Department of Electricity, Optics, Solid State Physics and Material Structure of the Faculty of Physics, West University of Timisoara, Romania. She is an internationally well-known author and has published extensively in mathematical modeling in physics, chemistry, engineering and aerospace sciences. 



\section{Contents}

Preface XI

Chapter 1 Adaptive Fight Control Actuators and Mechanisms for Missiles, Munitions and Uninhabited Aerial Vehicles (UAVs) 1 Ronald Barrett

Chapter 2 Adaptive Backstepping Flight Control for Modern Fighter Aircraft 23

L. Sonneveldt, Q.P. Chu and J.A. Mulder

Chapter 3 Hybrid Adaptive Flight Control with Model Inversion Adaptation $\mathbf{5 3}$ Nhan Nguyen

Chapter 4 Application of Evolutionary Computing in Control Allocation 77

Hammad Ahmad, Trevor Young, Daniel Toal and Edin Omerdic

Chapter 5 Fault Tolerant Flight Control, a Physical Model Approach 93

Thomas Lombaerts, Ping Chu, Jan Albert (Bob) Mulder and Olaf Stroosma

Chapter 6 Design of Intelligent Fault-Tolerant Flight Control System for Unmanned Aerial Vehicles 117 Yuta Kobayashi and Masaki Takahashi

Chapter 7 Active Fault Diagnosis and Major Actuator Failure Accommodation: Application to a UAV 137 François Bateman, Hassan Noura and Mustapha Ouladsine

Chapter 8 Fault-Tolerance of a Transport Aircraft with Adaptive Control and Optimal Command Allocation 159

Federico Corraro, Gianfranco Morani and Adolfo Sollazzo 
Chapter 9 Acceleration-based 3D Flight Control for UAVs:

Strategy and Longitudinal Design 173

lain K. Peddle and Thomas Jones

Chapter 10 Autonomous Flight Control System

for Longitudinal Motion of a Helicopter 197

Atsushi Fujimori

Chapter 11 Autonomous Flight Control

for RC Helicopter Using a Wireless Camera 217

Yue Bao, Syuhei Saito and Yutaro Koya

Chapter 12 Hierarchical Control Design of a UAV Helicopter 239

Ali Karimoddini, Guowei Cai, Ben M. Chen, Hai Lin and Tong H. Lee

Chapter 13 Comparison of Flight Control System

Design Methods in Landing 261

S.H. Sadati, M.Sabzeh Parvar and M.B. Menhaj

Chapter 14 Oscillation Susceptibility of an Unmanned Aircraft whose Automatic Flight Control System Fails 275

Balint Maria-Agneta and Balint Stefan 


\section{Preface}

Nonlinear problems in flight control have stimulated cooperation among engineers and scientists from a range of disciplines. Developments in computer technology allowed for numerical solutions of nonlinear control problems, while industrial recognition and applications of nonlinear mathematical models in solving technological problems is increasing.

The aim of the book Advances in Flight Control is to bring together reputable researchers from different countries in order to provide a comprehensive coverage of advanced and modern topics in flight control not yet reflected by other books. This product comprises 14 contributions submitted by 38 authors from 11 different countries and areas. It covers most of the currents main streams of flight control researches, ranging from adaptive flight control mechanism, fault tolerant flight control, acceleration based flight control, helicopter flight control, comparison of flight control systems and fundamentals. According to these themes the 14 contributions are grouped in six categories, corresponding to six parts of the book.

The first part of the book is a collection of four chapters $(1,2,3,4)$ dedicated to the adaptive flight control.

In Chapter 1 the purpose is to introduce the technical community to some of the adaptive flight control mechanisms and structures which have either lead directly to or actually flown in various classes of missiles, munitions and unhabited aircraft. Although many programs are not open for publication, glimpses of a select flow have made it to the public arena at various level. This chapter is centered on airing several supersystem-level advances to flight-proven missiles, munitions and UAVs. Toward that end, basic models were used to lay out proof-of-concept flight hardware which was then fabricated, bench and/or ground tested and incorporated in flight vehicles. In the early years the adaptive aircraft were often simply flown, just to prove the concept worked. More recently, aircraft using adaptive flight control mechanisms have been flown off against conventional benchmark aircraft so as to demonstrate systematic superiority, thereby proving that flight control systems employing adaptive aerostructures result in some combination of lower power consumption, higher bandwidth, reduction in total aircraft empty weight, greater flight-speed, shock resistance, lower part count, lower cost etc. On normal occasions, adaptive aerostructures have ever been shown to be "enabling" that is, the aircraft class would not be able to fly without them.

In Chapter 2 an integrated, though cascaded Lyapunov-based adaptive backstepping approach is taken and used to design a flight path controller for nonlinear high-fidelity 
F-16 model. Adaptive backstepping allows assuming that the aerodynamic force and moment models may not be known exactly, and even that they may change in flight due to causes as structural damage and control actuator failness. To simplify the mathematical approximation partition the flight envelope into multiple connecting operating regions, called hyperboxes, is proposed. In each hyperbox a locally valid linearin-parameters nonlinear model is defined. The coefficients of these local models can be estimated using the update laws of the adaptive backstepping control laws. The number and size of the hyperboxes should be based on a priori information on the physical properties of the vehicle on hand, and may be defined in terms of state variables as Mach number, angle of attack and engine thrust. To interpolate between the local models to ensure smooth model transitions B-spline neural networks are used. Numerical simulations of various maneuvers with aerodynamic uncertainties in the model and actuator failures are presented. The maneuvers are performed at several flight conditions to demonstrate that the control laws are valid for the entire flight envelope.

In Chapter 3 a hybrid adaptive flight control method as another possibility to reduce the effect of high-gain control, is investigated. The hybrid adaptive control blends both direct and indirect adaptive control in a model inversion flight control architecture. The blending of both direct and indirect adaptive control is sometimes known as composite adaptation. The indirect adaptive control is used to update the model inversion controller by two parameter estimation techniques: 1) an indirect adaptive law based on the Lyapunov theory and 2) a recursive least-squares indirect adaptive law. The model inversion controller generates a command signal using estimates of the unknown plant dynamics to reduce the model inversion error. This directly leads to a reduced tracking error. Because the direct adaptive control only needs to adapt to a residual uncertainity, its adaptive gain can be reduced in order to improve stability robustness. Simulations of the hybrid adaptive control for a damaged generic transport aircraft and a pilot-inloop flight simulator study show that the proposed method is quite effective in providing improved command tracking performance for a flight control system.

In Chapter 4 the purpose is to design a compensator using an evolutionary computing technique (i.e. generic algorithms) to compensate the interaction between control allocation and actuator dynamics.

The interaction of first order actuator dynamics and control allocation and the structure of the compensator is established for first order and second order actuator dynamics. The tuning of the compensator parameters using generic algorithm is described. Simulation and results for tuned compensator are shown for a range of first and second order actuator dynamics. At the end conclusions are established.

The second part of this book consists on four chapters $(5,6,7,8)$ dedicated to the fault tolerant flight control.

In Chapter 5 a physical modular approach, where focus is placed on the use of mathematical representation based on flight dynamics., is used. All variables and quantities which appear in the model have a physical meaning and thus are interpretable in this approach, and on avoids so called black and grey box models, where the content has no clear physical meaning. Besides the fact that this is a more transparent approach, allowing the designers and engineers to interpret data of each step, it is assumed that these physical models will facilitate certification for eventual future real 
life applications, since monitoring of data is more meaningfull. Adaptive nonlinear dynamic inversion is selected as the preferred adaptive control method in this modular or indirect approach. The advantage of dynamic inversion is the absence of any need for gain scheduling and an input-output decoupling of all control channels. Adaptation of the controller is achieved by providing up-to-date aerodynamic model information which is collected in a separate identification module.

In Chapter 6 an intelligent flight control system is presented that can discriminate between faults and natural disturbances in order to evaluate and deal with the situation. In the control system, an evaluator of flight conditions is designed on the basis of the dynamics of a controlled object. Moreover, to deal with the situation adaptively, a new flight-path-planning generator is introduced on the basis of the evaluation. In the study each system is designed by neural network. The learning based systematical design method is developed that uses evaluation functions for the subsystems. A six-degreeof-freedom nonlinear simulation is carried out.

In Chapter 7 a nonlinear UAV model, which allows simulating asymmetrical control surface failures is presented. In fault-free mode, a nominal control law based on an Eigenstructure assignment strategy is designed. As the control surface positions are not measured, a diagnosis system is performed with a bank of observers able to estimate the unknown inputs. However, as the two ailerons offer redundant effects, isolating a fault on these actuators requires an active diagnosis method. In the last part, a pre-computed F.T.C. strategy, dedicated to accommodate for a ruddervator failure, is depicted.

In Chapter 8 a scheme of a fault-tolerant flight control system is proposed. It is composed by the core control laws, based on the DAMF technique, to achieve both robustness and reconfiguration capabilities and the CA system, based on the active set method, to properly allocate the control effort on the healthy actuators. Numerical results of a case study with a detailed model of a large transport aircraft are reported to show the effectiveness of the proposed fault-tolerant control scheme.

The third part of this book consists on one chapter (9) dedicated to the acceleration based flight control.

In Chapter 9 the manoeuvre autopilot solution presented moves away from the more mainstream methods, recently reported in the literature and instead returns to the concept of acceleration control which has been commonly used in missile applications, and to a limited extent in aircraft applications, for a number of decades. However, whereas acceleration control has traditionally been used within the framework of linearized flight control (the aircraft or missile dynamics are linearized, typically about a straight and level flight condition), the algorithms and mathematics presented in this chapter extend the fundamental acceleration controller for operate equally effectively over the entire 3D flight envelope. The result of this extension is that the aircraft then reduces to a point mass with a steerable acceleration vector from a 3D guidance perspective. This abstraction which is now valid over the entire flight envelope is the key to significantly reducing the complexity involved in solving the manoeuvre flight control problem.

The fourth part of this book consists on three chapters $(10,11,12)$ dedicated to helicopter flight control. 
In chapter 10 a flight control design for the longitudinal motion of helicopter is presented. The flight mission considered here is that a helicopter hovers at a start position, moves to goal position with keeping a specified cruise velocity and hovers again at the goal. The characteristics of the linearized equation of the helicopter are changed during this flight mission because the trim values of the equation are widely varied. Gain scheduling is one of candidates to stabilize the vehicle for the entire flight region. The flight control system is constructed as a double loop control system, which consists of an inner-loop controller and outer-loop controller. The former is needed for stabilizing the controlled plant, while the latter is used for tracking the reference which is given to accomplish the flight mission. To design the inner-loop controller, the longitudinal motion of a helicopter is modelled by a linear interpolative polytopic model whose varying parameter is the flight velocity. The outer-loop controller is designed by taking into consideration the steady-state of the controlled variable.

In chapter 11 an autonomous flight of a RC helicopter with small-wireless camera and a simple artificial marker which is set on the ground is analyzed. It is thought that a more wide-ranging flight is possible if the natural feature points are detected from the image obtained by the camera on RC.

In chapter 12 an analytical approach to design and analyze the whole system including the inner-loop and outer-loop controllers for a small-scale UAV helicopter is presented. Here, in the proposed hierarchical structure, the inner-loop is responsible for the internal stabilization of the UAV in the hovering state and control of the linear velocities and heading angle angular velocity whereas the outer-loop is used to drive the system, which is already stabilized by the inner-loop, to follow a desired path while keeping the system close to the hovering state. This strategy is an intuitive way of controlling such a complex system. Another reason that compels to employ such a control system is that the UAV model cannot be fully linearized. In practice, the heading angle of the UAV cannot be restricted to a small range of variation, it depends on the mission and could be in any direction. This imposes nonlinearity, which can be modelled by a transformation. To handle this semi-linearized model, the linear and nonlinear parts are separated, and then the linear part is controlled by the inner-loop and the nonlinear part by the outer-loop.

The fifth part of this book consists on one chapter (13) dedicated to the comparison of flight control systems.

In chapter 13 an overview concerning conventional, fuzzy logic-based, neural netbased adaptive control techniques is provided to the reader. Practical control schemes applicable in the area of control system design are introduced. The control laws are demonstrated on a three-degree-of-freedom simulation with linearized aerodynamic and engine models. The chapter is focused on aircraft landing manoeuvres. This part of flight needs to be strongly assisted by human pilot.

The sixths part of this book consists on one chapter (14) dedicated to the oscillation susceptibility when the control system fails.

In chapter 14 the oscillation susceptibility of an unmanned aircraft whose automatic flight control system fails, is analysed. The analysis is focused on the research of oscillatory movement around the centre of mass in a longitudinal flight with constant 
forward velocity (mainly in the final approach and landing phase). The analysis is made in a mathematical model defined by a system of three nonlinear ordinary differential equations, which govern the aircraft movement around its centre of mass, in such a flight. This model is deduced from a set of nine nonlinear differential equations, governing the movement of the aircraft around its centre of mass. It is shown that in a longitudinal flight with constant forward velocity, if the elevator deflection outruns some limits, oscillatory movement appears. This is proved by means of coincidence degree theory and Mawhin's continuation theorem. Numerical results concern an Aero Data Model in Research Environment and consist in: identification of the range of the elevator deflection for which steady state exists; computation of the manifold of steady states; identification of stable and unstable steady states; simulation of successful and unsuccessful manoeuvres; simulation of oscillatory movements.

I would like to express my sincere gratitude to all chapter authors for the time and effort they generously gave to the book. I would like to thank the publisher, InTech Education and Publishing, for cooperation in publication. Special thanks go to Ms. Ivana Lorkovic, process manager, who initiated this project.

February 26, 2011.

Agneta M. Balint

West University of Timisoara

Faculty of Physics

Timisoara, 300223,

Romania 



\title{
Adaptive Fight Control Actuators and Mechanisms for Missiles, Munitions and Uninhabited Aerial Vehicles (UAVs)
}

\author{
Ronald Barrett \\ The University of Kansas, Lawrence, Kansas
}

USA

\section{Introduction}

The purpose of this chapter is to introduce the technical community to some of the adaptive flight control mechanisims and structures which have either lead directly to or actually flown in various classes of missiles, munitions and uninhabited aircraft. Although many programs are not open for publication, glimpses of a select few have made it to the public arena at various levels.

This chapter is centered on airing several supersystem-level advances to flight-proven missiles, munitions and UAVs. Toward that end, basic models were typically used to lay out proof-of-concept flight hardware which was then fabricated, bench and/or ground tested, and incorporated in flight vehicles. In the early years, the adaptive aircraft were often simply flown, just to prove the concept worked. More recently, aircraft using adaptive flight control mechanisms have been flown off against conventional benchmark aircraft so as to demonstrate systemic superiority, thereby proving that flight control systems employing adaptive aerostructures result in some combination of lower power consumption, higher bandwidth, reduction in total aircraft empty weight, greater flight speed, shock resistance, lower part count, lower cost etc. On several occasions, adaptive aerostructures have even been shown to be "enabling;" that is, the aircraft class would not be able to fly without them.

Although adaptive materials have been known for more than 120 years, the Aerospace industry has only more recently become aware of their basic characteristics. Starting in the mid 1980's Ed Crawley's group at MIT laid the foundations of what would become an active and vibrant branch of aerospace technology. With simple experiments on bending and extension-twist coupled plates, this group demonstrated that airloads on aerodynamic surfaces could be actively manipulated by using conventionally attached piezoelectric actuators. ${ }^{1-3}$ Although the structures resulting from these projects were not incorporated in flightworthy aircraft, their significance cannot be overstated as they introduced the technical community to the possibility of aircraft flight control with adaptive aerostructures and by doing so effectively started the entire field. Table 1 summarizes a collection of programs which have lead directly to a series of flight proven adaptive subscale aerospace systems. 


\begin{tabular}{|c|c|c|}
\hline $\begin{array}{l}\text { Project } p=\text { piezoelectric, } s=\text { shape memory alloy } \\
c=\text { component testing only } f=\text { flight tested } \\
v=\text { entire vehicle configuration tested with adaptive device }\end{array}$ & & $\begin{array}{l}\text { Agency/ } \\
\text { Sponsor }\end{array}$ \\
\hline 1 Bending-Twist Coupled Aeroelastic PZT Plate (1985-87) & $c, p$ & MIT \\
\hline 2 Adaptive Flap (1987-89) & $c, p$ & MIT \\
\hline 3 Twist-Active Subsonic DAP Missile Wing (1989-90) & $c, p$ & $A R O$ \\
\hline 4 Twist-Active DAP Rotor (1990-91) & $c, p$ & $A R O$ \\
\hline 5 Aeroservoelastic Twist-Active Wing (1990-92) & $c, p$ & Purdue \\
\hline 6 Twist-Active Supersonic DAP Wing (1991-92) & $c, p$ & KU \\
\hline 7 Constrained Spar Torque-Plate Missile Fin (1991-92) & $c, p$ & $K U$ \\
\hline 8 Free-Spar DAP Torque-Plate Fin (1992-93) & $c, p$ & $K U$ \\
\hline 9 Pitch-Active DAP Torque-Plate Rotor (1992-93) & $c, p$ & $K U$ \\
\hline 10 Subsonic Twist-Active DAP Wing (1993-94) & $c, p$ & WL/MNAV \\
\hline 11 Subsonic Twist-Active SMA Wing (1993-94) & $c, s$ & WL/MNAV \\
\hline 12 Subsonic Camber-Active DAP Wing (1993-94) & $c, p$ & WL/MNAV \\
\hline 13 Subsonic Camber-Active SMA Wing (1993-94) & $c, s$ & WL/MNAV \\
\hline 14 Supersonic Twist-Active DAP Wing (1993-94) & $c, p$ & WL/MNAV \\
\hline 15 Supersonic Twist-Active SMA Wing (1993-94) & $c, s$ & WL/MNAV \\
\hline 16 Supersonic Camber-Active DAP Wing (1993-94) & $c, p$ & WL/MNAV \\
\hline 17 Supersonic Camber-Active SMA Wing (1993-94) & $c, s$ & WL/MNAV \\
\hline 18 UAV with Flexspar Stabilator (Mothra 1993-94) & $v, f, p$ & $A A L$ \\
\hline 19 Flexspar TOW-2B Wing (1993-94) & $v, p$ & NSF \\
\hline 20 Solid State Adaptive Rotor (SSAR) (1994-95) & $c, p$ & NSF \\
\hline 21 Aeroservoelastic Flexspar Fin (1994-95) & $c, p$ & $A A L$ \\
\hline 22 UAV with Solid State Adaptive Servopaddle Rotor (95-96) & $v, f, p$ & NSF \\
\hline 23 MAV with Flexspar Stabilator (1994-97) & $v, f, p$ & DoD CDTO \\
\hline 24 Barrel-Launched Adaptive Munition (1995-97) & $v, p$ & AFOSR \\
\hline 25 Smart Compressed Reversed Adaptive Munition (1995-97) & $v, p$ & WL/MNAV \\
\hline 26 Rotationally Active Linear Actuator (RALA 1995-97) & $c, p$ & WL/Boeing \\
\hline 27 Pitch-Active Torque-Plate Wing (1997-98) & $c, p$ & $A A L$ \\
\hline 28 Range-Extended Adaptive Munition (1998-99) & $v, p$ & DARPA \\
\hline 29 Hypersonic Interceptor Test Technology (1998-2000) & $v, p$ & SMDC/Schafer \\
\hline 30 Coleopter MAV with Flexspar Stabilators (1998-2001) & $v, f, p$ & $D A R P A$ \\
\hline 31 UAVs with Pitch-Active SMA Wings (2000-01) & $v, f, s$ & $A A L$ \\
\hline 32 Light Fighter Lethality MicroFlex Actuator (2000-01) & $v, p$ & TACOMARDEC \\
\hline 33 Pitch-Active Curvilinear Fin Actuator (2001-02) & $c, s$ & AMCOM \\
\hline 34 SC Range-Ex Adaptive Munition (SCREAM) (2000-03) & $v, p$ & TACOM ARDEC \\
\hline 35 Thunder Multilaminate RALA Fin (2000-03) & $c, p$ & $A F R L / M N A V$ \\
\hline 36 Centerline Precompression RALA Fin (2000-03) & $c, p$ & AFRL/MNAV \\
\hline 37 Center Pivot Flexspar Fin (2002-03) & $c, p$ & $A R L$ \\
\hline 38 PBP StAB (2003-) & $v, f, p$ & TU Delft/TNO \\
\hline 39 Convertible UAV with PBP Grid Fin (2003-) & $v, f, p$ & TU Delft/KU \\
\hline 40 Convertible UAV with PBP Turning Vane Flaps(2003-) & $v, f, p$ & TU Delft/KU \\
\hline 41 Extended-Range Adaptive Gravity Weapons (2003-) & $v, f, p$ & AFRL/Boeing \\
\hline 42 PBP Supersonic NAV (2004-) & $v, f, p$ & Lutronix \\
\hline 43 PBP Morphing Wing UAV (2005-) & $v, f, p$ & TU Delft/KU \\
\hline
\end{tabular}

Table 1. Summary of Adaptive Aerostructures Projects with Direct Connections to Flightworthy Adaptive Uninhabited Aerial Vehicles 


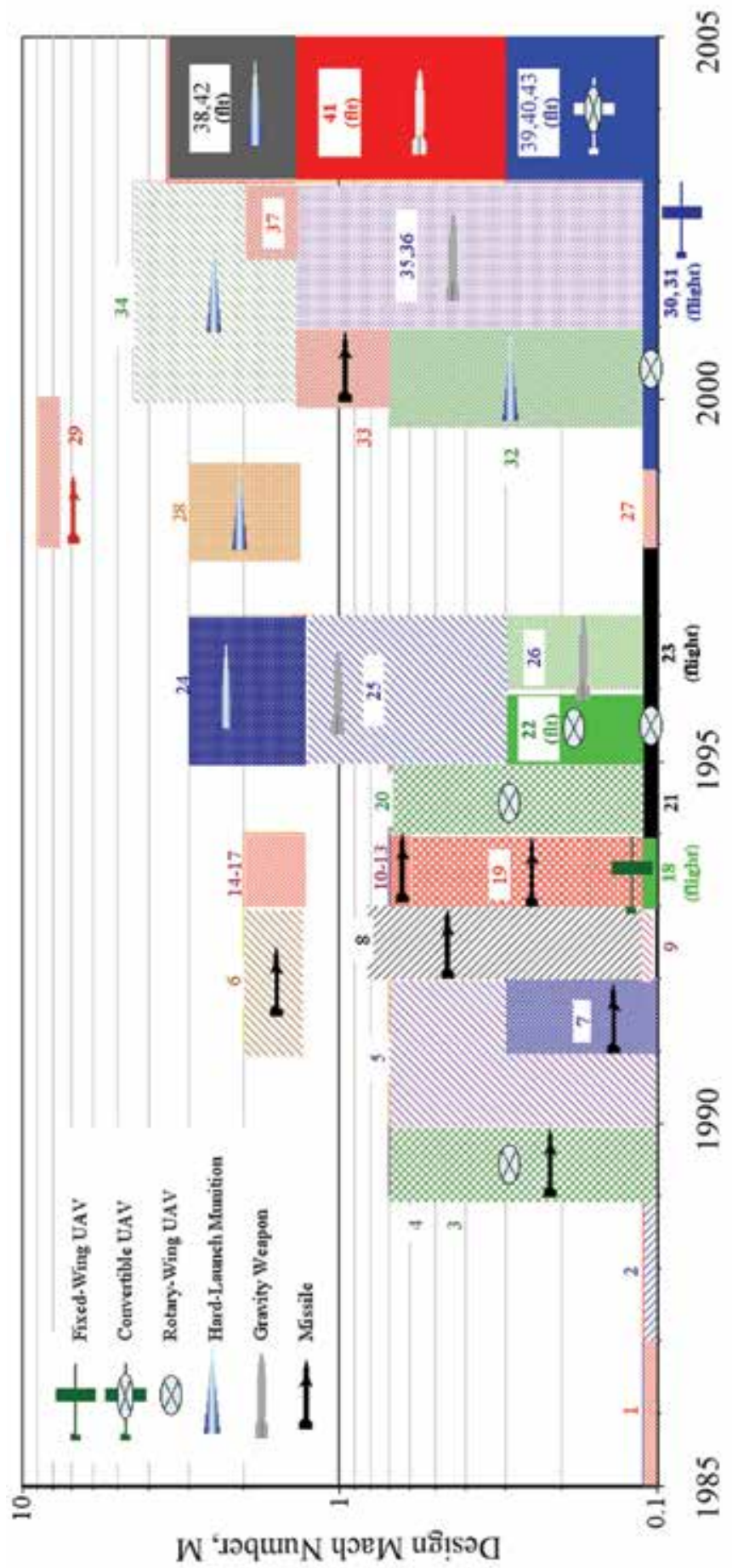

Fig. 1.1 Historical Overview of Adaptive Aerostructures Projects with Direct Connection to UAV Flight 


\section{Missile, munition and supersonic Uninhabited Aerial Vehicle (UAV) flight control}

In the late 1980's, up through 1990, a series of flight control mechanisms were being explored which could drive flaps with piezoelectric bender elements. ${ }^{4}$ Although these flight control devices were tested on a small scale, the goal of the investigators was to eventually transition these devices to full-scale, inhabited aircraft.

In 1990, a paradigm shift took was triggered which lead one branch of adaptive aerostructures technology down a large scale/inhabited aircraft path, the other to uninhabited aircraft. The driving philosophy behind the split was simple:

- Aircraft will benefit the most from this line of technology when adaptive materials can be integrated into aircraft primary structure.

- Because there is no current or planned FAA/MilHandbook5 database for materials certification, these materials -by law- will not be allowed in primary structure of FAR 23/25, 27/29 certified aircraft and will not allowed in the primary structure of most inhabited military aircraft because structural designers have no A- or B-basis mechanical properties to lay out designs with.

Because this philosophy split occured so long ago, the uninhabited branch of technology has had ample time to mature and be demonstrated in flight, thankfully, without the myriad of restrictions which begjile their inhabited counterparts. Among the uninhabited aircraft which were and still are being robustly pursued in this obscure corner of the adaptive aerostructures world are missiles, munitions and UAVs of many sizes. Because funding for military research was comparatively easier to obtain, some of the first applications development efforts were centered on missile and fin research. These eventually matured into system-level designs, bench test and eventually flight tests.

\subsection{Early fin and wing designs}

In 1990, an important advance in piezoelectric actuation was made. ${ }^{5-8}$ The invention of Directionally Attached Piezoelectric (DAP) actuators allowed otherwise isotropic piezoelectric elements to behave as if they were highly orthotropic. This was achieved by attaching piezoelectric elements along a longitudinal axis, leaving the sides free to expand or contract and thereby effectively nulling the lateral stiffness of the element while maintaining the longitudinal stiffness. This property was especially important as it allowed direct active manipulation of structures in twist, rather than just bending. Eventually, DAP actuators were developed with Orthotropy Ratios, OR in excess of 100 which makes them to this day one of the preferred actuator classes for twist activation, especially when cost, robustness and ease of manufacturing are considered. This high OR allowed both coupled and uncoupled structures to be directly manupulated in twist for the first time. If one considers an active laminate with an isotropic active material bonded to an uncoupled substrate, one can see clearly from laminated plate theory that the shear and twist activation terms are zero for CAP elements and non-zero for DAP elements: 
Conventionally Attached Piezoelectric Actuators:

$$
\left\{\frac{N}{M}\right\}_{C A P}=\left[\begin{array}{cccccc}
A_{11} & A_{12} & 0 & B_{11} & B_{12} & 0 \\
A_{12} & A_{11} & 0 & B_{12} & B_{11} & 0 \\
0 & 0 & 2 A_{66} & 0 & 0 & 2 B_{66} \\
B_{11} & B_{12} & 0 & D_{11} & D_{12} & 0 \\
B_{12} & B_{11} & 0 & D_{12} & D_{11} & 0 \\
0 & 0 & 2 B_{66} & 0 & 0 & 2 D_{66}
\end{array}\right]_{C A P}\left\{\begin{array}{c}
\Lambda \\
\Lambda \\
0 \\
0 \\
0 \\
0
\end{array}\right\}=\left\{\begin{array}{c}
\left(A_{11}+A_{12}\right) \Lambda \\
\left(A_{11}+A_{12}\right) \Lambda \\
0 \\
\left(B_{11}+B_{12}\right) \Lambda \\
\left(B_{11}+B_{12}\right) \Lambda \\
0
\end{array}\right\}_{C A P}
$$

Directionally Attached Piezoelectric Actuators:

$$
\left\{\frac{N}{M}\right\}_{D A P}=\left[\begin{array}{llllll}
A_{11} & A_{12} & 2 A_{16} & B_{11} & B_{12} & 2 B_{16} \\
A_{12} & A_{22} & 2 A_{26} & B_{12} & B_{22} & 2 B_{26} \\
A_{16} & A_{26} & 2 A_{66} & B_{16} & B_{26} & 2 B_{66} \\
B_{11} & B_{12} & 2 B_{16} & D_{11} & D_{12} & 2 D_{16} \\
B_{12} & B_{22} & 2 B_{26} & D_{12} & D_{22} & 2 D_{26} \\
B_{16} & B_{26} & 2 B_{66} & D_{16} & D_{26} & 2 D_{66}
\end{array}\right]_{D A P}\left\{\begin{array}{l}
\Lambda \\
\Lambda \\
0 \\
0 \\
0
\end{array}\right\}=\left\{\begin{array}{l}
\left(A_{11}+A_{12}\right) \Lambda \\
\left(A_{22}+A_{12}\right) \Lambda \\
\left(A_{16}+A_{26}\right) \Lambda \\
\left(B_{11}+B_{12}\right) \Lambda \\
\left(B_{22}+B_{12}\right) \Lambda \\
\left(B_{16}+B_{26}\right) \Lambda
\end{array}\right\}_{D A P}
$$

In the late 1980's it was not readily apparent which among the many approaches to adaptive flight control would actually work in a real flight environment. Several important papers relating aeroelastic tailoring, geometric sweep and aeroelastic coupling were authored, comparing CAP and DAP elements.9-11 These papers showed that with aeroelastic coupling, small deflections could be aeroelastically magnified to control authority levels which were consistent with aircraft flight control.

Although DAP elements were first integrated into a subscale missile wing in 1989, a new design incorporating DAP elements on a torque plate was conceived and reduced to practice in 1990.6-8 This approach allowed large rotations of an aerodynamic shell while the loads were taken up by a high strength internal structure. Figure 2 shows the Constrained Spar DAP Torque-Plate Missile Fin. Because the fin was intended for use in a TOW missile, it was capable of $\pm 5^{\circ}$ deflections with a corner frequency better than $30 \mathrm{~Hz}$ and a maximum power consumption under $50 \mathrm{~mW} .{ }^{12-14}$ During the this program, an important design philosophy was evolved that is still seen as critical advice for adaptive aerostructures designers to this day:

i. Minimize the amount of work done by adaptive materials on passive structure

ii. Employ aerodynamic and mass balancing principles so that the adaptive structures resist only transient external airloads and inertial loads.

iii. Use coefficient of thermal expansion mismatch to precompress piezoelectric actuator elements.

The US Air Force was generous in its support of this area after the concept was initially proven on the bench and the wind tunnel. The first study examining piezoelectric flight control for Air Force missiles was centered on low aspect ratio fin and wing manipulation. In 1993, Wright Laboratory commissioned a study examining DAP and CAP-activated surfaces. This was one of the first times that finite element methods were used to capture the behavior of subsonic and supersonic active lifting surfaces. figure 3 shows a FEM model of a supersonic double-circular arc camber-active DAP fin and NACA 0012 subsonic twist-active DAP fin. ${ }^{15}$ 


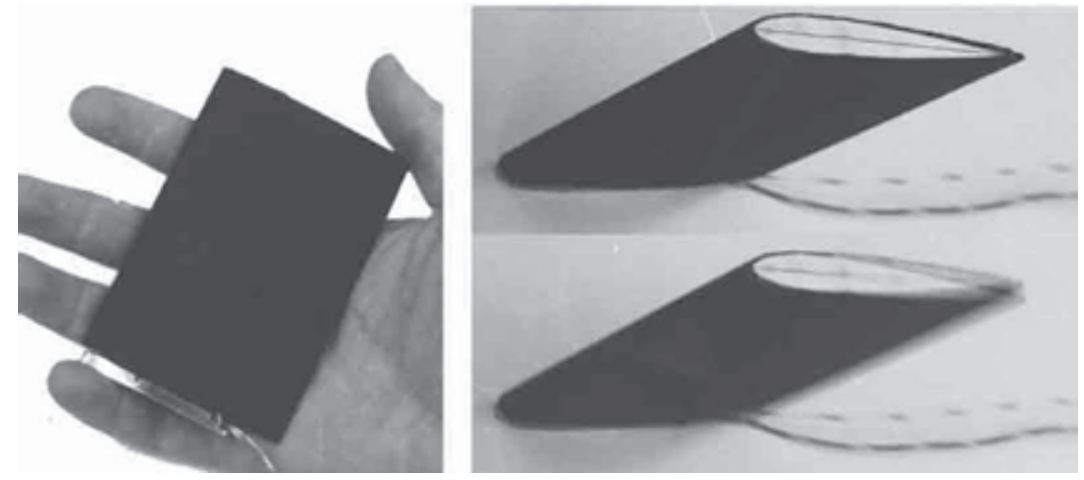

Fig. 2. Constrained Spar DAP Torque-Plate Missile Fin ${ }^{13}$
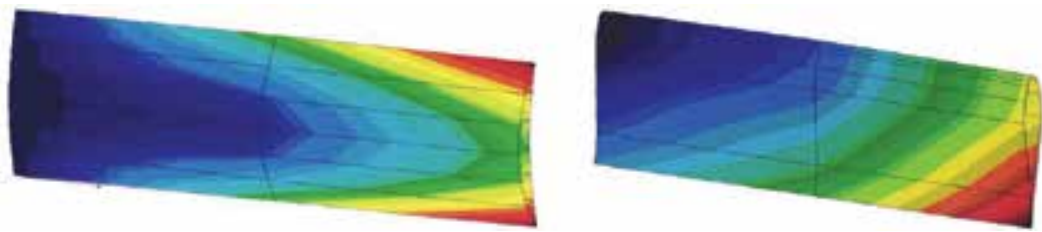

Fig. 3. Finitel Element Models of Active Supersonic \& Subsonic Fins ${ }^{15}$

Although the technology worked, at the time it did not work well enough as the levels of manipulation of low aspect ratio aerodynamic surfaces being actuated in twist and camber was simply far too low. Still, for higher aspect ratios, the design was shown not only to work, but work well. However, because most missiles employ low aspect ratio aerodynamic surfaces, a new approach was needed.

\subsection{Flexspar: The first flight enabling adaptive system}

In an effort to dramatically increase control surface deflections of low aspect ratio fins and in keeping with principles $i$. and ii above, a new flight control device was invented and reduced to practice. This flight control system would be shown to be one of the most useful and widely used in aircraft employing adaptive materials for flight control. Figure 4 shows the general layout of the device which was designed around a symmetric, balanced airfoil shell.

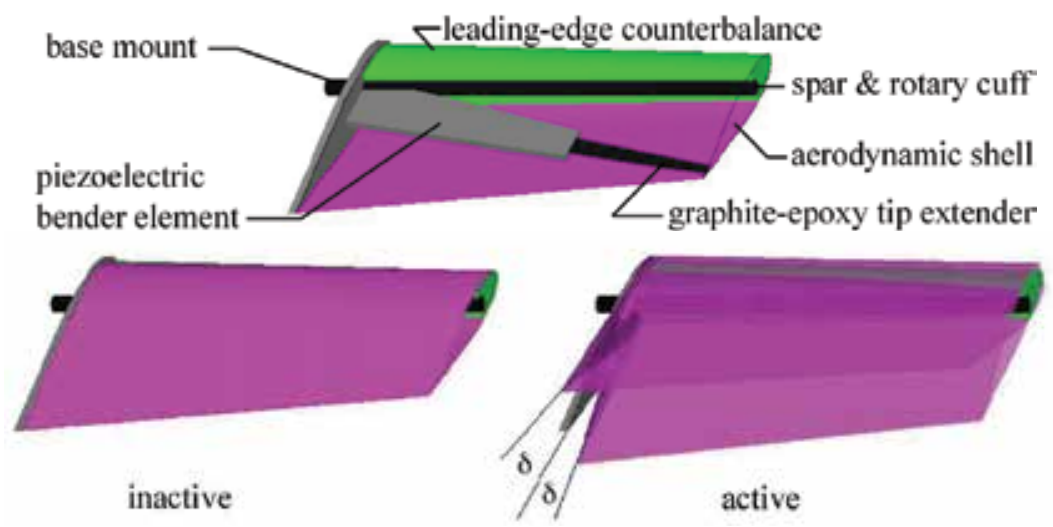

Fig. 4. Flexspar Fin Anatomy 


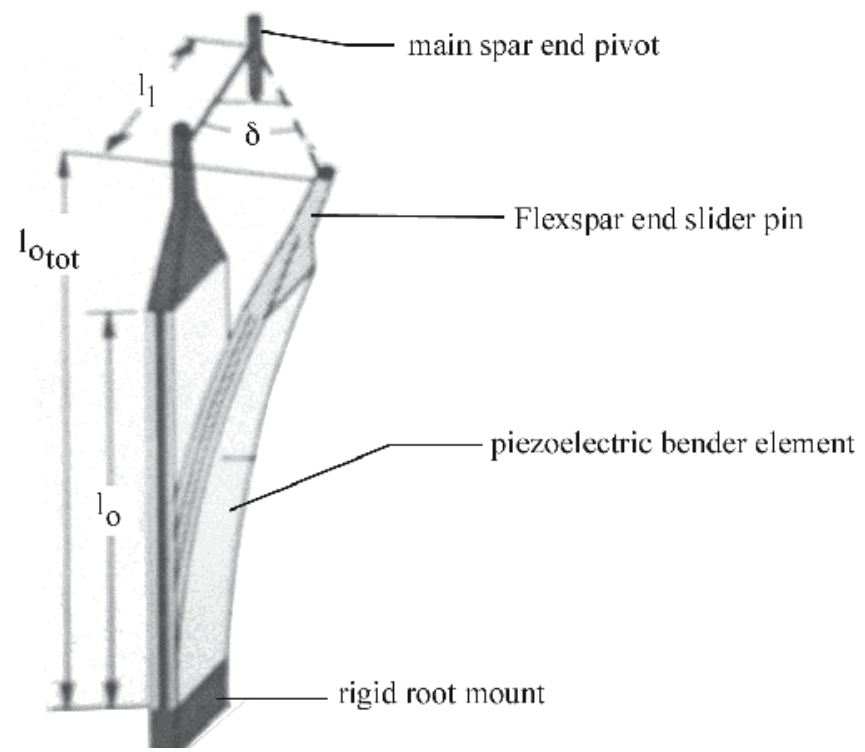

Fig. 5. Tip-Joint Flexspar Fin Geometric Parameters

If one examinies the basic construction of a piezoelectric bender element, then a simple expression can be laid out which relates laminate curvature, $\mathrm{k}$ to cure parameters, material characteristics and active free strain levels of the actuator, L. As has been shown over the past 20 years, it is important to use thermally induced precompression in all flightworthy adaptive aerostructures as shown in Equation 3. Equation 4 is the solution of the bending curvature considering a symmetric, balanced laminate composed of two sheets of CAP actuator material bonded on either side of an uncoupled substrate. By using the geometry of Figure 5, the curvatures commanded can be translated into control surface deflections, d. Of course these estimations assume a frictionless, balanced system operating without geometric binding (which typically set in on real systems for rotation angles in excess of $15^{\circ}$.

$$
\begin{aligned}
& {\left[\begin{array}{cc}
\mathrm{A}_{11}+\mathrm{A}_{12} & 0 \\
0 & \mathrm{D}_{11}+\mathrm{D}_{12}
\end{array}\right]_{\mathrm{lam}}\left\{\begin{array}{l}
\varepsilon \\
k
\end{array}\right\}\left[\begin{array}{cc}
\mathrm{A}_{11}+\mathrm{A}_{12} & 0 \\
0 & \mathrm{D}_{11}+\mathrm{D}_{12}
\end{array}\right]_{\mathrm{a}}\left\{\begin{array}{c}
x \Delta \mathrm{T} \\
0
\end{array}\right\}+} \\
& {\left[\begin{array}{cc}
\mathrm{A}_{11}+\mathrm{A}_{12} & 0 \\
0 & \mathrm{D}_{11}+\mathrm{D}_{12}
\end{array}\right]_{\mathrm{s}}\left\{\begin{array}{c}
\boldsymbol{c} \Delta \mathrm{T} \\
0
\end{array}\right\}+\left[\begin{array}{cc}
0 & \mathrm{~B}_{11}+\mathrm{B}_{12} \\
\mathrm{~B}_{11}+\mathrm{B}_{12} & 0
\end{array}\right]_{\mathrm{a}}\left\{\begin{array}{l}
\hat{0} \\
0
\end{array}\right\}} \\
& \kappa=\frac{E_{a}\left(t_{s} t_{a}+2 t_{b} t_{a}+t_{a}^{2}\right) \Lambda}{\frac{E_{s} t_{s}^{3}}{12}+E_{a}\left(\frac{\left(t_{s}+2 t_{b}\right)^{2} t_{a}}{2}+\left(t_{s}+2 t_{b}\right) t_{a}^{2}+\frac{2}{3} t_{a}^{3}\right)} \\
& \delta=2 \sin ^{-1}\left[\frac{1 / \kappa\left(1-\cos \left(\kappa l_{o}\right)\right)+\left(l_{o t o t}-1 / \kappa \sin \left(\kappa l_{o}\right)\right)}{2 l_{l}}\right]
\end{aligned}
$$

These expressions have been regularly used for more than a decade to predict Flexspar actuator deflection levels with experimental and predicted results typically within $5 \%$ of each other. ${ }^{16-18}$ 


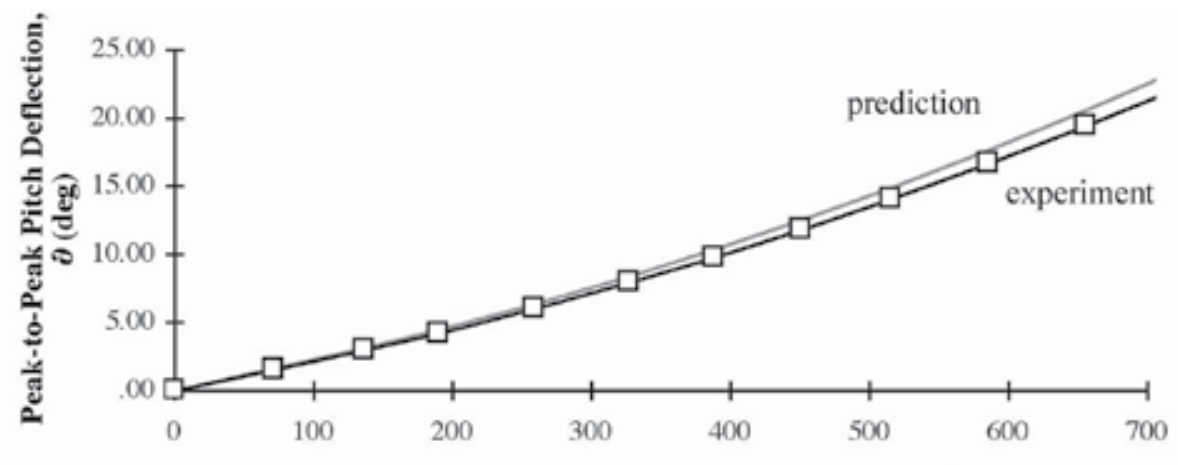

Static Half-Amplitude Actuation Field E31 (volts $/ \mathrm{mm}$ )

Fig. 6. Typical Flexspar Deflection s and Correlation Levels ${ }^{17}$

The Flexspar actuator configuration is still, to this day, one of the more well used actuation schemes for flight control. It comes in two major variants: the Tip-Joint Flexspar arrangement as shown in Fig. 5. This configuration is particularly well suited to low subsonic flight control using symmetric, balanced aerodynamic surfaces. A high moment configuration called a Shell-Joint Flexspar actuator is used for high subsonic and faster control surfaces. In the Fall of 1994 invention disclosures were submitted to Auburn University where the Flexspar was invented. Because the University failed to either file for patents or revert the rights to the inventors the Flexspar design can now be used royalty-free by one and all. The first missile system to incorporate the Flexspar design was the TOW-2B which used the Flexspar to manipulate wing deflections. Figure 7 shows the TOW-2B missle mounted in the wind tunnel during testing. Because the Flexspar wings were both aerodynamically and inertially balanced and they employed symmetric airfoils, the wing pitch deflections were not affected by airspeed.

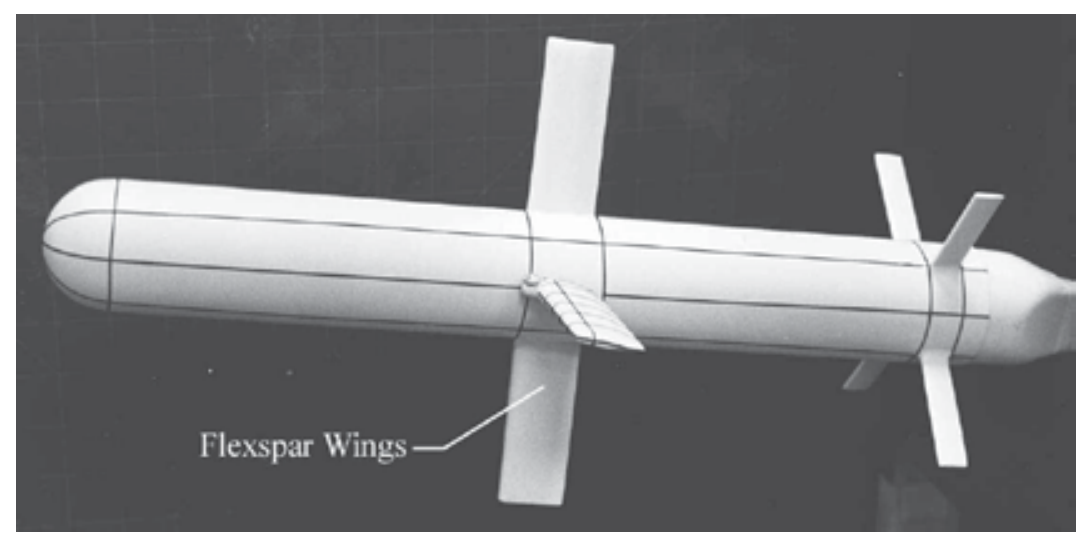

Fig. 7. Flexspar-Equipped TOW-2B Missile in Wind Tunnel

\subsection{Cruise missile and gravity weapon applications}

\subsubsection{Smart compressed reversed adaptive munition}

In 1995 the first of the gravity weapons programs was commissioned by the US Air Force employing adaptive flight control mechanisms. The program goal was to compress gravity 
weapons into bays the size of the weapon warheads. The driving factor in weapon compression came from the limited size of the F-22 internal weapon bays which were sized for AIM-120 air-to-air missiles, but not the existing slate of minimally compressed gravity weapons. Because conventionally guided gravity weapons of the time could not fit within the bay, a new approach was undertaken. Although a Flexspar configuration would have worked well, an antagonistic piezoelectric actuator was selected to drive a fin set as the design flight speed ranged from mid subsonic through low supersonic. Because of large shifts in position of center of pressure, the transonic flight regime is often the most challenging to flight control actuator designers as large rotations at high bandwidth against high moments are typically prescribed.

Because the designers were allowed to rearrange the weapon configuration itself, a new configuration was developed which took the most advantage of the 1940's-era Mk83 warhead design. This configuration called for a reversal in warhead direction such that the base of the warhead would fly first. This would allow for a stable bluff-body relase (important for weapon egress) and full strakes along the length of the weapon to maintain suitable levels of $\mathrm{C}_{\mathrm{Na}}$ and provide a housing to accommodate the antagonistic piezoelectric actuators. The entire weapon design took advantage of other artifacts including more than 80 in $^{3}$ of volume in large fuse well. Extensive bench and wind tunnel testing showed that full $\pm 10^{\circ}$ fin deflections could be resist all airloads without degradation through the transonic flight regime at frequencies in excess of $40 \mathrm{~Hz}$. Power consumption studies demonstrated that the actuators could be accommodated over the entire flight duration for less than 2cc of zinc-air batteries. ${ }^{19,20}$ Figure 8 shows the weapon configuration and during wind tunnel testing.
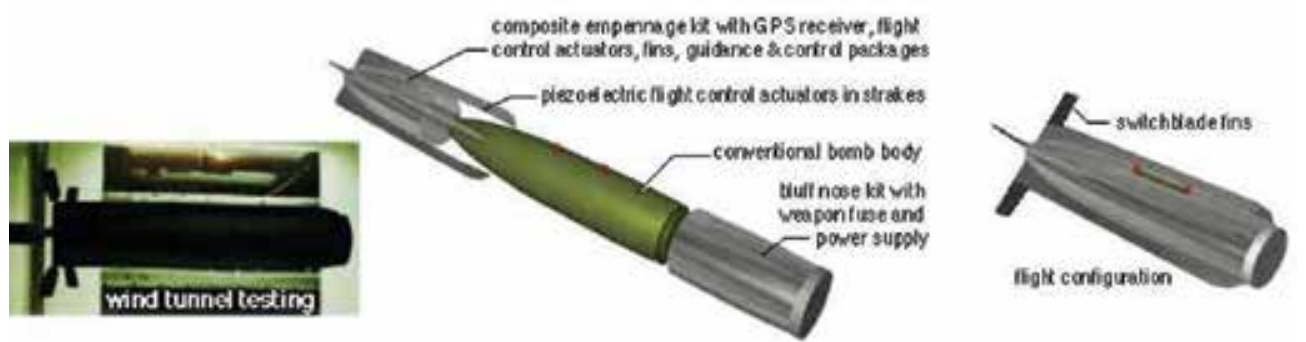

Fig. 8. Smart Compressed Reversed Adaptive Munition (SCRAM) ${ }^{19,20}$

Space constraints prevent the full chronicling of the program, but suffice it to say that this effort demonstrated that adaptive aerostructures could be used to increase weapon loadout by an order of magnitude.

\subsubsection{Weapon integration and design technology}

In 1997, following the success of the SCRAM program an effort was undertaken to provide vernier control for a new family of small penetrator weapons. The canard actuator used a modified form of a shell-joint Flexspar actuator called a Rotationally Active Linear Actuator (RALA). Although this unclassified program is many years old, details are not yet approved for public release.

The actuator set designed for the GBU-39 was intended to enhance terminal guidance and went through extensive bench and wind tunnel testing, showing full deflection capability through the transonic and low supersonic flight speeds. 22,23 


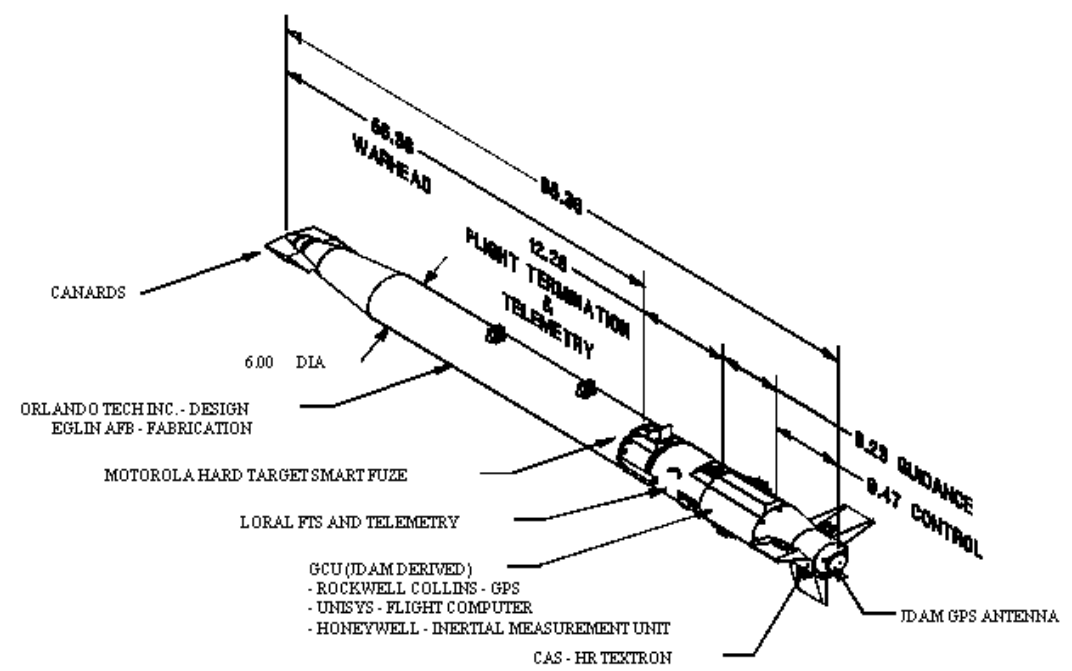

Fig. 9. GBU-39 Small Diameter Bomb with Adaptive Canards²1

\subsubsection{Miniature cruise missile airframe technology demonstrator}

Elements of the SCRAM and WIDT programs are currently alive and well in this USAF/Boeing project. Started in 2003, this effort is centered on demonstrating various advanced technologies including an adaptive on an extended range weapon system. As with the GBU-39 WIDT program, technical details have not yet been approved for release.

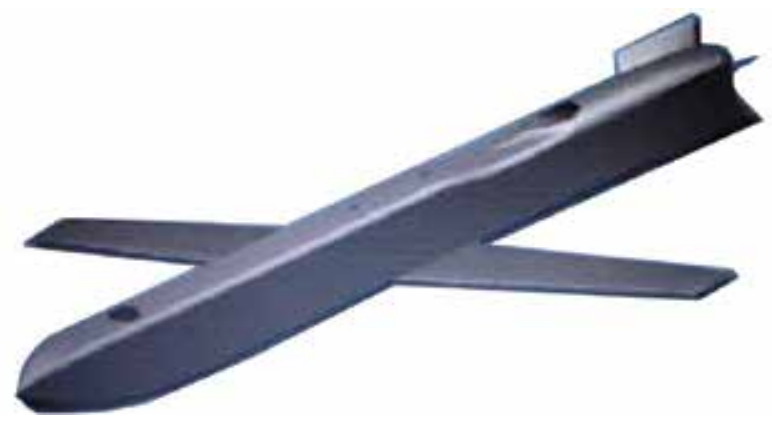

Fig. 10. Boeing/USAF Miniature Cruise Missile with Adaptive Wings²4

\subsection{Hard-launched munitions and supersonic Nano Aerial Vehicles (NAVs) 2.3.1 Barrel-Launched Adaptive Munition (BLAM) program}

In 1995 the Barrel-Launched Adaptive Munition (BLAM) program was initated to enhance aerial gunnery by increasing the hit probability and the probability of a kill given a hit in close-in aerial gunnery. To do this, a proof-of concept nontactical round was developed. Figure 11 shows the general arrangement of the test article.25-28

The most significant challenges that all hard-launched adaptive munition designs must overcome is clearly associated with launch loads. With respect to launch loads, all flight, storage and handling loads are trivial. In addition to launch loads, the round must also be able to deal with certain environmental factors that are also challenging for aerospace systems. The short summary below illustrates some of these challenges. 


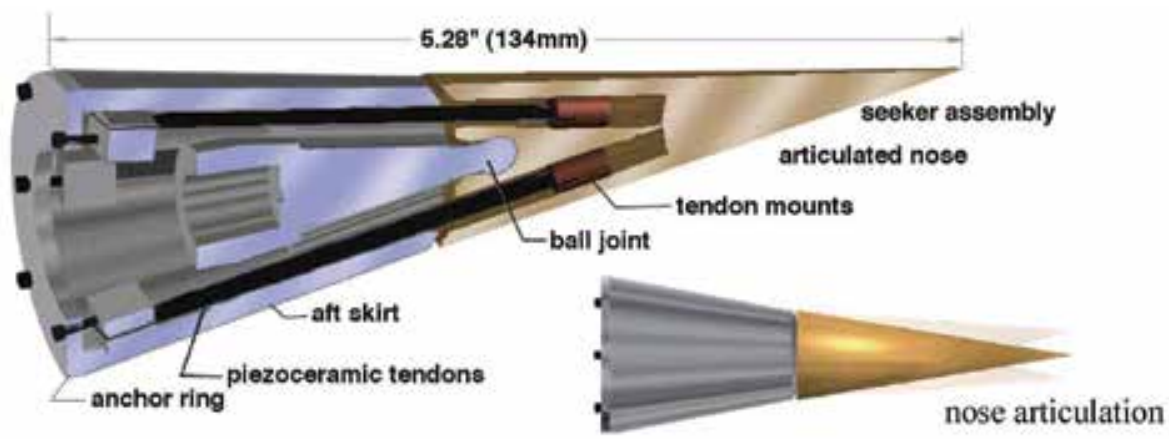

Fig. 11. Barrel-Launched Adaptive Munition (BLAM) Configuration

\subsubsection{Setback Accelerations}

Setback accelerations strongly influence structural layout and material choices and are the driving condition behind length limitations of actuators for hard-launched actuators. Although munitions designers use exacting profiles which are specific to a gun, round, muzzle velocity and charge type combination to predict peak setback accelerations, some basic boundaries can be gleaned from fundamental physics and empirical trends for firstorder design. If one assumes a constant acceleration along the length of a barrel (a travelingcharge profile), a round starting from 0 and exiting at a finite muzzle velocity, then a lower bound below which it is not possible to go:

$$
a_{\min }=V_{\text {muzzle }}^{2} / 2 L_{\text {barrel }}
$$

Because there is no upper bound which can be obtained by simple physics, generalized trends from interior ballistic profiles can be obtained. By examining the acceleration profiles of instrumented weapons like the Hypervelocity Weapon System, a rough upper bound can be gleaned for initial design purposes. ${ }^{29}$

$$
a_{\text {peak }} \cong 1.45 V_{\text {muzzle }}^{2} / \text { L }_{\text {barrel }}
$$

For larger caliber rounds which are currently fielded, setback accelerations on the order of $5,000-30,000$ g's are typical. The Navy's ERGM projectile is typical of the current families of guided 5" $(127 \mathrm{~mm})$ cannon shells and is designed for $12,000 \mathrm{~g}^{\prime}$ s of setback acceleration while the LCCM projectiles withstand 15,000g's. ${ }^{30}$

\subsubsection{Setforward, Balloting and Ringing}

Although secondary to setback accelerations, setforward accelerations have extremely detrimental effects on hard-launch round components and subsystems. Setforward accelerations are induced as the supersonic round exits the barrel into comparatively still air. This typically causes a large decelration force on most rounds with a pulse of approximately one order of magnitude lower than the setback acceleration. Setforward accelerations are the principal loads which induce buckling and end crush-out failure modes of many families of adaptive actuators. Reference 30 lists the design setforward accelerations for the ERGM round to be approximately 2,500g's. 


\subsubsection{Rotational Accelerations}

Most of the gun-launched munitions which are in use today are spin stabilized via the rifling in the barrel. Such rifling induces acceleration rates of several hundred thousand $\mathrm{rad} / \mathrm{s} 2$. As is the case with acceleration rates, Froude scaling principles hold when arriving at estimates for smaller (or larger) rounds, which indicates that lower caliber rounds will encounter acceleration levels as the reciprocal of the scale factor.

\subsubsection{Thermal Environment}

From Ref. 25 - 28, it can be seen that minimum operational and storage temperatures have a strong influence on the design of the actuator elements as they rely upon CTE mismatch to precompress actuator elements. Precompression levels at depressed temperatures must be carefully matched to thickness ratios and launch accelerations to ensure actuator survival of setback accelerations. Actuator material selection must be made with strong consideration of the operational and storage temperatures. Ref. 30 lists temperature environments which are typical of military munitions as: $-40^{\circ} \mathrm{C}\left(-40^{\circ} \mathrm{F}\right)$ to $+63^{\circ} \mathrm{C}\left(145^{\circ} \mathrm{F}\right)$ storage $-9^{\circ} \mathrm{C}\left(+15^{\circ} \mathrm{F}\right)$ to $+63^{\circ} \mathrm{C}\left(145^{\circ} \mathrm{F}\right)$ in a tactical/operational environment. References 30 - 36 lay out many other daunting environmental considerations which must be taken into account when laying out an adaptive munition.

\subsubsection{Current Progress}

The forefront of modern guided round research has progressed far beyond the BLAM which is now more than a decade old. These rounds range in size from just a few milimeters in caliber and up and employ several families of adaptive actuators, guiding rounds with control authorities of just over $1 \mathrm{~g}$ to many tens of g's. Not surprising, these projects are proprietary and/or restricted by ITARs and EARs. Several scattered efforts have intermittently surfaced, but most projects are still out of the public eye. ${ }^{36}$

\subsubsection{Supersonic Nano Aerial Vehicles (NAVs)}

The latest international incarnations of hard-launched aircraft comes in the form of supersonic Nano Aerial Vehicles (NAVs). Because conventional, subsonic NAVs are highly sensitive to the many adverse factors which become more severe with reduced scale, it is only logical that many of those problems can be skirted if the NAV is launched supersonically and flown for only a few seconds. The missions for these NAVs is nonlethal and primarily centered on reconnaissance. Figure 12 shows a CAD model of a supersonic NAV.

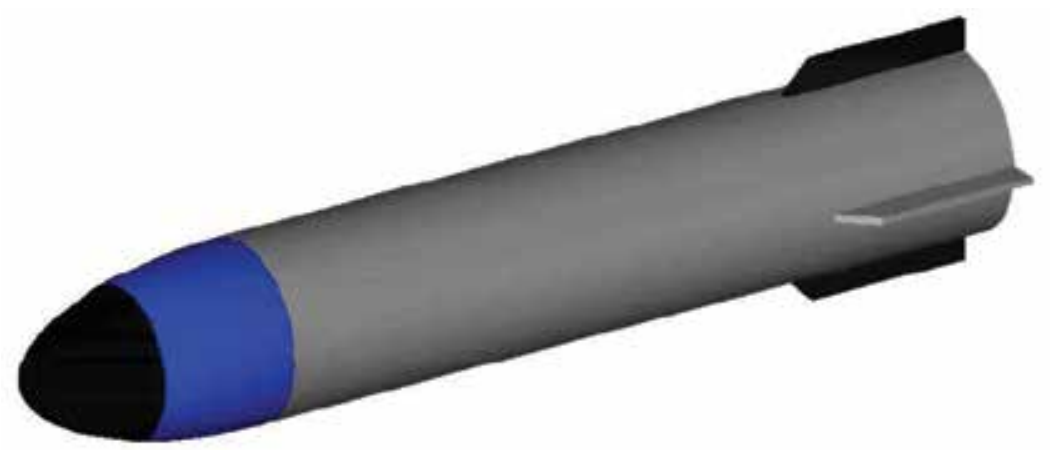

Fig. 12. Supersonic Nano-Aerial Vehicle (NAV) Design ${ }^{37}$ 
The flight control of these aircraft employ some of the latest adaptive actuators. These advanced "Post-Buckled Precompressed" (PBP) actuators have been shown to generate significantly higher deflections than conventional actuators and are ideal for small aircraft like NAVs. ${ }^{37}$

\section{Uninhabited Aerial Vehicle (UAV) \& Micro Aerial Vehicle (MAV) flight control}

Subsonic Uninhabited Aerial Vehicle (UAV) and Micro Aerial Vehicle (MAV) flight control with adaptive aerostructures draws its lineage back to some early experiments done on flight control devices which produced large changes in commanded lift coefficient. Although flight control mechanisms in rotary- and fixed-wing subsonic UAVs differ sharply, they share some common roots and even took advantage of some of the same families of actuators.

\subsection{Fixed-wing UAV beginnings}

As part of a National Science Foundation program investigating flight control with adaptive materials, the first fixed-wing aircraft using adaptive materials for all flight control was designed, built and flown in September of 1994. Using a Tip-Joint Flexspar configuration akin to the configuration shown in Figures 4 and 5, the aircraft executed basic maneuvers expected of micro-light aircraft using vertical and horizontal stabilator flight control.16
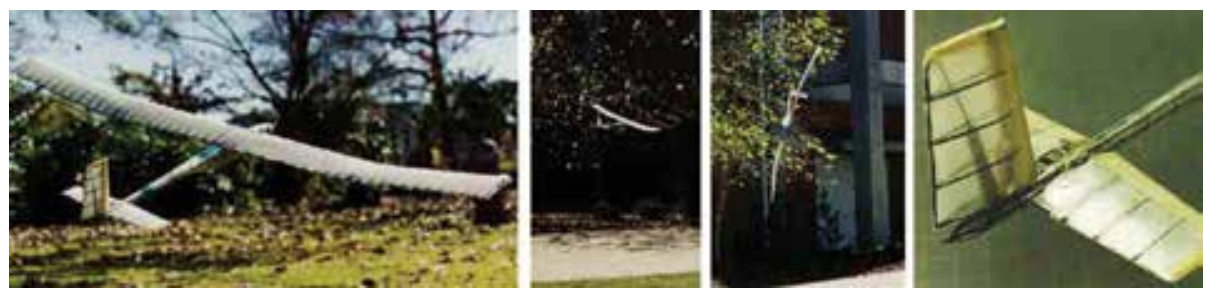

Fig. 13. Mothra, The First UAV with Flexspar Stabilators for Flight Control

\subsection{Foundations of rotary-wing UAV flight control}

The first serious attempts at achieving high control authority deflections of rotor systems was made in 1992. These early efforts employed the same class of torque-plates that drove missile fins, but in the roots of rotor blade systems. ${ }^{38}$ Although the first stages of the Solid State Adaptive Rotor (SSAR) was not selected for funding by the US Army, the founding experiments that went into the effort were instrumental in proving its feasibility. In 1994 the National Science Foundation picked up the project and supported it all the way through flight test. Figure 14 shows the earliest incarnation of the SSAR on a hover stand.

The first rotary-wing aircraft to fly using adaptive aerostructures for all flight control took to the air in December of 1996. Space constraints prevent its being chronicled completely, but it employed a pair of piezoelectric DAP servopaddles mounted on a teetering rotor system. The DAP servopaddles were driven by a brush contact assembly which allowed the rotor system to respond to basic cyclic commands at speeds in excess of $2.7 / \mathrm{rev}$. Flight tests were conducted against a benchmark aircraft, ultimately demonstrating maneuver authority nearly identical to the baseline aircraft. The big difference was that the aircraft shed $40 \%$ of its flight control system weight, leading to an $8 \%$ reduction in total gross weight, a $26 \%$ drop 
in parasite drag and a cut in part count from 94 components down to 5 . Figure 15 shows the SSAR aircraft Gamara in on the bench and during flight test. 40
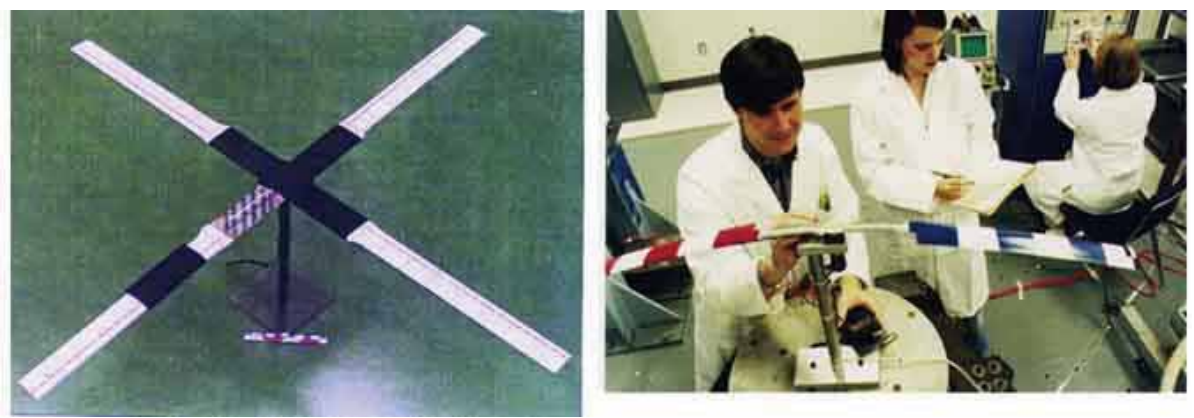

Fig. 14. Solid State Adaptive Rotor with Root Torque-Plate Actuator ${ }^{38,39}$
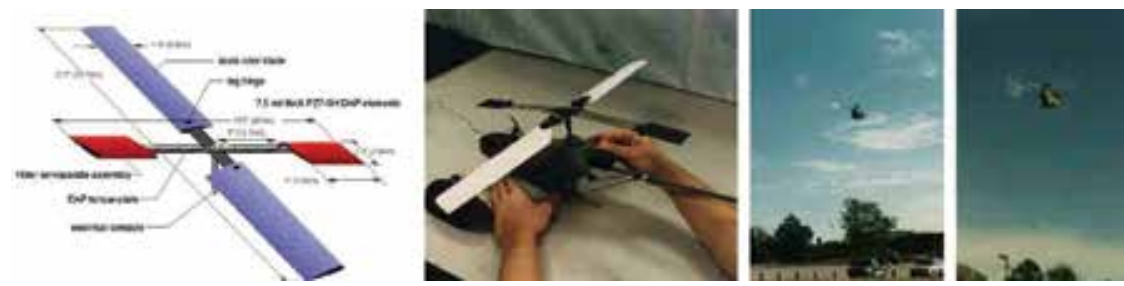

Fig. 15. Gamara, The First Rotary-Wing UAV to Fly with Adaptive Materials for All Flight Control,39

\subsection{The DoD's first MAV -- kolibri}

In 1994 the DoD CounterDrug Technology Office commissioned a program that would eventually lead to the US Military's first Micro Aerial Vehicle (MAV). In 1995, managers at Lutronix Corp. read about the success of the SSAR program in the technical literature and decided to fold the adaptive technology used in these various programs into their aircraft. The mission specification for the MAV called for a $24 \mathrm{hr}$ loiter with acoustic signature levels under $65 \mathrm{db}$ at $10 \mathrm{ft}$. Accordingly, an electric-tethered configuration was chosen. Because of the highly constrained rotor diameter and the limited adaptive materials manufacturing techniques of the day, it was decided that instead of a DAP-torque-plate rotor configuration, a Flexspar stabilator configuration would be used. These Flexspar stabilators would be placed in the rotor wash at the bottom of a graphite-truss frame fuselage counterrotating coleopter.
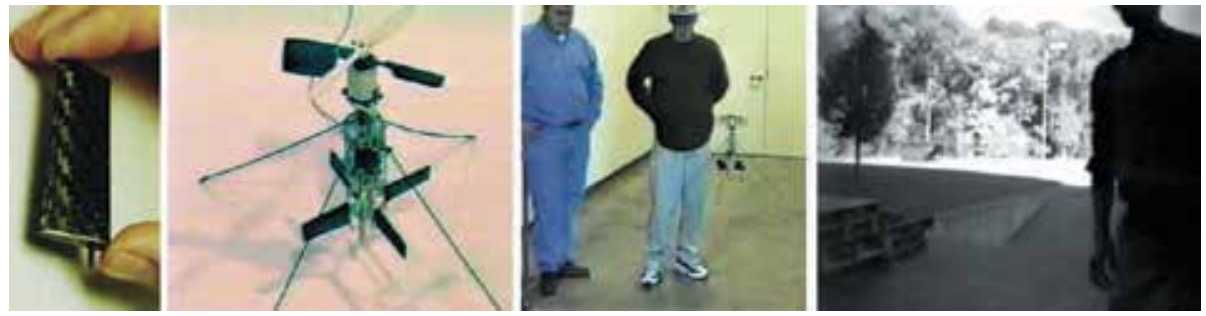

Fig. 16. DoD's First MAV -- Kolibri Stabilator, Aircraft \& in Flight 
Because the Kolibri was so severely weight-critical, any opportunity to shed weight was taken. Accordingly, the flight control system was a prime target for weight reduction. Because the aircraft body times to double amplitude were on the order of several tens of miliseconds, extremely fast actuators were a necessity. The conventional servoactuators on the open market were simply not fast enough to catch the aircraft and their weights were prohibitive. Flexspar actuators on the other hand were extremely lightweight with a mass of only 380mg each and exhibited a corner frequency of $47 \mathrm{~Hz}$-- almost double the bandwidth required to maintain flight. So for the first time, adaptive flight control mechanisms were not only enhancing technologies, but they actually enabled an entire class of aircraft to take to the air. Not surprisingly, the flight control system also included adaptive materials in the Tokin DO-16 piezoelectric gyros which were used to sense pitch, roll and yaw accelerations.

\subsection{The first free-flight rotary-wing MAV}

Following the success of the Kolibri, the DoDCDTO handed the program off to DARPA, thereby kicking off DARPA's much touted MAV program of the late '90's. Although the Kolibri satisfied the 24 hour hover endurance requirement with a tether, there was a strong desire to shed the tether. As a result a decision was made to go with an internal combustion engine. Although the boost in power was tremendous, the noise and structural vibrations were also boosted by an order of magnitude. As with the Kolibri, Flexspar stabilators and piezoelectric gyros allowed smooth flight in turbulent atmospheric conditions up through 18kt gusting winds. Figure 17 shows the aircraft overview and in flight. Ultimately, the aircraft was the only one of three finalist MAVs which successfully flew at DARPA's 3-day Fly-Off at Quantico Marine Corps Base, Virginia in September of 2000. Fly-offs were also conducted in several other locations including MacDill AFB, Florida, again, with the LuMAV appearing as the only rotary-wing/VTOL aircraft in the air. The aircraft performance specifications included a 15 minute endurance with all-weather capability including rain rates in excess of 12 " $(30 \mathrm{~cm}) / \mathrm{hr}$, dust and snow capability through $18 \mathrm{kt}$ gust fields, flight in $100^{\circ} \mathrm{F}, 100 \%$ humidity environments and $15 \mathrm{~g}$ wall-strikes. The aircraft was designed to carry a single submicrovideo camera an a GPS navigation suite. ${ }^{4}$
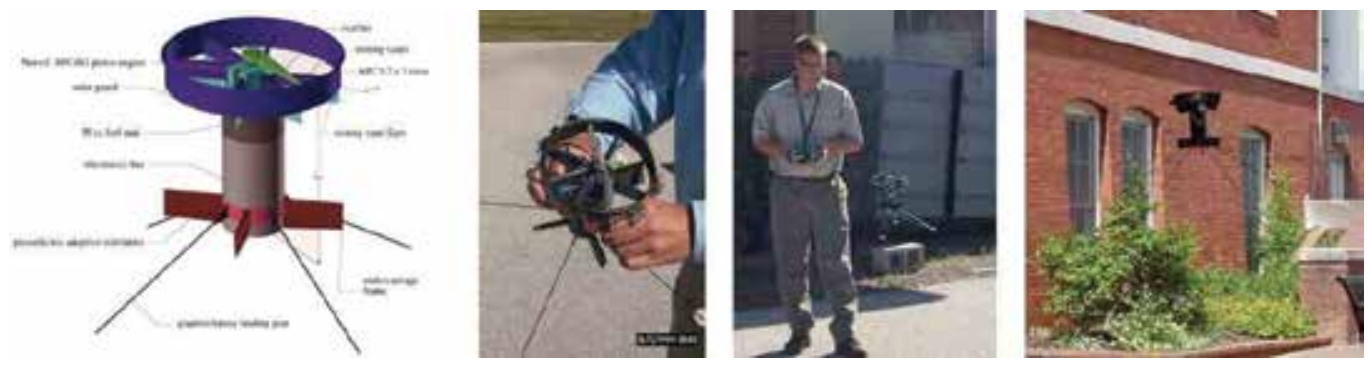

Fig. 17. Lutronix MAV Configuration \& Flying at MacDill AFB, Florida

\subsection{The XQ-138 convertible UAV}

As the LuMAV project came to a resoundingly successful conclusion, a follow-on design was sought. Although the LuMAV was clearly quite capable and flew circles around competitors, it was not selected for follow-on funding by DARPA. Instead, DARPA managers recommended approaching Boeing, which in turn recommended a new corporate partner on the Future Combat System (FCS) program, Singapore Technologies Engineering. 
A new aircraft configuration was independently conceived and reduced to practice in the summer of 2001 which employed the best of the rotary-wing and fixed-wing worlds. Impressed with the new aircraft performance and promise, ST Engineering purchased the rights to the aircraft and paid for its production. Initially, the XQ-138, a convertible coleopter, used conventional flight control actuators in its grid-fin empennage and turning vane flaps. Following component development efforts, these actuators were replaced by piezoelectric mechanisms. Figure XX18 shows the overall configuration of the XQ-138.45
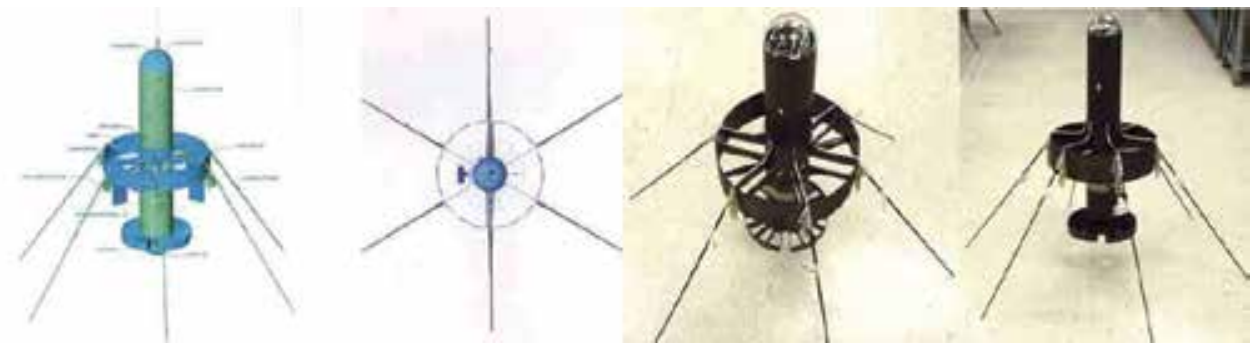

Fig. 18. The 11" Rotor Diameter XQ-138a Overall Configuration

More than 300 flight tests were conducted in all types of atmospheric conditions including gusts through $26 \mathrm{kts}$, rain at $9^{\prime \prime} / \mathrm{hr}, 100^{\circ} \mathrm{F}\left(38^{\circ} \mathrm{C}\right)$ heat at $100 \%$ humidity, winter flights in snow at $22^{\circ} \mathrm{F}\left(-6^{\circ} \mathrm{C}\right)$, dust, sand and finally flight in smoke plumes from exploded tanks. Figure 19 shows a sequence of photos of the aircraft flying off an FCS prototype on Redstone Arsenal, Alabama in April of 2002. These tests were followed by live-fire Battle-Damage Assessment (BDA) tests on the Hellfire Range of Eglin AFB in May of 2002. Although all variants of the aircraft used piezoelectric gyros at the core of its GNC package, the conversion of the aircraft to piezoelectric flight controls lent marked improvements in all aspects, eventually leading to a total empty weight savings in excess of $10 \%$ which allowed the range to be expanded by $30 \mathrm{nmi}$ to $100 \mathrm{nmi}$ and more than an hour and a half of endurance. Variants of the aircraft survive today as Singapore Technologies Engineering's FanTail UAV line of aircraft.

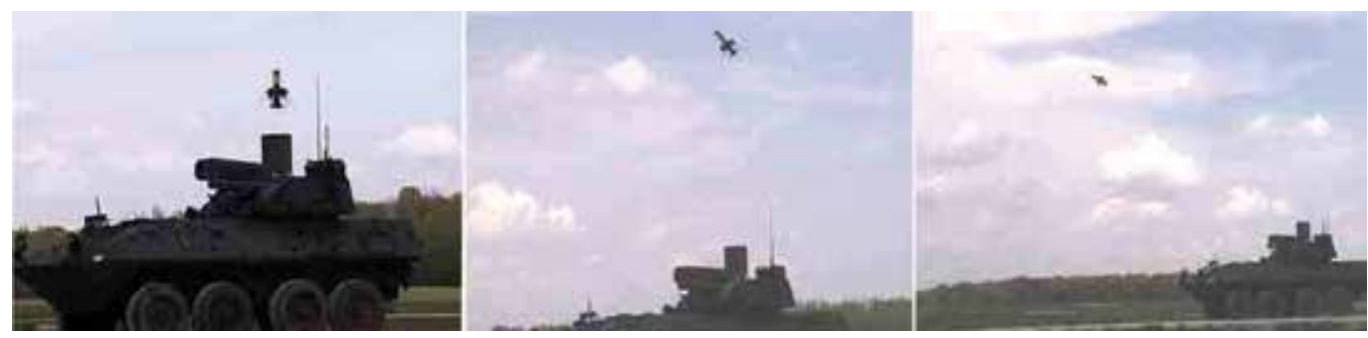

Fig. 19. The Piezoelectric FCS-Equipped XQ-138 Convertible Coleopter UAV

\subsection{Low and zero net passive stiffness structures}

In 2004 an important innovation was made which dramatically improved the performance of adaptive aerostructures. It was discovered how to simultaneously improve both deflection and force with minimal weight volume and cost penalties. ${ }^{46}$ This discovery was shown to dramatically improve flight control actuator performance and has been integrated into a number of flight control systems. ${ }^{47-53}$ Several variants of Low Net Passive Stiffness 
(LNPS), Zero Net Passive Stiffness (ZNPS) as Post-Buckled Precompressed (PBP) actuator elements have been built into aircraft which are currently undergoing development.

\section{Nomenclature}

\section{Symbol Description}

A,B,D in-plane, coupled, bending laminate stiffnesses

B actuator width

$\mathrm{D}_{\mathrm{p}} \quad$ actuator power density per unit mass, volume, cost

E stiffness

F applied end force

M Mach number

$\mathrm{M}$ applied moment vector

$\mathrm{N}$ applied force vector

OR Orthotropy Ratio $=\mathrm{E}_{\mathrm{L}} / \mathrm{E}_{\mathrm{T}}$

t thickness

y out of plane displacement dimension

$\mathrm{z} \quad$ through thickness dimension

$\alpha \quad$ angle of attack

$\delta \quad$ PBP beam angle

$\delta_{\mathrm{o}} \quad$ PBP end rotation angle

$\varepsilon \quad$ laminate in-plain strain

$\kappa \quad$ laminate curvature

$\Lambda$ piezoelectric free element strain

$\sigma \quad$ stress
Units

$\mathrm{N} / \mathrm{m}, \mathrm{N}, \mathrm{N}-\mathrm{m}$

$\mathrm{mm}$ (in)

$\mathrm{W} / \mathrm{g}, \mathrm{W} / \mathrm{cc}, \mathrm{W} / \mathrm{S}$

$\mathrm{GPa}(\mathrm{msi})$

$\mathrm{N}$ (lbf)

$\sim$

$\mathrm{N}-\mathrm{m} / \mathrm{m}$ (in-lb/in)

$\mathrm{N} / \mathrm{m}$ (lb/in)

$\sim$

$\mathrm{mm}$ (in)

$\mathrm{mm}$ (in)

$\mathrm{mm}$ (in)

deg

deg

deg

$\mu$ strain

$\mathrm{rad} / \mathrm{m}(\mathrm{rad} / \mathrm{in})$

ustrain

GPa (msi)

\section{Subscripts}

a actuator

b bond

c cost

ex external

$1 \quad$ laminate

L longitudinal

m mass

s substrate

$\mathrm{t}$ thermally induced

$\mathrm{T}$ transverse

v volume

\section{Acronyms}

AAL The Adaptive Aerostructures Laboratory

AFOSR US Air Force Office of Scientific Research,

AFRL Air Force Research Lab

AMCOM US Army Aviation and Missile Command

ARO US Army Research Office

DAP Directionally Attached Piezoelectric 
DARPA Defense Advanced Research Projects Agency

DoD CDTO Department of Defense CounterDrug Technology Office

FCS Future Combat System

LAV Light Armored Vehicle

MAV micro aerial vehicle

NAV nano aerial vehicle

NSF National Science Foundation

PZT lead zirconate titanate

SMDC Space and Missile Defense Command

TACOM-ARDEC US Army Tank-Automotive and Armaments

Command/Armament Research, Development and Engineering Center

TNO Toegepast Natuurwetenschappelijk Onderzoek

TU Delft The Technical University of Delft, Netherlands

UAV uninhabited aerial vehicle

WL Wright Laboratory

\section{References}

[1] Crawley, E., Lazarus, K. and Warkentin, D., "Embedded Actuation and Processing in Intelligent Materials," presented at the 2nd international Workshop on Composite Materials and Structures for Rotorcraft, Troy, NY, Sept., 1989.

[2] Lazarus, K., and Crawley, E., "Multivariable Active Lifting Surface Control using Strain Actuation: Analytical and Experimental Results," paper presented at the Third International Conference on Adaptive Structures, sponsored by the ASME, 9 - 11 November, 1992, San Diego.

[3] Lazarus, K. B., Crawley, E. F., and Bohlmann, J. D., "Static Aeroelastic Control Using Strain Actuated Adaptive Structures," Proceedings of the First Joint U.S./Japan Conference on Adaptive Structures, Maui, Hawaii, October, 1990.

[4] Spangler, R. L., and Hall, S. R., "Piezoelectric Actuators for Helicopter Rotor Control," Paper presented at the 31st Structures, Structural Dynamics and Materials Conference, Long Beach, California, April, 1990.

[5] Barrett, R., "Intelligent Rotor Blade Actuation through Directionally Attached Piezoelectric Crystals," 46th American Helicopter Society National Conference and Forum, Washington, D.C., May, 1990.

[6] Barrett, R., "Intelligent Rotor Blade and Structures Development using Piezoelectric Crystals," MS Thesis, the University of Maryland, College Park, Maryland 1990.

[7] Barrett, R., "Method and Apparatus for Sensing and Actuating in a Desired Direction," US Pat. 5,440,193, Aug. 1995.

[8] Barrett, R., "Actuation Strain Decoupling Through Enhanced Directional Attachment in Plates and Aerodynamic Surfaces," proceedings of the First European Conference on Smart Structures and Materials, Glasgow, Scotland, 12 - 14 May 1992, IOP Publishing, Bristol, UK 1992, pp. 383 - 386.

[9] Ehlers, S. M., and Weisshaar, T. A., "Static Aeroelastic Behavior of an Adaptive Laminated Piezoelectric Composite Wing," AIAA-90-1078-CP, April, 1990, pp. 1611-1623. 
[10] Ehlers, S. M., and Weisshaar, T., "Adaptive Wing Flexural Axis Control," paper presented at the Third International Conference on Adaptive Structures, sponsored by the ASME, 9 - 11 November, 1992, San Diego.

[11] Ehlers, S. M., and Weisshaar, T. A., "Effect of Material Properties on Static Aeroelastic Control," paper presented at the 33rd Structures, Structural Dynamics and Materials Conference, Dallas, Texas, 15 April, 1992.

[12] Barrett, R., "Active Plate and Missile Wing Development Using EDAP Elements," Journal of Smart Materials and Structures, Institute of Physics Publishing, Ltd., Techno House, Bristol, UK, Vol. 1, No. 3, pp. 214226, ISSN 096.

[13] Barrett, R., "Active Plate and Missile Wing Development Using DAP Elements," AIAA Journal,March, 1994.

[14] Barrett, R., "Active Composite Torque-Plate Fins for Subsonic Missiles," paper presented at the Dynamic Response of Composite Structures Conference, New Orleans, Louisiana, August 30 - September 1, 1993.

[15] Barrett, R., "Advanced Low-Cost Smart Missile Fin Technology Evaluation," Contractor Report to the United States Air Force Armament Directorate, Eglin Air Force Base, Florida, Contract No. F0 8630-93-C-0039, BAT, November 1993.

[16] Barrett, R., Brozoski, F., and Gross, R. S., "Design and Testing of a Subsonic All-Moving Adaptive Flight Control Surface," AIAA Journal, published by the AIAA, Reston, VA,Volume 35, No. 7, July 1997, pp. 1217 - 1219.

[17] Barrett, R. and Brozoski, F., "Missile Flight Control using Active Flexspar Actuators," Journal of Smart Materials and Structures, Institute of Physics Publishing, Ltd., Techno House, Bristol, UK, Vol. 5, No. 2, March 1996, pp. 121-128.

[18] Barrett, R., "Active Aeroelastic Tailoring of an Adaptive Flexspar Stabilator," Journal of Smart Materials and Structures, Vol. 5, No. 6 December 1996, Techno House, Bristol, UK, 1996, pp. 723 - 730.

[19] SCRAM Report Barrett, R., "Design, Construction and Testing of a Proof-of-Concept Smart Compressed Reversed Adaptive Munition," Final Report to the USAF Armament Directorate, Wright Laboratory, Eglin AFB, FL contract no. AF-FO863095-K-0079, September, 1996.

[20] Barrett, R., and Stutts, J., “Development of a Piezoceramic Flight Control Surface Actuator for Highly Compressed Munitions," proceedings of the 39th Structures, Structural Dynamics and Materials Conference 20 - 23 April 1998, Long Beach, CA, AIAA, Washington, D.C. 1998, paper no. AIAA-98-2034.

[21] Anon., "GBU-39 SDB - Small Diameter Bomb," http://www.globalsecurity.org/military/systems/munitions/sdb.htm, January 2006.

[22] Barrett, R., “Construction and Test Report for the Rotationally Active Linear Actuator (RALA) Adaptive Canard, Final Report for McDonnell Douglas, St. Louis, MO contract no. FO 8630-95-C-0009, August, 1997.

[23] Knowles, G., R. Barrett and M. Valentino, "Self-Contained High Authority Control of Miniature Flight Control Systems for Area Dominance," SPIE 11th International Symposium on Smart Structures and Materials, San Diego, CA, Mar. 2004. 
[24] Avila, C.A., "Precision Engagement," http://www.aviationnow.com/ conferences/html/ad03/avila_session_4b.pdf January 2006.

[25] Barrett, R., "Invention and Evaluation of the Barrel Launched Adaptive Munition (BLAM)," final report for USAF contract no. F-49620-93-C-0063, USAF Wright Laboratory Flight Vehicles Branch, WL/MNAV August, 1995.

[26] Barrett, R., and Stutts, J. "Barrel-Launched Adaptive Munition BLAM Experimental Round Research," final report for USAF contract no. F-49620-C-0063, USAF Wright Laboratory Flight Vehicles Branch, WL/MNAV February, 1997.

[27] Winchenbach, G., "Cone Aerodynamics Test", Aeroballistic Research Facility Ballistic Spark Range Technical Report, USAF Wright Laboratory Flight Vehicles Branch, WL/MNAV June 1996.

[28] Barrett, R. and Stutts, J., "Modeling, Design and Testing of a Barrel-Launched Adaptive Munition," proceedings of the 4" Annual SPIE Symposium on Smart Structures and Materials, San Diego, CA, 3-6 March 1997.

[29] Anon., "Actual Results and Analysis," Hypervelocity Weapon System (HVWS) Field Experiment 1 Final Report, Volume 1 Technical Report, published by Technology Gateways, Inc., Niceville, Florida, 1993.

[30] Hertlein, Robert and Mark Miner, "Extended Range Guided Munition (ERGM) Safe and Arm Device and Height-of-Burst Sensor," paper presented at the NDIA Fuse Conference, 9 April 2993, New Orleans, LA. (http://www.dtic.mil/ndia/2003fuze/hertlien.pdf

[31] Pike, J. "Trajectory Correctable Munition (TCM)," published by Global Security.org, Alexandria, Virginia, October, 2002. (http://www.globalsecurity.org/military/systems/munitions/tcm.htm).

[32] Anon., "M732A2 Proximity Fuse and M782 Multi-Option Fuse for Artillery (MOFA) Data Sheets," published by Alliant Techsystems, Inc. Edina, Minnesota, 2003. (http:/ / www.atk.com/productsPrecision/descriptions/products/fuses/ artilleryfuzes.htm)

[33] Lee, Gary, "Range-Extended Adaptive Munition (REAM)” Final Report from Lutronix Corporation to the Defense Advanced Research Projects Agency (DARPA), Del Mar, California, April 1999.

[34] Lee, Gary, “40/50 Caliber Range-Extended Adaptive Munition (REAM)” Final Report from Lutronix Corporation to the US Army TACOM-ARDEC Del Mar, California, May 2000.

[35] Lee, Gary, "Shipborne Countermeasure Range-Extended Adaptive Munition (SCREAM)," Final Report from Lutronix Corporation to the US Army TACOMARDEC, Del Mar, California, May 2003.

[36] Rabinovitch, O., and J. R., Vinson, “On the Design of Piezoelectric Smart Fins for Flight Vehicles," Journal of Smart Materials and Structures," IOP Publishing, Ltd., Techno House, Bristol, UK, Vol. 12, No. 5, pp. 686 - 695, Oct., 1993.

[37] Barrett, R. and P. Tiso, "PBP Adaptive Actuator Device and Embodiments," International Patent Application number PCT/NL2005/000054, via TU Delft, 18 February 2005. 
[38] Barrett, R., "High Bandwidth Electric Rotor Blade Actuator Study," Phase I SBIR proposal Submitted to the U.S. Army Aviation Systems Command, St. Louis, MO 27 June 1992.

[39] Barrett, R. and Stutts, J., "Design and Testing of a 1/12th Scale Solid State Adaptive Rotor," Journal of Smart Materials and Structures, Vol. 6, No. 4 August 1997, Techno House, Bristol, UK, 1997, pp. 491 - 497.

[40] Barrett, R., Frye, P., and Schliesman, M., “Design, Construction and Characterization of a Flightworthy Piezoelectric Solid State Adaptive Rotor," Journal of Smart Materials and Structures Vol. 7, No. 3, June 1998, pp. 422-431.

[41] Lee, G., "Design and Testing of the Kolibri Vertical Take-Off and Landing Micro Aerial Vehicle," final report for the Department of Defense CounterDrug Technology Office, November 1997.

[42] Barrett, R., and Howard, N., "Adaptive Aerostructures for Subscale Aircraft," refereed proceedings of the 20th Southeastern Conference on Theoretical and Applied Mechanics," Pine Mountain, GA, 17 April 2000.

[43] Barrett, R., Burger, C. and Melián J. P., "Recent Advances in Uninhabited Aerial Vehicle (UAV) Flight Control with Adaptive Aerostructures," $4^{\text {th }}$ European Demonstrators Conference, 10 - 15 Dec. 2001, Edinburgh, Scotland.

[44] Barrett, R. and Lee, G., "Design Criteria, Aircraft Design, Fabrication and Testing of Sub-Canopy and Urban Micro-Aerial Vehicles," AIAA/ AHS International Powered Lift Conference, Alexandria, Virginia, Nov. 2000.

[45] Barrett, R., “Convertible Vertical Take-Off and Landing Miniature Aerial Vehicle," US patent 6,502,787, 22 Feb. 2002.

[46] Barrett, R. and P. Tiso, "PBP Adaptive Actuator Device and Embodiments," International Patent Application number PCT/NL2005/000054, via TU Delft, 18 February 2005.

[47] Barrett, R., McMurtry,R., Vos, R., Tiso, P., and De Breuker, R., "Post-Buckled Precomprecompressed Piezoelectric Flight Control Acutator Design, Development and Demonstration," Journal of Smart Materials and Structures, Vol. 15, No. 5, October 2006, pp. 1323 - 1331.

[48] Vos, R., De Breuker, R., Barrett, R., and Tiso, P., “Morphing Wing Flight Control via Postbuckled Precopressed Piezoelectric Actuators, Journal of Aircraft, Vol. 44, No. 4, pp. 1060 - 1068, July-August 2007.

[49] Vos, R., Barrett, R., De Breuker, R. and Tiso, P., "Post-buckled Precompressed Elements: A New Class of Control Actuators for Morphing Wing UAVs," Journal of Smart Materials and Structures, Vol. 16, No. 3, June 2007, pp. 919 - 926.

[50] Barrett, R., "Post-Buckled Precompressed (PBP) Subsonic Micro Flight Control Actuators," Journal of Smart Materials and Structures, vol. 17, no. 5, 10pp., October 2008.

[51] Vos, R., and Barrett, R., "Dynamic Elastic Axis Shifting: An Important Enhancement of Piezoelectric Postbuckled Precompressed Actuators," The Journal of the American Institute of Aeronautics and Astronautics, Vol. 48, No. 3 March 2010. 
[52] Barrett, R., "Hypermaneuverability and Visual Cloaking; New Adaptive Aerostructures Technologies for Uninhabited Aerial Vehicles (UAVs)," The Aeronautical Journal, Royal Aeronautical Society, London, UK, Vol. 114, No. 1156, June 2010.

[53] Vos, R., and Barrett, R., "Post-Buckled Precompressed Techniques In Adaptive Aerostructures: An Overview," MD-08-1306 Journal of Mechanical Design, Vol. 132, Issue 3, March 2010. 


\title{
Adaptive Backstepping Flight Control for Modern Fighter Aircraft
}

\author{
L. Sonneveldt, Q.P. Chu and J.A. Mulder \\ Delft University of Technology \\ The Netherlands
}

\section{Introduction}

Inertial trajectory control is essential for UAVs which must follow predetermined paths through three-dimensional space (Healy and Liebard, 1993, Kaminer et al., 1998, Boyle et al., 1999, Singh et al., 2003, Tsach et al., 2003, Ren and Beard, 2004, Wegener et al., 2004, Ren and Atkins, 2005, No et al., 2005, Clough, 2005, Papadales et al., 2005, Narasimhan et al., 2006, Kaminer et al., 2007). Other applications of trajectory control include formation flight, aerial refueling, and autonomous landing maneuvers (Pachter et al., 1994, Proud et al., 1999, Fujimori et al. 2000, Singh et al., 2000, Pachter et al., 2001, Wang et al., 2008).

Two different approaches can be distinguished in the design of these trajectory control systems. The most popular approach is to separate the guidance and control laws: a given reference trajectory is converted by the guidance laws to velocity and attitude commands for the autopilot, which in turn generates the actuator signals (Ren and Beard, 2004, Pachter et al., 1994, Pachter et al., 2001). Usually, the assumption is made that the autopilot response to heading and airspeed commands is first order in nature to simplify the design.

The other design approach is to integrate the guidance and control laws into one system, in order to achieve better stability guarantees and improved performance. Kaminer et al. (1998) use an integrated guidance and control approach to trajectory tracking in which the trimmed flight conditions along the reference trajectory are the command input to the tracking controllers. Singh (2003) uses a combination of sliding-mode control and adaptive control.

In this chapter an integrated, though cascaded Lyapunov-based adaptive backstepping (Krstić et al., 1992, Singh and Steinberg 1996) approach is taken and used to design a flightpath controller for a nonlinear high-fidelity F-16 model. Adaptive backstepping allows assuming that the aerodynamic force and moment models may not be known exactly, and even that they may change in flight due to causes as structural damage and control actuator failures. There is much literature available on adaptive backstepping control system design for aircraft and missiles (see, for example, (Singh and Steinberg, 1996, Härkegård, 2003, Farrell et al., Kim et al., 2004, Shin and Kim, 2004, Farrell et al., 2005, Sonneveldt, et al., 2006, Sonneveldt, et al. 2007)). Most of these designs consider control of the aerodynamic angles $\mu$, $a$, and $\beta$. Due to the higher relative degree, however, the design of trajectory controllers as discussed here is much more complicated, as the required analytical calculation of the derivatives of the intermediate control variables leads to a rapid explosion of terms. This phenomenon is the main motivation for the authors of (Singh et al., 2003) to select a sliding- 
mode design for the outer feedback loops. Another disadvantage of (adaptive) backstepping flight control system design is that the contribution of the control-surface deflections to the aerodynamic forces cannot be taken into account. For these reasons, the constrained adaptive backstepping approach of (Farrell et al., 2005, Sonneveldt et al., 2007, Yip 1997) is used here. This method makes use of command filters to calculate the derivatives of the intermediate controls, which greatly simplifies the design. Additionally, these filters can be used to enforce magnitude and rate limits on the state and input variables.

To simplify the mathematical approximation of the unknown aerodynamic force and moment characteristics, we propose to partition the flight envelope into multiple connecting operating regions called hyperboxes. In each hyperbox a locally valid linear-in-theparameters nonlinear model is defined. The coefficients of these local models can be estimated using the update laws of the adaptive backstepping control laws. The number and size of the hyperboxes should be based on a priori information on the physical properties of the vehicle on hand, and may be defined in terms of state variables as Mach number, angle of attack and engine thrust. In this study we use B-spline neural networks (Cheng et al., 1999, Ward et al., 2003) to interpolate between the local models to ensure smooth model transitions. Numerical simulations of various maneuvers with aerodynamic uncertainties in the model and actuator failures are presented. The maneuvers are performed at several flight conditions to demonstrate that the control laws are valid for the entire flight envelope. The chapter is outlined as follows. First, the nonlinear dynamics of the aircraft model are introduced in Sec. II. In Sec. III the adaptive control system design is presented decomposed in four cascaded feedback-loop designs. The aerodynamic model identification process including the B-spline neural networks is discussed in Sec. IV. Section V validates the performance of the control laws using numerical simulations performed in MATLAB/Simulink. A summary of the results and the conclusions are given in Sec. VI. Finally, an appendix on the concept of constrained adaptive backstepping is included.

\section{Aircraft model description}

The aircraft model used in this study is that of an F-16 fighter aircraft with geometry and aerodynamic data as reported in (Nguyen et al., 1979). The aerodynamic data in tabular form have been obtained from wind-tunnel tests and are valid up to Mach 0.6 for the wide range of $-20 \mathrm{deg} \leq \alpha \leq 90 \mathrm{deg}$ and $-30 \mathrm{deg} \leq \beta \leq 30 \mathrm{deg}$. The control inputs of the model are the elevator, ailerons, rudder, and leading-edge flaps, as well as the throttle setting. The leading-edge flaps are not used in the control design. The control-surface actuators are modeled as first-order low-pass filters with rate and magnitude limits as given in (Sonneveldt et al., 2007). Before giving the equations of motion for the F-16 model, some reference frames to describe the aircraft motion are needed. The reference frames used in this paper are the Earth-fixed reference frame $F_{E}$, used as the inertial frame; the vehiclecarried local Earth reference frame $F_{O}$, with its origin fixed in the center of gravity of the aircraft, which is assumed to have the same orientation as $F_{E}$; the wind-axes reference frame $F_{W}$, obtained from $F_{O}$ by three successive rotations of $\chi, \gamma$, and $\mu$; the stability-axes reference frame $F_{S}$, obtained from $F_{W}$ by a rotation of $-\beta$; and the body-fixed reference frame $F_{B}$, obtained from $F_{S}$ by a rotation of $\alpha$, as is also indicated in Fig. 1 . The body-fixed reference frame $F_{B}$ can also be obtained directly from $F_{O}$ by three successive rotations of yaw angle $\psi$, pitch angle $\theta$, and roll angle $\phi$. More details and transformation matrices are given in, for example, (Lewis and Stevens, 1992, Cook, 1997). 


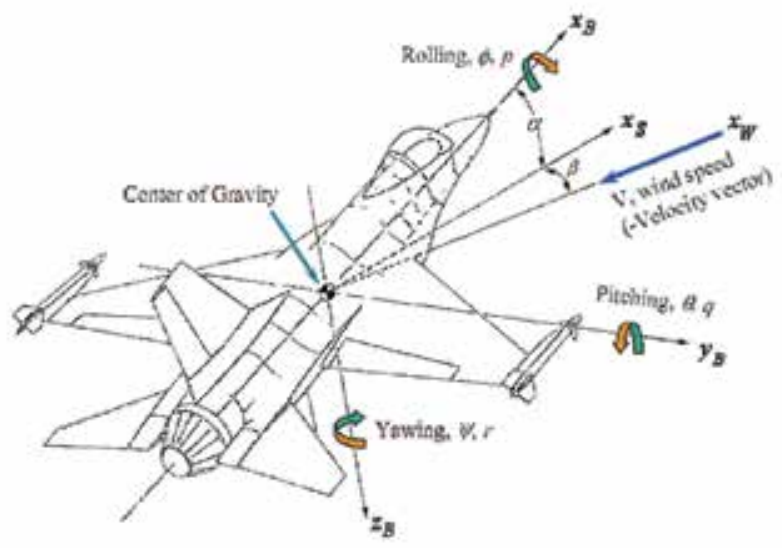

Fig. 1. Aircraft reference frames

Assuming that the aircraft has a rigid body, which is symmetric around the $X-Z$ body-fixed plane, the relevant nonlinear coupled equations of motion can be described by (Lewis and Stevens, 1992):

$$
\begin{aligned}
& \dot{X}_{0}=\left[\begin{array}{c}
V \cos \chi \cos \gamma \\
V \sin \chi \cos \gamma \\
-V \sin \gamma
\end{array}\right] \\
& \dot{X}_{1}=\left[\begin{array}{c}
\frac{1}{m}(-D+T \cos \alpha \cos \beta)-g \sin \gamma \\
\frac{1}{m V \cos \gamma}[L \sin \mu+Y \cos \mu+T(\sin \alpha \sin \mu-\cos \alpha \sin \beta \cos \mu)] \\
\frac{1}{m V}[L \cos \mu-Y \sin \mu+T(\cos \alpha \sin \beta \sin \mu+\sin \alpha \cos \mu)]-\frac{g}{V} \cos \gamma
\end{array}\right] \\
& \dot{X}_{2}=\left[\begin{array}{ccc}
\frac{\cos \alpha}{\cos \beta} & 0 & \frac{\sin \alpha}{\cos \beta} \\
-\cos \alpha \tan \beta & 1 & -\sin \alpha \tan \beta \\
\sin \alpha & 0 & -\cos \alpha
\end{array}\right] X_{3}+\left[\begin{array}{ccc}
0 & \sin \gamma+\cos \gamma \sin \mu \tan \beta & \cos \mu \tan \beta \\
0 & -\frac{\cos \gamma \sin \mu}{\cos \beta} & -\frac{\cos \mu}{\cos \beta} \\
0 & \cos \gamma \cos \mu & -\sin \mu
\end{array}\right] \dot{X}_{1} \\
& \dot{X}_{3}=\left[\begin{array}{l}
\left(c_{1} r+c_{2} p\right) q+c_{3} \bar{L}+c_{4}\left(\bar{N}+h_{e} q\right) \\
c_{5} p r-c_{6}\left(p^{2}-r^{2}\right)+c_{7}\left(\bar{M}-h_{e} r\right) \\
\left(c_{8} p-c_{2} r\right) q+c_{4} \bar{L}+c_{9}\left(\bar{N}+h_{e} q\right)
\end{array}\right]
\end{aligned}
$$

where $X_{0}=\left[\begin{array}{lll}x & y & z\end{array}\right]^{T}, \quad X_{1}=\left[\begin{array}{lll}V & \chi & \gamma\end{array}\right]^{T}, \quad X_{2}=\left[\begin{array}{lll}\mu & \alpha & \beta\end{array}\right]^{T}, \quad X_{3}=\left[\begin{array}{lll}p & q & r\end{array}\right]^{T}$, and the definition of the inertia terms $c_{i}(i=1, \cdots, 9)$ is given in, for example, (Sonneveldt et al., 2007). 
The engine angular momentum $h_{e}$ is assumed to be constant. These 12 differential equations are sufficient to describe the complete motion of the aircraft; other states such as the attitude angles $\phi, \theta$, and $\psi$ are functions of $X_{3}$, and their dynamics can be expressed as

$$
\left[\begin{array}{c}
\dot{\phi} \\
\dot{\theta} \\
\dot{\psi}
\end{array}\right]=\left[\begin{array}{ccc}
1 & \sin \phi \tan \theta & \cos \phi \tan \theta \\
0 & \cos \phi & -\sin \phi \\
0 & \frac{\sin \phi}{\cos \theta} & \frac{\cos \phi}{\cos \theta}
\end{array}\right] X_{3}
$$

The thrust model of (Nguyen et al., 1979) is implemented, which calculates the thrust as a function of altitude, Mach number, and throttle setting $\delta_{t}$. This model is given in tabular form. The aerodynamic forces $L, Y$, and $D$ (expressed in the wind reference frame $F_{W}$ ) and moments $\bar{L}, \bar{M}$, and $\bar{N}$ (expressed in body fixed frame $F_{B}$ ) are summations of the various aerodynamic contributions stored in lookup tables. As an example, the pitch moment $\bar{M}$ is given by

$$
\begin{aligned}
\bar{M}= & \bar{q} S \bar{c}\left\{C_{m}\left(\alpha, \beta, \delta_{e}\right)+C_{Z_{T}} \cdot\left(x_{c g_{r}}-x_{c g}\right)+\delta C_{m_{L E F}}\left(1-\frac{\delta_{L E F}}{25}\right)+\right. \\
& \left.+\frac{q \bar{c}}{2 V_{T}}\left(C_{m_{q}}(\alpha)+\delta C_{m_{q L E F}}(\alpha)\right)\left(1-\frac{\delta_{L E F}}{25}\right)+\delta C_{m}(\alpha)+\delta C_{m_{d s}}\left(\alpha, \delta_{e}\right)\right\}
\end{aligned}
$$

Other aerodynamic forces and moments are given in similar form; for a detailed discussion, see (Nguyen et al., 1979).

\section{Adaptive control design}

In this section we aim to develop an adaptive guidance and control system that

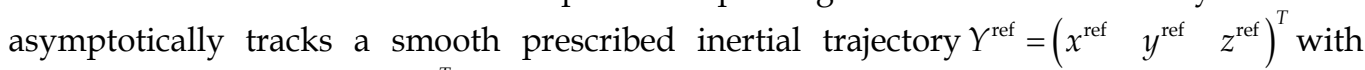
position states $X_{0}=\left(\begin{array}{lll}x & y & z\end{array}\right)^{T}$. Furthermore, the sideslip angle $\beta$ has to be kept at zero to enable coordinated turning. It is assumed that the reference trajectory $Y^{\text {ref }}=\left(\begin{array}{lll}x^{\text {ref }} & y^{\text {ref }} & z^{\text {ref }}\end{array}\right)^{T}$ satisfies

$$
\begin{aligned}
& \dot{x}^{\text {ref }}=V^{\text {ref }} \cos \chi^{\text {ref }} \\
& \dot{y}^{\text {ref }}=V^{\text {ref }} \sin \chi^{\text {ref }}
\end{aligned}
$$

with $V^{\text {ref }}, \chi^{\text {ref }}, z^{\text {ref }}$, and their derivatives continuous and bounded. It also assumed that the components of the total aerodynamic forces $L, Y$, and $D$ and moments $\bar{L}, \bar{M}$ and $\bar{N}$ are uncertain, and so these will have to be estimated. The available controls are the controlsurface deflections $\left(\begin{array}{lll}\delta_{e} & \delta_{a} & \delta_{r}\end{array}\right)^{T}$ and the engine thrust T. The Lyapunov-based control design based on (Farrell et al., 2005, Sonneveldt et al., 2007) is done in four feedback loops, starting at the outer loop. 


\subsection{Inertial position control}

We start the outer-loop feedback control design by transforming the tracking control problem into a regulation problem:

$$
Z_{0}=\left[\begin{array}{l}
z_{01} \\
z_{02} \\
z_{03}
\end{array}\right]=\left[\begin{array}{ccc}
\cos \chi & \sin \chi & 0 \\
-\sin \chi & \cos \chi & 0 \\
0 & 0 & 1
\end{array}\right]\left(X_{0}-Y^{\mathrm{ref}}\right)
$$

where we introduce a vehicle carried vertical reference frame with origin in the center of gravity and $\mathrm{X}$-axis aligned with the horizontal component of the velocity vector (Ren and Beard, 2004, Proud et al., 1999). Differentiating Eq. (9) now gives

$$
\dot{Z}_{0}=\left[\begin{array}{c}
V+z_{02} \dot{\chi}-V^{\mathrm{ref}} \cos \left(\chi-\chi^{\mathrm{ref}}\right) \\
-z_{01} \dot{\chi}+V^{\mathrm{ref}} \sin \left(\chi-\chi^{\mathrm{ref}}\right) \\
\dot{z}^{\mathrm{ref}}-V \sin \gamma
\end{array}\right]
$$

We want to control the position errors $Z_{0}$ through the flight-path angles $\chi$ and $\gamma$, and the total airspeed $V$. However, from Eq. (10) it is clear that it is not yet possible to do something about $z_{02}$ in this design step. Now we select the virtual controls

$$
\begin{gathered}
V^{\text {des }, 0}=V^{\text {ref }} \cos \left(\chi-\chi^{\text {ref }}\right)-c_{01} z_{01} \\
\gamma^{\text {des }, 0}=\arcsin \left(\frac{c_{03} z_{03}-\dot{z}^{\text {ref }}}{V}\right), \quad-\frac{\pi}{2}<\gamma<\frac{\pi}{2}
\end{gathered}
$$

where $c_{01}>0$ and $c_{03}>0$ are the control gains. The actual implementable virtual control signals $V^{\text {des }}$ and $\gamma^{\text {des }}$, as well as their derivatives, $\dot{V}^{\text {des }}$ and $\dot{\gamma}^{\text {des }}$, are obtained by filtering the virtual signals with a second-order low-pass filter. In this way, tedious calculation of the virtual control derivatives is avoided (Swaroop et al., 1997). An additional advantage is that the filters can be used to enforce magnitude or rate limits on the states (Farrell et al., 2003, 2007). As an example, the state-space representation of such a filter for $V^{\text {des, }, 0}$ is given by

$$
\begin{gathered}
{\left[\begin{array}{l}
\dot{q}_{1}(t) \\
\dot{q}_{2}(t)
\end{array}\right]=\left[2 \zeta_{V} \omega_{V}\left(S_{R}\left(\frac{\omega_{V}^{2}}{2 \zeta_{V} \omega_{V}}\left[S_{M}\left(V^{\text {des }, 0}\right)-q_{1}\right]\right)-q_{2}\right)\right]} \\
{\left[\begin{array}{l}
V^{\text {des }} \\
\dot{V}^{\text {des }}
\end{array}\right]=\left[\begin{array}{l}
q_{1} \\
q_{2}
\end{array}\right]}
\end{gathered}
$$

where $S_{M}(\cdot)$ and $S_{R}(\cdot)$ represent the magnitude and rate limit functions as given in (Farrell et al., 2007). These functions enforce the state $V$ to stay within the defined limits. Note that if 
the signal $V^{\text {des, } 0}$ is bounded, then $V^{\text {des }}$ and $\dot{V}^{\text {des }}$ are also bounded and continuous signals. When the magnitude and rate limits are not in effect, the transfer function from $V^{\text {des, } 0}$ to $V^{\text {des }}$ is given by

$$
\frac{V^{\text {des }}}{V^{\text {des }, 0}}=\frac{\omega_{V}^{2}}{s^{2}+2 \zeta_{V} \omega_{V}+\omega_{V}^{2}}
$$

and the error $V^{\text {des, }, 0}-V^{\text {des }}$ can be made arbitrarily small by selecting the bandwidth of the filter to be sufficiently large (Swaroop et al., 1997).

\subsection{Flight-path angle and airspeed control}

In this loop the objective is to steer $V$ and $\gamma$ to their desired values, as determined in the previous section. Furthermore, the heading angle $\chi$ has to track the reference signal $\chi^{\text {ref }}$, and we also have to guarantee that $z_{02}$ is regulated to zero. The available (virtual) controls in this step are the aerodynamic angles $\mu$ and $\alpha$, as well as the thrust $T$. The lift, drag, and side forces are assumed to be unknown and will be estimated. Note that the aerodynamic forces also depend on the control-surface deflections $U=\left[\begin{array}{lll}\delta_{e} & \delta_{a} & \delta_{r}\end{array}\right]^{T}$. These forces are quite small, because the surfaces are primarily moment generators. However, because the current control-surface deflections are available from the command filters used in the inner loop, we can still take them into account in the control design. The relevant equations of motion are given by

$$
\dot{X}_{1}=A_{1} F_{1}(X, U)+B_{1} G_{1}\left(X, U, X_{2}\right)+H_{1}(X)
$$

where

$$
A_{1}=\frac{1}{m V}\left[\begin{array}{ccc}
0 & 0 & -V \\
0 & \frac{\cos \mu}{\cos \gamma} & 0 \\
0 & -\sin \mu & 0
\end{array}\right], H_{1}=\left[\begin{array}{c}
-g \sin \gamma \\
\frac{T}{m V \cos \gamma} \cos \alpha \sin \beta \cos \mu \\
\frac{T}{m V} \cos \alpha \sin \beta \sin \mu-\frac{g}{V} \cos \gamma
\end{array}\right], B_{1}=\frac{1}{m V}\left[\begin{array}{ccc}
V \cos \alpha \cos \beta & 0 & 0 \\
0 & \frac{1}{\cos \gamma} & 0 \\
0 & 0 & 1
\end{array}\right]
$$

are known (matrix and vector) functions, and

$$
F_{1}=\left[\begin{array}{c}
L(X, U) \\
Y(X, U) \\
D(X, U)
\end{array}\right], \quad G_{1}=\left[\begin{array}{c}
T \\
(L(X, U)-T \sin \alpha) \sin \mu \\
(L(X, U)-T \sin \alpha) \cos \mu
\end{array}\right]
$$

are functions containing the uncertain aerodynamic forces. Note that the intermediate control variables $\alpha$ and $\mu$ do not appear affine in the $X_{1}$ subsystem, which complicates the design somewhat. Because the control objective in this step is to track the smooth reference signal $X_{1}^{\text {des }}=\left(\begin{array}{lll}V^{\text {des }} & \chi^{\text {des }} & \gamma^{\text {des }}\end{array}\right)^{T}$ with $X_{1}=\left(\begin{array}{lll}V & \chi & \gamma\end{array}\right)^{T}$, the tracking errors are defined as 


$$
Z_{1}=\left[\begin{array}{l}
z_{11} \\
z_{12} \\
z_{13}
\end{array}\right]=X_{1}-X_{1}^{\text {des }}
$$

To regulate $Z_{1}$ and $z_{02}$ to zero, the following equation needs to be satisfied (Kanayama et al., 1990):

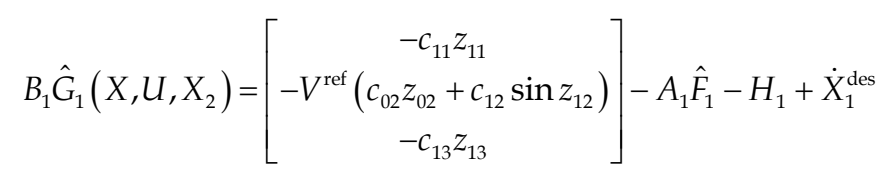

where $\hat{F}_{1}$ is the estimate of $F_{1}$ and

$$
\hat{G}_{1}\left(X, U, X_{2}\right)=\left[\begin{array}{c}
T \\
\left(\hat{L}_{0}(X, U)+\hat{L}_{\alpha}(X, U) \alpha+T \sin \alpha\right) \sin \mu \\
\left(\hat{L}_{0}(X, U)+\hat{L}_{\alpha}(X, U) \alpha+T \sin \alpha\right) \cos \mu
\end{array}\right]
$$

with the estimate of the lift force decomposed as $\hat{L}(X, U)=\hat{L}_{0}(X, U)+\hat{L}_{\alpha}(X, U) \alpha$

The estimate of the aerodynamic forces $\hat{F}_{1}$ is defined as

$$
\hat{F}_{1}=\Phi_{F_{1}}^{T}(X, U) \hat{\Theta}_{F_{1}}
$$

where $\Phi_{F_{1}}^{T}$ is a known (chosen) regressor function and $\hat{\Theta}_{F_{1}}$ is a vector with unknown constant parameters. It is assumed that there exists a vector $\Theta_{F_{1}}$ such that

$$
F_{1}=\Phi_{F_{1}}^{T}(X, U) \Theta_{F_{1}}
$$

This means the estimation error can be defined as $\tilde{\Theta}_{F_{1}}=\Theta_{F_{1}}-\hat{\Theta}_{F_{1}}$. We now need to determine the desired values $\alpha^{\text {des }}$ and $\mu^{\text {des }}$. The right-hand side of Eq. (18) is entirely known, and so the left-hand side can be determined and the desired values can be extracted. This is done by introducing the coordinate transformation

$$
\begin{aligned}
& x \equiv\left(\hat{L}_{0}(X, U)+\hat{L}_{\alpha}(X, U) \alpha+T \sin \alpha\right) \cos \mu \\
& y \equiv\left(\hat{L}_{0}(X, U)+\hat{L}_{\alpha}(X, U) \alpha+T \sin \alpha\right) \sin \mu
\end{aligned}
$$

which can be seen as a transformation from the two-dimensional polar coordinates

$$
\hat{L}_{0}(X, U)+\hat{L}_{\alpha}(X, U) \alpha+T \sin \alpha
$$

and $\mu$ to Cartesian coordinates $x$ and $y$. The desired signals $\left[\begin{array}{lll}T^{d e s, 0} & y_{0} & x_{0}\end{array}\right]^{T}$ are given by 


$$
B_{1}\left[\begin{array}{c}
T^{\text {des }, 0} \\
y_{0} \\
x_{0}
\end{array}\right]=\left[\begin{array}{c}
-c_{11} z_{11} \\
-V^{\text {ref }}\left(c_{02} z_{02}+c_{12} \sin z_{12}\right) \\
-c_{13} z_{13}
\end{array}\right]-A_{1} \hat{F}_{1}-H_{1}+\dot{X}_{1}^{\text {des }}
$$

Thus, the virtual control signals are equal to

$$
\hat{L}_{\alpha}(X, U) \alpha^{\text {des }, 0}=\sqrt{x_{0}^{2}+y_{0}^{2}}-\hat{L}_{0}(X, U)-T \sin \alpha
$$

and

$$
\mu^{\text {des }, 0}=\left\{\begin{array}{ccc}
\arctan \left(y_{0} / x_{0}\right) & \text { if } x_{0}>0 \\
\arctan \left(y_{0} / x_{0}\right)+\pi & \text { if } x_{0}<0 & \text { and } y_{0} \geq 0 \\
\arctan \left(y_{0} / x_{0}\right)-\pi & \text { if } x_{0}<0 & \text { and } y_{0}<0 \\
\pi / 2 & \text { if } x_{0}=0 & \text { and } y_{0}>0 \\
-\pi / 2 & \text { if } x_{0}=0 & \text { and } y_{0}<0
\end{array}\right.
$$

Filtering the virtual signals to account for magnitude, rate, and bandwidth limits will give the implementable virtual controls $\alpha^{\text {des }}, \mu^{\text {des }}$ and their derivatives. The sideslip-angle command was already defined as $\beta^{\text {ref }}=0$, and thus $X_{2}^{\text {des }}=\left[\begin{array}{lll}\mu^{\text {des }} & \alpha^{\text {des }} & 0\end{array}\right]^{T}$ and its derivative are completely defined. However, care must be taken because the desired virtual control $\mu^{\mathrm{des}, 0}$ is undefined when both $x_{0}$ and $y_{0}$ are equal to zero, making the system momentarily uncontrollable. This sign change of $\hat{L}_{0}(X, U)+\hat{L}_{\alpha}(X, U) \alpha+T \sin \alpha$ can only occur at very low or negative angles of attack. This situation was not encountered during the maneuvers simulated in this study. To solve the problem altogether, the designer could measure the rate of change for $x_{0}$ and $y_{0}$ and devise a rule base set to change sign when these terms approach zero. Furthermore, problems will also occur at high angles of attack when the control effectiveness term $\hat{L}_{\alpha}$ will become smaller and eventually change sign. Possible solutions include limiting the angle-of-attack commands using the command filters or proper trajectory planning to avoid high-angle-of-attack maneuvers. Also note that so far in the control design process, we have not taken care of the update laws for the uncertain aerodynamic forces; they will be dealt with when the static control design is finalized.

\subsection{Aerodynamic angle control}

Now the reference signal $X_{2}^{\text {des }}=\left[\begin{array}{lll}\mu^{\text {des }} & \alpha^{\text {des }} & \beta^{\text {des }}\end{array}\right]^{T}$ and its derivative have been found and we can move on to the next feedback loop. The available virtual controls in this step are the angular rates $X_{3}$. The relevant equations of motion for this part of the design are given by

where

$$
\dot{X}_{2}=A_{2} F_{1}(X, U)+B_{2}(X) X_{3}+H_{2}(X)
$$

$$
A_{2}=\frac{1}{m V}\left[\begin{array}{ccc}
\tan \beta+\tan \gamma \sin \mu & \tan \gamma \cos \mu & 0 \\
\frac{-1}{\cos \beta} & 0 & 0 \\
0 & 1 & 0
\end{array}\right], \quad B_{2}=\left[\begin{array}{ccc}
\frac{\cos \alpha}{\cos \beta} & 0 & \frac{\sin \alpha}{\cos \beta} \\
-\cos \alpha \tan \beta & 1 & -\sin \alpha \tan \beta \\
\sin \alpha & 0 & \cos \alpha
\end{array}\right]
$$




$$
H_{2}=\frac{1}{m V}\left[\begin{array}{c}
T(\sin \alpha \tan \gamma \sin \mu+\sin \alpha \tan \beta-\cos \alpha \sin \beta \tan \gamma \cos \mu)-\frac{g}{V} \tan \beta \cos \gamma \cos \mu \\
T \frac{\sin \alpha}{\cos \beta}+\frac{g}{V} \cos \gamma \cos \mu \\
-T \cos \alpha \cos \beta+\frac{g}{V} \cos \gamma \sin \mu
\end{array}\right]
$$

are known (matrix and vector) functions. The tracking errors are defined as

$$
Z_{2}=X_{2}-X_{2}^{\text {des }}
$$

To stabilize the $Z_{2}$ subsystem, a virtual feedback control $X_{3}^{\text {des }, 0}$ is defined as

$$
B_{2} X_{3}^{\text {des }, 0}=-C_{2} Z_{2}-A_{2} \hat{F}_{1}-H_{2}+\dot{X}_{2}^{\text {des }}, \quad C_{2}=C_{2}^{T}>0
$$

The implementable virtual control (i.e., the reference signal for the inner loop) $X_{3}^{\text {des }}$ and its derivative are again obtained by filtering the virtual control signal $X_{3}^{\text {des, } 0}$ with a second-order command-limiting filter.

\subsection{Angular rate control}

In the fourth step, an inner-loop feedback loop for the control of the body-axis angular rates $X_{3}=\left[\begin{array}{lll}p & q & r\end{array}\right]^{T}$ is constructed. The control inputs for the inner loop are the control-surface deflections $U=\left[\begin{array}{lll}\delta_{e} & \delta_{a} & \delta_{r}\end{array}\right]^{T}$. The dynamics of the angular rates can be written as

$$
\dot{X}_{3}=A_{3}\left(F_{3}(X, U)+B_{3}(X) U\right)+H_{3}(X)
$$

where

$$
A_{3}=\left[\begin{array}{ccc}
c_{3} & 0 & c_{4} \\
0 & c_{7} & 0 \\
c_{4} & 0 & c_{9}
\end{array}\right], \quad H_{3}=\left[\begin{array}{c}
\left(c_{1} r+c_{2} p\right) q \\
c_{5} p r-c_{6}\left(p^{2}-r^{2}\right) \\
\left(c_{8} p-c_{2} r\right) q
\end{array}\right]
$$

are known (matrix and vector) functions, and

$$
F_{3}=\left[\begin{array}{c}
\bar{L}_{0} \\
\bar{M}_{0} \\
\bar{N}_{0}
\end{array}\right], \quad B_{3}=\left[\begin{array}{lll}
\bar{L}_{\delta_{e}} & \bar{L}_{\delta_{a}} & \bar{L}_{\delta_{r}} \\
\bar{M}_{\delta_{e}} & \bar{M}_{\delta_{a}} & \bar{M}_{\delta_{r}} \\
\bar{N}_{\delta_{e}} & \bar{N}_{\delta_{a}} & \bar{N}_{\delta_{r}}
\end{array}\right]
$$

are unknown (matrix and vector) functions that have to be approximated. Note that for a more convenient presentation, the aerodynamic moments have been decomposed: for example,

$$
\bar{M}(X, U)=\bar{M}_{0}(X, U)+\bar{M}_{\delta_{e}} \delta_{e}+\bar{M}_{\delta_{a}} \delta_{a}+\bar{M}_{\delta_{r}} \delta_{r}
$$


where the higher-order control-surface dependencies are still contained in $\bar{M}_{0}(X, U)$. The control objective in this feedback loop is to track the reference signal $X_{3}^{\text {des }}=\left[\begin{array}{lll}p^{\text {ref }} & q^{\text {ref }} & r^{\text {ref }}\end{array}\right]^{T}$ with the angular rates $X_{3}$. Defining the tracking errors

$$
Z_{3}=X_{3}-X_{3}^{\text {des }}
$$

and taking the derivatives results in

$$
\dot{Z}_{3}=A_{3}\left(F_{3}(X, U)+B_{3}(X) U\right)+H_{3}(X)-\dot{X}_{3}^{\text {des }}
$$

To stabilize the system of Eq. (33), we define the desired control $U^{0}$ as

$$
A_{3} \hat{B}_{3} U^{0}=-C_{3} Z_{3}-A_{3} \hat{F}_{3}-H_{3}+\dot{X}_{3}^{\text {des }}, \quad C_{3}=C_{3}^{T}>0
$$

where $\hat{F}_{3}$ and $\hat{B}_{3}$ are the estimates of the unknown nonlinear aerodynamic moment functions $F_{3}$ and $B_{3}$, respectively. The F-16 model is not over-actuated (i.e., the $B_{3}$ matrix is square). If this is not the case, some form of control allocation would be required (Enns, 1998, Durham, 1993). The estimates are defined as

$$
\hat{F}_{3}=\Phi_{F_{3}}^{T}(X, U) \hat{\Theta}_{F_{3}}, \quad \hat{B}_{3_{i}}=\Phi_{B_{3_{i}}}^{T}(X) \hat{\Theta}_{B_{3_{i}}}, \quad \text { for } i=1, \cdots, 3
$$

where $\Phi_{F_{3}}^{T}$ and $\Phi_{B_{3 i}}^{T}$ are the known regressor functions, $\hat{\Theta}_{F_{3}}$ and $\hat{\Theta}_{B_{3_{i}}}$ are vectors with unknown constant parameters, and $\hat{B}_{3_{i}}$ represents the $i$ th column of $\hat{B}_{3}$. It is assumed that there exist vectors $\Theta_{F_{3}}$ and $\Theta_{B_{3_{i}}}$ such that

$$
F_{3}=\Phi_{F_{3}}^{T}(X, U) \Theta_{F_{3}}, \quad B_{3_{i}}=\Phi_{B_{3_{i}}}^{T}(X) \Theta_{B_{3_{i}}}
$$

This means that the estimation errors can be defined as $\tilde{\Theta}_{F_{3}}=\Theta_{F_{3}}-\hat{\Theta}_{F_{3}}$ and $\tilde{\Theta}_{B_{3 i}}=\Theta_{B_{3 i}}-\hat{\Theta}_{B_{3 i}}$. The actual control $U$ is found by applying a filter similar to Eq. (13) to $U^{0}$.

\subsection{Update laws and stability properties}

We have now finished the static part of our control design. In this section the stability properties of the control law are discussed and dynamic update laws for the unknown parameters are derived. Define the control Lyapunov function

$$
\begin{aligned}
V= & \frac{1}{2}\left(Z_{0}^{T} Z_{0}+z_{11}^{2}+\frac{2-2 \cos z_{12}}{c_{02}}+z_{13}^{2}+Z_{2}^{T} Z_{2}+Z_{3}^{T} Z_{3}\right)+ \\
& +\frac{1}{2}\left(\operatorname{trace}\left(\tilde{\Theta}_{F_{1}}^{T} \Gamma_{F_{1}}^{-1} \tilde{\Theta}_{F_{1}}\right)+\operatorname{trace}\left(\tilde{\Theta}_{F_{3}}^{T} \Gamma_{F_{3}}^{-1} \tilde{\Theta}_{F_{3}}\right)\right)+\sum_{i=1}^{3} \operatorname{trace}\left(\tilde{\Theta}_{B_{3_{i}}}^{T} \Gamma_{B_{3_{i}}}^{-1} \tilde{\Theta}_{B_{3_{i}}}\right)
\end{aligned}
$$

with the update gains matrices $\Gamma_{F_{1}}=\Gamma_{F_{1}}^{T}>0, \Gamma_{F_{3}}=\Gamma_{F_{3}}^{T}>0$, and $\Gamma_{B_{3_{i}}}=\Gamma_{B_{3 i}}^{T}>0$. Taking the derivative of $V$ along the trajectories of the closed-loop system gives 


$$
\begin{aligned}
\dot{V}= & -c_{01} z_{01}^{2}+z_{02} z_{01} \dot{\chi}+\left(V-V^{\text {des }, 0}\right) z_{01}+z_{02}\left(-z_{01} \dot{\chi}+V^{\text {ref }} \sin z_{12}\right)-c_{03} z_{03}^{2}-V\left(\sin \gamma-\sin \gamma^{\text {des }, 0}\right) z_{03}+ \\
& -c_{11} z_{11}^{2}-V^{\text {ref }}\left(\sin z_{12} z_{02}+\frac{c_{12}}{c_{02}} \sin ^{2} z_{12}\right)-c_{13} z_{13}^{2}+Z_{1}^{T}\left(A_{1} \Phi_{F_{1}}^{T} \tilde{\Theta}_{F_{1}}+B_{1}\left(G_{1}\left(X_{2}\right)-\hat{G}_{1}\left(X_{2}\right)\right)\right)+ \\
& +Z_{1}^{T} B_{1}\left(\hat{G}_{1}\left(X_{2}\right)-\hat{G}_{1}\left(X_{2}^{\text {des }, 0}\right)\right)-Z_{2}^{T} C_{2} Z_{2}+Z_{2}^{T} A_{2} \Phi_{F_{1}}^{T} \tilde{\Theta}_{F_{1}}+Z_{2}^{T} B_{2}\left(X_{3}-X_{3}^{\text {des }, 0}\right)-Z_{3}^{T} C_{3} Z_{3}+ \\
& +Z_{3}^{T} A_{3}\left(\Phi_{F_{3}}^{T} \tilde{\Theta}_{F_{3}}+\sum_{i=1}^{3} \Phi_{B_{3_{i}}}^{T} \tilde{\Theta}_{B_{3_{i}}} U_{i}\right)+Z_{3}^{T} A_{3} \hat{B}_{3}\left(U-U^{0}\right)-\operatorname{trace}\left(\dot{\hat{\Theta}}_{F_{1}}^{T} \Gamma_{F_{1}}^{-1} \tilde{\Theta}_{F_{1}}\right)-\operatorname{trace}\left(\dot{\hat{\Theta}}_{F_{3}}^{T} \Gamma_{F_{3}}^{-1} \tilde{\Theta}_{F_{3}}\right)+ \\
& -\sum_{i=1}^{3} \operatorname{trace}\left(\dot{\hat{\Theta}}_{B_{3_{i}}^{T}}^{T} \Gamma_{B_{3 i}}^{-1} \tilde{\Theta}_{B_{3_{i}}}\right)
\end{aligned}
$$

To cancel the terms in Eq. (38), depending on the estimation errors, we select the update laws

$$
\dot{\hat{\Theta}}_{F_{1}}=\Gamma_{F_{1}} \Phi_{F_{1}}\left(A_{1 a}^{T} Z_{1}+A_{2}^{T} Z_{2}\right), \quad \dot{\hat{\Theta}}_{F_{3}}=\Gamma_{F_{3}} \Phi_{F_{3}} A_{3}^{T} Z_{3}, \quad \dot{\hat{\Theta}}_{B_{3_{i}}}=\operatorname{proj}_{B_{3_{i}}}\left(\Gamma_{B_{3_{i}}} \Phi_{B_{3_{i}}} A_{3}^{T} Z_{3} U_{i}\right)
$$

with $A_{1 a} \Phi_{F_{1}}^{T} \tilde{\Theta}_{F_{1}}=A_{1} \Phi_{F_{1}}^{T} \tilde{\Theta}_{F_{1}}+B_{1}\left(G_{1}\left(X_{2}\right)-\hat{G}_{1}\left(X_{2}\right)\right)$

The update laws for $\hat{B}_{3}$ include a projection operator (Ioannou and Sun, 1995) to ensure that certain elements of the matrix do not change sign and full rank is maintained always. For most elements, the sign is known based on physical principles. Substituting the update laws in Eq. (38) leads to

$$
\begin{aligned}
\dot{V}= & -c_{01} z_{01}^{2}-c_{03} z_{03}^{2}-c_{11} z_{11}^{2}--V^{\text {ref }} \frac{c_{12}}{c_{02}} \sin ^{2} z_{12}-c_{13} z_{13}^{2}-Z_{2}^{T} C_{2} Z_{2}-Z_{3}^{T} C_{3} Z_{3}+\left(V-V^{\text {des }, 0}\right) z_{01}+ \\
& -V\left(\sin \gamma-\sin \gamma^{\text {des }, 0}\right) z_{03}+Z_{1}^{T} B_{1}\left(G_{1}\left(X_{2}\right)-\hat{G}_{1}\left(X_{2}^{\text {des }, 0}\right)\right)+Z_{2}^{T} B_{2}\left(X_{3}-X_{3}^{\text {des }, 0}\right)+Z_{3}^{T} A_{3} \hat{B}_{3}\left(U-U^{0}\right)
\end{aligned}
$$

where the first line is already negative semi-definite, which we need to prove stability in the sense of Lyapunov. Because our Lyapunov function $V$ equation (37) is not radially unbounded, we can only guarantee local asymptotic stability (Kanayama et al., 1990). This is sufficient for our operating area if we properly initialize the control law to ensure $z_{12} \leq \pm \pi / 2$. However, we also have indefinite error terms due to the tracking errors and due to the command filters used in the design. As mentioned before, when no rate or magnitude limits are in effect, the difference between the input and output of the filters can be made small by selecting the bandwidth of the filters to be sufficiently larger than the bandwidth of the input signal. Also, when no limits are in effect and the small bounded difference between the input and output of the command filters is neglected, the feedback controller designed in the previous sections will converge the tracking errors to zero (for proof, see (Farrell et al., 2005, Sonneveldt et al., 2007, Yip, 1997)).

Naturally, when control or state limits are in effect, the system will in general not track the reference signal asymptotically. A problem with adaptive control is that this can lead to corruption of the parameter-estimation process, because the tracking errors that are driving this process are no longer caused by the function approximation errors alone (Farrell et al., 2003). To solve this problem we will use a modified definition of the tracking errors in the update laws in which the effect of the magnitude and rate limits has been removed, as suggested in (Farrell et al., 2005, Sonneveldt et al., 2006). Define the modified tracking errors 


$$
\tilde{Z}_{1}=Z_{1}-\Xi_{1}, \quad \tilde{Z}_{2}=Z_{2}-\Xi_{2}, \quad \tilde{Z}_{3}=Z_{3}-\Xi_{3}
$$

with the linear filters

$$
\begin{aligned}
& \dot{\Xi}_{1}=-C_{1} \Xi_{1}+B_{1}\left(\hat{G}_{1}\left(X, U, X_{2}\right)-\hat{G}_{1}\left(X, U, X_{2}^{\text {des }, 0}\right)\right) \\
& \dot{\Xi}_{2}=-C_{2} \Xi_{2}+B_{2}\left(X_{3}-X_{3}^{\text {des }, 0}\right) \\
& \dot{\Xi}_{3}=-C_{3} \Xi_{3}+A_{3} \hat{B}_{3}\left(U-U^{0}\right)
\end{aligned}
$$

The modified errors will still converge to zero when the constraints are in effect, which means the robustified update laws look like

$$
\dot{\hat{\Theta}}_{F_{1}}=\Gamma_{F_{1}} \Phi_{F_{1}}\left(A_{1 a}^{T} Z_{1}+A_{2}^{T} Z_{2}\right), \quad \dot{\hat{\Theta}}_{F_{3}}=\Gamma_{F_{3}} \Phi_{F_{3}} A_{3}^{T} Z_{3}, \quad \dot{\hat{\Theta}}_{B_{3_{i}}}=\operatorname{proj}_{B_{3_{i}}}\left(\Gamma_{B_{3 i}} \Phi_{B_{3_{i}}} A_{3}^{T} Z_{3} U_{i}\right)
$$

To better illustrate the structure of the control system, a scheme of the adaptive inner-loop controller is shown in Fig. 2.

\section{Model identification}

To simplify the approximation of the unknown aerodynamic force and moment functions, thereby reducing computational load, the flight envelope is partitioned into multiple connecting operating regions called hyperboxes or clusters. This can be done manually using a priori knowledge of the nonlinearity of the system, automatically using nonlinear optimization algorithms that cluster the data into hyperplanar or hyperellipsoidal clusters (Babuška, 1998) or a combination of both. In each hyperbox a locally valid linear-in-theparameters nonlinear model is defined, which can be estimated using the update laws of the Lyapunov-based control laws. The aerodynamic model can be partitioned using different state variables, the choice of which depends on the expected nonlinearities of the system. In this study we use B-spline neural networks (Cheng et al., 1999, Ward et al., 2003) (i.e., radial basis function neural networks with B-spline basis functions) to interpolate between the local nonlinear models, ensuring smooth transitions. In the previous section we defined parameter update laws equation (43) for the unknown aerodynamic functions, which were written as

$$
\hat{F}_{1}=\Phi_{F_{1}}^{T}(X, U) \hat{\Theta}_{F_{1}}, \quad \hat{F}_{3}=\Phi_{F_{3}}^{T}(X, U) \hat{\Theta}_{F_{3}}, \quad \hat{B}_{3_{i}}=\Phi_{B_{3_{i}}}^{T}(X) \hat{\Theta}_{B_{3_{i}}}
$$

Now we will further define these unknown vectors and known regressor vectors. The total force approximations are defined as

$$
\begin{aligned}
& \hat{L}=\bar{q} S\left(\hat{C}_{L_{0}}(\alpha, \beta)+\hat{C}_{L_{\alpha}}\left(\beta, \delta_{e}\right) \alpha+\hat{C}_{L_{q}}(\alpha) \frac{q \bar{c}}{2 V}+\hat{C}_{L_{\delta_{e}}}(\alpha, \beta) \delta_{e}\right) \\
& \hat{Y}=\bar{q} S\left(\hat{C}_{Y_{0}}\left(\alpha, \beta, \delta_{e}\right)+\hat{C}_{Y_{p}}(\alpha, \beta) \frac{p b}{2 V}+\hat{C}_{Y_{r}}(\alpha, \beta) \frac{r b}{2 V}+\hat{C}_{Y_{\delta_{a}}}(\alpha, \beta) \delta_{a}+\hat{C}_{Y_{\delta_{r}}}(\alpha, \beta) \delta_{r}\right) \\
& \hat{D}=\bar{q} S\left(\hat{C}_{D_{0}}\left(\alpha, \beta, \delta_{e}\right)+\hat{C}_{D_{\delta_{e}}}(\alpha, \beta) \delta_{e}\right)
\end{aligned}
$$




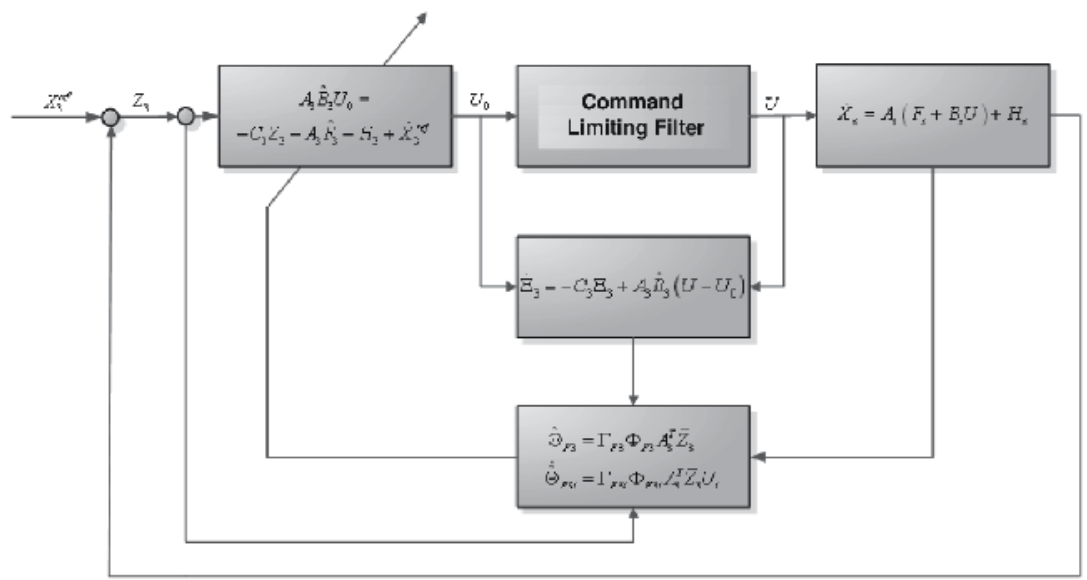

Fig. 2. Inner-loop control system

and the moment approximations are defined as

$$
\begin{aligned}
& \hat{\bar{L}}=\bar{q} S\left(\hat{C}_{\bar{L}_{0}}\left(\alpha, \beta, \delta_{e}\right)+\hat{C}_{\bar{L}_{p}}(\alpha, \beta) \frac{p b}{2 V}+\hat{C}_{\bar{L}_{r}}(\alpha, \beta) \frac{r b}{2 V}+\hat{C}_{\bar{L}_{\delta_{e}}}(\alpha, \beta) \delta_{e}+\hat{C}_{\bar{L}_{\delta_{a}}}(\alpha, \beta) \delta_{a}+\hat{C}_{\bar{L}_{\delta_{r}}}(\alpha, \beta) \delta_{r}\right) \\
& \hat{\bar{M}}=\bar{q} S\left(\hat{C}_{\bar{M}_{0}}(\alpha, \beta)+\hat{C}_{\bar{M}_{q}}(\alpha) \frac{q \bar{c}}{2 V}+\hat{C}_{\bar{M}_{\delta_{e}}}(\alpha, \beta) \delta_{e}\right) \\
& \hat{\bar{N}}=\bar{q} S\left(\hat{C}_{\bar{N}_{0}}\left(\alpha, \beta, \delta_{e}\right)+\hat{C}_{\bar{N}_{p}}(\alpha, \beta) \frac{p b}{2 V}+\hat{C}_{\bar{N}_{r}}(\alpha, \beta) \frac{r b}{2 V}+\hat{C}_{\bar{N}_{\delta_{e}}}(\alpha, \beta) \delta_{e}+\hat{C}_{\bar{N}_{\delta_{a}}}(\alpha, \beta) \delta_{a}+\hat{C}_{\bar{N}_{\delta_{r}}}(\alpha, \beta) \delta_{r}\right)
\end{aligned}
$$

Note that these approximations do not account for asymmetric failures that will introduce coupling of the longitudinal and lateral motions of the aircraft. If a failure occurs that introduces a parameter dependency that is not included in the approximation, stability can no longer be guaranteed. It is possible to include extra cross-coupling terms, but this is beyond the scope of this paper. The total nonlinear function approximations are divided into simpler linear-in-the parameter nonlinear coefficient approximations: for example,

$$
\hat{C}_{L_{0}}(\alpha, \beta)=\varphi_{C_{L_{0}}}^{T}(\alpha, \beta) \hat{\theta}_{C_{L_{0}}}
$$

where the unknown parameter vector $\hat{\theta}_{C_{L_{0}}}$ contains the network weights (i.e., the unknown parameters), and $\varphi_{C_{L_{0}}}$ is a regressor vector containing the B-spline basis functions (Sonneveldt et al., 2007). All other coefficient estimates are defined in similar fashion. In this case a two-dimensional network is used with input nodes for $\alpha$ and $\beta$. Different scheduling parameters can be selected for each unknown coefficient. In this study we used third-order B-splines spaced $2.5 \mathrm{deg}$ and one or more of the selected scheduling variables $\alpha, \beta$ and $\delta_{e}$. Following the notation of Eq. (47), we can write the estimates of the aerodynamic forces and moments as

$$
\begin{array}{ll}
\hat{L}=\Phi_{L}^{T}\left(\alpha, \beta, \delta_{e}\right) \hat{\Theta}_{L}, \quad \hat{Y}=\Phi_{Y}^{T}\left(\alpha, \beta, \delta_{e}\right) \hat{\Theta}_{Y}, \quad \hat{D}=\Phi_{D}^{T}\left(\alpha, \beta, \delta_{e}\right) \hat{\Theta}_{D} \\
\hat{\bar{L}}=\Phi_{\bar{L}}^{T}\left(\alpha, \beta, \delta_{e}\right) \hat{\Theta}_{\bar{L}}, \quad \hat{\bar{M}}=\Phi_{\bar{M}}^{T}\left(\alpha, \beta, \delta_{e}\right) \hat{\Theta}_{\bar{M}}, \quad \hat{\bar{N}}=\Phi_{\bar{N}}^{T}\left(\alpha, \beta, \delta_{e}\right) \hat{\Theta}_{\bar{N}}
\end{array}
$$


which is a notation equivalent to the one used in Eq. (44). Therefore, the update laws equation (43) can indeed be used to adapt the B-spline network weights. In practice nonparametric uncertainties such as 1) un-modeled structural vibrations 2) measurement noise, 3) computational round-off errors and sampling delays, and 4) time variations of the unknown parameters, can result in parameter drift. One approach to avoiding parameter drift taken here is to stop the adaptation process when the training error is very small (i.e. a dead zones (Babuška, 1998, Karason and Annaswamy, 1994)).

\section{Simulation results}

This section presents the simulation results from the application of the flight-path controller developed in the previous sections to the high-fidelity, six-degree-of-freedom F-16 model of Sec. 2. Both the control law and the aircraft model are written as C S-functions in MATLAB/Simulink. The simulations are performed at three different starting flight conditions with the trim conditions: 1) $h=5000 \mathrm{~m}, V=200 \mathrm{~m} / \mathrm{s}$, and $a=\theta=2.774 \mathrm{deg}$; $) ~ h=0 \mathrm{~m}$, $V=250 \mathrm{~m} / \mathrm{s}$, and $a=\theta=2.406 \mathrm{deg}$; and 3) $h=2500 \mathrm{~m}, V=150 \mathrm{~m} / \mathrm{s}$, and $a=\theta=0.447 \mathrm{deg}$; where $h$ is the altitude of the aircraft, and all other trim states are equal to zero.

Furthermore, two maneuvers are considered: 1) a climbing helical path and 2) a reconnaissance and surveillance maneuver. The latter maneuver involves turns in both directions and some altitude changes. The simulations of both maneuvers last $300 \mathrm{~s}$. The reference trajectories are generated with second-order linear filters to ensure smooth trajectories. To evaluate the effectiveness of the online model identification, all maneuvers will also be performed with a $\pm 30 \%$ deviation in all aerodynamic stability and control derivatives used by the controller (i.e., it is assumed that the onboard model is very inaccurate). Finally, the same maneuvers are also simulated with a lockup at $\pm 10 \mathrm{deg}$ of the left aileron.

\subsection{Control parameter tuning}

We start with the selection of the gains of the static control law and the bandwidths of the command filters. Lyapunov stability theory only requires the control gains to be larger than zero, but it is natural to select the largest gains of the inner loop. Larger gains will, of course, result in smaller tracking errors, but at the cost of more control effort. It is possible to derive certain performance bounds that can serve as guidelines for tuning (see, for example, Krstić, et al., 1993, Sonneveldt et al., 2007). However, getting the desired closed-loop response is still an extensive trial-and-error procedure. The control gains were selected as $c_{01}=0.1$, $c_{02}=10^{-5}, c_{03}=0.5, c_{11}=0.01, c_{12}=2.5, c_{13}=0.5, C_{2}=\operatorname{diag}(1,1,1), C_{3}=\operatorname{diag}(2,2,2)$.

The bandwidths of the command filters for the actual control variables $\delta_{e}, \delta_{a}$, and $\delta_{r}$ are chosen to be equal to the bandwidths of the actuators, which are given in (Sonneveldt et al., 2007). The outer-loop filters have the smallest bandwidths. The selection of the other bandwidths is again trial and error. A higher bandwidth in a certain feedback loop will result in more aggressive commands to the next feedback loop. All damping ratios are equal to 1.0. It is possible to add magnitude and rate limits to each of the filters. In this study magnitude limits on the aerodynamic roll angle $\mu$ and the flight-path angle $\gamma$ are used to avoid singularities in the control laws. Rate and magnitude limits equal to those of the actuators are enforced on the actual control variables. 
The selected command-filter parameters can be found back in Table 1. As soon as the controller gains and command-filter parameters have been defined, the update law gains can be selected. Again, the theory only requires that the gains should be larger than zero. Larger update gains means higher learning rates and thus more rapid changes in the Bspline network weights.

\subsection{Manoeuvre 1: upward spiral}

In this section the results of the numerical simulations of the first test maneuver, the climbing helical path, are discussed. For each of the three flight conditions, five cases are considered: nominal, the aerodynamic stability and control derivatives used in the control law perturbed with $+30 \%$ and with $-30 \%$ with respect to the real values of the model, a lockup of the left aileron at $+10 \mathrm{deg}$, and a lockup at $-10 \mathrm{deg}$. No actuator sensor information is used. In Fig. 3 the results are plotted of the simulation without uncertainty, starting at flight condition 1. The maneuver involves a climbing spiral to the left with an increase in airspeed. It can be seen that the control law manages to track the reference signal very well and closed-loop tracking is achieved. The sideslip angle does not become any larger than $\pm 0.02 \mathrm{deg}$. The aerodynamic roll angle does reach the limit set by the command filter, but this has no consequences for the performance. The use of dead zones ensures that the parameter update laws are indeed not updating during this maneuver without any uncertainties. The responses at the two other flight conditions are virtually the same, although less thrust is needed due to the lower altitude of flight condition 2 and the lower airspeed of flight condition 3 . The other control surfaces are also more efficient. This is illustrated in Tables 2-4, in which the mean absolute values (MAVs) of the outer-loop tracking errors, control-surface deflections, and thrust can be found. Plots of the parameter-estimation errors are not included. However, the errors converge to constant values, but not to zero, as is common with Lyapunov-based update laws (Sonneveldt et al., 2007, Page and Steinberg, 1999).

\begin{tabular}{lccc}
\hline Command variable & $\omega_{n}, \mathrm{rad} / \mathrm{s}$ & Magnitude limit & Rate limit \\
\hline$V^{\text {des }}$ & 5 & - & - \\
$\gamma^{\text {des }}$ & 3 & $\pm 80 \mathrm{deg}$ & - \\
$\mu^{\text {des }}$ & 8 & $\pm 80 \mathrm{deg}$ & - \\
$\alpha^{\text {des }}$ & 8 & - & - \\
$p^{\text {des }}$ & 20 & - & - \\
$q^{\text {des }}$ & 20 & - & - \\
$r^{\text {des }}$ & 10 & $\pm 25 \mathrm{deg}$ & $\pm 60 \mathrm{deg} / \mathrm{s}$ \\
$\delta_{e}$ & 40.4 & $\pm 21.5 \mathrm{deg}$ & $\pm 80 \mathrm{deg} / \mathrm{s}$ \\
$\delta_{a}$ & 40.4 & $\pm 30 \mathrm{deg}$ & $\pm 120 \mathrm{deg} / \mathrm{s}$ \\
$\delta_{r}$ & 40.4 & {$[1000-100,000] \mathrm{N}$} & $\pm 40,000 \mathrm{~N} / \mathrm{s}$ \\
$T$ & 10 & &
\end{tabular}

Table 1. Command-filter parameters

\begin{tabular}{lccc}
\hline Case & $\left(z_{01}, z_{00}, z_{00}\right)^{\mathrm{MAV}}, \mathrm{m}$ & $\left(\delta_{e}, \delta_{e}, \delta_{,}\right)^{\mathrm{MAV}}, \mathrm{deg}$ & $T^{\mathrm{MAV}, \mathrm{N}}$ \\
\hline Nominal & $(0.33,0.24,0.24)$ & $(4.63,0.12,0.10)$ & $5.59 e+04$ \\
$+30 \%$ uncertainty & $(4.56,3.75,1.07)$ & $(4.59,0.13,0.11)$ & $5.57 e+04$ \\
$-30 \%$ uncertainty & $(5.15,3.88,1.10)$ & $(4.68,0.16,0.11)$ & $5.62 e+04$ \\
$+10 \%$ deg, locked left aileron & $(0.39,0.32,0.78)$ & $(4.63,0.56,0.74)$ & $5.59 e+04$ \\
$-10 \%$ deg, locked left aileron & $(0.31,0.25,1.12)$ & $(4.63,0.46,1.16)$ & $5.59 e+04$ \\
\hline
\end{tabular}

Table 2. Manoeuvre 1 at flight condition 1: mean absolute value of tracking errors and control inputs 


\begin{tabular}{lccc}
\hline Case & $\left(z_{01}, z_{02}, z_{03}\right)^{\mathrm{MAV}}, \mathrm{m}$ & $\left(\delta_{e}, 8_{0}, 8_{r}\right)^{\mathrm{MAV}}, \mathrm{deg}$ & $T^{\mathrm{MAv}}, \mathrm{N}$ \\
\hline Nominal & $(0.30,0.23,0.21)$ & $(3.97,0.14,0.21)$ & $3.14 e+04$ \\
$+30 \%$ uncertainty & $(1.55,1.33,0.41)$ & $(3.96,0.15,0.23)$ & $3.14 e+04$ \\
-305 uncertainty & $(2.01,1.53,0.52)$ & $(3.98,0.15,0.20)$ & $3.14 e+04$ \\
$+10 \%$ deg, locked left aileron & $(0.36,0.33,0.72)$ & $(3.97,0.25,1.20)$ & $3.14 e+04$ \\
$-10 \%$ deg, locked left aileron & $(0.30,0.28,1.01)$ & $(3.96,0.40,1.52)$ & $3.14 e+04$ \\
\hline
\end{tabular}

Table 3. Manoeuvre 1 at flight condition 2: mean absolute value of tracking errors and control inputs

\begin{tabular}{lccc}
\hline Case & $\left(z_{01}, z_{02}, z_{03}\right)^{\mathrm{MAv}}, \mathrm{m}$ & $\left(\delta_{e}, \delta_{s}, \delta_{r}\right)^{\mathrm{Muv}}$, deg & $T^{\mathrm{Mav}}, \mathrm{N}$ \\
\hline Nominal & $(0.33,0.22,0.27)$ & $(3.37,0.08,0.08)$ & $4.41 e+04$ \\
$+30 \%$ uncertainty & $(2.01,1.43,0.61)$ & $(3.40,0.10,0.08)$ & $4.44 e+04$ \\
$-30 \%$ uncertainty & $(2.16,1.49,0.77)$ & $(3.38,0.09,0.08)$ & $4.41 e+04$ \\
$+10 \%$ deg, locked left aileron & $(0.32,0.33,0.29)$ & $(3.38,0.08,0.09)$ & $4.41 e+04$ \\
$-10 \%$ deg, locked left aileron & $(0.34,0.24,0.30)$ & $(3.38,0.08,0.09)$ & $4.41 e+04$ \\
\hline
\end{tabular}

Table 4. Manoeuvre 1 at flight condition 3: mean absolute value of tracking errors and control inputs

The response of the closed-loop system during the same maneuver starting at flight condition 1, but with $+30 \%$ uncertainties in the aerodynamic coefficients, is shown in Fig. 4 . It can be observed that the tracking errors of the outer loop are now much larger, but in the end, the steady-state tracking error converges to zero. The sideslip angle still remains within 0.02 deg. Some small oscillations are visible in Fig. 4j, but these stay well within the rate and magnitude limits of the actuators. In Tables 2-4 the MAVs of the tracking errors and control inputs are shown for all flight conditions. As was already seen in the plots, the average tracking errors increase, but the magnitude of the control inputs stays approximately the same. The same simulations have been performed for a $-30 \%$ perturbation in the stability and control derivatives used by the control law, and the results are also shown in the tables. It appears that underestimated initial values of the unknown parameters lead to larger tracking errors than overestimates for this maneuver. Finally, the maneuver is performed with the left aileron locked at \pm 10 deg [i.e., $\left.\delta_{a}^{\text {damaged }}=0.5\left(\delta_{a} \pm 10 \pi / 180\right)\right]$. Figure 5 shows the response at flight condition 3 with the aileron locked at $-10 \mathrm{deg}$.

Except for some small oscillations in the response of roll rate $p$, there is no real change in performance visible; this is confirmed by the numbers of Table 4 . However, from Tables 2 and 3 we observe that aileron and rudder deflections become larger with both locked aileron failure cases, whereas tracking performance hardly declines.

\subsection{Manoeuvre 2: reconnaissance}

The second maneuver, called reconnaissance and surveillance, involves turns in both directions and altitude changes, but airspeed is kept constant. Plots of the simulation at flight condition 3 with $-30 \%$ uncertainties are shown in Fig. 6. Tracking performance is again excellent and the steady-state tracking errors converge to zero. There are some small oscillations in the rudder deflection, but these are within the limits of the actuator. We compare the MAVs of the tracking errors and control inputs with those for the nominal case in Table 5 and observe that the average tracking errors have not increased much for this case. The degradation of performance for the uncertainty cases is somewhat worse at the other two flight conditions, as can be seen in Tables 6 and 7. The sideslip angle always remains within $0.05 \mathrm{deg}$ for all flight conditions and uncertainties. Corresponding with the 
results of maneuver 1, overestimation of the unknown parameters again leads to smaller tracking errors. Simulations of maneuver 2 with the locked aileron are also performed. Figure 7 shows the results for flight condition 1 with a locked aileron at_10 deg. Some very small oscillations are again visible in the roll rate, aileron, and rudder responses, but tracking performance is good and steady-state convergence is achieved.

Table 6 confirms that the results of the simulations with actuator failure hardly differ from the nominal case. There is only a small increase in the use of the lateral control surfaces. The same holds at the other flight conditions, as can be seen in Tables 5 and 7 .

\begin{tabular}{lccc}
\hline Case & $\left(z_{01}, z_{02}, z_{03}\right)^{\mathrm{MAv}}, \mathrm{m}$ & $\left(\delta_{e}, \delta_{a}, \delta_{s}\right)^{\mathrm{MAv}}, \mathrm{deg}$ & $T^{\mathrm{MAv}}, \mathrm{N}$ \\
\hline Nominal & $(0.49,0.40,0.56)$ & $(2.39,0.12,0.12)$ & $2.33 e+04$ \\
$+30 \%$ uncertainty & $(0.97,0.78,0.54)$ & $(2.39,0.12,0.13)$ & $2.33 e+04$ \\
$-30 \%$ uncertainty & $(0.97,0.56,0.85)$ & $(2.40,0.13,0.12)$ & $2.33 e+04$ \\
$+10 \%$ deg, locked left aileron & $(0.48,0.40,0.58)$ & $(2.39,0.12,0.13)$ & $2.33 e+04$ \\
$-10 \%$ deg, locked left aileron & $(0.49,0.40,0.56)$ & $(2.40,0.13,0.13)$ & $2.33 e+04$ \\
\hline
\end{tabular}

Table 5. Manoeuvre 2 at flight condition 3: mean absolute value of tracking errors and control inputs

\begin{tabular}{lccc}
\hline Case & $\left(z_{01}, z_{02}, z_{03}\right)^{\text {Mav }}, \mathrm{m}$ & $\left(\delta_{e}, \delta_{a}, \delta_{r}\right)^{\mathrm{MAv}}, \mathrm{deg}$ & $T^{\mathrm{MAV}, \mathrm{N}}$ \\
\hline Nominal & $(0.42,0.39,0.46)$ & $(3.17,0.16,0.13)$ & $2.25 e+04$ \\
$+30 \%$ uncertainty & $(2.69,2.30,1.13)$ & $(3.16,0.16,0.14)$ & $2.25 e+04$ \\
$-30 \%$ uncertainty & $(3.02 .2 .40,1.12)$ & $(3.19,0.18,0.14)$ & $2.25 e+04$ \\
$+10 \%$ deg, locked left aileron & $(0.43,0.40,0.45)$ & $(3.17,0.17,0.16)$ & $2.25 e+04$ \\
$-10 \%$ deg, locked left aileron & $(0.42,0.39,0.46)$ & $(3.17,0.17,0.15)$ & $2.25 e+04$ \\
\hline
\end{tabular}

Table 6. Manoeuvre 2 at flight condition 1: mean absolute value of tracking errors and control inputs

\begin{tabular}{lccc}
\hline Case & $\left(z_{01}, z_{00}, z_{03}\right)^{\mathrm{MAV}}, \mathrm{m}$ & $\left(\delta_{e}, \delta_{\alpha}, \delta_{f}\right)^{\mathrm{MAV}}, \mathrm{deg}$ & $T^{\mathrm{MAV}}, \mathrm{N}$ \\
\hline Nominal & $(0.58,0.49,0.34)$ & $(2.95,0.18,0.21)$ & $1.62 e+04$ \\
$+30 \%$ uncertainty & $(1.27,1.10 .0 .48)$ & $(2.95,0.19,0.22)$ & $1.62 e+04$ \\
$-30 \%$ uncertainty & $(1.73,1.24,0.55)$ & $(2.97,0.19,0.21)$ & $1.61 e+04$ \\
$+10 \%$ deg, locked left aileron & $(0.58,0.50 .0 .35)$ & $(2.95,0.20,0.22)$ & $1.62 e+04$ \\
$-10 \%$ deg, locked left aileron & $(0.59,0.51,0.34)$ & $(2.95,0.22,0.22)$ & $1.62 e+04$ \\
\hline
\end{tabular}

Table 7. Manoeuvre 2 at flight condition 2: mean absolute value of tracking errors and control inputs

\section{Conclusions}

In this paper a nonlinear adaptive flight-path control system is designed for a high-fidelity F-16 model. The controller is based on a backstepping approach with four feedback loops that are designed using a single control Lyapunov function to guarantee stability. The uncertain aerodynamic forces and moments of the aircraft are approximated online with Bspline neural networks for which the weights are adapted by Lyapunov-based update laws. Numerical simulations of two test maneuvers were performed at several flight conditions to verify the performance of the control law. Actuator failures and uncertainties in the stability and control derivatives are introduced to evaluate the parameter-estimation process. The results show that trajectory control can still be accomplished with these uncertainties and failures, and good tracking performance is maintained. Compared with other Lyapunov- 
based trajectory control designs, the present approach is much simpler to apply and the online estimation process is more robust to saturation effects. Future studies will focus on the actual trajectory generation and the extension to formation-flying control.

\section{Appendix constraint adaptive backstepping}

Backstepping [21] is a systematic, Lyapunov-based method for nonlinear control design, which can be applied to nonlinear systems that can be transformed into lower-triangular form, such as the system of Eq. (A.1):

$$
\dot{x}_{1}=f\left(x_{1}\right)+g\left(x_{1}\right) x_{2}
$$

The name "backstepping" refers to the recursive nature of the control law design procedure. Using the backstepping procedure, a control law is recursively constructed, along with a control Lyapunov function (CLF) to guarantee global stability. For the system Eq. (A.1), the aim of the design procedure is to bring the state vector $x_{1}$ to the origin. The first step is to consider $x_{2}$ as the virtual control of the scalar $x_{1}$ subsystem and to find a desired virtual control law $\alpha_{1}\left(x_{1}\right)$ that stabilizes this subsystem by using the control Lyapunov function $V_{1}\left(x_{1}\right)$ :

$$
V_{1}\left(x_{1}\right)=\frac{1}{2} x_{1}^{2}
$$

The time derivative of this CLF is negative definite

$$
\dot{V}_{1}\left(x_{1}\right)=\frac{\partial V_{1}\left(x_{1}\right)}{\partial x_{1}}\left[f\left(x_{1}\right)+g\left(x_{1}\right) \alpha_{1}\left(x_{1}\right)\right]<0, \quad x_{1} \neq 0
$$

If only the virtual control law

$$
x_{2}=\alpha_{1}\left(x_{1}\right)
$$

could be satisfied. The key property of backstepping is that we can now "step back" through the system. If the error between $x_{2}$ and its desired value is defined as

$$
z=x_{2}-\alpha_{1}\left(x_{1}\right)
$$

the system Eq. (6) can be rewritten in terms of this error state

$$
\begin{aligned}
& \dot{x}_{1}=f\left(x_{1}\right)+g\left(x_{1}\right)\left[\alpha_{1}\left(x_{1}\right)+z\right] \\
& \dot{z}=u-\frac{\partial \alpha_{1}\left(x_{1}\right)}{\partial x_{1}}\left(f\left(x_{1}\right)+g\left(x_{1}\right)\left[\alpha_{1}\left(x_{1}\right)+z\right]\right)
\end{aligned}
$$

The control Lyapunov function Eq. (A.2) can now be expanded with a term penalizing the error state $z$

$$
V_{2}\left(x_{1}, z\right)=V_{1}\left(x_{1}\right)+\frac{1}{2} z^{2}
$$


In simplified notation the time derivative of $V_{2}$ is equal to

$$
\begin{aligned}
\dot{V}_{2} & =\frac{\partial V_{1}}{\partial x_{1}}\left[f+g\left(\alpha_{1}+z\right)\right]+z\left(u-\frac{\partial \alpha_{1}}{\partial x_{1}}\left[f+g\left(\alpha_{1}+z\right)\right]\right) \\
& =\frac{\partial V_{1}}{\partial x_{1}}\left[f+g \alpha_{1}\right]+z\left(\frac{\partial V_{1}}{\partial x_{1}} g+u-\frac{\partial \alpha_{1}}{\partial x_{1}}\left[f+g\left(\alpha_{1}+z\right)\right]\right)
\end{aligned}
$$

which can be rendered negative definite with the control law

$$
u=-c z+\frac{\partial \alpha_{1}}{\partial x_{1}}\left[f+g\left(\alpha_{1}+z\right)\right]-\frac{\partial V_{1}}{\partial x_{1}} g, \quad c>0
$$

This design procedure can also be used for a system with a chain of integrators. The only difference is that there will be more virtual states to "backstep" through. Starting with the state "farthest" from the actual control, each step of the backstepping technique can be broken up into three parts: 1 . Introduce a virtual control $\alpha_{1}$ and an error state $z$, and rewrite the current state equation in terms of these; 2 . choose a CLF for the system, treating it as a final stage; 3 ) choose an equation for the virtual control that makes the CLF stabilizable.

The CLF is augmented at subsequent steps to reflect the presence of new virtual states, but the same three stages are followed at each step. Hence, backstepping is a recursive design procedure.

For systems with parametric uncertainties there exists a method called adaptive backstepping (Kannelakopoulos et al., 1991), which achieves boundedness of the closedloop states and convergence of the tracking error to zero.

Consider the parametric strict-feedback system

$$
\begin{array}{ccc}
\dot{x}_{1} & = & x_{2}+\varphi_{1}^{T}\left(x_{1}\right) \theta \\
\vdots & \vdots & \vdots \\
\dot{x}_{n-1} & = & x_{n}+\varphi_{n-1}^{T}\left(x_{1}, \cdots, x_{n-1}\right) \theta \\
\dot{x}_{n} & = & \beta(x) u+\varphi_{n}^{T}(x) \theta
\end{array}
$$

where $\beta(x) \neq 0$ for all $x \in \mathbb{R}^{n}, \theta$ is a vector of unknown constant parameters, and $\varphi_{i}$ are known (smooth) function vectors. The control objective is to asymptotically track a given reference $y_{r}(t)$ with $x_{1}$. All derivatives of $y_{r}(t)$ are assumed to be known.

The adaptive backstepping design procedure is similar to the normal backstepping procedure, only this time a control law (static part) and a parameter update law (dynamic part) are designed along with a control Lyapunov function to guarantee global stability. The control law makes use of a parameter estimate $\hat{\theta}$, which is constantly adapted by the dynamic parameter update law. Furthermore, the control Lyapunov function now contains an extra term that penalizes the parameter estimation error $\tilde{\theta}=\theta-\hat{\theta}$.

Theorem A.1 (Adaptive Backstepping Method): To stabilize the system Eq. (A.9) an error variable is introduced for each state

along with a virtual control law

$$
z_{i}=x_{i}-y_{r}^{(i-1)}-\alpha_{i-1}
$$


$\alpha_{i}\left(\bar{x}_{i}, \hat{\theta}, \bar{y}_{r}^{(i-1)}\right)=-c_{i} z_{i}-z_{i-1}-\omega_{i}^{T} \hat{\theta}+\sum_{k=1}^{i-1}\left(\frac{\partial \alpha_{i-1}}{\partial x_{k}} x_{k+1}+\frac{\partial \alpha_{i-1}}{\partial y_{r}^{(k-1)}} y_{r}^{(k)}\right)+\frac{\partial \alpha_{i-1}}{\partial \hat{\theta}} \Gamma \tau_{i}+\sum_{k=2}^{i-1} \frac{\partial \alpha_{i-1}}{\partial \hat{\theta}} \Gamma \omega_{i} z_{k}$

for $i=1,2, \cdots, n$, where the tuning function $\tau_{i}$ and the regressor vectors $\omega_{i}$ are defined as

$$
\tau_{i}\left(\bar{x}_{i}, \hat{\theta}, y_{r}^{(i-1)}\right)=\tau_{i-1}+\omega_{i} z_{i}
$$

and

$$
\omega_{i}\left(\bar{x}_{i}, \hat{\theta}, y_{r}^{(i-2)}\right)=\varphi_{i}-\sum_{k=1}^{i-1} \frac{\partial \alpha_{i-1}}{\partial x_{k}} \varphi_{k}
$$

where $\bar{x}_{i}=\left(x_{1}, x_{2}, \cdots, x_{i}\right), \bar{y}_{\dot{i}}^{(i)}=\left(y_{r}, \dot{y}_{r}, \cdots, y_{r}^{(i)}\right) . \quad c_{i}>0$ are design constants. With these new variables the control and adaptation laws can be defined as

$$
u=\frac{1}{\beta(x)}\left[\alpha_{n}\left(x, \hat{\theta}, \bar{y}_{r}^{(n-1)}\right)+y_{r}^{(n)}\right]
$$

and

$$
\dot{\hat{\theta}}=\Gamma \tau_{n}\left(x, \hat{\theta}, \bar{y}_{r}^{(n-1)}\right)=\Gamma W z
$$

where $\Gamma=\Gamma^{T}>0$ is the adaptation gain matrix and $W$ the regressor matrix

$$
W(z, \hat{\theta})=\left(\omega_{1}, \cdots, \omega_{i}\right)
$$

The control law Eq. (A.15) together with the update law Eq. (A.17) renders the derivative of the Lyapunov function

$$
V=\frac{1}{2} \sum_{i=1}^{n} z_{i}^{2}+\frac{1}{2} \tilde{\theta}^{T} \Gamma^{-1} \tilde{\theta}
$$

negative definite and thus this adaptive controller guarantees global boundedness of $x(t)$ and asymptotically tracking of a given reference $y_{r}(t)$ with $x_{1}$.

Proof of this theorem can be found in Sec. 4.3 of (Krstić et al., 1992).

The standard adaptive backstepping procedure as has been discussed so far has a number of drawbacks.

1. The analytic calculation of the virtual control derivatives is tedious, especially for large systems;

2. The procedure can only handle systems that can be transformed into a lower-triangular form;

3. Constraints on the inputs and states are not taken into account.

The third drawback can be a major problem when designing for flight control, because the actuators of an aircraft have rate, bandwidth, and magnitude constraints. When the control signal demanded by the backstepping controller cannot be generated by the actuators, that is, the actuators saturate, stability can no longer be guaranteed. The problem becomes worse 

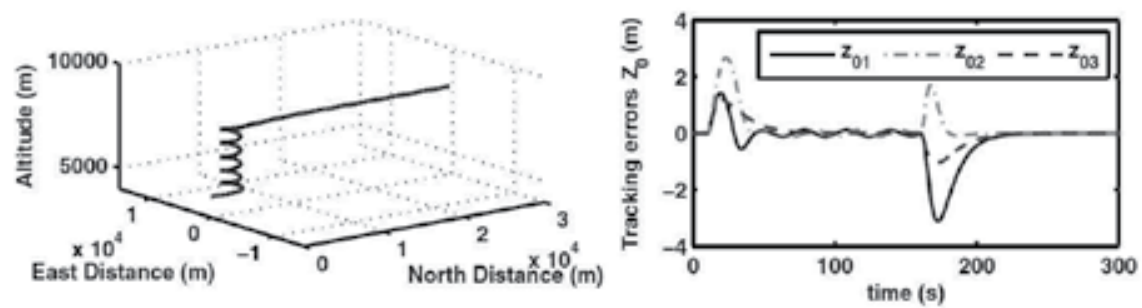

a)

b)
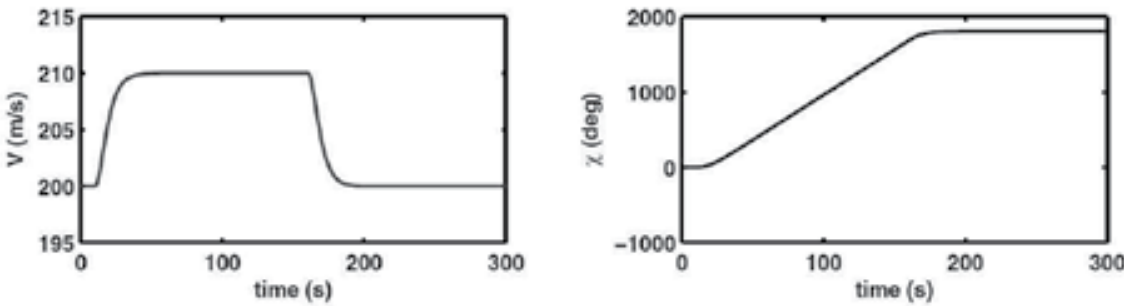

c)

d)
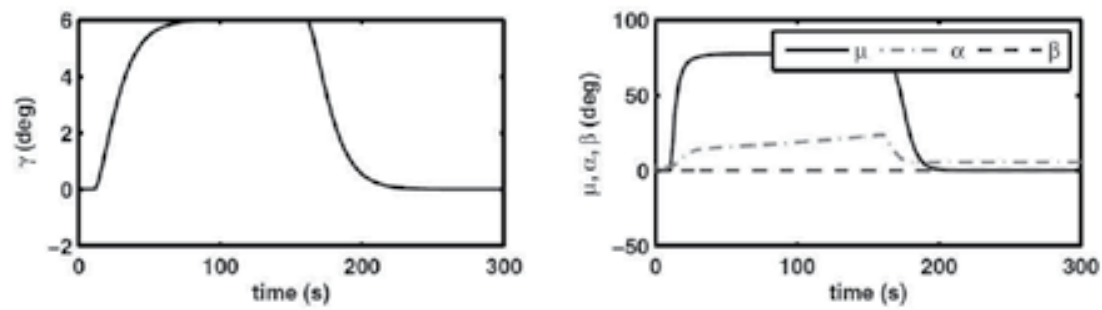

e)

ก
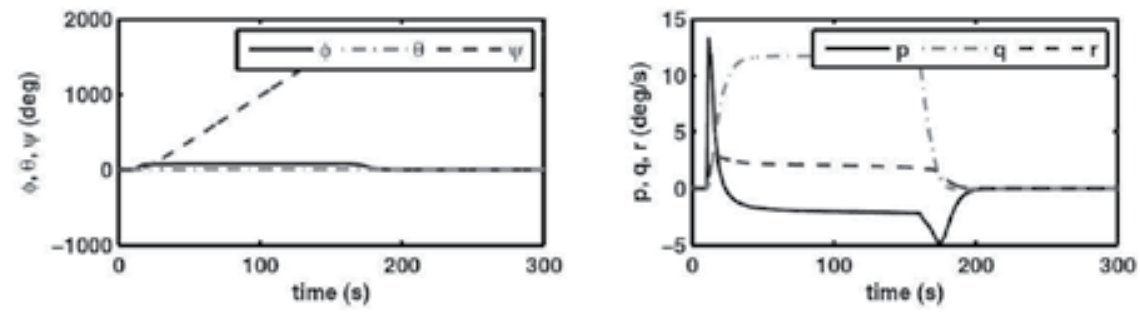

g)

h)
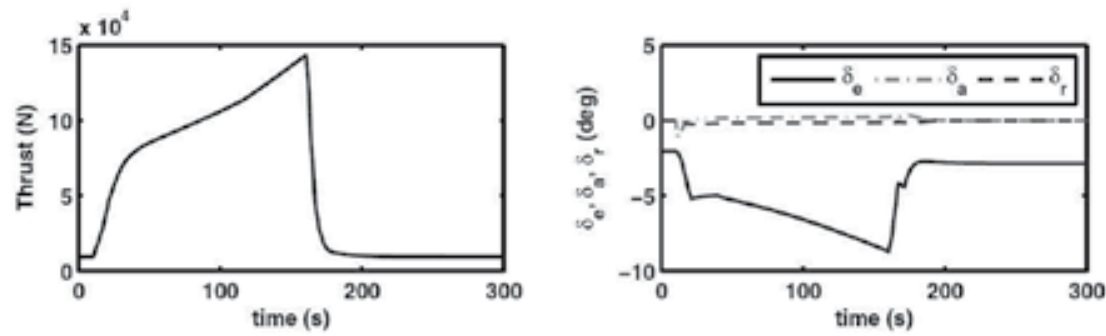

i)

j)

Fig. 3. Manoeuvre 1: climbing helical path performed at flight condition 1 without any uncertainty or actuator failures 

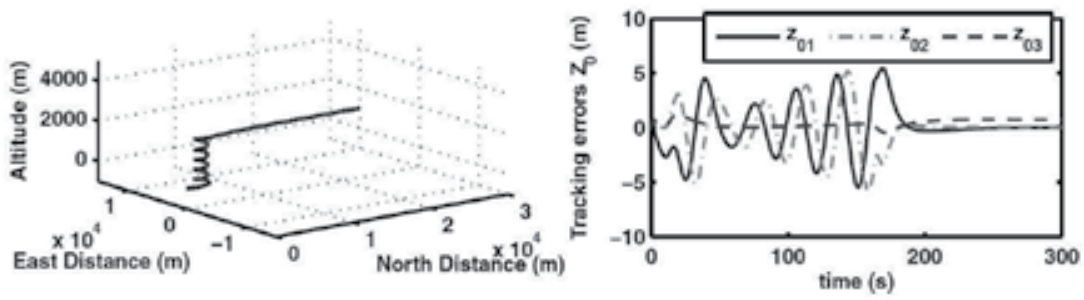

a)

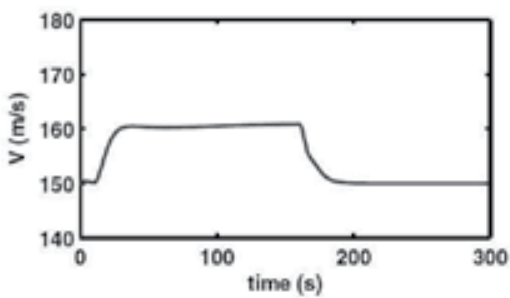

c)

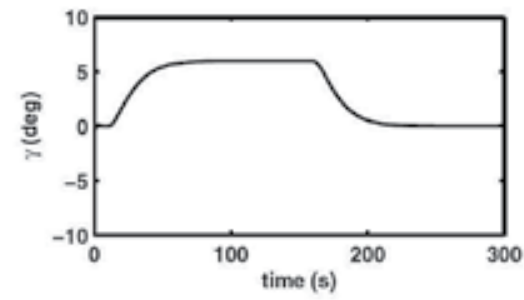

e)

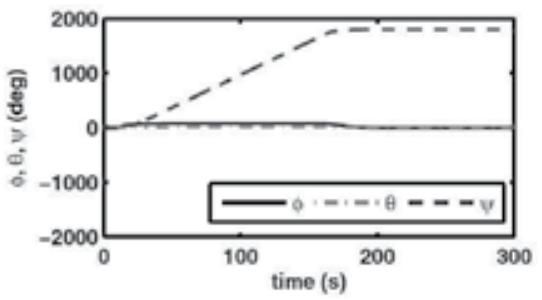

g)

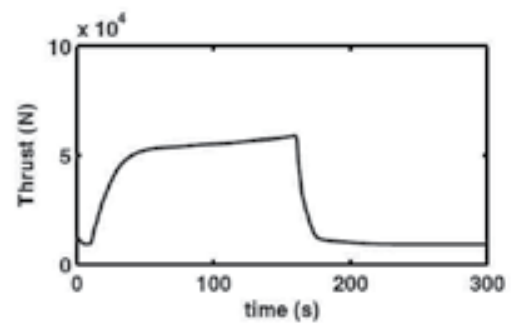

i) b)

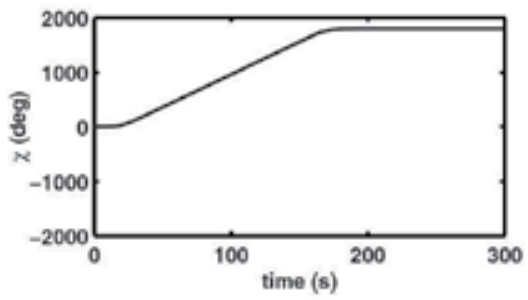

d)

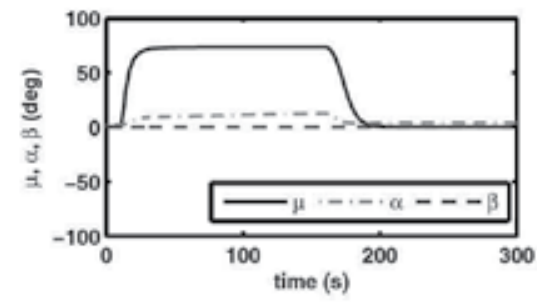

ก)

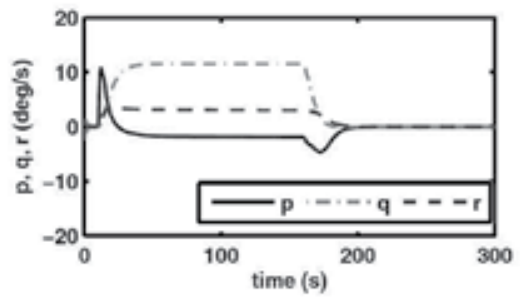

h)

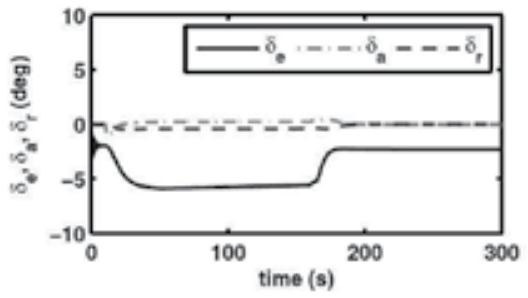

j)

Fig. 4. Manoeuvre 1: climbing helical path performed at flight condition 2 with $+30 \%$ uncertainties in the aerodynamic coefficients 

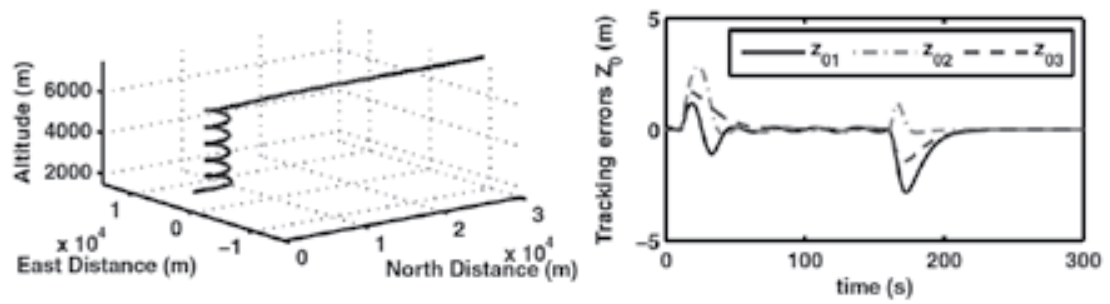

a)

b)
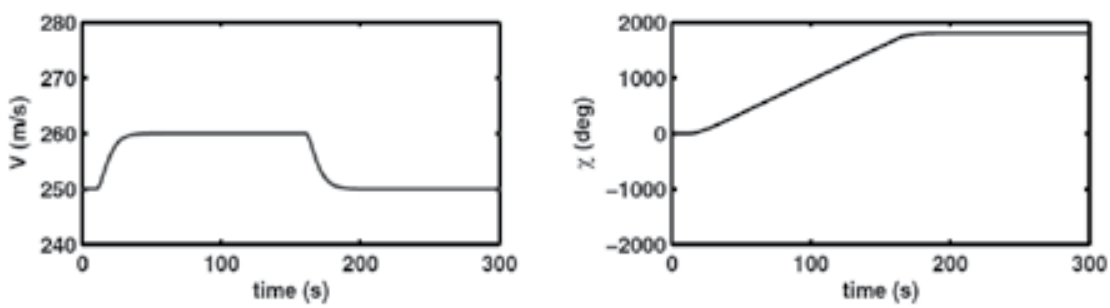

c)

d)
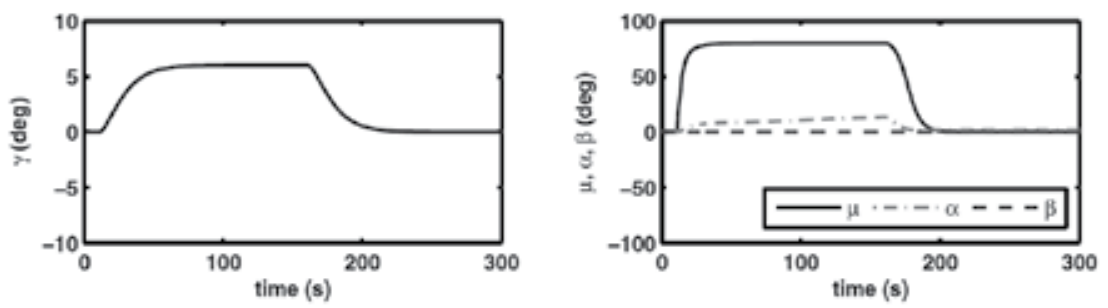

e)

)
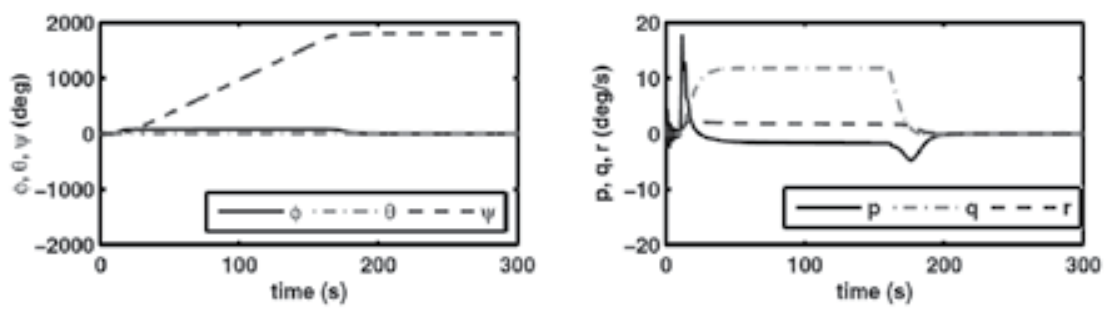

g)

h)
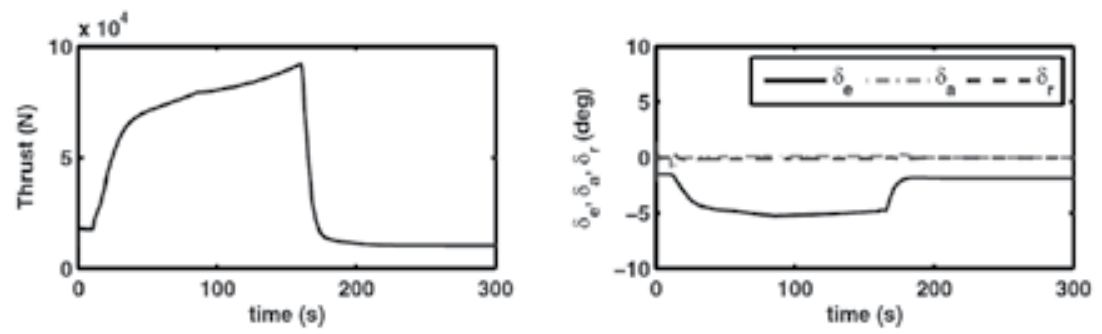

j)

Fig. 5. Manoeuvre 1: climbing helical path performed at flight condition 3 with left aileron locked at $-10 \mathrm{deg}$ 

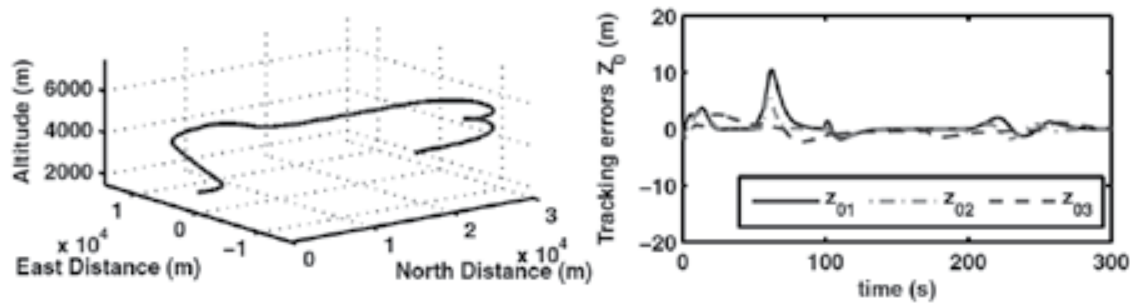

a)

b)
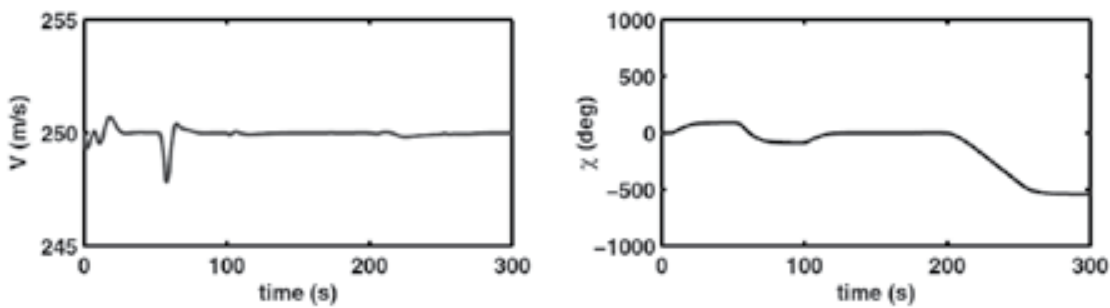

c)

d)
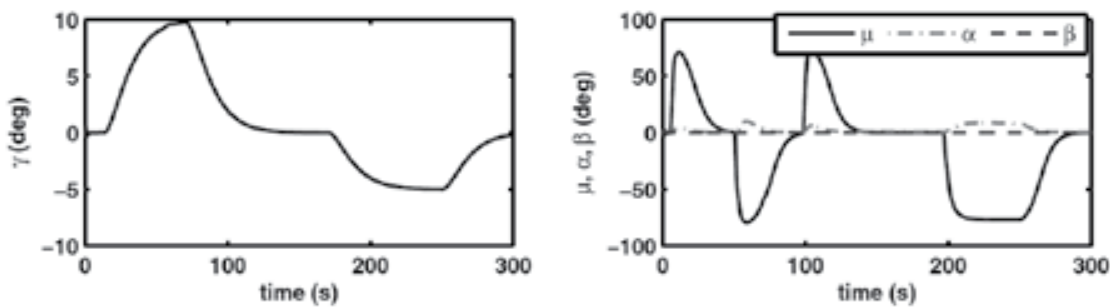

e)

f)
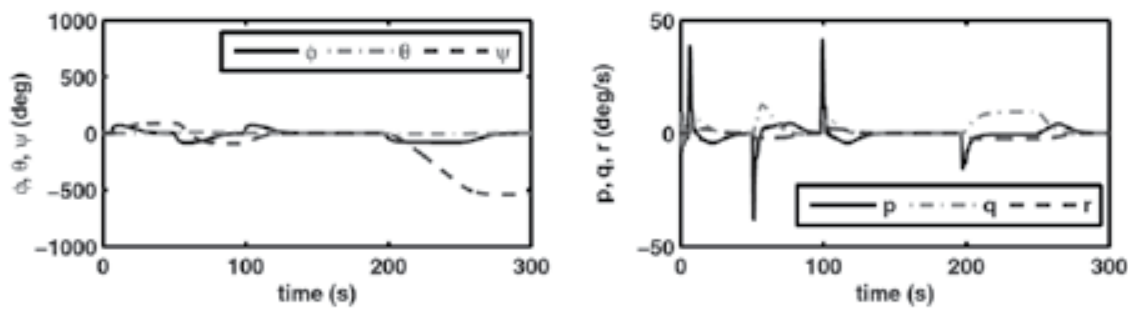

R)

h)

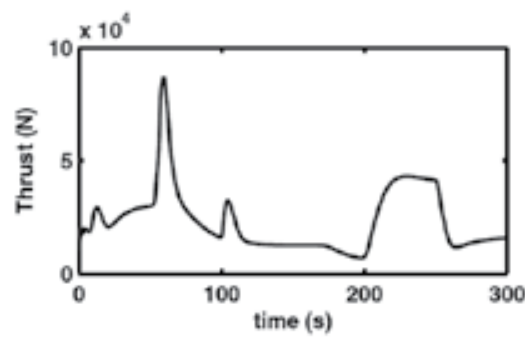

i)

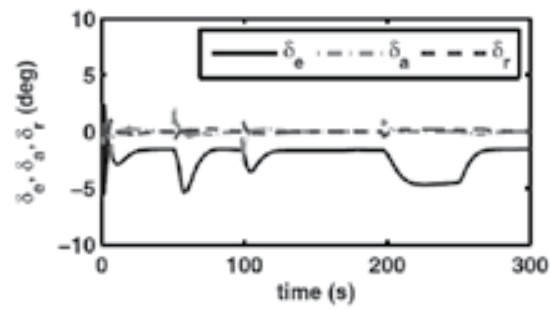

j)

Fig. 6. Manoeuvre 2: reconnaissance and surveillance performance at flight condition 3 with $-30 \%$ uncertainties in the aerodynamic coefficients 

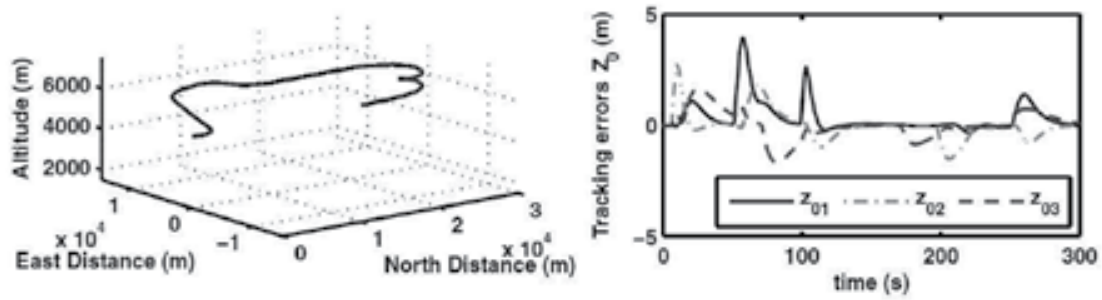

a)

b)
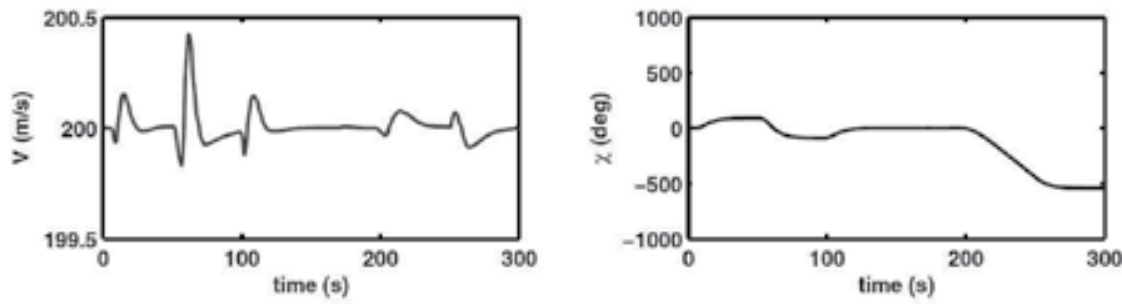

c)

d)
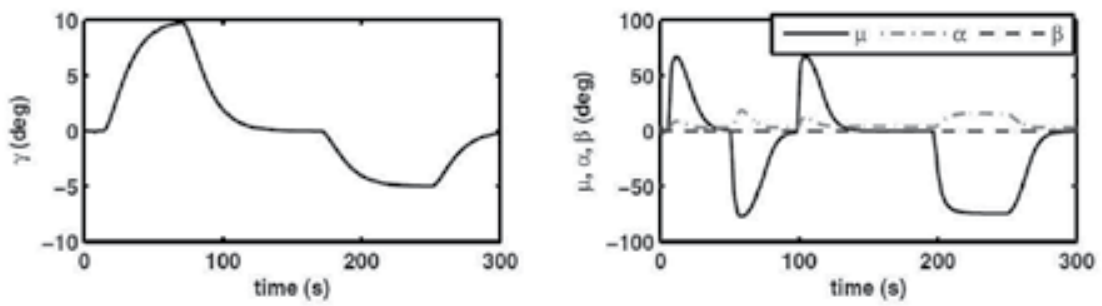

e)

ก
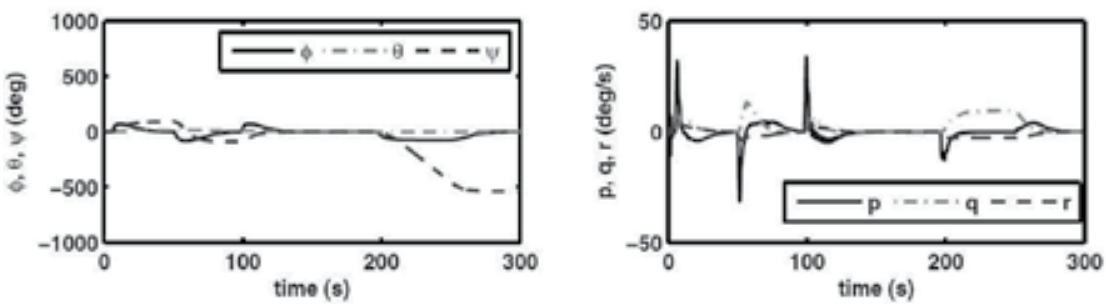

g)

h)
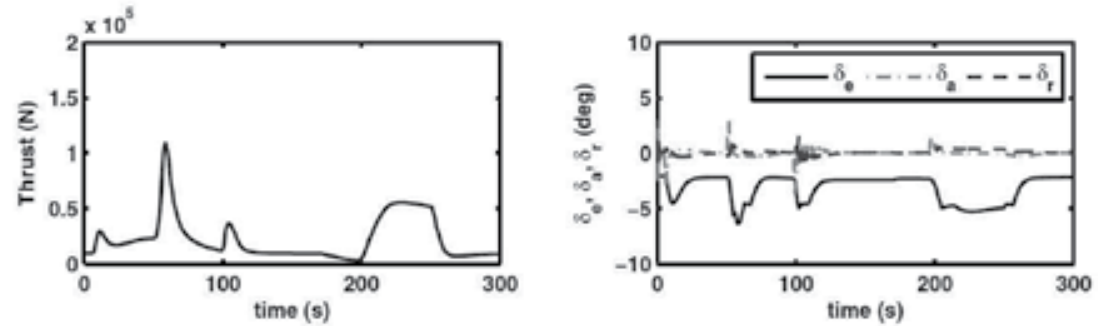

1)

j)

Fig. 7. Manoeuvre 2: reconnaissance and surveillance performance at flight condition 1 with left aileron locked at $+10 \mathrm{deg}$. 
when online parameter update laws are used, because these tend to be aggressive while seeking the desired tracking performance. Because the desired control signal is not achieved during saturation, the tracking error will increase. Because this tracking error is not just the result from the parameter estimation error, the update law may "unlearn" during these saturation periods.

In (Farrell et al., 2003, 2005) a method is proposed that fits within the recursive adaptive backstepping design procedure and deals with the constraints on both the control variables and the intermediate states used as virtual controls.An additional advantage of the method is that it also eliminates the two other drawbacks of the adaptive backstepping method, that is, the time consuming analytic computation of virtual control signal derivatives and the restriction to nonlinear systems of a lower-triangular form.

The proposed method extends the adaptive backstepping framework in two ways.

1. Command filters are used to eliminate the analytic computation of the time derivatives of the virtual controls. The command filters are designed as linear, stable, low-pass filters with unity gain from its input to its output. The inputs of these filters are the desired (virtual) control signals and the outputs are the actual (virtual) control signal and its time derivative. Using command filters to calculate the virtual control derivatives, it is still possible to prove stability in the sense of Lyapunov in the absence of constraints on the control input and state variables.

2. A stable parameter estimation process is ensured even when constraints on the control variables and states are in effect. During these periods the tracking error may increase because the desired control signal cannot be implemented due to these constraints imposed on the system. In this case the desired response is too aggressive for the system to be feasible and the primary goal is to maintain stability of the online function approximation. The command filters keep the control signal and the state variables within their mechanical constraints and operating limits, respectively. The effect these constraints have on the tracking errors can be estimated and this effect can be implemented in modified tracking error definitions. These modified tracking errors are only the result of parameter estimation errors as the effect of the constraints on the control input and state variables has been removed. These modified tracking errors can thus be used by the parameter update laws to ensure a stable estimation process.

The command filtered adaptive backstepping approach is summarized in the following theorem.

Theorem A.2 (Constrained Adaptive Backstepping Method): For the parameter strictfeedback system Eq. (15) the tracking errors are again defined as

$$
z_{i}=x_{i}-y_{r}^{(i-1)}-\alpha_{i-1}
$$

for $i=1,2, \cdots, n$. The nominal or desired virtual control laws can be defined as

$$
\alpha_{i}^{0}=-c_{i} z-\bar{z}_{i-1}-\varphi_{i}^{T} \hat{\theta}++\dot{\alpha}_{i-1}-\chi_{i+1}, \quad i=1,2, \cdots, n-1
$$

where

$$
\bar{z}_{i-1}=z_{i}-\chi_{i}, \quad i=1,2, \cdots, n
$$

are the modified tracking errors and where 


$$
\dot{\chi}_{i}=-c_{i} \chi_{i}+\left(\alpha_{i}-\alpha_{i}^{0}\right), \quad i=1,2, \cdots, n-1
$$

are the filtered versions of the effect of the state constraints on the tracking errors $z_{i}$. The nominal virtual control signals $\alpha_{i}^{0}$ are filtered to produce the magnitude, rate, and bandwidth limited virtual control signals $\alpha_{i}$ and its derivatives $\dot{\alpha}_{i}$ that satisfy the limits imposed on the state variables. This command filter can for instance be chosen as (Farrell et al., 2005)

$$
\left[\begin{array}{l}
\dot{q}_{1} \\
\dot{q}_{2}
\end{array}\right]=\left[2 \zeta \omega_{n}\left[S_{R}\left(\frac{\omega_{n}^{2}}{2 \zeta \omega_{n}}\left[S_{M}\left(\alpha_{i}^{0}\right)-q_{1}\right]-q_{2}\right)\right]\right], \quad\left[\begin{array}{c}
\alpha_{i} \\
\dot{\alpha}_{i}
\end{array}\right]=\left[\begin{array}{c}
q_{1} \\
q_{2}
\end{array}\right]
$$

where $S_{M}(\cdot)$ and $S_{R}(\cdot)$ represent the magnitude and rate limit functions, respectively. These saturation functions are defined similarly as

$$
S_{M}(x)=\left\{\begin{array}{c}
M \text { if } x \geq M \\
x \text { if }|x|<M \\
-M \text { if } x \leq-M
\end{array}\right.
$$

The effect of implementing the achievable virtual control signals instead of the desired ones is estimated by the $\chi_{i}$ filters. With these filters the modified tracking errors $\bar{z}_{i}$ can be defined. It can be seen from Eq. (A.21) that when the limitations on the states are not in effect the modified tracking error converges to the tracking error. The nominal control law is defined in a similar way as

$$
u^{0}=\frac{1}{\beta(x)}\left(-c_{n} z_{n}-\bar{z}_{n-1}-\varphi_{n}^{T} \hat{\theta}+\dot{\alpha}_{n-1}+y_{r}^{(n)}\right)
$$

which is again filtered to generate the magnitude, rate, and bandwidth limited control signal $u$. The effect of implementing the limited control law instead of the desired one can again be estimated with

$$
\dot{\chi}_{n}=-c_{n} \chi_{n}+\beta\left(u-u^{0}\right)
$$

Finally, the update law that now uses the modified tracking errors is defined as

$$
\dot{\hat{\theta}}=\Gamma \sum_{i=1}^{n} \varphi_{i} \bar{z}_{i}
$$

The resulting control law will render the derivative of the control Lyapunov function

$$
V=\frac{1}{2} \sum_{i=1}^{n} z_{i}^{2}+\frac{1}{2} \tilde{\theta}^{T} \Gamma^{-1} \tilde{\theta}
$$

negative definite, which means that the closed-loop system is asymptotically stable. 


\section{References}

Clough, B. T. (2005), “Unmanned Aerial Vehicles: Autonomous Control Challenges, a Researchers Perspective," Journal of Aerospace Computing, Information, and Communication, Vol. 2, No. 8, pp. 327-347, doi: 10.2514/1.5588.

Wegener, S., Sullivan, D., Frank, J., and Enomoto, F. (2004), "UAV Autonomous Operations for Airborne Science Missions," AIAA 3rd "Unmanned Unlimited" Technical Conference, Workshop and Exhibit, AIAA Paper 2004-6416.

Papadales, B., and Downing, M. (2005), "UAV Science Missions: A Business Perspective," Infotech@Aerospace, AIAA Paper 2005-6922.

Tsach, S., Chemla, J., and Penn, D. (2003), “UAV Systems Development in IAI-Past, Present and Future," 2nd AIAA "Unmanned Unlimited" Systems, Technologies, and Operations-Aerospace Land, and Sea Conference, AIAA Paper 2003-6535.

Kaminer, I., Pascoal, A., Hallberg, E., and Silvestre, C. (1998), "Trajectory Tracking for Autonomous Vehicles: An Integrated Approach to Guidance and Control," Journal of Guidance, Control, and Dynamics, Vol. 21, No. 1, pp. 29-38, doi:10.2514/2.4229.

Boyle, D. P., and Chamitof, G. E. (1999), "Autonomous Maneuver Tracking for Self-Piloted Vehicles," Journal of Guidance, Control, and Dynamics, Vol. 22, No. 1, pp. 58-67, doi: $10.2514 / 2.4371$.

Singh, S. N., Steinberg, M. L., and Page, A. B. (2003), "Nonlinear Adaptive and Sliding Mode Flight Path Control of F/A-18 Model," IEEE Transactions on Aerospace and Electronic Systems, Vol. 39, No. 4, pp. 1250-1262, doi: 10.1109/TAES.2003.1261125.

Ren, W., and Beard, R. W. (2004), "Trajectory Tracking for Unmanned Air Vehicles with Velocity and Heading Rate Constraints," IEEE Transactions on Control Systems Technology, Vol. 12, No. 5, pp. 706-716, doi:10.1109/TCST.2004.826956.

Ren, W., and Atkins, E. (2005), "Nonlinear Trajectory Tracking for Fixed Wing UAVs via Backstepping and Parameter Adaptation," AIAA Guidance, Navigation, and Control Conference and Exhibit, AIAA Paper 2005-6196.

No, T. S., Min, B. M., Stone, R. H., and K. C. Wong, J. E. (2005), “Control and Simulation of Arbitrary Flight Trajectory-Tracking," Control Engineering Practice, Vol. 13, No. 5, pp. 601-612, doi:10.1016/j.conengprac.2004.05.002.

Kaminer, I., Yakimenko, O., Dobrokhodov, V., Pascoal, A., Hovakimyan, N., Cao, C., Young, A., and Patel, V. (2007), "Coordinated Path Following for Time-Critical Missions of Multiple UAVs via L1 Adaptive Output Feedback Controllers," AIAA Guidance, Navigation, and Control Conference and Exhibit, AIAA Paper 2007-6409.

Pachter, M., D'Azzo, J. J., and J. L. Dargan (1994), "Automatic Formation Flight Control," Journal of Guidance, Control, and Dynamics, Vol. 17, No. 6, pp. 1380-1383.

Proud, A. W., Pachter, M., and D'Azzo, J. J. (1999), “Close Formation Flight Control,” AIAA Guidance, Navigation, and Control Conference, AIAA Paper 1999-4207.

Fujimori, A., Kurozumi, M., Nikiforuk, P. N., and Gupta, M. M. (2000), "Flight Control Design of an Automatic Landing Flight Experiment Vehicle," Journal of Guidance, Control, and Dynamics, Vol. 23, No. 2, pp. 373-376, doi:10.2514/2.4536.

Singh, S. N., Chandler, P., Schumacher, C., Banda, S., and Pachter, M. (2000), "Adaptive Feedback Linearizing Nonlinear Close Formation Control of UAVs," American Control Conference, Inst. of Electrical and Electronics Engineers, Piscataway, NJ, pp. 854-858. 
Pachter, M., D’Azzo, J. J., and Proud, A. W. (2001), “Tight Formation Control,” Journal of Guidance, Control, and Dynamics, Vol. 24, No. 2, pp. 246-254, doi:10.2514/2.4735.

Wang, J., Patel, V., Cao, C., Hovakimyan, N., and Lavretsky, E. (2008), “Novel L1 Adaptive Control Methodology for Aerial Refueling with Guaranteed Transient Performance," Journal of Guidance, Control, and Dynamics, Vol. 31, No. 1, pp. 182193, doi:10.2514/1.31199.

Healy, A., and Liebard, D. (1993), "Multivariable Sliding Mode Control for Autonomous Diving and Steering of Unmanned Underwater Vehicles," IEEE Journal of Oceanic Engineering, Vol. 18, No. 3, pp. 327-339, doi:10.1109/JOE.1993.236372.

Narasimhan, M., Dong, H., Mittal, R., and Singh, S. N. (2006), “Optimal Yaw Regulation and Trajectory Control of Biorobotic AUV Using Mechanical Fins Based on CFD Parametrization," Journal of Fluids Engineering, Vol. 128, No. 4, pp. 687-698, doi:10.1115/1.2201634.

Kannelakopoulos, I., Kokotović, P. V., and Morse, A. S. (1991), "Systematic Design of Adaptive Controllers for Feedback Linearizable Systems," IEEE Transactions on Automatic Control, Vol. 36, No. 11, pp. 1241-1253, doi:10.1109/9.100933.

Krstić, M., Kanellakopoulos, I., and Kokotović, P. V. (1992), “Adaptive Nonlinear Control Without Overparametrization," Systems and Control Letters, Vol. 19, pp. 177-185, doi:10.1016/0167-6911(92)90111-5.

Singh, S. N., and Steinberg, M. (1996), “Adaptive Control of Feedback Linearizable Nonlinear Systems With Application to Flight Control," AIAA Guidance, Navigation, and Control Conference, AIAA Paper 1996-3771.

Härkegård, O. (2003), "Backstepping and Control Allocation with Applications to Flight Control," Ph.D. Thesis, Linköping Univ., Linköping, Sweden.

Farrell, J., Polycarpou, M., and Sharma, M. (2003), “Adaptive Backstepping with Magnitude, Rate, and Bandwidth Constraints: Aircraft Longitude Control," American Control Conference, American Control Conference Council, Evanston, IL, pp. 3898-3903.

Kim, S. H., Kim, Y. S., and Song, C. (2004), "A Robust Adaptive Nonlinear Control Approach to Missile Autopilot Design," Control Engineering Practice, Vol. 12, No. 2, pp. 149-154, doi:10.1016/S0967-0661(03)00016-9.

Shin, D. H., and Kim, Y. (2004), "Reconfigurable Flight Control System Design Using Adaptive Neural Networks," IEEE Transactions on Control Systems Technology, Vol. 12, No. 1, pp. 87-100, doi:10.1109/TCST.2003.821957.

Farrell, J., Sharma, M., and Polycarpou, M. (2005), "Backstepping Based Flight Control with Adaptive Function Approximation," Journal of Guidance, Control, and Dynamics, Vol. 28, No. 6, pp. 1089-1102, doi:10.2514/1.13030.

Sonneveldt, L., Chu, Q. P., and Mulder, J. A. (2006), “Constrained Adaptive Backstepping Flight Control: Application to a Nonlinear F-16/MATV Model," AIAA Guidance, Navigation, and Control Conference and Exhibit, AIAA Paper 2006-6413.

Sonneveldt, L., Chu, Q. P., and Mulder, J. A. (2007), “Nonlinear Flight Control Design Using Constrained Adaptive Backstepping," Journal of Guidance, Control, and Dynamics, Vol. 30, No. 2, pp. 322-336, doi:10.2514/1.25834.

Yip, P.-C. P. (1997), “Robust and Adaptive Nonlinear Control Using Dynamic Surface Controller with Applications to Intelligent Vehicle Highway Systems," Ph.D. Thesis, Univ. of California at Berkeley, Berkeley, CA. 
Cheng, K. W. E., Wang, H., and Sutanto, D. (1999), “Adaptive B-Spline Network Control for Three-Phase PWM AC-DC Voltage Source Converter," IEEE 1999 International Conference on Power Electronics and Drive Systems, Inst. of Electrical and Electronics Engineers, Piscataway, NJ, pp. 467-472.

Ward, D. G., Sharma, M., Richards, N. D., and Mears, M. (2003), "Intelligent Control of UnManned Air Vehicles: Program Summary and Representative Results," 2nd AIAA Unmanned Unlimited Systems, Technologies and Operations Aerospace, Land and Sea, AIAA Paper 2003-6641.

Nguyen, L. T., Ogburn, M. E., Gilbert, W. P., Kibler, K. S., Brown, P. W., and Deal, P. L. (1979), "Simulator Study of Stall Post-Stall Characteristics of a Fighter Airplane with Relaxed Longitudinal Static Stability," NASA Langley Research Center, Hampton, VA.

Lewis, B. L., and Stevens, F. L. (1992), Aircraft Control and Simulation, Wiley, New York, pp. 1-54, 110-115.

Cook, M. V. (1997), Flight Dynamics Principles, Butterworth-Heinemann, London, pp. 1129.

Swaroop, D., Gerdes, J. C., Yip, P. P., and Hedrick, J. K. (1997), “Dynamic Surface Control of Nonlinear Systems," Proceedings of the American Control Conference.

Kanayama, Y. J., Kimura, Y., Miyazaki, F., and Noguchi, T. (1990), “A Stable Tracking Control Method for an Autonomous Mobile Robot," IEEE International Conference on Robotics and Automation, Inst. of Electrical and Electronics Engineers, Piscataway, NJ, pp. 384-389.

Enns, D. F. (1998), "Control Allocation Approaches," AIAA Guidance, Navigation, and Control Conference and Exhibit, AIAA Paper 1998-4109.

Durham, W. C. (1993), "Constrained Control Allocation," Journal of Guidance, Control, and Dynamics, Vol. 16, No. 4, pp. 717-725, doi:10.2514/3.21072.

Ioannou, P. A., and Sun, J. (1995), Stable and Robust Adaptive Control, Prentice-Hall, Englewood Cliffs, NJ, pp. 555-575.

Babuška, R. (1998), Fuzzy Modeling for Control, Kluwer Academic, Norwell, MA, pp. 49-52.

Karason, S. P., and Annaswamy, A. M. (1994), "Adaptive Control in the Presence of Input Constraints," IEEE Transactions on Automatic Control, Vol. 39, No. 11, pp. 23252330, doi:10.1109/9.333787.

Krstić, M., Kokotović, P. V., and Kanellakopoulos, I. (1993), “Transient Performance Improvement with a New Class of Adaptive Controllers," Systems and Control Letters, Vol. 21, No. 6, pp. 451-461, doi:10.1016/0167-6911(93)90050-G.

Sonneveldt, L., van Oort, E. R., Chu, Q. P., and Mulder, J. A. (2007), “Comparison of Inverse Optimal and Tuning Functions Designs for Adaptive Missile Control," AIAA Guidance, Navigation, and Control Conference and Exhibit, AIAA Paper 2007-6675.

Page, A. B., and Steinberg, M. L. (1999), "Effects of Control Allocation Algorithms on a Nonlinear Adaptive Design," AIAA Guidance, Navigation, and Control Conference and Exhibit, AIAA, Reston, VA, pp. 1664-1674; also AIAA Paper 19994282. 


\title{
Hybrid Adaptive Flight Control with Model Inversion Adaptation
}

\author{
Nhan Nguyen \\ NASA Ames Research Center \\ United States of America
}

\section{Introduction}

Adaptive flight control is a potentially promising technology that can improve aircraft stability and maneuverability. In recent years, adaptive control has been receiving a significant amount of attention. In aerospace applications, adaptive control has been demonstrated in many flight vehicles. For example, NASA has conducted a flight test of a neural net intelligent flight control system on board a modified F-15 test aircraft (Bosworth \& Williams-Hayes, 2007). The U.S. Air Force and Boeing have developed a direct adaptive controller for the Joint Direct Attack Munitions (JDAM) (Sharma et al., 2006). The ability to accommodate system uncertainties and to improve fault tolerance of a flight control system is a major selling point of adaptive control since traditional gain-scheduling control methods are viewed as being less capable of handling off-nominal flight conditions outside a normal flight envelope. Nonetheless, gain-scheduling control methods are robust to disturbances and unmodeled dynamics when an aircraft is operated as intended.

In spite of recent advances in adaptive control research and the potential benefits of adaptive control systems for enhancing flight safety in adverse conditions, there are several challenges related to the implementation of adaptive control technologies in flight vehicles to accommodate system uncertainties. These challenges include but are not limited to: 1) robustness in the presence of unmodeled dynamics and exogenous disturbances (Rohrs et al., 1985); 2) quantification of performance and stability metrics of adaptive control as related to adaptive gain and input signals; 3 ) adaptation in the presence of actuator rate and position limits; 4) cross-coupling between longitudinal and lateral-directional axes due to failures, damage, and different rates of adaptation in each axis; and 5) on-line reconfiguration and control reallocation using non-traditional control effectors such as engines with different rate limits.

The lack of a formal certification process for adaptive control systems poses a major hurdle to the implementation of adaptive control in future aerospace systems (Jacklin et al., 2005; Nguyen \& Jacklin, 2010). This hurdle can be traced to the lack of well-defined performance and stability metrics for adaptive control that can be used for the verification and validation of adaptive control systems. Recent studies by a number of authors have attempted to address metric evaluation for adaptive control systems (Annaswamy et al., 2008; Nguyen et al., 2007; Stepanyan et al., 2009; Yang et al., 2009). Thus, the development of verifiable metrics for 
adaptive control will be important in order to mature adaptive control technologies in the future.

Over the past several years, various model-reference adaptive control (MRAC) methods have been investigated (Cao \& Hovakimyan, 2008; Eberhart \& Ward, 1999; Hovakimyan et al., 2001; Johnson et al., 2000; Kim \& Calise, 1997; Lavretsky, 2009; Nguyen et al., 2008; Rysdyk \& Calise, 1998; Steinberg, 1999). The majority of MRAC methods may be classified as direct, indirect, or a combination thereof. Indirect adaptive control methods are based on identification of unknown plant parameters and certainty-equivalence control schemes derived from the parameter estimates which are assumed to be their true values (Ioannu \& Sun, 1996). Parameter identification techniques such as recursive least-squares and neural networks have been used in many indirect adaptive control methods (Eberhart \& Ward, 1999). In contrast, direct adaptive control methods adjust control parameters to account for system uncertainties directly without identifying unknown plant parameters explicitly. MRAC methods based on neural networks have been a topic of great research interest (Johnson et al., 2000; Kim \& Calise, 1997; Rysdyk \& Calise, 1998). Feedforward neural networks are capable of approximating a generic class of nonlinear functions on a compact domain within arbitrary tolerance (Cybenko, 1989), thus making them suitable for adaptive control applications. In particular, Rysdyk and Calise described a neural net direct adaptive control method for improving tracking performance based on a model inversion control architecture (Rysdyk \& Calise, 1998). This method is the basis for the intelligent flight control system that has been developed for the F-15 test aircraft by NASA. Johnson et al. introduced a pseudo-control hedging approach for dealing with control input characteristics such as actuator saturation, rate limit, and linear input dynamics (Johnson et al., 2000). Hovakimyan et al. developed an output feedback adaptive control to address issues with parametric uncertainties and unmodeled dynamics (Hovakimyan et al., 2001). Cao and Hovakimyan developed an $\mathcal{L}_{1}$ adaptive control method to address high-gain control (Cao \& Hovakimyan, 2008). Nguyen developed an optimal control modification scheme for adaptive control to improve stability robustness under fast adaptation (Nguyen et al., 2008).

While adaptive control has been used with success in many applications, the possibility of high-gain control due to fast adaptation can be an issue. In certain applications, fast adaptation is needed in order to improve the tracking performance rapidly when a system is subject to large uncertainties such as structural damage to an aircraft that could cause large changes in aerodynamic characteristics. In these situations, large adaptive gains can be used for adaptation in order to reduce the tracking error quickly. However, there typically exists a balance between stability and fast adaptation. It is well known that high-gain control or fast adaptation can result in high frequency oscillations which can excite unmodeled dynamics that could adversely affect stability of an MRAC law (Ioannu \& Sun, 1996). Recognizing this, some recent adaptive control methods have begun to address fast adaptation. One such method is the $\mathcal{L}_{1}$ adaptive control (Cao \& Hovakimyan, 2008) which uses a low-pass filter to effectively filter out any high frequency oscillation that may occur due to fast adaptation. Another approach is the optimal control modification that can enable fast adaptation while maintaining stability robustness (Nguyen et al., 2008).

This study investigates a hybrid adaptive flight control method as another possibility to reduce the effect of high-gain control (Nguyen et al., 2006). The hybrid adaptive control blends both direct and indirect adaptive control in a model inversion flight control architecture. The blending of both direct and indirect adaptive control is sometimes known as composite adaptation (Ioannu \& Sun, 1996). The indirect adaptive control is used to update the model 
inversion controller by two parameter estimation techniques: 1) an indirect adaptive law based on the Lyapunov theory, and 2) a recursive least-squares indirect adaptive law. The model inversion controller generates a command signal using estimates of the unknown plant dynamics to reduce the model inversion error. This directly leads to a reduced tracking error. Any residual tracking error can then be further reduced by a direct adaptive control which generates an augmented reference command signal based on the residual tracking error. Because the direct adaptive control only needs to adapt to a residual uncertainty, its adaptive gain can be reduced in order to improve stability robustness. Simulations of the hybrid adaptive control for a damaged generic transport aircraft and a pilot-in-the-loop flight simulator study show that the proposed method is quite effective in providing improved command tracking performance for a flight control system.

\section{Hybrid adaptive flight control}

Consider a rate-command-attitude-hold (RCAH) inner loop flight control design. The objective of the study is to design an adaptive law that allows an aircraft rate response to accurately follow a rate command. Assuming that the airspeed is regulated by the engine thrust, then the rate equation for an aircraft can be written as

$$
\dot{\omega}=\dot{\omega}^{*}+\Delta \dot{\omega}
$$

where $\boldsymbol{\omega}=\left[\begin{array}{ll}p & q \\ r\end{array}\right]^{\top}$ is the inner loop angular rate vector, $\Delta \dot{\boldsymbol{\omega}}$ is the uncertainty in the plant model which can include nonlinear effects, and $\dot{\omega}^{*}$ is the nominal plant model where

$$
\dot{\omega}^{*}=F_{1}^{*} \omega+F_{2}^{*} \sigma+G^{*} \delta
$$

with $F_{1}^{*}, F_{2}^{*}, G^{*} \in \mathbb{R}^{3 \times 3}$ as nominal state transition and control sensitivity matrices which are assumed to be known, $\sigma=[\Delta \phi \Delta \alpha \Delta \beta]^{\top}$ is the outer loop attitude vector which has slower dynamics than the inner loop rate dynamics, and $\delta=\left[\Delta \delta_{a} \Delta \delta_{e} \Delta \delta_{r}\right]^{\top}$ is the actuator command vector to flight control surfaces.

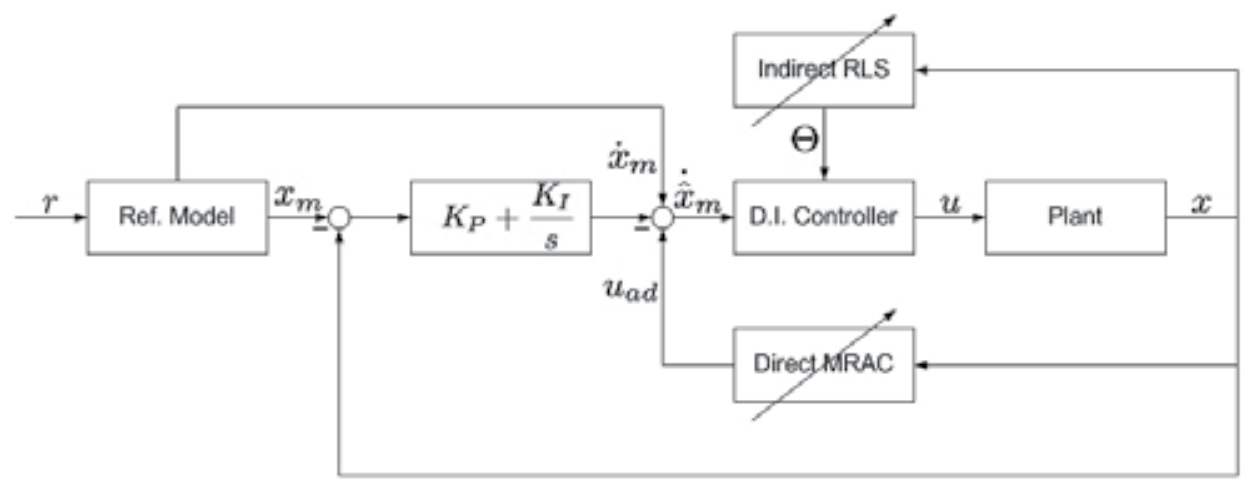

Fig. 1. Hybrid Adaptive Flight Control Architecture

Figure 1 illustrates the proposed hybrid adaptive flight control. The control architecture comprises: 1) a reference model that translates a rate command into a desired acceleration command, 2) a proportional-integral (PI) feedback control for rate stabilization and tracking, 
3) a model inversion controller that computes the actuator command using the desired acceleration command, 4) a neural net direct adaptive control augmentation, and 5) an indirect adaptive control that adjusts the model inversion controller to match the actual plant dynamics. The tracking error between the reference trajectory and the aircraft state is first reduced by the model inversion indirect adaptation. The neural net direct adaptation then further reduces the tracking error by estimating an augmented acceleration command to compensate for the residual tracking error. Without the model inversion indirect adaptation, the possibility of a high-gain control can exist with only the direct adaptation in use since a large adaptive gain needs to be used in order to reduce the tracking error rapidly. A high-gain control may be undesirable since it can lead to high frequency oscillations in the adaptive signal that can potentially excite unmodeled dynamics such as structural modes. The proposed hybrid adaptive control can improve the performance of a flight control system by incorporating a model inversion indirect adaptation in conjunction with a direct adaptation. The inner loop rate feedback control is designed to improve aircraft rate response characteristics such as the short period mode and the dutch roll mode. A second-order reference model is specified to provide desired handling qualities with good damping and natural frequency characteristics as follows:

$$
\begin{aligned}
& \left(s^{2}+2 \zeta_{p} \omega_{p} s+\omega_{p}^{2}\right) \phi_{m}=c_{p} \delta_{\text {lat }} \\
& \left(s^{2}+2 \zeta_{q} \omega_{q} s+\omega_{q}^{2}\right) \theta_{m}=c_{q} \delta_{l o n} \\
& \left(s^{2}+2 \zeta_{r} \omega_{r} s+\omega_{r}^{2}\right) r_{m}=c_{r} \delta_{r u d}
\end{aligned}
$$

where $\phi_{m}, \theta_{m}$, and $\psi_{m}$ are reference bank, pitch, and heading angles; $\delta_{l a t}, \delta_{l o n}$, and $\delta_{r u d}$ are the lateral stick input, longitudinal stick input, and rudder pedal input; $\omega_{p}, \omega_{q}$, and $\omega_{r}$ are the natural frequencies for desired handling qualities in the roll, pitch, and yaw axes; $\zeta_{p}, \zeta_{q}$, and $\zeta_{r}$ are the desired damping ratios; and $c_{p}, c_{q}$, and $c_{r}$ are stick gains.

Let $p_{m}=\dot{\phi}_{m}, q_{m}=\dot{\theta}_{m}$, and $r_{m}=\dot{\psi}_{m}$ be the reference roll, pitch, and yaw rates. Then the reference model can be represented as

$$
\dot{\omega}_{m}=-K_{p} \omega_{m}-K_{i} \int_{0}^{t} \omega_{m} d \tau+c \delta_{c}
$$

where $\boldsymbol{\omega}_{m}=\left[\begin{array}{lll}p_{m} & q_{m} & r_{m}\end{array}\right]^{\top}, K_{p}=\operatorname{diag}\left(2 \zeta_{p} \omega_{p}, 2 \zeta_{q} \omega_{q}, 2 \zeta_{r} \omega_{r}\right), K_{i}=\operatorname{diag}\left(\omega_{p}^{2}, \omega_{q}^{2}, \omega_{r}^{2}\right), c=$ $\operatorname{diag}\left(c_{p}, c_{q}, c_{r}\right)$, and $\delta_{c}=\left[\delta_{\text {lat }} \delta_{\text {lon }} \delta_{r u d}\right]^{\top}$.

A model inversion controller is computed to obtain an estimated control surface deflection command $\hat{\delta}$ to achieve a desired acceleration $\dot{\omega}_{d}$ as

$$
\hat{\delta}=\hat{G}^{-1}\left(\dot{\omega}_{d}-\hat{F}_{1} \omega-\hat{F}_{2} \sigma\right)
$$

where $\hat{F}_{1}, \hat{F}_{2}$, and $\hat{G}$ are the unknown plant matrices to be estimated by an indirect adaptive law which updates the model inversion controller; and moreover $\hat{G}$ is ensured to be invertible by verifying its matrix conditioning number. 
In order for the controller to track the reference acceleration $\dot{\omega}_{m}$, the desired acceleration $\dot{\omega}_{d}$ is computed as

$$
\dot{\omega}_{d}=\dot{\omega}_{m}+K_{p} \omega_{e}+K_{i} \int_{0}^{t} \omega_{e} d \tau-u_{a d}
$$

where $\omega_{e}=\omega_{m}-\omega$ is defined as a rate tracking error, and $u_{a d}$ is a direct adaptive signal designed to reduce the tracking error to small bound away from zero in order to provide stability robustness.

Because the true plant dynamics are unknown, the model inversion controller incurs a modeling error equal to

$$
\dot{\omega}-\dot{\omega}_{d}=\dot{\omega}-\dot{\omega}_{m}-K_{p} \omega_{e}-K_{i} \int_{0}^{t} \omega_{e} d \tau+u_{a d}
$$

but from Eq. (7) the model inversion controller is also equal to

$$
\dot{\omega}-\dot{\omega}_{d}=\epsilon-\left(\hat{F}_{1}-F_{1}^{*}\right) \omega-\left(\hat{F}_{2}-F_{2}^{*}\right) \sigma-\left(\hat{G}-G^{*}\right) \hat{\delta}
$$

where $\epsilon=\Delta \dot{\omega}$ is the unknown plant model error.

Comparing these two equations, the tracking error equation is formed as

$$
\dot{e}=A e+B u_{a d}+B \tilde{F}_{1} \omega+B \tilde{F}_{2} \sigma+B \tilde{G} \hat{\delta}-B \epsilon
$$

where $e=\left[\int_{0}^{t} \omega_{e} d \tau \omega_{e}\right]^{\top}$ is the tracking error, $\tilde{F}_{1}=\hat{F}_{1}-F_{1}^{*}, \tilde{F}_{2}=\hat{F}_{2}-F_{2}^{*}, \tilde{G}=\hat{G}-G^{*}$, and

$$
A=\left[\begin{array}{cc}
0 & I \\
-K_{i} & -K_{p}
\end{array}\right], B=\left[\begin{array}{l}
0 \\
I
\end{array}\right]
$$

The direct adaptive signal $u_{a d}$ is computed from a single-layer sigma-pi neural network

$$
u_{a d}=W^{\top} \Psi
$$

where $W \in \mathbb{R}^{m \times 3}$ is a neural network weight matrix, and $\Psi=\left[\begin{array}{llll}C_{1} & C_{2} & C_{3} & C_{4} \\ C_{5}\end{array}\right]^{\top} \in \mathbb{R}^{m \times 1}$ is a basis function with $C_{i}, i=1, \ldots, 5$, as inputs into the neural network consisting of control commands, sensor feedback, and bias terms; defined as follows

$$
\begin{gathered}
C_{1}=V^{2}\left[\begin{array}{lll}
\omega^{\top} \alpha \omega^{\top} \beta \omega^{\top}
\end{array}\right] \\
C_{2}=V^{2}\left[\begin{array}{lll}
1 \alpha \beta & \alpha^{2} & \beta^{2} \alpha \beta
\end{array}\right] \\
C_{3}=V^{2}\left[\begin{array}{lll}
\delta^{\top} & \alpha \delta^{\top} & \beta \delta^{\top}
\end{array}\right] \\
C_{4}=\left[\begin{array}{lll}
p \omega^{\top} & q \omega^{\top} & r \omega^{\top}
\end{array}\right] \\
C_{5}=\left[\begin{array}{lll}
1 & \theta \phi & \delta_{T}
\end{array}\right]
\end{gathered}
$$

where $\delta_{T}$ in $C_{5}$ is an engine throttle parameter.

These basis functions are designed to model the unknown nonlinearity that exists in the unknown plant model. For example, the aerodynamic force in the $x$-axis for an aircraft can be 
expressed as

$$
\begin{aligned}
F_{x}=\delta_{T} T_{\text {max }}+\frac{1}{2} \rho V^{2} S\left(C_{L_{0}}+C_{L_{\alpha}} \alpha\right. & \left.+C_{L_{\beta}} \beta+C_{L_{\boldsymbol{\omega}}} \boldsymbol{\omega}+C_{L_{\boldsymbol{\delta}}} \boldsymbol{\delta}\right) \alpha \\
& -\frac{1}{2} \rho V^{2} S\left(C_{D_{0}}+C_{D_{\alpha}} \alpha+C_{D_{\beta}} \beta+C_{D_{\boldsymbol{\omega}}} \boldsymbol{\omega}+C_{D_{\delta}} \boldsymbol{\delta}\right)
\end{aligned}
$$

where the engine thrust is replaced by $\delta_{T} T_{\max }$ and $T_{\max }$ is the maximum engine thrust.

Thus, $C_{1}, C_{2}$, and $C_{3}$ are designed to model the product terms of $\alpha, \beta, \omega$, and $\delta$ in the aerodynamic and propulsive forces. Similarly, $C_{4}$ models the cross-coupling terms of the aircraft rates in the moment equations, and $C_{5}$ models the effects the gravity and propulsive force. Alternatively, the basis function $\Psi$ can also be formed from a subset of $C_{i}, i=1,2, \ldots, 5$. The update law for the neural net weights $W$ is due to Rysdyk and Calise (Rysdyk \& Calise, 1998) and is given by

$$
\dot{W}=-\Gamma\left(\Psi e^{\top} P B+\mu\left\|e^{\top} P B\right\| W\right)
$$

where $\Gamma=\Gamma^{\top}>0 \in \mathbb{R}^{m \times m}$ is an adaptive gain matrix, $\mu>0 \in \mathbb{R}$ is an $e$-modification parameter (Narendra \& Annaswamy, 1987), $\|$.$\| is a Frobenius norm, and P=P^{\top}>0 \in \mathbb{R}^{6 \times 6}$ solves the Lyapunov equation

$$
P A+A^{\top} P=-Q
$$

for some positive-definite matrix $Q=Q^{\top}>0 \in \mathbb{R}^{6 \times 6}$.

The goal is to compute $\hat{F}_{1}, \hat{F}_{2}$, and $\hat{G}$ by a model inversion indirect adaptive law. The indirect adaptive law updates the estimates of $F_{1}, F_{2}$, and $G$ so that the model inversion controller $\hat{\delta}$ can accommodate as much as possible the effects of the unknown plant dynamics. Two approaches are considered: 1) an indirect adaptive law based on the Lyapunov's direct method, and 2) a recursive least-squares indirect adaptive law for parameter estimation of the unknown plant model. Both of these approaches are described as follows:

\subsection{Lyapunov-Based indirect adaptive law}

The hybrid adaptive control with model inversion adaptation can be implemented by the following indirect adaptive law

$$
\dot{\Phi}=-\Lambda\left(\Theta e^{\top} P B+\eta\left\|e^{\top} P B\right\| \Phi\right)
$$

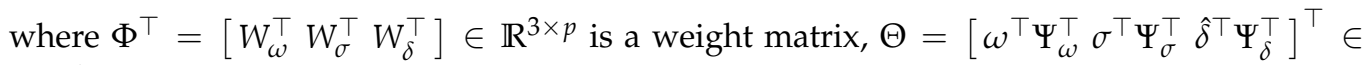
$\mathbb{R}^{p \times 1}$ is an input matrix of state and control vectors, $\Lambda=\operatorname{diag}\left(\Gamma_{\omega}, \Gamma_{\sigma}, \Gamma_{\delta}\right)>0 \in \mathbb{R}^{p \times p}$ is an adaptive gain matrix, and $\eta=\operatorname{diag}\left(\mu_{\omega} I, \mu_{\sigma} I, \mu_{\delta} I\right)>0 \in \mathbb{R}^{p \times p}$ is an $e$-modification parameter matrix.

Then the estimates of $F_{1}, F_{2}$, and $G$ can be computed as

$$
\begin{aligned}
& \hat{F}_{1}=F_{1}^{*}+W_{\omega}^{\top} \Psi_{\omega} \\
& \hat{F}_{2}=F_{2}^{*}+W_{\sigma}^{\top} \Psi_{\sigma} \\
& \hat{G}=G^{*}+W_{\delta}^{\top} \Psi_{\delta}
\end{aligned}
$$


The basis functions $\Psi_{\omega}, \Psi_{\sigma}$, and $\Psi_{\delta}$ are designed to model the nonlinearity in the plant model error. For example, if the plant model error is given by

$$
\epsilon=A_{1} \omega+A_{2} \alpha \omega+A_{3} \beta \omega
$$

then $W_{\omega}^{\top}=\left[\begin{array}{lll}A_{1} & A_{2} & A_{3}\end{array}\right]$ and $\Psi_{\omega}=\left[\begin{array}{lll}I & \alpha & \beta\end{array}\right]^{\top}$.

The tracking error then becomes

$$
\dot{e}=A e+B W^{\top} \Psi+B \Phi^{\top} \Theta-B \epsilon
$$

The indirect adaptive law (22) can be shown to provide a stable estimation of the unknown plant matrices $F_{1}, F_{2}$, and $G$ as follows:

Proof: The matrix $A$ is Hurwitz. Let $W=W^{*}+\tilde{W}$ and $\Phi=\Phi^{*}+\tilde{\Phi}$ where the asterisk symbol denotes the ideal weight matrices that cancel out the unknown plant model error $\epsilon$ and the tilde symbol denotes the weight deviations. The ideal weight matrices are unknown but they may be assumed constant and are bounded to stay within a $\Delta$-neighborhood of the plant model error $\epsilon$, assuming that the input or the command $\delta_{c} \in \mathcal{L}_{\infty}$ is bounded. Then

$$
\Delta=\sup _{\omega, \sigma, \delta}\left|W^{* \top} \Psi+\Phi^{* \top} \Theta-\epsilon\right|
$$

Choose the following Lyapunov candidate function

$$
V=e^{\top} P e+\operatorname{tr}\left(\tilde{W}^{\top} \Gamma^{-1} \tilde{W}+\tilde{\Phi}^{\top} \Lambda^{-1} \tilde{\Phi}\right)
$$

where $\operatorname{tr}($.$) denotes the trace operation.$

The time derivative of the Lyapunov candidate function is computed as

$$
\dot{V}=\dot{e}^{\top} P e+e^{\top} P \dot{e}+2 \operatorname{tr}\left(\tilde{W}^{\top} \Gamma^{-1} \dot{\tilde{W}}+\tilde{\Phi}^{\top} \Gamma^{-1} \dot{\tilde{\Phi}}\right)
$$

which upon substitution yields

$$
\begin{aligned}
\dot{V}=e^{\top}(P A & \left.+A^{\top} P\right) e+2 e^{\top} P B\left(W^{\top} \Psi+\Phi^{\top} \Theta-\epsilon\right) \\
& +2 \operatorname{tr}\left[-\tilde{W}^{\top}\left(\Psi e^{\top} P B+\mu\left\|e^{\top} P B\right\| W\right)-\tilde{\Phi}^{\top}\left(\Theta e^{\top} P B+\eta\left\|e^{\top} P B\right\| \Phi\right)\right]
\end{aligned}
$$

Utilizing the trace operation $\operatorname{tr}(X Y)=Y X$, where $X$ is a column vector and $Y$ is a row vector, then

$$
\begin{aligned}
& 2 \operatorname{tr}\left(-\tilde{W}^{\top} \Psi e^{\top} P B\right)=-2 e^{\top} P B \tilde{W}^{\top} \Psi \\
& 2 \operatorname{tr}\left(-\tilde{\Phi}^{\top} \Theta e^{\top} P B\right)=-2 e^{\top} P B \tilde{\Phi}^{\top} \Theta
\end{aligned}
$$

Completing the square yields

$$
\begin{aligned}
2 \operatorname{tr}\left[-\mu \tilde{W}^{\top}\left\|e^{\top} P B\right\|\left(W^{*}+\tilde{W}\right)\right]=-2 \mu\left\|e^{\top} P B\right\| & \left(\left\|\frac{W^{*}}{2}+\tilde{W}\right\|^{2}-\left\|\frac{W^{*}}{2}\right\|^{2}\right) \\
& \leq-\mu\left\|e^{\top} P B\right\|\left(\|\tilde{W}\|^{2}-\left\|W^{*}\right\|^{2}\right)
\end{aligned}
$$




$$
\begin{aligned}
2 \operatorname{tr}\left[-\tilde{\Phi}^{\top} \eta\left\|e^{\top} P B\right\|\left(\Phi^{*}+\tilde{\Phi}\right)\right] \leq-2\left\|e^{\top} P B\right\|\left[\lambda_{\min }(\eta)\left\|\left(\frac{\Phi^{*}}{2}+\tilde{\Phi}\right)\right\|^{2}\right. \\
\left.-\lambda_{\max }(\eta)\left\|\frac{\Phi^{*}}{2}\right\|^{2}\right] \leq-\left\|e^{\top} P B\right\|\left[\lambda_{\min }(\eta)\|\tilde{\Phi}\|^{2}-\lambda_{\max }(\boldsymbol{\eta})\left\|\Phi^{*}\right\|^{2}\right]
\end{aligned}
$$

where $\|$.$\| is a Frobenius norm, and \lambda_{\min }$ and $\lambda_{\max }$ are the maximum and minimum eigenvalues, respectively.

Then, substituting back into $\dot{V}$ gives

$$
\begin{aligned}
\dot{V} \leq-e^{\top} Q e+2 e^{\top} P B \Delta-\mu\left\|e^{\top} P B\right\| & \left(\|\tilde{W}\|^{2}-\left\|W^{*}\right\|^{2}\right) \\
& -\left\|e^{\top} P B\right\|\left[\lambda_{\min }(\eta)\|\tilde{\Phi}\|^{2}-\lambda_{\max }(\boldsymbol{\eta})\left\|\Phi^{*}\right\|^{2}\right]
\end{aligned}
$$

Since $\|B\|=1$, it can be established that

$$
\begin{aligned}
\dot{V} \leq-\lambda_{\min }(Q)\|e\|^{2}+\|P\|\|e\|\left[2\|\Delta\|-\mu\left(\|\tilde{W}\|^{2}-\left\|W^{*}\right\|^{2}\right)\right. & \\
& \left.-\lambda_{\min }(\eta)\|\tilde{\Phi}\|^{2}+\lambda_{\max }(\boldsymbol{\eta})\left\|\Phi^{*}\right\|^{2}\right]
\end{aligned}
$$

which can also be expressed as

$$
\begin{aligned}
\dot{V} \leq-\|e\|\left\{\lambda_{\min }(Q)\|e\|-\|P\|\left[2\|\Delta\|+\mu\left\|W^{*}\right\|^{2}+\lambda_{\max }(\boldsymbol{\eta})\left\|\Phi^{*}\right\|^{2}\right]\right. \\
\left.+\mu\|P\|\|\tilde{W}\|^{2}+\lambda_{\min }(\eta)\|P\|\|\tilde{\Phi}\|^{2}\right\}
\end{aligned}
$$

Let $\mathcal{S}$ be a compact set defined as

$$
\mathcal{S}=\left\{(e, \tilde{W}, \tilde{\Phi}): \lambda_{\min }(Q)\|e\|+\mu\|P\|\|\tilde{W}\|^{2}+\lambda_{\min }(\eta)\|P\|\|\tilde{\Phi}\|^{2} \leq r\right\}
$$

where

$$
r=\|P\|\left[2\|\Delta\|+\mu\left\|W^{*}\right\|^{2}+\lambda_{\max }(\boldsymbol{\eta})\left\|\Phi^{*}\right\|^{2}\right]
$$

Then $\dot{V} \leq 0$ outside the compact set $\mathcal{S}$. Also there exist functions $\varphi_{1}, \varphi_{2} \in \mathcal{K} \mathcal{R}$ where

$$
\begin{gathered}
\varphi_{1}(\|e\|,\|\tilde{W}\|,\|\tilde{\Phi}\|)=\lambda_{\min }(P)\|e\|^{2}+\lambda_{\min }\left(\Gamma^{-1}\right)\|\tilde{W}\|^{2}+\lambda_{\min }\left(\Lambda^{-1}\right)\|\tilde{\Phi}\|^{2} \\
\varphi_{2}(\|e\|,\|\tilde{W}\|,\|\tilde{\Phi}\|)=\lambda_{\max }(P)\|e\|^{2}+\lambda_{\max }\left(\Gamma^{-1}\right)\|\tilde{W}\|^{2}+\lambda_{\max }\left(\Lambda^{-1}\right)\|\tilde{\Phi}\|^{2}
\end{gathered}
$$

such that

$$
\varphi_{1}(\|e\|,\|\tilde{W}\|,\|\tilde{\Phi}\|) \leq V \leq \varphi_{2}(\|e\|,\|\tilde{W}\|,\|\tilde{\Phi}\|)
$$

Then, according to Theorem 3.4.3 of (Ioannu \& Sun, 1996), the solution is uniformly ultimately bounded. Therefore, the hybrid adaptive control results in stable and bounded tracking error; i.e., $e, \tilde{W}, \tilde{\Phi} \in \mathcal{L}_{\infty}$.

It should be noted that the bounds on $\|e\|,\|\tilde{W}\|$, and $\|\tilde{\Phi}\|$ depends on $\|\Delta\|$. To improve the tracking performance, the magnitudes of $\Delta$ must be kept small. This is predicated upon how well the neural network can approximate the nonlinear uncertainty in the plant dynamics. 
Increasing the adaptive gains $\Gamma$ and $\Lambda$ improves the tracking performance but at the same time degrades stability robustness. On the other hand, the values of $\mu$ and $\eta$ must also be kept sufficiently large to ensure stability robustness, but large values of $\mu$ and $\eta$ can degrade the tracking performance. Thus, there exists a trade-off between performance and robustness in selecting the adaptive gains $\Gamma$ and $\Lambda$ and the $e$-modification parameters $\mu$ and $\eta$.

To ensure that the indirect adaptive law will result in a convergence of the estimates $\hat{F}_{1}, \hat{F}_{2}$, and $\hat{G}$ to their steady state values, the input signals must be sufficiently rich to excite all frequencies of interest in the plant dynamics. This condition is known as a persistent excitation (PE) (Ioannu \& Sun, 1996).

\subsection{Recursive Least-squares indirect adaptive law}

The tracking error equation (11) can be expressed as

$$
\dot{e}=A e+B u_{a d}+B\left(\Phi^{\top} \Theta-\epsilon\right)
$$

Suppose the plant model error can be written as

$$
\epsilon=\dot{\hat{\omega}}-\dot{\omega}^{*}+\Delta \epsilon=\Phi^{\top} \Theta
$$

where $\Delta \epsilon$ is the estimation error of $\Delta \dot{\omega}$. Then, the estimated plant model error is

$$
\hat{\epsilon}=\dot{\hat{\omega}}-\dot{\omega}^{*}=\dot{\hat{\omega}}-F_{1}^{*} \omega-F_{2}^{*} \sigma-G^{*} \hat{\delta}
$$

where $\dot{\hat{\omega}}$ is the estimated acceleration.

The model inversion adaptation using the recursive least-squares indirect adaptive law is given by

$$
\begin{gathered}
\dot{\Phi}=\frac{1}{m^{2}} R \Theta\left(\hat{\epsilon}^{\top}-\Theta^{\top} \Phi\right) \\
\dot{R}=-\frac{1}{m^{2}} R \Theta \Theta^{\top} R
\end{gathered}
$$

where $R=R^{\top}>0 \in \mathbb{R}^{p \times p}$ is a positive definite covariance matrix and $m^{2}$ is a normalization factor

$$
m^{2}=1+\Theta^{\top} R \Theta
$$

The recursive least-squares indirect adaptive law can be derived as follows:

The estimation error can be minimized by considering the following cost function

$$
J(\Phi)=\frac{1}{2 m^{2}} \int_{0}^{t}\left\|\hat{\epsilon}^{\top}-\Theta^{\top} \Phi\right\|^{2} d \tau
$$

To minimize the cost function, the gradient of the cost function with respect to the weight matrix is computed and set to zero, thus resulting in

$$
\nabla J_{\Phi}^{\top}=-\frac{1}{m^{2}} \int_{0}^{t} \Theta\left(\hat{\epsilon}^{\top}-\Theta^{\top} \Phi\right) d \tau=0
$$

Equation (51) is then written as

$$
\frac{1}{m^{2}} \int_{0}^{t} \Theta \Theta^{\top} d \tau \Phi=\frac{1}{m^{2}} \int_{0}^{t} \Theta \hat{\epsilon}^{\top} d \tau
$$


Let

$$
R^{-1}=\frac{1}{m^{2}} \int_{0}^{t} \Theta \Theta^{\top} d \tau>0
$$

Differentiating Eq. (53) yields

$$
\frac{d R^{-1}}{d t}=\frac{1}{m^{2}} \Theta \Theta^{\top}
$$

It is noted that

$$
R^{-1} R=I \Rightarrow \frac{d R^{-1}}{d t} R+R^{-1} \dot{R}=0
$$

Solving for $\dot{R}$ yields Eq. (48).

Also, differentiating Eq. (52) yields

$$
R^{-1} \dot{\Phi}+\frac{1}{m^{2}} \Theta^{\top} \Phi=\frac{1}{m^{2}} \Theta \hat{\epsilon}^{\top}
$$

Solving for $\dot{\Phi}$ yields the recursive least-squares indirect adaptive law (47) .

The recursive least-squares indirect adaptive law can be shown to provide a stable estimation of the unknown plant matrices $F_{1}, F_{2}$, and $G$ as follows:

Proof: The steady state ideal weight matrix $\Phi^{*}$ is assumed to be bounded by a $\Delta_{\Phi}$-neighborhood where

$$
\bar{\Delta}=\sup _{\omega, \sigma, \delta}\left|\Phi^{* \top} \Theta-\hat{\epsilon}\right|
$$

The ideal weight matrix $W^{*}$ is assumed to be bounded inside a neighborhood where

$$
\Delta=\sup _{\omega, \sigma, \delta}\left|W^{* \top} \Psi+\Phi^{* \top} \Theta-\hat{\epsilon}-\Delta \epsilon\right| \leq \sup _{\omega, \sigma, \delta}\left|W^{* \top} \Psi-\Delta \epsilon\right|+\bar{\Delta}
$$

Choose the following Lyapunov candidate function

$$
L=e^{\top} P e+\operatorname{tr}\left(\tilde{W}^{\top} \Gamma^{-1} \tilde{W}+\tilde{\Phi}^{\top} R^{-1} \tilde{\Phi}\right)
$$

The only difference between $L$ and $V$ is in the last term. Then, the time rate of change of the Lyapunov candidate function is computed as

$$
\begin{aligned}
& \dot{L}=-e^{\top} Q e+2 e^{\top} P B\left(W^{\top} \Psi\right.\left.+\Phi^{\top} \Theta-\hat{\epsilon}-\Delta \epsilon\right) \\
&-2 \operatorname{tr}\left[\tilde{W}^{\top}\left(\Psi e^{\top} P B+\mu\left\|e^{\top} P B\right\| W\right)\right] \\
&+\operatorname{tr}\left[\frac{2}{m^{2}} \tilde{\Phi}^{\top} \Theta\left(\hat{\epsilon}^{\top}-\Theta^{\top} \Phi\right)+\tilde{\Phi}^{\top} \frac{d R^{-1}}{d t} \tilde{\Phi}\right]
\end{aligned}
$$

Further simplification yields

$$
\begin{aligned}
\dot{L} \leq-e^{\top} Q e+2 e^{\top} P B \Delta+2 e^{\top} P B \tilde{\Phi}^{\top} \Theta+\mu & \left\|e^{\top} P B\right\|\left(\left\|W^{*}\right\|^{2}-\|\tilde{W}\|^{2}\right) \\
& -\frac{1}{m^{2}} \Theta^{\top} \tilde{\Phi} \tilde{\Phi}^{\top} \Theta+\frac{2}{m^{2}}\left(\hat{\epsilon}^{\top}-\Theta^{\top} \Phi^{*}\right) \tilde{\Phi}^{\top} \Theta
\end{aligned}
$$


$\dot{L}$ is then bounded by

$$
\begin{aligned}
\dot{L} \leq-\lambda_{\min }(Q)\|e\|^{2}+\|P\|\|e\| & \left(2\|\Delta\|+2\left\|\tilde{\Phi}^{\top} \Theta\right\|+\mu\left\|W^{*}\right\|^{2}\right) \\
& -\mu\|P\|\|e\|\|\tilde{W}\|^{2}-\frac{1}{m^{2}}\left\|\tilde{\Phi}^{\top} \Theta\right\|^{2}+\frac{2}{m^{2}}\left\|\tilde{\Phi}^{\top} \Theta\right\|\|\bar{\Delta}\|
\end{aligned}
$$

which can also be expressed as

$$
\begin{aligned}
\dot{L} \leq-\|e\|\left[\lambda_{\min }(Q)\|e\|-\|P\|(2\right. & \left.\|\Delta\|+2\left\|\tilde{\Phi}^{\top} \Theta\right\|+\mu\left\|W^{*}\right\|^{2}\right) \\
& \left.+\mu\|P\|\|\tilde{W}\|^{2}\right]-\frac{1}{m^{2}}\left\|\tilde{\Phi}^{\top} \Theta\right\|\left(\left\|\tilde{\Phi}^{\top} \Theta\right\|-2\|\bar{\Delta}\|\right)
\end{aligned}
$$

$\dot{L}<0$ if

$$
\left\|\tilde{\Phi}^{\top} \Theta\right\|>2\|\bar{\Delta}\|
$$

and

$$
\begin{aligned}
\lambda_{\min }(Q)\|e\|+\mu\|P\|\|\tilde{W}\|^{2}>\|P\|\left(2\|\Delta\|+2\left\|\tilde{\Phi}^{\top} \Theta\right\|+\mu\left\|W^{*}\right\|^{2}\right) \\
>\|P\|\left(2\|\Delta\|+4\|\bar{\Delta}\|+\mu\left\|W^{*}\right\|^{2}\right)
\end{aligned}
$$

Let $\mathcal{C}$ be a compact set defined as

$$
\mathcal{C}=\left\{(e, \tilde{W}, \tilde{\Phi}): \lambda_{\min }(Q)\|e\|+\mu\|P\|\|\tilde{W}\|^{2} \leq \bar{r} \text { or }\left\|\tilde{\Phi}^{\top} \Theta\right\| \leq 2\|\bar{\Delta}\|\right\}
$$

where

$$
\bar{r}=\|P\|\left(2\|\Delta\|+4\|\bar{\Delta}\|+\mu\left\|W^{*}\right\|^{2}\right)
$$

Then $\dot{L} \leq 0$ outside the compact set $\mathcal{C}$, and so according to Theorem 3.4 .3 of (Ioannu \& Sun, 1996), the solution is uniformly ultimately bounded. Therefore, the hybrid adaptive control results in stable and bounded tracking error; i.e., $e, \tilde{W}, \tilde{\Phi} \in \mathcal{L}_{\infty}$. Thus, the recursive least-squares indirect adaptive law is stable.

The parameter convergence of the recursive least-squares depends on the persistent excitation condition on the input signals (Ioannu \& Sun, 1996). The update law for the covariance matrix $R$ has a very similar form to the Kalman filter with Eq. (48) as the differential Riccati equation for a zero-order plant dynamics. The recursive least-squares indirect adaptive law can also be implemented in a discrete time form with various modifications such as with an adaptive directional forgetting factor (Bobal et al., 2005) according to

$$
\begin{gathered}
\Phi_{i+1}=\Phi_{i}+\frac{1}{m_{i+1}^{2}} R_{i+1} \Theta_{i}\left[\hat{\epsilon}_{i+1}^{\top}-\Theta_{i}^{\top} \Phi_{i}\right] \\
R_{i+1}=R_{i}-\left(\psi_{i+1}^{-1}+\xi_{i+1}\right)^{-1} R_{i} \Theta_{i} \Theta_{i}^{T} R_{i}
\end{gathered}
$$

where $\psi$ and $\xi$ are defined as

$$
\begin{gathered}
\xi_{i+1}=m_{i+1}^{2}-1 \\
\psi_{i+1}=\varphi_{i+1}-\xi_{i}^{-1}\left(1-\varphi_{i+1}\right)
\end{gathered}
$$


The directional forgetting factor $\varphi$ is calculated as

$$
\varphi_{i+1}^{-1}=1+(1+\rho) \ln \left(1+\xi_{i+1}\right)+\left[\frac{\eta_{i+1}\left(1+\vartheta_{i+1}\right)}{1+\xi_{i+1}+\eta_{i+1}}-1\right] \frac{\xi_{i+1}}{1+\xi_{i+1}}
$$

where $\rho$ is a constant, and $\eta$ and $\vartheta$ are parameters with the following update laws

$$
\begin{gathered}
\eta_{i+1}=\lambda_{i+1}^{-1}\left\|\hat{\epsilon}_{i+1}-\Phi_{i}^{\top} \Theta_{i}\right\|^{2} \\
\vartheta_{i+1}=\varphi_{i+1}\left(1+\vartheta_{i}\right) \\
\lambda_{k+1}=\varphi_{i+1}\left[\lambda_{k}+\left(1+\xi_{i+1}\right)\left\|\hat{\epsilon}_{i+1}-\Phi_{i}^{\top} \Theta_{i}\right\|^{2}\right]
\end{gathered}
$$

\section{Flight control simulations}

\subsection{Generic transport model}

To evaluate the hybrid adaptive flight control method, a simulation was conducted using a NASA generic transport model (GTM) which represents a notional twin-engine transport aircraft as shown in Fig. 2 (Jordan et al., 2004). An aerodynamic model of the damaged aircraft is created using a vortex lattice method to estimate aerodynamic coefficients and derivatives. A damage scenario is modeled corresponding to a $28 \%$ loss of the left wing. The damage causes an estimated C.G. shift mostly along the pitch axis with $\Delta y=0.0388 \bar{c}$ and an estimated mass loss of $1.2 \%$. The principal moment of inertia about the roll axis is reduced by $12 \%$, while changes in the inertia values in the other two axes are not as significant. Since the damaged aircraft is asymmetric, the inertia tensor has all six non-zero elements. This means that all the three roll, pitch, and yaw axes are coupled together throughout the flight envelope.

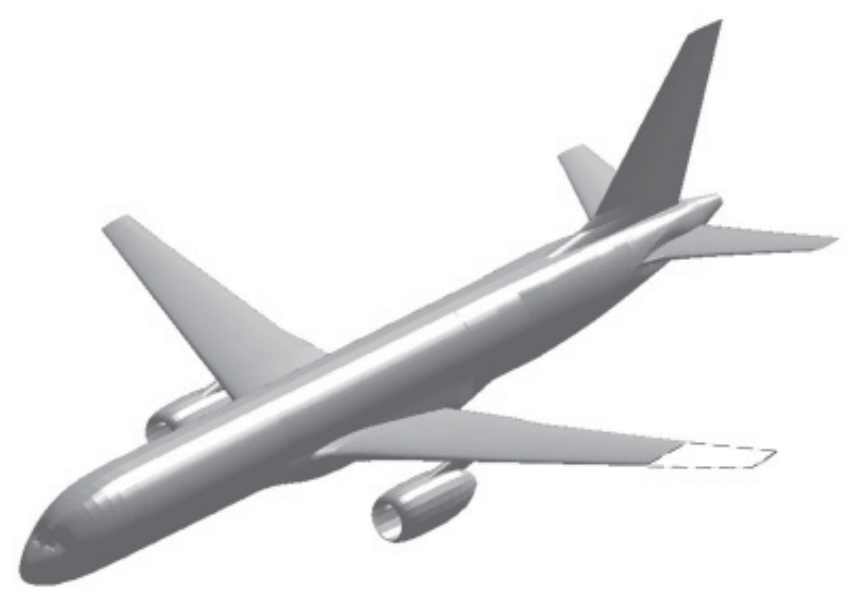

Fig. 2. Left Wing Damaged Generic Transport Model

A level flight condition of Mach 0.6 at $4572 \mathrm{~m}$ is selected. Upon damage, the aircraft is re-trimmed with $T=0.0705 \mathrm{~W}, \bar{\alpha}=5.9^{\circ}, \bar{\phi}=-3.2^{\circ}, \bar{\delta}_{a}=27.3^{\circ}, \bar{\delta}_{e}=-0.5^{\circ}, \bar{\delta}_{r}=-1.3^{\circ}$. The remaining right aileron is the only roll control effector available. In practice, some aircraft can control a roll motion with spoilers, which are not modeled in this study. The reference model is 
specified by $\omega_{p}=2.3 \mathrm{rad} / \mathrm{sec}, \omega_{q}=1.7 \mathrm{rad} / \mathrm{sec}, \omega_{r}=1.3 \mathrm{rad} / \mathrm{sec}$, and $\zeta_{p}=\zeta_{q}=\zeta_{r}=1 / \sqrt{2}$. slotine

The state space model of the damaged aircraft is given by

$$
\begin{aligned}
{\left[\begin{array}{l}
\dot{p} \\
\dot{q} \\
\dot{r}
\end{array}\right]=} & {\left[\begin{array}{ccc}
-1.3568 & -0.2651 & 0.5220 \\
-0.0655 & -0.8947 & 0.0147 \\
0.0836 & -0.0042 & -0.5135
\end{array}\right]\left[\begin{array}{l}
p \\
q \\
r
\end{array}\right] } \\
+ & {\left[\begin{array}{ccc}
0 & -10.9985 & -8.9435 \\
-0.0007 & -2.7041 & -0.0064 \\
0 & 0.1841 & 2.8822
\end{array}\right]\left[\begin{array}{c}
\Delta \phi \\
\Delta \alpha \\
\Delta \beta
\end{array}\right] } \\
& +\left[\begin{array}{ccc}
3.2190 & -0.0451 & 1.3869 \\
0.3391 & -3.4656 & 0.0245 \\
-0.0124 & 0.0007 & -2.2972
\end{array}\right]\left[\begin{array}{c}
\Delta \delta_{a} \\
\Delta \delta_{e} \\
\Delta \delta_{r}
\end{array}\right]
\end{aligned}
$$

$$
\begin{aligned}
& {\left[\begin{array}{c}
\Delta \dot{\phi} \\
\Delta \dot{\alpha} \\
\Delta \dot{\beta}
\end{array}\right]=\left[\begin{array}{ccc}
1 & 0 & 0.1024 \\
-0.0059 & 0.9723 & 0.0004 \\
-0.0031 & 0.0002 & -0.9855
\end{array}\right]\left[\begin{array}{l}
p \\
q \\
r
\end{array}\right] } \\
&+\left[\begin{array}{ccc}
0 & 0 & 0 \\
0.0028 & -0.4799 & 0.0235 \\
0.0507 & 0.0133 & -0.1751
\end{array}\right]\left[\begin{array}{c}
\Delta \phi \\
\Delta \alpha \\
\Delta \beta
\end{array}\right] \\
&+ {\left[\begin{array}{ccc}
0 & 0 & 0 \\
0.0240 & -0.0700 & -0.0011 \\
0.0019 & 0.0001 & 0.0588
\end{array}\right]\left[\begin{array}{l}
\Delta \delta_{a} \\
\Delta \delta_{e} \\
\Delta \delta_{r}
\end{array}\right] }
\end{aligned}
$$
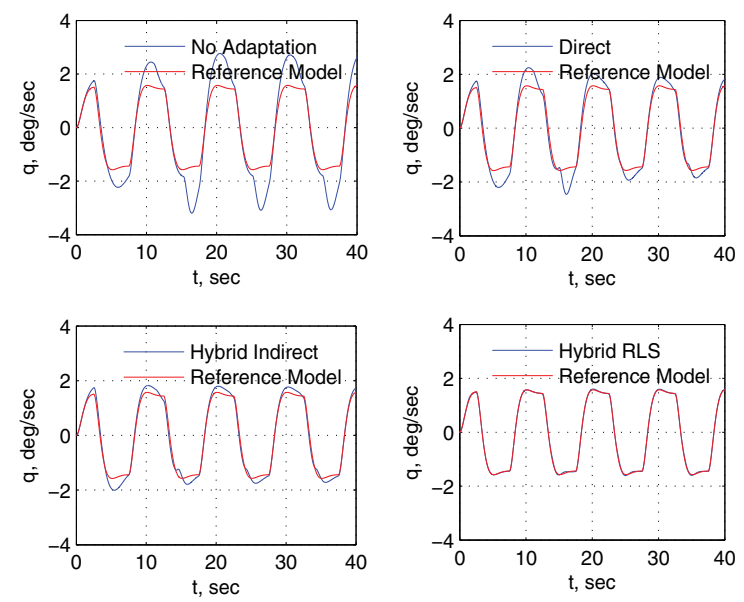

Fig. 3. Pitch Rate

The pilot pitch rate command is simulated with a series of ramp input longitudinal stick command doublets, corresponding to the reference pitch angle between $-3.1^{\circ}$ and $3.1^{\circ}$. The tracking performance of the baseline flight control with no adaptation versus the three 

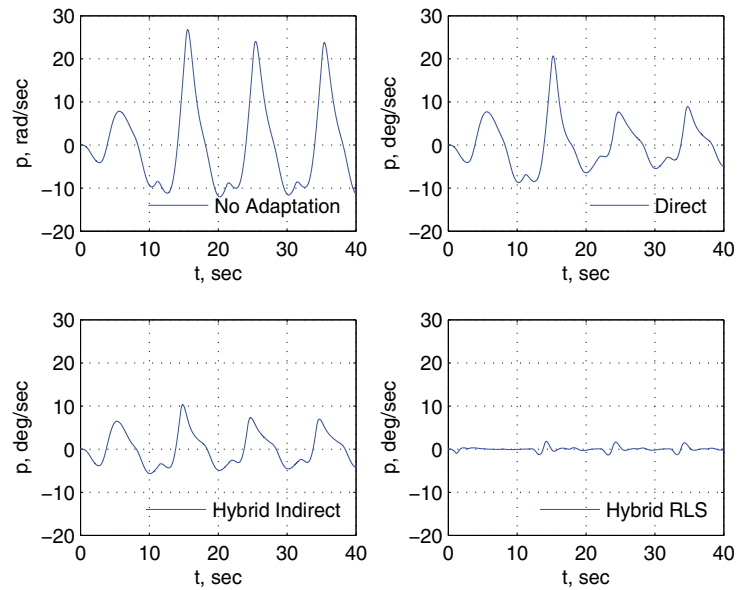

Fig. 4. Roll Rate

adaptive control methods is compared in Figs. 3 to 6 . With no adaptation, there is a significant overshoot in the ability for the baseline flight control system to follow the reference pitch rate as shown in Fig. 3. The performance progressively improves first with the direct adaptive control alone, then with the hybrid Lyapunov-based indirect adaptive control, and finally with the hybrid recursive least-squares (RLS) indirect adaptive control. The Lyapunov-based indirect adaptive control performs better than the direct adaptive control alone as expected, since the presence of the Lyapunov-based indirect adaptive law further enhances the ability for the flight control system to adapt to damage.
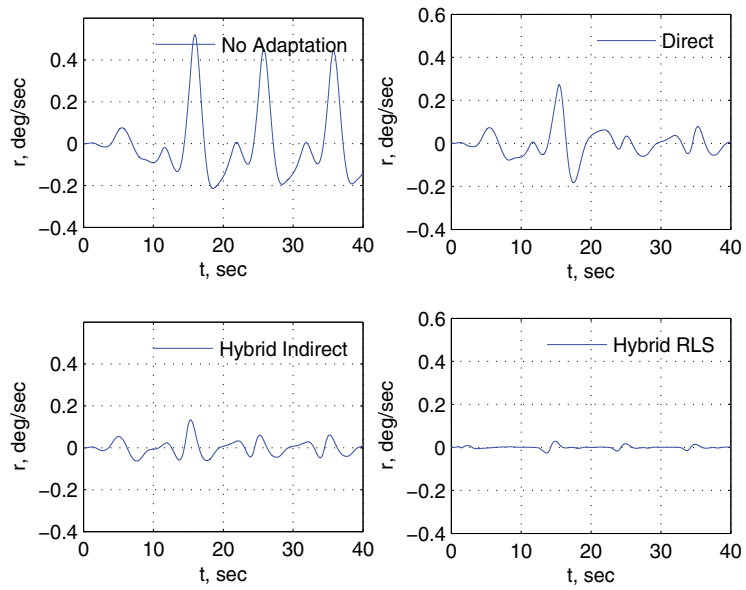

Fig. 5. Yaw Rate

The most drastic improvement is provided by the hybrid RLS indirect adaptive control which results in a very good tracking performance in all three control axes. In the pitch axis, the hybrid RLS indirect adaptive control tracks the reference pitch rate very accurately. In the roll and yaw axes, the roll and yaw rate responses are maintained close to zero. In contrast, both 

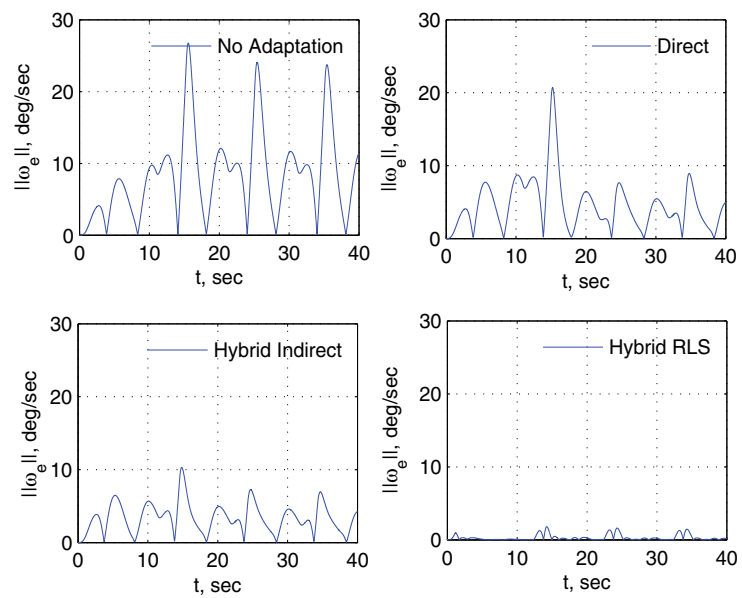

Fig. 6. Tracking Error Norm

the direct adaptive control and the hybrid Lyapunov-based indirect adaptive control improve the roll and yaw rate responses, but the response amplitudes are still significant and therefore can be objectionable particularly in the roll rate.

Figure 6 is the plot of the tracking error norm for all the three angular rates to demonstrate the effectiveness of the hybrid adaptive control method. The hybrid Lyapunov-based indirect adaptive control reduces the tracking error by roughly half of that with the direct adaptive control alone and by a factor of three when there is no adaptation. Moreover, the hybrid RLS indirect adaptive control drastically reduces the tracking error by more than an order of magnitude over those with the direct adaptive control and with the baseline flight control.
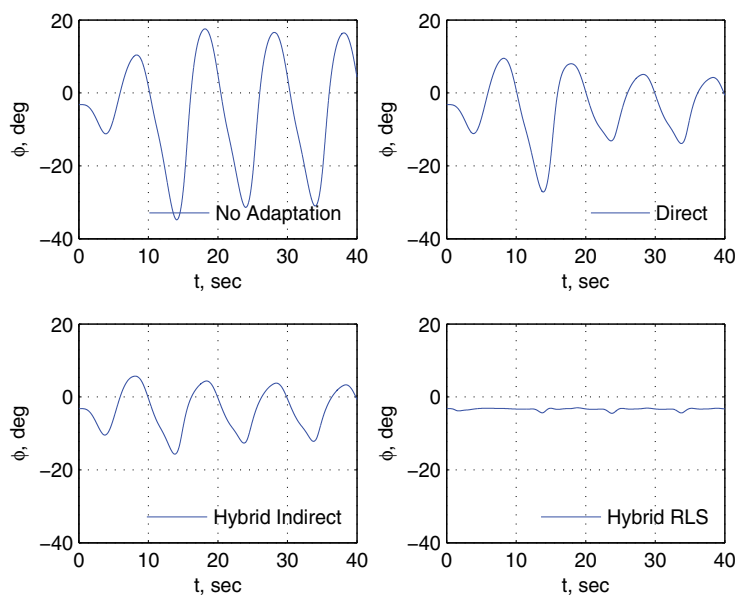

Fig. 7. Bank Angle

The attitude responses of the damaged aircraft are shown in Fig. 7 to 9. When there is no adaptation, the damaged aircraft exhibits a rather severe roll behavior with the bank angle ranging from almost $-40^{\circ}$ to $20^{\circ}$. The direct adaptive control improves the situation 

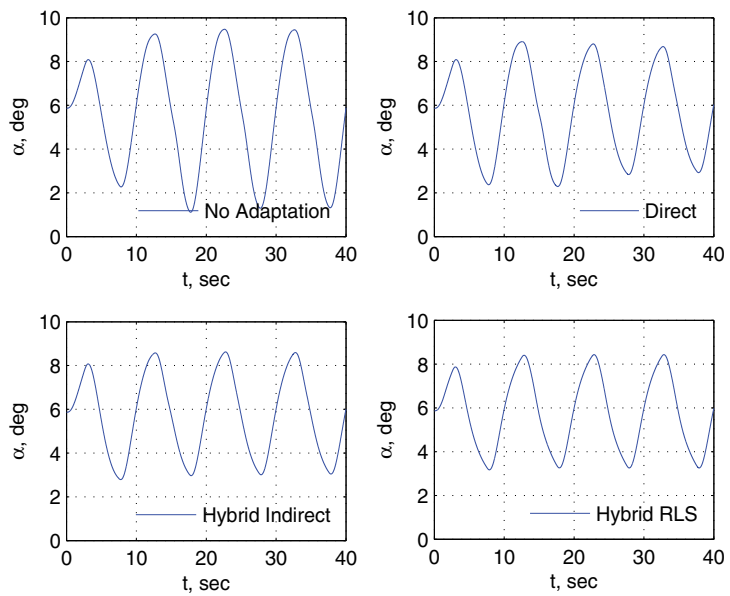

Fig. 8. Angle of Attack

significantly and cuts down the bank angle to a range between about $-30^{\circ}$ and $10^{\circ}$. With the hybrid RLS indirect adaptive control, the bank angle is essentially maintained at its trim value. The angle of attack as shown in Fig. 8 is in a reasonable range. The angle of attack when there is no adaptation goes through a large swing from $1^{\circ}$ to $9^{\circ}$, but the hybrid RLS indirect adaptive control reduces the angle of attack to a range between $3^{\circ}$ and $8^{\circ}$.

Figure 9 shows the plot of the sideslip angle. In general, flying with sideslip angle is not a recommended practice since a large sideslip angle can cause an increase in drag and more importantly a decrease in the yaw damping. With no adaptation, the largest negative sideslip angle is about $-3^{\circ}$. This is still within a reasonable limit, but the swing from $-3^{\circ}$ to $1^{\circ}$ can cause objectionable handling qualities. With the hybrid RLS indirect adaptive control, the sideslip angle is retained virtually at zero.
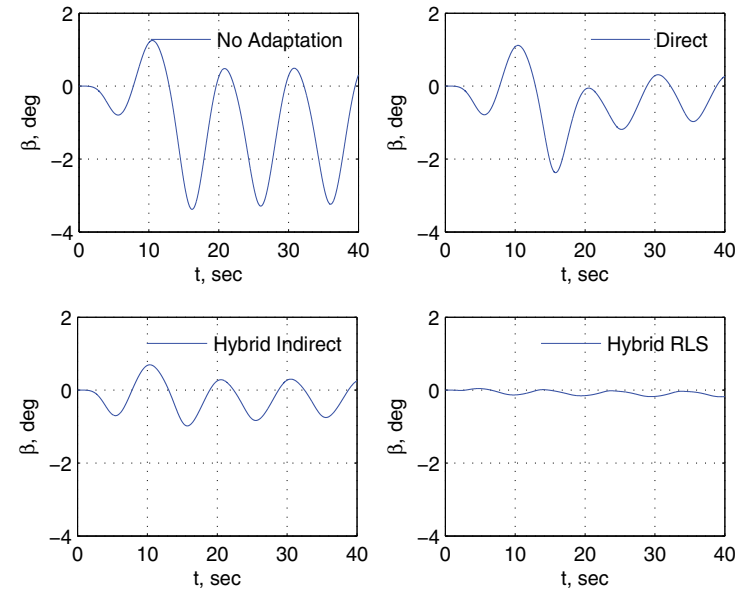

Fig. 9. Sideslip Angle 
The control surface deflections are plotted in Figs. 10 to 12. Because of the wing damage, the damaged aircraft has to be trimmed with a rather large aileron deflection. This causes the roll control authority to severely decrease. Any pitch maneuver can potentially run into a control saturation in the roll axis due to the pitch-roll coupling that exists in a wing damage scenario. With the maximum aileron deflection at $35^{\circ}$, it can be seen clearly that a roll control saturation is present in all cases, being the worst when there is no adaptation and the best with the hybrid RLS indirect adaptive control. The range of aileron deflection when there is no adaptation is quite large. As the aileron deflection hits the maximum position limit, it tends to over-compensate in the down swing because of the large pitch rate error produced by the control saturation. Both the direct adaptive control alone and the hybrid Lyapunov-based indirect adaptive control alleviate the situation somewhat but the control saturation is still present. The hybrid RLS indirect adaptive control is apparently very effective in dealing with the control saturation problem. As can be seen, it results in only a small amount of control saturation, and the aileron deflection does not vary widely. The hybrid RLS indirect adaptive control essentially enables the aileron to operate almost at its full authority, whereas with the other control methods, only partial control authority is possible.
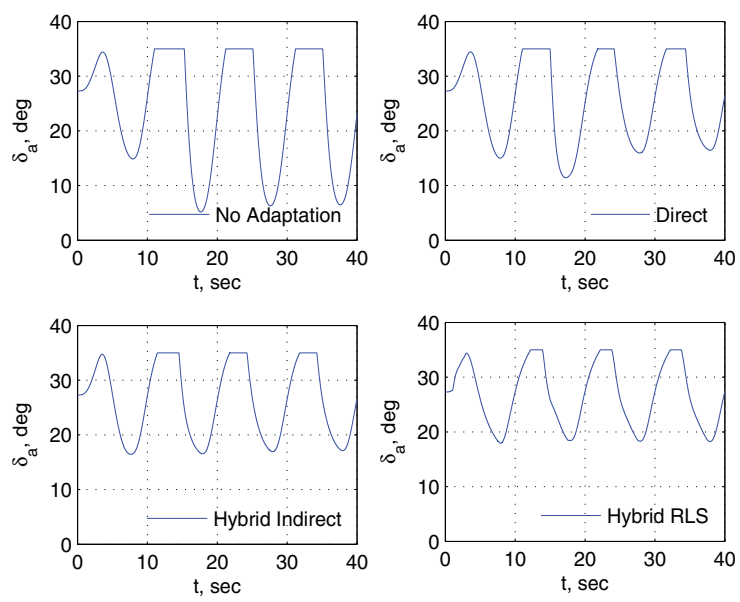

Fig. 10. Aileron Deflection

Figure 11 is the plot of the elevator deflection that shows similar elevator deflections to be within a range of few degrees for all the four different controllers. This implies that the roll control contributes mostly to the response of the damaged aircraft.

The rudder deflection is shown in Fig. 12. With no adaptation, the rudder deflection is quite active, going from $-5^{\circ}$ to $0^{\circ}$. While this appears small, it should be compared relative to the rudder position limit, which is usually reduced as the airspeed and altitude increase. The absolute rudder position limit is $\pm 10^{\circ}$ but in practice the actual rudder position limit may be less. Therefore, it is usually desired to keep the rudder deflection as small as possible. The direct adaptive control results in a maximum negative rudder deflection of $-4^{\mathrm{O}}$ and the hybrid Lyapunov-based indirect adaptive control further reduces it to $-2^{\circ}$. The hybrid RLS indirect adaptive control produces the smallest rudder deflection and keeps it to less than $\pm 0.5^{\circ}$ from the trim value. 

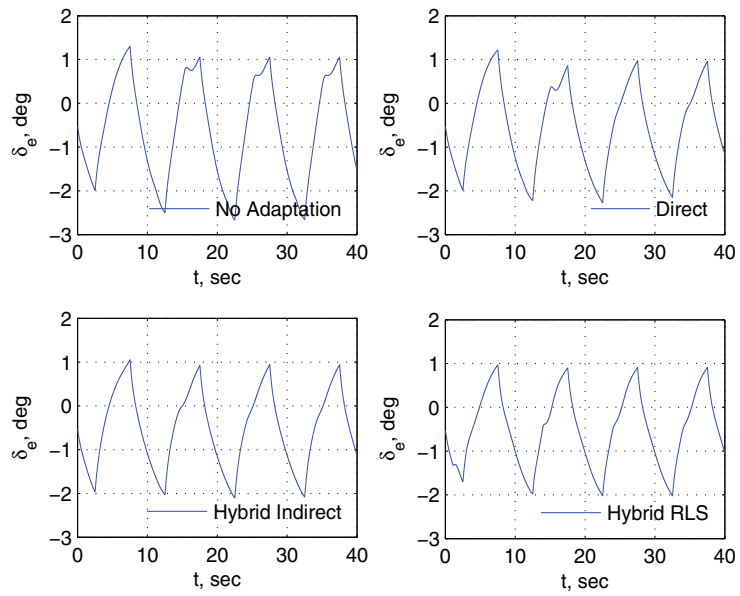

Fig. 11. Elevator Deflection
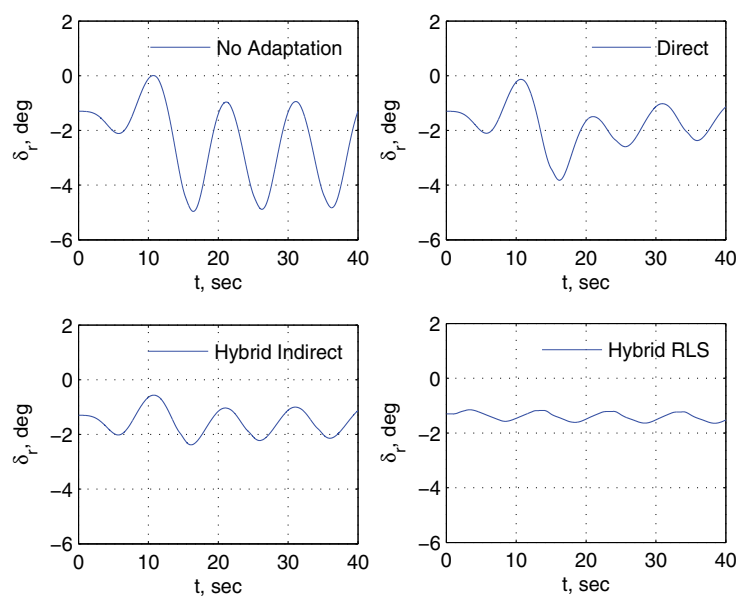

Fig. 12. Rudder Deflection

\subsection{Piloted flight simulator}

The Crew-Vehicle System Research Facility (CVSRF) at NASA Ames Research Center houses two motion-based flight simulators, the Advanced Concept Flight Simulator (ACFS) and the Boeing 747-400 Flight Simulator for use in human factor and flight simulation research. The ACFS has a highly customizable flight simulation environment that can be used to simulate a wide variety of transport-type aircraft. The ACFS employs advanced fly-by-wire digital flight control systems with modern features that can be found in today's modern aircraft. The flight deck includes head-up displays, a customizable flight management system, and modern flight instruments and electronics. Pilot inputs are provided by a side stick for controlling aircraft in pitch and roll axes.

Recently, a piloted study has been conducted in the ACFS to evaluate a number of adaptive control methods (Campbell et al., 2010). A high-fidelity flight dynamic model was developed 
to simulate a medium-range generic transport aircraft. The model includes aerodynamic models of various aerodynamic surfaces including flaps, slats, and other control surfaces. The aerodynamic database is based on Reynolds number corrected wind tunnel data obtained from wind tunnel testing of a sub-scale generic transport model. The ground model with landing gears as well as ground effect aerodynamic model are also included.

A number of failure and damage emulations were implemented including asymmetric damage to the left horizontal tail and elevator, flight control faults emulated by scaling the control sensitivity matrix (B-matrix failures), and combined failures. Eight different NASA test pilots were requested to participate in the study. For each failure emulation, each pilot was asked to provide Cooper-Harper Ratings (CHR) for a series of flight tasks, which included large amplitude attitude capture tasks and cross-wind approach and landing tasks.

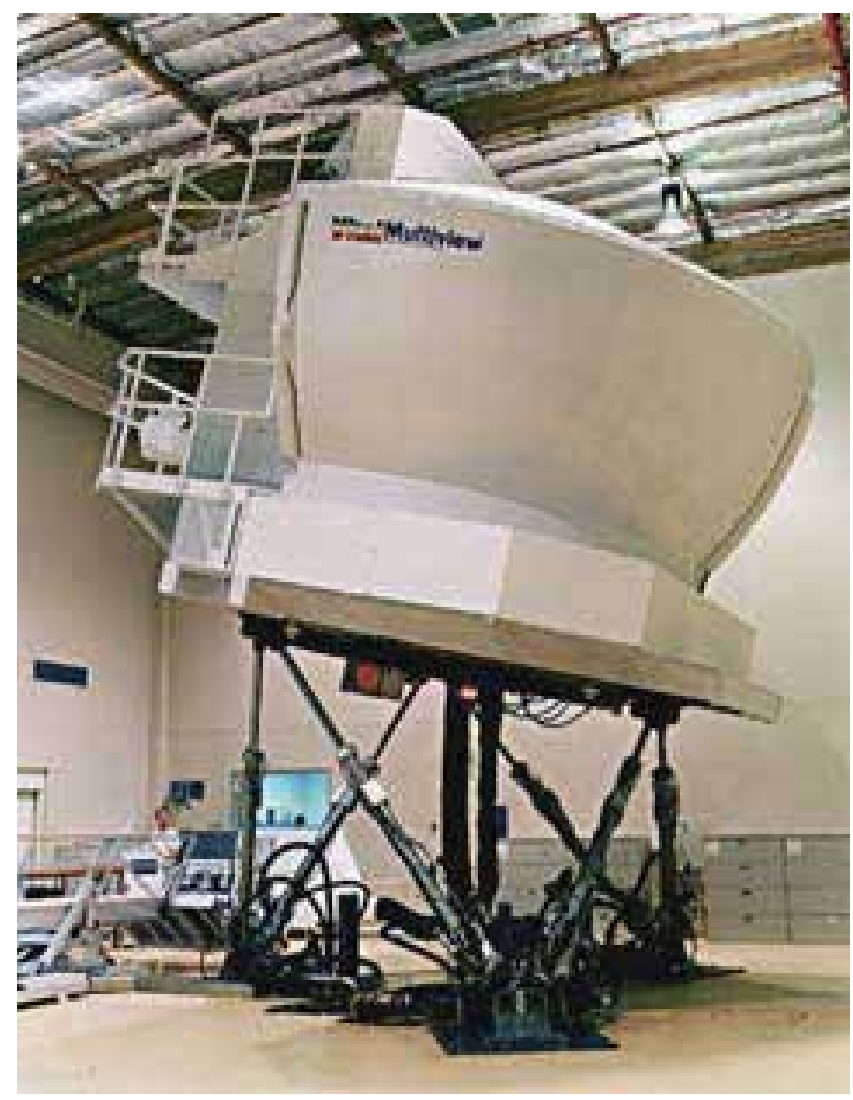

Fig. 13. Advanced Concept Flight Simulator at NASA Ames

Seven adaptive control methods were selected for the piloted study that include e-modification (Narendra \& Annaswamy, 1987), hybrid adaptive control (Nguyen et al., 2006), optimal control modification (Nguyen et al., 2008), metric-driven adaptive control using bounded linear stability method (Nguyen et al., 2007), $\mathcal{L}_{1}$ adaptive control (Cao \& Hovakimyan, 2008), adaptive loop recovery (Calise et al., 2009), and composite adaptive control (Lavretsky, 2009). This is by no means an exhaustive list of new advanced adaptive control methods that have been developed in the past few years, but this list provides an 


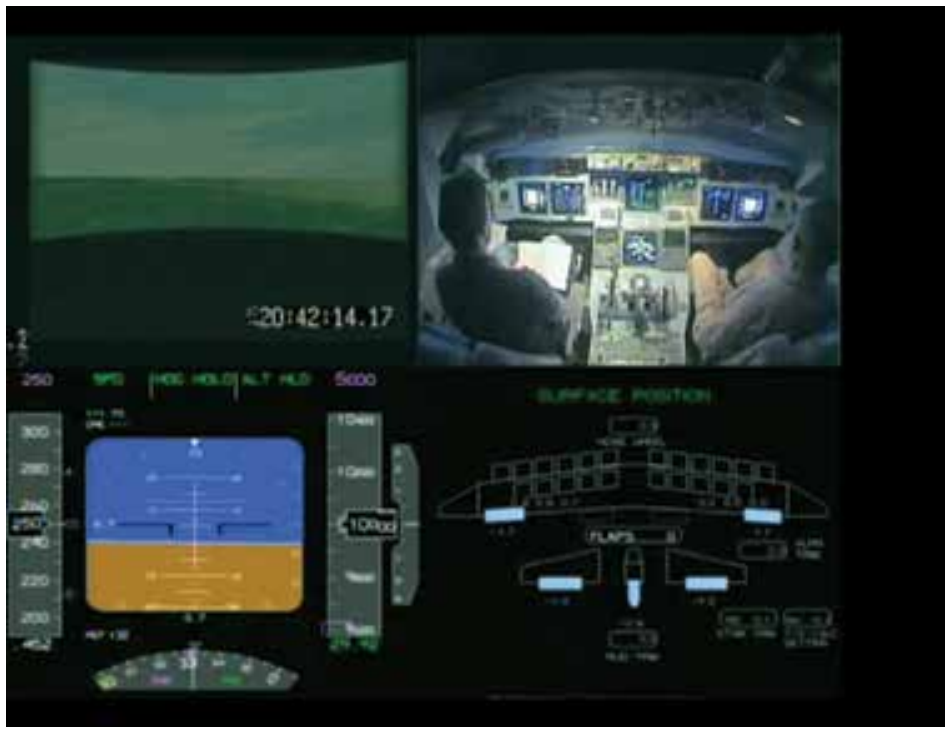

Fig. 14. Pilot Evaluation of Adaptive Flight Control

initial set of adaptive control methods that could be implemented under an existing NASA partnership with the industry and academia sponsored by the NASA Integrated Resilient Aircraft Control (IRAC) project.

The study generally confirms that adaptive control can clearly provide significant benefits to improve aircraft flight control performance in adverse flight conditions. The study also provides an insight of the role of pilot interactions with adaptive flight control systems. It was observed that many favorable pilot ratings were associated with those adaptive control methods that provide a measure of predictability, which is an important attribute of a flight control system design. Predictability can be viewed as a measure of how linear the aircraft response is to a pilot input. Being a nonlinear control method, some adaptive control methods can adversely affect linear behaviors of a flight control system more than others. Thus, while these adaptive control methods may appear to work well in a non-piloted simulation, they may present potential issues with pilot interactions in a realistic piloted flight environment. Thus, understanding pilot interaction issues is an important consideration in future research of adaptive flight control.

With respect to pilot handling qualities, among the seven adaptive flight controllers evaluated in the study, the optimal control modification, the adaptive loop recovery, and the composite adaptive control appeared to perform well over all flight conditions (Campbell et al., 2010). The hybrid adaptive control also performs reasonably well in most cases. For example, with the B-matrix failure emulation, the average $\mathrm{CHR}$ was 5 for 8 capture tasks with the baseline dynamic inversion flight controller. The average CHR number was improved to 3 with the hybrid adaptive control. In only one type of failure emulations that involved cross-coupling effects in aircraft dynamics, the performance of the hybrid adaptive flight controller fell below that for the $e$-modification which is used as the benchmark for comparison.

Future NASA research in advancing adaptive flight control will include flight testing of some of the new promising adaptive control methods. Previously, NASA conducted flight testing of the Intelligent Flight Control (IFC) on a NASA F-15 aircraft up until 2008 (Bosworth \& 


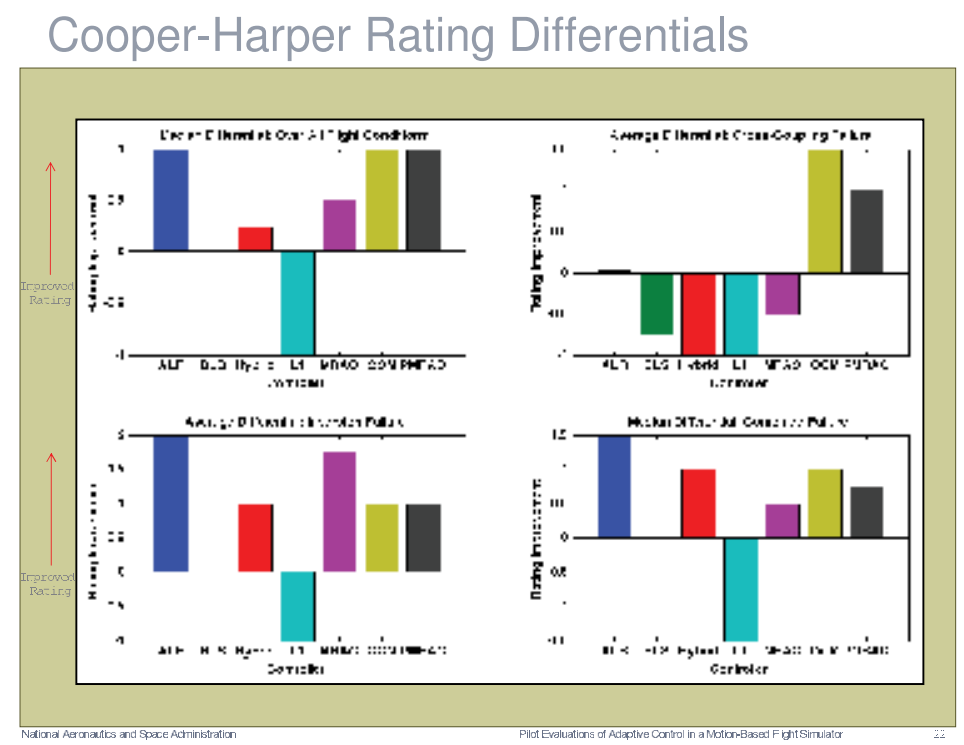

Fig. 15. Cooper-Harper Rating Improvement of Various Adaptive Control Methods

Williams-Hayes, 2007). In January of 2011, NASA has successfully completed a flight test program on a NASA F-18 aircraft to evaluate a new adaptive flight controller based on the Optimal Control Modification (Nguyen et al., 2008). Initial flight test results indicated that the adaptive controller was effective in improving aircraft's performance in simulated in-flight failures. Flight testing can reveal new observations and potential issues with adaptive control in various stages of the design implementation that could not be observed in flight simulation environments. Flight testing therefore is a critical part of validating any new technology such as adaptive control that will allow such a technology to transition into production systems in the future.

\section{Conclusions}

This study presents a hybrid adaptive flight control method that blends both direct and indirect adaptive control within a model inversion flight control architecture. Two indirect adaptive laws are presented: 1) a Lyapunov-based indirect adaptive law, and 2) a recursive least-squares indirect adaptive law. The indirect adaptive laws perform on-line parameter estimation and update the model inversion flight controller to reduce the tracking error. A direct adaptive control is incorporated within the feedback loop to correct for any residual tracking error.

A simulation study is conducted with a NASA wing-damaged transport aircraft model. The results of the simulation demonstrate that in general the hybrid adaptive control offers a potentially promising technique for flight control by allowing both direct and indirect adaptive control to operate cooperatively to enhance the performance of a flight control system. In particular, the hybrid adaptive control with the recursive least-squares indirect adaptive law is shown to be highly effective in controlling a damaged aircraft. Simulation results show that the hybrid adaptive control with the recursive least-squares indirect adaptive law is able to regulate the roll motion due to a pitch-roll coupling to maintain a nearly wing-level flight during a pitch maneuver. 
The issue of roll control saturation is encountered due to a significant reduction in the roll control authority as a result of the wing damage. The direct adaptive control and the hybrid adaptive control with the Lyapunov-based indirect adaptive law restore a partial roll control authority from the control saturation. On the other hand, the hybrid adaptive control with the recursive least-squares indirect adaptive law restores the roll control authority almost fully. Thus, the hybrid adaptive control with the recursive least-squares indirect adaptive law can demonstrate its effectiveness in dealing with a control saturation.

A recent piloted study of various adaptive control methods in the Advanced Concept Flight Simulator at NASA Ames Research Center confirmed the effectiveness of adaptive control in improving flight safety. The hybrid adaptive control was among the methods evaluated in the study. In general, it has been shown to provide an improved flight control performance under various types of failure emulations conducted in the piloted study.

In summary, the hybrid adaptive flight control is a potentially effective adaptive control strategy that could improve the performance of a flight control system when an aircraft operating in adverse events such as with damage and or failures.

\section{References}

Annaswamy, A.; Jang, J. \& Lavretsky, E. (2008). Stability Margins for Adaptive Controllers in the Presence of Time-Delay, AIAA Guidance, Navigation, and Control Conference, Honolulu, Hawaii, August 2008, AIAA 2008-6659.

Bobal, V.; BÃúhm, J.; Fessl, J. \& MachÃącek, J. (2005). Digital Self-Tuning Controllers: Algorithms, Implementation, and Applications, Springer-Verlag, ISBN 1852339802, London,

Bosworth, J. \& Williams-Hayes, P. (2007). Flight Test Results from the NF-15B IFCS Project with Adaptation to a Simulated Stabilator Failure, AIAA Infotech@Aerospace Conference, Rohnert Park, California, May 2007, AIAA-2007-2818.

Calise, A.; Yucelen, T.; Muse, J. \& Yang, B. (2009). A Loop Recovery Method for Adaptive Control, AIAA Guidance, Navigation, and Control Conference, Chicago, Illinois, August 2009, AIAA-2009-5967.

Campbell, S.; Kaneshige, J.; Nguyen, N. \& Krishnakumar, K. (2010). An Adaptive Control Simulation Study using Metrics and Pilot Handling Qualities Evaluations, AIAA Guidance, Navigation, and Control Conference, Toronto, Canada, August 2010, AIAA-2010-8013.

Cao, C. \& Hovakimyan, N. (2008). Design and Analysis of a Novel $\mathcal{L}_{1}$ Adaptive Control Architecture with Guaranteed Transient Performance. IEEE Transactions on Automatic Control, Vol. 53, No. 2, March 2008, pp. 586-591.

Cybenko, G. (1989). Approximation by Superpositions of a Sigmoidal Function. Mathematics of Control Signals Systems, Vol. 2, No. 4, 1989, pp. 303-314.

Eberhart, R. L. \& Ward, D. G. (1999). Indirect Adaptive Flight Control System Interactions. International Journal of Robust and Nonlinear Control, Vol. 9, No. 14, December 1999, pp. 1013-1031.

Hovakimyan, N.; Kim, N.; Calise, A. J.; Prasad, J.V. R. \& Corban, E. J. (2001). Adaptive Output Feedback for High-Bandwidth Control of an Unmanned Helicopter, AIAA Guidance, Navigation and Control Conference, Montreal, Canada, August 2001, AIAA-2001-4181.

Ioannu, P.A. \& Sun, J. (1996). Robust Adaptive Control, Prentice-Hall, ISBN 0134391004. 
Jacklin, S. A.; Schumann, J. M.; Gupta, P. P.; Richard, R.; Guenther, K. \& Soares, F. (2005). Development of Advanced Verification and Validation Procedures and Tools for the Certification of Learning Systems in Aerospace Applications, AIAA Infotech@Aerospace Conference, Arlington, VA, September 2005, AIAA-2005-6912.

Johnson, E. N.; Calise, A. J.; El-Shirbiny, H. A. \& Rysdyk, R. T. (2000). Feedback Linearization with Neural Network Augmentation Applied to X-33 Attitude Control, AIAA Guidance, Navigation, and Control Conference, Denver, Colorado, August 2000, AIAA-2000-4157.

Jordan, T. L.: Langford, W. M.; Belcastro, Christine M.; Foster, J. M.; Shah, G. H.; Howland, G. \& Kidd, R. (2004). Development of a Dynamically Scaled Generic Transport Model Testbed for Flight Research Experiments, AUVSI Unmanned Unlimited, Arlington, VA, 2004

Kim, B. S. \& Calise, A. J. (1997). Nonlinear Flight Control Using Neural Networks. AIAA Journal of Guidance, Control, and Dynamics, Vol. 20, No. 1, 1997, pp. 26-33.

Lavretsky, E. (2009). Combined / Composite Model Reference Adaptive Control, AIAA Guidance, Navigation, and Control Conference, Chicago, Illinois, August 2009, AIAA-2009-6065

Narendra, K. S. \& Annaswamy, A. M. (1987). A New Adaptive Law for Robust Adaptation Without Persistent Excitation. IEEE Transactions on Automatic Control, Vol. 32, No. 2, February 1987, pp. 134-145.

Nguyen, N.; Krishnakumar, K.; Kaneshige, J. \& Nespeca, P. (2006). Dynamics and Adaptive Control for Stability Recovery of Damaged Asymmetric Aircraft, AIAA Guidance, Navigation, and Control Conference,Keystone, Colorado, August 2006, AIAA-2006-6049.

Nguyen, N.; Bakhtiari-Nejad, M. \& Huang, Y. (2007). Hybrid Adaptive Flight Control with Bounded Linear Stability Analysis, AIAA Guidance, Navigation, and Control Conference, Hilton Head, South Carolina, August 2007, AIAA 2007-6422.

Nguyen, N.; Krishnakumar, K. \& Boskovic, J. (2008). An Optimal Control Modification to Model-Reference Adaptive Control for Fast Adaptation, AIAA Guidance, Navigation, and Control Conference, Honolulu, Hawaii, August 2008, AIAA 2008-7283.

Nguyen, N. \& Jacklin, S. (2010). Neural Net Adaptive Flight Control Stability, Verification and Validation Challenges, and Future Research, In: Applications of Neural Networks in High Assurance Systems, Schumann, J. \& Liu, Y., (Ed.), pp. 77-107, Springer-Verlag, ISBN 978-3-642-10689-7, Berlin.

Rohrs, C.E.; Valavani, L.; Athans, M. \& Stein, G. (1985). Robustness of Continuous-Time Adaptive Control Algorithms in the Presence of Unmodeled Dynamics. IEEE Transactions on Automatic Control, Vol. 30, No. 9, September 1985, pp. 881-889.

Rysdyk, R. T. \& Calise, A. J. (1998). Fault Tolerant Flight Control via Adaptive Neural Network Augmentation, AIAA Guidance, Navigation, and Control Conference, Boston, Massachusetts, August 1998, AIAA-1998-4483.

Sharma, M.; Lavretsky, E. \& and Wise, K. (2006). Application and Flight Testing of an Adaptive Autopilot On Precision Guided Munitions, AIAA Guidance, Navigation, and Control Conference, Keystone, Colorado, August 2006, AIAA-2006-6568.

Steinberg, M. L. (1999). A Comparison of Intelligent, Adaptive, and Nonlinear Flight Control Laws, AIAA Guidance, Navigation, and Control Conference, Portland, Oregon, August 1999, AIAA-1999-4044. 
Stepanyan, V.; Krishnakumar, K.; Nguyen, N. \& Van Eykeren, L. (2009). Stability and Performance Metrics for Adaptive Flight Control, AIAA Guidance, Navigation, and Control Conference, Chicago, Illinois, August 2009, AIAA-2009-5965.

Yang, B.-J.; Yucelen, T.; Calise, A. J. \& Shin, J.-Y. (2009). LMI-based Analysis of Adaptive Controller, American Control Conference, June 2009. 


\title{
Application of Evolutionary Computing in Control Allocation
}

\author{
Hammad Ahmad, Trevor Young, Daniel Toal and Edin Omerdic \\ University of Limerick, \\ Republic of Ireland
}

\section{Introduction}

This issue of interaction of control allocation and actuator dynamics and has been dealt with by very few researchers. What was not considered in most control allocation algorithms is the fact that the control surfaces are manipulated by either hydraulics or electric actuators, and constitute a dynamic system which cannot produce infinite accelerations. In other words, if a control was initially at rest, and later commanded to move at its maximum rate in some direction for a specified amount of time, it would gradually build up speed until it reached the commanded rate. The final position of the control would therefore not be the same as that calculated using the commanded rate and the time during which it was instructed to move (Bolling 1997). In this chapter, a method, which post-processes the output of a control allocation algorithm, is developed to compensate for actuator dynamics. The method developed is solved for a diagonal matrix of gain corresponding to individual actuators. This matrix is then multiplied with the commanded change in control effector settings as computed by the control allocator and actuators dynamics interactions. The basic premise of this method is to post process the output of the control allocation algorithm to overdrive the actuators so that at the end of a sampling interval the actual actuator positions are equivalent to the desired actuator positions (Oppenheimer and Doman 2004). The overdriving of the actuators is done by multiplying the change in commanded signal with the identified gain matrix which is called the compensator. This identification is done by using a soft computing technique (i.e. genetic algorithms). The simulation setup including control allocator block, compensator and actuator rig makes a non-linear set up. During the identification of the compensator using this setup by soft computing technique such as genetic algorithms, the likelihood of the solution being a global minimum is high as compared to other optimisation techniques. This is why genetic algorithms have been used in this analysis rather than other techniques such as linear programming. The main contribution is to design a compensator using an evolutionary computing technique (i.e. genetic algorithms) to compensate the interaction between control allocation and actuator dynamics. It should be mentioned that in this method the model of the actuator does not need to be known. The simulation setup consists of excitation signals, the control allocation block, the compensator and the actuators rig.

When designing control allocation typically the actuator dynamics are ignored because the bandwidth of the actuators is larger than the frequencies of the rigid body modes of the aircraft. Fig. 1 shows a control allocator with actuator dynamics neglected. If there is a case 
in which actuator frequencies are comparable with the bandwidth of the rigid body modes then the actuator dynamics cannot be neglected, as shown in Fig. 2.

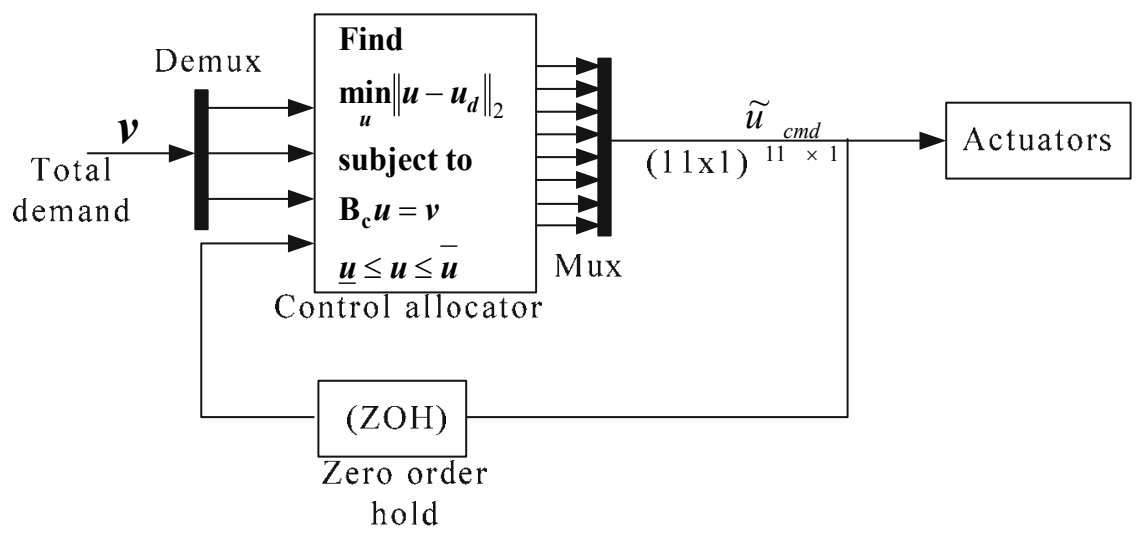

Fig. 1. Control allocation with actuator dynamics neglected

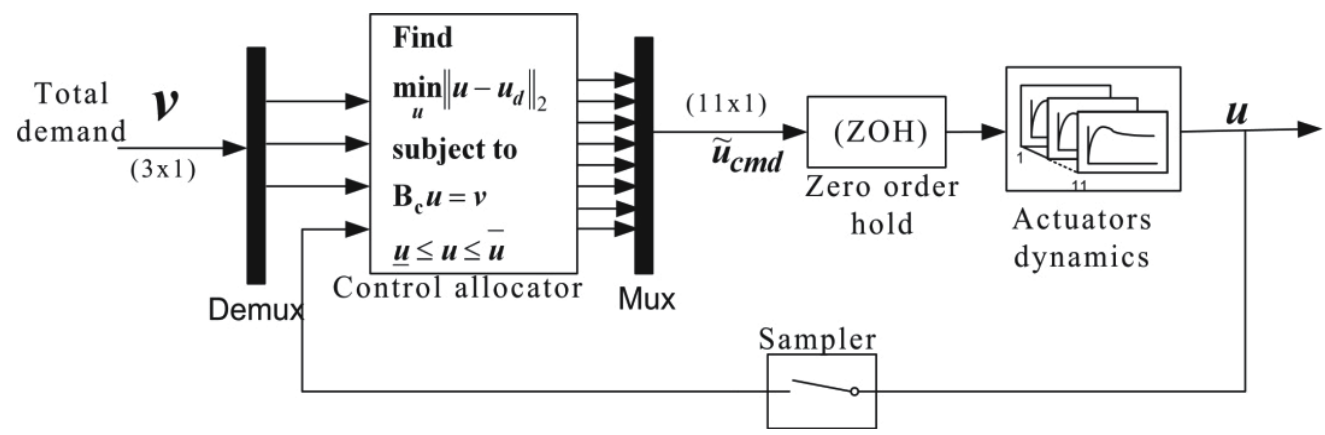

Fig. 2. Control allocation with actuator dynamics

In this case the output of the control allocator should match the output of the actuator dynamics. In reality the output of the control allocation is attenuated due to the presence of non-negligible actuator dynamics. The loss of the gain from the CA output signal is compensated by the scheme shown in Fig. 3. In the second order dynamics of the actuator the rate could be estimated using a Kalman filter if the rate sensing is not available. The Kalman filter is an efficient recursive filter that estimates the state of a dynamic system from a series of noisy measurements. The matrix of gains as shown in Fig. 3 is tuned offline using GA. The structure of the compensator is taken from (Oppenheimer and Doman 2004).

\subsection{Control allocation for aircraft: graphical illustration}

Control allocation is merely a mapping (i.e. linear or non-linear) from total virtual demands in terms of body angular accelerations to the control position setting subject to rate and position constraints. An illustration of control allocation is given in Fig. 4.

Section 2 describes the interaction of first order actuator dynamics and control allocation and the structure of the compensator is established in this section for first order actuator dynamics. Similarly, in section 2.2 the structure of the compensator is established for second 


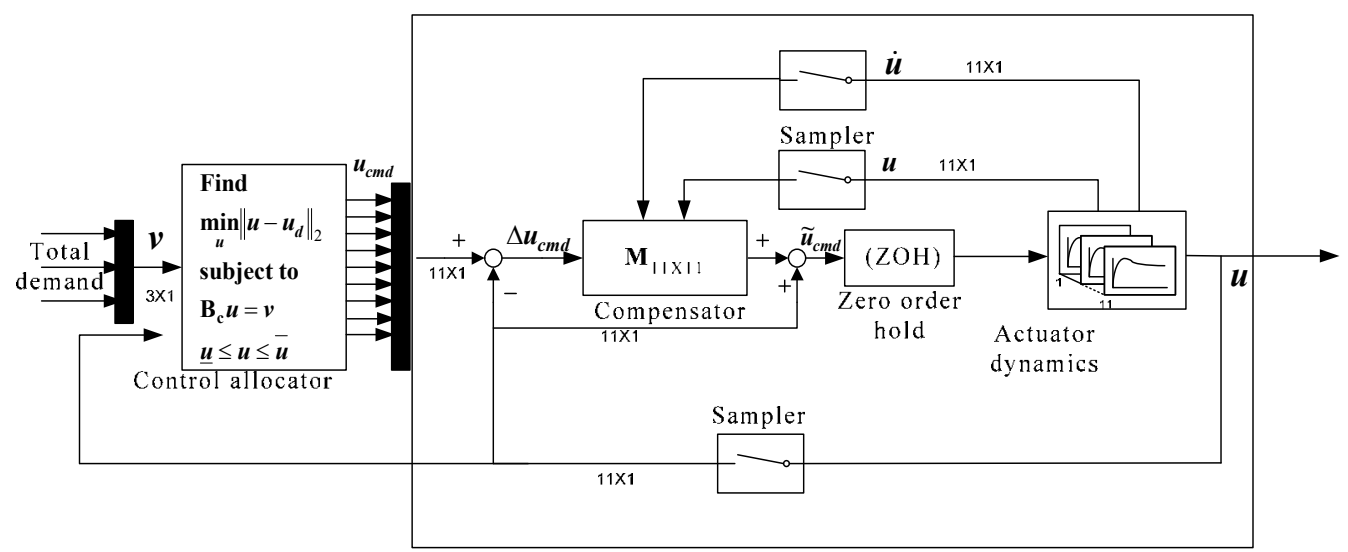

Fig. 3. Structure of compensator with actuator dynamics with diagonal gain matrix $\mathrm{M}$ of dimension (11X11)

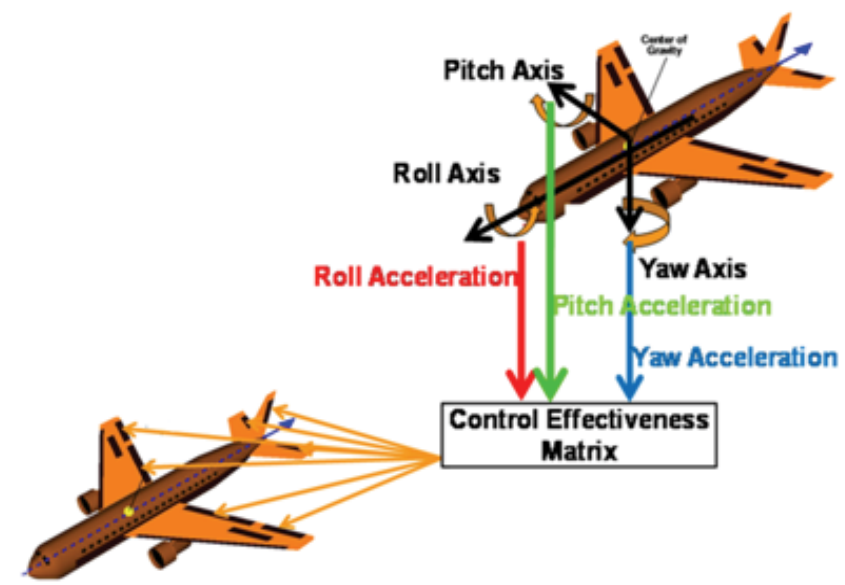

Fig. 4. Control allocation for aircraft: graphical illustration

order actuator dynamics. In section 3 tuning of the compensator parameters using genetic algorithm is described. In section 4 simulation and results for a tuned compensator are shown for a range of first and second order actuator dynamics. Finally, in section 5 some conclusions are established.

\section{First-order actuator dynamics interaction}

In this section, the effects of first-order actuator on the system are shown in Fig. 2 (Oppenheimer and Doman 2004). Let the dynamics of a single actuator be represented by a continuous time first order transfer function of the form

$$
\frac{u(s)}{\tilde{u}_{c m d}(s)}=\frac{a}{s+a}
$$

The discrete time solution to the first-order actuator dynamic equation for one sample period is given by 


$$
u(k T+T)=\mathrm{e}^{-a \mathrm{~T}} u(k T)+\int_{k T}^{k T+T} e^{-a(k T+T-\tau)} \tilde{u}_{c m d}(\tau) d \tau
$$

where $T$ is the sampling time. This result does not depend on the type of hold because $u$ is specified in terms of its continuous time history, $\tilde{u}_{c m d}(t)$ over a sample interval (Franklin $e t$ al. 1998). The most common hold element is zero-order hold ( $\mathrm{ZOH})$ with no delay, i.e.

Performing substitution

$$
\tilde{u}_{c m d}(\tau)=\tilde{u}_{c m d}(k T), \quad k T \leq \tau \leq k T+T
$$

in Eq. (2) yields

$$
\gamma=k T+T-\tau
$$

$$
u(k T+T)=\mathrm{e}^{-a \mathrm{~T}} u(k T)+\int_{0}^{T} e^{-a \gamma} \tilde{u}_{c m d}(k T) d \gamma
$$

Defining

$$
\begin{gathered}
\Phi=\mathrm{e}^{-a \mathrm{~T}} \\
\Gamma=\int_{0}^{T} e^{-a \gamma} d \gamma
\end{gathered}
$$

Eq. (5) can be written as a difference equation of standard form

$$
u(k+1)=\Phi u(k)+\Gamma \tilde{u}_{\mathrm{cmd}}(\mathrm{k})
$$

The signal $\tilde{u}_{c m d}(k)$, is held constant over each sampling period. The command to actuator is given by

$$
\tilde{u}_{c m d}(k)=\Delta u_{c m d}(k)+u(k)
$$

The command increment change in actuator position over one sample as shown in Fig. 5 is defined by

$$
\Delta u_{c m d}(k) \triangleq u_{c m d}(k)-u(k)
$$

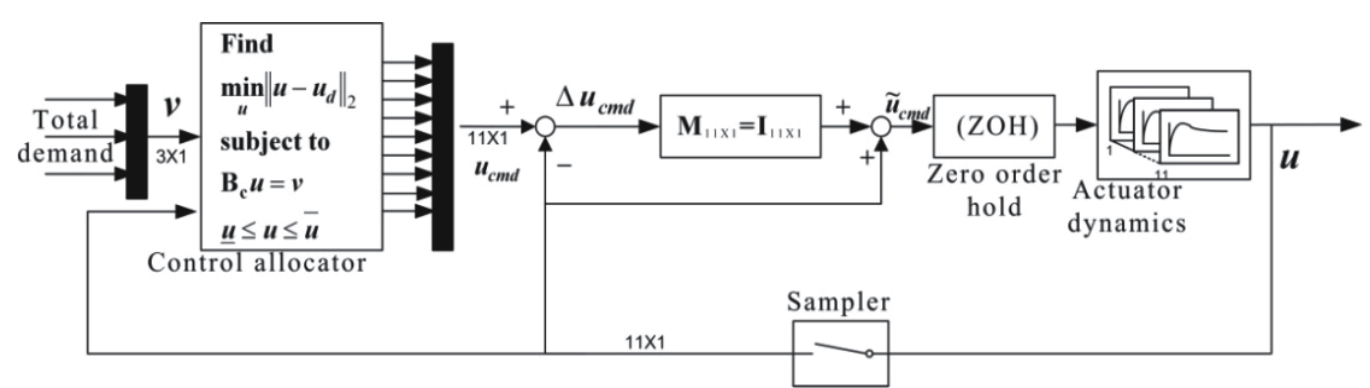

Fig. 5. Command increment change in actuator position with gain matrix $\mathrm{M}$ equal to Identity matrix I of dimension (11X11)

where $u_{c m d}$ is the actuator command coming from the control allocator. Since the effector commands are held constant for one sample period then $\Delta u_{c m d}(k)$ appear to be a step command from the measured position $u(k)$. Substituting Eq. (8) in Eq. (7) gives 


$$
u(k+1)=\Phi u(k)+\Gamma\left(\Delta u_{c m d}(k)+u(k)\right)
$$

If $\Gamma<1$, the incremental commanded signal from the control allocation algorithm, $\Delta u_{c m d}(k)$ is attenuated by the actuator dynamics, thus $u(k+1) \neq u_{c m d}$. The objective is to find the gain $\mathrm{M}$ that changes the output of the control allocation algorithm such that $u(k+1)=$ $u_{c m d}=\Delta u_{c m d}(k)+u(k)$ (Oppenheimer and Doman 2004). Hence

$$
u(k+1)=\Phi u(k)+\Gamma\left(\mathrm{M} \Delta u_{c m d}(k)+u(k)\right)
$$

The gain $M$ is tuned by using the genetic algorithm in section 3.2. If there is a bank of first order actuator dynamics, then the gain $\mathbf{M}$ is chosen to be a diagonal matrix $\mathbf{M}$ of dimensions (11x11), as shown in Fig. 3 and Fig. 5.

\subsection{Example showing effect of first-order actuator dynamics}

Let us consider an example with

$$
\mathbf{B}_{\mathbf{c}}=10^{-3}\left[\begin{array}{cccccccccrc}
-4.2 & 4.2 & -5.0 & 5.0 & 0 & 0 & 0 & 0 & 0 & 2.6 & 0.7 \\
-0.9 & -0.9 & -2.9 & -2.9 & -9.4 & -9.4 & -6.9 & -6.9 & -80.5 & -7.7 & 5.8 \\
-0.2 & 0.2 & -0.1 & 0.1 & 0 & 0 & 0 & 0 & 0 & 0 & 0
\end{array}\right]
$$

Position in (deg) and rate in (deg/s) constraints are defined as follows:

$$
\begin{gathered}
u_{\min }=\left[\begin{array}{llllllllllll}
-20 & -20 & -12 & -12 & -23 & -23 & -23 & -23 & -12 & -25 & -25
\end{array}\right]^{T} \\
u_{\max }=\left[\begin{array}{llllllllllll}
20 & 20 & 15 & 15 & 17 & 17 & 17 & 17 & 3 & 25 & 25
\end{array}\right]^{T} \\
\dot{\rho}_{\min }=\left[\begin{array}{llllllllllll}
-45 & -45 & -45 & -45 & -37 & -37 & -37 & -37 & -0.5 & -50 & -50
\end{array}\right]^{T} \\
\dot{\rho}_{\max }=\left[\begin{array}{lllllllllll}
45 & 45 & 45 & 45 & 37 & 37 & 37 & 37 & 0.5 & 50 & 50
\end{array}\right]^{T}
\end{gathered}
$$

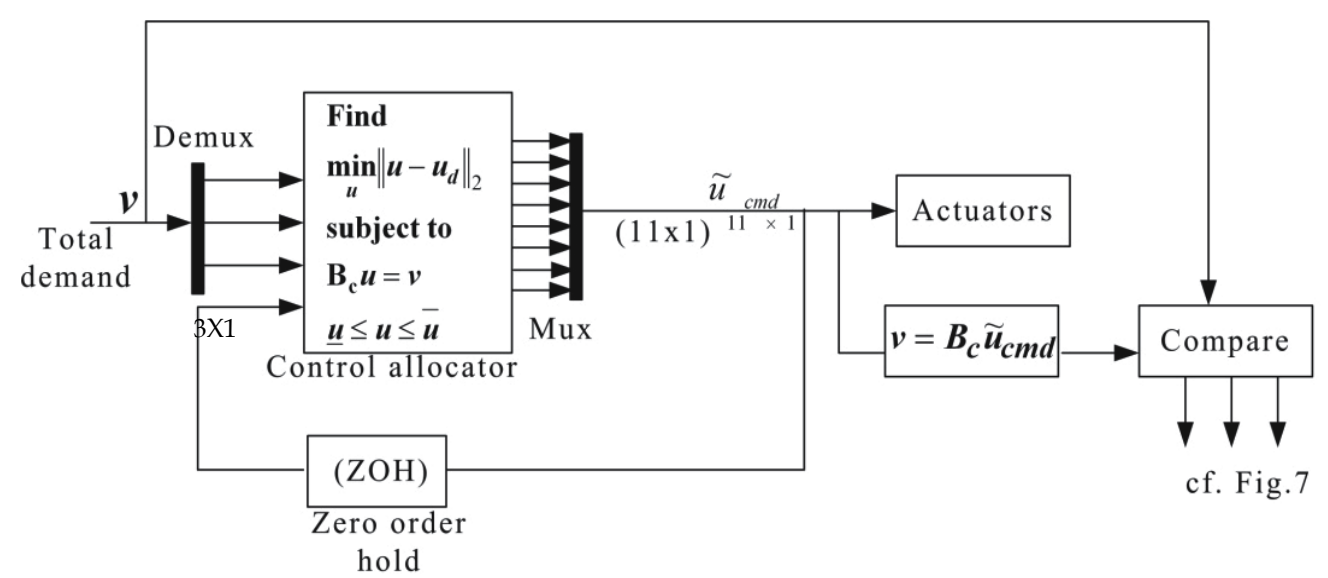

Fig. 6. Block diagram with desired demand produced by the control allocator and compared with the actual demand when there is no actuator dynamics included 1

First the time response of control allocation without actuator dynamics is shown in Fig. 6 and Fig 7. It can be seen that if the actuators are fast enough to cater for the rigid body

${ }^{1}$ cf. (from Latin confer) means compare 
modes, there is no need to consider the actuator dynamics and hence one to one mapping between the control allocator and control surfaces is sufficient. This would not be the case with the non aerodynamic actuators, so actuator dynamics cannot be ignored. It can be seen from the results shown in Fig. 8 and Fig. 9 that how the actuator dynamics affects the outcome of the control allocator. It can also be seen that how the control allocator command is attenuated. The first-order actuator dynamics used for this example are given as

$$
\frac{u(s)}{\tilde{u}_{c m d}(s)}=\frac{0.6128}{s+0.6128}
$$
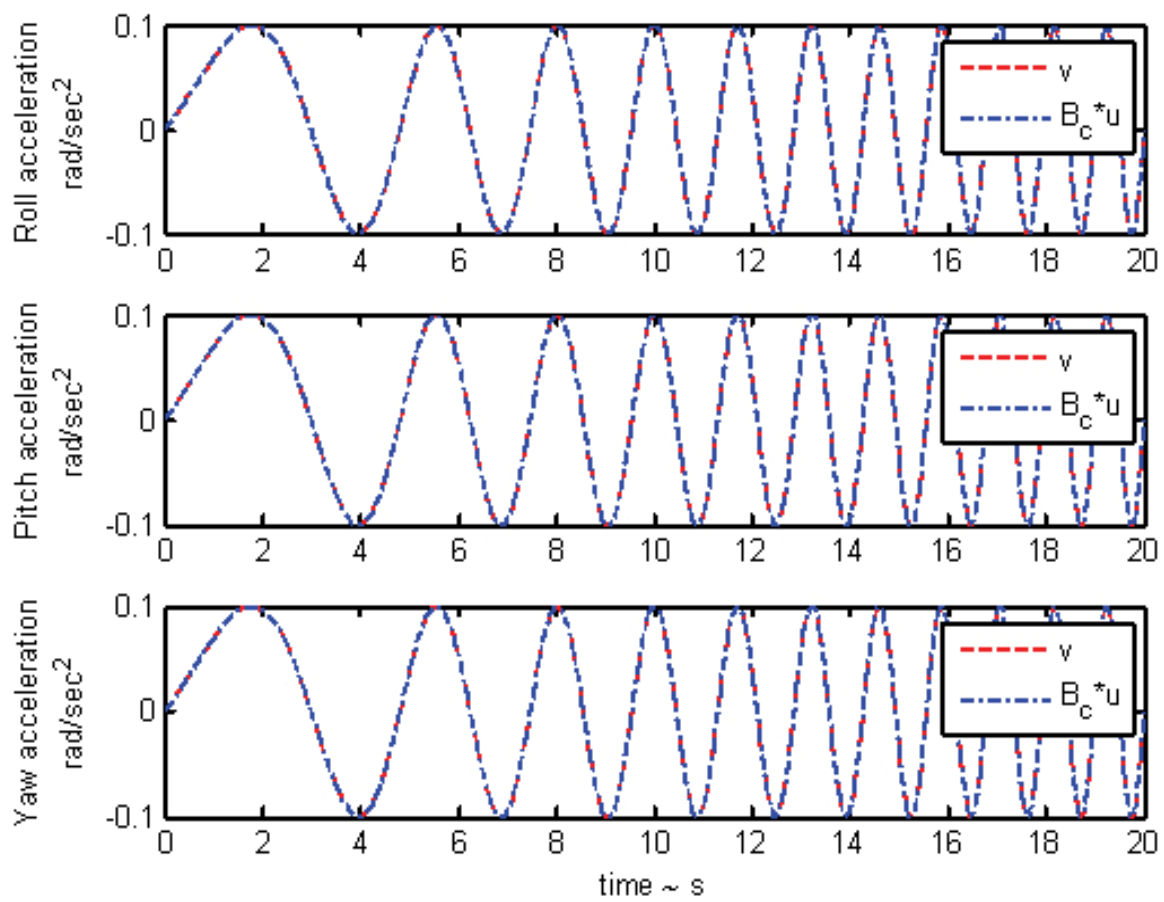

Fig. 7. Comparison of results with no dynamics of actuator involved

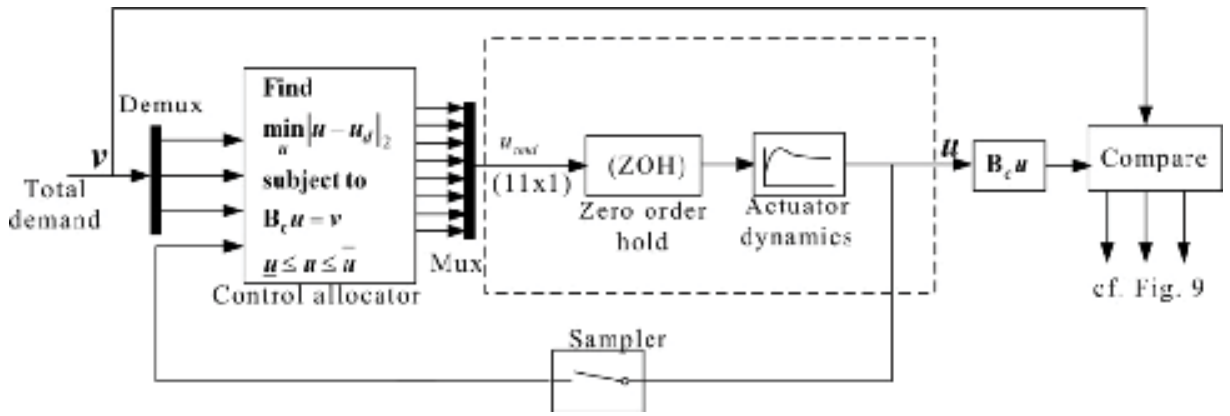

Fig. 8. Block diagram with desired demand produced by the control allocator and compared with the actual demand when there is actuator dynamics included 

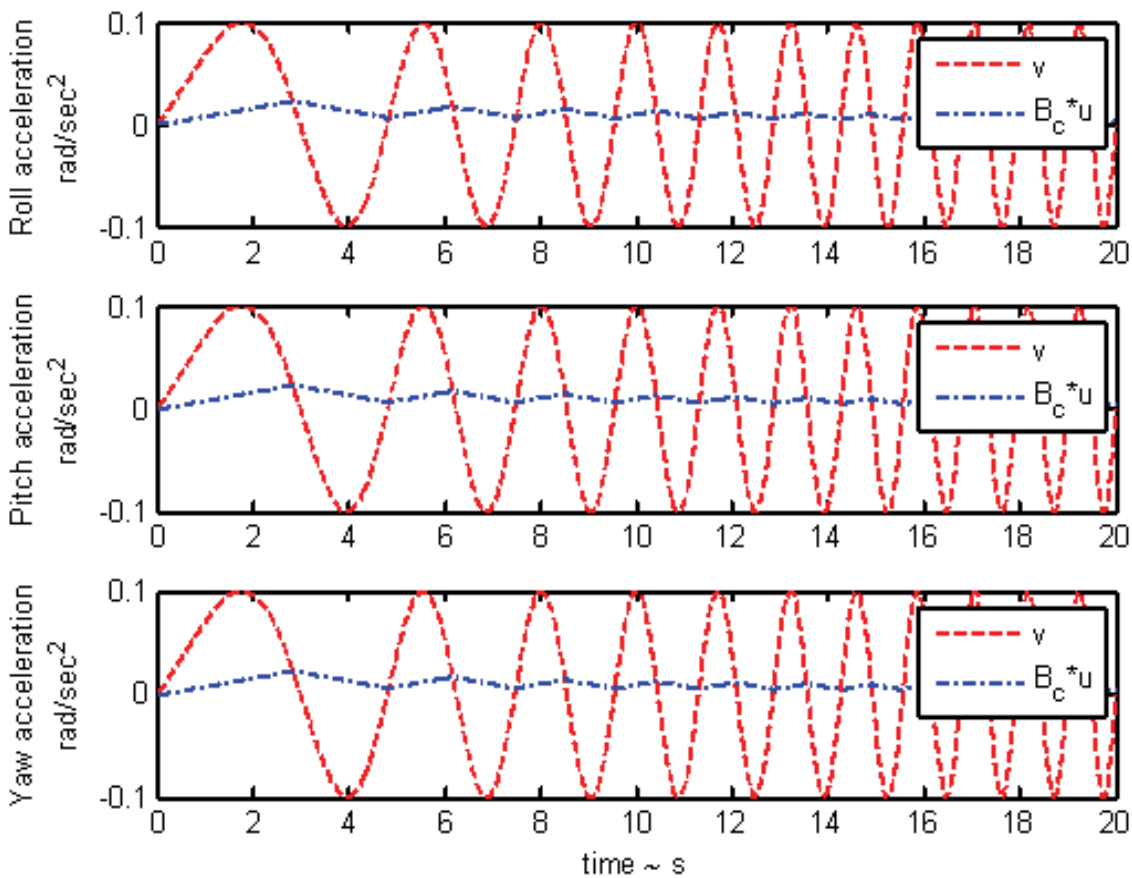

Fig. 9. Time responses of desired and actual responses of virtual demand with actuator dynamics included

In the following section the second order actuator dynamics are parameterised for the design of the compensator.

\subsection{Second-order model dynamics interaction}

In this section, the effects of second - order actuator on the system is shown in Fig. 2 (Oppenheimer and Doman 2004). Let the dynamics of a second actuator be represented by a continuous time second order function of the form

$$
\frac{u(s)}{\tilde{u}_{c m d}(s)}=\frac{k}{s^{2}+2 \zeta \omega_{n} s+\omega_{n}^{2}}
$$

The state space representation of this transfer function is given

$$
\begin{gathered}
{\left[\begin{array}{l}
\dot{u}(t) \\
\ddot{u}(t)
\end{array}\right]=\left[\begin{array}{cc}
0 & 1 \\
-\omega_{n}^{2} & -2 \zeta \omega_{n}
\end{array}\right]\left[\begin{array}{l}
u(t) \\
\dot{u}(t)
\end{array}\right]+\left[\begin{array}{l}
0 \\
k
\end{array}\right] \tilde{u}_{c m d}(t)} \\
{\left[\begin{array}{l}
\dot{u}(t) \\
\ddot{u}(t)
\end{array}\right]=\mathbf{A}\left[\begin{array}{l}
u(t) \\
\dot{u}(t)
\end{array}\right]+\mathbf{B} \tilde{u}_{c m d}(t)} \\
{\left[\begin{array}{l}
\dot{u}(t) \\
\ddot{u}(t)
\end{array}\right]=\left[\begin{array}{ll}
1 & 0 \\
0 & 1
\end{array}\right]\left[\begin{array}{l}
u(t) \\
\dot{u}(t)
\end{array}\right]=\mathbf{C}\left[\begin{array}{l}
u(t) \\
\dot{u}(t)
\end{array}\right]}
\end{gathered}
$$

The discrete time solution to the second-order actuator dynamic Eq. (16) to Eq. (17) for one sample period is given by 


$$
\left[\begin{array}{l}
u(k T+T) \\
\dot{u}(k T+T)
\end{array}\right]=\mathrm{e}^{\mathbf{A T}}\left[\begin{array}{l}
u(k T) \\
\dot{u}(k T)
\end{array}\right]+\int_{k T}^{k T+T} e^{\mathbf{A}(k T+T-\tau)} \mathbf{B} \tilde{u}_{c m d}(\tau) d \tau
$$

where $T$ is the sampling time. This result does not depend on the type of hold because $\tilde{u}_{c m d}$ is specified in terms of its continuous time history, $\tilde{u}_{c m d}(t)$ over a sample interval (Franklin et al. 1998). A zero-order hold ( $\mathrm{ZOH})$ with no delay is given by

$$
\tilde{u}_{c m d}(\tau)=\tilde{u}_{c m d}(k T), k T \leq \tau \leq k T+T
$$

Performing substitution

In Eq. (18) yields

$$
\gamma=k T+T-\tau
$$

$$
\left[\begin{array}{l}
u(k T+T) \\
\dot{u}(k T+T)
\end{array}\right]=\mathrm{e}^{\mathbf{A T}}\left[\begin{array}{c}
u(k T) \\
\dot{u}(k T)
\end{array}\right]+\int_{0}^{T} e^{\mathbf{A} \gamma} d \gamma \mathbf{B} \tilde{u}_{c m d}(k T)
$$

Defining,

$$
\begin{gathered}
\boldsymbol{\Phi}=\mathrm{e}^{\mathbf{A} \mathrm{T}}=\left[\begin{array}{ll}
\Phi_{1,1} & \Phi_{1,2} \\
\Phi_{2,1} & \Phi_{2,2}
\end{array}\right] \\
\boldsymbol{\Gamma}=\int_{0}^{T} e^{\mathbf{A} \gamma} d \gamma \mathbf{B} \\
{\left[\begin{array}{l}
u(k T+T) \\
\dot{u}(k T+T)
\end{array}\right]=\boldsymbol{\Phi}\left[\begin{array}{l}
u(k T) \\
\dot{u}(k T)
\end{array}\right]+\Gamma \tilde{u}_{c m d}(k T)}
\end{gathered}
$$

The first state variable $u(k T+T)$ equation can be written as

$$
u(k+1)=\left[\begin{array}{ll}
\Phi_{1,1} & \Phi_{1,2}
\end{array}\right]\left[\begin{array}{l}
u(k T) \\
\dot{u}(k T)
\end{array}\right]+\tilde{u}_{c m d}(k T) \int_{0}^{T} k \Phi_{1,2}(\gamma) d \gamma
$$

Parameterizing Eq. (24) will give

$$
u(k+1)=C_{1} u(k)+C_{2} \dot{u}(k)+C_{3} \tilde{u}_{c m d}(k)
$$

where $C_{1}=\Phi_{1,1}, C_{2}=\Phi_{1,2}$ and $C_{3}=\int_{0}^{T} k \Phi_{1,2}(\gamma) d \gamma$.

The objective is to find $\mathrm{M}$ to modify the $\Delta u_{c m d}(k)$, as shown in Fig. 3 such that $u(k+1)=$ $u_{c m d}(k)$.

$$
\Delta u_{c m d}(k)+u(k)=C_{1} u(k)+C_{2} \dot{u}(k)+C_{3}\left(\mathrm{M} \Delta u_{c m d}(k)+u(k)\right)
$$

Solving for M gives (Oppenheimer and Doman 2004)

$$
\mathrm{M}=\frac{\Delta u_{c m d}(k)+\left(1-C_{3}-C_{1}\right) u(k)-C_{2} \dot{u}(k)}{C_{3} \Delta u_{c m d}(k)}
$$

These parameters $C_{1}, C_{2}$ and $C_{3}$ are tuned using genetic algorithm optimisation. Here it is assumed that the positions and rate of change of actuators are available. If there is a bank of second order actuator dynamics then $\mathbf{M}$ is chosen to be a diagonal matrix $\mathbf{M}$ of dimension (11X11). 
In the following a stochastic evolutionary algorithm technique was discussed and applied to tune the parameters for the compensator design in section 3.

\section{Tuning of compensator to mitigate interaction using GAs}

The idea is to combine the design objective in the form of a cost function that is to be optimised using an optimizer such as a Genetic Algorithm. Where the cost function includes the time domain objective; the tracking error is transformed into the integrated square of error between the commanded signal and actual output $u$ of the actuators. In addition there is another design objective, exception handling (e.g. division by zero) and this is also included in the cost function. The schematic is shown in Fig. 10.

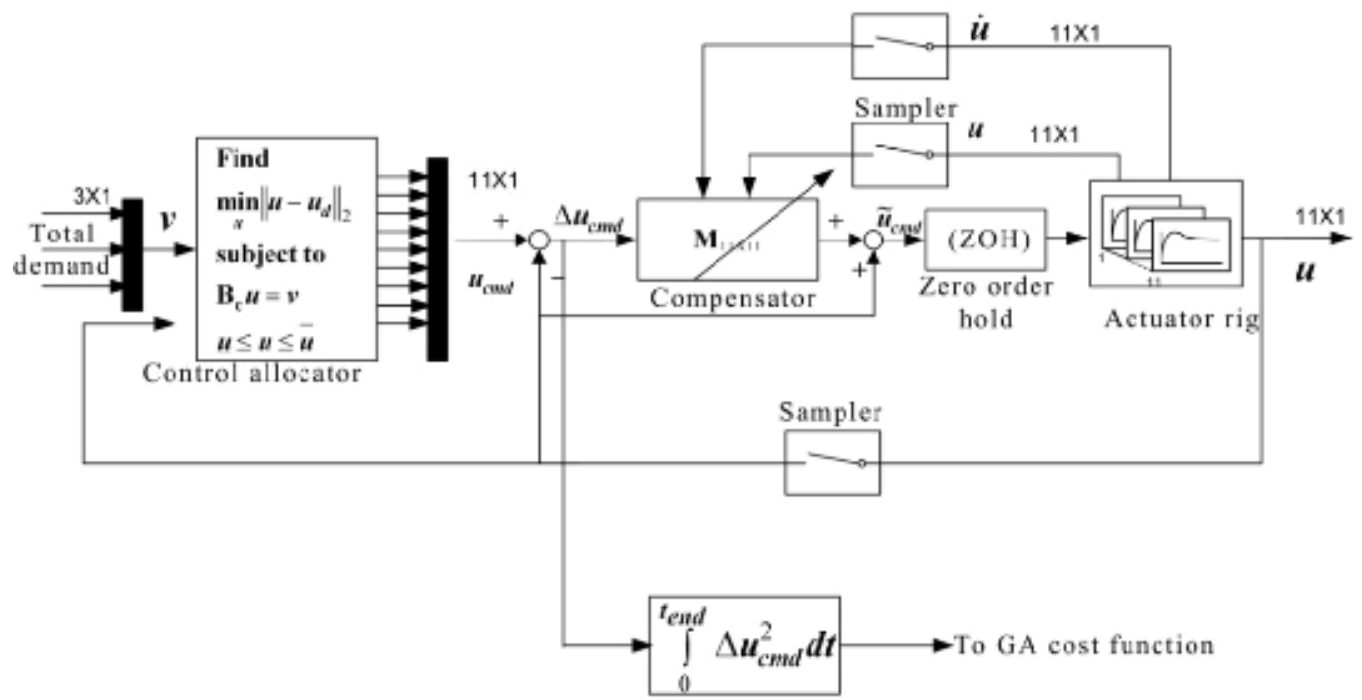

Fig. 10. Cost function error generated by the simulation

Numerically the cost function for tracking error is given by

$$
E_{\text {track }}=\int_{0}^{t_{\text {end }}} \Delta u_{c m d}^{2} d t
$$

where the $t_{\text {end }}$ is the simulation run time.

Numerically the cost function for exception handling is given by

$$
E_{\text {excep }}=\left\{\begin{array}{c}
\text { penalty if exception generated } \\
0 \text { if no exception }
\end{array}\right.
$$

In Eq. (29) the penalty is assigned as a large number like $10^{10}$ so that the individual generating this exception would most likely not be selected in the next generation because of having very low fitness value. Numerically the combined cost function is given as

$$
J=E_{\text {track }}+E_{\text {excep }}
$$

This cost function is then minimised to tune the parameters for the compensator. In the next section GA based optimisation details are given. 


\subsection{GA based optimisation}

Genetic Algorithms are a part of Evolutionary Computing which is a rapidly growing area of Artificial Intelligence. "GA take up the process of evaluating the relative fitness of the individuals of a large population called genes, to select for a new generation, and mimic mutations and crossing over (mixing genes from two parent genes to form offspring genes), the so called evolution phenomenon" (Lindenberg 2002). Unlike biological evolution, in GA the gene controls some other processes like compensator parameters in this work, and is evaluated by comparing properties of the process instead of simply computing some function on the gene space (Lindenberg 2002). Genes used in GA are encoded as bit strings, and their fitness (Lindenberg 2002) is a relation described by a real valued fitness function $\mathrm{f}$ on the set of bit strings $H=\left\{b_{0}, \ldots \ldots, b_{n-1}\right\}$ such that gene $a$ is fitter than gene $b$ if $f(b)<f(a)$.

Optimisation using GA begins with a set of solutions (represented by chromosomes) called the population. Solutions from one population (based on some selection criteria) are taken and used to form a new population. The flow chart of illustration of GA is shown in Fig. 11. After selection of an encoding method (binary encoding for this case) and fitness function (cost function value for an underlying gene), the algorithm proceeds in the following steps (Lindenberg 2002).

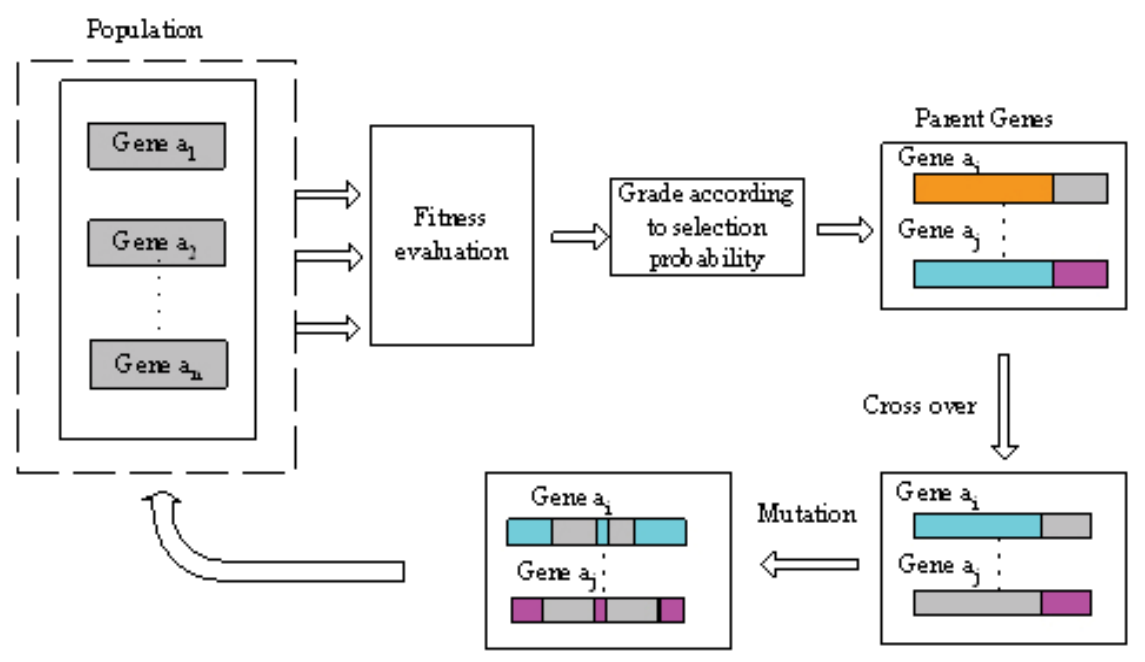

Fig. 11. Flow chart explanation of Genetic Algorithms

- $\quad$ Select an initial population indexed by $\mathrm{P}$ at random from some subset of $H$

- Repeat the following

- $\quad$ evaluate the fitness of all the $b_{P}$ and use them to assign selection probabilities $r_{P}$ to $b_{P}$

- $\quad$ select a new population, dropping genes of low fitness and duplicating fit ones, keeping index set $P$ (the size of population)

- apply the genetic operators of mutation and crossing over (after forming couples)

This is repeated until some condition (for instance maximum number of generations or improvement of the best solution) is satisfied. The main advantage (Leigh 2004) of GA over other optimizers is their parallelism, GA is travelling in a search space using more individuals so they are less likely to get stuck in a local minima. The most important 
attributes of GA are mutation and cross over. A good cross over rate is expected to take better parts of parent genes to the next generation. Mutation on the other hand changes the individuals and if it is kept to a safe low level it helps the population to avoid falling in local minima. This makes GA different from other optimisers, and particularly suitable for nonconvex optimisation problems like the compensator parameter optimisation in this research. The main disadvantage linked with GA is the higher computation time and required resources, but this can be avoided if there is a possibility to stop the GA anytime in the routine. Also with the ever increasing processing power of computers over time this constraint diminishes.

\subsection{Optimizing routine using GA}

Numerically the optimizing problem is given as "Find $\mathbf{M}$ by minimizing $J$ ".

$$
\min _{\mathbf{M}} J
$$

where $\mathbf{M}$ is a diagonal gain matrix of dimension (11X11). The GA optimising routine is formulated by using the MATLAB Genetic Algorithm Direct Search Toolbox. A flow chart representation of the optimisation routine is shown in Fig. 12.

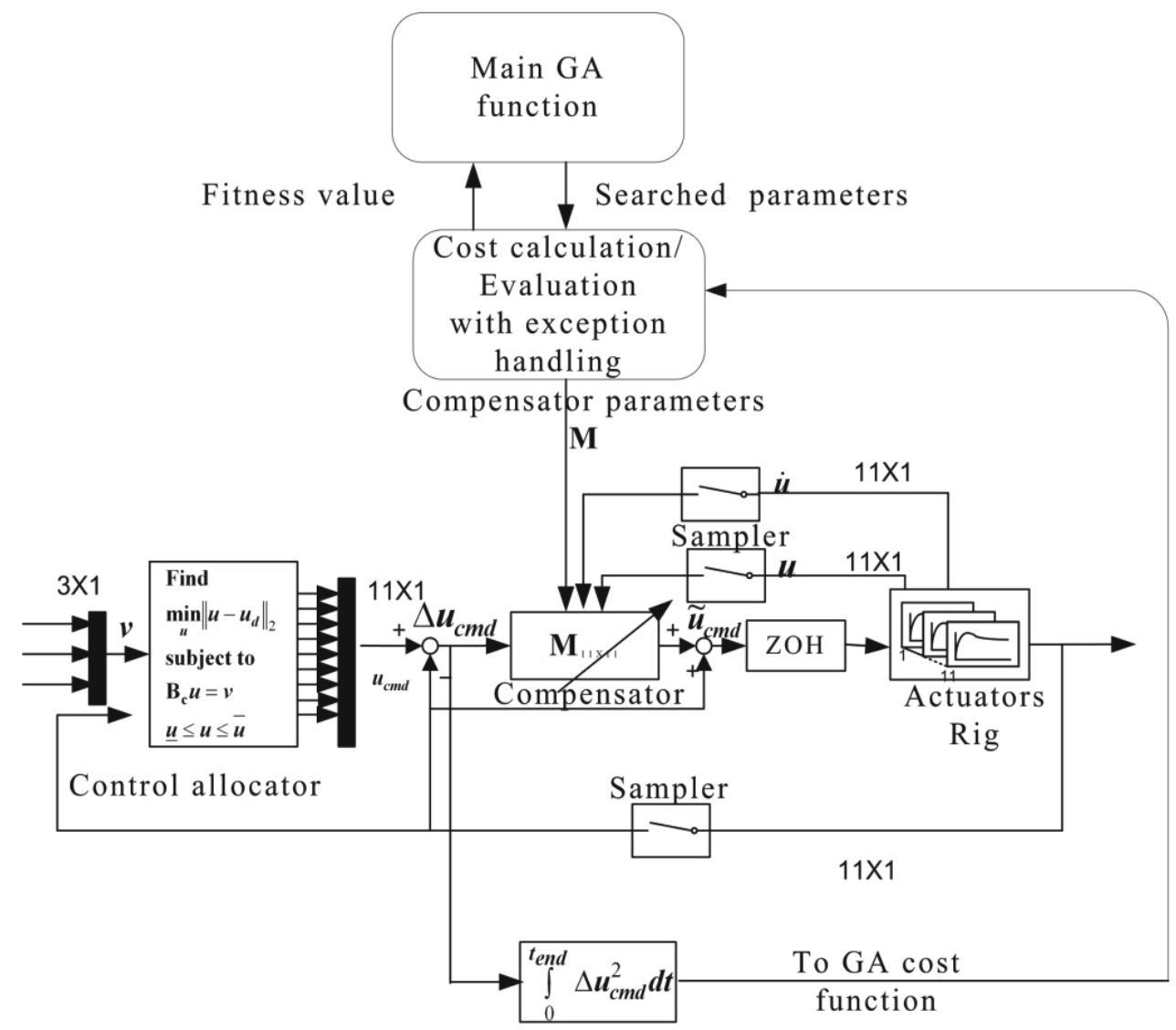

Fig. 12. Flow chart for tuning compensator parameters using GA 
The complete process shown in Fig. 11 can be summarised as:

- The GA main function calls the evaluation function, giving searched parameters to calculate compensator parameters

- The evaluation function calculates compensator parameters and calls the simulation model giving the parameters for the compensator

- The simulation model runs the simulation for the given compensator parameter (i.e. individual of population) and returns the value of error between $u_{c m d}$ and actual $u$

- The evaluation function calculates the cost function value for given errors and returns to the main GA function

- This is repeated for the total number of genes in one generation (population), and then one generation completes, and so the remaining generations are iteratively completed

- The above process is repeated until the cost function attains convergence, or the maximum number of generations is reached.

In the next section the simulation results are given to show how the compensator mitigates the interaction between the control allocation and actuator dynamics.

\section{Simulation results}

During simulation, a mixture of actuator dynamics was used. In the case of redundant control surfaces diagonal gain matrices were tuned by the GA. The control surfaces were approximated by the transfer functions as shown in Table 1.

\begin{tabular}{|l||c||c||}
\hline Control surfaces & Number of control surfaces & Transfer functions \\
\hline \hline Ailerons & 4 & $\frac{270}{s^{2}+22.19 s+270}$ \\
\hline \hline Elevators & 4 & $\frac{0.6128}{s+0.6128}$ \\
\hline \hline Stabilizer & 1 & $\frac{0.0087}{s+0.0087}$ \\
\hline \hline Rudders & 2 & $\frac{270}{s^{2}+22.19 s+270}$ \\
\hline
\end{tabular}

Table 1. Aerosurfaces actuator dynamics (Esteban and Balas 2003)

The virtual control signal, $\boldsymbol{v}$, consists of chirps of amplitude $0.1,0.15,0.1\left(\mathrm{rad} / \mathrm{s}^{2}\right)$ in roll, pitch and yaw angular accelerations respectively. The frequencies of chirps ranged from $0.1-1 \mathrm{~Hz}$ in 20 seconds. In the processing of the GA routine exception handling is carried out to avoid breaking the GA optimisation process. For example if there is an individual (i.e. gains in diagonal matrix) in the population that gives division by zero that would break the simulation. This is dealt with in an exception handling block, which will give a penalty to 
that individual without breaking the simulation. In the next generation that individual would not be selected.

Simulations are done with compensation (Fig. 13 and Fig. 14) and without compensator (Fig. 15 and Fig 16). As can be seen clearly from the results with no compensation there is serious attenuation and mismatch, but as soon as the compensation is turned on, $\mathbf{B}_{\mathbf{c}} \boldsymbol{u}=\boldsymbol{v}$ is achieved because sufficient control authority exists.

Deviations in the case of no compensation case means that the desired control surface positions coming out of the control allocator are different from the actual position of control surfaces. This interaction between the control allocator and the actuator dynamics results in serious consequence if the bandwidths of the actuators are not high or, in other words, the actuators are slow.

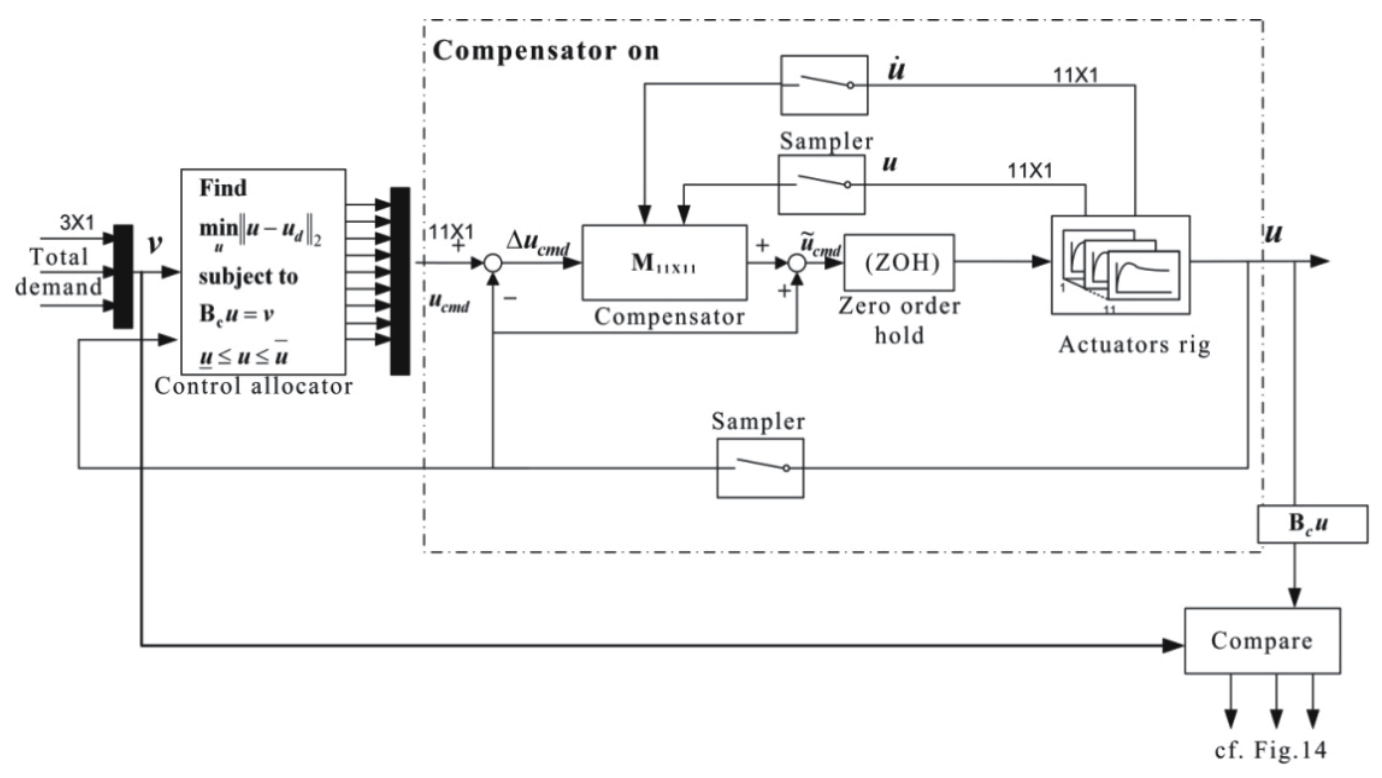

Fig. 13. Implementation scheme for compensator when the compensator is switched on 

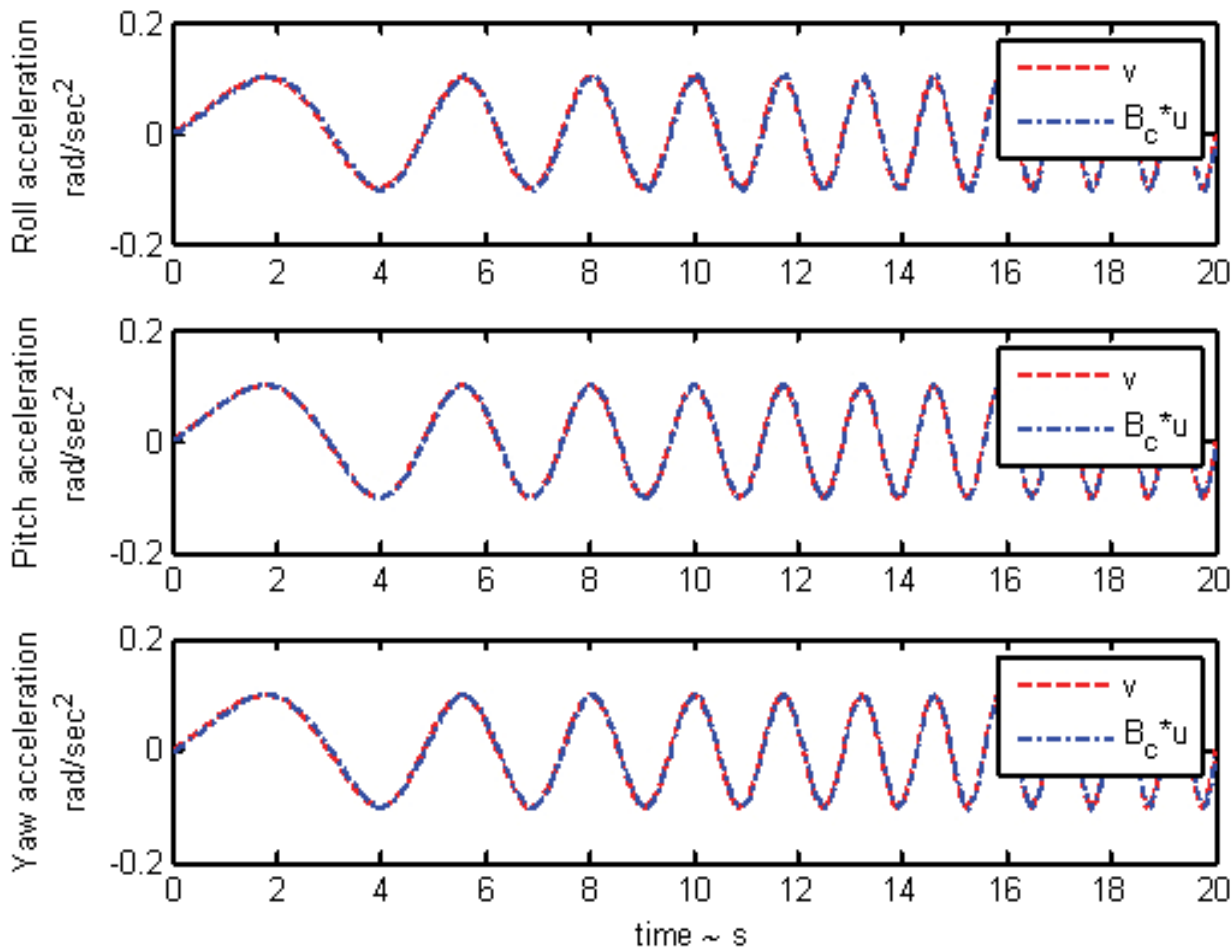

Fig. 14. Desired angular accelerations $(\boldsymbol{v})$ and actual angular acceleration $\left(\mathbf{B}_{\mathbf{c}} \boldsymbol{u}\right)$ in $\mathrm{rad} / \mathrm{s} 2$ when compensation is on 


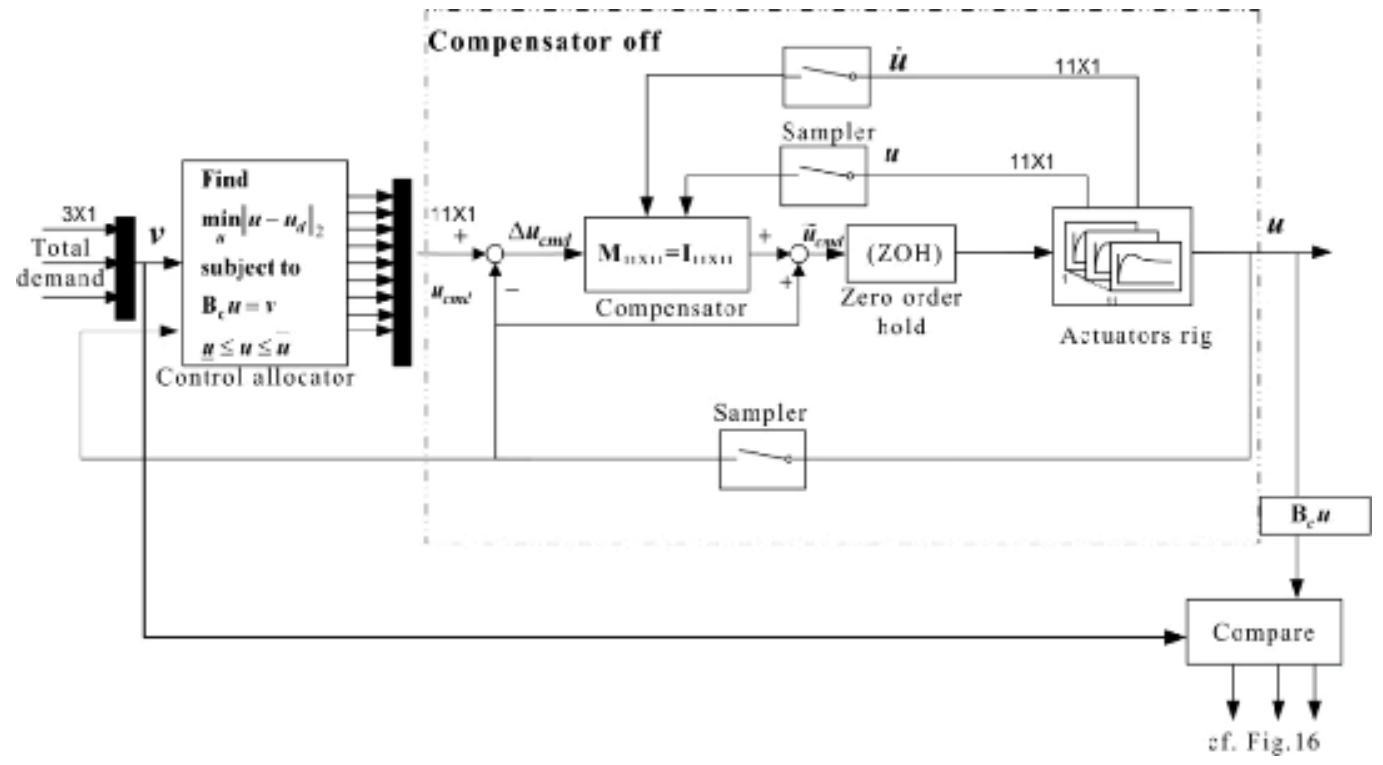

Fig. 15. Implementation scheme for compensator when the compensator is switched off
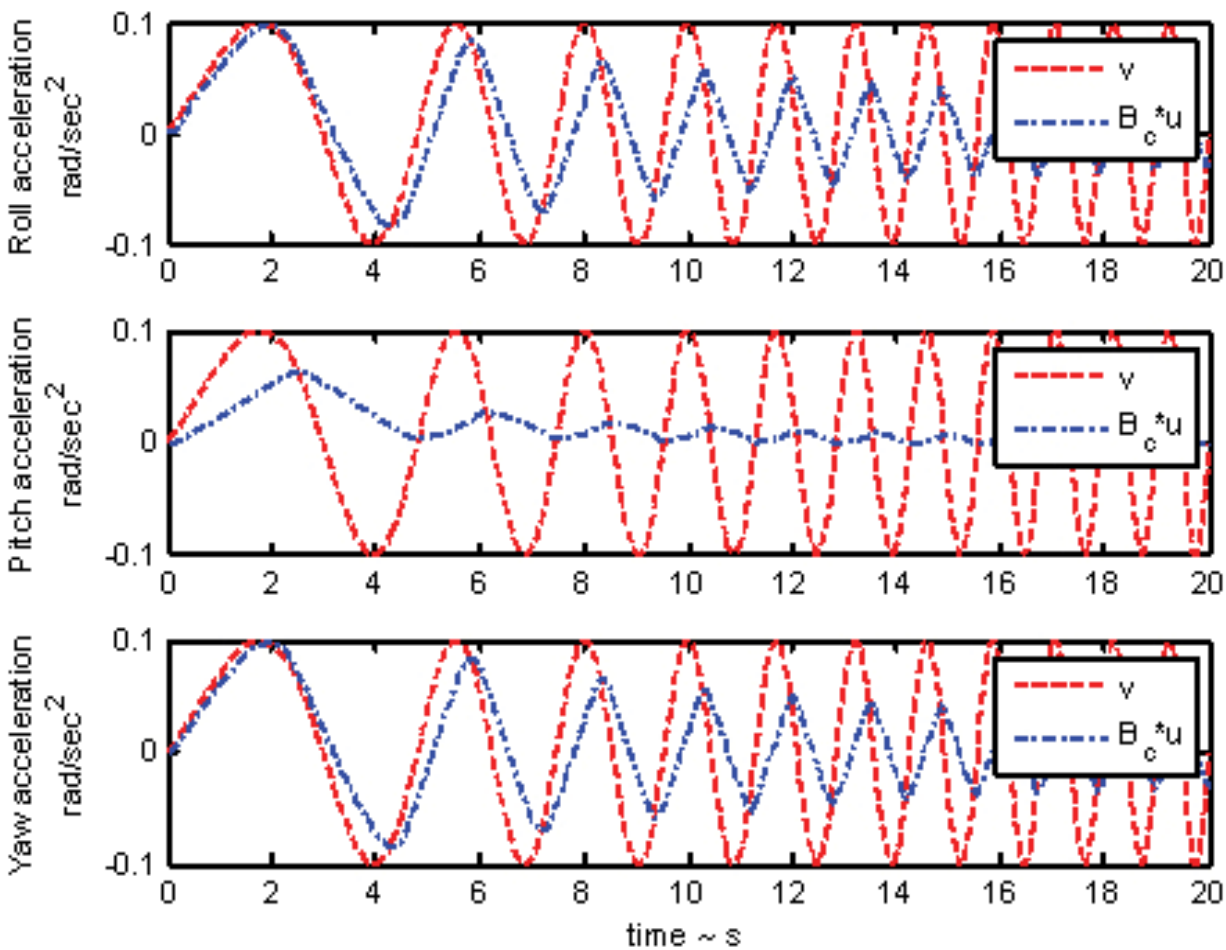

Fig. 16. Desired angular accelerations $(v)$ and actual angular acceleration $\left(\mathrm{B}_{\mathrm{c}} u\right)$ in rad/s2 when compensation is off 


\section{Conclusions}

This chapter details the application of genetic algorithms for the design and tuning of a compensator to alleviate the effects of control allocation and actuator dynamics interaction. The effects of non-negligible actuator dynamics have been investigated first. It was observed that, for the Boeing 747-200, the actuator dynamics cannot be ignored if the excitations are in the range of 0.1 to $1 \mathrm{~Hz}$, which normally depends on the pilot dynamics. Another observation suggests that the bandwidths of the actuators are smaller than the rigid body modes of the aircraft and should not be neglected. The benefit of using a soft-computing methodology for tuning the compensator gains is to avoid the optimisation converging to a local minima and it is seen that the likelihood of the genetic algorithms converging to local minima solution is less as compared to other techniques. In this methodology the model of the actuator is not needed to be known because this methodology was designed to be used on the actuator rig. In the case of the second order actuator, the rates should be either measured or observed. GAs are used offline and the band limited chirps signal is used as the excitation signal in the simulation. However, in the real system a band limited pseudorandom binary signal (PRBS) for this type of identification process could be used as an excitation signal rather than chirp because the later gives cyclic loading on the actuator, which could be problematic.

\section{References}

Bolling, J.G., (1997) Implementation of Constrained Control Allocation Techniques Using an Aerodynamic Model of an F-15 Aircraft, MSc. thesis, Virginia Polytechnic Institute and State University, Virginia, USA.

Esteban, A. M., Balas, G.J. (2003) A B747-100/200 aircraft fault tolerant and fault diagnostic benchmark, AEM-UoM 2003-1, Aerospace Engineering and Mechanics Department, University of Minnesota, USA.

Franklin, G.F., Powell, J.D. and Workman, M. (1998) Digital control of dynamic systems, 3 3 rd ed., Addison-Wesley Longman, Inc., California

Lindenberg, F.M. (2002) Adaptive Compute Systems lecture notes, Technical University Hamburg-Harburg Germany.

Oppenheimer, M.W. and Doman, D.B. (2004) 'Methods for compensating for control allocator and actuator interactions', Journal of Guidance, Control, and Dynamics, 27(5), pp. 922-927. 


\title{
Fault Tolerant Flight Control, a Physical Model Approach
}

\author{
Thomas Lombaerts, Ping Chu, \\ Jan Albert (Bob) Mulder and Olaf Stroosma \\ Delft University of Technology \\ the Netherlands
}

\section{Introduction}

Safety is of paramount importance in all transportation systems, but especially in civil aviation. Therefore, in civil aviation, a lot of developments focus on the improvement of safety levels and reducing the risks that critical failures occur. When one analyses recent aircraft accident statistics (Civil Aviation Safety Data 1993-2007 (2008); Smaili et al. (2006)), there are two major categories of accidents which can be attributed to a single primary cause, as illustrated in figure 1. The largest category is "collision with ground" (controlled flight into terrain, CFIT) where a fully functional aircraft hits terrain due to the loss of situational awareness by the pilot, which counts for as much as $26 \%$ of the accidents. This percentage is decreasing over the years thanks to the continuously evolving amount and manner of cockpit display information. The second major category is "loss of control in flight", which can be attributed to mistakes made by the pilot or a technical malfunctioning. This category counts for $16 \%$ of all aircraft accident cases and is not decreasing.

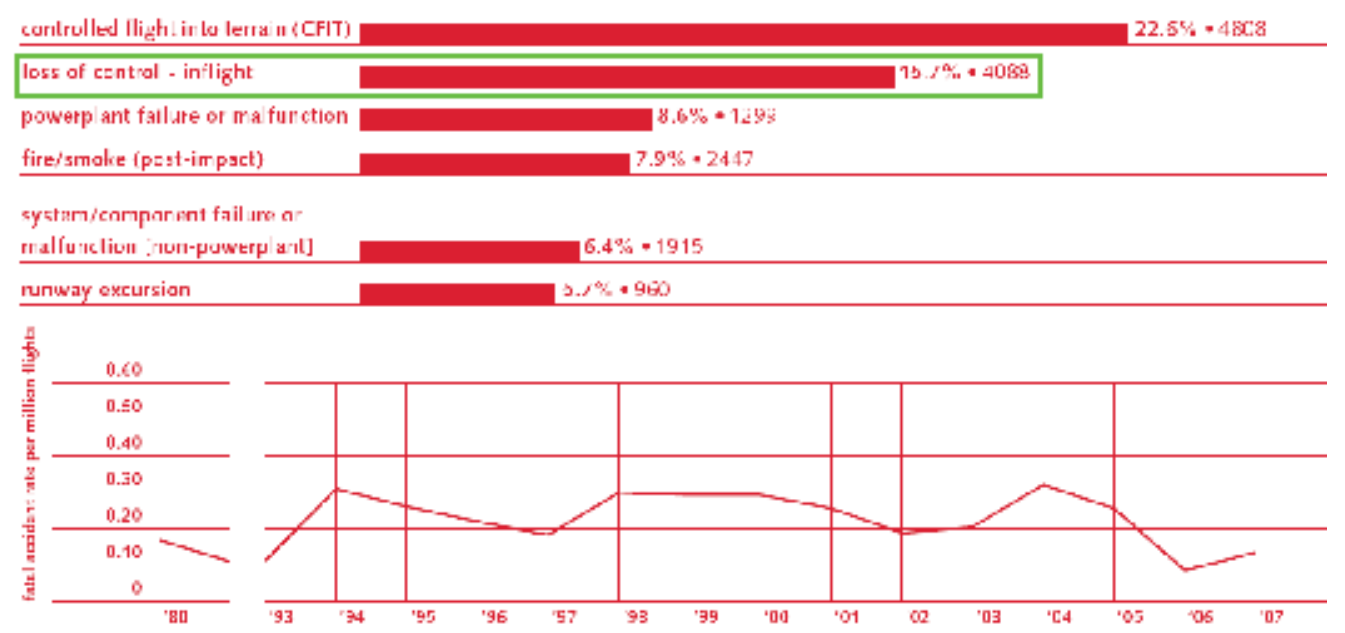

Fig. 1. Accident statistics, source: Civil Aviation Safety Data 1993-2007 (2008) 
Analysing a major part of the accidents in the latter category has led to a common conclusion: from a flight dynamics point of view, with the technology and computing power available at this moment, it might have been possible to recover the aircraft in many accident situations in this category, on the condition that non-conventional control strategies would have been available. These non-conventional control strategies involve the so-called concept of active fault tolerant flight control (FTFC), where the control system is capable to detect the change in the aircraft behaviour and to adapt itself so that it can handle the perturbed aircraft dynamics. Earlier research projects in FTFC involve the Self-Repairing Flight Control System (SRFCS) program (Corvin et al. (1991)), the MD-11 Propulsion Controlled Aircraft (PCA) (KrishnaKumar \& Gundy-Burlet (n.d.)), the Self-Designing Controller for the F-16 VISTA (Ward \& Barron (1995)), Reconfigurable Systems for Tailless Fighter Aircraft in the X-36 RESTORE program (Brinker \& Wise (1999); Calise et al. (2001)), the NASA Intelligent Flight Control System (IFCS) F-15 program (Intelligent Flight Control: Advanced Concept Program (1999)) and Damage Tolerant Flight Control Systems for Unmanned Aircraft by Athena/Honeywell (Gavrilets (2008)). There are many alternative control approaches to achieve FTFC. In all these control approaches, there remain some problems and limitations, varying from the limitation to a restricted number of failure cases to the limitation of the type and extent of damage which can be compensated for due to fixed model structures for identification. Another frequently encountered issue are convergence problems. Besides, black box structures like for neural networks reduce the transparency of the approach. Moreover, for many approaches it is not clear what will happen when the reference model behaviour is not achievable in post-failure conditions.

The research approach as elaborated in this chapter uses a physical modular approach, where focus is placed on the use of mathematical representations based on flight dynamics. All quantities and variables which appear in the model have a physical meaning and thus are interpretable in this approach, and one avoids so-called black and grey box models where the content has no clear physical meaning. Besides the fact that this is a more transparent approach, allowing the designers and engineers to interpret data in each step, it is assumed that these physical models will facilitate certification for eventual future real life applications, since monitoring of data is more meaningful.

Adaptive nonlinear dynamic inversion has been selected as the preferred adaptive control method in this modular or indirect approach. The advantages of dynamic inversion are the absence of any need for gain scheduling, and an effective input-output decoupling of all control channels. Adaptation of the controller is achieved by providing up-to-date aerodynamic model information which is collected in a separate identification module.

The structure of this chapter is as follows. Section 2 provides information on the high fidelity RECOVER simulation model which has been used in this research project. A global overview of the fault tolerant control architecture is given in section 3 , and further explanations of some of the individual modules are added in sections 4 and 5. Simulation results are discussed in section 4.2 for the aerodynamic model identification, section 5.3 for the autopilot and in section 5.5 for the manual control approach. Finally, section 6 presents some conclusions and recommendations for future research.

\section{The RECOVER benchmark simulation model}

The presented work is part of a research project by the Group for Aeronautical Research and Technology in Europe (GARTEUR). This group has established flight mechanics action group FM-AG(16) with the specific goal to investigate the possibilities of fault tolerant control in aeronautics and to compare the results of different reconfiguring control strategies applied to a reference benchmark flight trajectory. That benchmark scenario is inspired by the so-called 
Bijlmermeer disaster of EL AL flight 1862, where a Boeing 747-200 Cargo aircraft of Israel's national airline EL AL lost two engines immediately after take-off from Amsterdam airport Schiphol in the Netherlands and crashed into an apartment building in the neighbourhood while trying to return to the airport. A detailed simulation model of this damaged aircraft is available from the Dutch Aerospace Laboratory NLR. This RECOVER (REconfigurable COntrol for Vehicle Emergency Relief) benchmark model is discussed in detail in ref. Smaili et al. $(2008 ; 2006)$ and has been used (also in earlier versions) by a number of investigators and organizations (Maciejowski \& Jones (2003); Marcos \& Balas (2003); Szaszi et al. (2002)). More information about the reference benchmark scenario can be found in ref. Lombaerts et al. $(2005 ; 2006)$. Other control strategies and results applied to the same benchmark model as part of the framework of FM-AG(16) can be found in ref. Alwi (2008); Cieslak et al. (2008); Hallouzi \& Verhaegen (2008); Joosten et al. (2007; 2008). Related FDI work can be found in ref. Varga (2007); Varga \& Hecker (2004).

The simulation benchmark for evaluating fault tolerant flight controllers as discussed in ref. Smaili et al. (2006) contains six benchmark fault scenarios, enumerated in fig. 2(a). These failure cases have varying criticality. Fig. 2(b) shows the failure modes and structural damage configuration of the Flight 1862 accident aircraft, which is the most important fault scenario in the simulation benchmark.

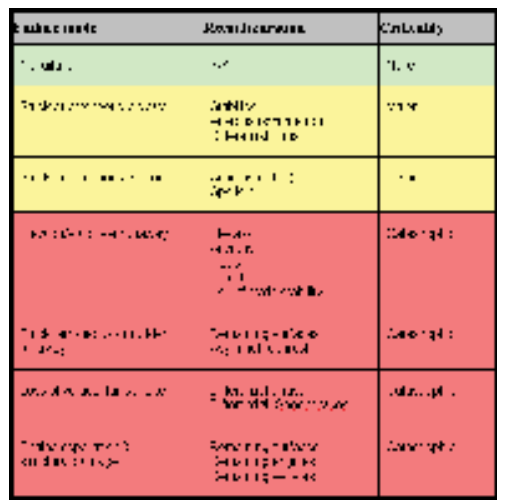

(a) GARTEUR FM-AG(16) RECOVER benchmark fault scenarios, source: Smaili et al. (2008)

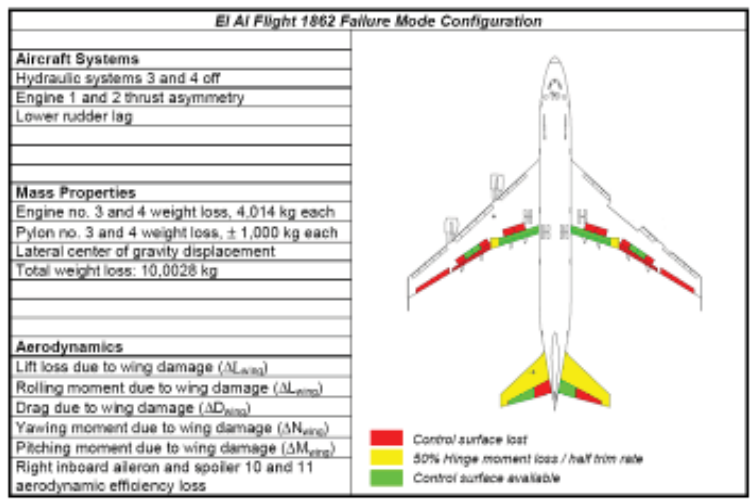

(b) Failure modes and structural damage configuration of the Flight 1862 accident aircraft, suffering right wing engine separation, partial loss of hydraulics and change in aerodynamics, source: Smaili et al. (2008)

Fig. 2. GARTEUR FM-AG(16) RECOVER benchmark fault scenarios and configuration

The rudder runaway, the vertical tail separation and the EL AL engine separation have been used as scenarios for this chapter. In the case of a rudder runaway (also called hardover), the rudder moves quickly to an extreme position. More precisely, the rudder deflects to the left, inducing a yawing tendency of the aircraft to the left. The rudder deflection limit in this scenario depends on the flight speed, since aerodynamic blowdown is taken into account in the RECOVER simulation model. As a result the maximum rudder deflection is slightly below $15^{\circ}$ for an airspeed around 270 knots, and even close to $25^{\circ}$ (the physical maximum deflection limit imposed by the rudder hardware structure) for an airspeed of $165 \mathrm{knots}$. The vertical tail separation leads to the loss of all rudder control surfaces as well as the loss of all damping in the roll and yaw axes. Mind that loss of hydraulics is not considered in this situation. The El $\mathrm{Al}$ engine separation scenario is an accurate simulation of flight 1862, validated by black box data of the accident, where the loss of hydraulics is taken into account. 


\section{Global overview of the physical modular approach}

Globally, the overall architecture of this modular approach consists of three major assemblies, namely the controlled system, the Fault Detection and Identification (FDI) assembly and the Fault Tolerant Flight Control (FTFC) assembly, as shown in fig. 3. The controlled system comprises the aircraft model and the actuator hardware. Possible failures in this controlled system are structural failures and actuator hardware failures in the latter. Sensor failures have not been considered in this research, since it has been assumed that effects of these failures can be minor thanks to sensor redundancy and sensor loss detection. However, the latter mechanism is recommended for future research.

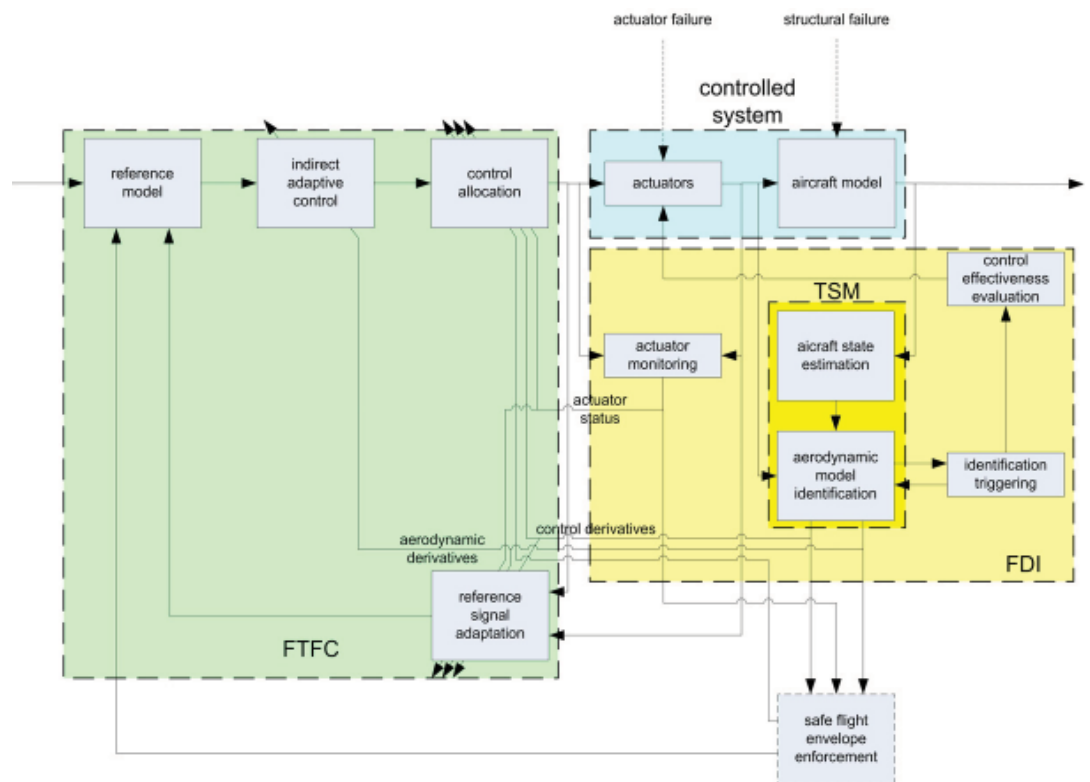

Fig. 3. Overview of the modular physical approach for fault tolerant flight control

The Fault Detection and Identification (FDI) architecture consists of several components. The core of this assembly is the two step method (TSM) module, described in section 4.1. This module consists of a separate aircraft state estimation step followed by an aerodynamic model identification step, where the latter is a joint structure selection and parameter estimation (SSPE) procedure. The state estimation step is a nonlinear problem solved by an Iterated Extended Kalman Filter. The preferred SSPE algorithm is Adaptive Recursive Orthogonal Least Squares. In case a structural failure occurs (in the aircraft structure or in one of the control surfaces), re-identification is triggered when the average square innovation exceeds a predefined threshold. For successful identification of the control derivatives of every individual control surface, control effectiveness evaluation is needed after failure. This can be done by inserting multivariate orthogonal input signals in the actuators. Although this must be done carefully such that the damaged aircraft cannot be destabilized, it is necessary in order to obtain sufficient control surface efficiency information for the control allocation module, to be discussed later. A valid approach might be to introduce these evaluation signals only when strictly needed, i.e. when successful reconfiguration is not possible due to a lack of information about this control efficiency. The two step method is ideally suited to deal with structural failures, but for the detection of actuator failures a separate actuator monitoring algorithm is needed, such as an Actuator Health Monitoring System (AHMS). 
Four other functions can be grouped to form the Fault Tolerant Flight Control (FTFC) assembly. The core for this group are indirect adaptive control and control allocation. Indirect adaptive control can be achieved by adaptive nonlinear dynamic inversion (ANDI) described in section 5.1. In this setup, the control structure consists of three inversion loops, as elaborated in section 5.2. This control structure relies on the principle of time scale separation. The advantages of ANDI are that it removes the need for gain scheduling over different operating conditions and it effectively decouples input-output relationships. Moreover, the NDI control algorithm automatically involves some form of control allocation, due to the structure of the control law. This structuring allows a clear separation how different failure types are dealt with. Structural failures independent of the control surfaces are detected by the TSM and this damage information is supplied to the ANDI algorithm by means of the aerodynamic derivatives. On the other hand control surface related failures, aerodynamics or actuator related, are identified by the TSM or the actuator monitoring algorithm respectively. This information is sent to the adaptive control allocation block. The preferred control allocation approach is found to be the control distributor concept (CDC), (Oppenheimer \& Doman (2006)), combined with the weighted pseudo inverse (WPI). This method assumes the presence of a large amount of similar control surfaces. This assumption holds for the type of aircraft considered in this research project, such as the Boeing 747. This approach fits in the modular setup of the global procedure, where the CDC principle takes into account aerodynamic changes and the WPI provides the adaptivity with reference to actuator failures. Furthermore, a reference model defines the reference signal that the closed loop configuration has to track. However, this reference model needs to be adaptive such that its signals are limited based upon the achievable performance of the damaged closed loop configuration. This reference signal adaptation can be achieved by Pseudo Control Hedging. This modulation is based upon the difference between the demanded input signal and the achieved input signal by the actuators. This reference signal adaptation is primarily driven by saturation effects. Besides, this hedging operation takes into account the updated model information from the FDI assembly. In this way, one makes sure that no unreachable reference signals are given to the closed loop configuration. This PCH operation can be considered as a first degree of safe flight envelope enforcement, based upon input saturation effects and updated model information. Experiments have shown that especially the throttle channel is prone to saturation effects.

During this research, it has been found that safe flight envelope enforcement is a crucial aspect in this control setup, and it is part of recommended future research. This protection algorithm, based upon the achievable performance which can be estimated based upon actuator status, aerodynamic derivatives and control derivatives, should contribute on two levels. On one hand, it has to assist the $\mathrm{PCH}$ algorithm by limiting the reference model output appropriately. Moreover, the output of the control allocation block, and thus the input to the actuators, should be limited based upon this reachable flight envelope information. A tabular overview of the preferred method for every component in the global overview of the fault tolerant flight control setup can be found in table 1 .

In the following sections, further explanations will be given about some of the individual modules.

\section{Fault detection and identification}

\subsection{Two step method}

The identification method considered in this study is the so-called two step method (Chu (2007); Chu et al. (1994); Laban (1994); Mulder (1986); Mulder et al. (1999)). There are many other identification algorithms mentioned in the literature like maximum likelihood 


\begin{tabular}{|c|c|}
\hline \hline component & preferred method \\
\hline \hline aircraft state estimation & Iterated Extended Kalman Filter \\
\hline $\begin{array}{c}\text { aerodynamic model } \\
\text { identification }\end{array}$ & $\begin{array}{c}\text { Adaptive Recursive } \\
\text { Orthogonal Least Squares }\end{array}$ \\
\hline identification triggering & average square innovation \& threshold \\
\hline control effectiveness evaluation & multivariate orthogonal input functions \\
\hline control allocation & $\begin{array}{c}\text { Control Distributor Concept } \\
\text { \& Weighted Pseudo Inverse }\end{array}$ \\
\hline indirect adaptive control & triple layer Adaptive \\
& Nonlinear Dynamic Inversion \\
\hline actuator monitoring & Actuator Health Monitoring System \\
\hline reference signal adaptation & Pseudo Control Hedging \\
\hline \hline
\end{tabular}

Table 1. Preferred methods for FTFC modules

identification (MLI) and other one step identification routines, but not all of them are applicable on line. One of the few procedures which can be implemented in real time is the so-called filtering method developed at German Aerospace Centre DLR, see ref. Jategaonkar (2006). This is a joint state and parameter estimation algorithm, but very complex. Another algorithm can be found in ref. Morelli (2000), which is frequency based. The advantage of the two step method is that it is easier to implement on-line. The aim is to update an a priori aerodynamic model (obtained by means of windtunnel tests and CFD calculations) by means of on-line flight data. The first step is called the Aircraft State Estimation phase, where the second one is the Aerodynamic Model Identification step. In the Aircraft State Estimation procedure, an Iterated Extended Kalman Filter is used to determine the aircraft states, by making use of the redundant but perturbed information from all sensors (air data, inertial, magnetic and GPS measurements). By means of this state information, it is possible to construct the combined aerodynamic and thrust forces and moments acting on the aircraft. Mass and inertia are considered as known constants in these calculations. In the absence of a structural failure, real time mass and inertia can be calculated by integrating fuel flow and subtracting it from the total take off values. Future research is aimed at taking into account changing masses and inertia in the presence of structural failures. Finally the aerodynamic derivatives can be deduced in the second step by means of structure selection and parameter estimation.

This second step involves the aerodynamic properties of the aircraft. Thanks to the separation of the kinematics, these aerodynamics become linear in the parameters. Model structure determination is the first concern, and a regression method can be applied in order to estimate the so-called aerodynamic model parameters. Simultaneous recursive structure selection and parameter estimation is performed in this phase, by means of so-called Adaptive Recursive Orthogonal Least Squares (AROLS), (Lombaerts et al. (2010c)). This method determines the model structure by means of an orthogonal QR-decomposition, which is extended recursively to make the method suitable for online applications. Special monitoring criteria verify continuously when the structure needs to be extended. For a detailed discussion of these criteria see ref. Lombaerts et al. (2010c).

The model structure selection procedure considers a set of candidate regressors based upon a Taylor series expansion with respect to the aircraft states and control inputs (control surface deflections and engine settings) relevant for the aerodynamic forces and moments. The candidate regressors are shown in table 2. 


\begin{tabular}{|c|c|}
\hline $\begin{array}{l}\text { dependent } \\
\text { variable }\end{array}$ & candidate regressors \\
\hline $\mathrm{X} ; \mathrm{Z} ; \mathrm{M}$ & $\begin{array}{c}1, \alpha, \alpha^{2}(\mathrm{X} \text { only }), \frac{q c}{V}, \delta_{e_{i}}, i_{h}, \delta_{s p_{i}}, \delta_{f_{i}}, T_{i} \\
\left\{\beta, \quad \frac{p b}{2 V}, \quad \frac{r b}{2 V}, \alpha^{m}, \alpha \frac{q \bar{c}}{V}, \alpha \delta_{e}, \alpha \beta, \alpha \beta^{2}, \alpha^{2} \beta, \alpha \beta^{3}, \alpha^{2} \beta^{3} \quad m=3, \ldots, 8\right\} \\
\left\{\alpha \frac{p b}{2 V}, \alpha \frac{r b}{2 V}, \alpha^{2} \frac{p b}{2 V}, \alpha^{2}, \frac{r b}{2 V}, \beta^{n} \quad n=2, \ldots, 5\right\}\end{array}$ \\
\hline Y; L; N & $\begin{array}{c}1, \beta, \frac{p b}{2 V}, \frac{r b}{2 V}, \delta_{a_{i}}, \delta_{r_{i}}, \delta_{s p_{i}},\left\{\alpha, \frac{q \bar{c}}{V}, T_{i}\right\} \\
\left\{\alpha^{m}, \alpha \frac{q \bar{c}}{V}, \alpha \delta_{e}, \alpha \beta, \alpha \beta^{2}, \alpha^{2} \beta, \alpha \beta^{3}, \alpha^{2} \beta^{3} \quad m=2, \ldots, 8\right\} \\
\left\{\alpha \frac{p b}{2 V}, \alpha \frac{r b}{2 V}, \alpha^{2} \frac{p b}{2 V}, \alpha^{2}, \frac{r b}{2 V}, \beta^{n} \quad n=2, \ldots, 5\right\}\end{array}$ \\
\hline
\end{tabular}

Table 2. States and control inputs which influence forces and moments

\begin{tabular}{rcccccr}
\multicolumn{1}{c}{$\beta$} & $p$ & $r$ & $\delta_{a}$ & $\delta_{r}$ & \\
\hline \hline \multirow{2}{*}{$Y$} & $C_{Y_{\beta}}<0$ & $C_{Y_{p}} \approx 0$ & $C_{Y_{r}}>0$ & $C_{Y_{\delta_{a}}} \approx 0$ & $C_{\delta_{\delta_{r}}}>0$ & nominal \\
& $C_{Y_{\beta}} \ll 0$ & $C_{Y_{p}} \approx 0$ & $C_{Y_{r}}>0$ & $C_{Y_{\delta_{a}}} \approx 0$ & $C_{Y_{\delta_{r}}}>0$ & engine separation \\
& $C_{Y_{\beta}} \leq 0$ & $C_{Y_{p}} \approx 0$ & $C_{Y_{r}} \geq 0$ & $C_{Y_{\delta_{a}}} \approx 0$ & $C_{Y_{\delta_{r}}}=0$ & tail loss \\
\hline \multirow{3}{*}{$L$} & $C_{l_{\beta}}<0$ & $C_{l_{p}}<0$ & $C_{l_{r}}>0$ & $C_{\delta_{\delta_{a}}}<0$ & $C_{l_{\delta_{r}}}>0$ & nominal \\
& $C_{l_{\beta}}<0$ & $C_{l_{p}} \leq 0$ & $C_{l_{r}} \gg 0$ & $C_{l_{\delta_{a}}}<0$ & $C_{\delta_{\delta_{r}}}>0$ & engine separation \\
& $C_{l_{\beta}} \leq 0$ & $C_{l_{p}}<0$ & $C_{l_{r}}>0$ & $C_{l_{\delta_{a}}}<0$ & $C_{\delta_{\delta_{r}}}=0$ & tail loss \\
\hline \multirow{2}{*}{$N$} & $C_{n_{\beta}}>0$ & $C_{n_{p}}<0$ & $C_{n_{r}}<0$ & $C_{n_{\delta_{a}}}>0$ & $C_{n_{\delta_{r}}}<0$ & nominal \\
& $C_{n_{\beta}} \gg 0$ & $C_{n_{p}}<0$ & $C_{n_{r}}<0$ & $C_{n_{\delta_{a}}}>0$ & $C_{n_{\delta_{r}}}<0$ & engine separation \\
& $C_{n_{\beta}} \leq 0$ & $C_{n_{p}}<0$ & $C_{n_{r}} \leq 0$ & $C_{n_{\delta_{a}}}>0$ & $C_{n_{\delta_{r}}}=0$ & tail loss \\
\hline \hline
\end{tabular}

Table 3. Predicted lateral aerodynamic derivative changes for asymmetric failure scenarios studied

In table $2,\{\ldots\}$ indicate regressor candidates for AROLS related to aerodynamic cross coupling effects in case of aircraft asymmetries as well as higher order aerodynamic effects, for example due to structural failures. The identified parameters contain valuable information about the physical state of the aircraft. For example, the parameters $C_{m_{\alpha}}$ and $C_{n_{\beta}}$ represent measures for static stability. In fact, $C_{n_{\beta}}$ is also known as Weathercock stability. The derivatives $C_{m_{\delta_{e}}}, C_{l_{\delta_{a}}}$ and $C_{n_{\delta_{r}}}$ represent "elevator-", "aileron-" and "rudder-effectiveness" respectively.

A structural failure will result in changing aerodynamic derivatives. Based upon flight dynamics theory (Mulder et al. (January 2006)), it is possible to predict the changes in the aerodynamic derivatives in the engine separation and tail loss scenarios, as illustrated in table 3. Each parameter is an aerodynamic derivative, e.g. $C_{Y_{\beta}}$ represents the change in lateral force $Y$ caused by a change in sideslip angle $\beta$. In the engine separation scenario for example, wing sweep combined with the loss of the leading edge results in a larger negative $Y$-force caused by a positive sideslip angle $\beta$.

Various validation tests by means of a posteriori batch process identification, least squares residual analysis and reconstruction of velocity and angular rate components have shown that this method works well and can in fact be used for identification purposes of damaged aircraft. Identification of component as well as structural failures is possible in this setting, see ref Lombaerts et al. (2007) for a more elaborate discussion of validation methods and results.

\subsection{Applications}

Two application examples are considered here. First, a static directional stability analysis is performed for the tail loss scenario. Thereafter, the model structure of the dimensionless 
aerodynamic force coefficient $C_{Z}$ is elaborated and its parameter values are estimated at the same moment in the engine separation scenario.

\subsubsection{Tail loss}

A natural consequence of the tail loss scenario is the huge reduction in directional static stability. This can be seen in the behaviour of the aerodynamic derivative $C_{n_{\beta}}$, as shown in fig. 4. A positive value for $C_{n_{\beta}}$ indicates static directional stability. From fig. 4 , it is clear that, while the nominal aircraft is stable, the damaged aircraft is observed to be directionally statically unstable.

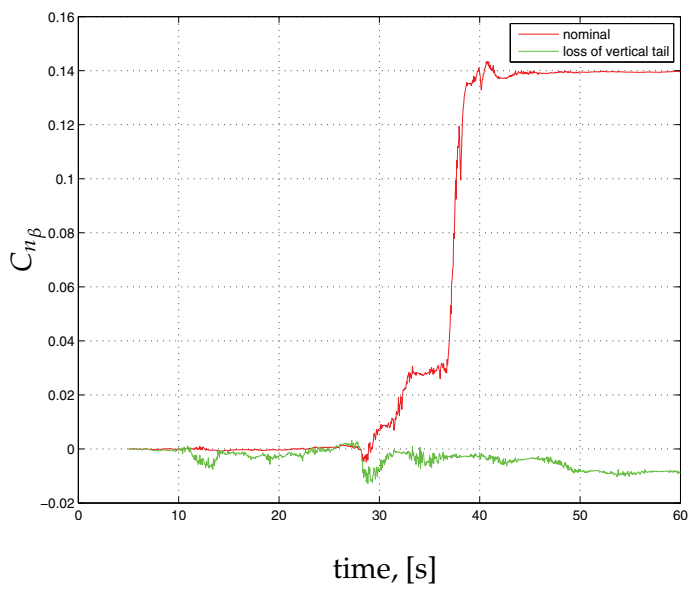

Fig. 4. Comparison of Weathercock stability derivative for undamaged and damaged aircraft with loss of vertical tail

\subsubsection{Engine separation}

In this example, the dimensionless aerodynamic force coefficient $C_{Z}$ is considered in the engine separation scenario. Applying the AROLS routine to this stretch of simulation data leads to the results as shown in fig. 5. Fig. 5(a) shows the results prior to failure, while fig. 5 (b) focuses on the post-failure situation. As can be seen in this figure, the fit is accurate in both cases. The optimal residual, which is updated over the entire time history at every time step, is minimal. The real residual, which is not updated a posteriori, gives an indication of the reduction in the residual over the time interval. Fig. 5(a) reveals the initial large residual due to initialization. Fig. 5(b) shows the initial large residual caused by the failure. This is minimized in very short term, but starts increasing thereafter due to slow dynamics which become dominant. Between 55s and 60s, the residual is again minimized, thanks to the incorporation of these slow dynamics in the aerodynamic model.

The parameter estimation results, together with their standard deviations, can be found in table 4. These results highlight the difference between the situations before and after failure. Because there is no anomaly before the failure, the important independent variables for the vertical force are the conventional ones, namely a constant, the angle of attack $\alpha$ and pitch rate $\frac{q \bar{c}}{V}$. However, after the failure a violent roll-dive manoeuvre follows. The influence of the damage on the change in aerodynamics is represented by additional contributions by sideslip $\beta$ and roll rate $\frac{p b}{2 V}$. These are a consequence of the asymmetric damage, as a result of which 

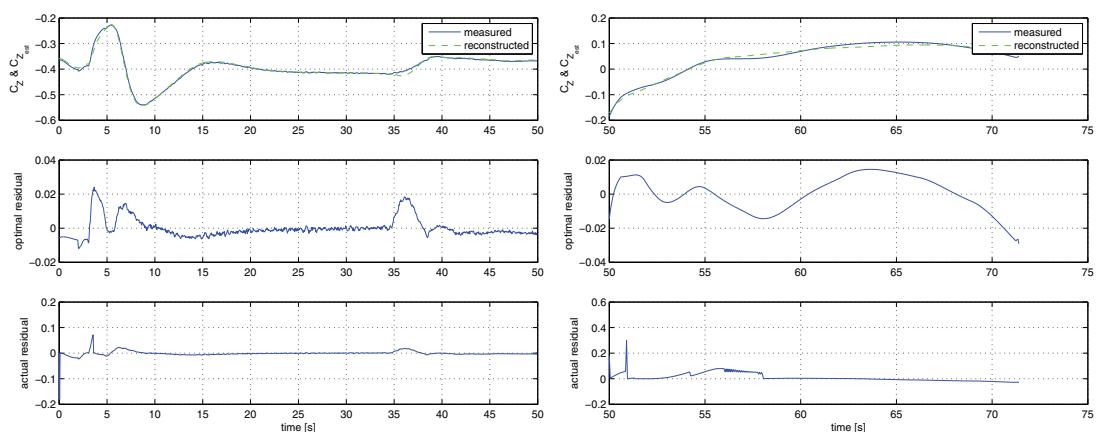

(a) AROLS structure selection and (b) AROLS structure selection and parameter estimation result for $C_{Z}$ prior parameter estimation result for $C_{Z}$ after to failure failure

Fig. 5. AROLS structure selection and parameter estimation results for $C_{Z}$ before and after the failure

the decoupling of longitudinal and lateral regressors does not hold anymore in this scenario. The standard deviations give an indication of the accuracy of the results.

\begin{tabular}{ccccc}
\hline \hline & \multicolumn{2}{c}{ prior failure } & \multicolumn{2}{c}{ post failure } \\
& estimated & $(\sigma)$ & estimated & $(\sigma)$ \\
\hline 1 & -0.1583 & $(1 \%)$ & -0.4131 & $(2 \%)$ \\
$\alpha$ & -4.4016 & $(\approx 0 \%)$ & 4.9047 & $(1 \%)$ \\
$q \bar{c}$ & -1.6124 & $(22 \%)$ & 105.1966 & $(1 \%)$ \\
$V$ & - & - & 4.2319 & $(1 \%)$ \\
$\beta$ & - & - & 9.4708 & $(\approx 0 \%)$ \\
$\frac{p b}{2 V}$ & - & - & - & - \\
$\frac{r b}{2 V}$ & - & - & - & - \\
all others & - & - & \\
\hline \hline
\end{tabular}

Table 4. SSPE results for $C_{Z}$ for engine separation scenario, before and after failure

A more elaborate discussion of this example can be found in ref. Lombaerts et al. (2010c).

\section{Fault Tolerant Control method}

Fault Tolerant Flight Control is achieved by making use of a triple layered adaptive nonlinear dynamic inversion algorithm. First, the basics of the control approach are explained. Thereafter, each of the three individual control loops is described. Finally, some application examples on a selection of failure scenarios will show the failure handing capabilities of this fault tolerant control setup.

\subsection{Control approach: Adaptive nonlinear dynamic inversion}

A major advantage of dynamic inversion is its ability to naturally handle changes of operating condition, which removes the need for gain scheduling, e.g. as is the case for classical control methods. This is especially advantageous for control of space re-entry vehicles, due to their extreme and wide operating conditions which include hypersonic speed during re-entry and subsonic regions during the terminal glide approach phase to the runway. Another advantage is its natural property of decoupling the control axes, i.e. no coupling effects remain between 
steering channels and the different degrees of freedom. NDI control has been implemented in the Lockheed F-35 Lightning II, (Balas (2003); Walker \& Allen (2002)).

Nonlinear dynamic inversion considers original nonlinear systems of the affine form:

$$
\dot{\mathbf{x}}=\mathbf{a}(\mathbf{x})+\mathbf{b}(\mathbf{x}) \mathbf{u}
$$

and provides a solution for the physical control input $\mathbf{u}$ by introducing an outerloop virtual control input $\nu$ :

$$
\mathbf{u}=\mathbf{b}^{-1}(\mathbf{x})[\boldsymbol{\nu}-\mathbf{a}(\mathbf{x})]
$$

which results in a closed-loop system with a decoupled linear input-output relation:

$$
\dot{\mathbf{x}}=\nu
$$

A linear outer loop control law is sufficient to enforce exponentially stable tracking dynamics, where the control gains can be determined by the required closed loop characteristics.

Dynamic inversion is a popular control method for flight control and aircraft guidance, (Balas et al. (1992); Campa et al. (2005); da Costa et al. (2003); Ramakrishna et al. (2001); Reiner et al. (1996); van Soest et al. (2006)) as well as reconfiguring control, (Ganguli et al. (2005; 2006); Oppenheimer \& Doman (2006); Ostroff \& Bacon (2002)).

The main assumption in NDI is that the plant dynamics are assumed to be perfectly known and therefore can be cancelled exactly. However, in practice this assumption is not realistic, not only with respect to system uncertainties but especially to unanticipated failures for the purpose of fault tolerant flight control. In order to deal with this issue, one can make use of robust control methods as outer loop control to minimize or suppress undesired behaviour due to plant uncertainties which cause imperfect plant dynamic cancellation. Other control methods such as neural networks also have been proposed in the literature, in order to augment the control signal as a compensation for the non-inverted dynamics, as explained previously. However, another solution to the weakness of classical NDI, namely its sensitivity to modelling errors, is the use of a real time identification algorithm, which supplies updated model information to the dynamic inversion controller. These augmented structures are called adaptive nonlinear dynamic inversion (ANDI). The latter procedure is the method of choice for this research which led to the results presented in this paper.

\subsection{Autopilot control loops}

Three consecutive inversion loops have been implemented, namely a body angular rate loop, an aerodynamic angle loop and a navigation loop. The developed control laws are described below.

\subsubsection{Body angular rate loop}

Consider the dynamic equation of an aircraft:

$$
\dot{\boldsymbol{\omega}}=\mathbf{I}^{-1} \mathbf{M}_{a}-\mathbf{I}^{-1} \boldsymbol{\omega} \times \mathbf{I} \boldsymbol{\omega}
$$

where $\boldsymbol{\omega}=\left[\begin{array}{lll}p & q & r\end{array}\right]^{T}$ are the rotational rates and $\mathbf{M}_{a}=\left[\begin{array}{lll}L_{a} & M_{a} & N_{a}\end{array}\right]^{T}$ the aerodynamic moments acting on the aircraft. The inertia matrix I stands for:

$$
\mathbf{I}=\left[\begin{array}{ccc}
I_{x x} & -I_{x y} & -I_{x z} \\
-I_{y x} & I_{y y} & -I_{y z} \\
-I_{z x} & -I_{z y} & I_{z z}
\end{array}\right]
$$


where the moments of inertia $I_{x y}, I_{y x}, I_{y z}$ and $I_{z y}$ are assumed to be zero.

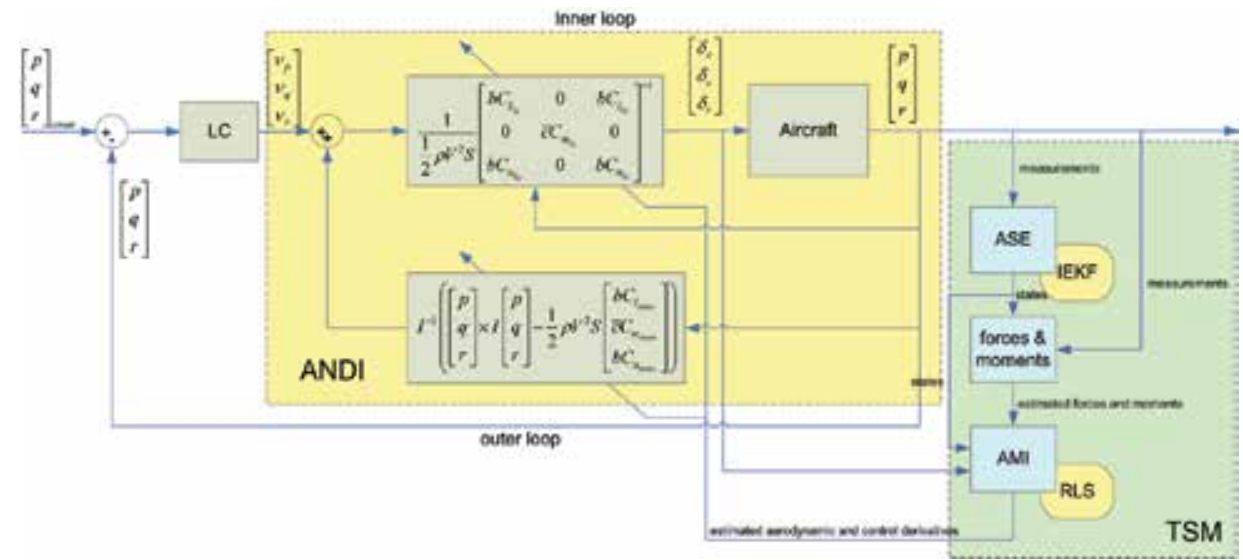

Fig. 6. NDI rate control inner loop

Rewriting the aircraft dynamics into the form of eq. (2):

$$
\begin{aligned}
{\left[\begin{array}{l}
\delta_{a} \\
\delta_{e} \\
\delta_{r}
\end{array}\right]=} & {\left[\begin{array}{ccc}
b \tilde{C}_{l_{\delta_{a}}} & 0 & b \tilde{C}_{l_{\delta_{r}}} \\
0 & \bar{c} \tilde{C}_{m_{\delta_{e}}} & 0 \\
b \tilde{C}_{n_{\delta_{a}}} & 0 & b \tilde{C}_{n_{\delta_{r}}}
\end{array}\right]^{-1} \cdot\left\{\left(\frac{1}{2} \rho V^{2} S\right)^{-1}\right.} \\
& \left.\left(\mathbf{I}\left[\begin{array}{c}
v_{p} \\
v_{q} \\
v_{r}
\end{array}\right]+\left[\begin{array}{c}
p \\
q \\
r
\end{array}\right] \times \mathbf{I}\left[\begin{array}{c}
p \\
q \\
r
\end{array}\right]\right)-\left[\begin{array}{c}
b C_{l_{\text {states }}} \\
\bar{c} C_{m_{\text {states }}} \\
b C_{n_{\text {states }}}
\end{array}\right]\right\}
\end{aligned}
$$

where the virtual inputs $\left[\begin{array}{lll}v_{p} & v_{q} & v_{r}\end{array}\right]^{T}$ are the time derivatives of the rotational rates of the aircraft. All control and state derivatives in this expression are estimated via the real time identification procedure.

\subsubsection{Aerodynamic angle loop}

The middle loop quantities are roll angle $\phi$, angle of attack $\alpha$ and sideslip angle $\beta$ :

$$
\begin{aligned}
{\left[\begin{array}{l}
p \\
q \\
r
\end{array}\right]=} & {\left[\begin{array}{ccc}
1 & \sin \phi \tan \theta & \cos \phi \tan \theta \\
-\frac{v_{b}}{\sqrt{V^{2}-w_{b}^{2}}} & \frac{u_{b}}{\sqrt{V^{2}-w_{b}^{2}}} & 0 \\
\frac{w_{b}}{\sqrt{V^{2}-v_{b}^{2}}} & 0 & \frac{-u_{b}}{\sqrt{V^{2}-v_{b}^{2}}}
\end{array}\right]^{-1} . } \\
& \left\{\left[\begin{array}{c}
v_{\dot{\phi}} \\
v_{\dot{\alpha}} \\
v_{\dot{\beta}}
\end{array}\right]-\left[\begin{array}{cc}
-\frac{1}{\sqrt{V^{2}-w_{b}^{2}}}\left(A_{z}+g \cos \theta \cos \phi\right) \\
\frac{1}{\sqrt{V^{2}-v_{b}^{2}}}\left(A_{y}+g \cos \theta \sin \phi\right)
\end{array}\right]\right\}
\end{aligned}
$$

\subsubsection{Navigation loop}

The procedure used in this step is inspired by the method used in ref. Holzapfel (2004), although the application for this study implies some important deviations compared to the conventional method, since an adaptive model needs to be taken into account. Main crux in this deviating approach is that this inversion loop consists of two separate steps. First, the 
kinematics based virtual inputs are transformed towards the roll angle and the symmetric aerodynamic forces through a physically interpretable nonlinear mapping. Consequently, the aforementioned force components are translated into commanded angle of attack and dimensionless thrust values via a classical NDI-setup as used before, which involves a local gradient determination step.

The kinematics based inversion is as follows:

$$
\begin{aligned}
F_{A_{X}} & =m(\dot{V}+g \sin \gamma) \\
F_{A_{Z}} & =-\cos \gamma \sqrt{m^{2}\left[\left(g+\frac{V \dot{\gamma}}{\cos \gamma}\right)^{2}+(V \dot{\chi})^{2}\right]-\left(\frac{F_{A_{Y}}}{\cos \gamma}\right)^{2}} \\
\mu & =\arctan \left(\frac{\dot{\chi} \cos \gamma}{\dot{\gamma}+g \frac{\cos \gamma}{V}}\right)
\end{aligned}
$$

where $F_{A_{Z}}$ corresponds to $\left|F_{\text {required }}\right|$ and $\mu$ is in fact $\mu_{\text {required }}$.

The subsequent aerodynamic forces based inversion is as follows:

$$
\left(\begin{array}{c}
\alpha \\
T_{C}
\end{array}\right)=\left(\begin{array}{ll}
F_{A_{X_{\alpha}}} & F_{A_{X_{T c}}} \\
F_{A_{Y_{\alpha}}} & F_{A_{Y_{T c}}} \\
F_{A_{Z_{\alpha}}} & F_{A_{Z_{\mathrm{T}}}}
\end{array}\right)^{\dagger}\left[\left(\begin{array}{c}
F_{A_{\mathrm{Xcomm}}} \\
F_{A_{Y}} \\
F_{A_{\mathrm{Zcomm}}}
\end{array}\right)-\left(\begin{array}{c}
F_{A_{\mathrm{X}_{0}}} \\
F_{A_{Y_{0}}} \\
F_{A_{Z_{0}}}
\end{array}\right)\right]
$$

where the symbol + denotes the left inverse.

More information about how these control laws have been derived in detail can be found in ref. Lombaerts (2010); Lombaerts et al. (2010b). Summarizing, the global setup of the third dynamic inversion loop can be found in figure 7 .

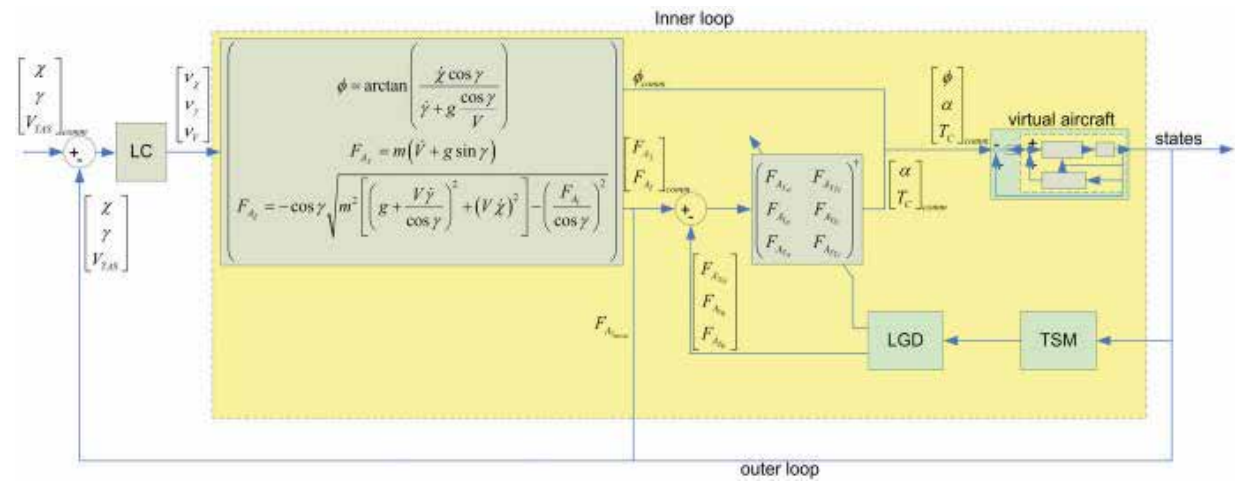

Fig. 7. Third NDI autopilot loop, featuring $V_{T A S}, \gamma$ and $\chi$ control. LGD stands for local gradient determination. TSM represents the two step method model identification block elaborated in section 4 .

Linear controllers act on each separate NDI loop, as indicated by "LC" in fig. 6 and 7. These linear controllers involve proportional and proportional-integral control, and gains have been selected to ensure favourable flying qualities by means of damping ratio $\zeta$ and natural frequency $\omega_{n}$ while complying with the time scale separation principle. Optimization of these gain values has been achieved by means of multi-objective parameter synthesis (MOPS) optimization, see ref. Lombaerts (2010); Looye (2007); Looye \& Joos (2006). 


\subsection{Evaluations of autopilot experiments on RECOVER}

Implementation of these control laws results in the controller performance illustrated in the following simulation results. Besides the unfailed scenario the following failures have been investigated: vertical tail loss and engine separation. In each scenario, the failure occurs exactly at $t=50$ s.

\subsubsection{Nominal unfailed}

A complex manoeuvre is performed to evaluate the performance of the fault tolerant controller. Three joint commands are given, more precisely a course change of $\Delta \chi=160^{\circ}$ at $t=70 \mathrm{~s}$, an altitude change towards $h=5000 \mathrm{~m}$ at $t=80 \mathrm{~s}$ and a velocity change of $\Delta V=50 \mathrm{~m} / \mathrm{s}$ around $t=110 \mathrm{~s}$. This scenario allows to evaluate the performance as well as eventual inadvertent couplings between the different channels. The tracking of the reference signals is satisfactory and couplings are minimal to even non-existent, as can be seen in fig. 8. In the control surface deflection time histories in figures 9 and 10(a), it can be seen that the elevators compensate first for the vertical lift component loss induced by the turn, and then initiate the altitude change. Ailerons and rudders cooperate in order to achieve a coordinated turn. It can be seen that the spoilers assist the ailerons to set the turn up. The specific forces in fig. 10(b) indicate an acceptable behaviour. The responses of the unfailed aircraft will serve as a comparison basis to evaluate the performance of the controller in failed situations.

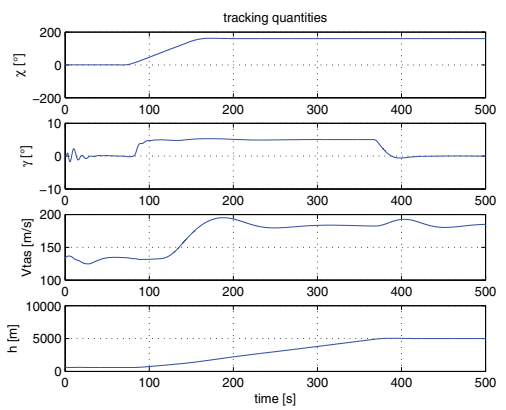

(a) tracking quantities

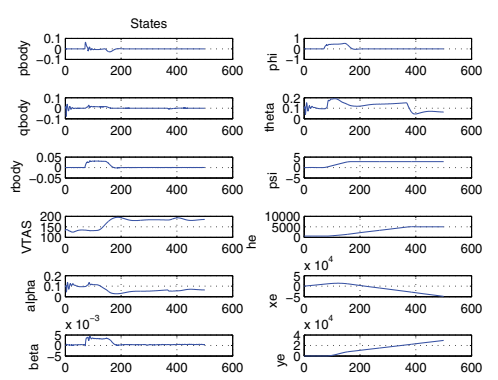

(b) states

Fig. 8. Tracking quantities and states for the unfailed scenario
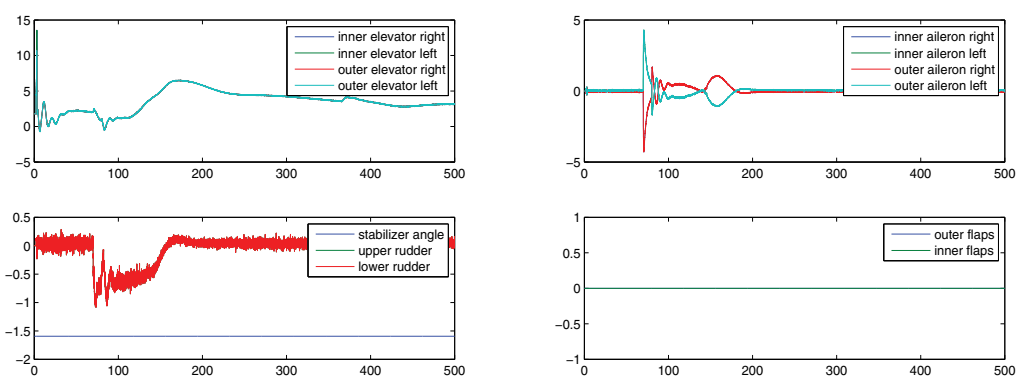

(a) deflections of elevators, stabilizer and rudders

(b) deflections of ailerons and flaps

Fig. 9. Deflections of elevators, stabilizer, rudders, ailerons and flaps for the unfailed scenario 

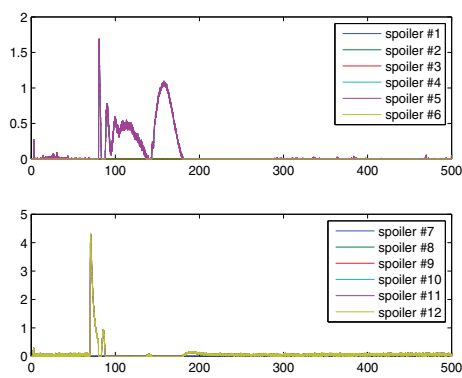

(a) deflections of spoilers
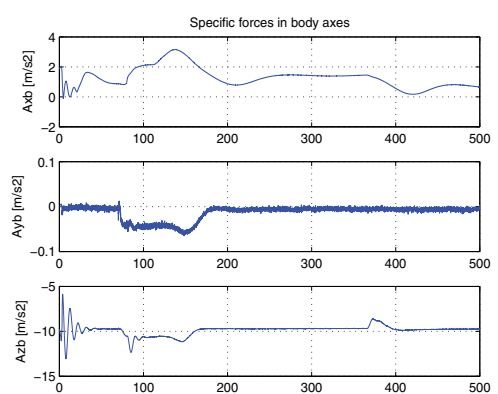

(b) specific forces

Fig. 10. Deflections of spoilers and specific forces for the unfailed scenario

\subsubsection{Vertical tail loss}

For this failure scenario, the reference signals have been slightly adapted. No speed change is requested here, because this makes it more demanding for the control surfaces. When the speed increase is maintained, the increasing aerodynamic damping reduces the disturbing effect of the failure. The altitude capture has been changed accordingly to $1500 \mathrm{~m}$, in order to prevent throttle saturation. Aerodynamic damping is the very reason why, in practice, control laws actually work with calibrated airspeed CAS, in this way the control actions are related to the dynamic pressure and control surface efficiency is maintained.

Figure 12(b) reveals the compensating behaviour of the ailerons which takes into account the loss of the vertical tail and the cessation of rudder functioning after failure, as can be seen in fig. 12(a). It can be observed that the ailerons effectively take over the function of yaw damper, assisted by the spoilers in fig. 13(a), since the rudders are lost. Figure 13(b) shows that the oscillation in the lateral specific force $A_{y}$ is only damped in the longer term, which can be explained by the fact that ailerons and spoilers have no direct influence on the yawing moment, but on the rolling moment.

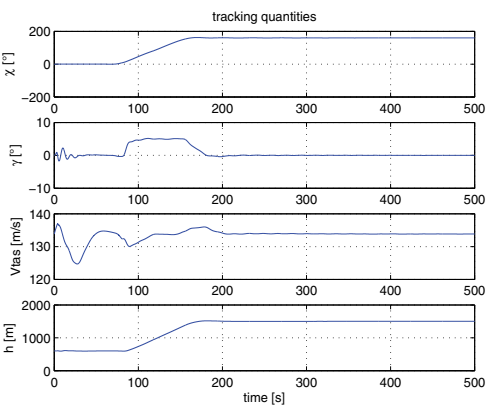

(a) tracking quantities

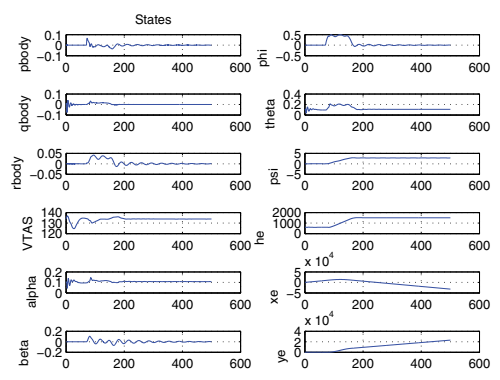

(b) states

Fig. 11. Tracking quantities and states for the tail loss scenario

\subsubsection{Engine separation}

The engine separation scenario is a very sensitive situation to combine commands in heading, altitude and speed simultaneously. Crucial in this context is to avoid engine throttle saturation. Therefore, in this experiment only a heading change has been considered, as shown 

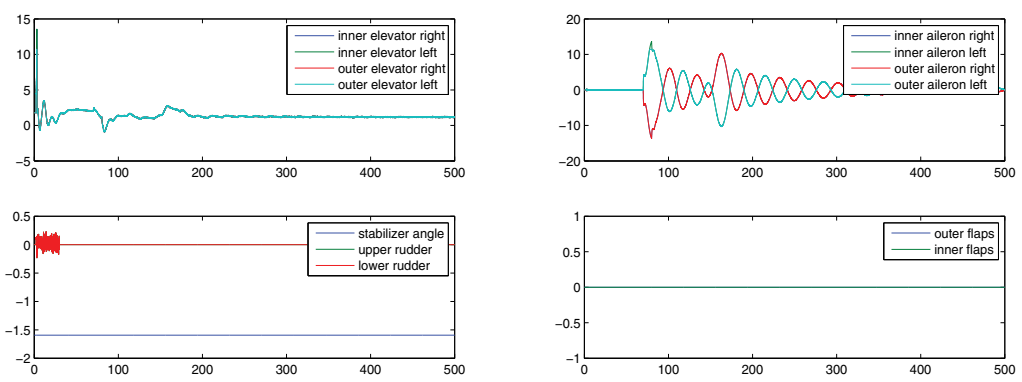

(a) deflections of elevators, stabilizer and rudders

(b) deflections of ailerons and flaps

Fig. 12. Deflections of elevators, stabilizer, rudders, ailerons and flaps for the tail loss scenario
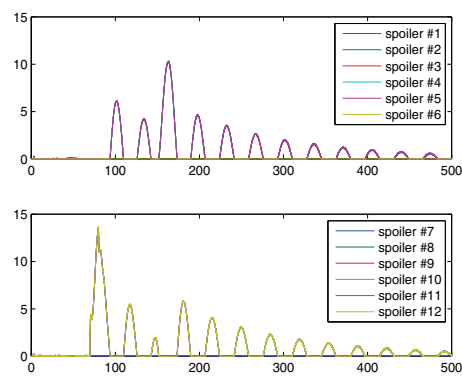

(a) deflections of spoilers
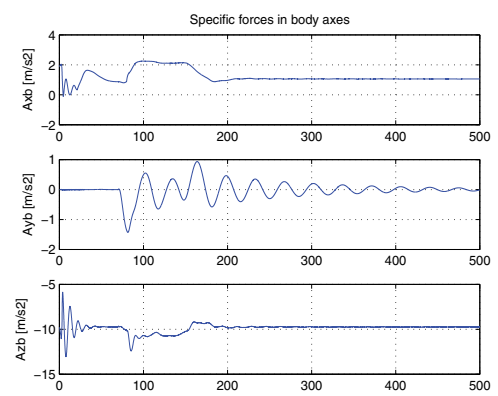

(b) specific forces

Fig. 13. Deflections of spoilers and specific forces for the tail loss scenario

in fig. 14(a). Moreover, a limited maximum roll angle has been imposed, due to the restricted safe flight envelope as explained in section 3. It has been found that altitude and speed changes are also feasible separately, but these are not discussed in this section.

The time histories of the states in fig. 14(b) reveal that the aircraft in post failure conditions flies with a small nonzero roll angle and sideslip angle, due to the asymmetric damage, despite a zero commanded sideslip angle. The control surface deflections in figures 15 and 16(a) confirm the cessation of functioning of the control surfaces which are powered by the hydraulic circuits connected to engines number 3 and 4 , as illustrated in fig. 2(b). The remaining operative surfaces are successful in keeping the aircraft in equilibrium and under control, although with restricted authority. The nonzero lateral specific force in fig. 16(b) is a consequence of the sideslipping flight.

Two additional interesting quantities to investigate are the throttle setting and the average square innovation, which triggers the re-identification routine as explained in ref. Lombaerts et al. (2009; 2010a). Figure 17(a) confirms that the throttle setting does not saturate, however the remaining control margins in order to remain inside the safe flight envelope are severely restricted. This is due to the asymmetric thrust which needs to be compensated by the control surfaces. The spike at $t=50 \mathrm{~s}$ is caused by the feedforward path in the controller, which is needed to compensate for the instantaneous speed loss of the two dead engines. Figure 17(b) depicts the values for the average square innovation for each force and moment channel separately. At $t=50$ s, it can be seen that the threshold for $\bar{\Delta}_{X}$ is exceeded, and a 


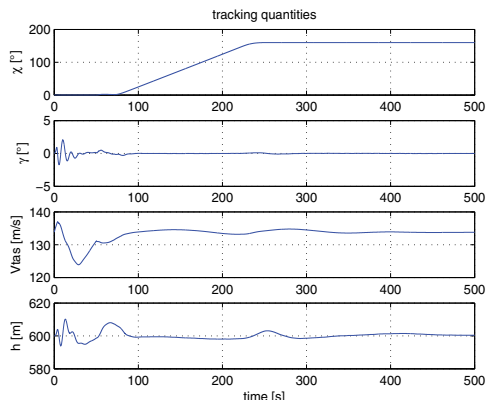

(a) tracking quantities

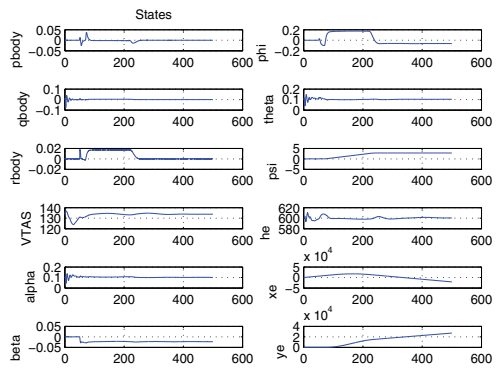

(b) states

Fig. 14. Tracking quantities and states for the engine separation scenario
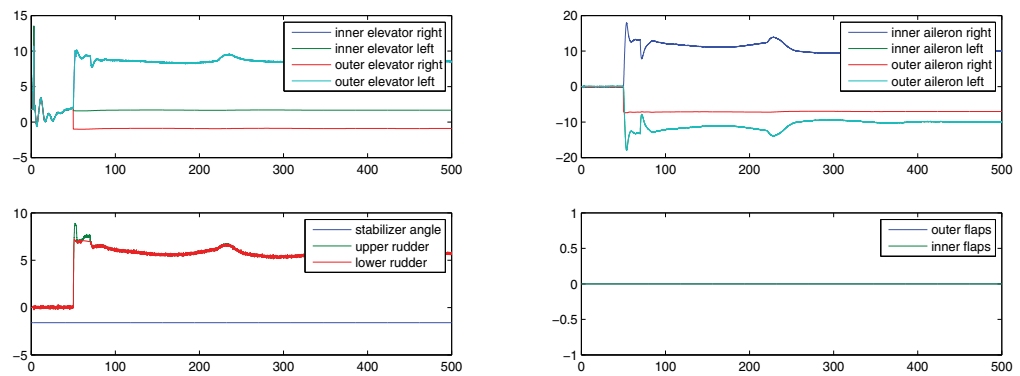

(a) deflections of elevators, stabilizer and rudders

(b) deflections of ailerons and flaps

Fig. 15. Deflections of elevators, stabilizer, rudders, ailerons and flaps for the engine separation scenario
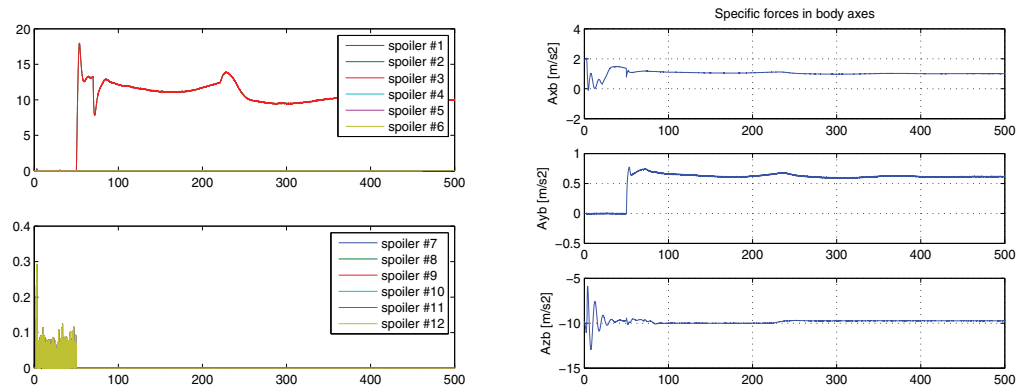

(a) deflections of spoilers

(b) specific forces

Fig. 16. Deflections of spoilers and specific forces for the engine separation scenario 
re-identification procedure is triggered for $C_{X}$. It has become necessary to include the sideslip angle $\beta$, which has become significant due to the sideslipping flight, as an additional regressor in the identification procedure. This leads to a successful new identification procedure which is performed extremely quickly as can be seen in this figure. This result confirms the beneficial contribution from the identification routine in this fault tolerant flight control setup.

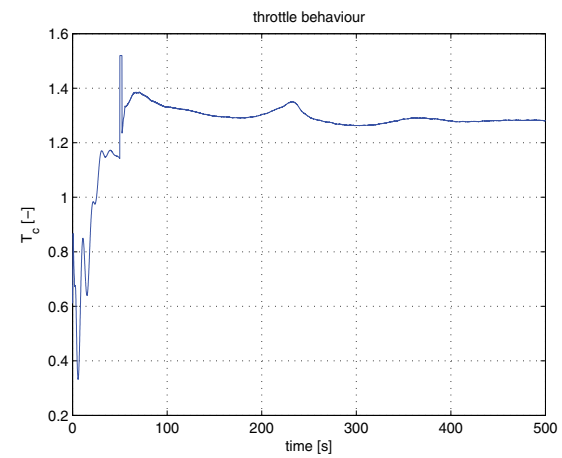

(a) throttle behaviour

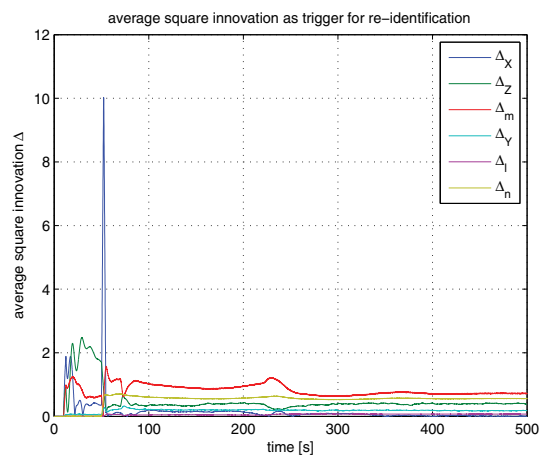

(b) average square innovation as trigger for re-identification

Fig. 17. Spoilers and specific forces for the engine separation scenario

\subsection{Manual control loops}

A manual variant of this fault tolerant controller has been developed as well. This variant consists of the body angular rate inner loop as described in section 5.2.1, augmented by the sideslip $\beta$ coordination axis only from the aerodynamic angle middle loop as explained in section 5.2.2. Throttle control is by the conventional autothrottle. As a result, the pilot steers roll rate $p$ by means of the control wheel, pitch rate $q$ with the control columns, and finally the pedals can be used for creating a nonzero sideslipping flight, although this is rarely used. Since dynamic inversion is used in all control loops, these steering channels are effectively decoupled.

\subsection{Simulator evaluation of manual controller}

This manual control setup has been applied in the SIMONA (SImulation, MOtion and NAvigation) Research Simulator (SRS), see fig.18(a). It is a pilot-in-the-loop flight simulator developed, built and operated by Delft University of Technology. It provides researchers with a flexible powerful tool that can be adapted to various uses, see ref. Stroosma et al. (2003). The simulator's flexible software architecture and high-fidelity cueing environment allows the integration of a variety of aircraft simulation models, such as the aforementioned Boeing 747 benchmark simulation model from ref. Smaili et al. (2006). Its inputs and outputs were standardized to fit the SRS software environment and the SIMULINK ${ }^{\mathrm{TM}}$ simulation model as well as NDI-controller were converted to C code using Real-Time Workshop. Finally the models were integrated with the pilot controls, aircraft instruments (Figure 18(b)) and other cueing devices of the SRS (i.e. outside visual and motion systems). On the flight deck of the SRS the evaluation pilot was presented with flight instruments representative of a large transport aircraft, a control column with large transport aircraft feel system dynamics, a central pedestal with dual engine controls and a wide collimated view on a virtual outside world. The simulator's motion system was tuned to give the pilot realistic inertial motion 
cues in nominal and failure conditions. The test pilots were four Boeing 747 captains (one retired) and one other wide body captain on Airbus A330 and Boeing 767. All were familiar with the research simulator practices used for this investigation.

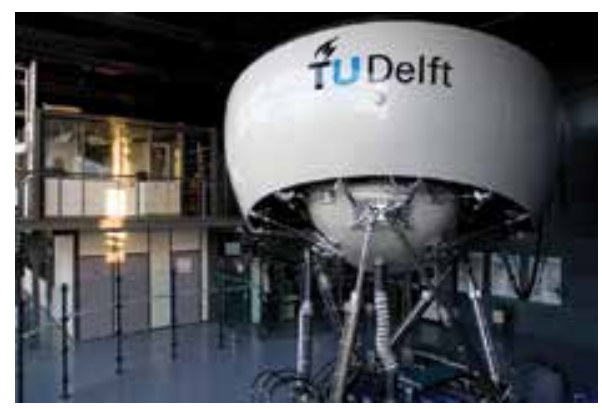

(a) outside view

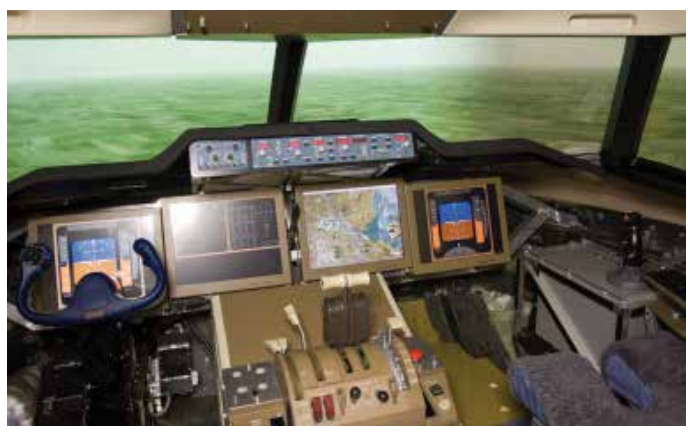

(b) cockpit view

Fig. 18. The SIMONA (SImulation, MOtion and NAvigation) Research Simulator (SRS) at Delft University of Technology, photo by Joost Ellerbroek

The adaptive NDI control system has been validated on two failure scenarios, namely the engine separation failure and the rudder runaway scenarios. Fig. 19 shows the evaluation trajectory during the piloted simulation runs in SIMONA. The trajectory consists of four main phases, namely altitude capture, bank angle capture, localizer intercept and glideslope intercept. For every phase, required and adequate performance specifications have been defined for the relevant longitudinal as well as lateral quantities. The scheme presented in fig. 20 assists the pilot in rating the handling qualities (Cooper \& Harper (1969)) of the aircraft while taking into account the performance of the aircraft with respect to the aforementioned requirements. Fig. 21 shows the time histories of a selection of the most important aircraft states. These confirm the evaluation trajectory as shown in fig. 19. Moreover, altitude and roll angle plots show altitude and roll angle captures which have been executed by the test pilot in order to evaluate the post-failure handling qualities of the aircraft.

The handling qualities results for the algorithm show that, especially for the El Al Flight 1862 scenario, conventional flight control was restored to acceptable levels while physical and mental workload were reduced significantly. This is illustrated in Figure 22 where an example is given of lateral handling quality pilot ratings for the localizer capture task. It can be seen that, for this task, both the baseline and fault-tolerant fly-by-wire (FBW) aircraft were rated Level 1 (Rating 1-3). After separation of the right-wing engines (Figure 22), lateral handling qualities degraded to Level 2 for the conventional aircraft with the classical control system. The reconfigured aircraft (FBW) shows about Level 1 handling qualities after incurring significant damage due to the loss of the right-wing engines. This was substantiated by measured pilot control activities, representative of workload, indicating no pilot compensation after reconfiguration. For the rudder runaway failure, however, Level 2 handling qualities remained after reconfiguration despite the fact that no sustained pilot compensation was required. The difference was most probably caused by the fact that this initial setup is a rate control and hold loop instead of a rate control attitude hold type. As a consequence, angular rate disturbances are corrected for automatically by the controller but subsequent disturbances from the equilibrium attitude had to be compensated for by the pilot himself. The use of a rate control attitude hold setup will solve this issue.

Figure 23 illustrates the physical workload analysis results by depicting the average pilot forces. In the graph, a distinction is made between roll, pitch and yaw channel, as illustrated 


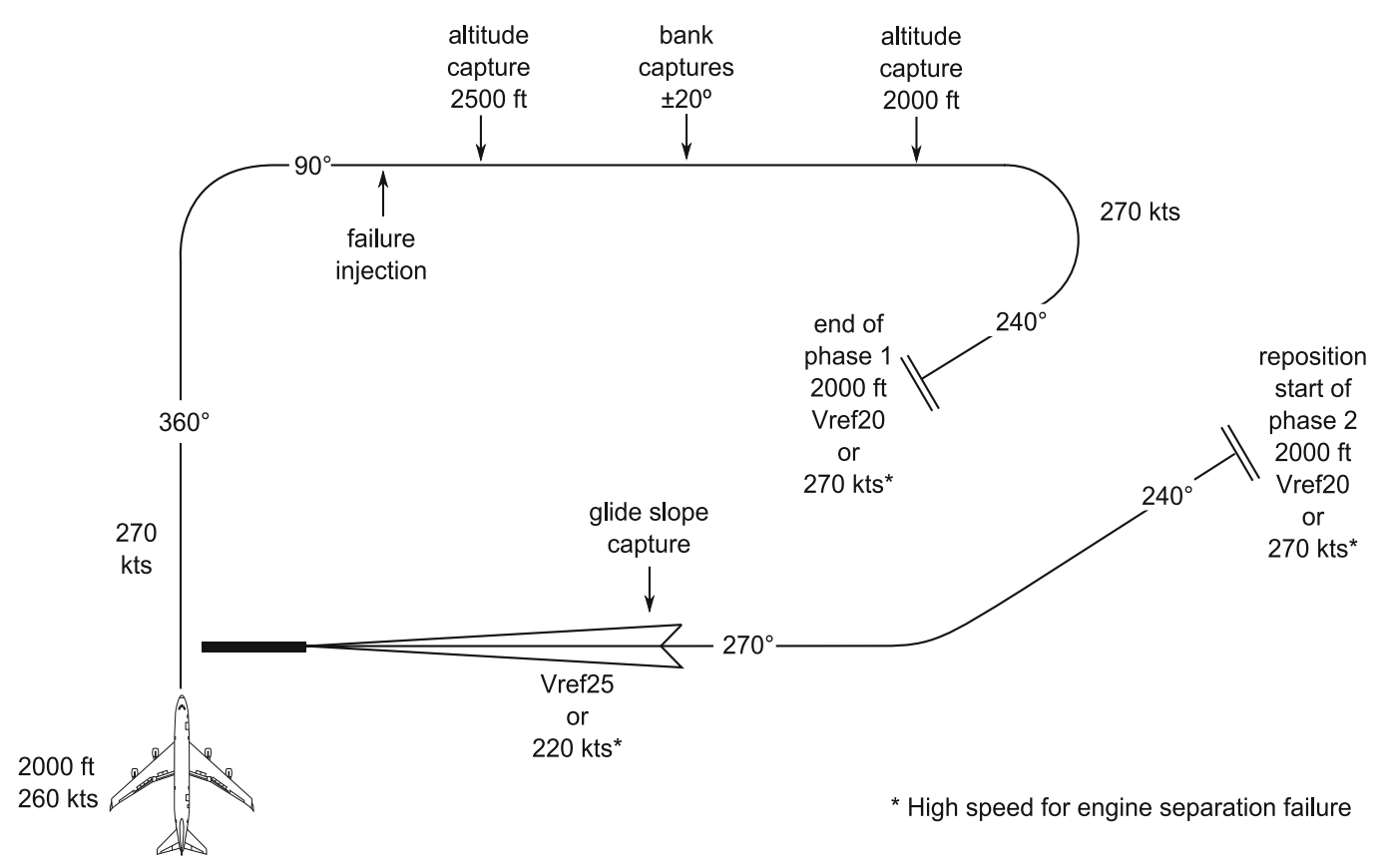

Fig. 19. Trajectory of the piloted simulation runs in SIMONA.

by the three graphs separated vertically. In each control channel, six cases have been studied, namely unfailed, engine separation and rudder runaway, each time with classical and fault tolerant control. In each case, the workload figure of each of the five pilots is represented individually by means of bar plots, after which the mean and standard deviations are superimposed on these bar plots for every case, in order to facilitate mutual comparisons.

First of all, the unfailed conditions confirm that this is a good comparison basis between classic and FTFC, since both have the same ratings. Comparing classic control with FTFC for failed configurations shows that overall values for average manual control forces over all pilots decrease for FTFC in the failure scenarios. In addition, in the failure scenarios the standard deviations also reduce from classic control towards FTFC. At first sight this seems not the case for the pedal forces. Closer inspection of the experimental data, however, reveals that this is caused by the deviating performance of pilot no 2 (probably due to misconception of the control principle within the fault tolerant controller). Finally, searching for overlap of the errorbars between classic and FTFC shows that this overlap does not occur. This observation makes the trends significant, despite the limited number of experiment subjects.

As a global conclusion, which is supported by the graphs above, it can be stated that this fault tolerant flight controller improves the handling qualties and reduces physical pilot workload considerably in failure conditions.

\section{Conclusions and future work}

Summarizing, it can be stated that, following numerous experiments, fault tolerant flight control using a physical modular approach is successful in recovering damaged aircraft. The designed methods are capable to accommodate the damage scenarios which have been investigated in this project. It has been found that the engine separation scenario, based upon 


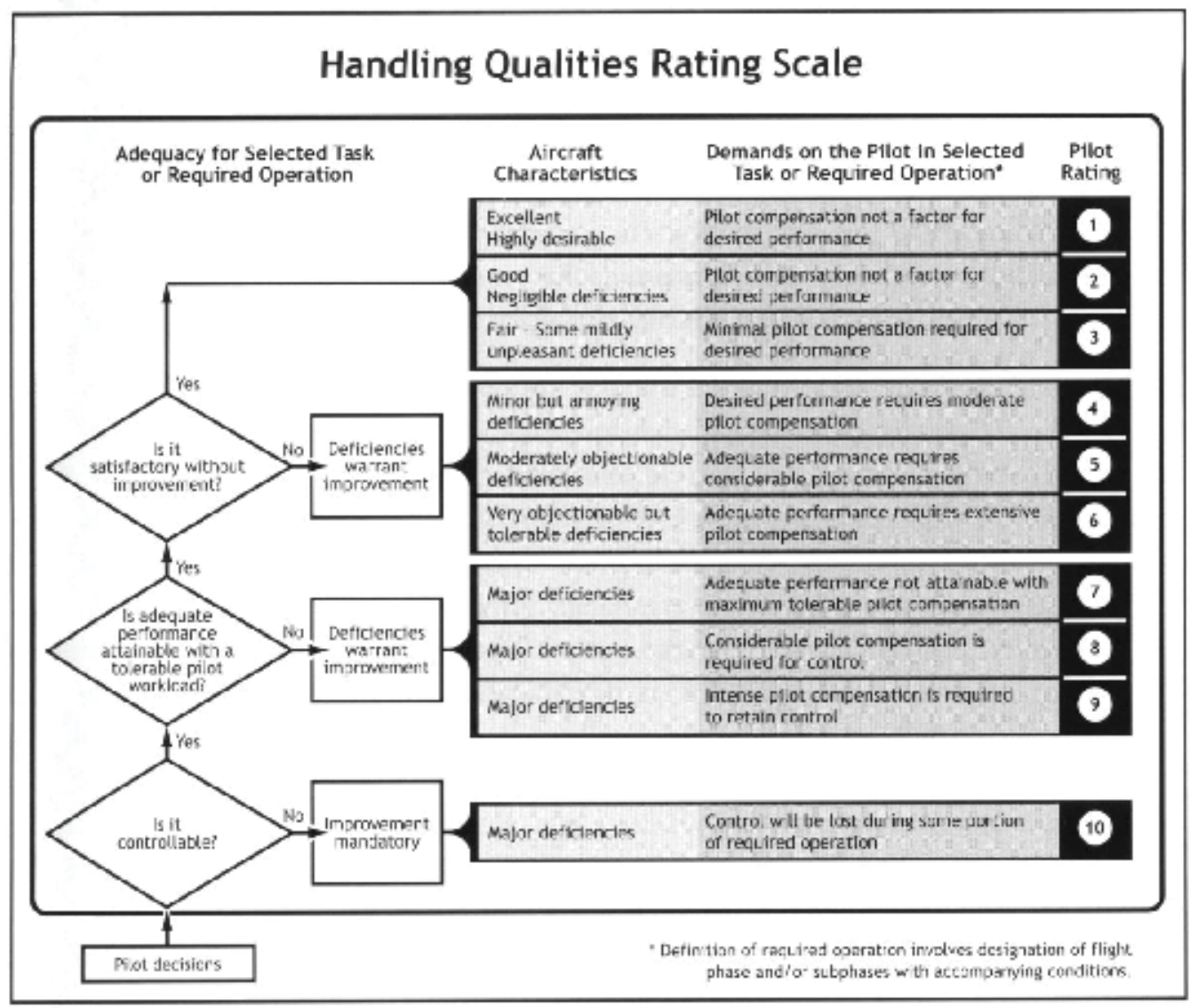

Fig. 20. Cooper Harper Handling Qualities Rating Scale, source: Cooper \& Harper (1969)
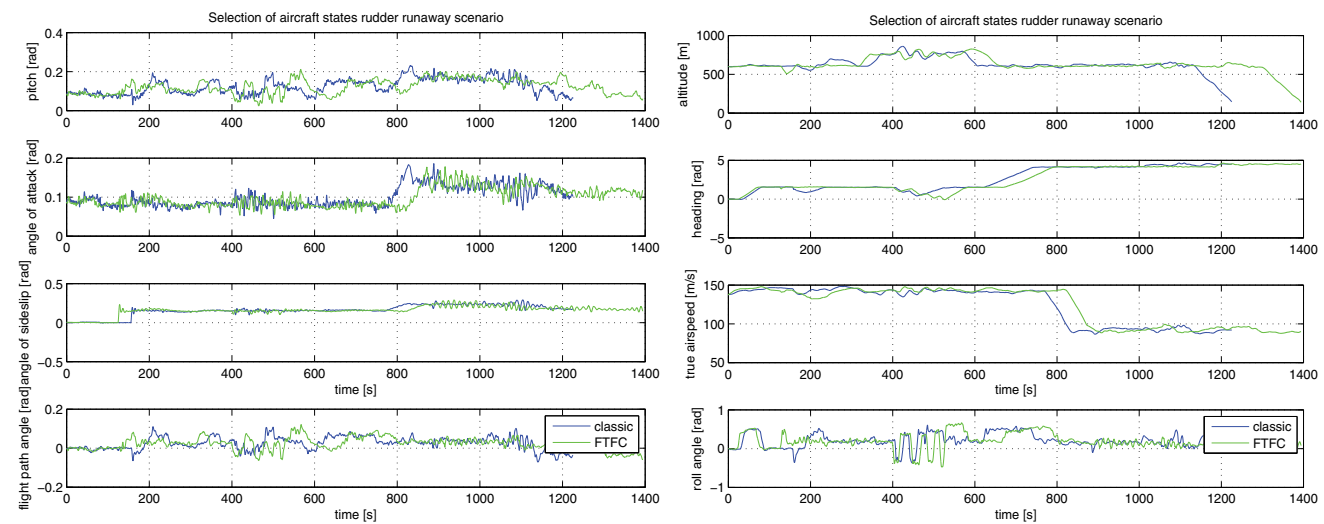

Fig. 21. Comparison of a selection of aircraft states for the rudder runaway scenario 


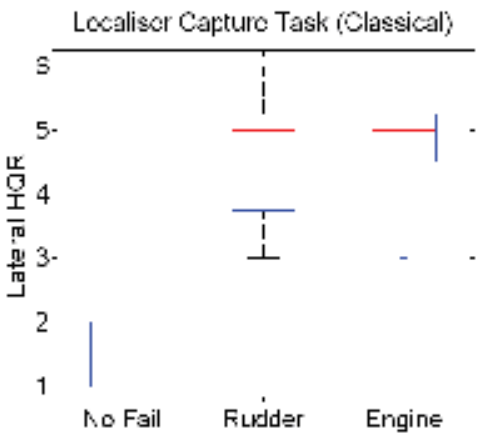

(a) classical control

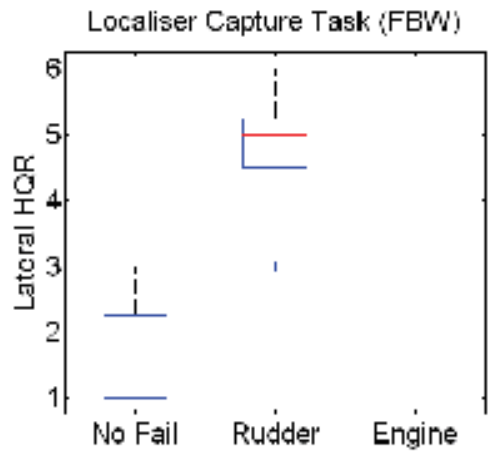

(b) fault tolerant control

Fig. 22. Localizer capture task handling qualities ratings for classical control and fault tolerant control
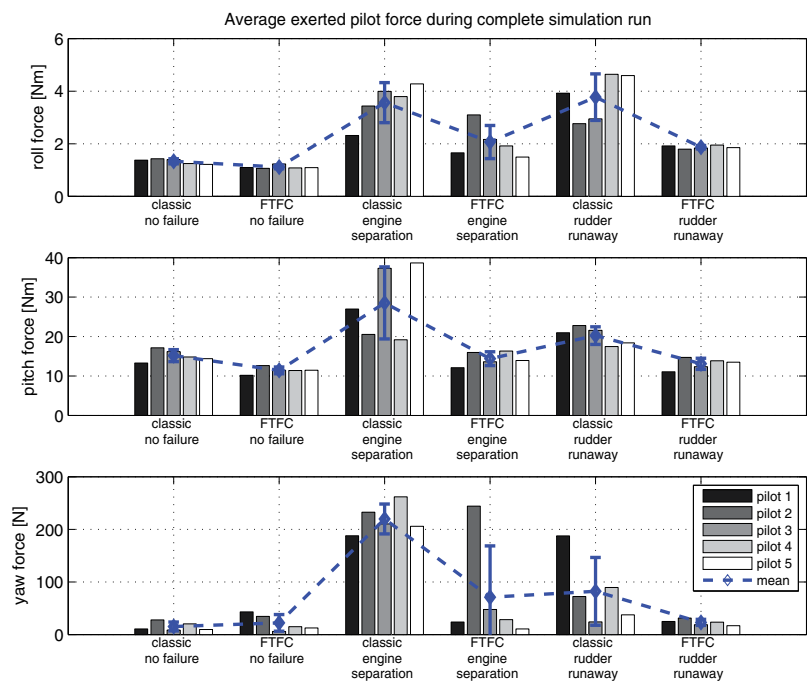

Fig. 23. Total average manual control forces during the simulation runs

El Al flight 1862, is survivable with adaptive control techniques. Experiments have also shown that the two step method is successful for real time identification of damaged aircraft models, including a real time static stability analysis. Autopilot control based upon adaptive nonlinear dynamic inversion shows good failure handling capabilities.

An important aspect which has not been considered in this research is sensor loss detection. Despite the presence of redundant sensors, recent aircraft accidents (Lombaerts (2010)) have shown that sensor loss detection cannot be avoided and current monitoring techniques are not always sufficient. More elaborate flight envelope protection algorithms, taking into account a.o. minimum control airspeed limits, are another important topic for future research. Finally, an important next step in the development of fault tolerant flight control technologies is to validate them in real flight on board of manned as well as unmanned research aircraft. This is one of the major challenges for the future. 


\section{References}

Alwi, H. (2008). Fault Tolerant Sliding Mode Control Schemes with Aerospace Applications, PhD thesis, University of Leicester.

Balas, G. (2003). Flight control law design: An industry perspective, European Journal of Control, special issue 9(2-3): 207-226.

Balas, G., Garrard, W. \& Reiner, J. (1992). Robust dynamic inversion control laws for aircraft control, Proceedings of the AIAA Guidance, Navigation and Control Conference, AIAA, Washington, DC, pp. 192-205.

Brinker, J. \& Wise, K. (1999). Nonlinear simulation analysis of a tailless advanced fighter aircraft reconfigurable flight control law, AIAA Guidance, Navigation and Control Conference and Exhibit, number AIAA-99-4040, Portland, OR.

Calise, A. J., Lee, S. \& Sharma, M. (2001). Development of a reconfigurable flight control law for the $\mathrm{x}-36$ tailless fighter aircraft, AIAA Journal of Guidance, Control and Dynamics 24(5): 896-902.

Campa, G., Seanor, B., Gu, Y. \& Napolitano, M. R. (2005). Nldi guidance control laws for close formation flight, American Control Conference, Portland, OR, USA.

Chu, Q. (2007). Lecture Notes AE4-394, Modern Flight Test Technologies and System Identi cation, Delft University of Technology, Faculty of Aerospace Engineering.

Chu, Q., Mulder, J. \& Sridhar, J. (1994). Decomposition of aircraft state and parameter estimation problems, Proceedings of fhe 10th IFAC Sympium on System Identi ation, Vol. Vol. 3, pp. pp. 61-66.

Cieslak, J., Henry, D., Zolghadri, A. \& Goupil, P. (2008). Development of an active fault-tolerant flight control strategy, AIAA Journal of Guidance, Control and Dynamics 31: 135-147.

Civil Aviation Safety Data 1993-2007 (2008). Technical report, Civil Aviation Authority of the Netherlands (CAA-NL).

Cooper, G. \& Harper, R. J. (1969). The use of pilot rating in the evaluation of aircraft handling qualities, Technical Report TN D-5153, NASA.

Corvin, J. H., Havern, W. J., Hoy, S. E., Norat, K. F., Urnes, J. M. \& Wells, E. A. (1991). Self-repairing flight control systems, volume i: Flight test evaluation on an f-15 aircraft, Final Report WL-TR-91-3025.

da Costa, R., Chu, Q. P. \& Mulder, J. A. (2003). Re-entry flight controller design using nonlinear dynamic inversion, Journal of Spacecraft and Rockets 40(1): 64-71.

Ganguli, S., Papageorgiou, G., Glavaski, S. \& M., E. (2005). Piloted simulation of fault detection, isolation and reconfiguration algorithms for a civil transport aircraft, AIAA Guidance, Navigation and Control Conference and Exhibit, number AIAA-2005-5936, San Francisco, CA.

Ganguli, S., Papageorgiou, G., Glavaski, S. \& M., E. (2006). Aircraft fault detection, isolation and reconfiguration in the presence of measurement errors, AIAA Guidance, Navigation and Control Conference and Exhibit, number AIAA-2006-6551, Keystone, Co.

Gavrilets, V. (2008). Damage tolerant flight control systems for unmanned aircraft, ICAS/ATIO Conference.

Hallouzi, R. \& Verhaegen, M. (2008). Fault-tolerant subspace predictive control applied to a boeing 747 model, AIAA Journal of Guidance, Control and Dynamics 31: 873-883.

Holzapfel, F. (2004). Nichtlineare adaptive Regelung eines unbemannten Fluggerätes, PhD thesis, Lehrstuhl für Flugmechanik und Flugregelung, Technische Universität München.

Intelligent Flight Control: Advanced Concept Program (1999). Final Report BOEING-STL 99P0040, The Boeing Company. 
Jategaonkar, R. (2006). Flight Vehicle System Identi cation: A Time Domain Methodology, Vol. 216 of Progress in Astronautics and Aeronautics Series, first edn, AIAA.

Joosten, D., van den Boom, T. \& Lombaerts, T. (2007). Effective control allocation in fault-tolerant flight control with mpc and feedback linearization, Proceedings of the European Conference on Systems and control, Kos, Greece, pp. 3552-3559.

Joosten, D., van den Boom, T. \& Lombaerts, T. (2008). Fault-tolerant control using dynamic inversion and model-predictive control applied to an aerospace benchmark, the Proceedings of the 17th IFAC world congress, Vol. 17, pp. 12030-12035.

KrishnaKumar, K. \& Gundy-Burlet, K. (n.d.). Intelligent control approaches for aircraft applications, Technical report, NeuroEngineering Laboratory, NASA Ames Research Center.

Laban, M. (1994). Online aircraft aerodynamic model identi cation, PhD thesis, Delft University of Technology.

Lombaerts, T. (2010). Fault Tolerant Flight Control. A Physical Model Approach, PhD thesis, Delft University of Technology.

Lombaerts, T., Breeman, J., Joosten, D., van den Boom, T., Chu, Q., Mulder, J. \& Verhaegen, M. (2005). Specifications modelling document for Garteur AG16 fault tolerant control, Technical report, Delft University of Technology.

Lombaerts, T., Chu, Q., Mulder, J. \& Joosten, D. (2007). Real time damaged aircraft model identification for reconfiguring control, Proceedings of the AIAA AFM Conference and Exhibit, number AIAA-2007-6717, Hilton Head, SC.

Lombaerts, T., Chu, Q.-P., Mulder, J. A. \& Joosten, D. (2009). Flight control reconfiguration based on a modular approach, Proceedings of the 7th IFAC SAFEPROCESS Symposium on Fault Detection, Supervision and Safety of Technical Processes, pp. 259-264.

Lombaerts, T., Chu, Q.-P., Mulder, J. A. \& Joosten, D. (2010a). Flight control reconfiguration based on a modular approach, Control Engineering Practice . under review.

Lombaerts, T., Joosten, D., J.H.Breemand, Smaili, H., Chu, Q., van den Boom, T., Mulder, J. \& Verhaegen, M. (2006). Assessment criteria as specifications for reconfiguring control, proceedings of the AIAA Guidance, Navigation, and Control Conference and Exhibit, number AIAA-2006-6331, Keystone, CO.

Lombaerts, T., Looye, G., Chu, Q. P. \& Mulder, J. A. (2010b). Pseudo control hedging and its application for safe flight envelope protection, AIAA Guidance, Navigation and Control Conference and Exhibit.

Lombaerts, T., Van Oort, E., Chu, Q. P., Mulder, J. A. \& Joosten, D. (2010c). Online aerodynamic model structure selection and parameter estimation for fault-tolerant control, Journal of Guidance, Control and Dynamics . to be published.

Looye, G. (2007). An Integrated Approach to Aircraft Modelling and Flight Control Law Design, PhD thesis, Delft University of Technology.

Looye, G. \& Joos, H.-D. (2006). Design of autoland controller functions with multiobjective optimization, AIAA Journal of Guidance, Control and Dynamics 29(2): 475-484.

Maciejowski, J. \& Jones, C. N. (2003). Mpc fault-tolerant flight control case study: Flight 1862, Proceedings of the 5th IFAC Symposium on Fault Detection, Supervision and Safety of Technical Processes SAFEPROCESS, Washington DC, USA, pp. 121-126.

Marcos, A. \& Balas, G. (2003). A boeing 747Ü100/200 aircraft fault tolerant and diagnostic benchmark, Technical Report AEMUUoMU2003U1, Department of Aerospace and Engineering Mechanics, University of Minnesota.

Morelli, E. (2000). Real-time parameter estimation in the frequency domain, Journal of Guidance, Control and Dynamics 23(5): 812-818. 
Mulder, J. (1986). Design and evaluation of dynamic ight test manoeuvers, PhD thesis, TU Delft, Faculty of Aerospace Engineering.

Mulder, J., Chu, Q., Sridhar, J., Breeman, J. \& Laban, M. (1999). Non-linear aircraft flight path reconstruction review and new advances, Progress in Aerospace Sciences 35(7): 673-726.

Mulder, J., van Staveren, W., van der Vaart, J. \& de Weerdt, E. (January 2006). Lecture Notes AE3-302, Flight Dynamics, Delft University of Technology, Faculty of Aerospace Engineering, Delft, The Netherlands.

Oppenheimer, M. \& Doman, D. (2006). Efficient reconfiguration and recovery from damage for air vehicles, Proceedings of the AIAA Guidance, Navigation and Control Conference and Exhibit, number AIAA-2006-6552.

Ostroff, A. J. \& Bacon, B. J. (2002). Enhanced ndi strategies for reconfigurable flight control, Proceedings of the American Control Conference, Anchorage, AK.

Ramakrishna, V., Hunt, L. \& Meyer, G. (2001). Parameter variations, relative degree, and stable inversion, Automatica 37: 871-880.

Reiner, J., Balas, G. J. \& Garrard, W. L. (1996). Flight control design using robust dynamic inversion and time-scale separation, Automatica 32(11): 1493-1504.

Smaili, H., Breeman, J. \& Lombaerts, T. (2008). A simulation benchmark for aircraft survivability assessment, Proceedings of the International Congress of Aeronautical Sciences, number 2008-9.3.2.

Smaili, M., Breeman, J., Lombaerts, T. \& Joosten, D. (2006). A simulation benchmark for integrated fault tolerant flight control evaluation, Proceedings of the AIAA Modelling and Simulation Technologies Conference and Exhibit, number AIAA-2006-6471.

Stroosma, O., van Paassen, M. \& Mulder, M. (2003). Using the simona research simulator for human-machine interaction research, AIAA Modeling and Simulation Technologies Conference.

Szaszi, I., Ganguli, S., Marcos, A., Balas, G. J. \& Bokor, J. (2002). Application of fdi to a nonlinear boeing 747 aircraft, 10th Mediterranean Conference on Control and Automation, Lisbon, Portugal.

van Soest, W., Chu, Q. P. \& Mulder, J. A. (2006). Combined feedback linearization and model predictive control for re-entry flight, AIAA Journal of Guidance, Control and Dynamics 29(2): 427-434.

Varga, A. (2007). Design of least order residual generators for fault detection and isolation with application to monitoring actuator/surface faults for a boeing 747-100/200 aircraft, Technical report, German Aerospace Center (DLR).

Varga, A. \& Hecker, S. (2004). Methods for threshold selection for robust residual evaluation, Technical report, German Aerospace Center (DLR).

Walker, G. \& Allen, D. (2002). X-35b stovl flight control law design and flying qualities, Proceedings of the Biennial International Powered Lift Conference and Exhibit, number AIAA-2002-6018.

Ward, D. \& Barron, R. (1995). A self-designing receding horizon optimal flight controller, Proceedings of the American Control Conference, Seattle, Washington. 


\title{
Design of Intelligent Fault-Tolerant Flight Control System for Unmanned Aerial Vehicles
}

\author{
Yuta Kobayashi and Masaki Takahashi \\ Keio University \\ Japan
}

\section{Introduction}

Recently, unmanned aerial vehicles (UAVs) have gained worldwide attention. Because the safety of people on board does not need to be considered, small UAVs can easily be made for low-cost. Therefore, a UAV can be used to observe disasters, to surveil for a long time, and so on. However, it also has several disadvantages such as unreliability and worse performance in unexpected situations. Because small UAVs must be easily made for lowcost, adding a redundant on-board actuator or sensor in order to deal with unexpected situations is unsuitable. Thus, several researchers have proposed a flight control system using a software redundancy approach.

For fault detection, methods using multiple-model adaptive estimation (MMAE) (Guillaume Ducard \& Hans P. Geering, 2008), and system parameters (Mohammad Azam et al, 2005) have been proposed. However, because these methods design a model or parameters for only each assumed fault in designing, unexpected faults cannot be detected. On the other hand, another method discriminates between faults and natural disturbances like gusts of wind. (Jovan D. Boskobic et al, 2005) However, this is not easy because the expected disturbances are assumed in designing. Currently, the demand for a UAV flight control system is to discriminate between faults and natural disturbances fundamentally with a simple algorithm.

In this research, an intelligent flight control system was developed that can discriminate between faults and natural disturbances in order to evaluate and deal with the situation. In the proposed control system, an evaluator of flight conditions was designed on the basis of the dynamics of a controlled object. Moreover, to deal with the situation adaptively, a new flight-path-planning generator was introduced on the basis of the evaluation. In this study, each subsystem was designed by a neural network. Moreover, the learning-based systematical design method was developed that uses evaluation functions for the subsystems. To verify the effectiveness of the proposed flight control system, a six-degreeof-freedom nonlinear simulation was carried out.

\section{Aircraft motion}

The UAV treated in this research is a double-delta-wing UAV shown in Fig. 1. The coordinate system is defined in Table 1. The motion equation of an aircraft is derived from Newtonian dynamics. Six-degree-of-freedom nonlinear equation of motion is shown in Eq. (1). 


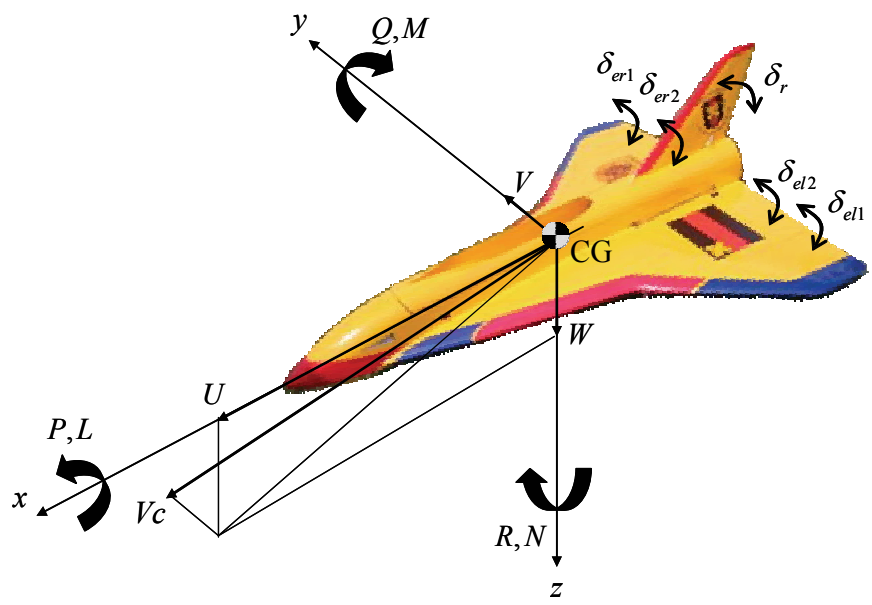

Fig. 1. Body axis

$$
\begin{aligned}
& X=m(\dot{U}+Q W-V R+g \sin \Theta) \\
& Y=m(\dot{V}+U R-P W-g \cos \Theta \sin \Phi) \\
& Z=m(\dot{W}+P V-U Q-g \cos \Theta \cos \Phi) \\
& L=\dot{P} I_{x}-\dot{R} J_{x z}+Q R\left(I_{z}-I_{y}\right)-P Q J_{x z} \\
& M=\dot{Q} I_{y}+P R\left(I_{x}-I_{z}\right)+\left(P^{2}-R^{2}\right) J_{x z} \\
& N=\dot{R} I_{z}-\dot{P} J_{x z}+P Q\left(I_{y}-I_{x}\right)+Q R J_{x z}
\end{aligned}
$$

In Eq. (1), $X, Y$, and $Z$ indicate each axis's external force term except for gravitational force (including aerodynamic force, thrust force). In addition, $\Phi, \Theta$, and $\Psi$ indicate Euler angle of each axis. The proper nonlinear model shown in Eq. (1) is used in the numerical simulation. In contrast, the linearized model based on Eq. (1) is used to design the controller. Many of parameters of motion equation are decided on the basis of the wind-tunnel experiment. The parameters that cannot be acquired in the experiment are estimated by the method using nonlinear function. (Kato et al, 1982)

Each elevon steerage angle of the double-delta-wing UAV is expressed in Eq. (2) by using elevator steerage angle $\delta_{e}$ and aileron steerage angle $\delta_{a}$.

$$
\begin{aligned}
& \delta_{e l_{1}}=\delta_{e l_{2}}=1 / 2\left(\delta_{e}+\delta_{a}\right) \\
& \delta_{e r_{1}}=\delta_{e r_{2}}=1 / 2\left(\delta_{e}-\delta_{a}\right)
\end{aligned}
$$

\section{Fault-tolerant system}

The block diagram of the proposed intelligent fault-tolerant flight control system is shown in Fig. 2. It is composed of fault detection, fault identification, and fault accommodation (FDIA). In this section, the brief summary of each system is represented. 


\begin{tabular}{c|c|c|c}
\hline \hline & $\begin{array}{c}\text { Velocity \& } \\
\text { Angular velocity }\end{array}$ & $\begin{array}{c}\text { External force \& } \\
\text { Moment }\end{array}$ & Distance \\
\hline Forward & $U$ & $X$ & $x$ \\
Starboard & $V$ & $Y$ & $y$ \\
Down & $W$ & $Z$ & $z$ \\
Roll & $P$ & $L$ & \\
Pitch & $Q$ & $M$ & \\
Yaw & $R$ & $N$ &
\end{tabular}

Table 1. Coordinate system and symbol

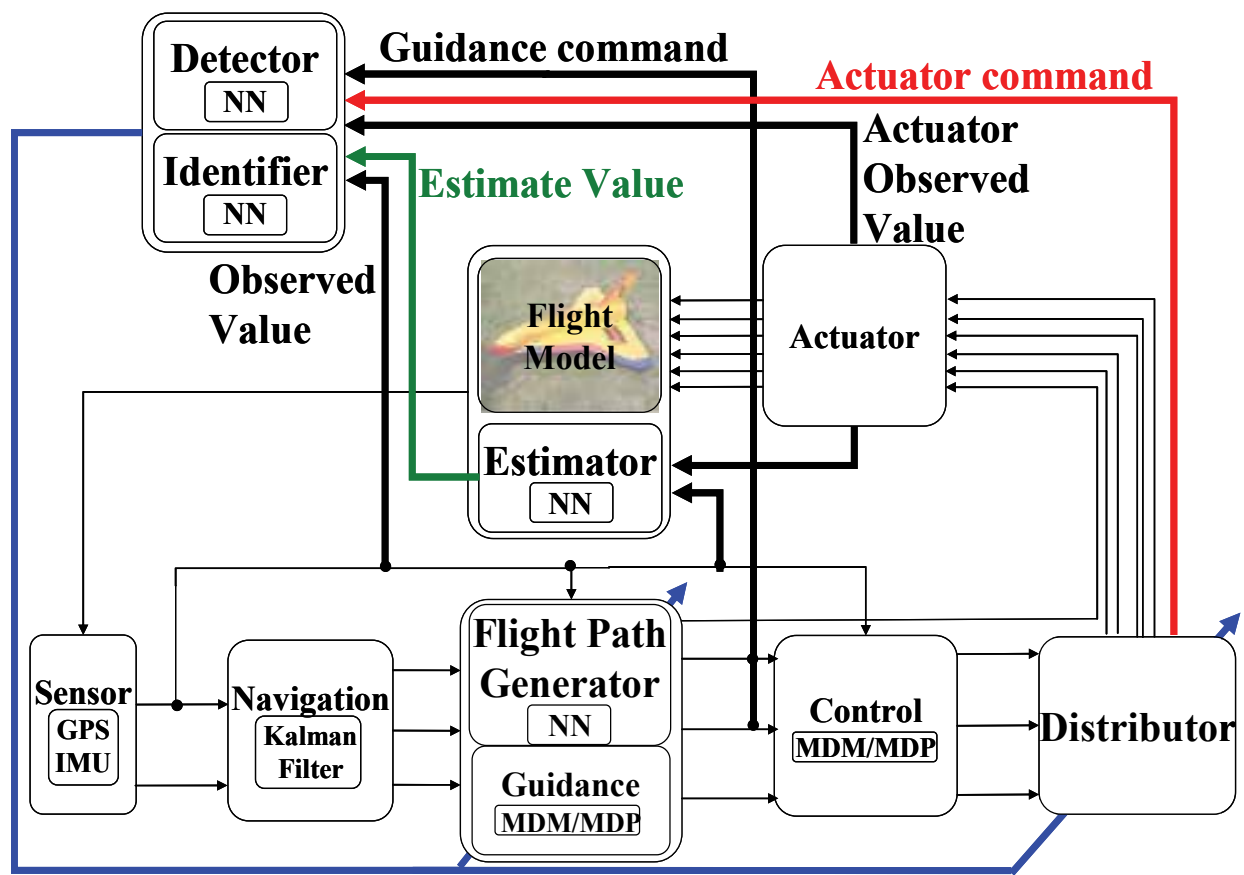

Flight Condition, Failure Position

Fig. 2. Block diagram of proposed flight control system

\subsection{Fault detection}

Fault detection is to distinguish faults from natural phenomena like gusts of wind. To achieve this, this research focused on how each influence on the dynamics of an aircraft, and then an estimator and a detector were designed. The estimator can estimate the ideal state of an UAV. The detector evaluates the flight conditions of an UAV by using the error information between the observed and estimated values.

\subsection{Fault identification}

Fault identification is to locate a broken actuator. To achieve this, an identifier was designed. Generally, to identify a fault, a method is used that sets a threshold value of error 
information between an actuator command and a steerage value. However, this method depends on designer's thought, and inevitably the design work gets into trial and error. By contrast, this research focused on the nonlinear mapping ability of a neural network to flexibly respond to changes.

\subsection{Fault accommodation}

Fault accommodation is to stabilize the flight conditions of an UAV when a fault emerges. To achieve this, a distributor and a flight path generator were designed. The distributor switches the distribution matrix that sends a control command to actuators on the basis of the location of a broken actuator. This countermeasure results in the maximum application of the remaining actuators. The flight path generator generates a new flight path which automatically takes account of both flight stability and following capability of mission trajectory on the basis of the evaluation result.

\section{Specific design of each component}

\subsection{Guidance and control}

In this research, a coupled motion between longitudinal and lateral-directional is controlled. This is because the roll angular velocity is controlled by limiting the derivative value of the bank angle command. Therefore, the motion of UAV can be separated into longitudinal and lateral-directional motions. In the guidance and control system, longitudinal guidance, lateral-directional guidance, longitudinal control, and lateral-directional control were designed separately. The guidance and control laws were designed by multiple delay model and multiple design point (MDM/MDP) method. The block diagram of longitudinal guidance, lateral-directional guidance, longitudinal control, and lateral-directional control are shown in Figs. 3 to 6.

\subsection{Estimator}

The estimator achieves nonlinear dynamics of the UAV approximately by using nonlinear mapping ability of feedforward-type neural network. It estimates next state vectors of the UAV from previous state vectors and actuator steerage commands.

The structure is three-layer neural network shown in Fig. 7. Input layer has 15 neurons, hidden layer has 18, and output layer has 9. In Fig. 4, the index of "obs" means the observed value, the index of "cmd" means the actuator steerage command, and the index of "est"

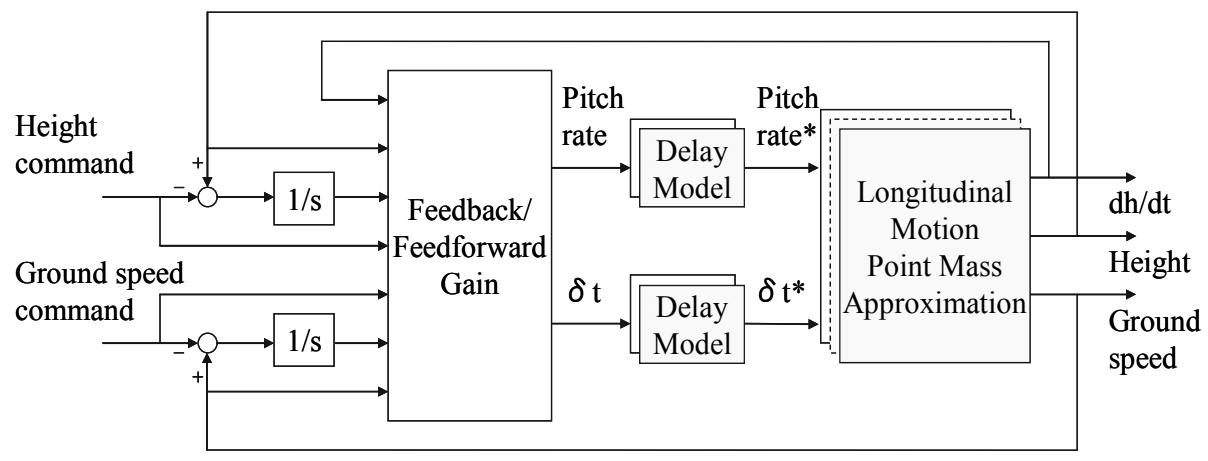

Fig. 3. Block diagram of longitudinal guidance system 


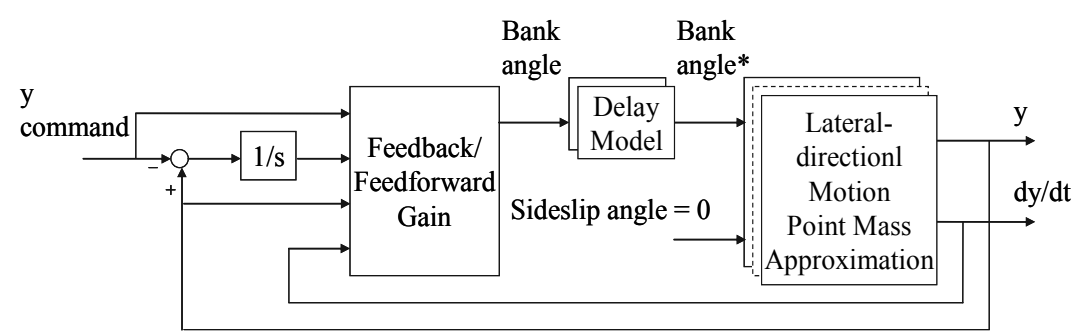

Fig. 4. Block diagram of lateral-directional guidance system

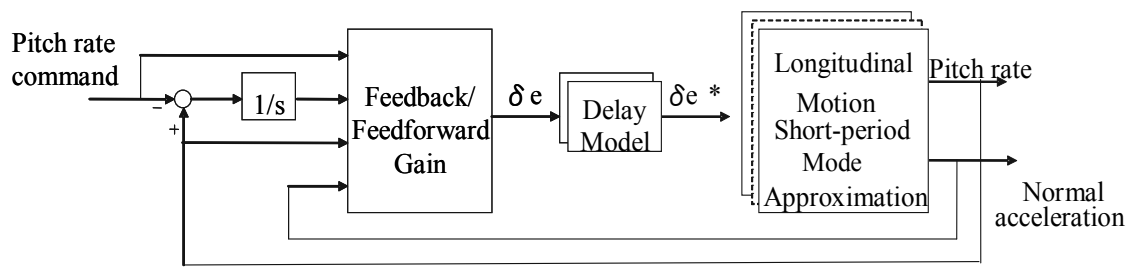

Fig. 5. Block diagram of longitudinal control system

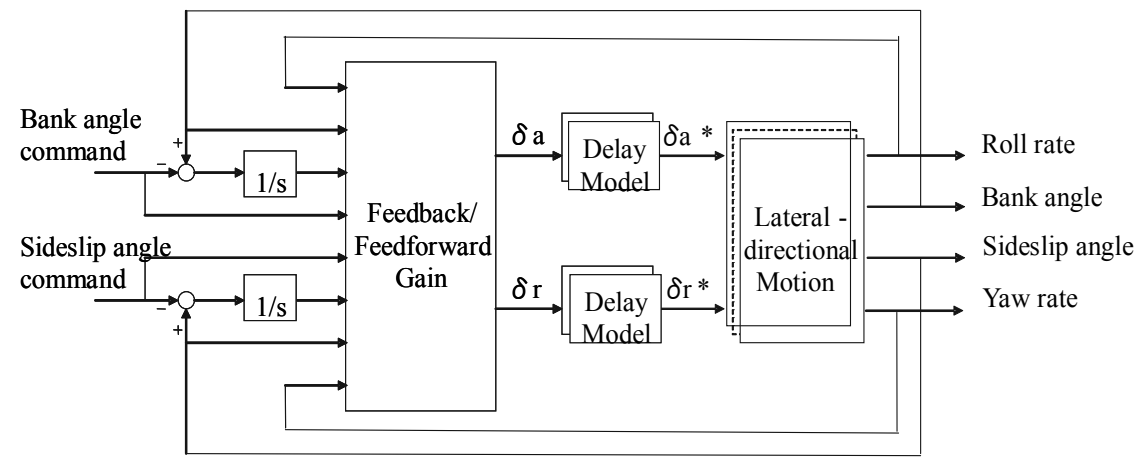

Fig. 6. Block diagram of lateral-directional control system

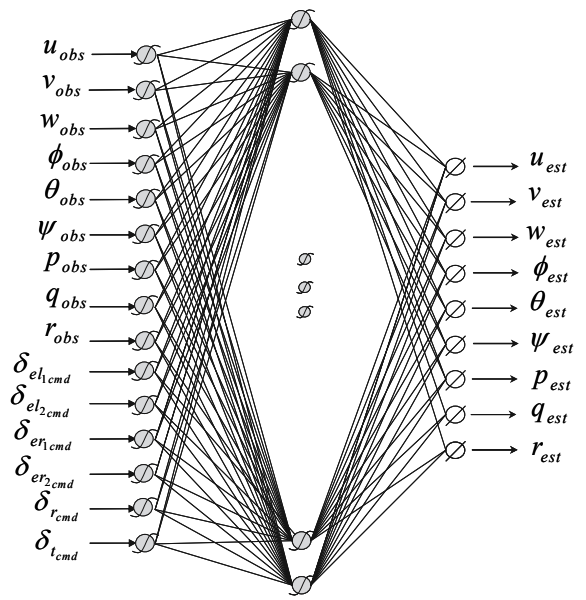

Fig. 7. Structure of estimator neural network 
means the estimated value. The transfer functions of each layer are shown in Eqs. (3) to (5), where each "tansig" and "purelin" means tangent-sigmoid function, linear function shown in Eqs. (6) and (7) respectively. In Eqs. (3) to (5), net ${ }_{\mathrm{i}}$, net $\mathrm{t}_{\mathrm{j}}$, and net $\mathrm{t}_{\mathrm{k}}$ mean the input of input layer, hidden layer, and output layer respectively.

In addition, back propagation (BP) is applied for the learning of neural network. The flight data acquired with the six-degree-of-freedom nonlinear simulation is used as a teach signal.

$$
\begin{gathered}
f_{i}=\operatorname{tansig}\left(\text { net }_{i}\right) \\
f_{j}=\operatorname{tansig}\left(\text { net }_{j}\right) \\
f_{k}=\operatorname{purelin}\left(\text { net }_{k}\right) \\
f(x)=\frac{2}{1+\exp (-2 \cdot x)}-1 \\
f(x)=x
\end{gathered}
$$

\subsection{Detector}

The detector discriminates the influence of fault on the UAV from that of natural disturbance such as gusts of wind by focusing on the impact on the dynamics of the UAV. It uses the error between observed value and estimated value as the information about the dynamics of the UAV. Moreover, the error between actuator steerage command and the real actuator steerage angle is used for the evaluation of the flight condition. The derivative of bank angle is also used.

Because input-output characteristic is unknown, the structure of the detector is three-layer neural network shown in Fig. 8. Input layer has 10 neurons, hidden layer has 20, and output layer has 1. The transfer functions of each layer are shown in Eqs. (8) to (10).

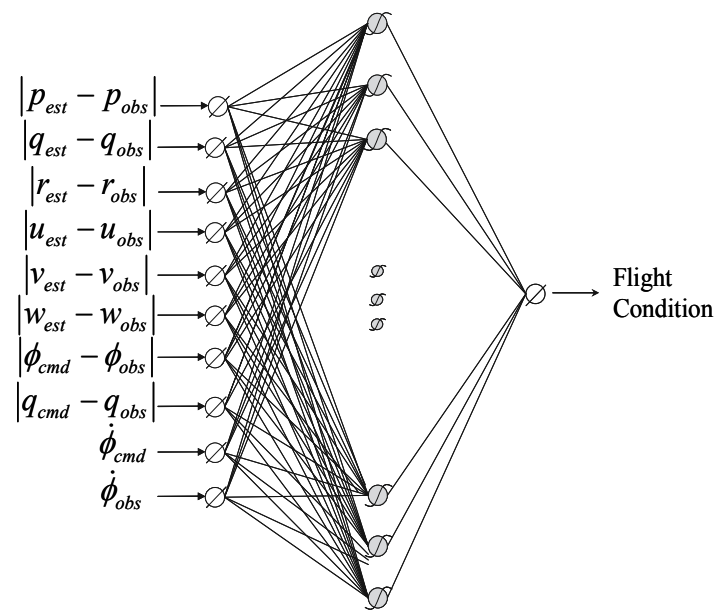

Fig. 8. Structure of detector neural network 


$$
\begin{gathered}
f_{i}=\operatorname{purelin}\left(\text { net }_{i}\right) \\
f_{j}=\operatorname{tansig}\left(\text { net }_{j}\right) \\
f_{k}=\operatorname{purelin}\left(\text { net }_{k}\right)
\end{gathered}
$$

Because there is no explicit teach signal, a genetic algorithm (GA) was applied for the learning of neural network. In GA, 50 individuals that encode the connection weight of a neural network were prepared. Both fitness proportionate and elite selection strategies were used. Moreover, with repeating random crossover and mutation, the individual that had the highest fitness was acquired. 3 cases about gust in different directions and 9 cases about left elevon-1 fault in different angles are used as the simulation case. Both gusts of wind and fault are occurred in horizontal flight. The fitness function is shown in Eq. (11), where $t_{d}$ is the detection time, $t_{\text {failure }}$ is the initiation time of fault, and $a_{d}$ is the constant value of detector for evaluation. In the evaluation, to detect the fault more quickly has higher score. In addition, in the gusts of wind cases, when the detector did false detection, the value of fitness function becomes zero.

$$
J=\left\{\begin{array}{l}
0 \quad\left(t_{d}<t_{\text {failure }}\right) \\
\exp \left(-a_{d} \cdot\left(t_{d}-t_{\text {failure }}\right)\right) \quad\left(t_{d} \geq t_{\text {failure }}\right)
\end{array}\right.
$$

\subsection{Identifier}

The identifier locates where the broken actuator is by using the information of both actuator steerage command and actuator steerage angle. Neural network shown in Fig. 9 is located in each actuator and the location of broken actuator is identified by the outputs of each neural network.

Because input-output characteristic is unknown, the structure of the identifier is three-layer neural network. Input layer has 3 neurons, hidden layer has 18, and output layer has 1 . The

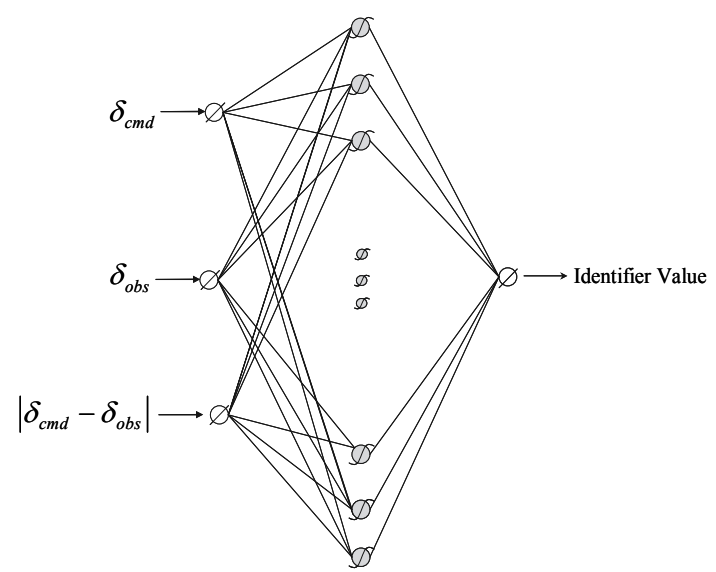

Fig. 9. Structure of identifier neural network 
transfer functions of each layer are shown in Eqs. (12) to (14). GA is applied for the learning of neural network. As the simulation case, 9 cases about left elevon- 1 fault in different angles happened in horizontal flight are used. Equation (15) is the fitness function, where $t_{i}$ is the identification time, $t_{\text {failure }}$ is the initiation time of fault, and $a_{i}$ is the constant value of identifier for evaluation. In the evaluation, to identify the location of broken actuator more quickly has higher score.

$$
\begin{gathered}
f_{i}=\operatorname{purelin}\left(\text { net }_{i}\right) \\
f_{j}=\operatorname{tansig}\left(\text { net }_{j}\right) \\
f_{k}=\operatorname{purelin}\left(\text { net }_{k}\right) \\
J=\left\{\begin{array}{l}
0\left(t_{i}<t_{\text {failure }}\right) \\
\exp \left(-a_{i} \cdot\left(t_{i}-t_{\text {failure }}\right)\right)\left(t_{i} \geq t_{\text {failure }}\right)
\end{array}\right.
\end{gathered}
$$

\subsection{Distributor}

The distributor switches the distribution matrix by using the outputs of the detector and the identifier. When the distribution matrix was changed, the elevator, aileron, and rudder commands from the control system are divided into 5 actuator commands (left elevon-1, left elevon-2, right elevon-1, right elevon-2, and rudder) to separate the broken actuator. The switching algorithm is to change the command for the broken actuator to zero and to realize the maximum use of the remaining actuators. The structure of the distributor is shown in Fig. 10.

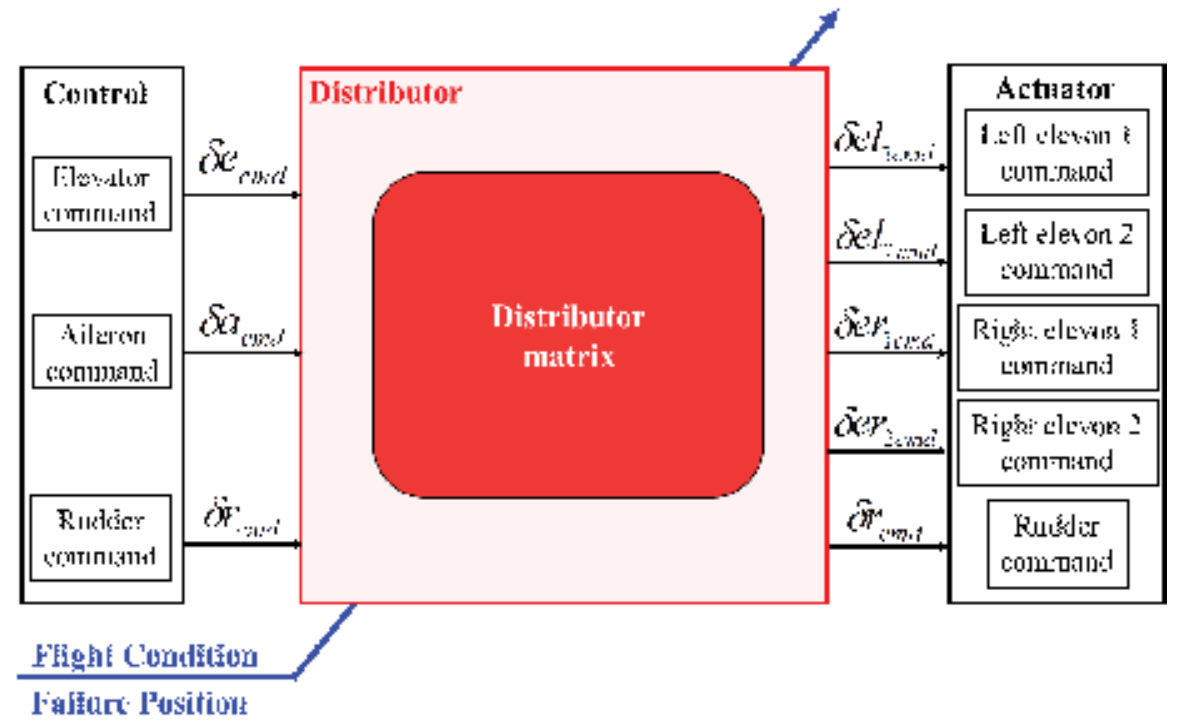

Fig. 10. Structure of distributor 


\subsection{Flight path generator}

The flight path generator is located in parallel with the guidance system. It generates a new flight path which considers both flight stability and following capability of mission trajectory under the condition where the elevon fault is occurred.

In general, there are two turning methods. One is to use a bank angle and the other a sideslip angle. To assure robustness against the rudder fault, the turning method using the bank angle was adopted in the guidance system. On the other hand, to assure robustness against the elevon fault, the turning method using the sideslip was adopted in the flight path generator.

Generally, because drag increases when the sideslip angle is allowed to changes in the turning flight, too much energy is used. However, the emergency situation such as an elevon fault is an exception because keeping the flight stable is more important than saving energy. Therefore, the flight path generator has been designed that enables the sideslip angle to change.

The flight path generator calculates the desired sideslip angle command by using Eq. (16),

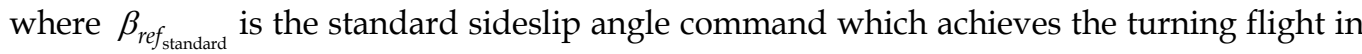
mission trajectory. To generate a new flight path by changing the radius adaptively, the flight path generator calculates $K_{\beta}$ depending on the fault level.

$$
\beta_{\text {ref }}{ }^{\prime}=K_{\beta} \cdot \beta_{\text {ref standard }}
$$

Because input-output characteristic is unknown, the structure of the flight path generator is three-layer neural network shown in Fig.11. Input layer has 6 neurons, hidden layer has 1, and output layer has 1 . The input signals of the flight path generator are the signals from both the detector and the identifier which are integrated in a given time. The transfer functions of each layer are shown in Eqs. (17) to (19), where shift $t_{i}$ is the width of parallel shift. Equation (17) is the symmetric double sigmoid function. (Akihiko Shimura \& Kazuo Yoshida, 2001)

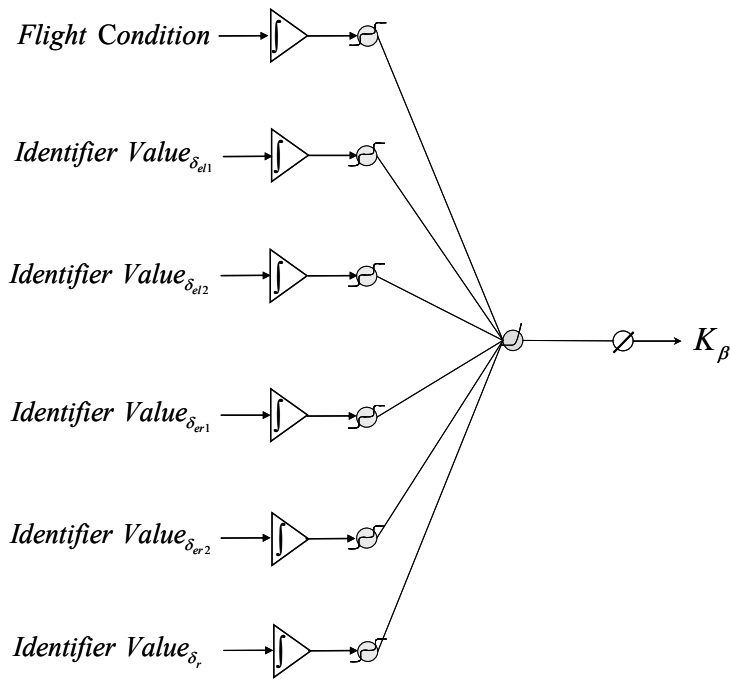

Fig. 11. Structure of flight path generator 


$$
\begin{gathered}
f_{i}=\frac{1}{2}\left\{\operatorname{tansig}\left(\text { net }_{i}-\text { shift }_{i}\right)+\operatorname{tansig}\left(\text { net }_{i}+\text { shift }_{i}\right)\right\} \\
f_{j}=\exp \left(\text { net }_{j}\right) \\
f_{k}=\operatorname{purelin}\left(\text { net }_{k}\right)
\end{gathered}
$$

GA is applied for the learning of neural network. As the simulation case, 7 cases about conducting the turning flight after left elevon-1 fault in different angles happened in horizontal flight are used. The termination conditions of each simulation case are as follows.

(A) $120<\psi[\mathrm{deg}]<300 \cap x[\mathrm{~m}]<-500$

(B) height $<0.18$

(C) $\alpha[\mathrm{deg}]<-4.9 \cup 29<\alpha[\mathrm{deg}]$

(D) $\beta[\mathrm{deg}]<-9.9 \cup 9.9<\beta[\mathrm{deg}]$

Equation (20) is the fitness function, where $a_{r e f 1}$ and $a_{r e f 2}$ are the constant value for the following capability of mission trajectory. $Y_{\text {ref }}$ is the y-direction target value of mission trajectory. $Y_{r e f}^{\prime}$ is the y-direction target value generated by the flight path generator. time,

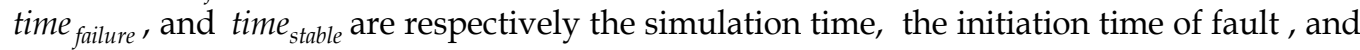
the time when the error value between the real height and that of mission trajectory is controlled within the constant value. In the evaluation, both the following capability of mission trajectory and the flight stability are evaluated.

In addition to Eq. (20), the termination conditions are also evaluated. When the simulation was stopped because of the termination condition except for (A), the value of fitness function becomes zero because the stability is lost.

$$
\begin{array}{r}
J=a_{\text {ref } 1} \cdot \exp \left(-a_{\text {ref } 2} \cdot\left|Y_{\text {ref }}{ }^{\prime}-Y_{\text {ref }}\right|\right) \\
+\exp \left(\frac{\text { time }_{\text {stable }}}{\left.(\text { time-failure time })_{\text {time }}\right)}\right)
\end{array}
$$

\section{Numerical simulation}

\subsection{Simulation condition}

The effectiveness of the proposed intelligent fault-tolerant flight control system was verified with the six-degree-of-freedom nonlinear simulation. The airframe model, external environment model, and guidance/control law were considered as a mathematical model in the simulation. In the airframe model, the actuator characteristic was expressed using the second order time delay model with restrictions of position and velocity. In addition, the characteristic of sensor was assumed to be ideal that there were no errors in both static and dynamic conditions. As the external environment model, only wind was used. The constant wind model was constructed by using the MIL-F-9490D method applied to the ALFLEX simulation. (NAL/NASDA ALFLEX Group, 1994) It considered the difference of the 
airframe size by multiplying the scale-dependent constant value. The actuator-fixed fault such as Lock-in-place, Hard-over and Float was adopted as the actuator fault model. (Jovan D. Boskobic et al, 2005)

As the mission trajectory, turning above devastated district in constant height to observe was applied. It is shown in Fig. 12. In this mission, the UAV flaw at the height of $30 \mathrm{~m}$ in the velocity of $20 \mathrm{~m} / \mathrm{s}$ and the constant wind was from $+x$ direction. The gusts of wind was expressed by changing the scale-dependent constant value of constant wind in 3 seconds. (Kohichiroh Yoshida et al, 1994) In addition, not only the learned fault, left elevon-1 fault, but also non-learned fault, rudder fault, was considered. The conditions of fault and gust are represented in Table 2 .

In the simulation, the proposed intelligent fault-tolerant flight control system and the flight control system designed by MDM/MDP method were compared.

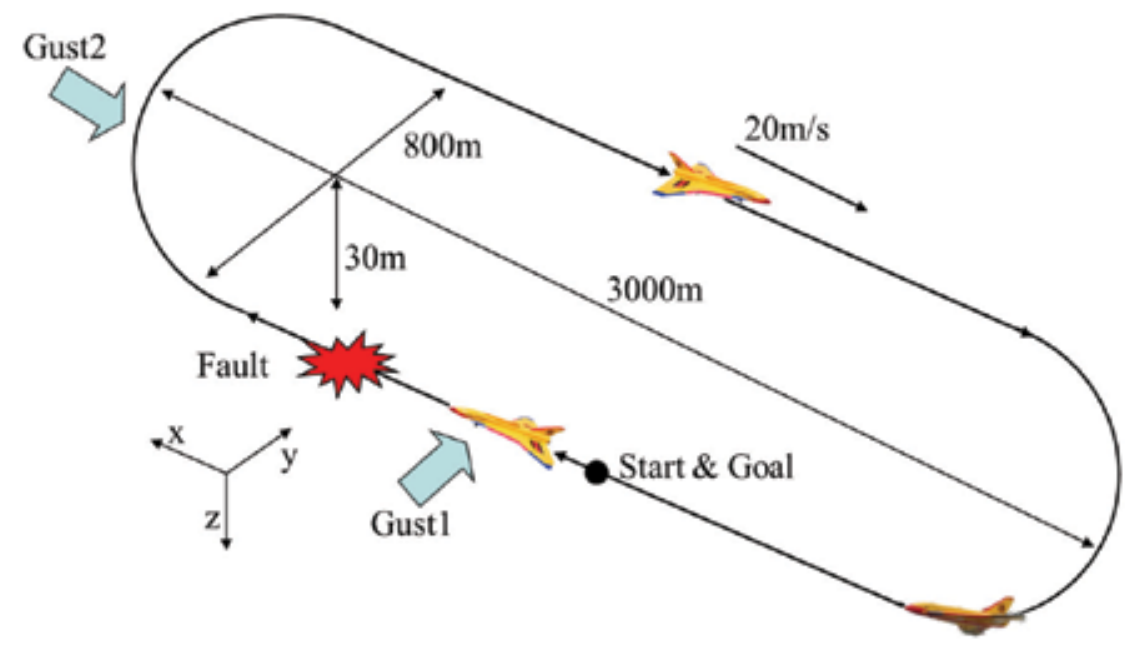

Fig. 12. Mission trajectory

\begin{tabular}{c|c|c}
\hline \hline Condition & Time & Direction \\
\hline Gust1 & $15 \mathrm{~s}$ & From y minus \\
\hline Failure & $30 \mathrm{~s}$ & \\
\hline Gust2 & $90 \mathrm{~s}$ & From x plus
\end{tabular}

Table 2. Conditions of disturbance

\subsection{Simulation results}

In this section, the simulation results under the condition shown in section 5.1 are represented.

First, Figs. 13 to 19 respectively show the results under the conditions where the left elevon1 was fixed at 9 degree for the flight trajectory, the time history of bank angle, sideslip angle, and actuator steerage. In addition, Table 3 shows the effective area.

Second, the results for detection, identification, and accommodation are shown. The output of the detector and the identifier are respectively shown in Figs. 20 and 21. Figure 22 shows the relationship between the fixed angle of broken elevon and the y-direction target value 
generated by the flight path generator. Moreover, the coherence functions between the observed value and the estimated value for velocity $u$ and angular velocity $q$ are compared under the conditions of a fault and gust of wind in Figs. 23 and 24.

Finally, Figs. 25 and 26 show the results under the condition where the rudder was fixed at 8 degree for the flight trajectory and the time history of actuator steerage.

\subsection{Evaluation}

From the results in Figs. 13 to 19, we confirmed how each method deals with the fault in which the elevon is fixed at the angle.

The conventional system generates a bank angle command and achieves a turning flight by using an elevon. On the other hand, the proposed flight control system stabilizes the airframe by using redundant elevon in horizontal flight as soon as the fault happens. After that, it generates a sideslip angle command and achieves a turning flight by using a rudder.

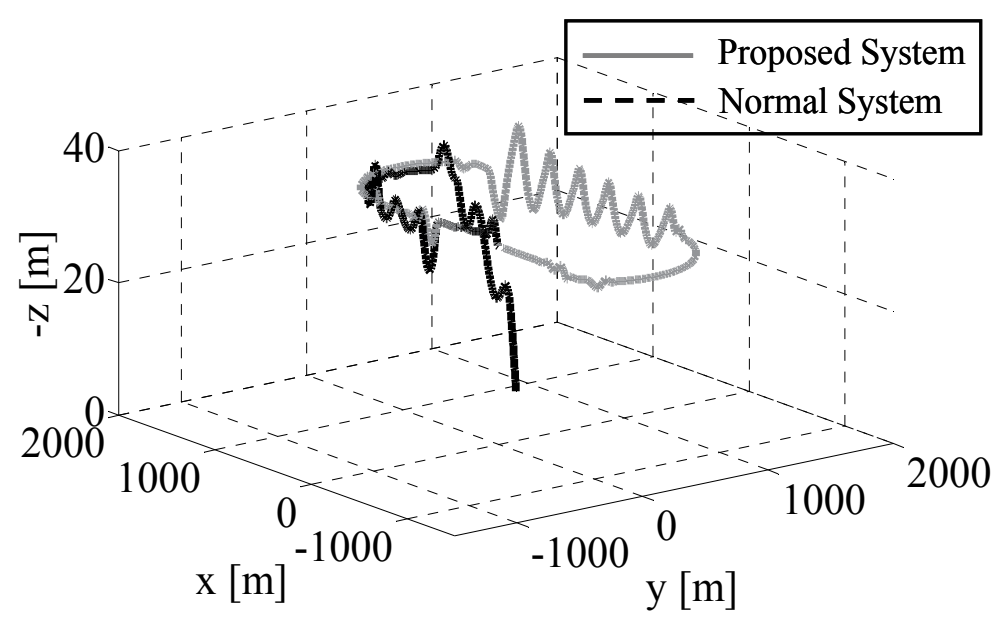

Fig. 13. Flight trajectory (left elevon-1 fault)

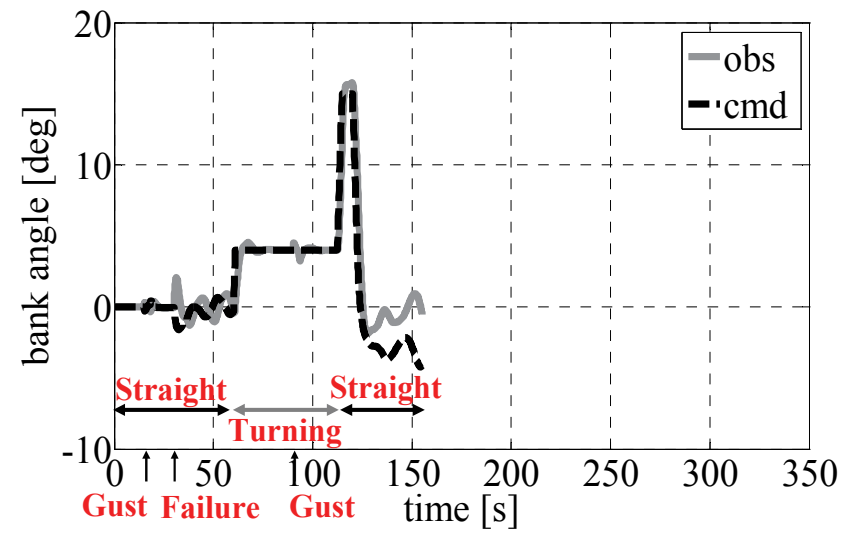

Fig. 14. Time history of bank angle (normal system) 


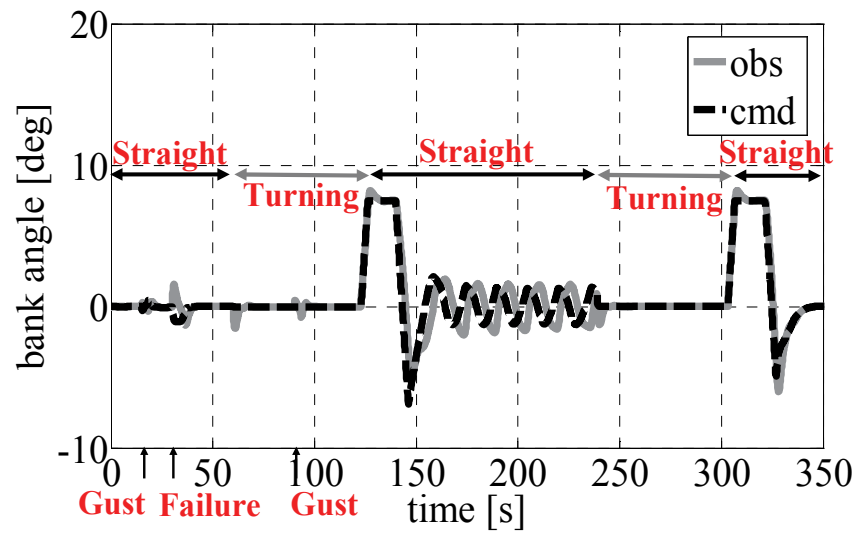

Fig. 15. Time history of bank angle (proposed system)

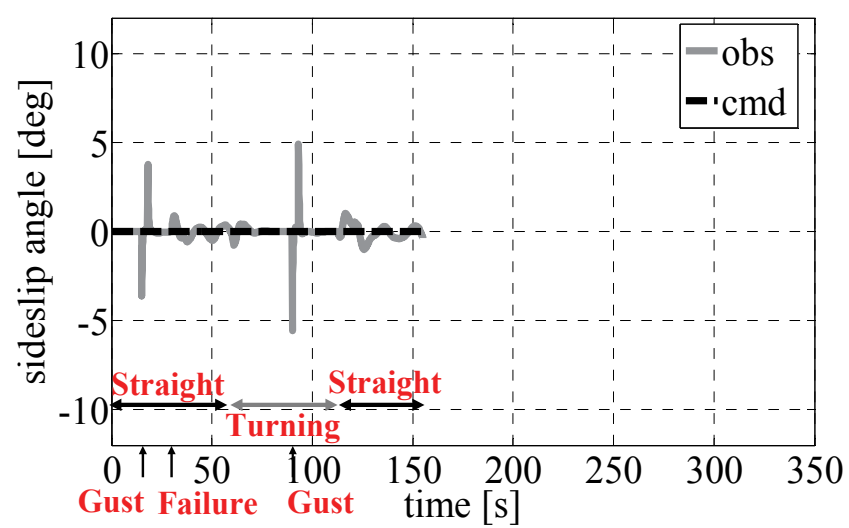

Fig. 16. Time history of sideslip angle (normal system)

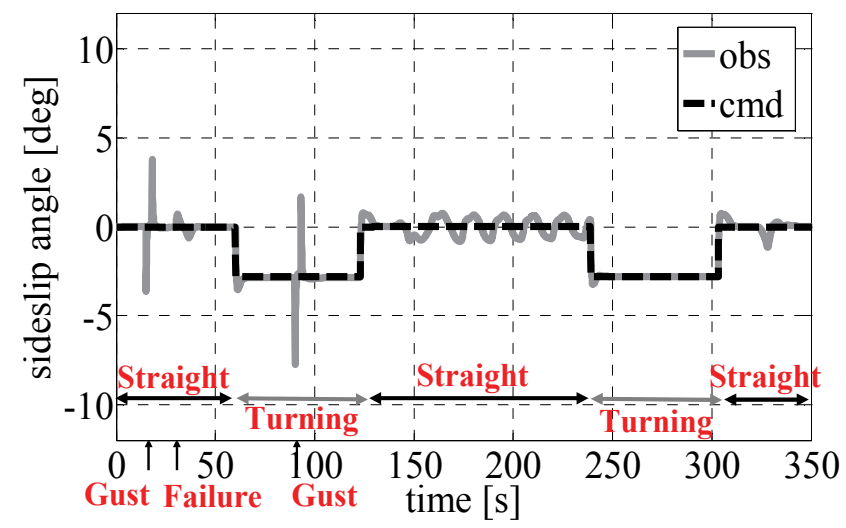

Fig. 17. Time history of sideslip angle (proposed system) 


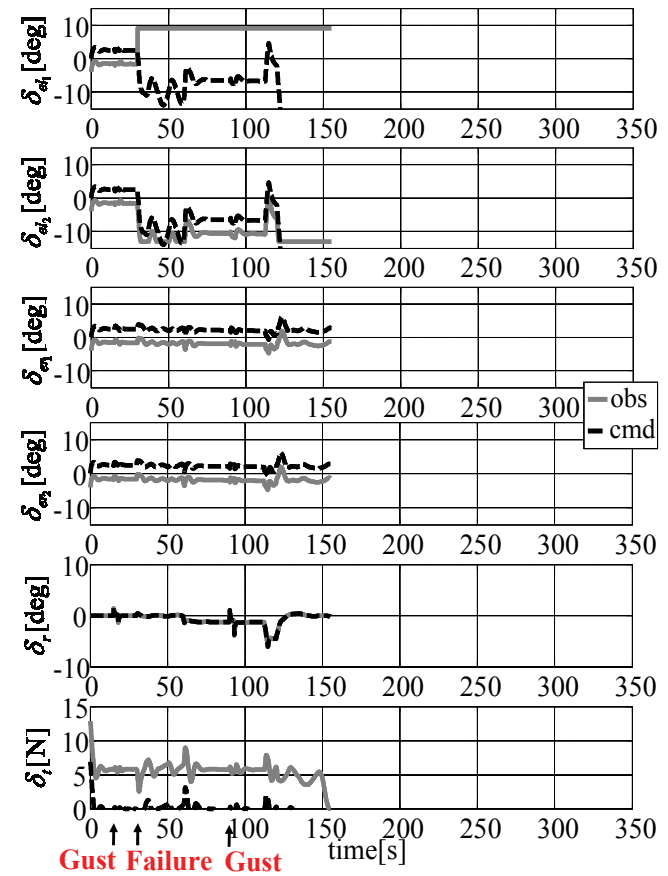

Fig. 18. Time history of actuator steerage (normal system)

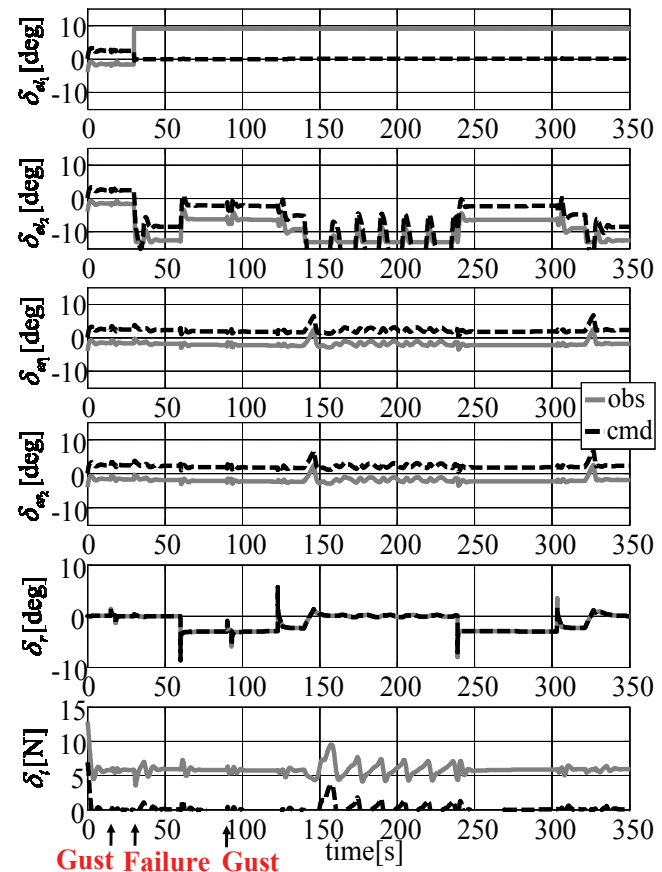

Fig. 19. Time history of actuator steerage (proposed system) 


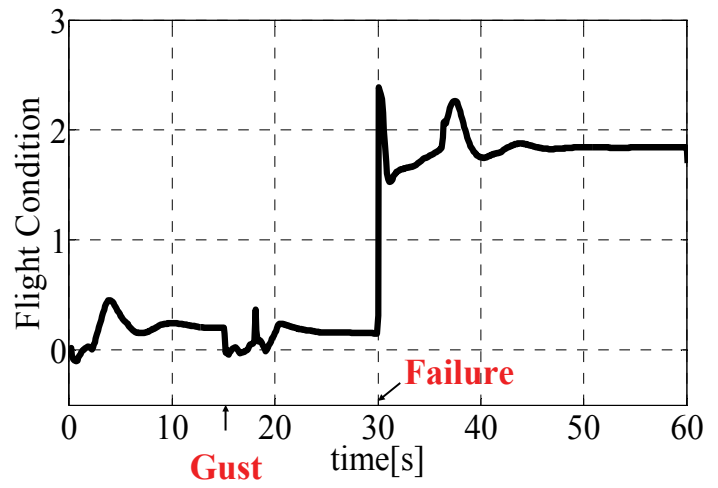

Fig. 20. Output of detector neural network
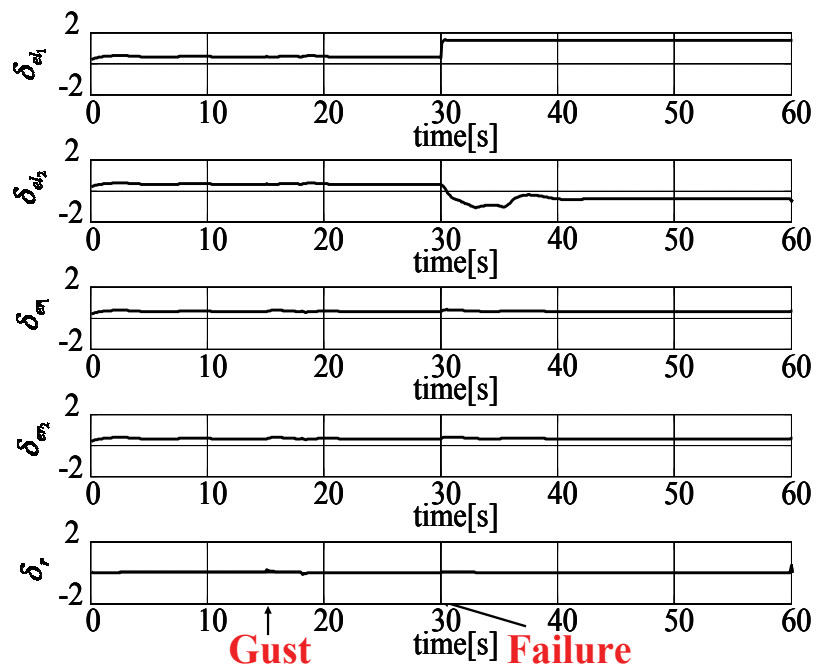

Fig. 21. Output of identifier neural network

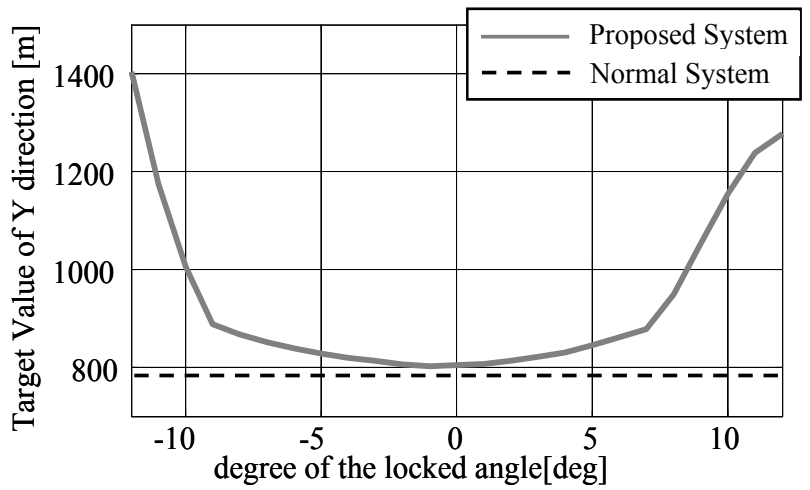

Fig. 22. Target value generated by flight path generator 


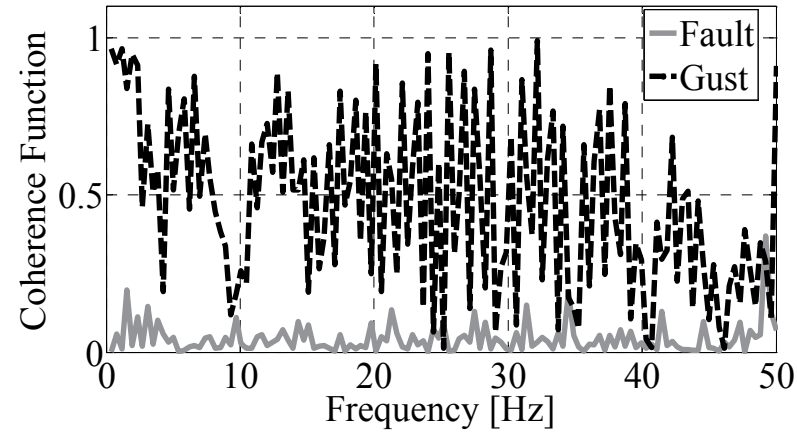

Fig. 23. Coherence function, $u$

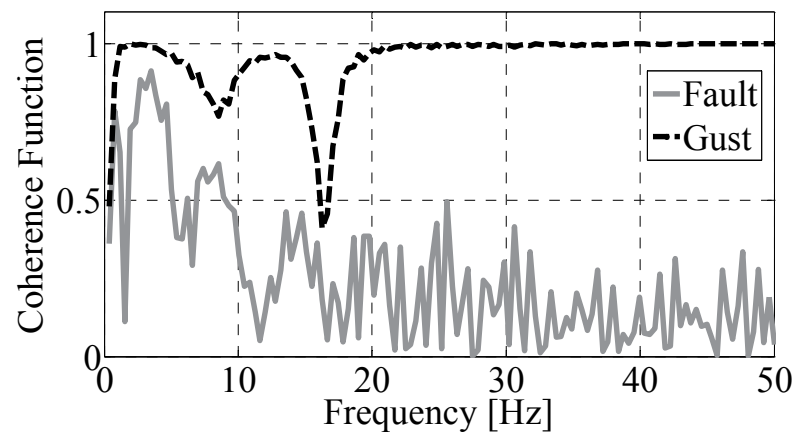

Fig. 24. Coherence function, $q$

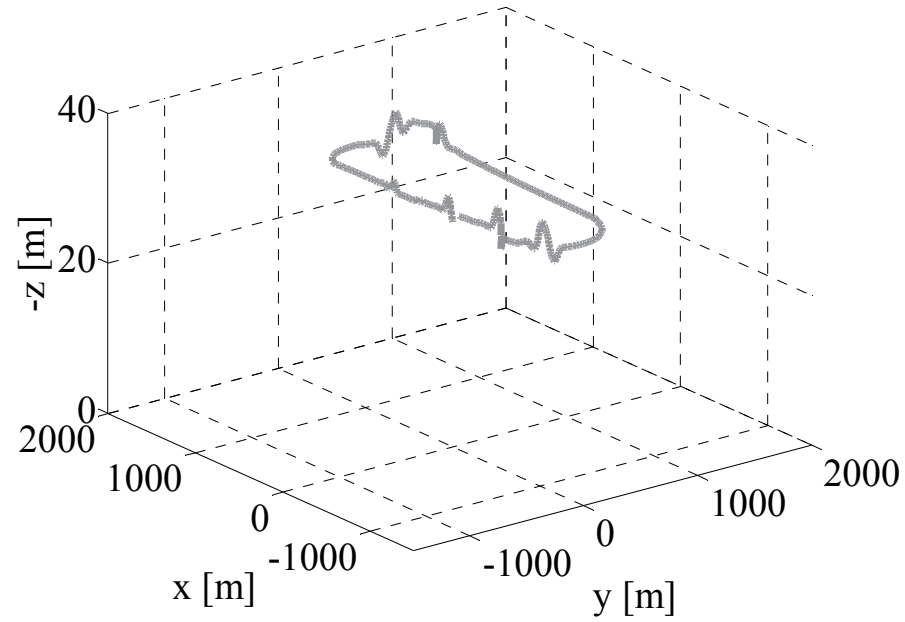

Fig. 25. Flight trajectory (rudder fault) 

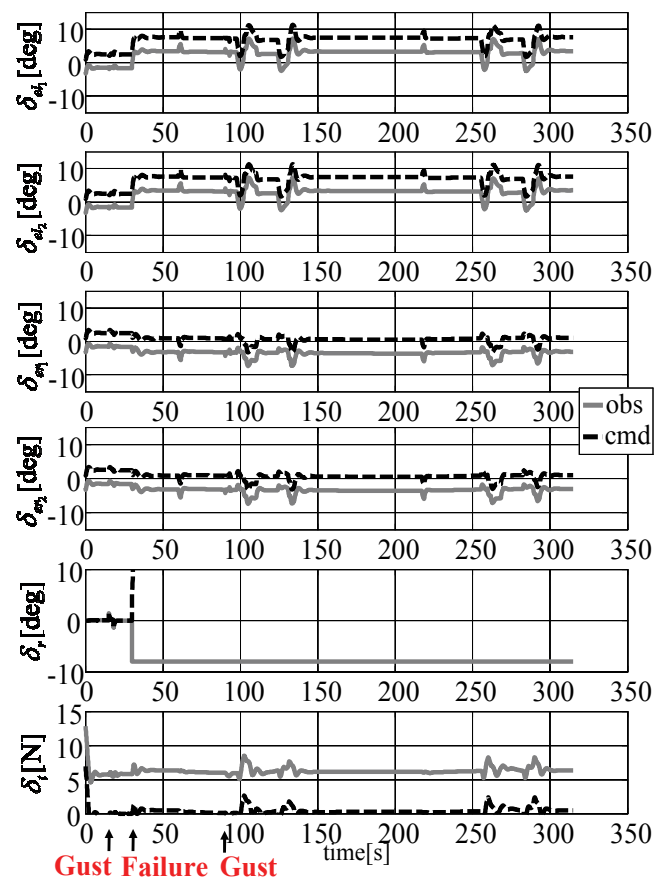

Fig. 26. Time history of actuator steerage (rudder fault)

\begin{tabular}{c|c|c}
\hline \hline Fault position & Elevon & Rudder \\
\hline Range of movement [deg] & $-13 \sim 13$ & $-8 \sim 8$ \\
Effective area[deg] & $-13 \sim 9$ & $-8 \sim 8$
\end{tabular}

Table 3. Effective area of proposed system 
The results in Figs. 13, 15, 17, and 19, confirm that the vibration motion is generated in the horizontal flight after turning flight by using the proposed method. This vibration frequency is about $0.067 \mathrm{~Hz}$. This is because the resonation with the vibration occurs at the longitudinal short cycle mode and the lateral-directional dutchroll mode when the turning flight is changed to the horizontal flight in order to deal with the fault. However, this vibration fits into the stable area of both an attack angle and a sideslip angle that is established when designed and shown in section 4.6 as the termination conditions. Therefore, the vibration is considered to be an allowable range.

From the results in Figs. 25 and 26, we confirmed that the proposed flight control system generates a bank angle command and achieves a turning flight by using an elevon when a rudder fault happens.

These results confirm that the proposed system can detect, identify and accommodate both learned and non-learned faults.

From the simulation results, we confirmed that the proposed flight control system can stabilize the airframe in fault situations shown in Table 3.

Figure 20 shows the output of a detector which means the evaluation value of a flight condition. We confirmed that the detector can distinguish the fault from the gust of wind. The flight control system can distinguish between the fault and the gusts from various directions because a number of directional gusts are considered in the learning of neural network. Figures 23 and 24 show that the gust has a wider range of frequency where the coherence function takes the value of approximately 1 than the fault. If the disturbance is estimated, the motion of the system is the same as the model assumed when the control system is designed. On the other hand, the motion of the system with the fault is different from the assumed model. Therefore, the proposed model-based detector can accurately detect faults.

Figure 21 shows the output of an identifier which means the evaluation value of the fault position. It was confirmed that the proposed identifier can identify the fault position because only the broken actuator indicates the abnormal value.

Figure 22 shows the performance of a flight path generator. The horizontal axis indicates a fixed angle of a broken elevon and the vertical axis indicates a new target value of $y$ direction that is calculated by the flight path generator. The results confirm that the higher the level of a fault, the gentler the turning based on a new target value generated by the flight path generator. In this research, the actuator error between the stable and the broken conditions means the fault level. Moreover, the error from a mission trajectory is considered in the evaluation function. Therefore, the proposed flight control system can generate a suitable target value of turning in accordance with the situation.

The proposed flight control system focuses on the change in dynamics caused by a fault. It is designed by considering the elevon fault that enormously influences the airframe because an elevon plays the roles of both an aileron and an elevator. The simulation results confirm the proposed system can perform well in both learned and non-learned fault situations.

\section{Conclusion}

This research aimed at proposing an intelligent fault-tolerant flight control system for an unmanned aerial vehicle (UAV). In particular, the flight control system was developed that 
has estimator, detector, identifier, distributor, and flight path generator. The proposed system distinguishes a fault from a disturbance like a gust of wind and automatically generates a new flight path suited to the fault level. To verify the effectiveness of the proposed method, a six-degree-of-freedom nonlinear simulation was carried out. In the simulation, we assumed that the fault in left elevon-1, which was learned in designing each neural network, or the fault in the rudder, which was not learned, would be generated in a horizontal flight. The simulation results confirm that the proposed flight control system can detect, identify and accommodate the fault and keep a flight stable. Moreover, the proposed system can distinguish a fault from a gust and keep a flight stable automatically. It is expected that the proposed design method can be used in broader flight areas by expanding the learning area.

\section{References}

Akihiko Shimura and Kazuo Yoshida, Non-Linear Neuro Control for Active Steering for Various Road Condition, The Japan Society of Mechanical and Engineers, Vol. 67, No. 654(2001), pp. 407-413.

Brian L. Steavens and Frank L. Lewis, Aircraft Control and Simulation 2nd Edition, JOHN WILEY \& SONS, INC. (2003)

Guillaume Ducard and Hans P. Geering, Efficient Nonlinear Actuator Fault Detection and Isolation System for Unmanned Aerial Vehicles, AIAA, Journal of Guidance, Control, and Dynamics, Vol. 31, No.1 (2008), pp. 225-237.

Jovan D. Boskovic, Sarah E. Bergstrom , and Raman K. Mehra, Robust Integrated Flight Control Design Under Failures, Damage, and State-Depenndent Disturbances, AIAA, Journal of Guidance, Control, and Dynamics, Vol. 28, No.5 (2005), pp. 902-916.

Kanichiro Kato, Akio Oya, and Kenzi Karasawa, Introduction of Aircraft Dynamics, University of Tokyo Press, (1982).

Kohichiroh Yoshida, kazumichi Mototsuna and Yasushi Kumakura, Elementary knowledge of marine technology, Seizandou,(1994)

Masaki Takahashi, Teruma Narukawa and Kazuo Yoshida, Robustness and Fault-Tolerance of Cubic Neural Network Intelligent Control Method : Comparison with Sliding Mode Control, The Japan Society of Mechanical and Engineers, Vol. 69, No. 682(2003), pp. 1579-1586.

Mohammad Azam, Krishana Pattipati, Jeffrey Allanach, Scott Poll, and Ann Patterson-Hine, In-flight Fault Detection and Isolation in Aircraft Flight Control Systems, Aerospace Conference, 2005 IEEE, (2005), pp. 3555- 3565.

NAL/NASDA ALFLEX Group, Flight simulation model for Automatic Landing Flight Experiment (Part I : Free Flight and Ground Run Basic Model), Technical Report of National Aerospace Laboratory, Vol. 1252 (1994).

Taro Tsukamoto, Masaaki Yanagihara, and Takanobu Suito, Feasibility Study of Lateral/Directional Control of Winged Re-entry Vehicle with Split Elevons, Technical Report of National Aerospace Laboratory, Vol. 1379 (1999). 
Toshinari Shiotsuka, Kazusige Ohta, Kazuo Yoshida and Akio Nagamatsu, Identification and Control of Four-Wheel-Steering Car by Neural Network, The Japan Society of Mechanical and Engineers, Vol. 59, No. 559(1993), pp. 708-713.

Tsuyoshi Hatake, Junichiro Kawaguchi, and Tatsushi Izumi, Control in Aerospace, CORONA PUBLISHING CO., LTD. (1999). 


\title{
Active Fault Diagnosis and Major Actuator Failure Accommodation: Application to a UAV
}

\author{
François Bateman ${ }^{1}$, Hassan Noura $^{2}$ and Mustapha Ouladsine ${ }^{3}$ \\ ${ }^{1}$ French Air Force Academy, Salon de Provence \\ ${ }^{2}$ United Arab Emirates University, Al-Ain \\ ${ }^{3}$ Paul Cezanne University, Marseille \\ 1,3 France \\ ${ }^{2}$ United Arab Emirates
}

\section{Introduction}

Interest in Unmanned Aerial Vehicles (UAVs) is growing worldwide. Nevertheless there are numerous issues that must be overcome as a precondition to their routine and safe integration in military and civilian airspaces. Chief among these are absence of certification standards and regulations addressing UAV systems, poor reliability record of UAV systems and operations. Standards and regulations for airworthiness certification and flight operations in the military and civilian airspaces are being studied (Brigaud, 2006). In this respect, the USAR standard suggests a mishap rate of one catastrophic mishap per one million hours (Brigaud, 2006). To reach such performances, upcoming technologies have the promise of significantly improving the reliability of UAVs.

In this connection, a detailed study (OSD, 2003) shows that most of the breakdowns are due to system failures such as propulsion, data link and Flight Control Systems (FCS). These latter include all systems contributing to the aircraft stability and control such as avionics, air data system, servo-actuators, control surfaces/servos, on-board software, navigation, and other related subsystems. As regards FCS, it is recommended in (OSD, 2003) to incorporate emerging technologies such as Self-Repairing Flight Control Systems (SRFCS) which have the capability to diagnose and to repair malfunctions.

In this respect, Fault-tolerant control (FTC) are control systems that have the ability to accommodate failures automatically in order to maintain system stability and a sufficient level of performance. FTC are classified into passive and active methods. The analytical fault-tolerant control operation can be achieved passively by the use of a control law designed to guarantee an acceptable degree of performance in fault-free case and to be insensitive to some faults. However, the passive methods are unsuitable to deal with a significant number of faults. In particular, for an aircraft, it may be tricky to design an a priori controller able to accommodate the whole of the faults affecting the control surfaces. By contrast, an active FTC consists of adjusting the controllers on-line according to the fault magnitude and type, in order to maintain the closed-loop performance of the system. To do so, a fault detection and isolation (FDI) module which provides information about the fault is required (Noura et al., 2009). Active FTC mechanisms may be implemented either via pre-computed control laws or via on-line automatic redesign. 
In this respect, FDI and FTC applied to aeronautical systems have received considerable attention in the literature. However, regarding the control surface failures, some problematics tackled in this chapter are underlined:

- severe failures are considered and the control surfaces may abruptly lock in any position in their deflection range,

- each control surface being driven indenpendently, an actuator failure produces aerodynamical couplings between the longitudinal and the lateral axis,

- the UAV is equipped with an autopilot which masks the failure effects,

- the aircraft studied is a small UAV and the control surface positions are not measured, which makes the fault detection difficult,

- the control surfaces have redundant effects, which complicates the fault isolation,

- the FTC must take into account the bounds existing on the control surface deflections and the flight envelope.

In this chapter, a nonlinear UAV model which allows to simulate assymetrical control surface failures is presented (Bateman et al., 2009). In fault-free mode, a nominal control law based on an Eigenstructure Assignment (EA) strategy is designed. As the control surface positions are not measured, a diagnosis system is performed with a bank of observers able to estimate the unkown inputs. However, as the two ailerons offert redundant effects, isolating a fault on these actuators requires an active diagnosis method (Bateman et al., 2008a). In the last part, a precomputed FTC strategy dedicated to accommodate for a ruddervator failure is depicted (Bateman et al., 2008b).

All the models can be simulated with MATLAB-SIMULINK. Files and tutorial can be downloaded at http:/ / www.lsis.org/bateman/UAV.zip.

\section{Aircraft model}

The aircraft studied in this paper and shown in Fig.1 is an inverted V-tail UAV. It is assumed that its controls are fully indenpendent: $\delta_{x}$ is the throttle, $\delta_{a r}, \delta_{a l}, \delta_{f r}, \delta_{f l}, \delta_{e r}, \delta_{e l}$ control the right and left ailerons, the right and left flaps, the right and left inverted $\mathrm{V}$ tail control surfaces respectively. These latter controls are named ruddervators because they combine the tasks of the elevators and rudder. In the fault-free mode, the ailerons and the ruddervators are known as the primary control surfaces, they produce the roll, the pitch and the yaw. As far as the flaps are concerned, in the fault-free mode, they are only used to produce a lift increment during takeoff and a drag increment during landing. They are known as the secondary control surfaces.

It is assumed that each one of the primary control surfaces may lock at any arbitrary position on its deflection range. To compensate for the fault, the FTC exploits the redundancies provided by the remaining control surfaces. In this pespective, the UAV model has to consider the aerodynamic effects produced by each control surface.

The following dynamic model of the aircraft is presented in the case of a rigid-body aircraft, the weight $m$ is constant and the centre of gravity c.g. is fixed position. Let $R_{E}=\left(O, \mathbf{x}_{\mathbf{E}}, \mathbf{y}_{\mathbf{E}}, \mathbf{z}_{\mathbf{E}}\right)$ be a right-hand inertial frame such that $\mathbf{z}_{\mathrm{E}}$ is the vertical direction downwards the earth, $\boldsymbol{\xi}=$ $(x, y, z)$ denotes the position of $c . g$. in $R_{E}$. Let $R_{b}=\left(c . g ., \mathbf{x}_{\mathbf{b}}, \mathbf{y}_{\mathbf{b}}, \mathbf{z}_{\mathbf{b}}\right)$ be a right-hand body fixed frame for the UAV, at $t=0 R_{E}$ and $R_{b}$ coincide. The linear velocities $\boldsymbol{\mu}=(u, v, w)$ and the angular velocities $\Omega=(p, q, r)$ are expressed in the body frame $R_{b}$ where $p, q, r$ are roll, pitch and yaw respectively. The orientation of the rigid body in $R_{E}$ is located with the bank angle 


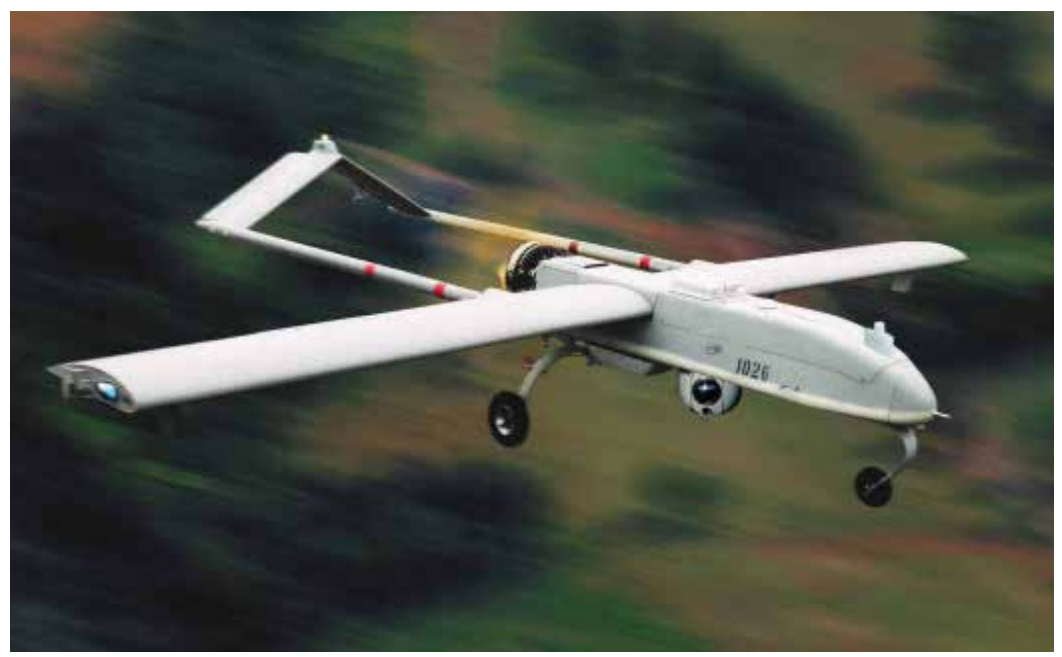

Fig. 1. The RQ7A Shadow $200 \mathrm{UAV}$

$\phi$, the pitch angle $\theta$ and the heading angle $\psi$. The transformation from $R_{b}$ to $R_{E}$ is given by a transformation matrix $\mathbf{T}_{\mathrm{bE}}$ :

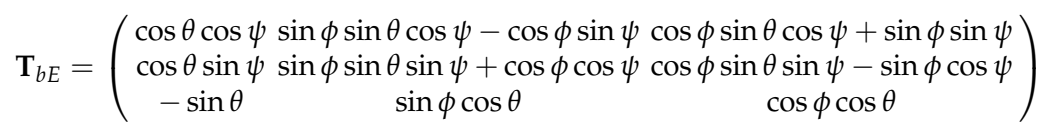

Forces $F_{x}^{R_{b}}, F_{y}^{R_{b}}, F_{z}^{R_{b}}$ acting on the aircraft are expressed in $R_{b}$, they originate in gravity $\mathbf{F}_{\text {grav }}$, propulsion $\mathbf{F}_{\text {prop}}$, and aerodynamic effects $\mathbf{F}_{\text {aero }}$. According to Newton's second law:

$$
\left(\begin{array}{c}
\dot{u} \\
\dot{v} \\
\dot{w}
\end{array}\right)=\frac{1}{m}\left(\begin{array}{c}
F_{x}^{R_{b}} \\
F_{y}^{R_{b}} \\
F_{z}^{R_{b}}
\end{array}\right)-\left(\begin{array}{c}
p \\
q \\
r
\end{array}\right) \wedge\left(\begin{array}{c}
u \\
v \\
w
\end{array}\right)
$$

where $\wedge$ denotes the cross product. Let $R_{w}=\left(c . g_{.}, \mathbf{x}_{\mathbf{w}}, \mathbf{y}_{\mathbf{w}}, \mathbf{z}_{\mathbf{w}}\right)$ be the wind reference frame where $\mathbf{x}_{\mathbf{w}}$ is aligned with the true airspeed $V$. The orientation of the body reference frame in the wind reference frame is located with the angle of attack $\alpha$ and the sideslip $\beta$. The transformation from $R_{b}$ to $R_{w}$ is given by a transformation matrix $\mathbf{T}_{\mathbf{b w}}$ :

$$
\mathbf{T}_{b w}=\left(\begin{array}{ccc}
\cos \alpha \cos \beta & \sin \beta & \sin \alpha \cos \beta \\
-\cos \alpha \sin \beta & \cos \beta & -\sin \alpha \sin \beta \\
-\sin \alpha & 0 & \cos \alpha
\end{array}\right)
$$

Furthermore, the aerodynamic state variables $(V, \alpha, \beta)$ and their time derivatives can be formulated using $\mathbf{T}_{\mathbf{b w}}$ from $\boldsymbol{\mu}$ (Rauw, 1993).

$$
\begin{aligned}
& V=\sqrt{u^{2}+v^{2}+w^{2}} \\
& \alpha=\arctan \left(\frac{w}{u}\right) \\
& \beta=\arctan \left(\frac{v}{\sqrt{u^{2}+w^{2}}}\right)
\end{aligned}
$$

For the sake of clarity, the forces are written in the reference frame where their expressions are the simplest. They are transformed into the desired frame by means of the matrices $\mathbf{T}_{\mathbf{b E}}$ and 
$\mathbf{T}_{\mathbf{b w}}$ or their inverse.

$$
\begin{aligned}
& \mathbf{F}_{\text {grav }}{ }^{R_{E}}=\left(\begin{array}{lll}
0 & 0 & g
\end{array}\right)^{T} \\
& \mathbf{F}_{\text {prop }} R_{b}=\left(\frac{k \rho(z)}{V} \delta_{x} \quad 000\right)^{T} \\
& \mathbf{F}_{\text {aero }}{ }^{R_{w}}=\bar{q} S\left(-C_{D} C_{y}-C_{L}\right)^{T}
\end{aligned}
$$

The model of the engine propeller is given in (Boiffier, 1998), $\rho$ is the air density, $k$ is a constant characteristic of the propeller engine, $\bar{q}=\frac{1}{2} \rho V^{2}$ and $S$ denote the aerodynamic pressure and a reference surface. The aerodynamic force coefficients are expressed as linear combination of the state elements and control inputs. The values of these aerodynamic coefficients can be found in the attached MATLAB files.

$$
\begin{aligned}
C_{D} & =C_{D 0}+\frac{S}{\pi b^{2}} C_{L}^{2}+C_{D \delta_{a r}}\left|\delta_{a r}\right|+C_{D \delta_{a l}}\left|\delta_{a l}\right|+C_{D \delta_{f r}}\left|\delta_{f r}\right|+C_{D \delta_{f l}}\left|\delta_{f l}\right|+C_{D \delta_{e r}}\left|\delta_{e r}\right|+C_{D \delta_{e l}}\left|\delta_{e l}\right| \\
C_{y} & =C_{y \beta} \beta+C_{y \delta_{a r}} \delta_{a r}+C_{y \delta_{a l}} \delta_{a l}+C_{y \delta_{f r}} \delta_{f r}+C_{y \delta_{f l}} \delta_{f l}+C_{y \delta_{e r}} \delta_{e r}+C_{y \delta_{e l}} \delta_{e l} \\
C_{L} & =C_{L 0}+C_{L \alpha} \alpha+C_{L \delta_{a r}} \delta_{a r}+C_{L \delta_{a l}} \delta_{a l}+C_{L \delta_{f r}} \delta_{f r}+C_{L \delta_{f l}} \delta_{f l}+C_{L \delta_{e r}} \delta_{e r}+C_{L \delta_{e l}} \delta_{e l}
\end{aligned}
$$

The relationships between the angular velocities, their derivatives and the moments $\mathcal{M}_{x}^{R_{b}}$, $\mathcal{M}_{y}^{R_{b}}, \mathcal{M}_{z}^{R_{b}}$ applied to the aircraft originate from the general moment equation. $\mathrm{J}$ is the inertia matrix.

$$
\left(\begin{array}{c}
\dot{p} \\
\dot{q} \\
\dot{r}
\end{array}\right)=\mathbf{J}^{-1}\left[\left(\begin{array}{c}
\mathcal{M}_{x}^{R_{b}} \\
\mathcal{M}_{y}^{R_{b}} \\
\mathcal{M}_{z}^{R_{b}}
\end{array}\right)-\left(\begin{array}{c}
p \\
q \\
r
\end{array}\right) \wedge \mathbf{J}\left(\begin{array}{c}
p \\
q \\
r
\end{array}\right)\right]
$$

The moments are expressed in $R_{b}$, they are due to aerodynamic effects and are modeled as follows:

$$
\left(\mathcal{M}_{x}^{R_{b}} \mathcal{M}_{y}^{R_{b}} \mathcal{M}_{z}^{R_{b}}\right)=\bar{q} S\left(b C_{l} \bar{c} C_{m} b C_{n}\right)
$$

where $\bar{c}$ and $b$ are the mean aerodynamic chord and the wing span. The aerodynamic moment coefficients are expressed as a linear combination of state elements and control inputs as

$$
\begin{aligned}
& C_{l}=C_{l \beta} \beta+C_{l p} \frac{b p}{2 V}+C_{l r} \frac{b r}{2 V}+C_{l \delta_{a r}} \delta_{a r}+C_{l \delta_{a l}} \delta_{a l}+C_{l \delta_{e r}} \delta_{e r}+C_{l \delta_{e l}} \delta_{e l}+C_{l \delta_{f r}} \delta_{f r}+C_{l \delta_{f l}} \delta_{f l} \\
& C_{m}=C_{m 0}+C_{m \alpha} \alpha+C_{m q} \frac{\bar{c} q}{2 V}+C_{m \delta_{a r}} \delta_{a r}+C_{m \delta_{a l}} \delta_{a l}+C_{m \delta_{e r}} \delta_{e r}+C_{m \delta_{e l}} \delta_{e l}+C_{m \delta_{f r}} \delta_{f r}+C_{m \delta_{f l}} \delta_{f l} \\
& C_{n}=C_{n \beta} \beta+C_{n p} \frac{b p}{2 V}+C_{n r} \frac{b r}{2 V}+C_{n \delta_{a r}} \delta_{a r}+C_{n \delta_{a l}} \delta_{a l}+C_{n \delta_{e r}} \delta_{e r}+C_{n \delta_{e l}} \delta_{e l}+C_{n \delta_{f r}} \delta_{f r}+C_{n \delta_{f l}} \delta_{f l}
\end{aligned}
$$

Equations (6) and (9) make obvious the aerodynamic forces and moments produced by each control surface. This is useful to model the fault effects and the redundancies provided by the healthy control surfaces.

The FDI/FTC problem is first an attitude control problem, thus the heading angle $\psi$, the $x$ and $y$ coordinates are not studied in the sequel.

With regard to the kinematic relations, the bank angle and the pitch angle time derivatives are (Boiffier, 1998):

$$
\begin{aligned}
\dot{\phi} & =p+q \sin \phi \tan \theta+r \cos \phi \tan \theta \\
\dot{\theta} & =q \cos \phi-r \sin \phi
\end{aligned}
$$


The relationship between the time derivative of the position of the aircraft's centre of gravity $\boldsymbol{\xi}$, the transformation matrix $\mathbf{T}_{\mathbf{b E}}$ and the linear velocities $\boldsymbol{\mu}$ allows to write:

$$
\dot{z}=-u \sin \theta+v \sin \phi \cos \theta+w \cos \phi \cos \theta
$$

Let $\mathbf{X}=\left(\begin{array}{llllllllll}\phi & \theta & V & \alpha & \beta & p & q & r & z\end{array}\right)^{T}$ the state vector and $\mathbf{U}=\left(\begin{array}{lllll}\delta_{x} & \delta_{a r} & \delta_{a l} & \delta_{f r} & \delta_{f l}\end{array}\right.$ $\left.\delta_{e r} \delta_{e l}\right)^{T}$ the control vector. All the state vector is measured and $\mathbf{Y}$ is the measure vector. From above, the model of the UAV can be written as a nonlinear model affine in the control

$$
\begin{aligned}
& \dot{\mathbf{X}}=f(\mathbf{X})+g(\mathbf{X}) \mathbf{U} \\
& \mathbf{Y}=\mathbf{C} \mathbf{X}
\end{aligned}
$$

Practically, the nonlinear aircraft model has been implemented with MATLAB in a sfunction. In the fault-free mode, for a given operating point $\left\{\mathbf{X}_{\mathbf{e} 0}, \mathbf{U}_{\mathbf{e} 0}\right\}$, where $\mathbf{U}_{\mathbf{e} 0}$ denotes the trim positions of the controls, the linearized model of the aircraft can be written as

$$
\begin{aligned}
& \dot{\mathbf{x}}=\mathbf{A x}+\mathbf{B u} \\
& \mathbf{y}=\mathbf{C x}
\end{aligned}
$$

\subsection{Fault model}

In the fault-free mode, the control surface deflections are constrained: asymmetrical aileron deflections produce the roll control, pitch is achieved through deflecting both ruddervators in the same direction and yaw is achieved through deflecting both ruddervators in opposite direction. As for the flaps, their deflection is symmetrical.

The faults considered are stuck control surfaces. For $t \geq t_{f}$, the faulty control vector $\mathbf{U}^{\mathbf{f}}(t)=\mathcal{U}^{\mathbf{f}}$, where $t_{f}$ is the fault-time and $\mathcal{U}^{\mathbf{f}}$ are the stuck control surface positions. For the simulations, a fault is modeled as a rate limiter response to a step. The slew rate is chosen equal to the maximum speed of the actuators. Let $\mathbf{U}^{\text {h }}$ be the remaining surfaces, then the state equation (12) in faulty mode becomes:

$$
\dot{\mathbf{X}}=f(\mathbf{X})+g^{f}(\mathbf{X}) \mathbf{U}^{\mathbf{f}}+g^{h}(\mathbf{X}) \mathbf{U}^{\mathbf{h}}
$$

\section{The nominal controller}

A linear state feedback controller with reference tracking is designed. It is based on an EA method which allows to set the aircraft handling qualities (Magni et al., 1997). This method allows to set the modes of the closed-loop (CL) aircraft with respect to the standards (MIL-HDBK-1797, 1997) and to decouple some state and control variables from some modes. The design is based on the fault-free linearized model (13) which modal analysis shows that the spiral mode is open-loop (OL) unstable.

Let $\tilde{\mathbf{Y}}=(\phi \beta V z)$ the tracked vector and $\tilde{\mathbf{Y}}_{\text {ref }}$ the reference vector. The autopilot is depicted in figure 2 and the nominal control law is:

$$
\mathbf{u}=\mathbf{L} \zeta+\mathbf{K x}
$$

where $\dot{\boldsymbol{\zeta}}=\tilde{\mathbf{Y}}_{\text {ref }}-\tilde{\mathbf{Y}}$ augments the state vector with the state variables which have to be tracked to zero and

$$
\dot{\zeta}=\left(\begin{array}{llll}
\varepsilon_{\phi} & \varepsilon_{\beta} & \varepsilon_{V} & \varepsilon_{z}
\end{array}\right)^{T}
$$




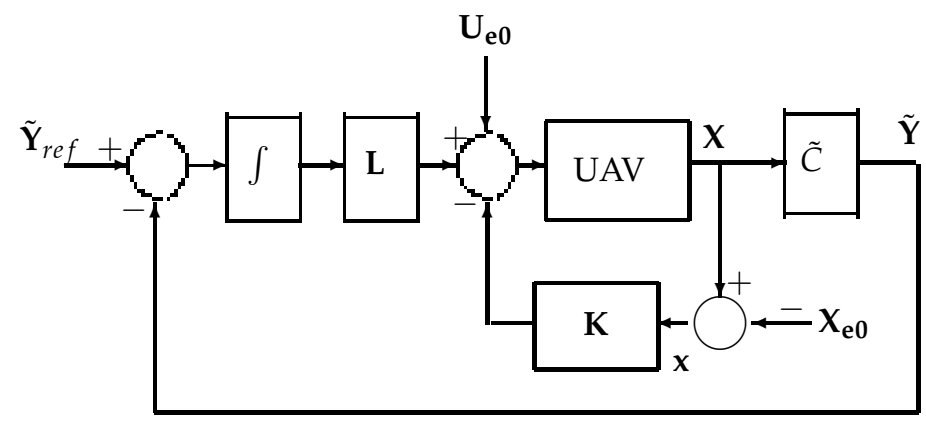

Fig. 2. The UAV control law

For a straight and level flight stage, Table 1 illustrates the EA strategy. A 0 means that the mode and the state variable or the control are decoupled. On the contrary, an $\times$ means that they are coupled.

In the fault-free mode, the ruddervators produce the pitch and the yaw, thus the longitudinal and the lateral axis are coupled. To take this into account, the autopilot is designed by considering the complete linearized model of the aircraft (13). For example, a coupling has been set between the spiral mode and the angle of attack which are a lateral mode and a longitudinal state variable respectively. This is illustrated by the highlighted cells in Table 1 .

This approach significantly differs from the classical method which consists in designing two autopilots, one for the longitudinal axis, another one for the lateral axis. However, from the FTC point of view, a control surface failure upsets the equilibrium of forces and moments and produce significant couplings between longitudinal and lateral axis. Because of this, the method adopted to design the nominal autopilot could be used to design the fault-tolerant controllers. From (13), (16) and (17)

$$
\left(\begin{array}{c}
\dot{\mathbf{x}} \\
\dot{\boldsymbol{\zeta}}
\end{array}\right)=\left(\begin{array}{cc}
\mathbf{A}+\mathbf{B K} & \mathbf{B L} \\
-\tilde{\mathbf{C}} & \mathbf{0}
\end{array}\right)\left(\begin{array}{l}
\mathbf{x} \\
\boldsymbol{\zeta}
\end{array}\right)+\left(\begin{array}{l}
\mathbf{0} \\
\mathbf{I}
\end{array}\right)\left(\tilde{Y}_{r e f}\right)
$$

with $\tilde{\mathbf{C}} \in \mathbb{R}^{4 \times 9}$ and $\tilde{\mathbf{C}}(i, j)=1$ for $\{i, j\}=\{1,1\},\{2,5\},\{3,3\},\{4,9\}$ else $\tilde{\mathbf{C}}(i, j)=0$.

Matrices $\mathbf{K} \in \mathbb{R}^{7 \times 9}$ and $\mathbf{L} \in \mathbb{R}^{7 \times 4}$ are computed in order to set the state space matrix's eigenvalues and eigenvectors in (18). These latter define the aircraft's modes. The state space matrix in (18) also writes:

$$
\left(\begin{array}{cc}
\mathbf{A} & \mathbf{0} \\
-\tilde{\mathbf{C}} & \mathbf{0}
\end{array}\right)+\left(\begin{array}{l}
\mathbf{B} \\
\mathbf{0}
\end{array}\right)(\mathbf{K} \mathbf{L})=\mathbf{F}+\mathbf{G}(\mathbf{K} \mathbf{L})
$$

Let $\lambda_{i}$ the $i^{\text {th }}$ eigenvalue (or closed-loop pole) corresponding to the eigenvector $\overrightarrow{\mathbf{v}}^{i} \in \mathbb{R}^{13}$, next

$$
(\mathbf{F}+\mathbf{G}(\mathbf{K ~ L})) \overrightarrow{\mathbf{v}}^{i}=\lambda_{i} \overrightarrow{\mathbf{v}}^{i}
$$

let

$$
\overrightarrow{\mathbf{w}}^{i}=(\mathbf{K} \mathbf{L}) \overrightarrow{\mathbf{v}}^{i} \in \mathbb{R}^{7}
$$

then

$$
\left(\mathbf{F}-\lambda_{i} \mathbf{I} \mathbf{G}\right)\left(\begin{array}{c}
\overrightarrow{\mathbf{v}}^{i} \\
\overrightarrow{\mathbf{w}}^{i}
\end{array}\right)=\overrightarrow{\mathbf{0}}
$$




\begin{tabular}{|c|c|c|c|c|c|c|c|c|}
\hline mode & short period & phugoid & throttle & roll & dutchroll & spiral, $\varepsilon_{\phi}$ & $\varepsilon_{\beta}$ & $\varepsilon_{V}, \varepsilon_{z}$ \\
\hline OL poles & $-4.2 \pm 9.6 i$ & $-0.03 \pm 0.5 i$ & -0.0002 & -21 & $-1.7 \pm 5.6 i$ & 0.073 & & \\
\hline CL poles & $-10 \pm 10 i$ & $-2 \pm 2 i$ & -1 & -100 & $-5 \pm 5 i$ & $-1 \pm .25 i$ & -1.5 & $-1 \pm .5 i$ \\
\hline eigenvector & $\vec{v}^{1,2}$ & $\vec{v}^{3,4}$ & $\vec{v}^{5}$ & $\vec{v}^{6}$ & $\vec{v}^{7,8}$ & $\vec{v}^{9,10}$ & $\vec{v}^{11}$ & $\vec{v}^{12,13}$ \\
\hline$\phi$ & 0 & 0 & 0 & $\times$ & $\times$ & $\times$ & $\times$ & $\times$ \\
\hline$\theta$ & $\times$ & $\times$ & $\times$ & 0 & 0 & $\times$ & 0 & $\times$ \\
\hline$V$ & $\times$ & $\times$ & $\times$ & 0 & 0 & 0 & 0 & $\times$ \\
\hline$\alpha$ & $\times$ & $\times$ & $\times$ & 0 & 0 & $\times$ & 0 & $\times$ \\
\hline$\beta$ & 0 & 0 & 0 & $\times$ & $\times$ & $\times$ & $\times$ & $\times$ \\
\hline$p$ & 0 & 0 & 0 & $\times$ & $\times$ & $\times$ & $\times$ & 0 \\
\hline$q$ & $\times$ & $\times$ & $\times$ & 0 & 0 & $\times$ & 0 & $\times$ \\
\hline$r$ & 0 & 0 & 0 & $\times$ & $\times$ & $\times$ & $\times$ & 0 \\
\hline$z$ & $\times$ & $\times$ & $\times$ & $\times$ & $\times$ & 0 & $\times$ & $\times$ \\
\hline$\varepsilon_{\Phi}$ & $\times$ & $\times$ & $\times$ & $\times$ & $\times$ & $\times$ & $\times$ & $\times$ \\
\hline$\varepsilon_{\beta}$ & $\times$ & $\times$ & $\times$ & $\times$ & $\times$ & 0 & $\times$ & $\times$ \\
\hline$\varepsilon_{V}$ & $\times$ & $\times$ & $\times$ & $\times$ & $\times$ & $\times$ & $\times$ & $\times$ \\
\hline$\varepsilon_{z}$ & $\times$ & $\times$ & $\times$ & $\times$ & $\times$ & $\times$ & $\times$ & $\times$ \\
\hline$\delta_{x}$ & $\times$ & $\times$ & $\times$ & $\times$ & $\times$ & $\times$ & $\times$ & $\times$ \\
\hline$\delta_{a r}$ & 0 & 0 & 0 & $\times$ & $\times$ & $\times$ & $\times$ & 0 \\
\hline$\delta_{a l}$ & 0 & 0 & 0 & $\times$ & $\times$ & $\times$ & $\times$ & 0 \\
\hline$\delta_{f r}$ & 0 & 0 & 0 & 0 & 0 & 0 & 0 & 0 \\
\hline$\delta_{f l}$ & 0 & 0 & 0 & 0 & 0 & 0 & 0 & 0 \\
\hline$\delta_{e r}$ & $\times$ & $\times$ & $\times$ & $\times$ & $\times$ & 0 & $\times$ & $\times$ \\
\hline$\delta_{e l}$ & $\times$ & $\times$ & $\times$ & $\times$ & $\times$ & 0 & $\times$ & $\times$ \\
\hline
\end{tabular}

Table 1. Eigenstructure assignment strategy, airspeed $25 \mathrm{~ms}^{-1}$, height $200 \mathrm{~m}$

$\left(\mathbf{F}-\lambda_{i} \mathbf{I} \mathbf{G}\right)$ is a $13 \times 20$ matrix and the size of its null space is equal to the number of control inputs, here seven. In order to set the eigenvector structure (Table 1) while reducing the solution space, some constraints are added. To hide the $i^{\text {th }}$ mode to the $j^{\text {th }}$ state variable or control input, a mask is introduced such that

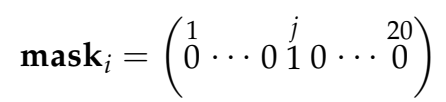

In order to find a unique solution vector, for each eigenvalue $\lambda_{i}$, six masks are defined and the system to solve writes

$$
\left(\begin{array}{c}
\mathbf{F}-\lambda_{i} \mathbf{I} \mathbf{G} \\
\mathbf{m a s k}_{\mathbf{i} 1} \\
\cdots \\
\text { mask }_{\mathbf{i 6} 6}
\end{array}\right)\left(\begin{array}{c}
\overrightarrow{\mathbf{v}}^{i} \\
\overrightarrow{\mathbf{w}}^{i}
\end{array}\right)=\overrightarrow{\mathbf{0}}
$$

Let $\mathbf{P}=\left(\overrightarrow{\mathbf{v}}^{1} \ldots \overrightarrow{\mathbf{v}}^{13}\right)$ and $\mathbf{Q}=\left(\overrightarrow{\mathbf{w}}^{1} \ldots \overrightarrow{\mathbf{w}}^{7}\right)$, then according to (21), matrices $\mathbf{K}$ and $\mathbf{L}$ write:

$$
(\mathbf{K} \mathbf{L})=\mathbf{Q P}^{-1}
$$

Fig. 4 shows the nominal autopilot functionning in the $[0 s, 16 s]$ fault-free time interval.

\section{Fault diagnosis}

The class of faults addressed here are stuck control surfaces. However, the proposed diagnosis system can also deal with actuator the loss of efficiency. 
To process for the faults, the diagnosis system could be realized by measuring the actuator positions. This approach which requires potentiometers, wiring and acquisition board is complex to implement and induces an increase of weight. Without these measurements, the control inputs appear as unknown inputs which have to be estimated. This can be achieved by the use of observers able to estimate the unknown inputs of a system.

In this connection, the problem of unknown, constant or slowy varying input estimation using banks of Kalman filters is discussed in (Kobayashi \& Simon, 2003), (Ducard \& Geering, 2008). The unknown inputs are declared as state variables, under the condition that the system is observable, the problem consists in estimating an augmented state vector.

However, to catch for the actuator fault transients, the observer has to estimate time varying inputs. Such an estimation is possible if these inputs are observable. The input observability problem was addressed by Patton in (Hou \& Patton, 1998) who gave some necessary and sufficient conditions to prove input observability for linear time invariant systems with unknown initial conditions.

As far as the observer is concerned, Xiong (Xiong \& Saif, 2003) proposed an Unknown Input Decoupled Functionnal Observer (UIDFO) which has no boundedness conditions as for time varying inputs and does not require differentiation of the measured outputs.

The fact remains that control surfaces offer redundancies that make aileron failures not isolable. In these conditions, an active diagnosis strategy has to be considered.

Input observability of the UAV is studied in subsection 4.1, the UIDFO is briefly described in subsection 4.2 and a diagnosis system based on a bank of UIDFO is detailed in subsection 4.3. The active diagnosis strategy is presented in subsection 4.4 .

\subsection{Input observability}

Given, the linearized model (13).

Definition 1. The input $u(t)$ is said to be observable if $y(t)=0$ for $t \geq 0$ implies $u(t)=0$ for $t>0$ (Hou \& Patton, 1998).

Let $\lambda \in \mathbb{C}, \Sigma_{\text {sys }}$ and $\Sigma_{\mathrm{AC}}$ refer to the system matrix and the observability pencil respectively, they write:

$$
\boldsymbol{\Sigma}_{\text {sys }}=\left(\begin{array}{ll}
\mathbf{A} & \mathbf{B} \\
\mathbf{C} & \mathbf{D}
\end{array}\right)-\lambda\left(\begin{array}{ll}
\mathbf{I} & 0 \\
\mathbf{0} & \mathbf{0}
\end{array}\right) \text { and } \boldsymbol{\Sigma}_{\mathrm{AC}}=\left(\begin{array}{l}
\mathbf{A} \\
\mathbf{C}
\end{array}\right)-\lambda\left(\begin{array}{l}
\mathbf{I} \\
\mathbf{0}
\end{array}\right)
$$

Kroenecker's theory of singular pencils shows that any pencil $\lambda \mathbf{M}-\mathbf{N}$ with dimension $m \times n$ can be brought into the canonical quasidiagonal form:

$$
\mathbf{P}(\lambda \mathbf{M}-\mathbf{N}) \mathbf{Q}=\left(\begin{array}{cccc}
\lambda \mathbf{M}_{\mathbf{c}}-\mathbf{N}_{\mathbf{c}} & \times & \times & \times \\
& \lambda \mathbf{I}-\mathbf{N}_{\mathbf{f}} & \times & \times \\
& & \lambda \mathbf{M}_{\infty}-\mathbf{I} & \times \\
& & & \lambda \mathbf{M}_{\mathbf{r}}-\mathbf{N}_{\mathbf{r}}
\end{array}\right)
$$

- $\mathbf{P}$ and $\mathbf{Q}$ are nonsingular constant matrices with dimensions $m \times m$ and $n \times n$.

- the finite eigenvalues are in the square and regular pencil $\lambda \mathbf{I}-\mathbf{J}_{\mathbf{f}}$ and $\mathbf{J}_{\mathbf{f}}$ is in Jordan canonical form. It is built with Jordan blocks $\mathbf{J}_{\mathbf{f}_{\mathbf{i}}}$ with dimension $i \times i$.

- the infinite eigenvalues are in the square and regular pencil $\lambda \mathbf{J}_{\infty}-\mathbf{I}$ and $\mathbf{J}_{\infty}$ is compound with Jordan blocks $\mathbf{J}_{\infty_{\mathbf{i}}}$ of size $i \times i$.

- $\lambda \mathbf{M}_{\mathbf{r}}-\mathbf{N}_{\mathbf{r}}$ is a singular pencil and has a block diagonal structure, each block takes the form $\lambda \mathbf{M}_{\mathbf{r}_{\mathbf{i}}}-\mathbf{N}_{\mathbf{r}_{\mathbf{i}}}=\lambda\left(\begin{array}{c}\mathbf{I} \\ \mathbf{0}^{T}\end{array}\right)-\left(\begin{array}{c}\mathbf{0}^{T} \\ \mathbf{I}\end{array}\right)$ with dimension $r_{i+1} \times r_{i} . \mathbf{0}$ stands for zero vector and $\mathbf{0}^{T}$ denotes its transpose. The $r_{i}$ are called Kroenecker's row indices. 
- $\lambda \mathbf{M}_{\mathbf{c}}-\mathbf{N}_{\mathbf{c}}$ is a singular pencil and has a block diagonal structure, each block takes the form $: \lambda \mathbf{M}_{\mathbf{c}_{\mathbf{j}}}-\mathbf{N}_{\mathbf{c}_{\mathbf{j}}}=\lambda(\mathbf{I} \mathbf{0})-(\mathbf{0} \mathbf{I})$. The $c_{j} \times c_{j+1}$ are called Kroenecker's column indices.

This decomposition is applied to $\Sigma_{\text {sys }}$ and $\Sigma_{\text {AC }}$. The following theorem contains a numerically effective test for input observability:

Theorem 1. System (13) is input observable if and only if the block $\lambda \mathbf{M}_{\mathbf{c}_{\text {sys }}}-\mathbf{N}_{\mathbf{c}_{\text {sys }}}$ vanishes in equation (27) and $\operatorname{dim}\left(\mathbf{J}_{\mathbf{f}_{\text {sys }}}\right)=\operatorname{dim}\left(\mathbf{J}_{\mathbf{f}_{\mathrm{AC}}}\right)$ (Hou et Patton)(Hou $\mathcal{E}$ Patton, 1998).

Nevertheless, due to the numerical unreliability of the computation, this form is not suitable and the staircase form, computed with the GUPTRI algorithm, is used to exhibit the Kroenecker form (Demmel \& Kågstrom, 1993).

All the state vector is measured and theorem 1 is applied to assess the ruddervator observability. For this outpout vector, the matrix system and the observability pencil have the following structures:

- $\Sigma_{\mathrm{AC}}$ has no finite eigenvalue, nine singular pencils with Kronecker raw indice equal to one,

- $\boldsymbol{\Sigma}_{\text {sys }}$ has no finite eigenvalue, seven singular pencils with Kronecker raw indice equal to one and two $2 \times 2$ jordan blocks containing infinite eigenvalues.

According to theorem 1 the ruddervator positions are observable. With the same measured outputs, theorem 1 is applied to assess the aileron observability. The matrix system and the observability pencil have the following structure:

- $\Sigma_{\text {AC }}$ is unchanged

- $\Sigma_{\text {sys }}$ has no finite eigenvalue, one singular pencil with Kronecker column indice equal to one, eight singular pencils with Kronecker raw indice equal to one and one $2 \times 2$ jordan block containing infinite eigenvalues.

Due to the presence of the singular pencil with Kronecker column equal to one, the aileron positions are not both observable. Thus, if one of the ailerons breaks down, the faulty control is not isolable. This is depicted in Fig. 3 where a down lock position of the left aileron has the same effects as a top lock position of the right aileron. In this case, an active diagnosis must be used to discriminate the faulty control surface.

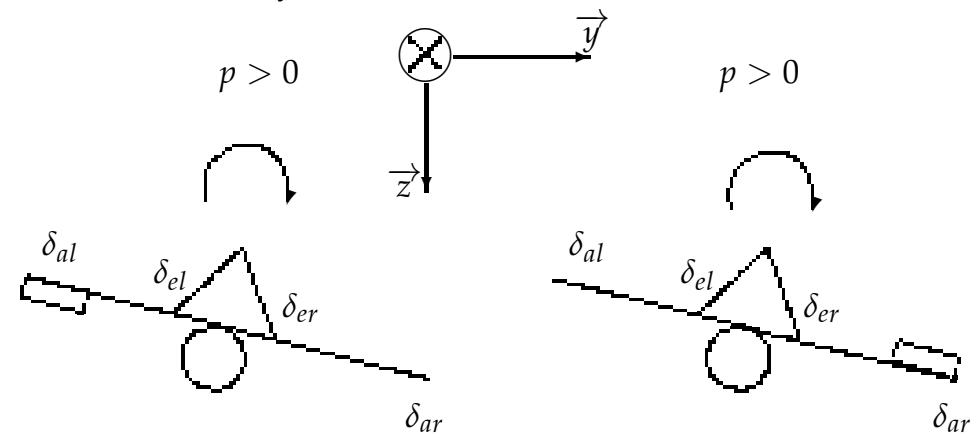

Fig. 3. Aerodynamic effects induced by right and left aileron failures 


\subsection{The unknown input decoupled functional observer}

In this part, results established in (Xiong \& Saif, 2003) are recalled. The following dynamic system driven by both known and unknown inputs is considered

$$
\begin{aligned}
& \dot{\mathbf{x}}=\mathbf{A x}+\mathbf{B u}+\mathbf{G d} \\
& \mathbf{y}=\mathbf{C x}
\end{aligned}
$$

where $\mathbf{x} \in \mathbb{R}^{n}$ is the state vector, $\mathbf{u} \in \mathbb{R}^{m}$ is the known input vector, $\mathbf{d} \in \mathbb{R}^{\ell}$ is the unknown input vector and $\mathbf{y} \in \mathbb{R}^{o}$ is the output vector. $\mathbf{A}, \mathbf{B}, \mathbf{G}$ and $\mathbf{C}$ are matrices with appropriate dimensions, $\mathbf{C}$ and $\mathbf{G}$ are assumed to be full rank.

The UIDFO detailed in (Xiong \& Saif, 2003) provides an estimation $\hat{\mathbf{d}}$ of the unknown input $\mathbf{d}$ and an estimation $\mathbf{z}$ of linear combination of state $\mathbf{T x}$. Theoretically, no boundedness conditions are required for the unknown inputs and their derivatives.

$$
\begin{aligned}
& \dot{\mathbf{z}}=\mathbf{F z}+\mathbf{H y}+\mathbf{T B u}+\mathbf{T G} \hat{\mathbf{d}} \\
& \hat{\mathbf{d}}=\gamma(\mathbf{W y}-\mathbf{E z}) \text { with } \gamma \in \mathbb{R}^{*+}
\end{aligned}
$$

Matrices $\mathbf{F}, \mathbf{H}, \mathbf{T}, \mathbf{W}$ and $\mathbf{E}$ are all design parameters. in order to satisfy the following conditions

$$
\begin{cases}\mathbf{F T}-\mathbf{T A}+\mathbf{H C}=0 & \mathbf{F} \text { is stable, } \\ \mathbf{E}=(\mathbf{T G})^{T} \mathbf{P} & \text { with } \mathbf{P} \text { solution of: } \mathbf{P F}+\mathbf{F}^{T} \mathbf{P}=-\mathbf{Q} \\ \mathbf{E T}=\mathbf{G}^{T} \mathbf{T}^{T} \mathbf{P T}=\mathbf{W C} & \text { and } \mathbf{Q}, \text { a semi-positive definite matrix, } \\ \operatorname{rank}(\mathbf{T G})=\operatorname{rank}(\mathbf{G})=\ell & \end{cases}
$$

These matrices exist if and only if

(i) $\operatorname{rank}(\mathbf{C G})=\operatorname{rank}(\mathbf{G})$,

(ii) all unstable transmission zeros of system $(\mathbf{A}, \mathbf{G}, \mathbf{C})$ are unobservable modes of $(\mathbf{A}, \mathbf{C})$.

To prove their existence and to calculate them, system (28) is transformed into "Special Coordinate Basis" (SCB) (X.Liu et al., 2005).

\subsection{Diagnosis system performed with a bank of UIDFO}

The right aileron, the left aileron, the right ruddervator and the left ruddervator positions are unknown. In order to estimate each one of these unknown input positions, a bank of four UIDFOs is implemented. However, it has been proven above that the ailerons are not both input observable. It means that, if one of these two controls is faulty, one of the two estimations is wrong whereas the other is right, but in any case it is impossible to know which one is which.

Let $\hat{\delta}_{a r}, \hat{\delta}_{a l}, \hat{\delta}_{e r}$ and $\hat{\delta}_{e l}$ the positions estimated by each UIDFO, $\delta_{a r}, \delta_{a l}, \delta_{e r}$ and $\delta_{e l}$ the known control inputs computed by the nominal controller and $\bar{\delta}_{a r}, \bar{\delta}_{a l}, \bar{\delta}_{e r}$ and $\bar{\delta}_{e l}$ the unknown actual control surface positions. In the fault-free mode, the actual control surface positions are equal to those computed by the controller and to their estimations. When an actuator breaks down, the faulty actual control surface position is equal to its estimation but differs from that computed by the controller. Residuals are obtained by processing the differences between the positions computed by the controller with the estimated positions. The fault detection and isolation process consists in monitoring residuals by comparing them with thresholds. 
The first UIDFO estimates the unknown right aileron actual position $\bar{\delta}_{a r}$ by processing the measurement vector $\mathbf{y}$ and the known input $\mathbf{u}_{\mathbf{1}}=\left(\delta_{a l}, \delta_{e r}, \delta_{e l}\right)^{T}$. Let $b_{\delta_{i}}$ the column of the control matrix $B$ associated with the $\delta_{i}$ control input, then $\mathbf{B}_{\mathbf{1}}=\left(b_{\delta_{a l}}, b_{\delta_{e r}}, b_{\delta_{e l}}\right)^{T}$ and $\mathbf{G}_{\mathbf{1}}=$ $\left(b_{\delta_{a r}}\right)$,

$$
\begin{aligned}
\dot{\mathbf{z}}_{\mathbf{1}} & =\mathbf{F}_{\mathbf{1}} \mathbf{z}_{\mathbf{1}}+\mathbf{H}_{\mathbf{1}} \mathbf{y}+\mathbf{T}_{\mathbf{1}} \mathbf{B}_{\mathbf{1}}\left(\delta_{a l}, \delta_{e r}, \delta_{e l}\right)^{T}+\mathbf{T}_{\mathbf{1}} \mathbf{G}_{\mathbf{1}} \hat{\delta}_{a r} \\
\hat{\delta}_{a r} & =\gamma_{1}\left(\mathbf{W}_{\mathbf{1}} \mathbf{y}-\mathbf{E}_{\mathbf{1}} \mathbf{z}_{\mathbf{1}}\right)
\end{aligned}
$$

The other three ones UIDFO equations write

$$
\begin{aligned}
& \dot{\mathbf{z}}_{\mathbf{2}}=\mathbf{F}_{\mathbf{2}} \mathbf{z}_{\mathbf{2}}+\mathbf{H}_{\mathbf{2}} \mathbf{y}+\mathbf{T}_{\mathbf{2}} \mathbf{B}_{\mathbf{2}}\left(\delta_{a r}, \delta_{e r}, \delta_{e l}\right)^{T}+\mathbf{T}_{\mathbf{2}} \mathbf{G}_{\mathbf{2}} \hat{\delta}_{a l} \\
& \hat{\delta}_{a l}=\gamma_{2}\left(\mathbf{W}_{\mathbf{2}} \mathbf{y}-\mathbf{E}_{\mathbf{2}} \mathbf{z}_{\mathbf{2}}\right)
\end{aligned}
$$

with $\mathbf{B}_{\mathbf{2}}=\left(b_{\delta_{a r}}, b_{\delta_{e r}}, b_{\delta_{e l}}\right)^{T}$ and $\mathbf{G}_{\mathbf{2}}=\left(b_{\delta_{a l}}\right)$,

$$
\begin{aligned}
& \dot{\mathbf{z}}_{\mathbf{3}}=\mathbf{F}_{3} \mathbf{z}_{\mathbf{3}}+\mathbf{H}_{3} \mathbf{y}+\mathbf{T}_{3} \mathbf{B}_{\mathbf{3}}\left(\delta_{a r}, \delta_{a l}, \delta_{e l}\right)^{T}+\mathbf{T}_{\mathbf{3}} \mathbf{G}_{3} \hat{\delta}_{e r} \\
& \hat{\delta}_{e r}=\gamma_{3}\left(\mathbf{W}_{3} \mathbf{y}-\mathbf{E}_{3} \mathbf{z}_{\mathbf{3}}\right)
\end{aligned}
$$

with $\mathbf{B}_{\mathbf{3}}=\left(b_{\delta_{a r}}, b_{\delta_{a l}}, b_{\delta_{e l}}\right)^{T}$ and $\mathbf{G}_{\mathbf{3}}=\left(b_{\delta_{e r}}\right)$,

$$
\begin{aligned}
& \dot{\mathbf{z}}_{4}=\mathbf{F}_{4} \mathbf{z}_{\mathbf{4}}+\mathbf{H}_{4} \mathbf{y}+\mathbf{T}_{\mathbf{4}} \mathbf{B}_{4}\left(\delta_{a r}, \delta_{a l}, \delta_{e r}\right)^{T}+\mathbf{T}_{4} \mathbf{G}_{4} \hat{\delta}_{e l} \\
& \hat{\delta}_{e l}=\gamma_{4}\left(\mathbf{W}_{4} \mathbf{y}-\mathbf{E}_{4} \mathbf{z}_{\mathbf{4}}\right)
\end{aligned}
$$

with $\mathbf{B}_{4}=\left(b_{\delta_{a r}}, b_{\delta_{a l}}, b_{\delta_{e r}}\right)^{T}$ and $\mathbf{G}_{\mathbf{4}}=\left(b_{\delta_{e l}}\right)$.

For all the UIDFOs, condition (i) is assessed and condition (ii) is checked by computing the staircase forms of the system matrices $\left(\mathbf{A}, \mathbf{G}_{\mathbf{j}}, \mathbf{C}, \mathbf{0}\right)$ with $j=\{1, \ldots, 4\}$ and the observability pencil $(\mathbf{A}, \mathbf{C})$ with the GUPTRI algorithm.

Error signals are generated by comparison between the control positions $\delta_{i}$ and the estimated positions $\hat{\delta}_{i}$ where $\delta_{i} \in\left\{\delta_{a r}, \delta_{a l}, \delta_{e r}, \delta_{e l}\right\}$. In order to avoid false alarm that may arise from the transient behavior, these signals are integrated on a duration $\tau$ to produce residuals $r_{\delta_{i}}$ such that

$$
r_{\delta_{i}}=\left|\int_{t}^{t+\tau} \hat{\delta}_{i}(\theta)-\delta_{i}(\theta) d \theta\right|
$$

Let $\sigma_{\delta_{i}}$ the corresponding threshold and $\mu_{\delta_{i}}$ a logical state such that $\mu_{\delta_{i}}=1$ if $r_{\delta_{i}}>\sigma_{\delta_{i}}$ else $\mu_{\delta_{i}}=0$. Then, to detect and to partially isolate the faulty control surface, an incidence matrix is defined as follows:

\begin{tabular}{|c||c|c|c|c|}
\hline Faulty control & $\mu_{\delta_{a r}}$ & $\mu_{\delta_{e \mid} \mid}$ & $\mu_{\delta_{a \mid}}$ & $\mu_{\delta_{e r}}$ \\
\hline right aileron & 1 & 0 & 1 & 0 \\
\hline left aileron & 1 & 0 & 1 & 0 \\
\hline right ruddervator & 0 & 0 & 0 & 1 \\
\hline left ruddervator & 0 & 1 & 0 & 0 \\
\hline
\end{tabular}

Table 2. The incidence matrix

This matrix reveals that fault on right aileron and fault on left aileron are not isolable.

In order to illustrate the above-mentioned concepts, three failure scenarios are studied: a non critical ruddervator loss of efficiency $50 \%$, a catastrophic ruddervator locking and a non critical aileron locking. For the three cases, the fault occurs at faulty time $t_{f}=16 \mathrm{~s}$ whereas the UAV is turning and changing its airspeed (see Fig.4, Fig.7, Fig.10). These two manoeuvres involve both ailerons and ruddervators. 


\subsubsection{Ruddervator loss of efficiency}

For the right ruddervator, a $50 \%$ loss of efficiency is simulated. The nominal controller is robust enough to accommodate for the fault as it is depicted in Fig.4. The actual control surface positions and their estimations are shown in Fig. 5. As far as the right ruddervator is concerned, after time $t_{f}$, its control signal differs from its estimated position and this difference renders the fault obvious. The residuals are depicted in Fig. 6, with respect to (35) and to the incidence matrix Tab. 2, the right ruddervator is declared to be faulty.

\subsubsection{Ruddervator locking}

At time $t_{f}$, the right ruddervator is stuck at position $0^{\circ}$. As it is illustrated in Fig.7, the nominal controller cannot accommodate for the fault and the UAV is lost. The actual control surface positions and their estimations are shown in Fig.8, As regards the right ruddervator, its control signal differs from its estimated position and the residual analysis Fig. 9 allows to declare this control surface to be faulty. However, the control and measurement vectors diverge quickly, thus the signal acquisition of the estimated positions has to be sampled fast.

\subsubsection{Aileron locking}

In the event of an aileron failure, the nominal controller is robust enough to accommodate for the fault. However, the incidence matrix shows that faults on right and left ailerons cannot be isolated. This is due to the redundancies offered by these control surfaces that are not input observable. This aspect is depicted in Fig. 10, 11, 12 where the left aileron locks at position $+5^{\circ}$ at time $t_{f}=16 \mathrm{~s}$. Fig. 10 shows that this fault is non critical (it is naturally accommodated by the right aileron). However, as it is shown in Fig. 11, both estimations of aileron positions are consistent and the corresponding residuals exceed the thresholds. As a consequence, it is not possible to isolate the faulty aileron.

\subsection{Active diagnosis}

To overcome this problem, an active fault diagnosis strategy is proposed. It consists in exciting one of the aileron (here the right aileron) with a small-amplitude sinusoidal signal. If the left aileron is stuck, the measures contain a sinusoidal component which is detected with a selective filter. If the right aileron is stuck, the sinusoidal excitation cannot affect the state vector and the measures do not contain the sinusoidal components. This point is depicted in Fig. 13, a fault is simulated on the left aileron next to the right aileron. In the first case, the left aileron is stuck at $-5^{\circ}$, after the fault has been detected, the right aileron is excited with a $1^{\circ} \sin (20 t)$ signal. This component distinctly appears in the estimation of the left aileron position and allows to declare the left aileron faulty. In the second case, the right aileron is stuck at $+5^{\circ}$, after the fault has been detected, the right aileron is excited with the same sinusoidal signal. As this control surface is stuck, the sinusoidal signal does not appear in the estimation of the right aileron position and this control surface is declared faulty. Note that this method allows only to detect stuck ailerons. To deal with a loss of efficiency, three control surfaces are excited: the right aileron, the right and the left flaps. The excitation signals are such that they little affect the state and the measurement vectors. This is achieved by choosing the amplitudes of these excitations in the nullspace of the $\left(b_{\delta_{a r}}, b_{\delta_{f r}}, b_{\delta_{f l}}\right)$ matrix or if the nullspace does not exist, the excitation vector can be chosen as the right singular vector corresponding with the smallest singular value of the $\left(b_{\delta_{a r}}, b_{\delta_{f r}}, b_{\delta_{f l}}\right)$ matrix. If the right aileron is faulty, the excitation is not fulfilled and the measures contain a sinusoidal component. On the contrary, if the left aileron is faulty, the right aileron and the flaps fulfill the exctitation 

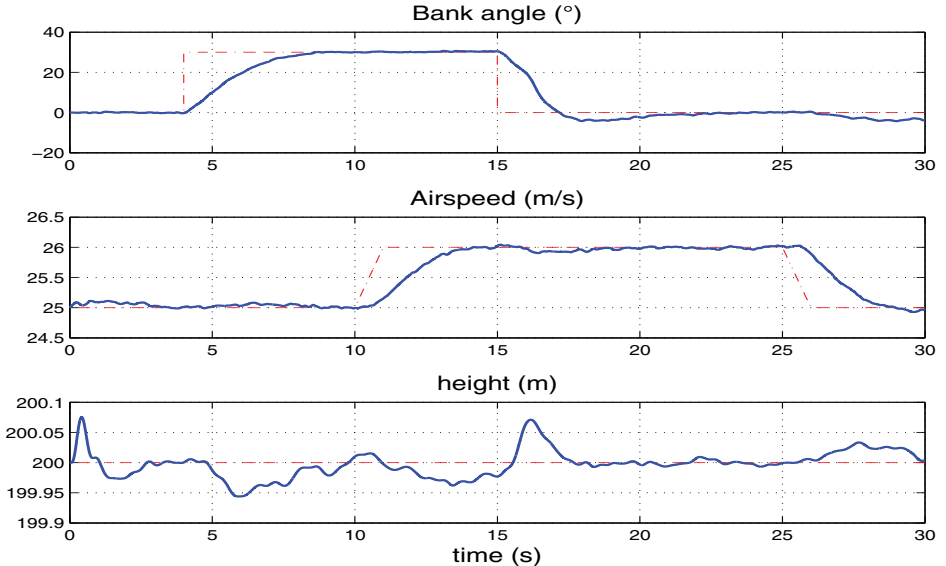

Fig. 4. Right ruddervator loss of efficiency: the tracked state variables
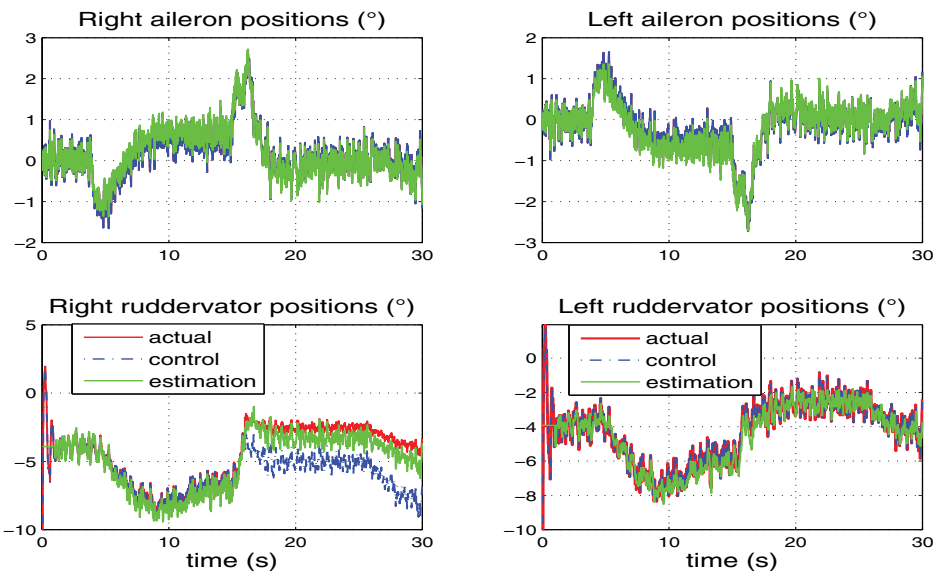

Fig. 5. Right ruddervator failure: the estimation process
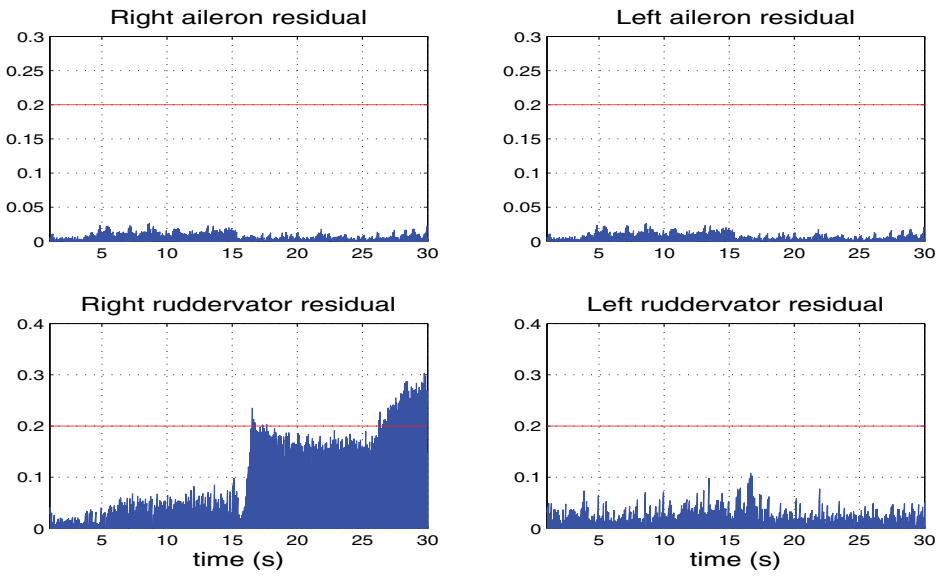

Fig. 6. Right ruddervator failure: the fault detection and isolation process 

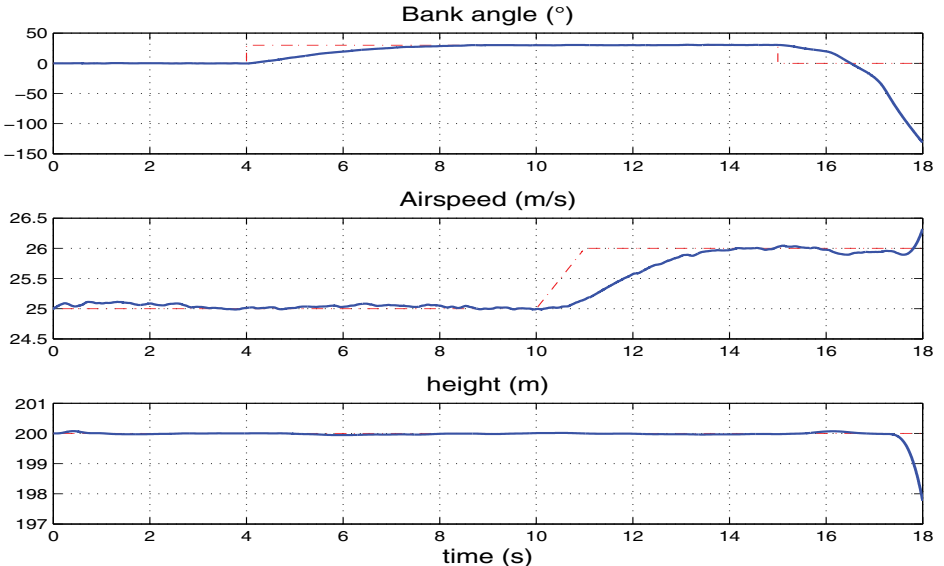

Fig. 7. Right ruddervator stuck: the tracked state variables
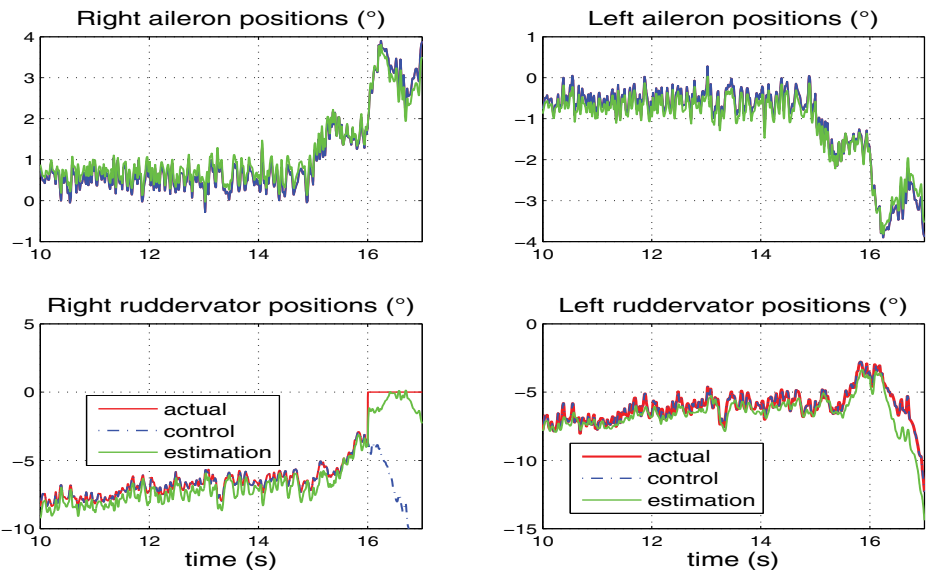

Fig. 8. Right ruddervator failure: the estimation process
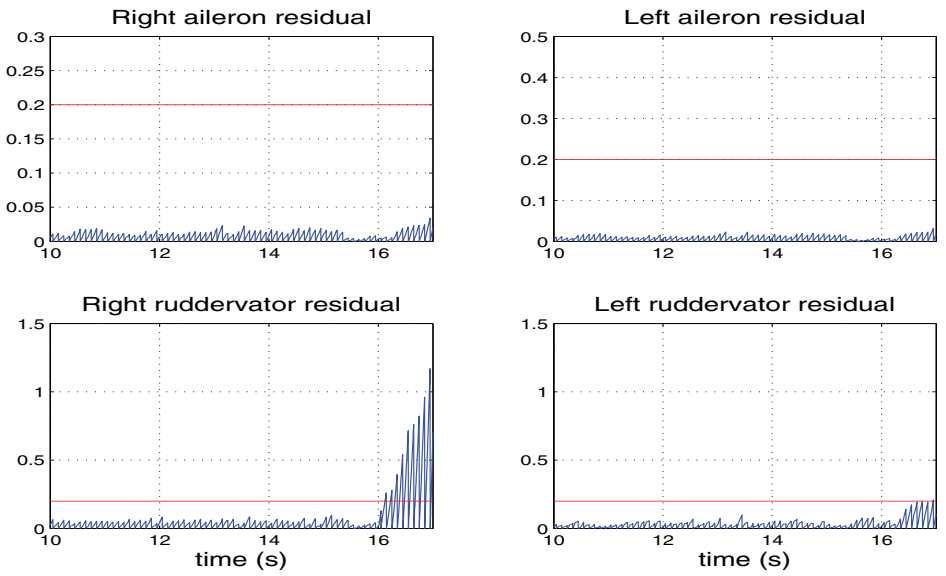

Fig. 9. Right ruddervator failure: the fault detection and isolation process 

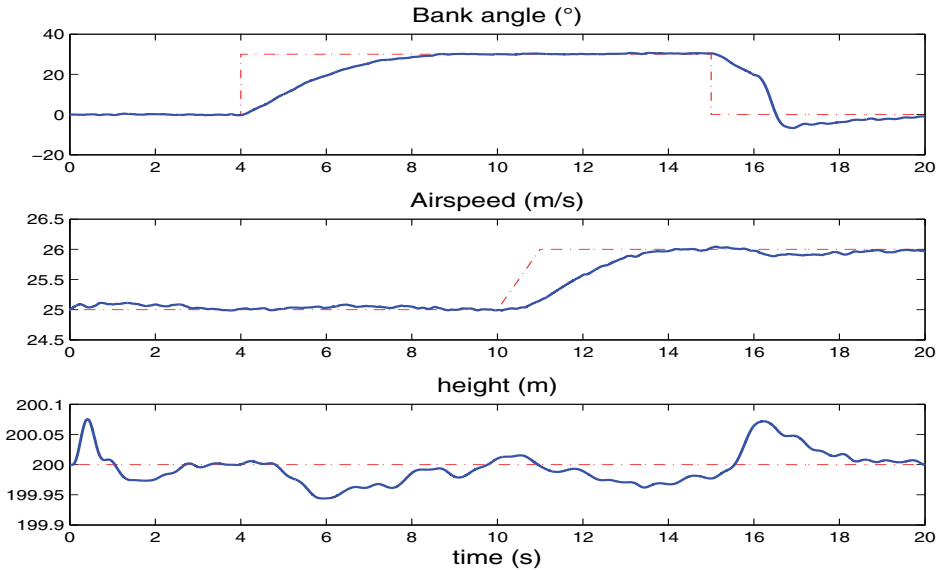

Fig. 10. Left aileron stuck: the tracked state variables
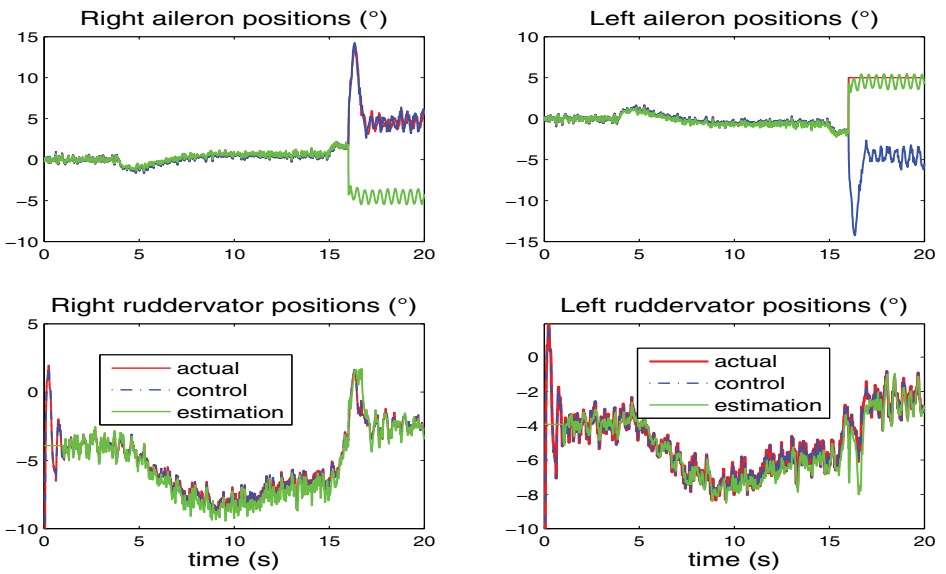

Fig. 11. Left aileron failure: the estimation process
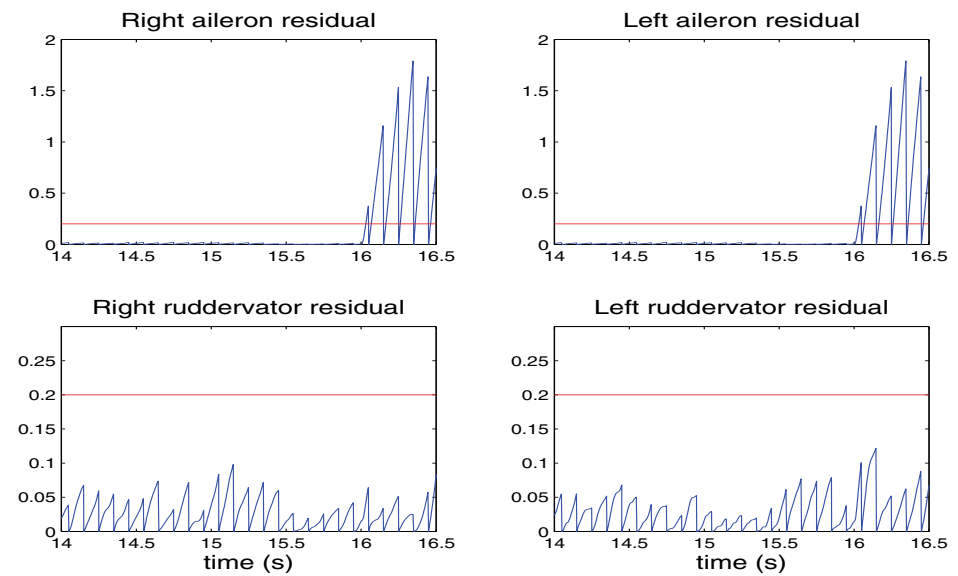

Fig. 12. Left aileron failure: the fault detection and isolation process 

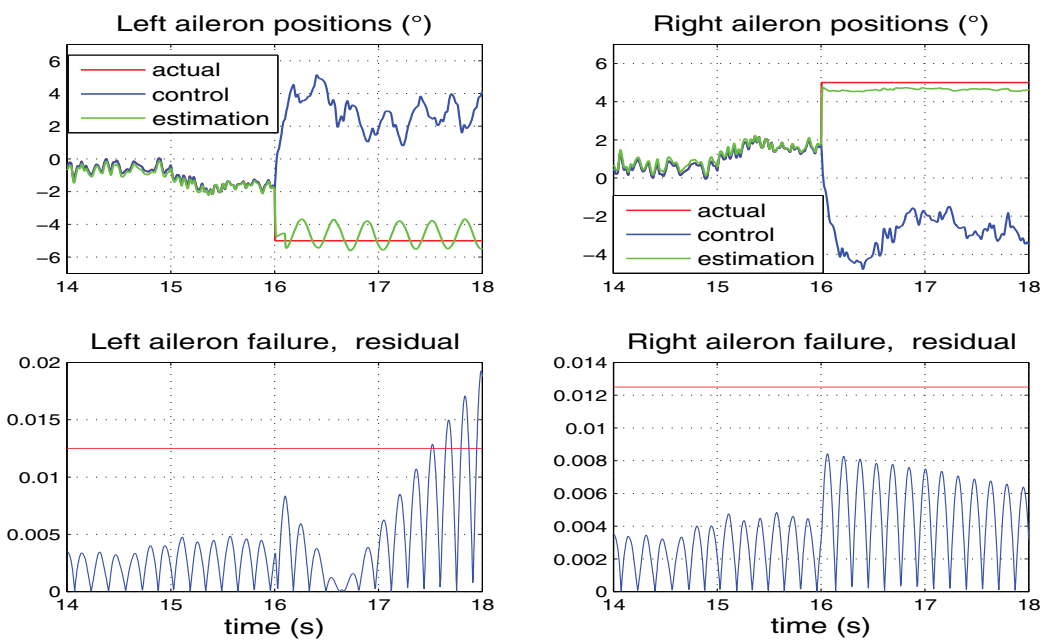

Fig. 13. Left or right aileron stuck: the active diagnosis method

signals, as these latter have no effect on the state vector, the measures do not contain sinusoidal components.

\section{Fault-tolerant control}

The faults considered are asymmetric stuck control surfaces. When one or several control surfaces are stuck, the balance of forces and moments is broken, the UAV moves away from the fault-free mode operating point and there is a risk of losing the aircraft. This risk is all the more so critical that it affects the ruddervators, these latter producing the pitch and the yaw moments.

So a fault may be accommodated only if an operating point exists and the design of the FTC follows this scheme.

1. It is assumed that the faulty control surface and the fault magnitude are known. This information is provided by the fault diagnosis system described above.

2. The deflection constraints of the remaining control surfaces are released e.g symmetrical deflections for flaps, asymmetrical deflections for ailerons.

3. For the considered faulty actuator and its fault position, a new operating point is computed.

4. For this new operating point a linear state feedback controller is designed with an EA strategy. This controller aims to maintain the aircraft handling qualities at their fault-free values.

5. The accommodation is achieved by implementing simultaneously the new operating point and the fault-tolerant controller.

\subsection{Operating point computation}

The operating point exists if the healthy controls offer sufficient redundancies and its value depends on:

- the considered flight stage, 
- the faulty control surface,

- the fault magnitude.

In the following $\left\{\mathbf{X}_{\mathbf{e}}, \mathbf{U}_{\mathbf{e}}\right\}$ denote the operating point in faulty mode, $\mathbf{U}_{\mathbf{e}}^{\text {h }}$ the trim positions of the remaining controls and $\mathbf{U}^{\mathrm{f}}$ the faulty controls. According to (15) and when $k$ control surfaces are stuck, computing an operating point is equivalent to solve the algebraic equation:

$$
0=f\left(\mathbf{X}_{\mathbf{e}}\right)+g^{h}\left(\mathbf{X}_{\mathbf{e}}\right) \mathbf{U}_{\mathbf{e}}^{\mathbf{h}}+g^{f}\left(\mathbf{X}_{\mathbf{e}}\right) \mathbf{U}^{\mathbf{f}}
$$

To take into account the flight stage envelope and the remaining control surface deflections, the operating point computation is achieved with an optimization algorithm. This latter aims at minimizing the cost function:

$$
J=q_{V}\left(V-V_{e_{0}}\right)^{2}+q_{\alpha}\left(\alpha-\alpha_{e_{0}}\right)^{2}+q_{\beta}\left(\beta-\beta_{e_{0}}\right)^{2}
$$

under the following equality and inequality constraints:

- a control surface is stuck

$$
\mathbf{U}^{\mathrm{f}}=\mathcal{U}^{\mathrm{f}}
$$

- the flight envelope and the healthy control deflections are bounded:

$$
\left\{\begin{array}{l}
\boldsymbol{X}_{\min } \leq \mathbf{X} \leq \mathbf{X}_{\max } \\
\mathbf{U}_{\min }^{\mathrm{h}} \leq \mathbf{U}^{\mathbf{h}} \leq \mathbf{U}_{\max }^{\mathrm{h}}
\end{array}\right.
$$

- according to the desired flight stage, some equality constraints are added

- flight level

$$
\left\{\begin{array}{l}
\dot{\phi}=\dot{\theta}=\dot{V}=\dot{\alpha}=\dot{\beta}=\dot{p}=\dot{r}=\dot{q}=\dot{z}=0 \\
\phi=p=q=r=0
\end{array}\right.
$$

- climb or descent with a flight path equal to $\gamma$

$$
\left\{\begin{array}{l}
\dot{\phi}=\dot{\theta}=\dot{V}=\dot{\alpha}=\dot{\beta}=\dot{p}=\dot{r}=\dot{q}=0 \\
\phi=p=q=r=0, \quad \theta-\alpha=\gamma
\end{array}\right.
$$

- turn

$$
\left\{\begin{array}{l}
\dot{\phi}=\dot{\theta}=\dot{V}=\dot{\alpha}=\dot{\beta}=\dot{p}=\dot{r}=\dot{q}=\dot{z}=0 \\
p=q=0, \quad \phi=\phi_{e}, \quad r=r_{e}
\end{array}\right.
$$

This strategy aims at keeping the operating point in faulty mode the closest to its fault-free value. As the linearized model i.e. the state space and the control matrices strongly depends of the operating point, the open-loop poles (and consequently the open-loop handling qualities) are little modified.

The computation of an operating point for a faulty ruddervator is described in the sequel. The right ruddervator is stuck on its whole deflection range $\left[-20^{\circ},+20^{\circ}\right]$ and the remaining controls are trimmed in order to maintain the UAV flight level with an airspeed close to $25 \mathrm{~m} / \mathrm{s}$ and an height equal to $200 \mathrm{~m}$. The results of the computation are illustrated in Fig 14 . They show that an operating point exists in the $\left[-13^{\circ},+3^{\circ}\right]$ interval. However, for some fault positions, the actuator positions are close to their saturation positions. This will drastically limit the aircraft performance. For example, a fault in the $1^{\circ}$ position can be compensated with a throttle trimmed at $90 \%$. It is obvious that this value will limit the turning performance. 
Indeed, during the turn, due to the bank angle, the lift force decreases and to keep a constant height, increasing the throttle control is necessary. As the throttle range is limited, the bank angle variations will be reduced. This is all the more critical that the aircraft has a lateral unstable mode. Note that, from now on, there are couplings between the longitudinal and the lateral axes. Indeed, to obtain these faulty operating points, the longitudinal and the lateral state variables are coupled e.g. the sideslip angle must differ from zero to achieve a flight level stage.

For each fault position in the $\left[-13^{\circ},+3^{\circ}\right]$ interval, the operating point and the related linearized model are computed. The root locus is depicted in Fig. 15 and shows that the open-loop poles are little scattered.

To complete this work, similar studies should be conducted for the left ruddervator, the right and left ailerons.
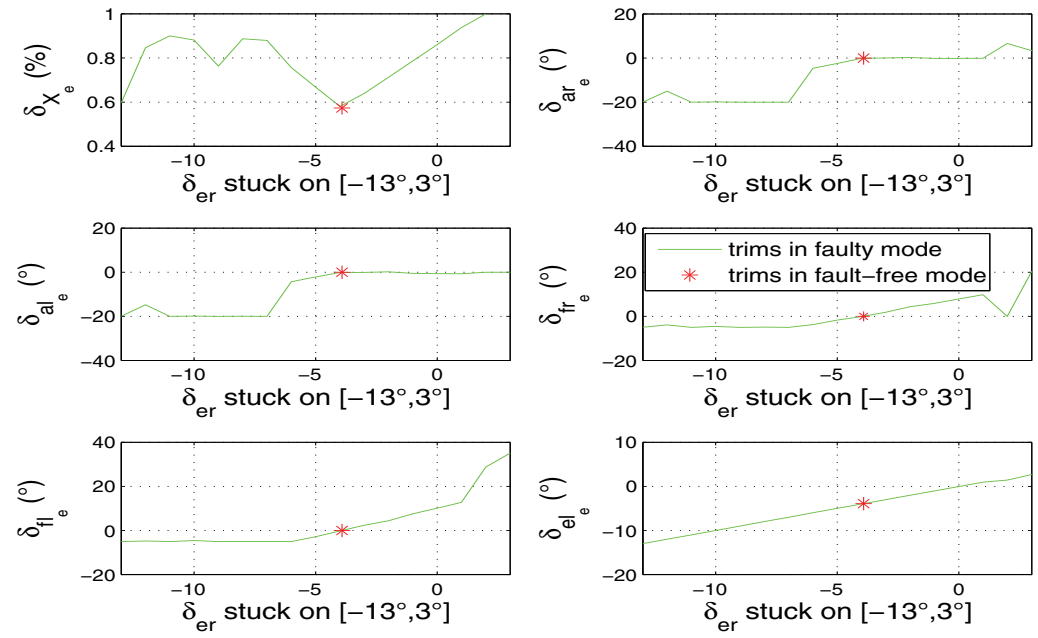

Fig. 14. Right ruddervator stuck, the remaining control trim positions

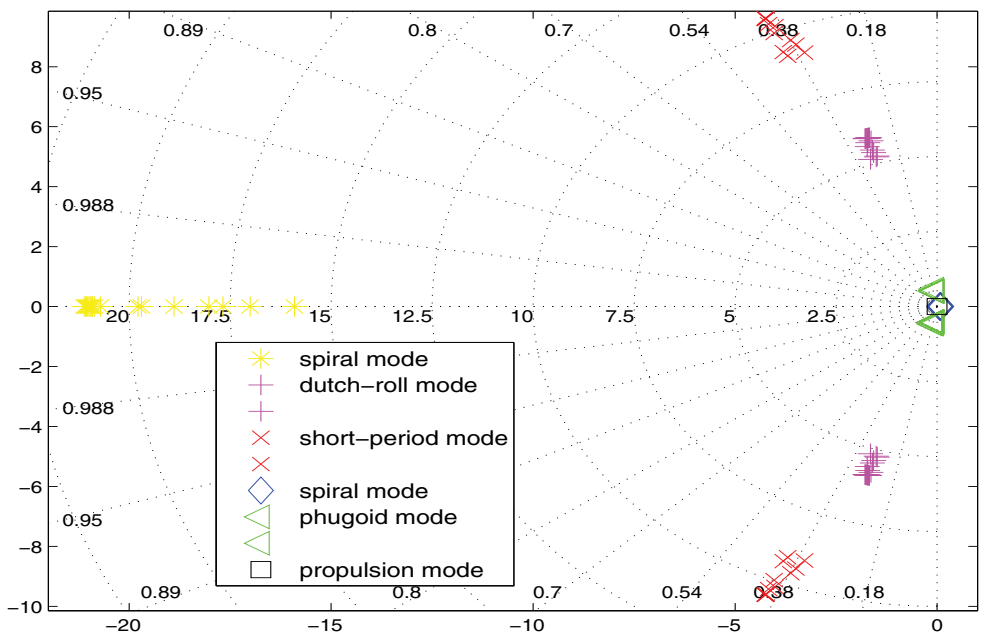

Fig. 15. Right ruddervator stuck, the poles's map 


\subsection{Fault-tolerant controller design}

Fault-tolerant control (FTC) strategy has received considerable attention from the control research community and aeronautical engineering in the last two decades (Steinberg, 2005). An exhaustive and recent bibliographical review for FTC is presented in (Zhang \& Jiang, 2008). Even though different methods use different design strategies, the design goal for reconfigurable control is in fact the same. That is, the design objective of reconfigurable control is to design a new controller such that post-fault closed-loop system has, in certain sense the same or similar closed-loop performance to that of the pre-fault system (Zhang \& Jiang, 2006). In this work, the FTC objective consists in keeping the faulty UAV handling qualities identical to those existing in fault-free mode. Moreover, the tracked outputs $(\phi, \beta, V, z)$ should have the same dynamics that in fault-free mode. As the computation of the faulty operating point induced couplings between the longitudinal and the lateral axes, and as each healthy actuator is driven separately, FTC controllers identical to the nominal controller are kept, i.e. linear state feedback fault-tolerant controllers which design is based on an EA strategy. This is illustrated in Tab. 3 where the proposed EA strategy aims at accommodating a right ruddervator failure. Note that with respect to Tab. 1, the closed-loop poles are unchanged, but the eigenvectors are modified, particularly to decouple the modes from the faulty control. The design of the

\begin{tabular}{|c|c|c|c|c|c|c|c|c|}
\hline mode & short period & phugoid & throttle & roll & dutchroll & spiral, $\varepsilon_{\phi}$ & $\varepsilon_{\beta}$ & $\varepsilon_{V}, \varepsilon_{z}$ \\
\hline CL poles & $-10 \pm 10 i$ & $-2 \pm 2 i$ & -1 & -100 & $-5 \pm 5 i$ & $-1 \pm .25 i$ & -1.5 & $-1 \pm .5 i$ \\
\hline eigenvector & $\vec{v}^{1,2}$ & $\vec{v}^{3,4}$ & $\vec{v}^{5}$ & $\vec{v}^{6}$ & $\vec{v}^{7,8}$ & $\vec{v}^{9,10}$ & $\vec{v}^{11}$ & $\vec{v}^{12,13}$ \\
\hline$\phi$ & $\times$ & 0 & $\times$ & $\times$ & $\times$ & $\times$ & $\times$ & $\times$ \\
\hline$\theta$ & $\times$ & $\times$ & $\times$ & 0 & $\times$ & $\times$ & 0 & $\times$ \\
\hline$V$ & $\times$ & $\times$ & $\times$ & 0 & 0 & 0 & 0 & $\times$ \\
\hline$\alpha$ & $\times$ & $\times$ & $\times$ & 0 & $\times$ & $\times$ & $\times$ & $\times$ \\
\hline$\beta$ & 0 & 0 & 0 & $\times$ & $\times$ & $\times$ & $\times$ & $\times$ \\
\hline$p$ & 0 & 0 & 0 & $\times$ & $\times$ & $\times$ & $\times$ & 0 \\
\hline$q$ & $\times$ & $\times$ & $\times$ & 0 & $\times$ & $\times$ & 0 & $\times$ \\
\hline$r$ & 0 & 0 & 0 & $\times$ & $\times$ & $\times$ & $\times$ & 0 \\
\hline$z$ & $\times$ & $\times$ & $\times$ & $\times$ & 0 & 0 & $\times$ & $\times$ \\
\hline$\varepsilon_{\Phi}$ & $\times$ & $\times$ & $\times$ & $\times$ & $\times$ & $\times$ & $\times$ & $\times$ \\
\hline$\varepsilon_{\beta}$ & $\times$ & $\times$ & $\times$ & $\times$ & $\times$ & $\times$ & $\times$ & $\times$ \\
\hline$\varepsilon_{V}$ & $\times$ & $\times$ & $\times$ & $\times$ & $\times$ & $\times$ & $\times$ & $\times$ \\
\hline$\varepsilon_{z}$ & $\times$ & $\times$ & $\times$ & $\times$ & $\times$ & $\times$ & $\times$ & $\times$ \\
\hline$\delta_{x}$ & 0 & $\times$ & $\times$ & $\times$ & $\times$ & $\times$ & $\times$ & $\times$ \\
\hline$\delta_{a r}$ & $\times$ & 0 & 0 & $\times$ & $\times$ & $\times$ & $\times$ & 0 \\
\hline$\delta_{a l}$ & $\times$ & 0 & 0 & $\times$ & $\times$ & $\times$ & $\times$ & 0 \\
\hline$\delta_{f r}$ & 0 & $\times$ & $\times$ & 0 & 0 & 0 & 0 & $\times$ \\
\hline$\delta_{f l}$ & $\times$ & $\times$ & $\times$ & 0 & 0 & 0 & 0 & 0 \\
\hline$\delta_{e r}$ & 0 & 0 & 0 & 0 & 0 & 0 & 0 & 0 \\
\hline$\delta_{e l}$ & $\times$ & $\times$ & 0 & $\times$ & $\times$ & $\times$ & $\times$ & $\times$ \\
\hline
\end{tabular}

Table 3. Fault-tolerant controller, EA strategy for a ruddervator failure

FTC is similar to those presented in section 3. Similar studies could be conducted for the other control surfaces.

\subsection{Fault-tolerant controller implementation}

A fault is described by the type of control surface and its fault magnitude. This information is provided by the FDI system studied above. For a given fault, a new operating point and a FTC controller must be theoretically computed. As far as the operating points are concerned, they 
are precomputed, tabulated and selected with respect to the fault. In the same way, a FTC should be designed for each operating point and its corresponding linearized model. This method has been adopted to compensate for right ruddervator failures. Practically, it enables to accommodate for them in the $\left[-5^{\circ}, 0^{\circ}\right]$ interval with a $1^{\circ}$ step study. Consequently, six fault-tolerant controllers should be designed.

In order to reduce this number and for the six faulty linearized models, a single fault-tolerant controller is kept, the one which minimizes the scattering of the poles. For a right ruddervator failure, this fault-tolerant controller is the one designed for $a-2^{\circ}$ fault position.

Outside this interval, the faults are too severe to be accommodated.

\subsection{Results of simulations}

Results of simulations are depicted in Fig. 16. The right ruddervator is stuck in the $0^{\circ}$ position at time $t_{f}=16 \mathrm{~s}$. This case is similar to the one studied in the paragraph 4.3.2. After time $t_{f}$, the fault is detected, isolated and its magnitude is estimated, then the fault-tolerant controller efficiently compensates for the fault and the aircraft can continue to fly and to maneuver. However, for the reasons explained in subsection 5.1, the bank angle cannot exceed $10^{\circ}$ and the nonlinear effects due to the throttle saturation (see. Fig. 16) affects the dynamics of the airspeed.

The same fault-tolerant controller is tested for various stuck positions simulated in the $\left[-5^{\circ}, 0^{\circ}\right]$ interval and occuring at time $t_{f}=16 \mathrm{~s}$. As it is shown in Fig. 18, all these faults are accommodated with this unique fault-tolerant controller.
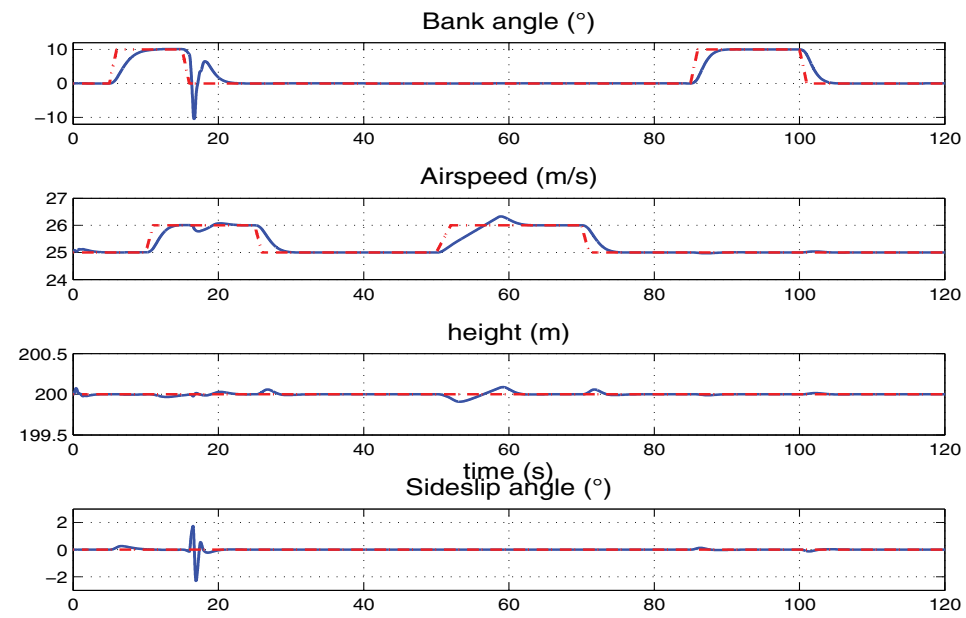

Fig. 16. Right ruddervator stuck, the tracked state variables

\section{Conclusion}

A UAV model has been designed to deal with asymmetrical control surfaces failures that upset the equilibrium of moments and produce couplings between the longitudinal and the lateral axes. The nominal controller aims at setting the UAV handling qualities and it is based on an eigenstructure assignment strategy. Control surface positions are not measured and, in order to diagnose faults on these actuators, input observability has been studied. It has proven that faults on the ailerons are not isolable. Next, a bank of Unknown Input Decoupled Functional 


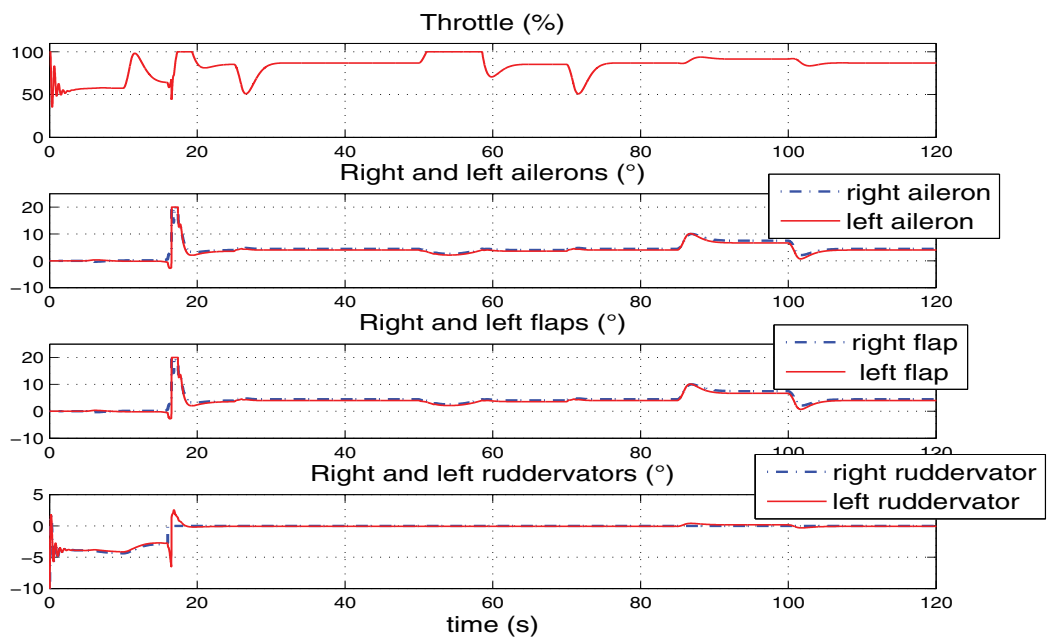

Fig. 17. Right ruddervator stuck, the controls
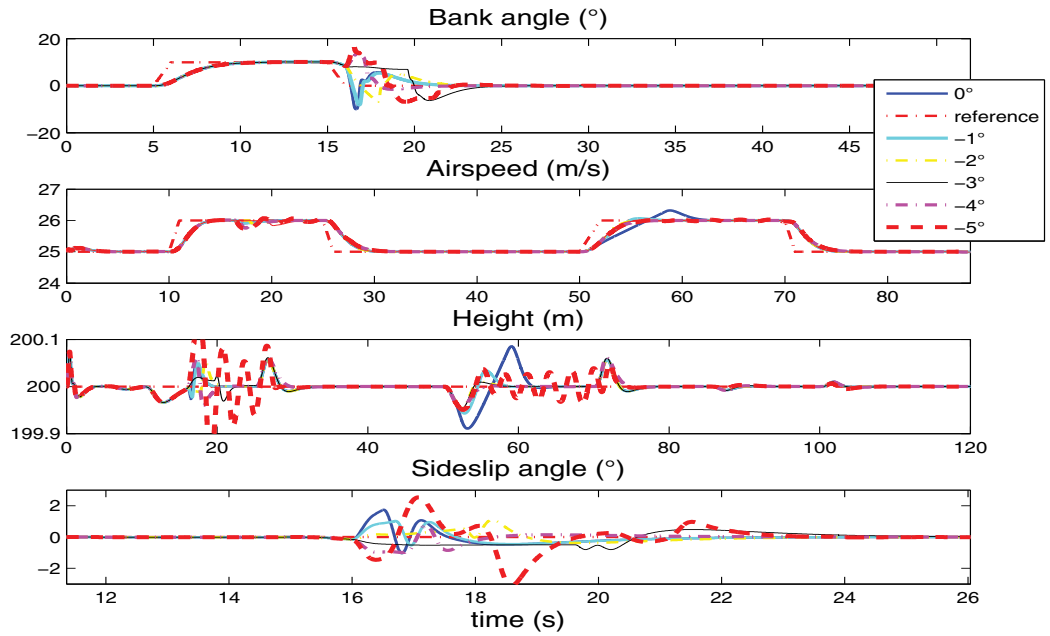

Fig. 18. Right ruddervator stuck, the ruddervator actual position, estimation, control position and the residual

Observers has been implemented in order to detect, isolate and estimate faults. To process with the faulty ailerons, an isolation method based on a signal processing method has been presented. Future works should also take into account the redundancies provided by the right and left flaps. The fault accommodation consists in computing a new operating and a related fault-tolerant controller. For this latter, the objectives of the settings are identical to those pursued in the fault-free mode. However, the results of simulations show the importance of actuator saturations, especially in faulty mode, where to compensate for the fault, the remaining actuator strokes may be significantly reduced and may affect the control stability. Our present works deal with FTC designs which aim at setting the handling qualities while sizing the stability domain with respect to the flight envelope by considering the actuator saturations. 
The first author would like to acknowledge Pr. T. Hermas for proofreading the initial manuscript.

\section{References}

Bateman, F., Noura, H. \& Ouladsine, M. (2008a). An active fault tolerant procedure for an uav equipped with redundant control surfaces, 16th Mediterranean Conference on Control and Automation, Ajaccio, France.

Bateman, F., Noura, H. \& Ouladsine, M. (2008b). A fault tolerant control strategy for an unmanned aerial vehicle based on a sequential quadratic programming algorithm, Conference on Decision and Control, Cancun, Mexico.

Bateman, F., Noura, H. \& Ouladsine, M. (2009). Fault tolerant control strategy for an unmanned aerial vehicle, 7th IFAC SafeProcess, Barcelona, Spain.

Boiffier, J. (1998). The dynamics of $\square$ ight, Wiley.

Brigaud, R. (2006). Working towards a usar stanag, Euro UAV, Paris, France.

Demmel, J. \& Kågstrom, B. (1993). The generalized schur decomposition of an arbitrary pencil a - zb: robust software with error bounds and applications, ACM Transaction on Mathematical Software 19(2): 175-201.

Ducard, G. \& Geering, H. (2008). Efficient nonlinear actuator fault detection and isolation for unmanned aerial vehicles, Journal of Guidance, Control and Dynamlics 31(1): 225-237.

Hou, M. \& Patton, R. (1998). Input observability and input reconstruction, Automatica 34(6): 789-794.

Kobayashi, T. \& Simon, D. (2003). Application of a bank of kalman filters for aircraft engine fault diagnostics, Technical Report NASA Report 212526, NASA.

Magni, J. F., Bennami, S. \& Terlouw, J. (1997). Robust Flight Control, a design challenge, Springer.

MIL-HDBK-1797 (1997). U.s. military handbook mil-hdbk-1797, Technical report, U.S Department Of Defense.

Noura, H., Theilliol, D., Ponsart, J. \& Chamsedinne, A. (2009). Fault-tolerant Flight Control Systems, Springer.

OSD (2003). Unmanned aerial vehicle reliability study, Technical report, Office of the Secretary of Defense.

Rauw, M. (1993). A Simulink environment for $\square$ ight dynamics and control analysis, $\mathrm{PhD}$ thesis, Delft University of Technology, Faculty of Aerospace Engineering.

Steinberg, M. (2005). Historical review of research in reconfigurable flight control, Journal of Aerospace Engineering 219(4): 263-275.

Xiong, Y. \& Saif, M. (2003). Unknown disturbance inputs estimation based on state functional observer design, Automatica 39: 1390-1398.

X.Liu, Chen, B. \& Lin, Z. (2005). Linear system toolkit in matlab : structural decomposition and their applications, Journal of Control, theory and application 3: 287-294.

Zhang, Y. \& Jiang, J. (2006). Issues on integration of fault diagnosis and reconfigurable control in active fault-tolerant control systems, IFAC Safe Process, Beijing, China.

Zhang, Y. \& Jiang, J. (2008). Bibliographical Review on Reconfigurable Fault Tolernat Control, Annual Reviews in Control 32(2): pp. 229-252. 


\title{
Fault-Tolerance of a Transport Aircraft with Adaptive Control and Optimal Command Allocation
}

\author{
Federico Corraro, Gianfranco Morani and Adolfo Sollazzo \\ Italian Aerospace Research Centre (CIRA) \\ Italy
}

\section{Introduction}

How to achieve high performance and reliability against various unforeseen events, uncertainties and other changes in plant dynamics has been a very challenging issue for control system design in recent years. Reconfigurable flight controls aim to guarantee greater survivability in all the cases in which the systems to be controlled may be poorly modelled or the parameters of the systems may be subjected to large variations with respect to the operating environment. A suitable approach to the problem of flight control reconfiguration consists in redesigning its own structure and/or re-computing control gains in the case of unexpected events or large model and environmental uncertainties. A number of different approaches have been proposed and developed in the past years (Patton, 1997). In this chapter a Direct Adaptive Model Following (DAMF) algorithm has been used for reconfiguration purposes. It is possible to find in literature a great amount of proposed techniques to implement (Bodson \& Groszkiewicz, 1997; Calise et al., 2001; Boskovic \& Mehra, 2002; Kim et al., 2003; Tandale \& Valasek, 2003). The Lyapunov theory described in (Kim et al., 2003; Tandale \& Valasek, 2003) has very attractive features both in terms of effectiveness and implementation and it has been used to develop the fault-tolerant scheme described in this chapter.

Another important matter in flight control reconfiguration is the Control Allocation (CA) problem. It concerns the possibility to exploit actuators redundancy with respect to the variables to be controlled in order to redistribute the control effort among the available control effectors. In this way the control commands needed to attain the desired moments can be computed even in presence of actuator failures, while also dealing with position and rate limits of the control effectors. A great amount of techniques for control allocation are available in literature (Virnig \& Bodden, 2000; Enns, 1998; Buffington \& Chandler, 1998; Durham \& Bordignon, 1995; Burken et al., 2001). The technique used in this chapter is the one introduced by Harkegard (Harkegard, 2002) based on active set methods, which is very effective for real-time applications and converge in a finite number of steps.

Therefore in this chapter a scheme of a fault-tolerant flight control system is proposed. It is composed by the core control laws, based on the DAMF technique, to achieve both robustness and reconfiguration capabilities, and the CA system, based on the active set method, to properly allocate the control effort on the healthy actuators. Numerical results of 
a case study with a detailed model of a large transport aircraft are reported to show the effectiveness of the proposed fault-tolerant control scheme.

The chapter is organized as follows. Sec. 2 explains the proposed flight control system architecture and all its features. Sec. 3 and 4 report detailed descriptions of each element composing the Flight Control System (FCS). Sec. 5 contains the most meaningful results of the numerical evaluation. Finally, Sec. 6 reports the main outcomes and the conclusions about the current results of the research project.

\section{The fault-tolerant flight controller scheme}

The logical scheme of the full fault-tolerant flight controller is reported in Fig. 1. The picture shows four main elements, the autopilot $(\mathrm{A} / \mathrm{P})$, the actuators health monitor, the Stability and Controllability Augmentation System (SCAS), based on the DAMF, and the Control Allocation module. The last two elements represent the Fault-Tolerant Control System (FTCS) that is the object of this chapter.

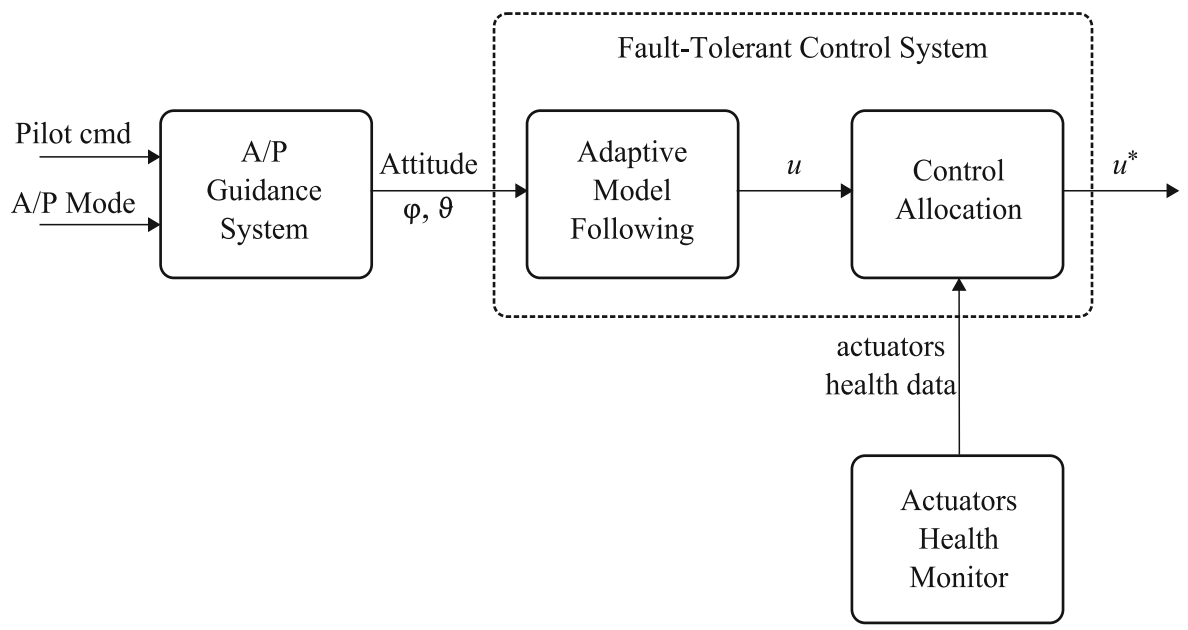

Fig. 1. The first layer scheme of the full Flight Control System

Although adaptive control exhibits great reconfiguration capabilities, in case of in-flight faults, abrupt and dramatic changes in control effectiveness and/or plant dynamics may occur, such that the adaptive controller may not be able to recover the vehicle. Therefore, an adaptive controller could take advantage of a control allocation module to ensure the generation of the demanded moments by the optimal control system, both in healthy and faulty conditions of the actuation system.

The remaining two elements are not the focus of this work and they are developed with classic techniques. In details, the $\mathrm{A} / \mathrm{P}$ is designed by means of the classic sequential loop closures, implementing the typical guidance modes for the aircraft (see Table. 1).

\begin{tabular}{ll}
\hline \hline Longitudinal & Lateral \\
\hline Altitude Hold/Select & Heading Hold/Select \\
GlideSlope Intercept & Localizer Intercept \\
Approach Lon & Approach Lat \\
\hline \hline
\end{tabular}

Table 1. List of Autopilot modes 
Also the health monitoring of actuators is a very trivial system based on the comparison between the input and the output of each actuators. In the numerical validation it is supposed to use a monitoring system with the capability to detect an actuator fault within 10 seconds, and to pass the binary information healthy/faulty to the control allocation system. In the following two sections the elements of the FTCS are briefly recalled.

\section{Adaptive control system}

The core module of the whole flight control system is the SCAS that is in charge of guaranteeing vehicle attitude control and stability. As already said, the proposed algorithm for this module has been designed using a Direct Adaptive Model-Following method (Boskovic \& Mehra, 2002; Kim et al., 2003; Tandale \& Valasek, 2003), having the advantage of strong robustness against model parameter uncertainty, and a good capability of reacting to system parameters' variation. Moreover, the model following strategy lets the designer to define in a clear and simple way the reference dynamics for the system, thus making this control strategy very attractive among other available robust control techniques. In the following some recalls about the DAMF are given.

\subsection{Theoretical recalls}

DAMF is a Model Reference Control Strategies and it earns its robustness properties by means of a direct adaptation of control loop gains to asymptotically reach two objectives. The first is a null error between the output of the reference model and the one of real plant. The second objective is to minimize the control effort. As already said, the proposed adaptation algorithm is based on the Lyapunov theory. A mathematical description of the method, fully reported in (Kim et al., 2003), follows. Let us consider the linear model of a plant:

$$
\begin{aligned}
& \dot{x}=A x+B u+d \\
& y=C x
\end{aligned}
$$

with $x \in \mathfrak{R}^{n}$ the state vector, $y \in \mathfrak{R}^{l}$ the output vector, $u \in \mathfrak{R}^{m}$ the control vector, $A \in \mathfrak{R}^{n \times n}$, $B \in \mathfrak{R}^{n \times m}, C \in \mathfrak{R}^{l \times n}$ and the term $d$ represents the trim data. The reference system dynamics is written in term of desired input-output behaviour:

$$
\dot{y}_{m}=A_{m} y_{m}+B_{m} r
$$

where $y_{m}$ is the desired output for the plant, $r$ is the given demand, $A_{m}$ and $B_{m}$ represent the reference linear system. The control laws structure is defined as follows:

$$
u=C_{0}\left(G_{0} x+v+r+K_{0} y_{m}\right)
$$

where $G_{0}, C_{0}$ and $v$ are proper terms generated by the adaptation rules, instead $K_{0}$ is a feedforward gain matrix off-line computed. It is now possible to calculate the error function as follows:

$$
e=y-y_{m}
$$

and to evaluate the error dynamics, in terms of the plant parameters and the reference system dynamics: 


$$
\dot{e}=\left(C A+C B C_{0} G_{0}\right) x+C B C_{0} r+C B C_{0} v+C B C_{0} K_{0} y_{m}+C d-A_{m} y_{m}-B_{m} r
$$

Assuming a desired error system dynamics, expressed as:

$$
\dot{e}=A_{e} e+\Phi
$$

where $A_{e}$ is a stable and properly chosen matrix, and $\Phi$ represents a bounded forcing function, it is possible to write the following identities:

$$
\begin{aligned}
& C A+C B C_{0}^{*} G_{0}^{*}=A_{e} C \\
& C B C_{0}^{*}=B_{m} \\
& C B C_{0}^{*} v^{*}=-C d \\
& C B C_{0}^{*} K_{0}=A_{m}-A_{e}
\end{aligned}
$$

Equations 7 allow to write the expressions of the optimal terms $G_{0}{ }^{*}, C_{0}{ }^{*}, v^{*}$ and $K_{0}$ to obtain a perfect model inversion that guarantees the asymptotical stability of the closed loop system and the asymptotical null error.

$$
\begin{aligned}
& G_{0}^{*}=B_{m}^{-1}\left(A_{e} C-C A\right) \\
& C_{0}^{*}=(C B)^{-1} B_{m} \\
& v^{*}=-B_{m}^{-1} C d \\
& K_{0}=B_{m}^{-1}\left(A_{m}-A_{e}\right)
\end{aligned}
$$

In order to evaluate the left hand terms (the gains of the controller), Equations 8 require matrix $B_{m}$ to be invertible and $C B$ matrix to be pseudo-invertible. While the former is a design parameter, the latter, called high frequency gain, is a structural characteristic of the plant. Anyway, modern aircrafts have typically a sufficient redundancy order for the control surfaces, thus ensuring not to lose rank order even in the case of single and often double actuators failure. Concerning the $C$ matrix, no sensor failure cases are addressed in this chapter, anyway the device redundancy or several techniques, available in literature (f.i. Kalman filtering), may ensure a full state feedback, even though each signal may lose accuracy in case of sensor failure.

It should be anyway noted that the control parameters of Equation 8 do not take into account the system parameters variation. However, the system parameters uncertainties can be modelled by a proper variation of the matrices in Equation 1. Finally, a set of adaptation rules is necessary to react to the system parameters variation and uncertainty, Lyapunov theory furnishes a very efficient solution. First of all, let us define the differences between the actual adaptive parameters and the optimal ones:

$$
\begin{aligned}
& \Delta G=G_{0}-G_{0}^{*} \\
& \Delta \Psi=C_{0}^{*-1}-C_{0}^{-1} \\
& \Delta \nu=v_{0}-v_{0}^{*}
\end{aligned}
$$

After some manipulations (Kim et al., 2003), here left out for the sake of brevity, it is now possible to write the real expression of the error dynamics taking into account a parameters variation: 


$$
\dot{e}=A_{e} e+B_{m} \Delta G \cdot x+B_{m} \Delta \Psi \cdot u+B_{m} \Delta v
$$

It is allowed to impose the Lyapunov stability condition for the error system. So, let us consider the Lyapunov candidate function:

$$
V=e^{T} P e+\operatorname{tr}\left\{\frac{\Delta G^{T} \Delta G}{\gamma_{1}}\right\}+\operatorname{tr}\left\{\frac{\Delta \Psi^{T} \Delta \Psi}{\gamma_{2}}\right\}+\frac{\Delta v^{T} \Delta v}{\gamma_{3}}
$$

where $P$ is the matrix solution of Lyapunov equation:

$$
A_{e}^{T} P+P A_{e}=-Q
$$

with $Q$ is a positive definite weighting matrix. By calculating the time derivative of the Lyapunov candidate function and by casting it to get null, the following conditions can be found, that represent the adaptation rules for the control laws parameters.

$$
\begin{aligned}
& \dot{G}_{0}=-\gamma_{1} B_{m}^{T} P e x^{T} \\
& \dot{C}_{0}=-\gamma_{2} C_{0} B_{m}^{T} P e u^{T} C_{0} \\
& \dot{v}_{0}=-\gamma_{3} B_{m}^{T} P e
\end{aligned}
$$

Moreover by taking into account the Equations 10, 11 and 13 it is possible to demonstrate the non-positiveness of Lyapunov candidate function derivative:

$$
\dot{V}=-e^{T} P e \leq 0
$$

which assures the asymptotical stability for the error dynamic system.

\subsection{Implementation of the SCAS module}

The SCAS module is made of two nested sub-modules, taking advantage of the dynamics separation principle, being the angular rate dynamics sufficiently faster than those of the attitude ones. This two modules architecture also leads to a relevant reduction of the overall complexity (in terms of states number) of the adaptive algorithm. The detailed structure of each Multi Input Multi Output (MIMO) controller is reported in Fig. 2. The design of both inner and outer loops consists in tuning some parameters. First of all, the matrices $A_{m}$ and $B_{m}$, representing the dynamics of the Reference Model, must be selected with the limitation that the former must trivially be chosen with negative eigenvalues and the latter must be chosen invertible. These two matrices actually define the control system performance requirements. For both attitude and rates regulators, a couple of very simple reference models made of two diagonal systems ( 1 st order and decoupled systems) have been chosen. The desired error dynamics are chosen through the matrix $A_{e}$ by which, it is also possible to modify the system capability to reject noise and disturbances.

The matrix $Q$, used in the Equation 12 for the calculation of $P$, has the meaning of a weighting matrix. By fine tuning this matrix, it is possible to give more or less relevance to the tracking requirement of one or more output variables with respect to the others. Finally, the three parameters $\gamma_{1}, \gamma_{2}$ and $\gamma_{3}$ (evaluated by means of a trial and error procedure) are used to regulate the adaptive capability. As a reminder, in Table 2 all the design parameters are reported. 


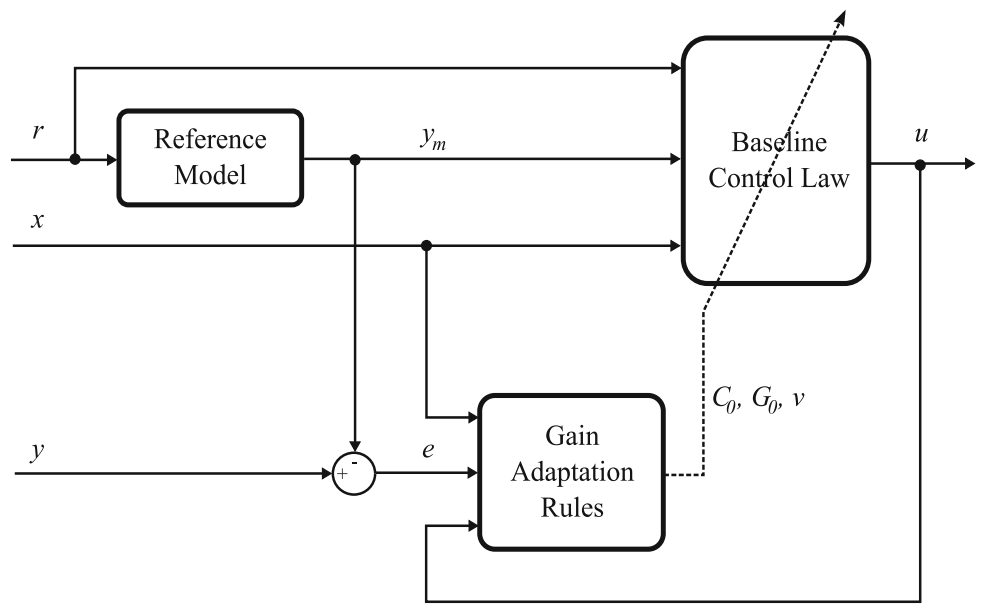

Fig. 2. SCAS sub-module logical architecture

\begin{tabular}{|c|c|c|c|c|}
\hline & $A_{m}, B_{m}$ & $A_{e}$ & $Q$ & $\gamma_{1}, \gamma_{2}, \gamma_{3}$ \\
\hline 站 & {$\left[\begin{array}{ccc}-1 & 0 & 0 \\
0 & -1 & 0 \\
0 & 0 & -1\end{array}\right],\left[\begin{array}{lll}1 & 0 & 0 \\
0 & 1 & 0 \\
0 & 0 & 1\end{array}\right]$} & {$\left[\begin{array}{ccc}-4.5 & 0 & 0 \\
0 & -4.5 & 0 \\
0 & 0 & -4.5\end{array}\right]$} & {$\left[\begin{array}{ccc}0.8 & 0 & 0 \\
0 & 1.2 & 0 \\
0 & 0 & 1\end{array}\right]$} & {$\left[\begin{array}{c}0.06 \\
0.1 \\
0.1\end{array}\right]$} \\
\hline 站 & {$\left[\begin{array}{cc}-0.5 & 0 \\
0 & -0.5\end{array}\right],\left[\begin{array}{cc}0.5 & 0 \\
0 & 0.5\end{array}\right]$} & {$\left[\begin{array}{cc}-1.5 & 0 \\
0 & -0.75\end{array}\right]$} & {$\left[\begin{array}{cc}1 & 0 \\
0 & 0.5\end{array}\right]$} & {$\left[\begin{array}{l}0.01 \\
0.01 \\
0.2\end{array}\right]$} \\
\hline
\end{tabular}

Table 2. SCAS module parameters

\section{The control allocation module}

As mentioned in the introduction a control allocation algorithm can be very useful for control reconfiguration purposes due to its ability of managing actuator redundancy, so to redistribute control effort after a failure event. Moreover, it may be a great support for an optimal control strategy, such as the DAMF that works well in the case of limited faults (i.e. the plant dynamics do not change dramatically) and in any case it does not take into account the limited range and limited rate of the control variables.

\subsection{Theoretical background}

In this section the control allocation problem is briefly introduced. Given a control vector $u_{p} \in \mathfrak{R}^{m}$ as computed by the control system (see Equation 3), a desired moment vector $v_{\text {des }} \in \mathfrak{R}^{l}$ can be defined as:

$$
v_{\text {des }}=C B u_{p}
$$

where the matrix product $C B$ is the high frequency gain of the healthy system (no faults). In the event of one or more faults, system defined in Equation 1 becomes: 


$$
\begin{aligned}
& \dot{x}=A x+B_{\text {fault }} u+B u_{\text {lock }}+d \\
& y=C x
\end{aligned}
$$

where $B_{\text {fault }}$ is the control matrix of the failed plant which can be expressed as:

$$
B_{\text {fault }}=B \Pi
$$

where $\Pi$ is a diagonal matrix with the elements $\pi_{i}=0$ for $i$-th actuator failed and $\pi_{i}=1$ for a healthy actuator. This matrix accounts for the fact that failed actuators cannot be used anymore to change the system's dynamics. The term $B u_{\text {lock }}$ accounts for a residual moment due to an actuator locked in a fixed position, so we set $u_{\text {lock }}(j)=0$ if $j$-th actuator is healthy and $u_{\text {lock }}(j)=u_{j}$ if $j$-th actuator is locked at $u_{j}$. After this setting, the residual moment to be attained by the failed system is defined as

$$
\Delta v=v_{\text {des }}-C B u_{\text {lock }}
$$

which is the moment to be attained with the failed high frequency gain matrix $B_{\text {fault }}$. Therefore the goal of control allocation is to find a control $\bar{u} \in \mathfrak{R}^{m}$ such that $C B_{\text {fault }} \bar{u}=\Delta v$. The new control vector shall also satisfy the constraints on maximum and minimum values, which can be computed at each instant depending on the actual position and rate limits, that is, $u_{\min } \leq \bar{u} \leq u_{\max }$.

Generally speaking a solution to the above problem may not exist or it may be not unique depending on the rank of matrix $C B_{\text {fault }}$. If there are more solutions, the exceeding control authority can be exploited to choose the solution which is the nearest to a reference control vector $u_{p} \in \mathfrak{R}^{m}$ (for example the one computed by the control system). A common approach to solve the control allocation problem is based on the following weighted least square formulation (Harkegard, 2002):

$$
u_{w}=\min _{u_{\min } \leq u \leq u_{\max }}\left\|W_{u}\left(u-u_{p}\right)\right\|^{2}+\gamma\left\|W_{v}\left(C B_{\text {fault }} u-\Delta v\right)\right\|^{2}
$$

where $\|\cdot\|^{2}$ is the $L_{2}$-norm, $u_{p}$ is a reference control vector, $W_{u}$ and $W_{v}$ are non-singular weighting matrices. The first term of the above minimization problem allows to choose, among all the feasible control vectors which minimize the $L_{2}$-norm of the error $C B_{\text {faul }} u-\Delta v$, the one minimizing the norm of $\left(u-u_{p}\right)$. The weighting factor $\gamma$ defines the relative degree of importance between the moments error $C B_{\text {faul }} u-\Delta v$ and the control error $\left(u-u_{p}\right)$. Obviously $\gamma$ should be chosen large enough to ensure the minimization of the error in attaining the desired moments.

In this chapter we will address the problem of control allocation with the use of a technique based on the active set method and described in (Harkegard, 2002). Active set methods are very common in constrained quadratic programming. They only consider active constraints (equality constraints) and disregard inactive constraints. Therefore active set algorithms move on the surface defined by the set of the active constraints (named Working Set) to get an improved solution. The working set is continuously updated during the execution of the algorithm. In fact, whenever a new constraint is violated, it is added to the current working set. On the other hand, if a feasible solution has been found by the algorithm, but a Lagrange multiplier related to the current working set is negative, the corresponding active constraint is dropped in order to get an improved solution. An exhaustive description of the 
control allocation algorithm used in this chapter can be found in (Harkegard, 2002). Some recalls are given in the section below.

\subsection{Control allocation algorithm}

For each iteration step of the algorithm the following optimization problem is solved:

$$
\begin{aligned}
& \min _{p}\left\|A\left(p+u_{k}\right)-b\right\|^{2} ; \\
& p_{i}=0, i \in W_{k}, \quad A=\left(\begin{array}{c}
W_{v} B \\
W_{u}
\end{array}\right), b=\left(\begin{array}{c}
W_{v} v \\
W_{u} u_{p}
\end{array}\right)
\end{aligned}
$$

where $u_{k}$ is the starting solution at the iteration step $k$, the set $W_{k}$ is the current working set, that is, the set containing the active constraints (i.e. saturated controls) which are expressed through the equality $p_{i}=0$, while the remaining inequality constraints are disregarded.

Solution to the least square problem of Equation 21 consists of finding the optimal perturbation $p$ which can be obtained by using a simple pseudo inversion method (Harkegard, 2002). Once the constraints on actuator limits have been set

$$
\begin{aligned}
& C u \geq U ; \\
& C=\left(\begin{array}{l}
+I \\
-I
\end{array}\right), U=\left(\begin{array}{l}
+u_{\max } \\
-u_{\min }
\end{array}\right)
\end{aligned}
$$

( $I m \times m$ is the identity matrix), if the solution $u_{k}+p$ is feasible, the Lagrange multipliers $\lambda_{i}$ associated with the active constraints are computed. If they are non-negative, the optimum solution is obtained otherwise the $i$-th constraint is dropped from the active set because a better solution can be found according to the meaning of Lagrange multipliers (Luenberger, 1989). In the case that $u_{k}+p$ is not feasible, the maximum step $\alpha$ is calculated such that $u_{k}+\alpha p$ is still feasible and a new constraint is added to the working set. This iterative procedure is then repeated until a suitable solution and a working set with negative related Lagrange multiplier are found.

\subsection{Remarks}

As above described control allocation algorithm has the aim of redistribute the control effort among the "healthy" surfaces to achieve the moments needed to keep the system along reference trajectory. In view of these considerations we argue that control allocation can be very useful, when used in conjunction with a direct adaptive control in those critical failure scenarios which can be hardly handled by the only use of the adaptive controller. Nevertheless, in order to be effective for reconfiguration purposes, control allocation needs a Fault Detection (FD) system, which gives information about the health of the surfaces' actuators. This aspect could make unfeasible the use of a CA scheme. Anyway, in the following sections it will be shown that, in order to obtain a satisfactory performance of the CA module, only limited failure information are needed. In fact, also a very simple monitoring algorithm, based on the actuator model and on the surface actual position, can be sufficient to establish whether an actuator is failed or not. The results show that the use of a CA scheme allows significant improvements of the control system performances also in the event of very critical failures and it only needs limited information about actuators' 
health. These features make the proposed control architecture very appealing for reconfiguration purposes.

\section{Numerical validation}

The FCS has been applied in a case study with a large transport aircraft. The works has been performed within the GARTEUR Action Group 16, project focused on Fault-Tolerant Control. In that project a benchmark environment (Smaili et al., 2006) has been developed modelling a bunch of surface actuators faulty conditions. A brief summary of all these conditions is given in Table 3, while a detailed explanation of the benchmark can be found in (Smaili et al., 2006).

Several manoeuvres are considered in the benchmark to be accomplished in the various faulty conditions. The test results are here shown both in terms of time histories of the state variables and with a visual representation of the trajectories performed by the airplane.

\section{Stuck Ailerons: \\ Both inboard and outboard ailerons are stuck. \\ Stuck Elevators: \\ Both inboard and outboard elevators are stuck. \\ Stabilizer Runaway: \\ The stabilizer goes at the maximum speed toward the maximum deflection. \\ Rudder Runaway: \\ The upper and lower rudders go at the maximum speed toward the maximum deflection. \\ Loss of Vertical Tail: \\ The vertical tail separates from the aircraft.}

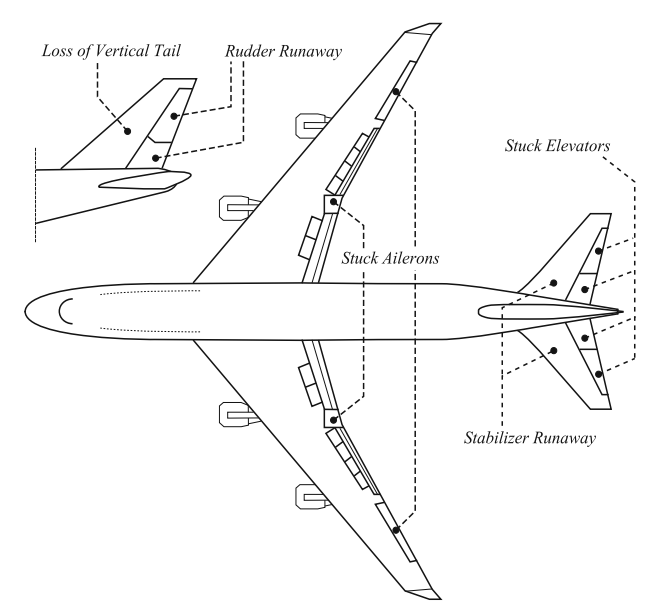

Table 3. Failures considered in the test campaign

Only the most meaningful conditions are here reported and discussed. To better demonstrate the improvement of fault-tolerance achieved by adopting the adaptive control in conjunction with the Control Allocation, comparison is made between three versions of the FCS, the first is a baseline SCAS developed with classic control techniques. The two remaining FCS are based on the adaptive SCAS with and without the CA respectively. As above said, only limited FD information are supposed to be provided, that is, the information about whether an actuator is failed or not but the current position of the failed actuator will be considered as unknown. The CA parameters have been set to:

$$
\begin{aligned}
& \gamma=10^{6} \\
& W_{u}=I^{3 \times 3} \\
& W_{v}=I^{3 \times 3}
\end{aligned}
$$




\subsection{Straight flight with stabilizer failure}

In this condition, while in straight and levelled flight, the aircraft experiences a stabilizer runaway to maximum defection that generates a pitching down moment. The initial flight condition data are summarized in Table 4.

\begin{tabular}{ccccc}
\hline \hline $\begin{array}{c}\text { Altitude } \\
{[\mathrm{m}]}\end{array}$ & $\begin{array}{c}\text { True Airspeed } \\
{[\mathrm{m} / \mathrm{s}]}\end{array}$ & $\begin{array}{c}\text { Heading } \\
{[\mathrm{deg}]}\end{array}$ & $\begin{array}{c}\text { Mass } \\
{[\mathrm{kg}]}\end{array}$ & $\begin{array}{c}\text { Flaps } \\
{[\mathrm{deg}]}\end{array}$ \\
\hline 600 & 92.6 & 180 & 263,000 & 20 \\
\hline \hline
\end{tabular}

Table 4. Flight condition data

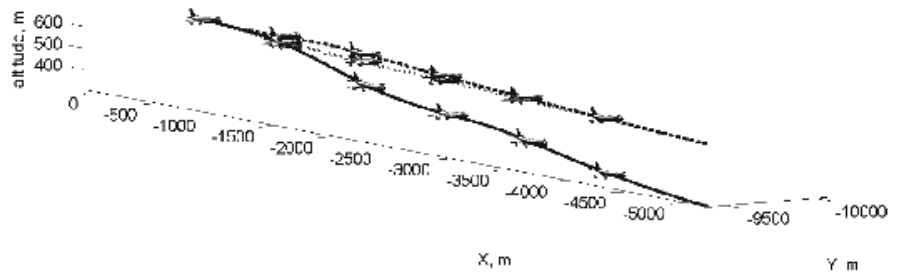

(a) Trajectories
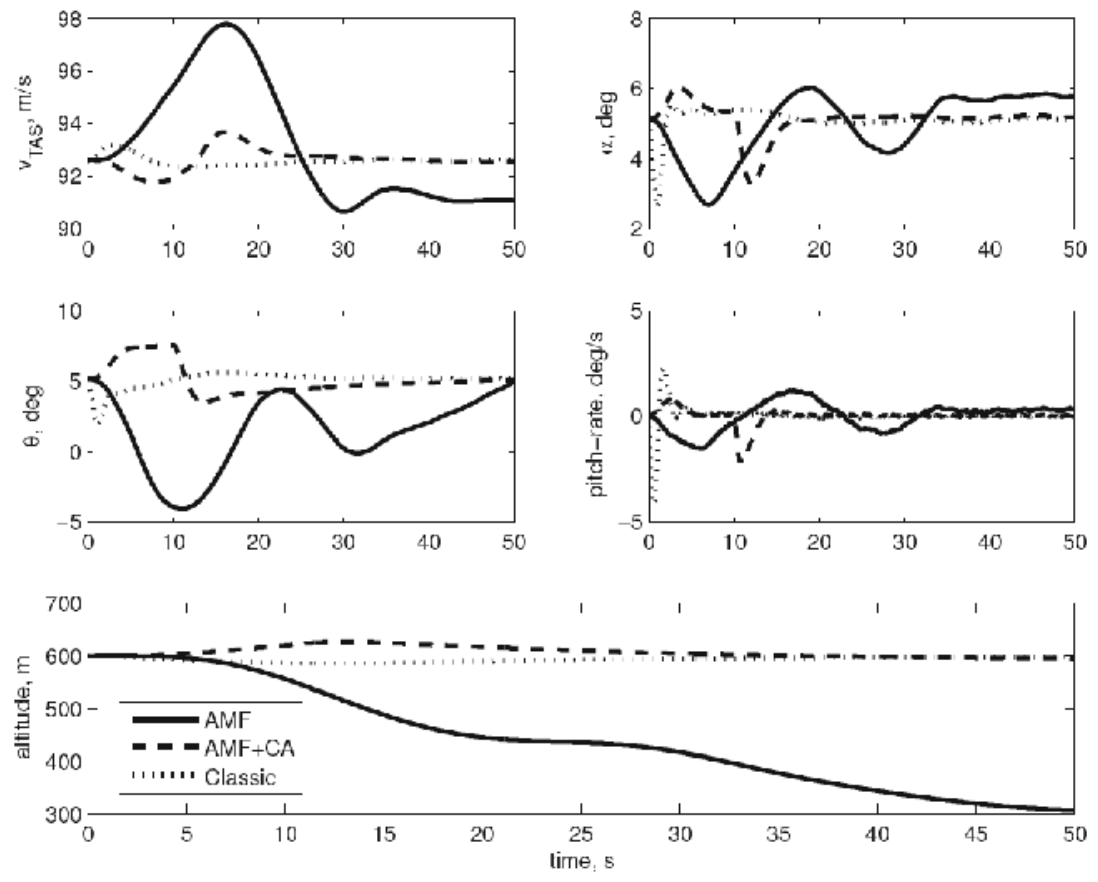

(b) Time plots

Fig. 3. Straight flight with stabilizer runaway with classic technique (dotted line), DAMF (solid line) and DAMF+CA (dashed line)

Fig. 3 (a) shows the great improvement achieved thanks to the adoption of the control allocation. Note that the classic technique, for this failure condition, shows adequate robustness. This is caused by its structure. In fact, the longitudinal control channel (PI for 
pitch-angle above proportional pitch-rate SAS) affects only the elevators, while the stabilizer is supposed to be operated by the pilot separately. In this way, the stabilizer runway results to be a strong, but manageable disturbance. Instead, the DAMF tries to recover the attitude lavishing stronger control effort on the faulty stabilizer, the most effective surface, with bad results. The awareness of the fault on the stabilizer gives the chance to the CA technique to compensate by moving the control effort from this surface to the elevators, thus achieving the same results of the classical technique. As it is also evident in the time plots of Fig. 3 (b) when the failure is detected and isolated (here it is supposed to be done in $10 \mathrm{sec}$ after the failure occurs), the aircraft recovers a more adequate attitude to carry out properly the manoeuvre.

\subsection{Right turn and localizer intercept with rudder runaway}

This manoeuvre consists in the interception of the localizer beam, parallel to initial flight path, but opposite in versus. So, in the early stage of the manoeuvre, a right turn is performed, and then the capture and the tracking of the localizer beam are carried out. The fault, instead, consists in a runaway of both upper and lower rudder surfaces, so giving a strong yawing moment opposite to the desired turn. The initial flight condition data are summarized in Table 4.

In this failure case, a classical technique is totally inadequate to face such a failure, so leading the aircraft to crash into the ground. Instead, the DAMF shows to be robust enough to deal with this failure condition and it makes the aircraft to accomplish the manoeuvre, even though with reduced performance. The control allocation technique, instead, shows a sensible improvement of the robustness (see Fig. 4), if compared to the DAMF technique. The awareness of the fault (detected $10 \mathrm{sec}$ after it actually occurs) allows the control laws to fully exploit all the efficient effectors, thus accomplishing the manoeuvre smoothly. It is worth noting that in this case the DAMF without CA is robust enough to accomplish the manoeuvre, even though with degraded performances.

\subsection{Right turn and localizer intercept with loss of vertical tail}

The manoeuvre, here considered, is the same described in the previous subsection, but the failure scenario consists in the loss of the vertical tail (Smaili et al., 2006). The initial flight condition data are summarized in Table 4 . This is both a structural and actuation failure, in fact, the loss of the rudders strongly affects the lateral-directional aerodynamics and stability, compromising the possibility to damp the rotations about the roll and yaw axes. In this case (see Fig. 5), the classical technique is not able to reach lateral stability. Instead, no significant differences are evidenced between the two versions of the adaptive FCS (with and without CA). In fact, the information about the efficiency of the differential thrust is already available to the DAMF, due to the linear model of the bare Aircraft. Thus, as the tracking errors increase, the core control laws raise the control effort for both the rudders (failed) and the differential thrust. The latter is efficient enough to ensure the manoeuvrability.

\section{Conclusions}

In this chapter a fault-tolerant FCS architecture has been proposed. It exploits the main features of two different techniques, the adaptive control and the control allocation. The contemporaneous usage of these two techniques, the former for the robustness, and the latter for the explicit actuators failure treatment, has shown significant improvements in terms of fault-tolerance if compared to a simple classical controller and to the only adaptive 


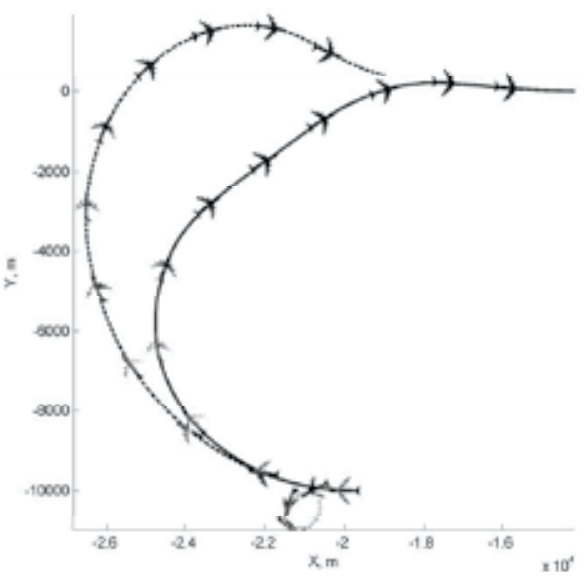

(a) Trajectories
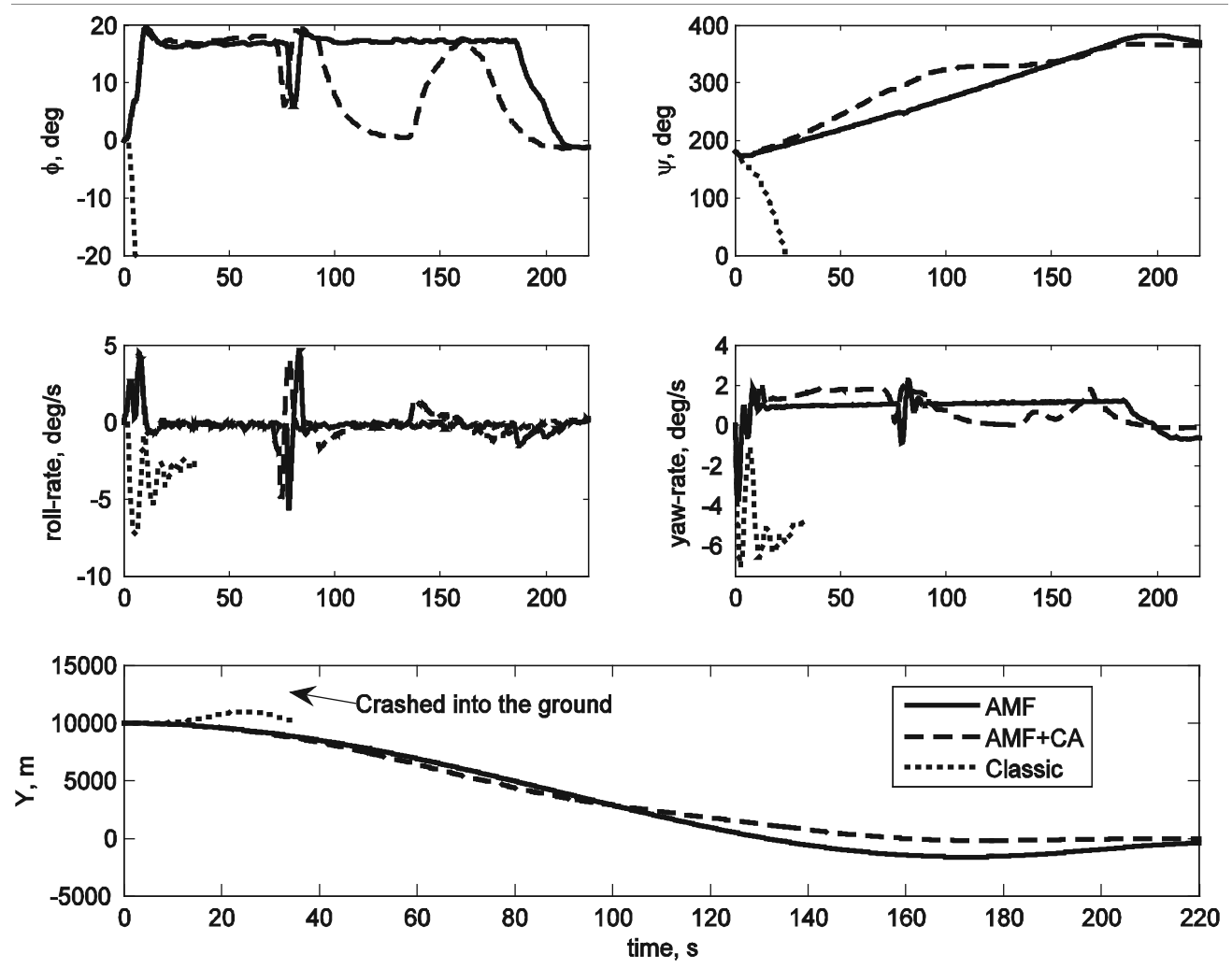

(b) Time plots

Fig. 4. Right turn and localizer intercept with rudder runaway with classic technique (dotted line), DAMF (solid line) and DAMF+CA (dashed line) 


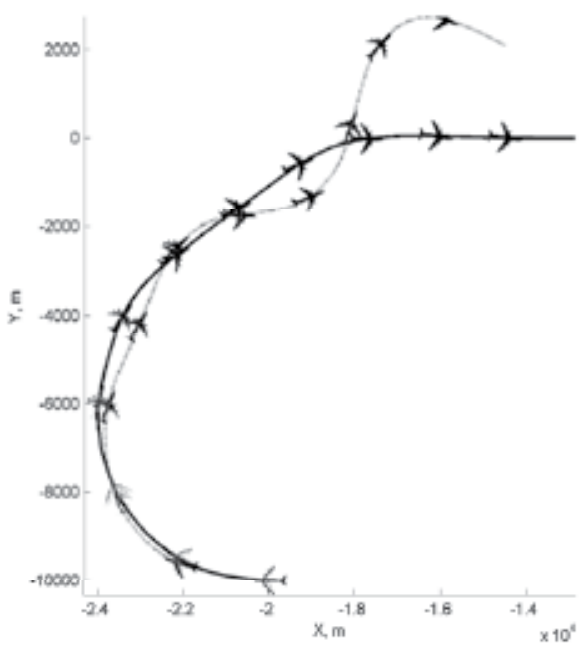

(a) Trajectories
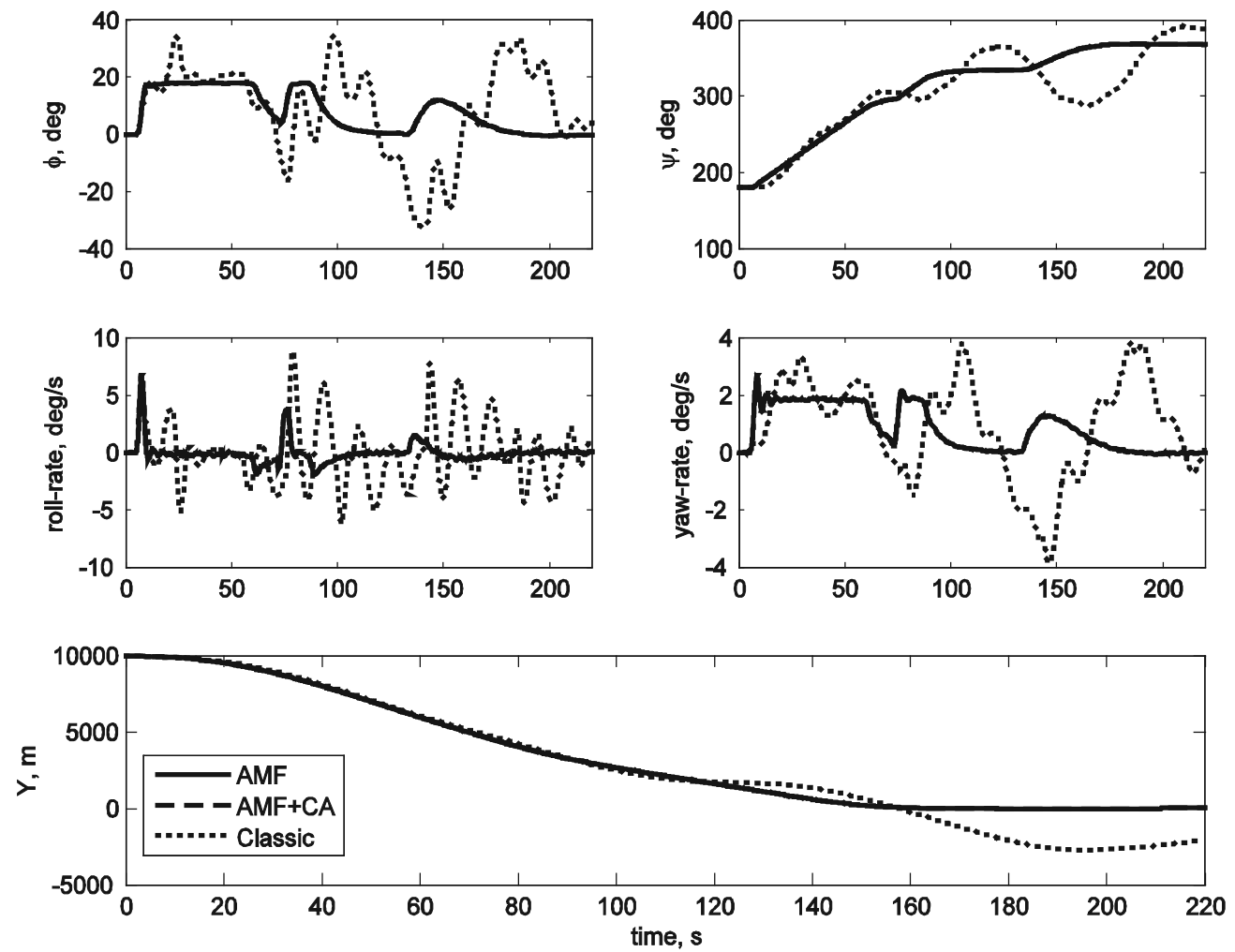

(b) Time plots

Fig. 5. Loss of vertical tail failure scenario, while performing a right turn \& localizer intercept runaway with classic technique (dotted line), DAMF (solid line) and DAMF+CA (dashed line) 
controller. The ability of the DAMF to on-line re-compute the control gains guarantees both robustness and performance, as shown in the proposed test cases. However, the contemporary usage of a control allocation scheme allowed improving significantly the fault-tolerance capabilities, at the only expense of requiring some limited information about the vehicle actuators' health. Therefore the proposed fault-tolerant scheme appears to be very promising to deal with drastic off-nominal conditions as the ones induced by severe actuators failure and damages thus improving the overall adaptive capabilities of a reconfigurable flight control system.

\section{References}

Bodson, M. \& Groszkiewicz, J. E. (1997). Multivariable Adaptive Algorithms for Reconfigurable Flight Control, IEEE Transaction on Control Systems Technology, Vol. 5, No. 2, pp. 217-229.

Boskovic, J. D. \& Mehra, R. K. (2002), Multiple-Model Adaptive Flight Control Scheme for Accommodation of Actuator Failures, Journal of Guidance, Control, and Dynamics, Vol. 25, No. 4, pp. 712-724.

Buffington, J. \& Chandler, P. (1998), Integration of on-line system identification and optimization-based control allocation, Proceedings of AIAA Guidance, Navigation, and Control Conference and Exhibit, Boston, MA.

Burken, J. J., Lu, P., Wu, Z. \& Bahm, C. (2001), Two Reconfigurable Flight-Control Design Methods: Robust Servomechanism and Control Allocation, Journal of Guidance, Control, and Dynamics, Vol. 24, No. 3, pp. 482-493.

Calise, A. J., Hovakimyan, N. \& Idan, M. (2001). Adaptive output feedback control of nonlinear systems using neural networks, Automatica, Vol. 37, No. 8, pp. 1201-1211.

Durham, W. C. \& Bordignon, K. A. (1995), Closed-Form Solutions to Constrained Control Allocation Problem, Journal of Guidance, Control and Dynamics, Vol. 18, No. 5, pp. 1000-1007.

Enns, D. (1998), Control Allocation Approaches, Proceedings of the AIAA Guidance, Navigation and Control Conference, Boston, MA.

Harkegard, O. (2002), Efficient Active Set Algorithms for Solving Constrained Least squares Problems in Aircraft Control Allocation, Proceedings. of the 41st IEEE Conference on Decision and Control, Vol. 2, pp. 1295-1300.

Kim, K. S., Lee, K. J. \& Kim, Y. (2003), Reconfigurable Flight Control System Design Using Direct Adaptive Method, Journal of Guidance, Control, and Dynamics, Vol. 26, No. 4, pp. 543-550.

Luenberger, D. G. (1989), Linear and Nonlinear Programming, 2nd ed., Addison-Welsey, 1989, Chapter 11.

Patton, R. J. (1997). Fault-Tolerant Control Systems: The 1997 Situation, Proceedings of the IFAC Symposium on Fault Detection, Supervision and Safety for Technical Processes, Vol. 2, pp. 1033-1055.

Smaili, M. H., Breeman, J., Lombaerts, T. J. \& Joosten, D. A. (2006), A Simulation Benchmark for Integrated Fault Tolerant Flight Control Evaluation, Proceedings of AIAA Modeling and Simulation Technologies Conference and Exhibit, Keystone, CO.

Tandale, M. \& Valasek, J. (2003), Structured Adaptive Model Inversion Control to Simultaneously Handle Actuator failure and Actuator Saturation, Proceedings. of the AIAA Guidance, Navigation and Control Conference, Austin, TX.

Virnig, J. \& Bodden, D. (2000), Multivariable Control Allocation and Control Law Conditioning when Control Effector Limit, Proceedings of the AIAA Guidance, Navigation and Control Conference, Denver, CO. 


\title{
Acceleration-based 3D Flight Control for UAVs: Strategy and Longitudinal Design
}

\author{
Iain K. Peddle and Thomas Jones \\ Stellenbosch University \\ South Africa
}

\section{Introduction}

The design of autopilots for conventional flight of UAVs is a mature field of research. Most of the published design strategies involve linearization about a trim flight condition and the use of basic steady state kinematic relationships to simplify control law design (Blakelock, 1991);(Bryson, 1994). To ensure stability this class of controllers typically imposes significant limitations on the aircraft's allowable attitude, velocity and altitude deviations. Although acceptable for many applications, these limitations do not allow the full potential of most UAVs to be harnessed. For more demanding UAV applications, it is thus desirable to develop control laws capable of guiding aircraft though the full 3D flight envelope. Such an autopilot will be referred to as a manoeuvre autopilot in this chapter.

A number of manoeuvre autopilot design methods exist. Gain scheduling (Leith \&. Leithead, 1999) is commonly employed to extend aircraft velocity and altitude flight envelopes (Blakelock, 1991), but does not tend to provide an elegant or effective solution for full 3D manoeuvre control. Dynamic inversion has recently become a popular design strategy for manoeuvre flight control of UAVs and manned aircraft (Bugajski \& Enns, 1992); (Lane \& Stengel, 1998);(Reiner et al., 1996);(Snell et al., 1992) but suffers from two major drawbacks. The first is controller robustness, a concern explicitly addressed in (Buffington et al., 1993) and (Reiner et al., 1996), and arises due to the open loop nature of the inversion and the inherent uncertainty of aircraft dynamics. The second drawback arises from the slightly Non Minimum Phase (NMP) nature of most aircraft dynamics, which after direct application of dynamic inversion control, results in not only an impractical controller with large counterintuitive control signals (Hauser et al., 1992) (Reiner et al., 1996), but also in undesired internal dynamics whose stability must be investigated explicitly (Slotine \& $\mathrm{Li}$, 1991). Although techniques to address the latter drawback have been developed (AlHiddabi \& McClamroch, 2002);(Hauser et al., 1992), dynamic inversion is not expected to provide a very practical solution to the $3 \mathrm{D}$ flight control problem and should ideally only be used in the presence of relatively certain minimum phase dynamics.

Receding Horizon Predictive Control (RHPC) has also been applied to the manoeuvre flight control problem (Bhattacharya et al., 2002);(Miller \& Pachter, 1997);(Pachter et al., 1998), and similarly to missile control (Kim et al., 1997). Although this strategy is conceptually very promising the associated computational burden often makes it a practically infeasible solution for UAVs, particularly for lower cost UAVs with limited processing power. 
The manoeuvre autopilot solution presented in this chapter moves away from the more mainstream methods described above and instead returns to the concept of acceleration control which has been commonly used in missile applications, and to a limited extent in aircraft applications, for a number of decades (see (Blakelock, 1991) for a review of the major results). However, whereas acceleration control has traditionally been used within the framework of linearised flight control (the aircraft or missile dynamics are linearised, typically about a straight and level flight condition), the algorithms and mathematics presented in this chapter extend the fundamental acceleration controller to operate equally effectively over the entire 3D flight envelope. The result of this extension is that the aircraft then reduces to a point mass with a steerable acceleration vector from a $3 \mathrm{D}$ guidance perspective. This abstraction which is now valid over the entire flight envelope is the key to significantly reducing the complexity involved in solving the manoeuvre flight control problem.

The chapter thus begins by presenting the fundamental ideas behind the design of gross attitude independent specific acceleration controllers. It then highlights how these inner loop controllers simplify the design of a manoeuvre autopilot and motivates that they lead to an elegant, effective and robust solution to the problem. Next, the chapter presents the detailed design and associated analysis of the acceleration controllers for the case where the aircraft is constrained to the vertical plane. A number of interesting and useful novel results regarding aircraft dynamics arise from the aforementioned analysis. The 2D flight envelope illustrates the feasibility of the control strategy and provides a foundation for development to the full 3D case.

\section{Autopilot design strategy for 3D manoeuvre flight}

For most UAV autopilot design purposes, an aircraft is well modelled as a six degree of freedom rigid body with specific and gravitational forces and their corresponding moments acting on it. The specific forces typically include aerodynamic and propulsion forces and arise due to the form and motion of the aircraft itself. On the other hand the gravitational force is universally applied to all bodies in proportion to their mass, assuming an equipotential gravitational field. The sum of the specific and gravitational forces determines the aircraft's total acceleration. It is desirable to be able to control the aircraft's acceleration as this would leave only simple outer control loops to regulate further kinematic states.

Of the total force vector, only the specific force component is controllable (via the aerodynamic and propulsion actuators), with the gravitational force component acting as a well modelled bias on the system. Thus, with a predictable gravitational force component, control of the total force vector can be achieved through control of the specific force vector. Modelling the specific force vector as a function of the aircraft states and control inputs is an involved process that introduces almost all of the uncertainty into the total aircraft model. Thus, to ensure robust control of the specific force vector a pure feedback control solution is desirable. Regulation techniques such as dynamic inversion are thus avoided due to the open loop nature of the inversion and the uncertainty associated with the specific force model.

Considering the specific force vector in more detail, the following important observation is made from an autopilot design simplification point of view. Unlike the gravitational force vector which remains inertially aligned, the components that make up the specific force vector tend to remain aircraft aligned. This alignment occurs because the specific forces arise 
as a result of the form and motion of the aircraft itself. For example, the aircraft's thrust vector acts along the same aircraft fixed action line at all times while the lift vector tends to remain close to perpendicular to the wing depending on the specific angle of attack. The observation is thus that the coordinates of the specific force vector in a body fixed axis system are independent of the gross attitude of the aircraft. This observation is important because it suggests that if gross attitude independent measurements of the specific force vector's body axes coordinates were available, then a feedback based control system could be designed to regulate the specific force vector independently of the aircraft's gross attitude. Of course, appropriately mounted accelerometers provide just this measurement, normalized to the aircraft's mass, thus practically enabling the control strategy through specific acceleration instead.

With gross attitude independent specific acceleration controllers in place, the remainder of a full 3D flight autopilot design is greatly simplified. From a guidance perspective the aircraft reduces to a point mass with a fully steerable acceleration vector. Due to the acceleration interface, the guidance dynamics will be purely kinematic and the only uncertainty present will be that associated with gravitational acceleration. The highly certain nature of the guidance dynamics thus allows among others, techniques such as dynamic inversion and RHPC to be effectively implemented at a guidance level. In addition to the associated autopilot simplifications, acceleration based control also provides for a robust autopilot solution. All aircraft specific uncertainty remains encapsulated behind a wall of high bandwidth specific acceleration controllers. Furthermore, high bandwidth specific acceleration controllers would be capable of providing fast disturbance rejection at an acceleration level, allowing action to be taken before the disturbances manifest themselves into position, velocity and attitude errors.

With the novel control strategy and its associated benefits conceptually introduced the remainder of this chapter focuses on the detailed development of the inner loop specific acceleration controllers for the case where the aircraft's motion is constrained to the $2 \mathrm{D}$ vertical plane. No attention will be given to outer guidance level controllers in the knowledge that control at this level is simplified enormously by the inner loop controllers. The detailed design of the remaining specific acceleration controllers to complete the set of inner loop controllers for full 3D flight are presented in (Peddle, 2008).

\section{Modelling}

To take advantage of the potential of regulating the specific acceleration independently of the aircraft's gross attitude requires writing the equations of motion in a form that provides an appropriate mathematical hold on the problem. Conceptually, the motion of the aircraft needs to be split into the motion of a reference frame relative to inertial space (to capture the gross attitude and position of the aircraft) and the superimposed rotational motion of the aircraft relative to the reference frame. With this mathematical split, it is expected that the specific acceleration coordinates in the reference and body frames will remain independent of the attitude of the reference frame. An obvious and appropriate choice for the reference frame is the commonly used wind axis system (axial unit vector coincides with the velocity vector). Making use of this axis system, the equations of motion are presented in the desired form below. The dynamics are split into the point mass kinematics (motion of the wind axis system through space), 


$$
\begin{gathered}
\dot{\Theta}_{W}=-\left(C_{W}+g \cos \Theta_{W}\right) / \bar{V} \\
\dot{\bar{V}}=A_{W}-g \sin \Theta_{W} \\
\dot{P}_{N}=\bar{V} \cos \Theta_{W} \\
\dot{P}_{D}=-\bar{V} \sin \Theta_{W}
\end{gathered}
$$

and the rigid body rotational dynamics (attitude of the body axis system relative to the wind axis system),

$$
\begin{gathered}
\dot{Q}=M / I_{y y} \\
\dot{\alpha}=Q+\left(C_{W}+g \cos \Theta_{W}\right) / \bar{V}
\end{gathered}
$$

with, $\Theta_{W}$ the flight path angle, $\bar{V}$ the velocity magnitude, $P_{N}$ and $P_{D}$ the north and down positions, $g$ the gravitational acceleration, $Q$ the pitch rate, $M$ the pitching moment, $I_{y y}$ the pitch moment of inertia, $\alpha$ the angle of attack and $A_{W}$ and $C_{W}$ the axial and normal specific acceleration coordinates in wind axes respectively. Note that the point mass kinematics describe the aircraft's position, velocity magnitude and gross attitude over time, while the rigid body rotational dynamics describes the attitude of the body axis system with respect to the wind axis system (through the angle of attack) as well as how the torques on the aircraft affect this relative attitude. It must be highlighted that the particular form of the equations of motion presented above is in fact readily available in the literature (Etkin, 1972), albeit not appropriately rearranged. However, presenting this particular form within the context of the proposed manoeuvre autopilot architecture and with the appropriate rearrangements will be seen to provide a novel perspective on the form that explicitly highlights the manoeuvre autopilot design concepts. Expanding now the specific acceleration terms with a commonly used pre-stall flight aircraft specific force and moment model yields,

$$
\begin{aligned}
& A_{W}=(T \cos \alpha-D) / m \\
& C_{W}=-(T \sin \alpha+L) / m
\end{aligned}
$$

with,

$$
\begin{gathered}
\dot{T}=-T / \tau_{T}+T_{C} / \tau_{T} \\
L=q S C_{L} \\
D=q S C_{D} \\
M=q S C_{m}
\end{gathered}
$$

where $m$ is the aircraft's mass, $\tau_{T}$ the thrust time constant, $S$ the area of the wing, $C_{L}, C_{D}$ and $C_{m}$ the lift, drag and pitching moment coefficients respectively and, 


$$
q=\rho \bar{V}^{2} / 2
$$

the dynamic pressure. Expansion of the aerodynamic coefficients for pre-stall flight (Etkin \& Reid, 1995) yields,

$$
\begin{gathered}
C_{L}=C_{L_{0}}+C_{L_{\alpha}} \alpha+C_{L_{Q}}(\bar{c} / 2 \bar{V}) Q+C_{L_{\delta_{E}}} \delta_{E} \\
C_{D}=C_{D_{0}}+C_{L}^{2} / \pi A e \\
C_{m}=C_{m_{0}}+C_{m_{\alpha}} \alpha+C_{m_{Q}}(\bar{c} / 2 \bar{V}) Q+C_{m_{\delta_{E}}} \delta_{E}
\end{gathered}
$$

where $A$ is the aspect ratio, $e$ the Oswald efficiency factor and standard non-dimensional stability derivative notation is used. Note that it is assumed in this chapter that the nondimensional stability derivatives above are independent of both the point mass and rigid body rotational dynamics states. Although in reality the derivatives do change somewhat with the system states, for many UAVs operating under pre-stall flight conditions this change is small. Furthermore, with the intention being to design a feedback based control system to regulate specific acceleration, the adverse effect of the modelling errors will be greatly reduced thus further justifying the assumption.

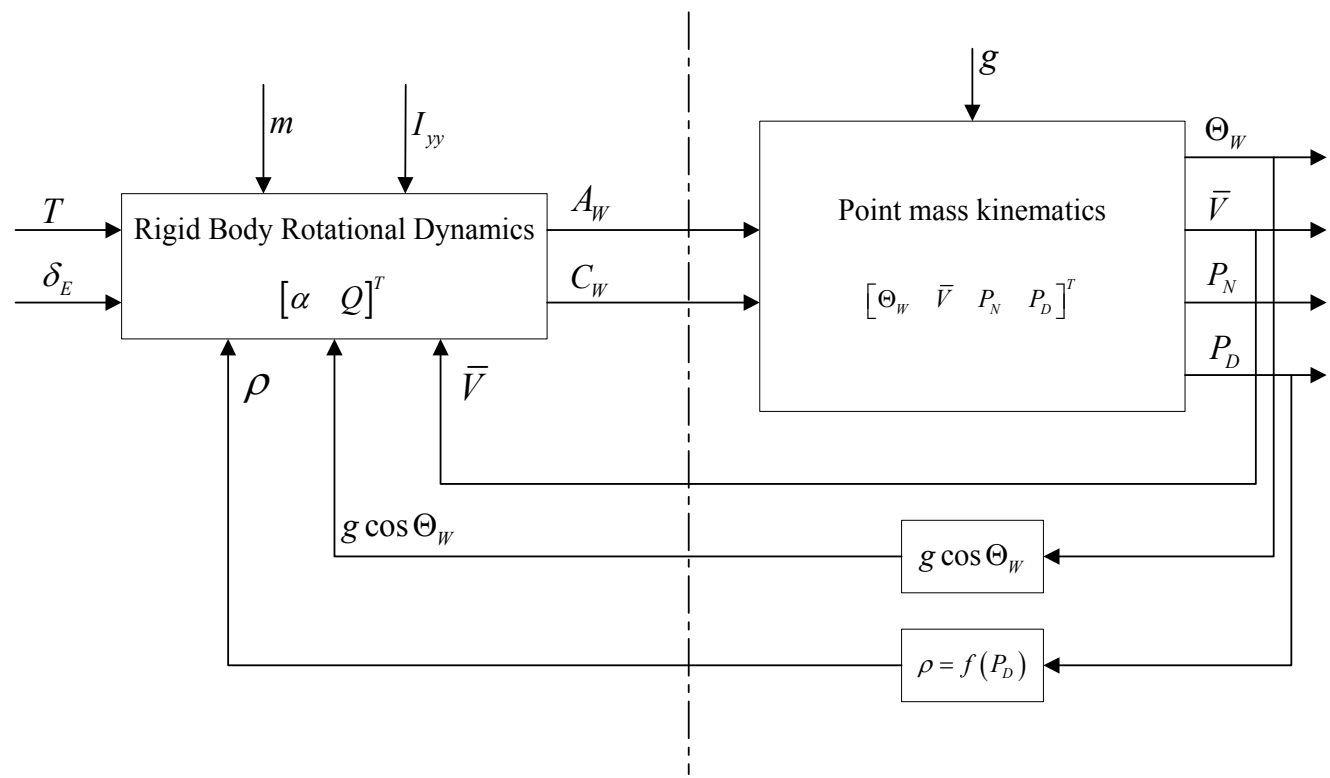

Fig. 1. Split between the rigid body rotational dynamics and the point mass kinematics.

Figure 1 provides a graphical overview of the particular form of the dynamics presented here. The dash-dotted vertical line in the figure highlights a natural split in the aircraft dynamics into the aircraft dependent rigid body rotational dynamics on the left and the aircraft independent point mass kinematics on the right. It is seen that all of the aircraft specific uncertainty resides within the rigid body rotational dynamics, with gravitational acceleration being the only inherent uncertainty in the point mass kinematics. Of course left 
unchecked, the aircraft specific uncertainty in the rigid body rotational dynamics would leak into the point mass kinematics via the axial and normal specific acceleration, thus motivating the design of feedback based specific acceleration controllers.

Continuing to analyze Figure 1, the point mass kinematics are seen to link back into the rigid body rotational dynamics via the velocity magnitude, air density (altitude) and flight path angle. If it can be shown that the aforementioned couplings do not strongly influence the rigid body rotational dynamics, then the rigid body rotational dynamics would become completely independent of the point mass kinematics and thus the gross attitude of the aircraft. This in turn would provide the mathematical platform for the design of gross attitude independent specific acceleration controllers.

Investigating the feedback couplings, the velocity magnitude and air density couple into the rigid body rotational dynamics primarily through the dynamic pressure, which is seen in equations (10) through (12) to scale the magnitude of the aerodynamic forces and moments. However, the dynamics of most aircraft are such that the angle of attack and pitch rate dynamics operate on a timescale much faster than that of the velocity magnitude and air density dynamics. Thus, assuming that a timescale separation either exists or can be enforced through feedback control, the dynamic coupling is reduced to a static dependence where the velocity magnitude and air density are treated as parameters in the rigid body rotational dynamics.

The flight path angle is seen to couple only into the angle of attack dynamics, via gravitational acceleration. The flight path angle coupling term in equation (6) represents the tendency of the wind axis system to rotate under the influence of the component of gravitational acceleration normal to the velocity vector. The rotation has the effect of changing the relative attitude of the body and wind axis system as modelled by the angle of attack dynamics. However, the normal specific acceleration (shown in parenthesis next to the gravity term in equation (6)) will typically be commanded by an outer loop guidance controller to cancel the gravity term and then further to steer the aircraft as desired in inertial space. Thus, the effect of the flight path coupling on the angle of attack dynamics is expected to be small. However, to fully negate this coupling, it will be assumed that a dynamic inversion control law can be designed to reject it, the details of which will be discussed in a following section. Note however, that dynamic inversion will only be used to reject the arguably weak flight path angle coupling, with the remainder of the control solution to be purely feedback based.

With the above timescale separation and dynamic inversion assumptions in place, the rigid body rotational dynamics become completely independent of the point mass kinematics and thus provide the mathematical platform for the design of gross attitude independent specific acceleration controllers. With all aircraft specific uncertainty encapsulated within the inner loop specific acceleration controllers and disturbance rejection occurring at an acceleration level, the design is argued to provide a robust solution to the manoeuvre flight control problem. The remainder of this article focuses of the design and simulation of the axial and normal specific acceleration controllers, as well as the associated conditions for their implementation.

\section{Decoupling the axial and normal dynamics}

Control of the axial and normal specific acceleration is dramatically simplified if the rigid body rotational dynamics can be decoupled into axial and normal dynamics. This 
decoupling would allow the axial and normal specific acceleration controllers to be designed independently. To this end, consider equations (7) and (8), and notice that for small angles of attack and typical lift to drag ratios, the equations can be well approximated as follows,

$$
\begin{gathered}
A_{W} \approx(T-D) / m \\
C_{W} \approx-L / m
\end{gathered}
$$

With these simplifying assumptions, the thrust no longer couples into the normal dynamics whose states and controls include the angle of attack, pitch rate and elevator deflection. On the other hand, the normal dynamics states still drive into the axial dynamics through the drag coupling of equation (17). However, through proper use of the bandwidth-limited thrust actuator the drag coupling can be rejected up to some particular frequency. Assuming that effective low frequency disturbance rejection can be achieved up to the open loop bandwidth of the thrust actuator, then only drag disturbance frequencies beyond this remain of concern from a coupling point of view.

Considering now the point mass kinematics, it is clear from equation (2) that the axial specific acceleration drives solely into the velocity magnitude dynamics. Thus uncompensated high frequency drag disturbances will result in velocity magnitude disturbances which in turn will couple back into the rest of the rigid body rotational dynamics both kinematically and through the dynamic pressure. However, the natural integration process of the velocity magnitude dynamics will filter the high frequency part of the drag coupling. Thus, given acceptable deviations in the velocity magnitude, the thrust actuator need only reject enough of the low frequency portion of the drag disturbance for its total effect on the velocity magnitude to be acceptable. By acceptable it is meant that the velocity magnitude perturbations are small enough to result in a negligible coupling back into the rigid body rotational dynamics.

To obtain a mathematical hold on the above arguments, consider the closed loop transfer function from the normalized drag input to the axial specific acceleration output,

$$
S_{D}(s) \equiv \frac{A_{W}(s)}{D(s) / m}
$$

Through proper control system design, the gain of the sensitivity transfer function above can be kept below a certain threshold within the controller bandwidth. The bandwidth of the axial specific acceleration controller will however typically be limited to that of the thrust actuator for saturation reasons. For frequencies above the controller bandwidth, the sensitivity transfer function will display some form of transient and then settle to unity gain. Considering the velocity magnitude dynamics of equation (2), the total transfer function of the normalized drag input to velocity magnitude is then,

$$
\frac{\bar{V}(s)}{D(s) / m}=\frac{S_{D}(s)}{s}
$$

Note that the integrator introduced by the natural velocity dynamics will result in diminishing high frequency gains. Equation (20) can be used to determine whether drag 
perturbations will result in acceptable velocity magnitude perturbations. Conversely, given the expected drag perturbation spectrum and the acceptable level of velocity magnitude perturbations, the specifications of the sensitivity transfer function can be determined. To ease the process of determining acceptable levels of velocity magnitude perturbations and expected levels of drag perturbations, it is convenient to write these both in terms of normal specific acceleration. The return disturbance in normal specific acceleration due to a normal specific acceleration perturbation can then be used to specify acceptable coupling levels. Relating first normalized drag to normal specific acceleration, use of equation (18) provides the following result,

$$
\frac{D / m}{C_{W}}=-\frac{1}{R_{L D}}
$$

where use has been made of the fact that lift is related to drag through the lift to drag ratio $R_{L D}$. Then, equation (18) can be used to capture the dominant relationship between velocity perturbations and the resulting normal specific acceleration perturbations. Partially differentiating equation (18) with respect to the velocity magnitude yields the desired result,

$$
\frac{\partial C_{W}}{\partial \bar{V}}=\frac{\partial}{\partial \bar{V}} \frac{-q S C_{L}}{m} \approx \frac{-\rho \bar{V} S C_{L}}{m}=2 \frac{C_{W}}{\bar{V}}
$$

Combining equations (20) to (22) yields the return disturbance sensitivity function,

$$
\begin{aligned}
S_{C_{W}}(s) & \equiv \frac{\partial C_{W}}{\partial \bar{V}} \cdot \frac{V(s)}{D(s) / m} \cdot \frac{D / m}{C_{W}} \\
& =\left(-2 \frac{C_{W}}{\bar{V} R_{L D}} \frac{1}{s}\right) S_{D}(s)
\end{aligned}
$$

Given an acceptable return disturbance level, the specifications of the sensitivity function of equation (19) can be determined for a particular flight condition. With the velocity magnitude and lift to drag ratio forming part of the denominator of equation (23), the resulting constraints on the sensitivity function are mild for low operating values of normal specific acceleration. Only during very high acceleration manoeuvres, does the sensitivity specification become more difficult to practically realize. This is illustrated through an example calculation at the end of section 5 .

Given the above arguments, the drag coupling into the axial specific acceleration dynamics can be ignored if the associated sensitivity function constraint is adhered to when designing the axial specific acceleration controller. With the coupling of the drag term ignored, the axial dynamics become independent of the normal dynamics allowing the controllers to be designed separately. Furthermore, note that the axial dynamics also become independent of the velocity magnitude and the air density. Thus, unlike the normal specific acceleration controller, there is no need for the axial specific acceleration controller to operate on a timescale much faster than these variables. This greatly improves the practical viability of designing an axial specific acceleration control system since most thrust actuators are significantly bandwidth limited. The axial and normal dynamics can thus be decoupled as follows, 
Axial Dynamics:

Normal Dynamics:

$$
\begin{aligned}
& \dot{T}=\left[-1 / \tau_{T}\right] T+\left[1 / \tau_{T}\right] T_{C} \\
& A_{W}=[1 / m] T+[-D / m]
\end{aligned}
$$

$$
\begin{aligned}
& {\left[\begin{array}{c}
\dot{\alpha} \\
\dot{Q}
\end{array}\right]=\left[\begin{array}{cc}
-\frac{L_{\alpha}}{m \bar{V}} & 1-\frac{L_{Q}}{m \bar{V}} \\
\frac{M_{\alpha}}{I_{y y}} & \frac{M_{Q}}{I_{y y}}
\end{array}\right]\left[\begin{array}{l}
\alpha \\
Q
\end{array}\right]+\left[\begin{array}{c}
-\frac{L_{\delta_{E}}}{m \bar{V}} \\
\frac{M_{\delta_{E}}}{I_{y y}}
\end{array}\right] \delta_{E}+\left[\begin{array}{c}
\frac{g \cos \Theta_{W}}{\bar{V}}-\frac{L_{0}}{m \bar{V}} \\
\frac{M_{0}}{I_{y y}}
\end{array}\right]} \\
& C_{W}=\left[\begin{array}{rr}
-\frac{L_{\alpha}}{m} & -\frac{L_{Q}}{m}
\end{array}\right]\left[\begin{array}{l}
\alpha \\
Q
\end{array}\right]+\left[-\frac{L_{\delta_{E}}}{m}\right] \delta_{E}+\left[-\frac{L_{0}}{m}\right]
\end{aligned}
$$

where dimensional stability and control derivative notation has been used to remove clutter. Finally, notice that the normal dynamics are simply the classical short period mode approximation (Etkin \& Reid, 1995) but have been shown here to be valid for all point mass kinematics states (i.e. all gross attitudes) with the flight path angle coupling term acting as a disturbance input. Intuitively this makes sense since the physical phenomena that manifest themselves into what is classically referred to as the short period mode are not dependent on the gross attitude of the aircraft. Whether an aircraft is flying straight and level, inverted or climbing steeply, its short period motion remains unchanged.

\section{Axial specific acceleration controller}

In this section a controller capable of regulating the axial specific acceleration is designed. Attention will also be given to the closed loop sensitivity function constraint of equation (23) for a specific return disturbance level. With reference to the axial dynamics of equations (24) and (25) define the following Proportional-Integrator (PI) control law with enough degrees of freedom to allow for arbitrary closed loop pole placement,

$$
\begin{gathered}
T_{c}=-K_{A} A_{W}-K_{E} E_{A} \\
\dot{E}_{A}=A_{W}-A_{W_{R}}
\end{gathered}
$$

where $A_{W_{R}}$ is the reference axial specific acceleration command. The integrator in the controller is essential for robustness towards uncertain steady state drag and thrust actuator offsets. It is straightforward to show that given the desired closed loop characteristic equation,

$$
\alpha_{c}(s)=s^{2}+\alpha_{1} s+\alpha_{0}
$$

the feedback gains that will fix the closed loop poles are,

$$
K_{A}=m\left(\tau_{T} \alpha_{1}-1\right)
$$




$$
K_{E}=m \tau_{T} \alpha_{0}
$$

These simple, closed form solution gains will ensure an invariant closed loop axial specific acceleration dynamic response as desired. The controller design freedom is reduced to that of selecting appropriate closed loop poles bearing in mind factors such as actuator saturation and the sensitivity function constraint of the previous section. Investigation of the closed loop sensitivity function for this particular control law yields the following result,

$$
S_{D}(s)=-\frac{s}{\tau_{T} \alpha_{0}}\left(\frac{\tau_{T} s+1}{1} \frac{\alpha_{0}}{s^{2}+\alpha_{1} s+\alpha_{0}}\right)
$$

For actuator saturation reasons the closed loop axial dynamics bandwidth is typically limited to being close to that of the open loop thrust actuator and thus for reasonable closed loop damping ratios the second order term in parenthesis above can be well approximated by a first order model to simplify the sensitivity function as follows,

$$
S_{D}(s) \approx-\frac{s}{\tau_{T} \alpha_{0}} \frac{1}{\tau_{A} s+1}
$$

Here, $\tau_{A}=1 /\left(\tau_{T} \alpha_{0}\right)$ is the approximating time constant calculated to match the high frequency sensitivity function asymptotes. Substituting equation (34) into equation (23) yields the return disturbance transfer function,

$$
S_{C_{W}}(s)=2 \frac{C_{W}}{\bar{V} R_{L D} \tau_{T} \alpha_{0}} \frac{1}{\tau_{A} s+1}
$$

Given the maximum allowable gain of the return disturbance transfer function $\gamma$, a lower bound constraint on the natural frequency $\left(\omega_{n}\right)$ of the closed loop axial control system is calculated by satisfying the inequality

$$
\frac{\omega_{n}}{\omega_{T}} \geq \sqrt{\left[\frac{2 C_{W} \tau_{T}}{\bar{V} R_{L D} \gamma}\right]_{\max }}
$$

where, $\omega_{T}=1 / \tau_{T}$ is the open loop bandwidth of the thrust actuator and the subscript max denotes the maximum value of the term in parenthesis. The following example illustrates the practical feasibility of adhering to the sensitivity function constraint. Consider a UAV that is to fly with a minimum velocity of $20 \mathrm{~m} / \mathrm{s}$, with a maximum normal specific acceleration of $4 \mathrm{~g}$ and a minimum lift to drag ratio of 10 . Then, for more than $20 \mathrm{~dB}$ of return disturbance rejection, the natural frequency of the closed loop system should have the following relationship to the open loop thrust bandwidth,

$$
\omega_{n} / \omega_{T} \geq 2 \sqrt{\tau_{T}}
$$

For this specific example, thrust actuators with a bandwidth of below $4 \mathrm{rad} / \mathrm{s}$ (time constant of greater than $0.25 \mathrm{~s}$ ) will require that the closed loop natural frequency is greater than that of the thrust actuator. Despite the fairly extreme nature of this example (low velocity magnitude, high acceleration and low lift to drag ratio), thrust time constants on the order 
of $0.25 \mathrm{~s}$ are still practically feasible for UAVs. The deduction is thus that the axial specific acceleration controller will be practically applicable to most UAVs.

\section{Normal specific acceleration controller}

This section presents the design and associated analysis of a closed form normal specific acceleration controller that yields an invariant dynamic response for all point mass kinematics states. The design is based on the linear normal dynamics of equations (26) and (27) with the velocity magnitude and air density considered parameters and the flight path angle coupling rejected using dynamic inversion. To consider the velocity magnitude and air density as parameters requires a timescale separation to exist between these two quantities and the normal dynamics. Therefore, it is important to investigate any upper limits on the allowable bandwidth of the normal dynamics as this will in turn clamp the upper bandwidth of the velocity magnitude and the air density (altitude) dynamics. Furthermore, it is important to investigate the eligibility of the normal dynamics for effective dynamic inversion of the flight path angle coupling term. Thus, before continuing with the normal specific acceleration controller design, the natural normal dynamics are analyzed in detail.

\subsection{Natural normal specific acceleration dynamics}

Consider the dynamics from the elevator control input to the normal specific acceleration output. The direct feed-through term in the normal specific acceleration output implies that the associated transfer function has as many zeros as it does poles. The approximate characteristic equation for the poles is easily be shown to be,

$$
p(s)=s^{2}+\left(\frac{L_{\alpha}}{m \bar{V}}-\frac{M_{Q}}{I_{y y}}\right) s-\left(\frac{L_{\alpha}}{m \bar{V}} \frac{M_{Q}}{I_{y y}}+\frac{M_{\alpha}}{I_{y y}}\right)
$$

where use has been made of the commonly used simplifying assumption (Etkin \& Reid, 1995),

$$
\left|L_{Q} / m \bar{V}\right| \ll 1
$$

Considering equation (38) it is important to note that the normal dynamics poles are not influenced by the lift due to pitch rate or elevator deflection. The importance of this will be made clear later on in this section. The zeros from the elevator input to the normal specific acceleration output can be shown, after some manipulation, to be well approximated by the roots of the characteristic equation,

$$
s^{2}-L_{Q}\left(l_{T}-l_{D}\right) / I_{y y} s-L_{\alpha}\left(l_{T}-l_{N}\right) / I_{y y}=0
$$

with the following characteristic lengths defined,

$$
\begin{gathered}
l_{N} \equiv-M_{\alpha} / L_{\alpha} \\
l_{T} \equiv-M_{\delta_{E}} / L_{\delta_{E}}
\end{gathered}
$$




$$
l_{D} \equiv-M_{Q} / L_{Q}
$$

where, $l_{N}$ is the length to the neutral point, $l_{T}$ is the effective length to the tail-plane and $l_{D}$ is the effective damping arm length. Note that only the simplifying assumption of equation (39) has been used in obtaining the novel characteristic equation for the zeros above. Completing the square to find the roots of equation (40) gives,

$$
\left[s-L_{Q}\left(l_{T}-l_{D}\right) / 2 I_{y y}\right]^{2}=\left[L_{Q}\left(l_{T}-l_{D}\right) / 2 I_{y y}\right]^{2}+L_{\alpha}\left(l_{T}-l_{N}\right) / I_{y y}
$$

For most aircraft the effective length to the tail-plane and effective damping arm lengths are very similar. This is because most of the damping arises from the tail-plane which is also typically home to the elevator control surface. Thus the moment arm lengths for pitch rate and elevator deflection induced forces are very similar. As a result, the first term on the right hand side of equation (44) is most often negligibly small and to a good approximation, the zeros from elevator to normal specific acceleration are,

$$
z_{1,2} \approx L_{Q}\left(l_{T}-l_{D}\right) / 2 I_{y y} \pm \sqrt{L_{\alpha}\left(l_{T}-l_{N}\right) / I_{y y}}
$$

Analysis of equation (45) reveals that the only significant effect of the lift due to pitch rate derivative on the zeros is that of producing an offset along the real axis. As previously argued, the effective tail-plane and damping arm lengths are typically very similar and as a result, even this effect is usually small. Thus, it can be seen that to a good approximation, the lift due to pitch rate plays no role in determining the elevator to normal specific acceleration dynamics.

On the other hand, the effective length to the tail-plane and the length to the neutral point typically differ significantly. With this difference scaled by the lift due to angle of attack (which is usually far greater than the lift due to pitch rate) it can be seen that the second term in equation (45) will dominate the first in determining the zero positions. Thus although the lift due to elevator deflection played no role in determining the system poles, it plays a large role in deter-mining the zeros. Knowing the position of the zeros is important from a controller design point of view because not only do they affect the dynamic response of the system but they also impose controller independent limitations on the system's practically achievable dynamic response. These limitations are mathematically described by Bode's sensitivity and complementary sensitivity integrals as discussed in (Freudenberg \& Looze, 1985); (Goodwin et al., 2001).

Expanding on the above point, it is noted that for most aircraft the effective length to the tail-plane is far greater than the length to the neutral point and so the zeros are real and of opposite sign. The result, as intuitively expected, is that the dynamics from the elevator to normal specific acceleration are NMP since a Right Half Plane (RHP) zero exists. A RHP zero places severe, controller independent restrictions on the practically attainable upper bandwidth of the closed loop normal specific acceleration dynamics. Furthermore, designing a dynamic inversion control law in a system with NMP dynamics, particularly when the NMP nature of the system is weak, tends to lead to an impractical solution with internal dynamics that may or may not be stable (Hauser et al., 1992); (Hough, 2007).

Since this NMP dynamics case is by far the most common for aircraft, the limits imposed by it shall be investigated further in the following subsection. The goal of the investigation is to seek 
a set of conditions under which the effects of a RHP zero become negligible, equivalently allowing the NMP nature of a system to be ignored. With these conditions identified and satisfied, the design of the normal specific acceleration controller can continue based on a set of simplified dynamics that do not capture the NMP nature of the system.

\subsection{Analysis of the NMP dynamics case}

Ignoring the typically negligible real axis offset term in equation (45), the transfer function from the elevator to normal specific acceleration is of the form,

$$
G(s)=k \frac{\omega_{n}^{2}}{s^{2}+2 \zeta \omega_{n} s+\omega_{n}^{2}} \frac{z_{0}-s}{z_{0}}
$$

where $z_{0}$ is the RHP zero position. Note that the effect of the left half plane zero has been neglected because it is most often largely negated through pole-zero cancellation by unmodelled dynamics such as those introduced through servo lag. The transfer function of equation (46) can be written as follows,

$$
G(s)=G_{n}(s)-G_{n}(s) s / z_{0}
$$

where,

$$
G_{n}(s)=k \frac{\omega_{n}^{2}}{s^{2}+2 \zeta \omega_{n} s+\omega_{n}^{2}}
$$

is a nominal second order system with no zeros. Equation (48) makes it clear that as the position of the zero tends towards infinity, so the total system transfer function converges towards $G_{n}(s)$. The purpose of the analysis to follow is to investigate more precisely, the conditions under which $G(s)$ can be well approximated by $G_{n}(s)$. To this end, a time response analysis method is employed. Consider the Laplace transform of the system's step response,

$$
Y(s)=G_{n}(s) / s-G_{n}(s) / z_{0}
$$

Equation (49) makes it clear that the total step response is the nominal system step response less the impulse response of the system scaled by the inverse of the RHP zero frequency. Since the nominal response gradient is always zero at the time of the step, the system must exhibit undershoot. The level of undershoot will depend of the damping, speed of response of the system and the zero frequency. If the level of undershoot is small relative to unity then it is equivalent to saying that the second term of equation (47) has a negligible effect. Thus, by investigating the undershoot further, conditions can be developed under which the total system response is well approximated by the nominal system response.

A closed form solution for the exact level of undershoot experienced in response to a step command for a system of the form presented in equation (46) is provided below,

$$
y_{\min }=k\left[1-(\sin \theta / \sin \phi) e^{-(\theta-\phi) / \tan \theta}\right]
$$

where, 


$$
\begin{gathered}
\theta=\cos ^{-1}(\zeta) \\
\phi=\tan ^{-1}\left(\sqrt{1-\zeta^{2}} /(\zeta+r)\right) \\
r=\omega_{n} / z_{0}
\end{gathered}
$$

Derivation of this novel result involves inverse Laplace transforming equation (49) and finding the time response minima through calculus. The above equations make it clear that the undershoot is only a function of the ratio between the system's natural frequency and the zero frequency $(r)$ and the system's damping ratio $(\zeta)$. Figure 2 below provides a plot of the maximum percentage undershoot as a function of $r^{-1}$ for various damping ratios.

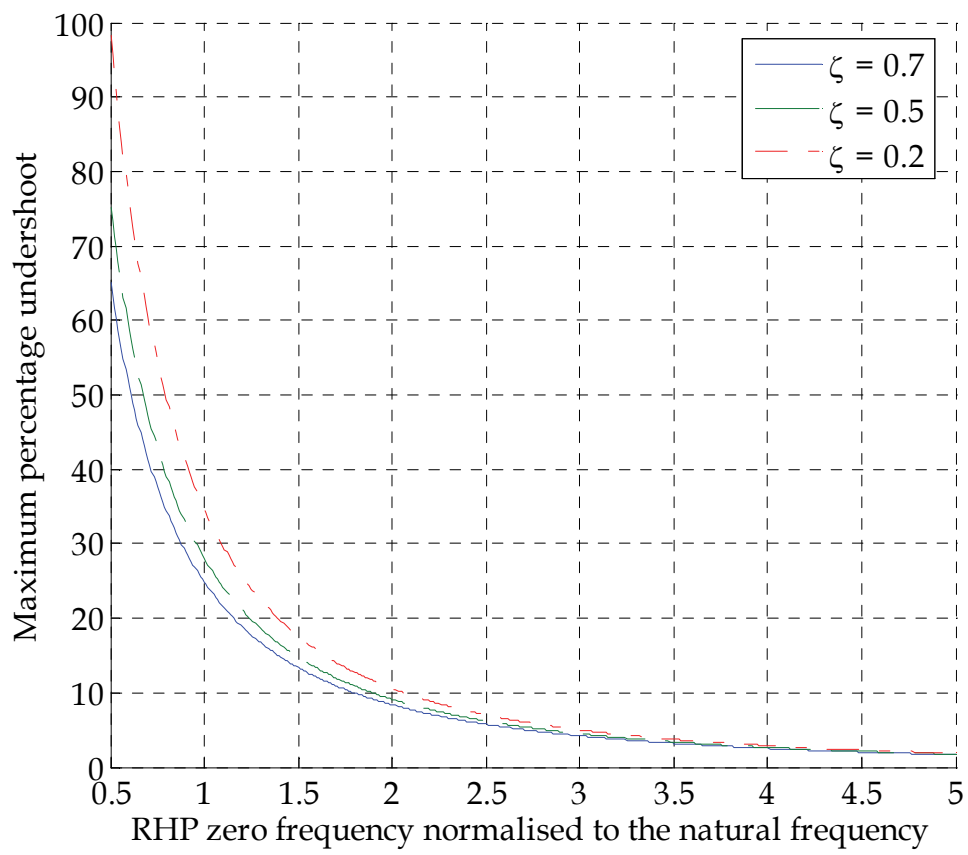

Fig. 2. Maximum undershoot of a 2nd order system as a function of normalized RHP zero frequency for various damping ratios.

It is clear from Figure 2 that for low percentage undershoots, the damping ratio has little influence. Thus, the primary factor determining the level of undershoot is the ratio of the system's natural frequency to that of the zero frequency. Furthermore, it is clear that for less than $5 \%$ maximum undershoot, the natural frequency should be at least three times lower than that of the zero. With only $5 \%$ undershoot the response of the total system will be well approximated by the response of the nominal system with no zero. Thus by making use of the maximum undershoot as a measure of the NMP nature of a system the following novel frequency domain design rule is developed,

$$
\omega_{n}<z_{0} / 3
$$


for the NMP nature of the system to be considered negligible. This rule implies that the system poles must lie within a circle of radius $z_{0} / 3$ in the s-plane. Thus, an upper bound is placed on the natural frequency of the system if its NMP nature is to be ignored.

\subsection{Frequency bounds on the normal specific acceleration controller}

Given the results of the previous two subsections, the upper bound on the natural frequency of the normal specific acceleration controller becomes,

$$
\omega_{n}<\left|\sqrt{L_{\alpha}\left(l_{T}-l_{N}\right) / I_{y y}}\right| / 3
$$

where the typically negligible offset in the zero positions in equation (45) has been ignored. Adhering to this upper bound will allow the NMP nature of the system to be ignored and will thus ensure both practically feasible dynamic inversion of the flight path angle coupling and no large sensitivity function peaks (Goodwin et al., 2001) in the closed loop system. Note that given the physical meaning of the characteristic lengths defined in equations (41) through (43), the approximate zero positions and thus upper frequency bound can easily be determined by hand for a specific aircraft.

It is important to note that the upper bound applies to both the open loop and closed loop normal specific acceleration dynamics. If the open loop poles violate the condition of equation (55) then moving them through control application to within the acceptable frequency region will require taking into account the effect of the system zeros. Thus, for an aircraft to be eligible for the normal specific acceleration controller of the next subsection, its open loop normal dynamics poles must at least satisfy the bound of equation (55). If they do not then an aircraft specific normal specific acceleration controller would have to be designed. However, most aircraft tend to satisfy this bound in the open loop because open loop poles outside the frequency bound of equation (55) would yield an aircraft with poor natural flying qualities i.e. the aircraft would be too statically stable and display significant undershoot and lag when performing elevator based manoeuvres. Interestingly, the frequency bound can thus also be utilized as a design rule for determining the most forward centre of mass position of an aircraft for good handling qualities.

In term of lower bounds, the normal dynamics must be timescale separated from the velocity magnitude and air density (altitude) dynamics. Of these two signals, the velocity magnitude typically has the highest bandwidth and is thus considered the limiting factor. Given the desired velocity magnitude bandwidth (where it is assumed here that the given bandwidth is achievable with the available axial actuator), then as a practical design rule the normal dynamics bandwidth should be at least five times greater than this for sufficient timescale separation. Note that unlike in the upper bound case, only the closed loop poles need satisfy the lower bound constraint. However, if the open loop poles are particularly slow, then it will require a large amount of control effort to meet the lower bound constraint in the closed loop. This may result in actuator saturation and thus a practically infeasible controller. However, for typical aircraft parameters the open loop poles tend to already satisfy the timescale separation lower bound.

With the timescale separation lower bound and the NMP zero upper bound, the natural frequency of the normal specific acceleration controller is constrained to lying within a circular band in the s-plane as shown in Figure 3 (poles would obviously not be selected in the RHP for stability reasons). The width of the circular band in Figure 3 is an indication of 
the eligibility of a particular airframe for the application of the normal specific acceleration controller to be designed in the following subsection.

For most aircraft this band is acceptably wide and the control system to be presented can be directly applied. For less conventional aircraft, the band can become very narrow and the two constraint boundaries may even cross. In this case, the generic control system to be presented cannot be directly applied. One solution to this problem is to design an aircraft specific normal specific acceleration controller. However, this solution is typically not desirable since the closeness of the bounds suggests that the desired performance of the particular airframe will not easily be achieved practically. Instead, redesign of the airframe and/or reconsideration of the outer loop performance bandwidths will constitute a more practical solution.

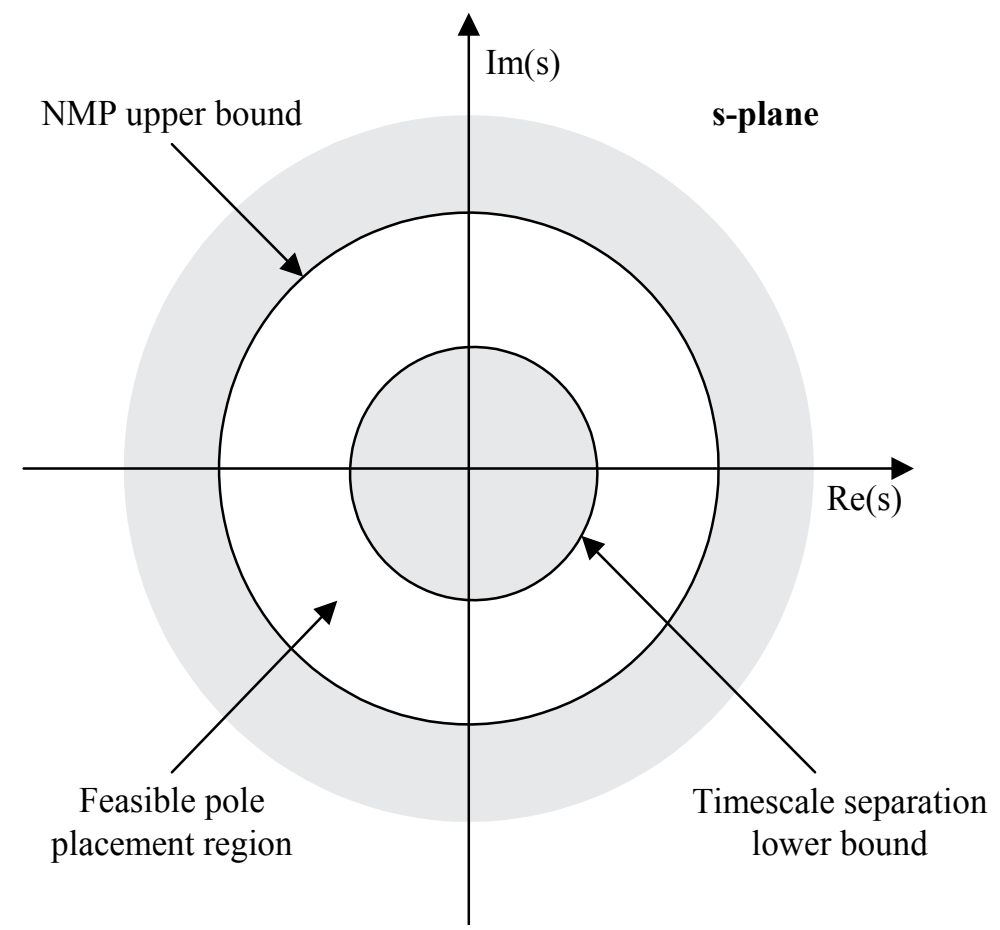

Fig. 3. NMP upper bound and timescale separation lower bound outlining feasible pole placement region.

\subsection{Normal specific acceleration controller design}

Assuming that the frequency bounds of the previous section are met, the design of a practically feasible normal specific acceleration controller can proceed based on the following reduced normal dynamics,

$$
\left[\begin{array}{c}
\dot{\alpha} \\
\dot{Q}
\end{array}\right]=\left[\begin{array}{cc}
-\frac{L_{\alpha}}{m \bar{V}} & 1 \\
\frac{M_{\alpha}}{I_{y y}} & \frac{M_{Q}}{I_{y y}}
\end{array}\right]\left[\begin{array}{l}
\alpha \\
Q
\end{array}\right]+\left[\begin{array}{c}
0 \\
\frac{M_{\delta_{E}}}{I_{y y}}
\end{array}\right] \delta_{E}+\left[\begin{array}{c}
\frac{g \cos \Theta_{W}}{\bar{V}}-\frac{L_{0}}{m \bar{V}} \\
\frac{M_{0}}{I_{y y}}
\end{array}\right]
$$




$$
C_{W}=\left[\begin{array}{ll}
-\frac{L_{\alpha}}{m} & 0
\end{array}\right]\left[\begin{array}{l}
\alpha \\
Q
\end{array}\right]+[0] \delta_{E}+\left[-\frac{L_{0}}{m}\right]
$$

The simplifications in the dynamics above arise from the analysis of subsection 6.1 where it was shown that to a good approximation, the lift due to pitch rate and elevator deflection only play a role in determining the zeros from elevator to normal specific acceleration. Under the assumption that the upper bound of equation (55) is satisfied, the zeros effectively move to infinity and correspondingly these two terms become zero. Thus, the simplified normal dynamics above will yield identical approximated poles to those of equation (38), but will display no finite zeros from elevator to normal specific acceleration.

To dynamically invert the effect of the flight path angle coupling on the normal specific acceleration dynamics requires differentiating the output of interest until the control input appears in the same equation. The control can then be used to directly cancel the undesirable terms. Differentiating the normal specific acceleration output of equation (57) once with respect to time yields,

$$
\dot{C}_{W}=\left[-\frac{L_{\alpha}}{m \bar{V}}\right] C_{W}+\left[-\frac{L_{\alpha}}{m}\right] Q+\left[-\frac{L_{\alpha} g \cos \Theta_{W}}{m \bar{V}}\right]
$$

where the angle of attack dynamics of equation (6) have been used in the result above. Differentiating the normal specific acceleration a second time gives,

$$
\begin{aligned}
\ddot{C}_{W} & =\left[\frac{M_{Q}}{I_{y y}}-\frac{L_{\alpha}}{m \bar{V}}\right] \dot{C}_{W}+\left[\frac{M_{\alpha}}{I_{y y}}+\frac{L_{\alpha} M_{Q}}{m \bar{V} I_{y y}}\right] C_{W} \\
& +\left[-\frac{L_{\alpha} M_{\delta_{E}}}{m I_{y y}}\right] \delta_{E}+\left[\frac{L_{0} M_{\alpha}}{m I_{y y}}-\frac{L_{\alpha} M_{0}}{m I_{y y}}\right]+\left[\frac{L_{\alpha} g}{m \bar{V}}\left(\frac{M_{Q}}{I_{y y}} \cos \Theta_{W}+\dot{\Theta}_{W} \sin \Theta_{W}\right)\right]
\end{aligned}
$$

where use has been made of equations (56) to (58) in obtaining the result above. The elevator control input could now be used to cancel the effect of the flight path angle coupling terms on the normal specific acceleration dynamics. However, the output feedback control law to be implemented will make use of pitch rate feedback. Upon analysis of equation (6), it is clear that pitch rate feedback will reintroduce flight path angle coupling terms into the normal specific acceleration dynamics. Thus, the feedback control law is first defined and substituted into the dynamics, and then the dynamic inversion is carried out. A PI control law with enough degrees of freedom to place the closed loop poles arbitrarily and allow for dynamic inversion (through $\delta_{E_{D I}}$ ) is defined below,

$$
\begin{gathered}
\delta_{E}=-K_{Q} Q-K_{C} C_{W}-K_{E} E_{C}+\delta_{E_{D I}} \\
\dot{E}_{C}=C_{W}-C_{W_{R}}
\end{gathered}
$$

with $C_{W_{R}}$ the reference normal specific acceleration command. The integral action of the control law is introduced to ensure that the normal specific acceleration is robustly tracked with zero steady state error. Offset disturbance terms such as those due to static lift and pitching moment can thus be ignored in the design to follow. It is best to remove the effect 
of terms such as these with integral control since they are not typically known to a high degree of accuracy and thus cannot practically be inverted along with the flight path angle coupling. Upon substitution of the control law above into the normal specific acceleration dynamics of equation (59), the closed loop normal dynamics become,

$$
\begin{gathered}
\ddot{C}_{W}=\left[\frac{L_{\alpha} M_{\delta_{E}}}{m I_{y y}} K_{E}\right] E_{C}+\left[\frac{M_{Q}}{I_{y y}}-\frac{L_{\alpha}}{m \bar{V}}-\frac{M_{\delta_{E}}}{I_{y y}} K_{Q}\right] \dot{C}_{W} \\
+\left[\frac{M_{\alpha}}{I_{y y}}+\frac{L_{\alpha} M_{Q}}{m \bar{V} I_{y y}}+\frac{L_{\alpha} M_{\delta_{E}}}{m I_{y y}} K_{C}-\frac{L_{\alpha} M_{\delta_{E}}}{m \bar{V} I_{y y}} K_{Q}\right] C_{W} \\
\dot{E}_{C}=C_{W}-C_{W_{R}}
\end{gathered}
$$

when,

$$
\delta_{E_{D I}}=\frac{g}{\bar{V}}\left[\left(\frac{M_{Q}}{M_{\delta_{E}}}-K_{Q}\right) \cos \Theta_{W}+\left(\frac{I_{y y}}{M_{\delta_{E}}} \dot{\Theta}_{W}\right) \sin \Theta_{W}\right]
$$

and the static offset terms are ignored. Note that the dynamic inversion part of the control law is still a function of the yet to be determined pitch rate feedback gain. Given the desired closed loop characteristic equation for the normal dynamics,

$$
\alpha_{c}(s)=s^{3}+\alpha_{2} s^{2}+\alpha_{1} s+\alpha_{0}
$$

the closed form solution feedback gains can be calculated by matching characteristic equation coefficients to yield,

$$
\begin{gathered}
K_{Q}=\frac{I_{y y}}{M_{\delta_{E}}}\left(\alpha_{2}+\frac{M_{Q}}{I_{y y}}-\frac{L_{\alpha}}{m \bar{V}}\right) \\
K_{C}=-\frac{m I_{y y}}{L_{\alpha} M_{\delta_{E}}}\left(\alpha_{1}+\frac{M_{\alpha}}{I_{y y}}-\frac{L_{\alpha}}{m \bar{V}}\left(\alpha_{2}-\frac{L_{\alpha}}{m \bar{V}}\right)\right) \\
K_{E}=-\frac{m I_{y y}}{L_{\alpha} M_{\delta_{E}}} \alpha_{0}
\end{gathered}
$$

Substituting the pitch rate feedback gain into equation (64) gives,

$$
\delta_{E_{D I}}=\frac{g}{\bar{V}} \frac{I_{y y}}{M_{\delta_{E}}}\left[\left(\frac{L_{\alpha}}{m \bar{V}}-\alpha_{2}\right) \cos \Theta_{W}-\left(\frac{C_{W}+g \cos \Theta_{W}}{\bar{V}}\right) \sin \Theta_{W}\right]
$$

where use has been made of equation (1) to remove the flight path angle derivative. The controller design freedom is reduced to that of placing the three poles that govern the closed loop normal dynamics. The control system will work to keep these poles fixed for all point mass kinematics states and in so doing yield a dynamically invariant normal specific acceleration response at all times. 


\section{Simulation}

To verify the controller designs of the previous subsections, they are applied to an off-theshelf scale model aerobatic aircraft, the 0.90 size CAP232, used for research purposes at Stellenbosch University. In the simulations and analysis to follow, the aircraft is operated about a nominal velocity magnitude of $30 \mathrm{~m} / \mathrm{s}$ and a nominal sea level air density of 1.225 $\mathrm{kg} / \mathrm{m}^{3}$. The modelling parameters for the aircraft are listed in the table below and were obtained from (Hough, 2007).

\begin{tabular}{|c|c|c|c|}
\hline$m=5.0 \mathrm{~kg}$ & $A=5.97$ & $C_{L_{0}}=0.0$ & $C_{m_{0}}=0.0$ \\
\hline$I_{y y}=0.36 \mathrm{kgm}^{2}$ & $\tau_{T}=0.25 \mathrm{~s}$ & $C_{L_{\alpha}}=5.1309$ & $C_{m_{\alpha}}=-0.2954$ \\
\hline$c=0.30 \mathrm{~m}$ & $e=0.85$ & $C_{L_{Q}}=7.7330$ & $C_{m_{Q}}=-10.281$ \\
\hline$S=0.50 \mathrm{~m}^{2}$ & $C_{D_{0}}=0.02$ & $C_{L_{\delta E}}=0.7126$ & $C_{m_{\delta E}}=-1.5852$ \\
\hline
\end{tabular}

Table 1. Model parameters for the Stellenbosch University aerobatic UAV

Given that the scenario described in the example at the end of section 5 applies to the aerobatic UAV in question, the closed loop natural frequency of the axial specific acceleration controller should be greater than or equal to the bandwidth of the thrust actuator (4 rad/s) for a return disturbance of $-20 \mathrm{~dB}$. Selecting the closed loop poles at $\{-4 \pm 3 i\}$, provides a small buffer for uncertainty in the actuator lag, without overstressing the thrust actuator. Figure 4 provides a Bode plot of the actual and approximated return disturbance transfer functions for this design, i.e. equation (23), with the actual and approximated sensitivity functions of equations (33) and (34) substituted respectively. Also plotted are the actual and approximated sensitivity functions themselves as well as the term in parenthesis in equation (23), i.e. the normalized drag to normalized velocity perturbation transfer function. Figure 4 clearly illustrates the greater than $20 \mathrm{~dB}$ of return disturbance rejection obtained over the entire frequency band due to the appropriate selection of the closed loop poles. The figure also shows how the return disturbance rejection is contributed towards by the controller at low frequencies and the natural velocity magnitude dynamics at high frequencies. The plot thus verifies the mathematics of the decoupling analysis done in section 4 .

Open loop analysis of the aircraft's normal dynamics reveals the actual and approximated poles (shown as crosses) in Figure 5 and the actual and approximated elevator to normal specific acceleration zeros of $\{54.7,-46.7\}$ and $\{54.5,-46.6\}$ respectively. The closeness of the poles in Figure 5 and the similarity of the numerical values above verify equations (38) and (40). The approximate zero positions are used in equation (55) to determine the upper NMP frequency bound shown in Figure 5. The lower timescale separation bound arises as a result of a desired velocity magnitude bandwidth of $1 \mathrm{rad} / \mathrm{s}$ (a feasible user selected value). Notice both the large feasible pole placement region and the fact that the open loop poles naturally satisfy the NMP frequency constraint, implying good open loop handling qualities.

The controller of subsection 6.4 is then applied to the system with desired closed loop complex poles selected to have a constant damping ratio of 0.7 as shown in Figure 5. The desired closed loop real pole is selected equal to the real value of the complex poles. The corresponding actual closed loop poles are illustrated in Figure 5. Importantly, the locus of actual closed loop poles is seen to remain similar to that of the desired poles while the upper NMP frequency bound is adhered to. Outside the bound the actual poles are seen to diverge quickly from the desired values. 


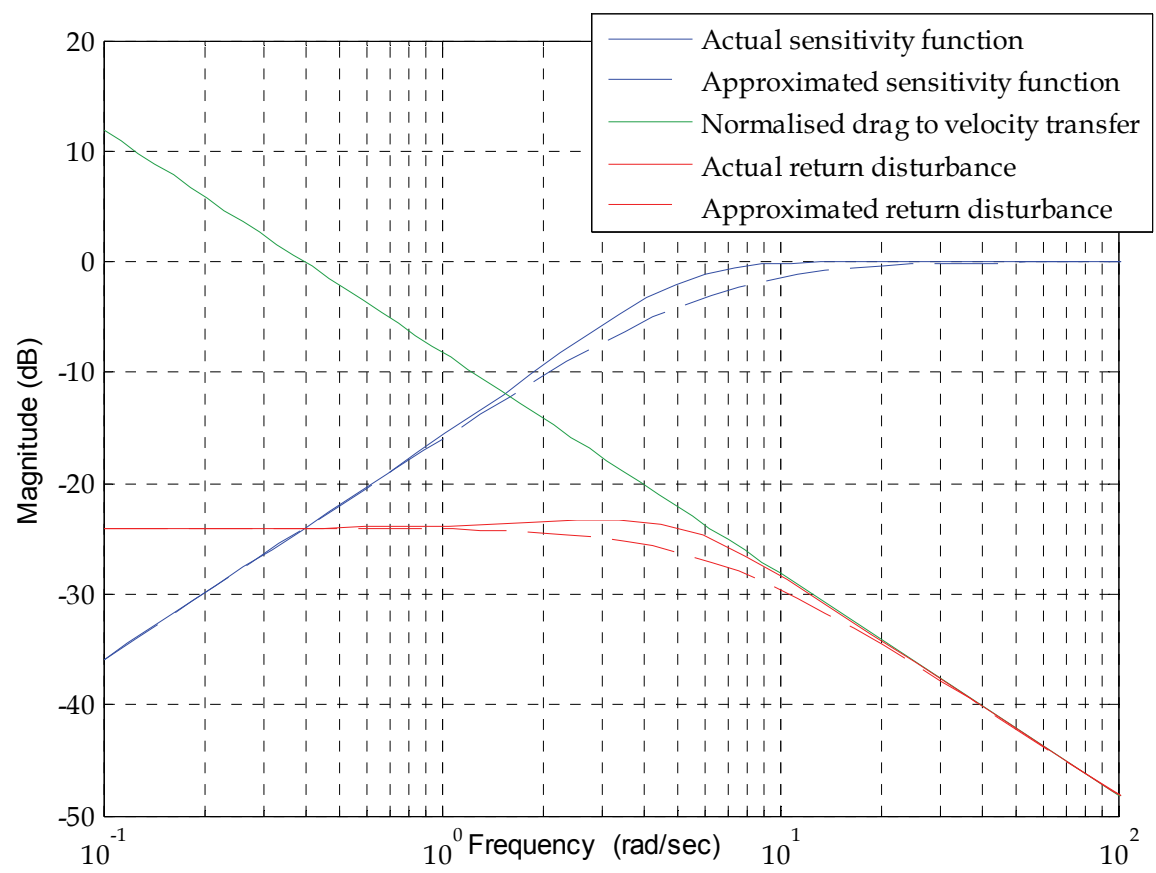

Fig. 4. NMP upper bound and timescale separation lower bound outlining feasible pole placement region.

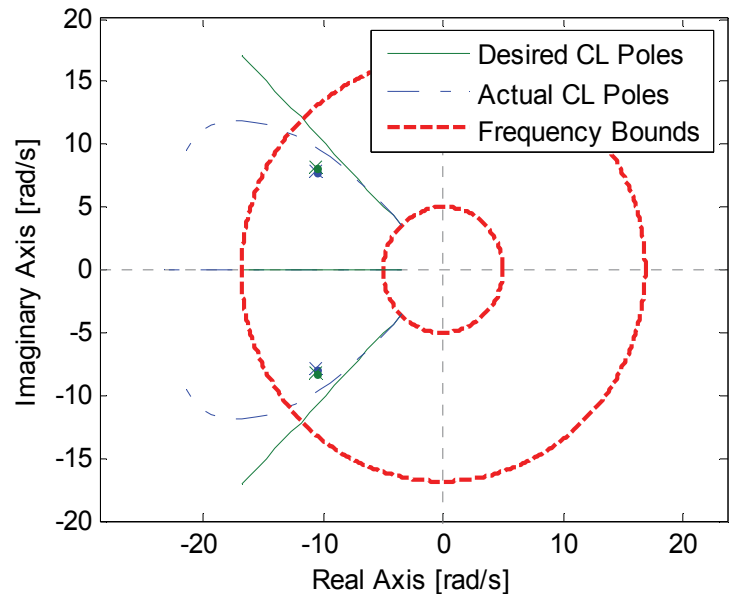

Fig. 5. Actual and approximated open loop (CL) poles, actual and desired closed loop poles and upper and lower frequency bounds.

The controller of subsection 6.4 is then applied to the system with desired closed loop complex poles selected to have a constant damping ratio of 0.7 as shown in Figure 5 . The desired closed loop real pole is selected equal to the real value of the complex poles. The corresponding actual closed loop poles are illustrated in Figure 5. Importantly, the locus of 
actual closed loop poles is seen to remain similar to that of the desired poles while the upper NMP frequency bound is adhered to. Outside the bound the actual poles are seen to diverge quickly from the desired values.

Figure 6 shows the corresponding feedback gains plotted as a function of the RHP zero position normalized to the desired natural frequency $\left(r^{-1}\right)$. The feedback gains are normalized such that their maximum value shown is unity. Again, it is clear from the plot that the feedback gains start to grow very quickly, and consequently start to become impractical, when the RHP zero is less than 3 times the desired natural frequency. The results of Figures 5 and 6 verify the design and analysis of section 6 .

Given the analysis above, the desired normal specific acceleration closed loop poles are selected at $\{-10 \pm 8 \mathrm{i},-10\}$. The desired closed loop natural frequency is selected close to that of the open loop system in an attempt to avoid excessive control effort. With the axial and normal specific acceleration controllers designed, a simulation based on the full, nonlinear dynamics of section 3 was set up to test the controllers. Figure 7 provides the simulation results.

The top two plots on the left hand side of the figure show the commanded (solid black line), actual (solid blue line) and expected/desired (dashed red line) axial and normal specific acceleration signals during the simulation. The normal specific acceleration was switched between -1 and $-2 \quad g$ 's (negative sign implies 'pull up' acceleration) during the simulation while the axial specific acceleration was set to ensure the velocity magnitude remained within acceptable bounds at all times.

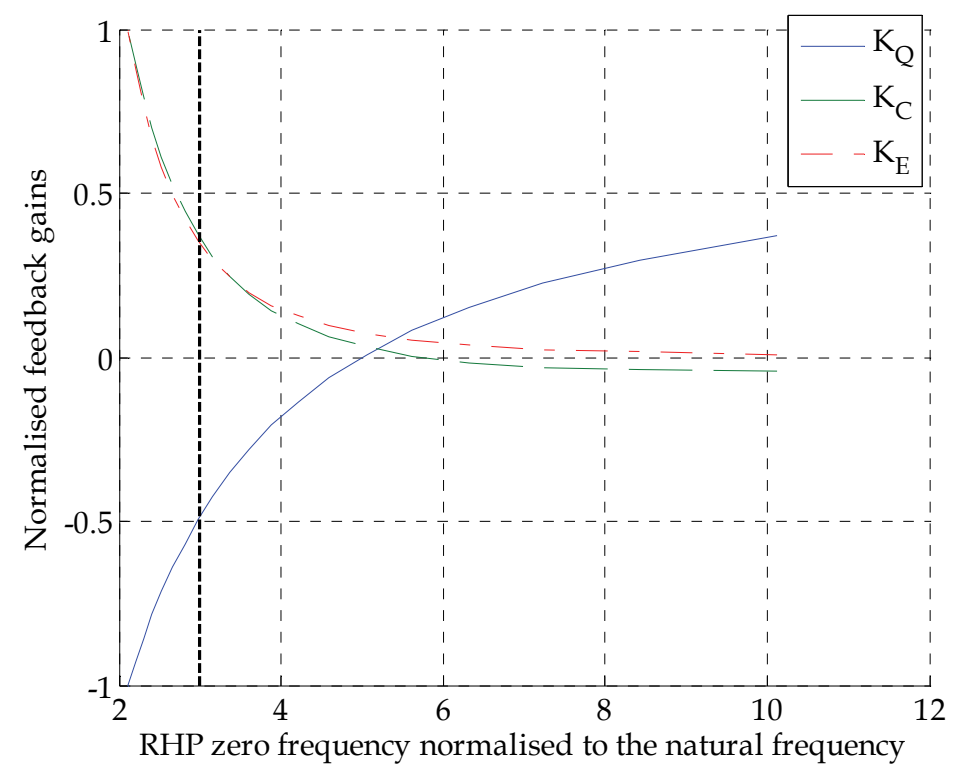

Fig. 6. Normalized controller feedback gains as a function of the RHP zero position normalized to the desired natural frequency.

Importantly, note how the axial and normal specific acceleration remain regulated as expected regardless of the velocity magnitude and flight path angle, the latter of which varies dramatically over the course of the simulation. As desired, the specific acceleration controllers are seen to regulate their respective states independently of the aircraft's velocity 
magnitude and gross attitude. The angle of attack, pitch rate, elevator deflection and normalized thrust command are shown on the right hand side of the figure. The angle of attack remains within pre-stall bounds and the control signals are seen to be practically feasible.

Successful practical results of the controllers operating on the aerobatic research aircraft and other research aircraft at Stellenbosch University have recently been obtained. These results will be made available in future publications.
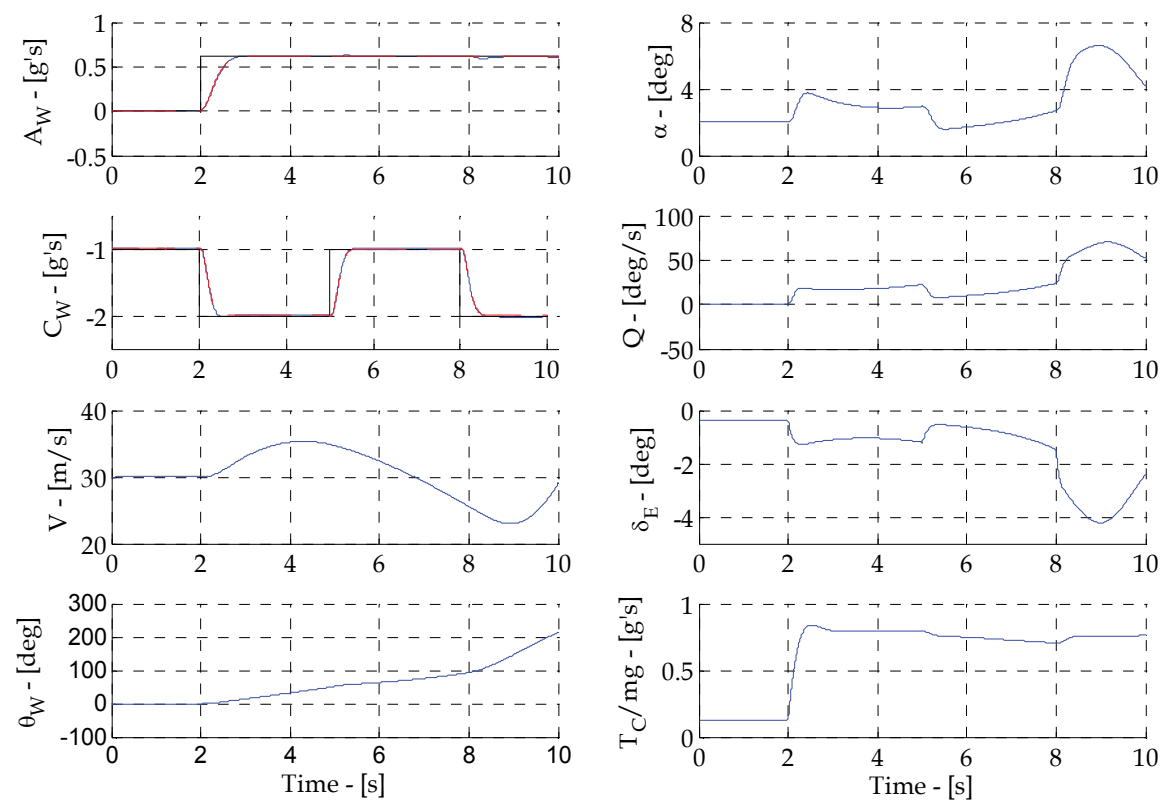

Fig. 7. Simulation results illustrating gross attitude independent regulation of the axial and normal specific acceleration.

\section{Conclusion and future work}

An acceleration based control strategy for the design of a manoeuvre autopilot capable of guiding an aircraft through the full 3D flight envelope was presented. The core of the strategy involved the design of dynamically invariant, gross attitude independent specific acceleration controllers. Adoption of the control strategy was argued to provide a practically feasible, robust and effective solution to the 3D manoeuvre flight control problem and the non-iterative nature of the controllers provides for a computationally efficient solution.

The analysis and design of the specific acceleration controllers for the case where the aircraft's flight was constrained to the $2 \mathrm{D}$ vertical plane was presented in detail. The aircraft dynamics were shown to split into aircraft specific rigid body rotational dynamics and aircraft independent point mass kinematics. With a timescale separation and a dynamic inversion condition in place the rigid body rotational dynamics were shown to be dynamically independent of the point mass kinematics, and so provided a mathematical foundation for the design of the gross attitude independent specific acceleration controllers. Under further mild conditions and a sensitivity function constraint the rigid body rotational 
dynamics were shown to be linear and decouple into axial and normal dynamics. The normal dynamics were seen to correspond to the classical Short Period mode approximation dynamics and illustrated the gross attitude independent nature of this mode of motion.

Feedback based, closed form pole placement control solutions were derived to regulate both the axial and normal specific accelerations with invariant dynamic responses. Before commencing with the design of the normal specific acceleration controller, the elevator to normal specific acceleration dynamics were investigated in detail. Analysis of these dynamics yielded a novel approximating equation for the location of the zeros and revealed the typically NMP nature of this system. Based on a time domain analysis a novel upper frequency bound condition was developed to allow the NMP nature of the system to be ignored, thus allowing practically feasible dynamic inversion of the flight path angle coupling.

Analysis and simulation results using example data verified the functionality of the specific acceleration controllers and validated the assumptions upon which their designs were based. Future research will involve extending the detailed control system design to the full 3D flight envelope case based on the autopilot design strategy presented in section 2 . Intelligent selection of the closed loop poles will also be the subject of further research. Possibilities include placing the closed loop poles for maximum robustness to parameter uncertainty as well as scheduling the closed loop poles with flight condition to avoid violation of the NMP frequency bound constraint.

\section{References}

Al-Hiddabi, S.A. \& McClamroch, N.H. (2002). Aggressive longitudinal aircraft trajectory tracking using nonlinear control. Journal of Guidance, Control and Dynamics, Vol. 25, No. 1, January 2002, pp. 26-32, ISSN 07315090.

Bhattacharya, R.; Balas, G.J.; Kaya, M.A. \& Packard, A (2002). Nonlinear receding horizon control of an F-16 aircraft. Journal of Guidance, Control and Dynamics, Vol. 25, No. 5, September 2002, pp. 924-931, ISSN 07315090.

Blakelock, J.H. (1991). Automatic Control of Aircraft and Missiles (2nd Ed.), John Wiley and Sons, ISBN 0471506516, New York.

Buffington, J.; Adams, R. \& Banda, S. (1993). Robust, nonlinear, high angle-of-attack control design for a super-manoeuvrable vehicle, Proceedings of the AIAA Guidance, Navigation and Control Conference, pp. 690-700, Monterey, August 1993.

Bugajski, D.J. \& Enns, D.F. (1992). Nonlinear control law with application to high angle-ofattack flight. Journal of Guidance, Control and Dynamics, Vol. 15, No. 3, May 1992, pp. 761-767, ISSN 07315090.

Etkin, B. (1972). Dynamics of Atmospheric Flight, John Wiley and Sons, ISBN-10 0471246204 , New York.

Etkin, B. \& Reid, L.D. (1995). Dynamics of Flight Stability and Control (3rd Ed.), John Wiley and Sons, ISBN 0471034185, New York.

Freudenberg, J.S. \& Looze, D.P. (1985). Right-half plane poles and zeros and design tradeoffs in feedback systems. IEEE Transactions on Automatic Control, Vol. 30, No. 6, June 1985, pp. 555-565, ISSN 00189286.

Goodwin, G.C.; Graebe, S.F. \& Salgado, M.E. (2001). Control System Design, Prentice Hall, ISBN-10 0139586539, Upper Saddle River. 
Hauser, J.; Sastry, S. \& Meyer, G. (1992). Nonlinear control design for slightly non-minimum phase systems: Application to V/STOL aircraft. Automatica, Vol. 28, No. 4, April 1992, pp. 665-679, ISSN 00051098.

Hough, W.J. (2007). Autonomous Aerobatic Flight of a Fixed Wing Unmanned Aerial Vehicle, Masters thesis, Stellenbosch University.

Kim, M.J.; Kwon, W.H.; Kim, Y.H. \& Song, C. (1997). Autopilot design for bank-to-turn missiles using receding horizon predictive control scheme. Journal of Guidance, Control and Dynamics, Vol. 20, No. 6, November 1997, pp. 1248-1254, ISSN 07315090.

Lane, S.H. \& Stengel, R.F. (1988). Flight control design using non-linear inverse dynamics. Automatica, Vol. 24, No. 4, April 1988, pp. 471-483, ISSN 00051098.

Leith, D.J. \& Leithead, W.E. (2000). Survey of gain scheduling analysis \& design, International Journal of Control, Vol. 73, No. 11, July 2000, pp. 1001-1025, ISSN 00207179.

Miller, R.B. \& Pachter, M. (1997). Manoeuvring flight control with actuator constraints. Journal of Guidance, Control and Dynamics, Vol. 20, No. 4, July 1997, pp. 729-734, ISSN 07315090.

Pachter, M.; Chandler P.R. \& Smith, L. (1998). Manoeuvring flight control. Journal of Guidance, Control and Dynamics, Vol. 21, No. 3, May 1998, pp. 368-374, ISSN 07315090 .

Peddle, I.K. (2008). Acceleration Based Manoeuvre Flight Control System for Unmanned Aerial Vehicles, PhD thesis, Stellenbosch University.

Reiner, J.; Balas, G.J. \& Garrard, W.L. (1996). Flight control design using robust dynamic inversion and time-scale separation. Automatica, Vol. 32, No. 11, November 1996, pp. 1493-1504, ISSN 00051098.

Slotine, J.E. \& Li, W. (1991). Applied Nonlinear Control, Prentice Hall, ISBN-10 0130408905 , Upper Saddle River.

Snell, S.A.; Enns, D.F. \& Garrard, W.L. (1992). Nonlinear inversion flight control for a supermanoeuvrable aircraft. Journal of Guidance, Control and Dynamics, Vol. 15, No. 4, July 1992, pp. 976-984, ISSN 07315090. 


\title{
Autonomous Flight Control System for Longitudinal Motion of a Helicopter
}

\author{
Atsushi Fujimori \\ University of Yamanashi \\ Japan
}

\section{Introduction}

Recently, unmanned aerial vehicles (UAVs) have been developed for the purposes of scientific observations, detecting disasters, surveillance of traffic and army objectives (Wilson, 2007; Langelaan \& Rock, 2005; Cho et al., 2005). This paper presents an autonomous flight control design to give insights for developing helicopter-type UAVs.

A Helicopter is generally an unstable aircraft. Once it is stalled, it is not easy to recover its attitude. A control system is therefore needed to keep the vehicle stable during flight (Bramwell, 1976; Padfield, 1996; Johnson \& Kannan, 2005). This paper presents a flight control design for the longitudinal motion of helicopter to establish autopilot techniques of helicopters. The flight mission considered in this paper is that a helicopter hovers at a start position, moves to a goal position with keeping a specified cruise velocity and hovers again at the goal. The characteristics of the linearized equation of the helicopter is changed during this flight mission because the trim values of the equation are widely varied. Gain scheduling (GS) is one of candidates to stabilize the vehicle for the entire flight region. In this paper, a flight control system is designed as follows. The flight control system is constructed as a double loop control system (Fujimori et al., 1999; Fujimori et al., 2002) which consists of an inner-loop controller and an outer-loop controller. The former is needed for stabilizing the controlled plant, while the latter is used for tracking the reference which is given to accomplish the flight mission. To design the inner-loop controller, the longitudinal motion of a helicopter is first modeled by a linear interpolative polytopic model whose varying parameter is the flight velocity. A GS state feedback law is then designed by linear matrix inequality (LMI) (Boyd et al., 1994; Fujimori et al., 2007) so as to stabilize the polytopic model for the entire flight region. On the other hand, the outer-loop controller is designed by taking into consideration the steady-state of the controlled variable.

The rest of this paper is organized as follows. Section 2 shows equations of the longitudinal motion of helicopter. Section 3 gives a flight mission and shows a double loop control system adopted in this paper. The details of the controller designs are presented in Section 4. Section 5 shows computer simulation in Matlab/Simulink to evaluate the proposed flight control system. Concluding remarks are given in Section 6. 


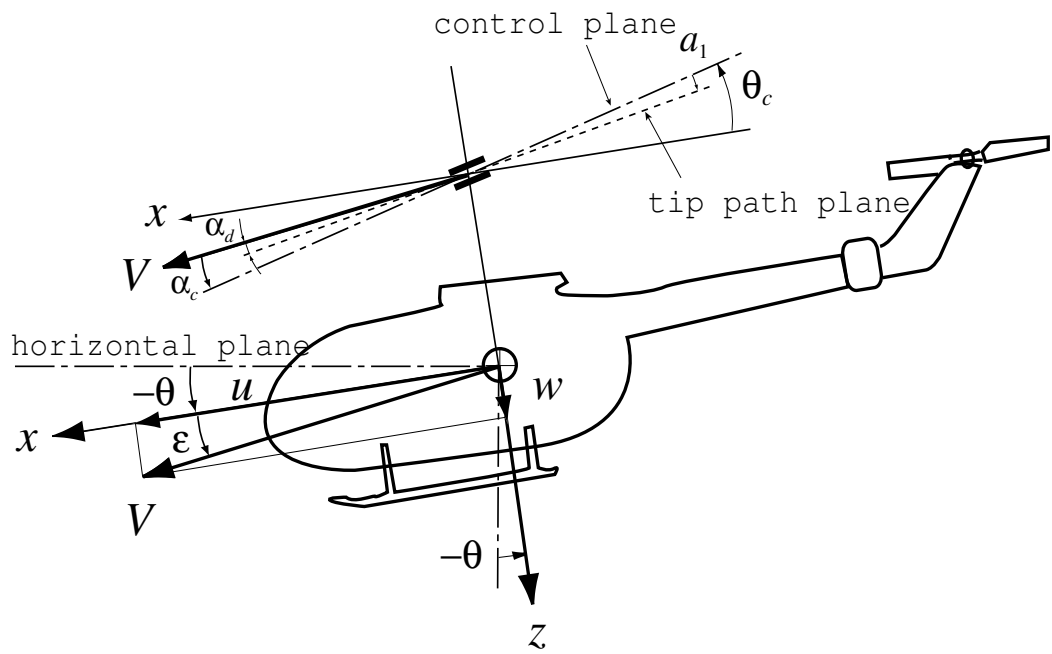

Fig. 1. Helicopter in forward flight

\section{Equation of longitudinal motion of helicopter}

Figure 1 shows a helicopter considered in this paper. The angular velocity of the main rotor is $\Omega$. The main rotor produces the thrust $T$ which is needed for not only lifting the vehicle against the gravity but also moving transitionally and rotationally. It depends on the tilting angle of the control plane.

$(x, y, z)$ represent the body-fixed-axes whose origin is located at the center of gravity of the vehicle. The forward velocity is $V$ whose $x$ - and $z$-axes elements are $u$ and $w$, respectively. The longitudinal motion of helicopter consists of the transitional motion with respect to $x$ and $z$-axes and the rotational motion around $y$-axis; that is, the pitch angle denoted as $\theta$ and its derivative $q(=\dot{\theta})$. It is represented as the following equations (Bramwell, 1976; Padfield, 1996):

$$
\begin{gathered}
m(\dot{u}+q w)=X-m g \sin \theta \\
m(\dot{w}-q u)=Z+m g \cos \theta \\
I_{y y} \dot{q}=M
\end{gathered}
$$

where $m$ and $I_{y y}$ are respectively the mass and the moment of inertia of the vehicle. $g$ is the gravity acceleration. The external forces $X, Z$ and the moment $M$ are given by

$$
\begin{gathered}
X=T \sin \left(\theta_{c}-a_{1}\right)-D \cos \varepsilon \\
Z=-T \cos \left(\theta_{c}-a_{1}\right)-D \sin \varepsilon \\
M=-T h_{R} \sin \left(\theta_{c}-a_{1}\right)
\end{gathered}
$$

where $\varepsilon$ is defined as $\varepsilon \tan . \theta_{c}$ is the cyclic pitch angle which is one of the control inputs for the longitudinal motion of the helicopter. $a_{1}$ is the angle between the control plane and the tip path plane. $h_{R}$ is the distance of the hub from the center of gravity. $D$ is the drag of the vehicle and is given by

$$
D=\frac{1}{2} \rho V^{2} S C_{D}
$$


where $\rho$ is the atmospheric density, $S$ the representative area and $C_{D}$ the drag coefficient. The thrust $T$ can be calculated by integrating the lift over the whole blade. This results in the following expression for the thrust coefficient:

$$
C_{T} \triangleq \frac{T}{\rho(\Omega R)^{2} \pi R^{2}}=\frac{N c}{4 \pi R} C_{l \alpha}\left\{\left(\frac{2}{3}+\mu^{2}\right) \theta_{0}-\lambda_{c}-\lambda_{i}\right\}
$$

where

$$
\begin{aligned}
\mu \triangleq \frac{V}{\Omega R} \cos \alpha_{c}, & \lambda_{c} \triangleq \frac{V}{\Omega R} \sin \alpha_{c}, \quad \lambda_{i} \triangleq \frac{v_{i}}{\Omega R} \\
\alpha_{c} \triangleq \theta_{c}-\varepsilon &
\end{aligned}
$$

$R$ is the radius of the rotor blades, $c$ the chord length, $N$ the number of the blades and $C_{l \alpha}$ the lift slope of the blades. $v_{i}$ is the induced velocity through the rotor. $\theta_{0}$ is the collective pitch angle which is another control input. According to Van Hoydonck (2003), the dimensionless induced velocity $\lambda_{i}$ through the rotor is approximated by

$$
\tau \dot{\lambda}_{i}=C_{T}-C_{T_{G l}}
$$

where $C_{T_{G l}}$ is the thrust coefficient which is given by Glauert's hypotheses. $\tau$ is a time constant of $\lambda_{i}$.

Summarizing the above equations, define the state and the input vectors as

$$
x_{p} \triangleq\left[\begin{array}{lllll}
u & w & q & \theta & \lambda_{i}
\end{array}\right]^{T} \in \Re^{5}, \quad u_{p} \triangleq\left[\begin{array}{ll}
\theta_{0} & \theta_{c}
\end{array}\right]^{T} \in \Re^{2} .
$$

The equation of the longitudinal motion of the helicopter is then written as

$$
\dot{x}_{p}=f_{p}\left(x_{p}, u_{p}\right) \text {. }
$$

Equation (13) is referred as the nonlinear plant $P_{n l}$ hereafter.

Letting $x_{e}$ and $h_{e}$ be horizontal and the vertical positions of the helicopter from the start, they are given by

$$
\begin{aligned}
& \dot{x}_{e}=u \cos \theta+w \sin \theta \\
& \dot{h}_{e}=u \sin \theta-w \cos \theta .
\end{aligned}
$$

Defining $\xi_{p}$ as $\xi_{p} \triangleq\left[\begin{array}{ll}x_{e} & h_{e}\end{array}\right]^{T}$, they are compactly given as

$$
\dot{\xi}_{p}=g_{p}\left(x_{p}\right) \text {. }
$$

In this paper, numerical values of the Eurocopter Deutschland Bo105 (Padfield, 1996) were used in simulation. They are listed in Table 1. Since this paper considers hovering and forward flight, the trim condition is given in level flight. Letting $\bar{x}_{p}$ and $\bar{u}_{p}$ be the state and the input in trim, respectively, $f\left(\bar{x}_{p}, \bar{u}_{p}\right)=0$ holds. Figure 2 shows variations of $\bar{x}_{p}$ and $\bar{u}_{p}$ with respect to the flight velocity $V$. It is seen that all trim values are changed in the range of $V \in[0,60]$ $[\mathrm{m} / \mathrm{s}]$. 

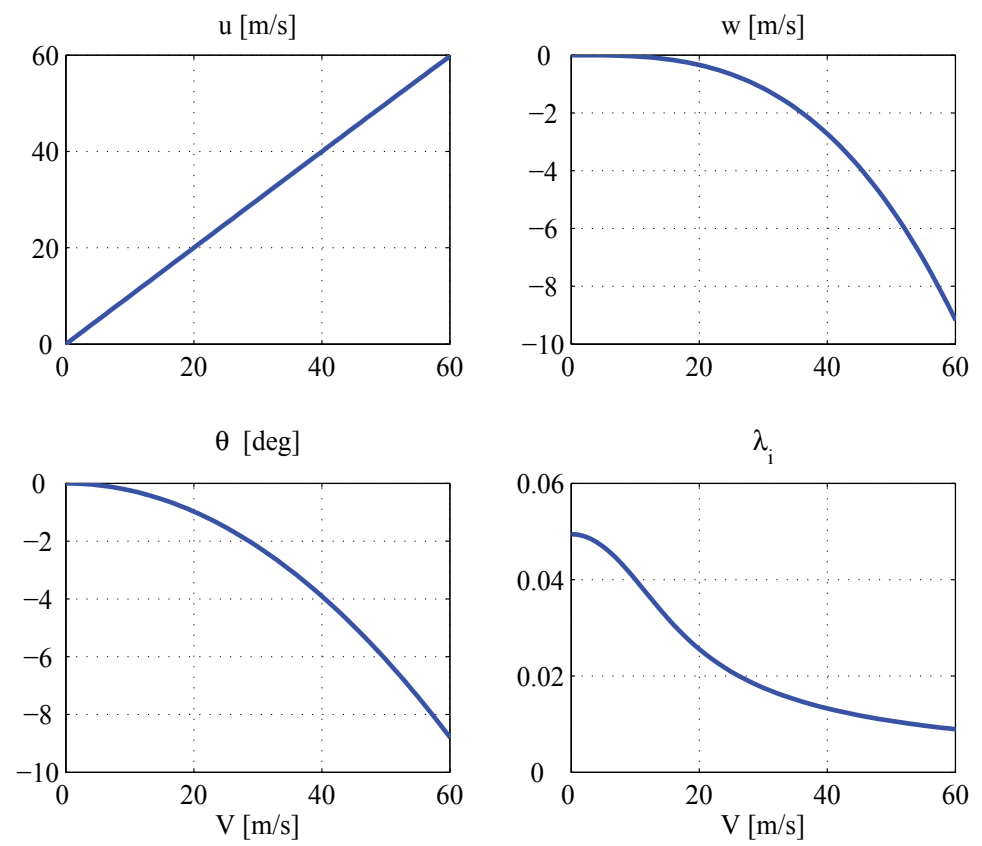

(a) States in trim $\bar{x}_{p}$
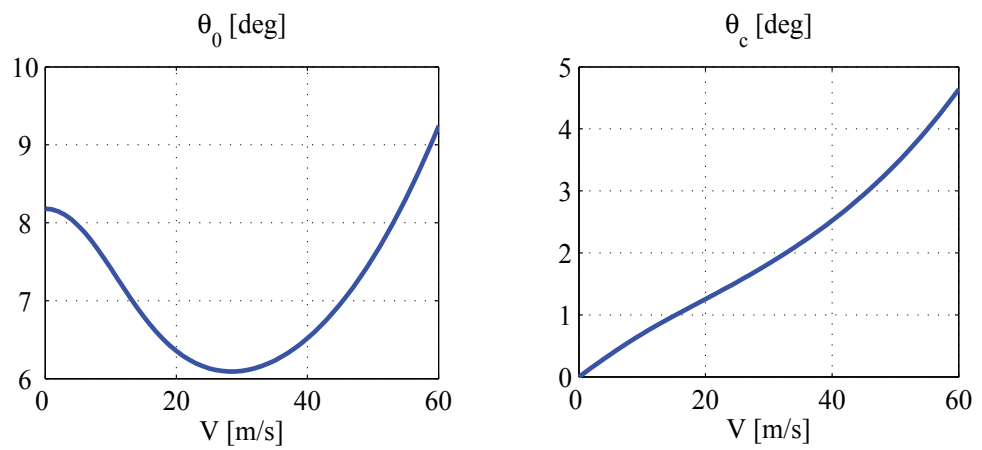

(b) Inputs in trim $\bar{u}_{p}$

Fig. 2. Trim values with respect to forward velocity $V$ 


\begin{tabular}{ccc}
\hline parameter & value & unit \\
\hline$C_{l \alpha}$ & 6.113 & {$[1 / \mathrm{rad}]$} \\
$c$ & 0.27 & {$[\mathrm{~m}]$} \\
$m$ & 2200 & {$[\mathrm{~kg}]$} \\
$I_{b l}$ & 231.7 & {$\left[\mathrm{kgm}^{2}\right]$} \\
$I_{y y}$ & 4973.0 & {$\left[\mathrm{kgm}^{2}\right]$} \\
$R$ & 4.91 & {$[\mathrm{~m}]$} \\
$\Omega$ & 44.4 & {$[\mathrm{rad} / \mathrm{s}]$} \\
$N$ & 4 & {$[-]$} \\
$C_{D} S$ & 1.5 & {$\left[\mathrm{~m}^{2}\right]$} \\
$h_{R}$ & 1.48 & {$[\mathrm{~m}]$} \\
\hline
\end{tabular}

Table 1. Parameters of Bo105 (Padfield, 1996)

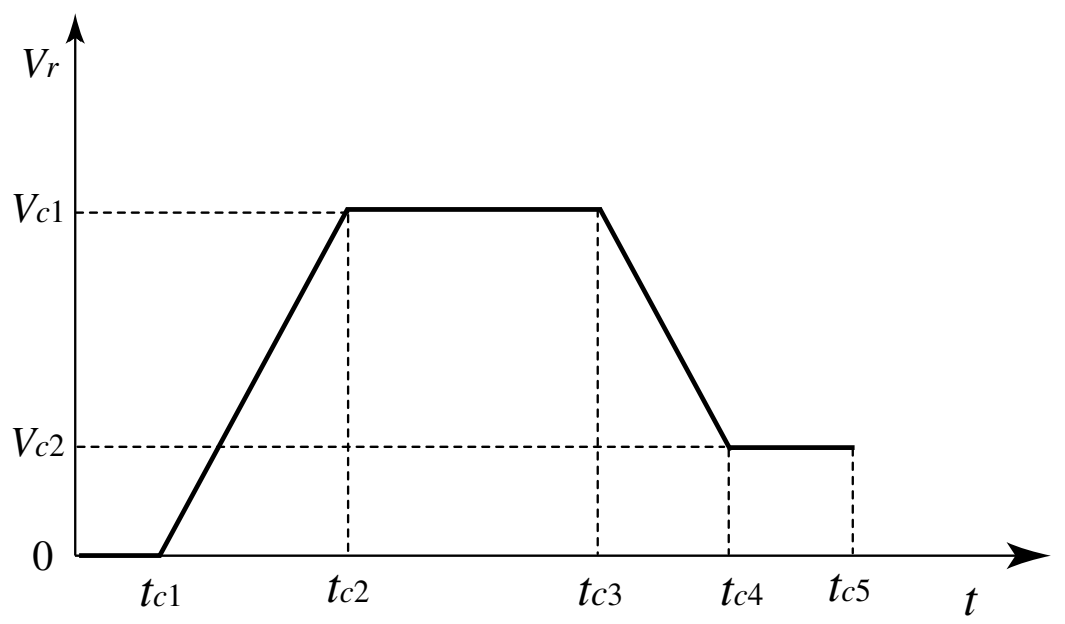

Fig. 3. Flight velocity profile $V_{r}$

\section{Construction of flight control system}

Let the start position be the origin of the coordinates $\left(x_{e}, h_{e}\right)$. A flight mission considered in this paper is to navigate the helicopter from the start $(0,0)$ to the goal, denoted as $\left(x_{r}, h_{r}\right)$, with keeping its attitude stable. To design a control system, the followings are assumed to be satisfied:

(i) The motion in $y$-axis direction is not taken into account.

(ii) $x_{p}$ is measurable.

(iii) The trim values $\bar{x}_{p}$ and $\bar{u}_{p}$ are known in advance.

To realize the flight mission, this paper constructs a tracking control system whose controlled variable is the flight velocity. The flight region is divided into six phases with respect to the flight velocity as shown in Fig. 3. They are referred as follows. 


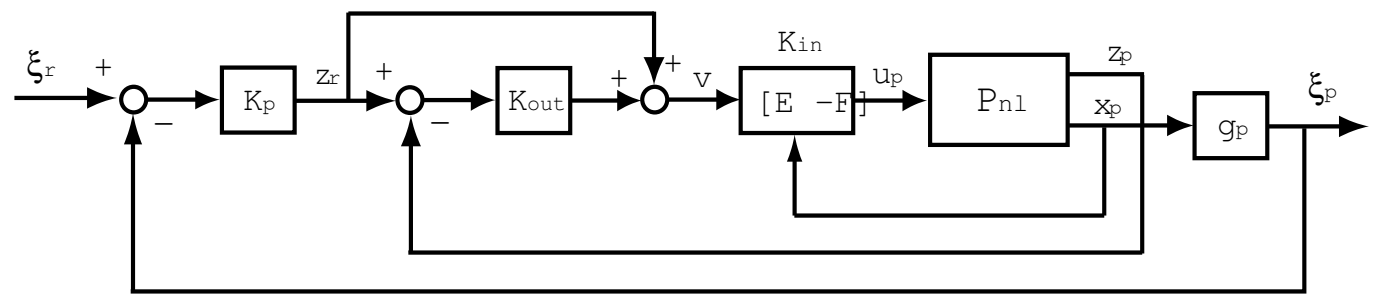

Fig. 4. Flight control system

$$
\begin{aligned}
& 0 \leq t<t_{c 1}: \text { initial hovering phase } \\
& t_{c 1} \leq t<t_{c 2}: \text { acceleration phase } \\
& t_{c 2} \leq t<t_{c 3}: \text { cruise phase } \\
& t_{c 3} \leq t<t_{c 4}: \text { deceleration phase } \\
& t_{c 4} \leq t<t_{c 5}: \text { low speed phase } \\
& t_{c 5} \leq t: \quad \text { approach phase }
\end{aligned}
$$

From the initial hovering phase to the low speed phase, the reference of the flight velocity is given by $V_{r}$ shown in Fig. 3. In this paper, the total time of the flight is not cared. But the integrated value of $V_{r}$ for $t \in\left[0, t_{c 5}\right]$ must be less than $x_{r}$ not to overtake the goal before the approach phase. In the approach phase, the reference is generated to meet the position of the helicopter $\xi_{p}=\left[\begin{array}{ll}x_{e} & h_{e}\end{array}\right]^{T}$ with the goal $\xi_{r}=\left[\begin{array}{ll}x_{r} & h_{r}\end{array}\right]^{T}$.

Taking into consideration the above, a double loop control system (Fujimori et al., 1999; Fujimori et al., 2002) is used as a flight control system in this paper. It is shown in Fig. 4. $P_{n l}$ represents the nonlinear helicopter dynamics given by Eq. (13), $K_{i n}$ is the inner-loop controller, $K_{\text {out }}$ is the outer-loop controller and $K_{p}$ is a gain. The controlled variable from the initial hovering phase to the low speed phase is given by $z_{p} \triangleq[u w]^{T}$ and its reference is given by $z_{r} \triangleq\left[u_{r} w_{r}\right]^{T}$. In the approach phase, another loop is added outside of $\left(z_{r}-z_{p}\right)$-loop, where $\xi_{p} \triangleq\left[x_{e} h_{e}\right]^{T}$ is the controlled variable and $\xi_{r} \triangleq\left[x_{r} h_{r}\right]^{T}$ is its reference. $K_{\text {in }}$ consists of

$$
K_{\text {in }}=[E-F]
$$

where $E$ is a feedforward gain for tracking the reference, while $F$ is a feedback gain for stabilizing the plant. Since the trim values are widely varied as shown in Fig. 2, the characteristics of the linearized plant is also varied. Then, $F$ is designed by a GS technique in terms of LMI formulation (Boyd et al., 1994).

The reference $z_{r}$ from the initial hovering phase to the low speed phase is generated by the flight velocity profile shown in Fig. 3, and $z_{r}$ in the approach phase is derived from the positional error $\xi_{r}-\xi_{p}$. The switch of the reference is done at $t=t_{c 5}$.

\section{Design of control system}

\subsection{Linear interpolative polytopic model}

The objective of flight control in this paper is that the controlled variable is regulated to the specified trim condition. Linearized models along with the trim is therefore used for controller design. Letting $\bar{x}_{p}(V), \bar{u}_{p}(V)$ be respectively the state and the input in trim where the flight 
velocity is $V$, the perturbed state and the input are defined as

$$
\delta x_{p}(t) \triangleq x_{p}(t)-\bar{x}_{p}(V), \quad \delta u_{p}(t) \triangleq u_{p}(t)-\bar{u}_{p}(V)
$$

The linearized equation of Eq. (13) is then given as

$$
\delta \dot{x}_{p}(t)=A_{p}(V) \delta x_{p}(t)+B_{p}(V) \delta u_{p}(t)
$$

where

$$
A_{p}(V) \triangleq \frac{\partial f_{p}\left(\bar{x}_{p}, \bar{u}_{p}\right)}{\partial x_{p}^{T}}, \quad B_{p}(V) \triangleq \frac{\partial f_{p}\left(\bar{x}_{p}, \bar{u}_{p}\right)}{\partial u_{p}^{T}} .
$$

Although matrices $A_{p}$ and $B_{p}$ are functions with respect to $V$, it is hard to get their explicit representations because of complicated dependence of $V$ as described in Section 2. Then, $A_{p}$ and $B_{p}$ are approximated by interpolating multiple linearized models in the trim condition. For the range of the flight velocity $V \in\left[0, V_{u}\right], r$ points $\left\{V_{1}, \cdots, V_{r}\right\}$, called the operating points, are chosen as

$$
0 \leq V_{1}<\cdots<V_{r} \leq V_{u} .
$$

The linearized model for $V=V_{i}$ is a local LTI model representing the plant near the $i$-th operating point. Linearly interpolating them, a global model over the entire range of the flight velocity is constructed as

$$
\left\{\begin{array}{l}
\delta \dot{x}_{p}(t)=A_{p}(V) \delta x_{p}(t)+B_{p}(V) \delta u_{p}(t) \\
z_{p}(t)=C_{p} \delta x_{p}(t)+\bar{z}_{p}(V)
\end{array}\right.
$$

where

$$
\begin{aligned}
& A_{p}(V)=\sum_{i=1}^{r} \mu_{i}(V) A_{p i}, \quad B_{p}(V)=\sum_{i=1}^{r} \mu_{i}(V) B_{p i}, \\
& C_{p}=\left[\begin{array}{lllll}
1 & 0 & 0 & 0 & 0 \\
0 & 1 & 0 & 0 & 0
\end{array}\right] .
\end{aligned}
$$

$\mu_{i}(V)$ satisfies the following relations.

$$
\begin{gathered}
0 \leq \mu_{i}(V) \leq 1 \quad(i=1, \cdots, r) \\
\sum_{i=1}^{r} \mu_{i}(V)=1
\end{gathered}
$$

Equation (22) with Eq. (23) is called the linear interpolative polytopic model in this paper.

\subsection{Design of $K_{\text {in }}$}

Under assumption (ii), consider a state feedback law

$$
\delta u_{p}(t)=-F(V) \delta x_{p}(t)+E(V) v(t)
$$


where $v$ is a feedforward input for tracking $z_{r}$ and is given by $v=z_{r}-\bar{z}_{p}$ when designing $K_{i n}$. The closed-loop system combining Eq. (26) with Eq. (22) is given by

$$
\begin{aligned}
& \left\{\begin{array}{l}
\delta \dot{x}_{p}(t)=A_{F}(V) \delta x_{p}(t)+B_{p}(V) E(V) v(t) \\
z_{p}(t)=C_{p}(V) \delta x_{p}(t)+D_{p}(V) E(V) v(t)+\bar{z}_{p}(V)
\end{array}\right. \\
& A_{F}(V) \triangleq A_{p}(V)-B_{p}(V) F(V) \text {. }
\end{aligned}
$$

The steady-state controlled variable is given by

$$
z_{p}(\infty)=-C_{p} A_{F}^{-1} B_{p} E v+\bar{z}_{p} .
$$

$v$ is then given so as to meet $z_{p}(\infty)$ with the reference $z_{r}$; that is, $z_{p}(\infty) \rightarrow z_{r}$. E is designed as

$$
E=-\left(C_{p} A_{F}^{-1} B_{p}\right)^{-1} .
$$

Next, $F(V)$ is designed so that the closed-loop system is stable over the entire flight range and $\mathcal{H}_{2}$ cost is globally suppressed (Fujimori et al., 2007). The controlled plant is newly given by

$$
\left\{\begin{array}{l}
\delta \dot{x}_{p}(t)=A_{p}(V) \delta x_{p}(t)+B_{p}(V) \delta u_{p}(t)+B_{1}(V) w_{1}(t) \\
z_{1}(t)=C_{1}(V) \delta x_{p}(t)+D_{1}(V) \delta u_{p}(t)
\end{array}\right.
$$

where $z_{1}$ and $w_{1}$ are respectively the input and the output variable for evaluating $\mathcal{H}_{2}$ cost. $B_{1}(V), C_{1}(V)$ and $D_{1}(V)$ are matrices corresponding to $z_{1}$ and $w_{1}$. Substituting Eq. (26) without $v$ into Eq. (30), the closed-loop system is

$$
\begin{gathered}
\left\{\begin{array}{c}
\delta \dot{x}_{p}(t)=A_{F}(V) \delta x_{p}(t)+B_{1}(V) w_{1}(t) \\
z_{1}(t)=C_{1 F}(V) \delta x_{p}(t)
\end{array}\right. \\
C_{1 F}(V) \triangleq C_{1}(V)-D_{1}(V) F(V) .
\end{gathered}
$$

In this paper, $F(V)$ is designed so as to minimize the integration of $\mathcal{H}_{2}$ cost over $V \in\left[0, V_{u}\right]$. That is, the objective is to find $F(V)$ such that (Boyd et al., 1994).

$$
\begin{gathered}
\inf _{F, P, W} \int_{0}^{V_{u}} \operatorname{tr} W(V) d V \text { subject to } \\
{\left[\begin{array}{cc}
P(V) P(V) B_{1}(V) \\
(\star) & W(V)
\end{array}\right]>0,} \\
{\left[\begin{array}{cr}
\operatorname{He}\left(P(V) A_{F}(V)\right)+\dot{V} \frac{d P}{d V} & (\star) \\
C_{1 F}(V) & -I_{q}
\end{array}\right]<0}
\end{gathered}
$$

$\mathrm{He}(A)$ is defined as $\mathrm{He}(A) \triangleq A+A^{T}$ where $(\star)$ means the transpose of the element located at the diagonal position. $P(V)>0$ is the parameter dependent Lyapunov variable. To derive finite number of inequalities from Eq. (32), the following procedures are performed. First define new variables $X(V) \triangleq P^{-1}(V)$ and $M(V) \triangleq F(V) X(V) . A_{p}(V)$ and $B_{p}(V)$ are given 
by the polytopic forms Eq. (23). $B_{1}(V), C_{1}(V), D_{1}(V), X(V), M(V)$ and $W(V)$ are also given by similar polytopic forms. The range of $\dot{V} \triangleq a_{V}$ is given as $a_{V} \in\left[\underline{a_{V}}, \overline{a_{V}}\right]$. Furthermore, $d P / d V$ are approximated as

$$
\begin{gathered}
\frac{d P}{d V}=-X^{-1} \frac{d X}{d V} X^{-1} \\
\frac{d X}{d V} \simeq \frac{X_{i+1}-X_{i}}{V_{i+1}-V_{i}} \triangleq \frac{\Delta X_{i}}{\Delta V_{i}}
\end{gathered}
$$

Pre- and post-multiplying $\operatorname{diag}\{X, I\}$ to Eq. (32) and using the polytopic forms, the following LMIs are derived as a sufficient condition.

$$
\begin{gathered}
\inf _{M_{i}, X_{i}, W_{i}} \sum_{i=1}^{r-1} \operatorname{tr}\left(W_{i} \Delta V_{i}\right) \quad \text { subject to } \\
{\left[\begin{array}{cc}
X_{i} & B_{1 i} \\
(\star) & W_{i}
\end{array}\right]>0 \quad(i=1, \cdots, r),} \\
{\left[\begin{array}{cc}
\operatorname{He}\left(A_{p i} X_{i}-B_{p i} M_{i}\right)-a_{V} \frac{\Delta X_{i}}{\Delta V_{i}} & (\star) \\
C_{1 i} X_{i} D_{1 i} M_{i} & -I_{q}
\end{array}\right]<0,} \\
{\left[\begin{array}{cc}
\operatorname{He}\left(A_{p j} X_{j}-B_{p j} M_{j}\right)-a_{V} \frac{\Delta X_{i}}{\Delta V_{i}} & (\star) \\
C_{1 j} X_{j} D_{1 j} M_{j} & -I_{q}
\end{array}\right]<0,} \\
{\left[\begin{array}{cc}
\operatorname{He}\left(A_{p i} X_{j}-B_{p i} M_{j}\right. & (\star) \\
\left.+A_{p j} X_{i}-B_{p j} M_{i}\right)-2 a_{V} \frac{\Delta X_{i}}{\Delta V_{i}} & \\
C_{1 i} X_{j}-D_{1 i} M_{j} & -2 I_{q} \\
+C_{1 j} X_{i}-D_{1 j} M_{i} &
\end{array}\right]<0} \\
(i=1, \cdots, r-1, j=i+1), a_{V}=\underline{a_{V}}, \overline{a_{V}}
\end{gathered}
$$

If $M_{i}, X_{i}$ and $W_{i}(i=1, \cdots, r)$ satisfying the above LMIs, $F(V)$ is given by

$$
F(V)=\left(\sum_{i=1}^{r} \mu_{i}(V) M_{i}\right)\left(\sum_{i=1}^{r} \mu_{i}(V) X_{i}\right)^{-1} .
$$

\subsection{Design of $K_{\text {out }}$}

Since $v$ in Eq. (26) is a feedforward input from the flight velocity reference, the tracking error will be occurred by model uncertainties and/or disturbances. Let us evaluate this in the LTI representation. Let $T_{z_{p} v}(s)$ be a transfer function from $v$ to $z_{p} . z_{p}$ converges to a constant $z_{r}$ if there are no model uncertainties in $T_{z_{p} v}(s)$ because $T_{z_{p} v}(0)=I$. If $T_{z_{p} v}(0)$ is varied as $T_{z_{p} v}(0)=$ $I+\Delta$ due to model uncertainties, we have the following steady-state error:

$$
e_{0} \triangleq z_{r}-z_{p}(\infty)=-\Delta z_{r}
$$




\begin{tabular}{lcc}
\hline Model & $V_{i}[\mathrm{~m} / \mathrm{s}]$ & GS-SF \\
\hline$P_{\text {poly-1 }}$ & $\{0,50\}$ & $F_{g s-1}$ \\
$P_{\text {poly }-2}$ & $\{0,25,50\}$ & $F_{g s-2}$ \\
$P_{\text {poly-3 }}\{0,10,15,40,50\}$ & $F_{g s-3}$ \\
\hline
\end{tabular}

Table 2. Operating points of polytopic models

\begin{tabular}{ccc}
\hline Model & $V_{d}[\mathrm{~m} / \mathrm{s}]$ & Fixed-SF \\
\hline$P_{l t i-1}$ & 0 & $F_{f i x-1}$ \\
$P_{l t i-2}$ & 25 & $F_{f i x-2}$ \\
$P_{l t i-3}$ & 50 & $F_{f i x-3}$ \\
\hline
\end{tabular}

Table 3. Design points of LTI models

To reduce the error, a feedback from $z_{p}$; that is, an outer-loop is added as shown in Fig. 4. The transfer function from $z_{r}$ to $z_{p}$ is given by

$$
T_{z_{p} z_{r}}(s)=\left(I+T_{z_{p} v}(s) K_{\text {out }}(s)\right)^{-1} T_{z_{p} v}(s)\left(I+K_{\text {out }}(s)\right)
$$

The steady-state error is then

$$
\begin{aligned}
e_{1} & \triangleq z_{r}-z_{p}(\infty)=z_{r}-\lim _{s \rightarrow 0} s T_{z_{p} z_{r}}(s) \frac{1}{s} z_{r} \\
& =-\left(I+T_{z_{p} v}(0) K_{\text {out }}(0)\right)^{-1} \Delta z_{r} .
\end{aligned}
$$

This means that the steady-state error $e_{1}$ with the outer-loop is reduced by (I+ $\left.T_{z_{p} v}(0) K_{\text {out }}(0)\right)^{-1}$. Summarizing the above, the design requirements of $K_{o u t}$ are given as follows:

(i) $K_{\text {out }}$ must stabilize $T_{z_{p} v}$.

(ii) The amplitude of $\left(I+T_{z_{p} v}(j \omega) K_{o u t}(j \omega)\right)^{-1}$ should be small in the low frequency region.

\section{Simulation}

To evaluate the proposed flight control system, a flight simulator was built on MATLAB/Simulink. For design and discussion hereafter, the notations about plant models are given as follows: $P_{l p v}(V)$ is a linear parameter varying (LPV) model obtained by linearizing $P_{n l}$. $P_{\text {poly }}(V)$ is the linear interpolative polytopic model given by Eq. (22) with Eq. (23). $P_{l t i}\left(V_{d}\right)$ is an LTI model where the flight velocity is fixed at $V_{d}$.

Two cases of flight control system with respect to the state feedback gain $F$ were compared in simulation. One is that $F$ was designed by GS where the plant model was $P_{\text {poly }}(V)$. Another is that $F$ was designed by LQR where the plant model was $P_{l t i}\left(V_{d}\right)$. The former is referred to as GS-SF, while the latter is referred to as Fixed-SF. The parameter values of the flight velocity profile in Fig. 3 were given as follows:

$$
\begin{aligned}
& \left(x_{r}, h_{r}\right)=(3000,0)[\mathrm{m}], \quad V_{c 1}=50, \quad V_{c 2}=15[\mathrm{~m} / \mathrm{s}] \\
& t_{c 1}=5, \quad t_{c 2}=30, t_{c 3}=60, \quad t_{c 4}=80, \quad t_{c 5}=100[\mathrm{~s}] .
\end{aligned}
$$




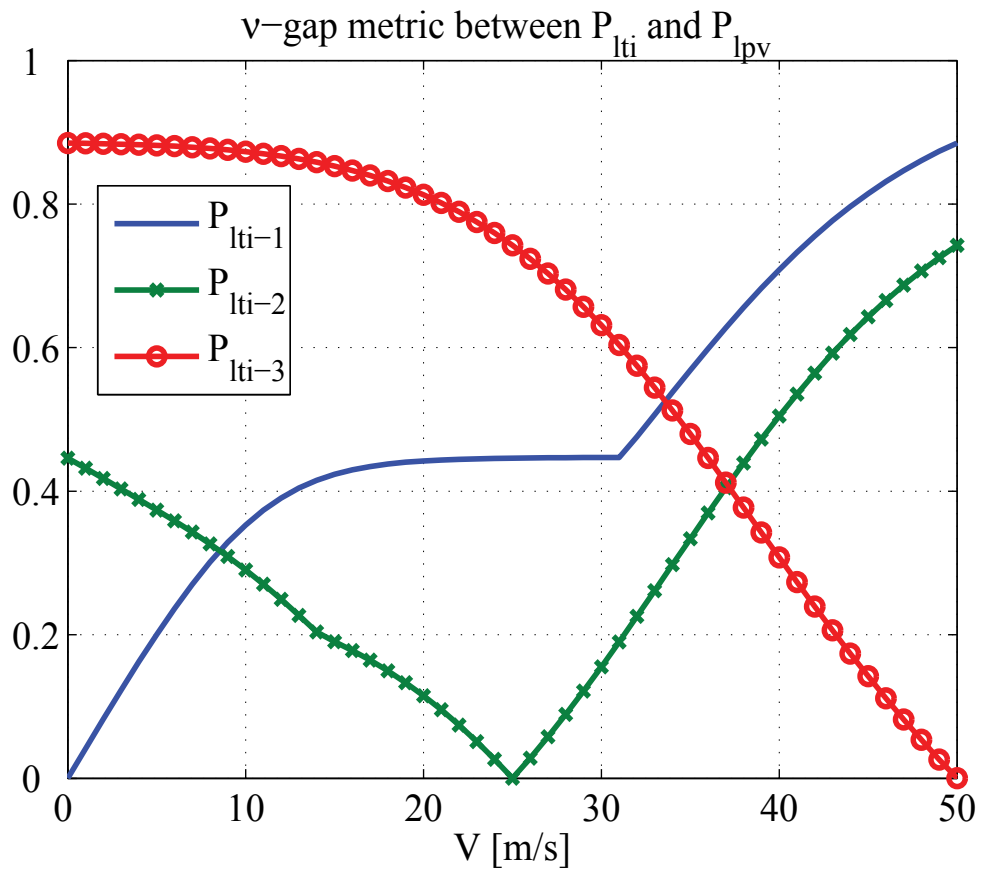

(a) LTI models

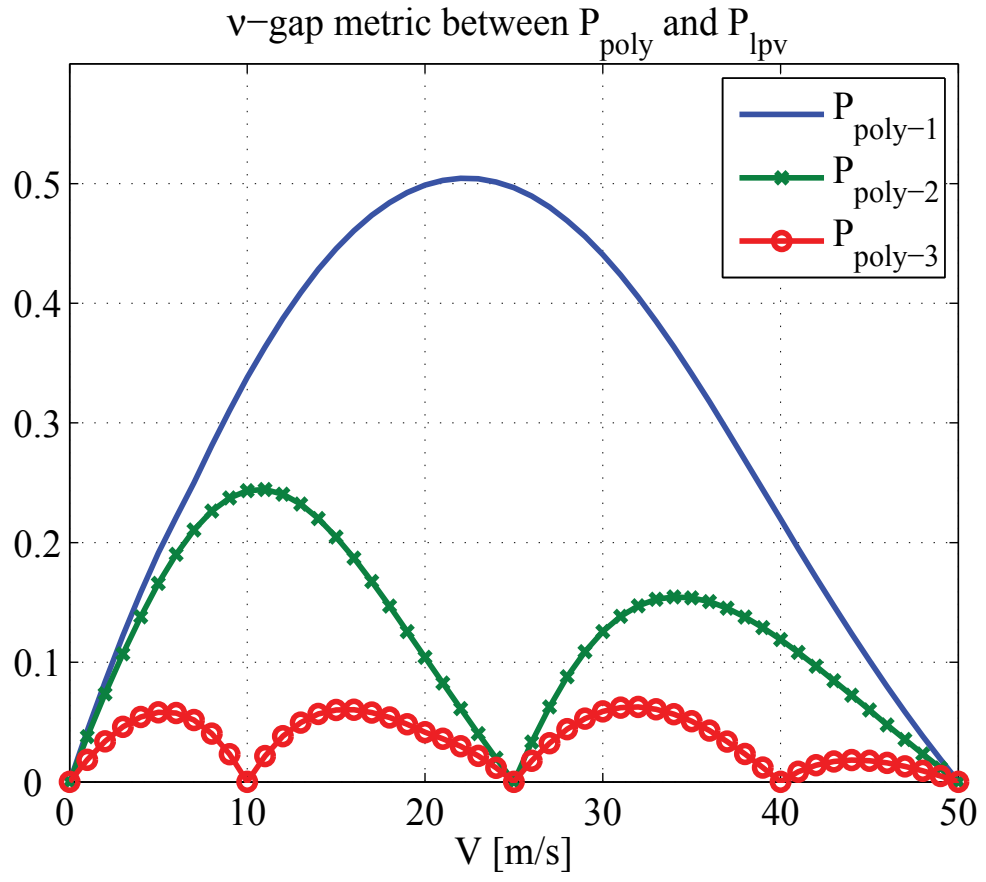

(b) polytopic models

Fig. 5. v-gap metric 


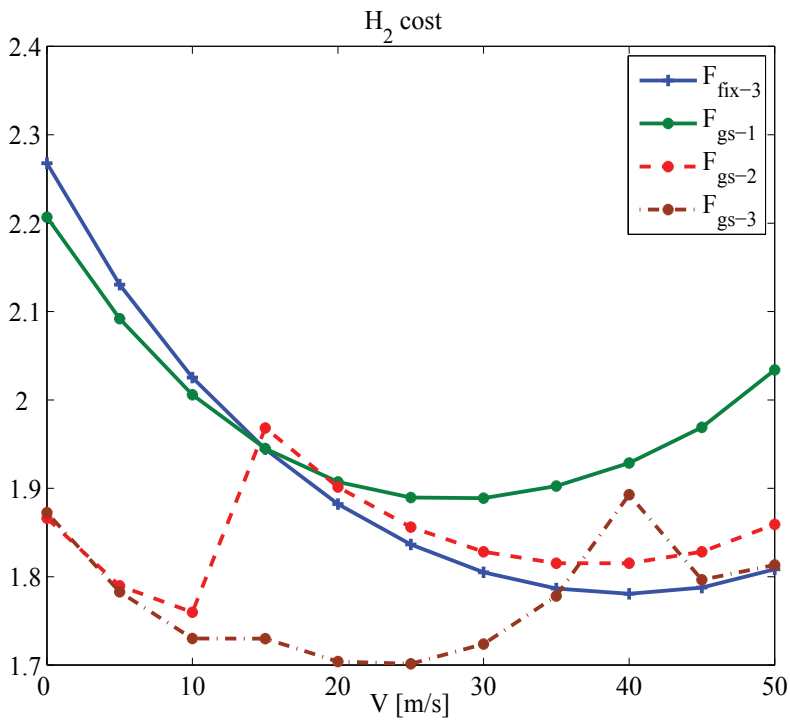

Fig. 6. $\mathcal{H}_{2}$ cost

\subsection{Evaluation of design models}

According to Section 4.1, three linear interpolative polytopic models were obtained. Table 2 shows the operating points chosen for the models. While, the design points $V_{d}$ of three LTI models are shown in Table 3. The $v$-gap metric is one of criteria measuring the model error in the frequency domain. It had been introduced in robust control theories associated with the stability margin (Vinnicombe, 2001). The $v$-gap metric between two LTI models, $P_{1}(s)$ and $P_{2}(s)$, is defined as

$$
\delta_{v}\left(P_{1}, P_{2}\right) \triangleq\left\|\left(I+P_{2} P_{2}^{*}\right)^{-1 / 2}\left(P_{1}-P_{2}\right)\left(I+P_{1} P_{1}^{*}\right)^{-1 / 2}\right\|_{\infty}
$$

The range is $\delta_{v} \in[0,1]$. A large $\delta_{v}$ means that the model error is large. The $v$-gap metric is used for evaluating the model $P_{\text {poly }}(V)$ and $P_{l t i}\left(V_{d}\right)$. Figure 5 shows $v$-gap metric between $P_{l t i}\left(V_{d}\right)$ and $P_{l p v}(V)$ and between $P_{p o l y}(V)$ and $P_{l p v}(V) . \delta_{v}\left(P_{l t i}(V), P_{l p v}\left(V_{d}\right)\right)$ was rapidly increased when $V$ was shifted from $V_{d}$. On the other hand, the maximum of $\delta_{v}\left(P_{p o l y}(V), P_{l p v}(V)\right)$ was reduced according to the number of the operating points. It was seen that $P_{\text {poly }}(V)$ appropriately approximated $P_{l p v}(V)$ over the entire range of the flight velocity.

\subsection{Design of $F$ and $\mathcal{H}_{2}$ cost}

The design parameters for designing $F$ in Eq. (30) were given as follows.

$$
B_{1}=\left[\begin{array}{cc}
-6.039 & 10.977 \\
-154.03 & 49.188 \\
3.954 & -7.187 \\
0 & 0 \\
0.38495 & -0.12395
\end{array}\right], \quad C_{1}=\left[\begin{array}{c}
0.001 I_{5} \\
0_{2 \times 5}
\end{array}\right], \quad D_{1}=\left[\begin{array}{c}
0_{5 \times 2} \\
I_{2}
\end{array}\right]
$$



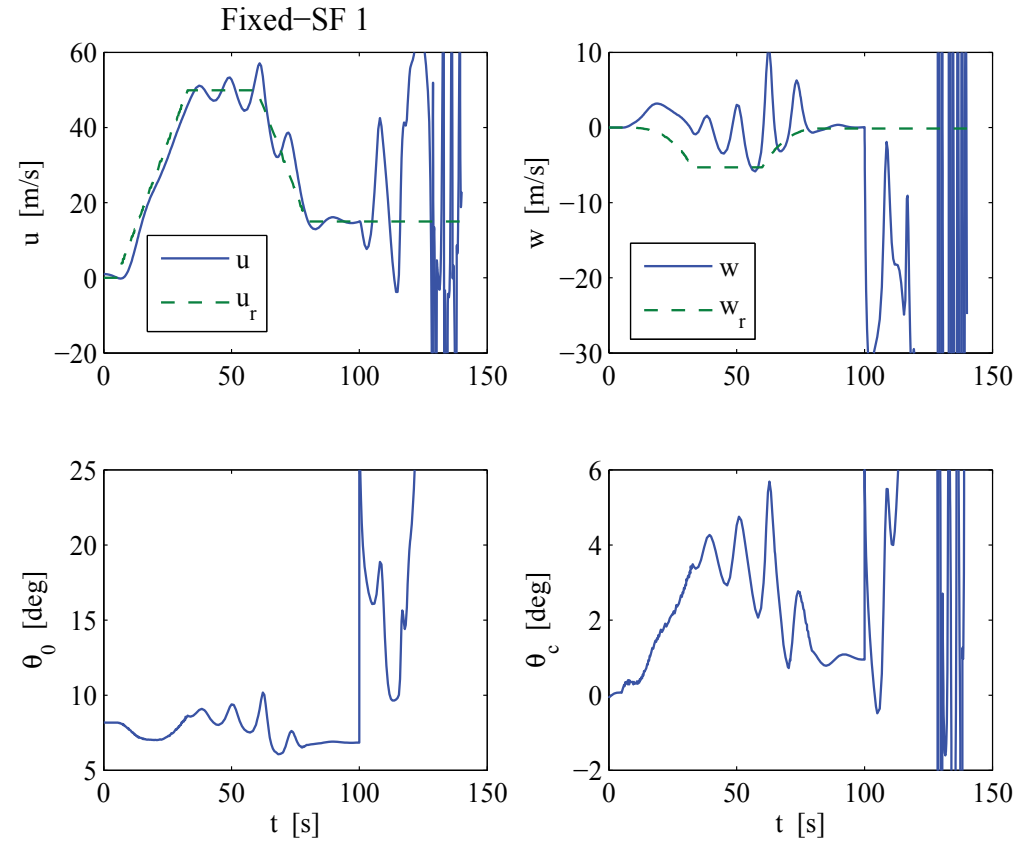

(a) Controlled variables and inputs

Fixed-SF 1
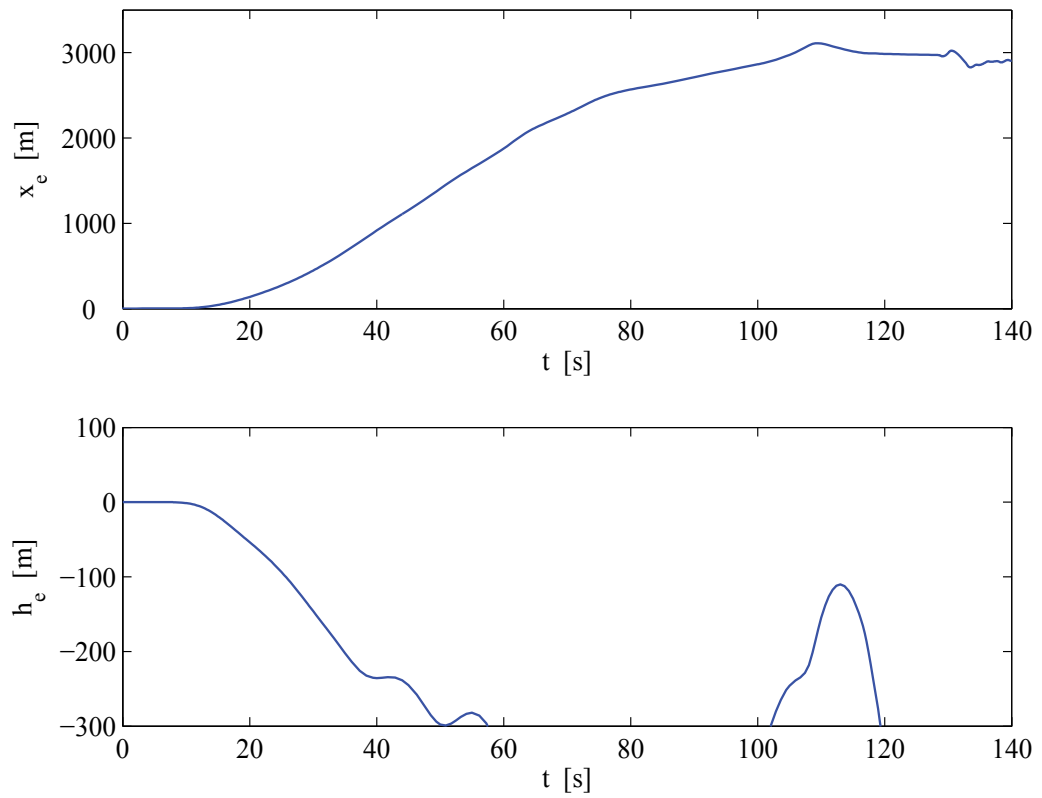

(b) Positions

Fig. 7. Time responses using $F_{f i x-1}$ 

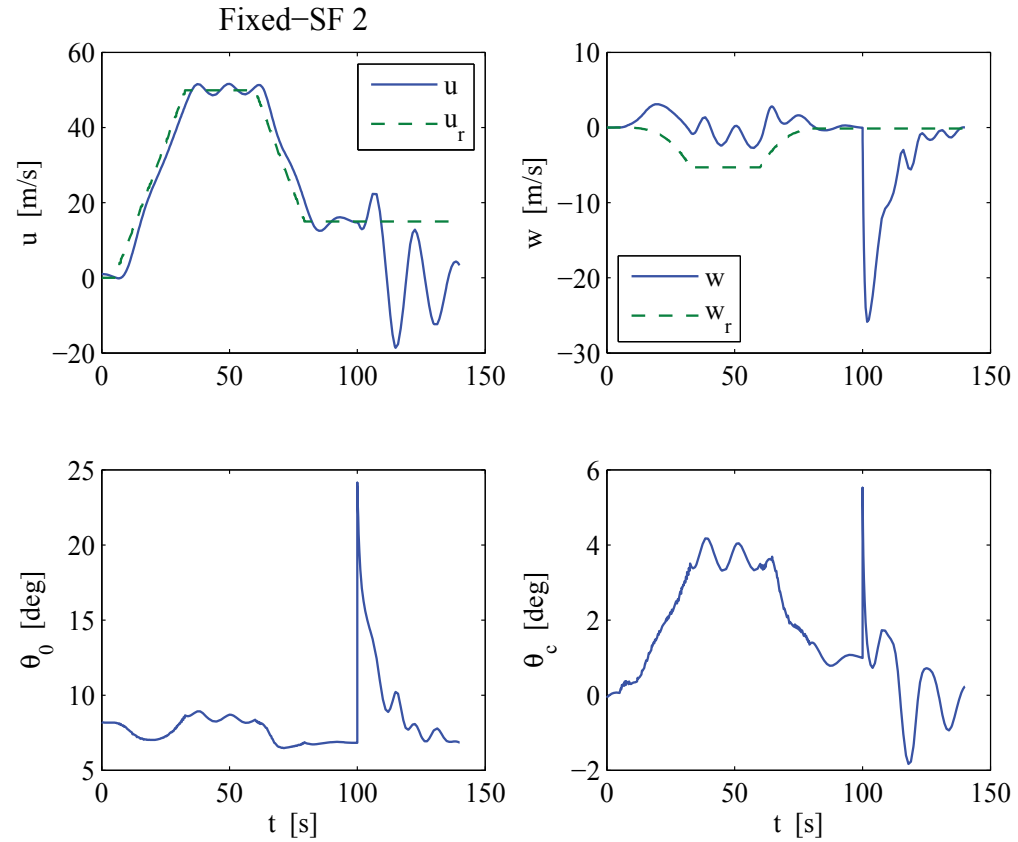

(a) Controlled variables and inputs

Fixed-SF 2
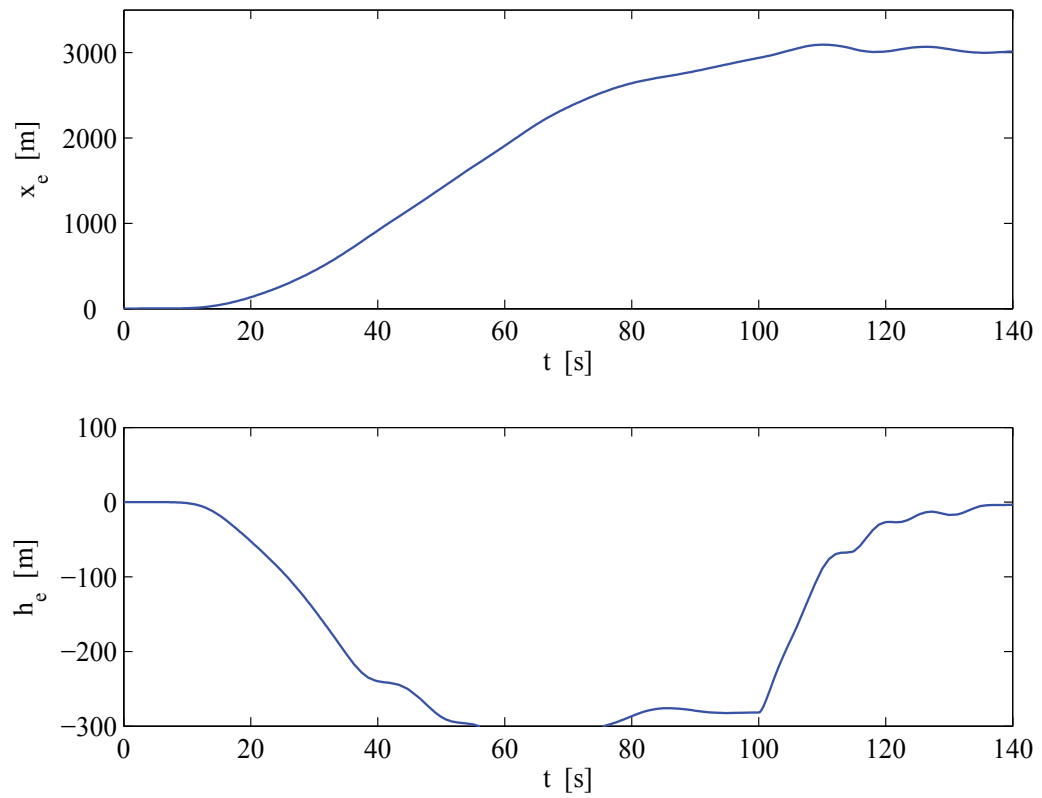

(b) Positions

Fig. 8. Time responses using $F_{f i x-2}$ 

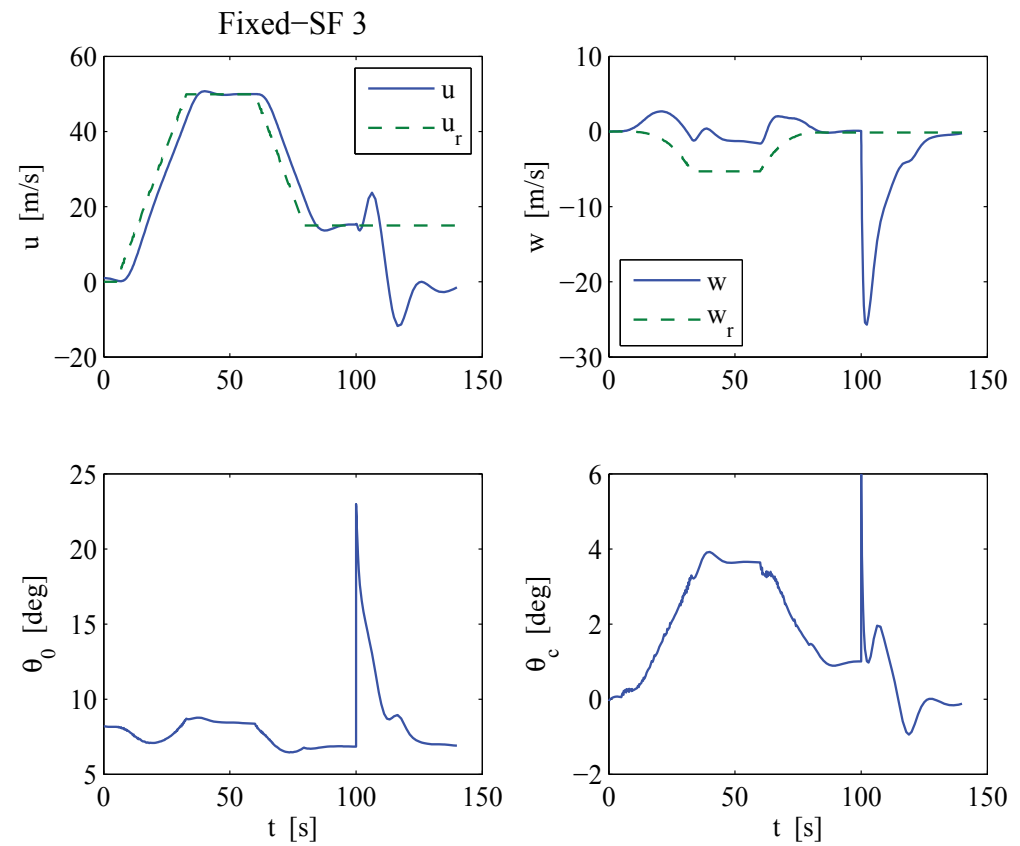

(a) Controlled variables and inputs

Fixed-SF 3
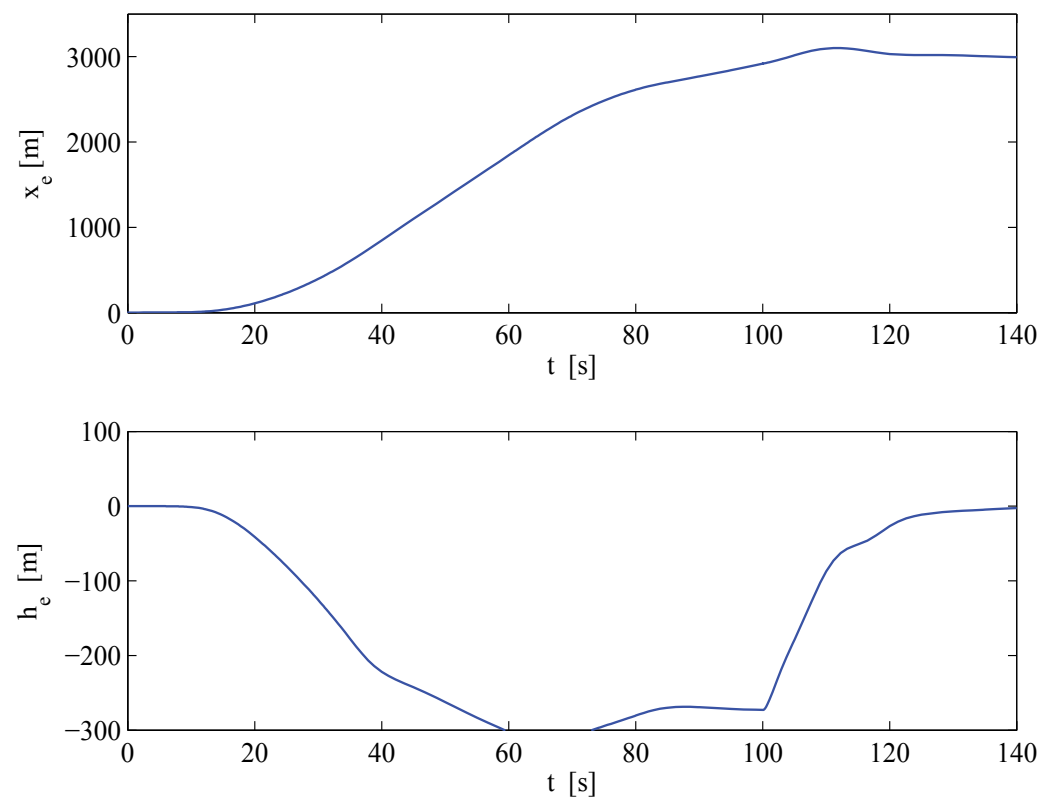

(b) Positions

Fig. 9. Time responses using $F_{f i x-3}$ 

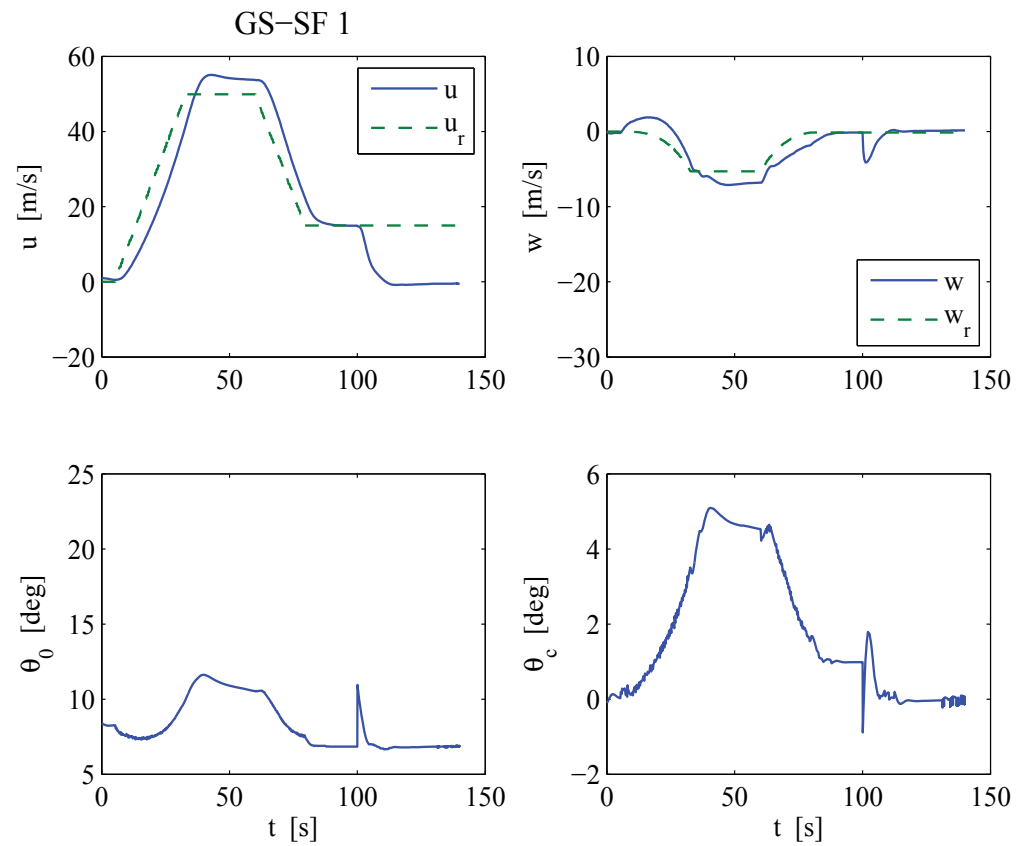

(a) Controlled variables and inputs
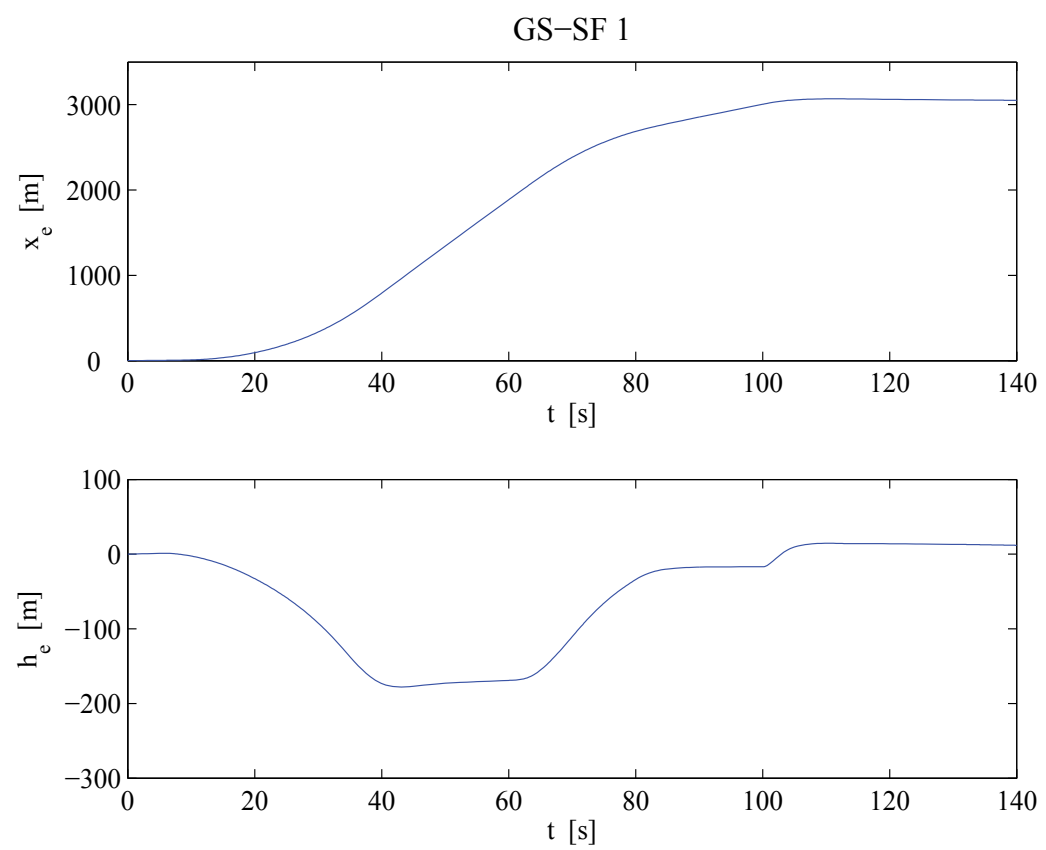

(b) Positions

Fig. 10. Time responses using $F_{g s-1}$ 

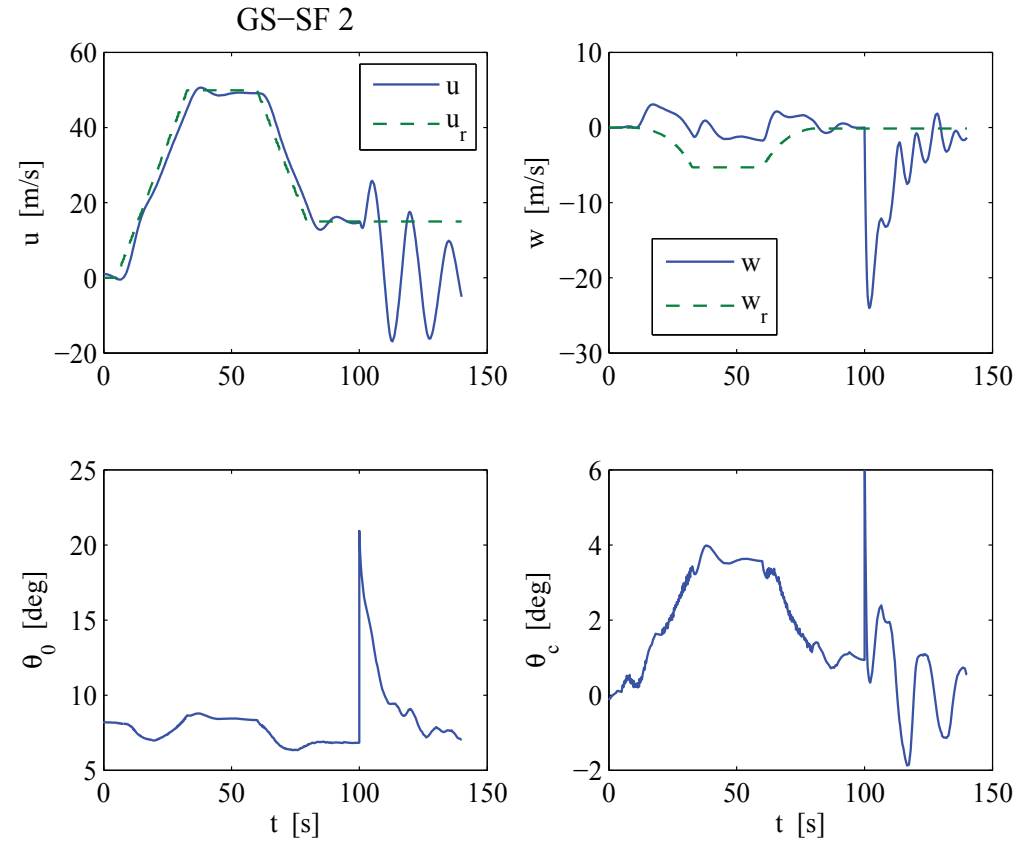

(a) Controlled variables and inputs

GS-SF 2
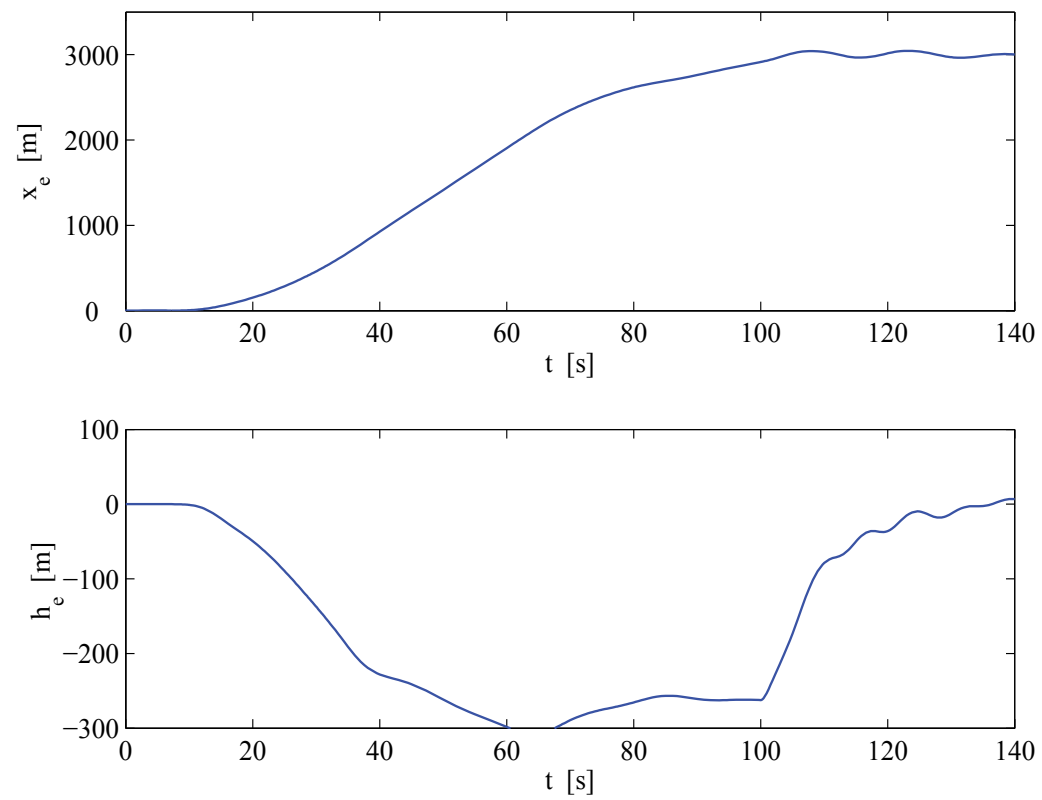

(b) Positions

Fig. 11. Time responses using $F_{g s-2}$ 

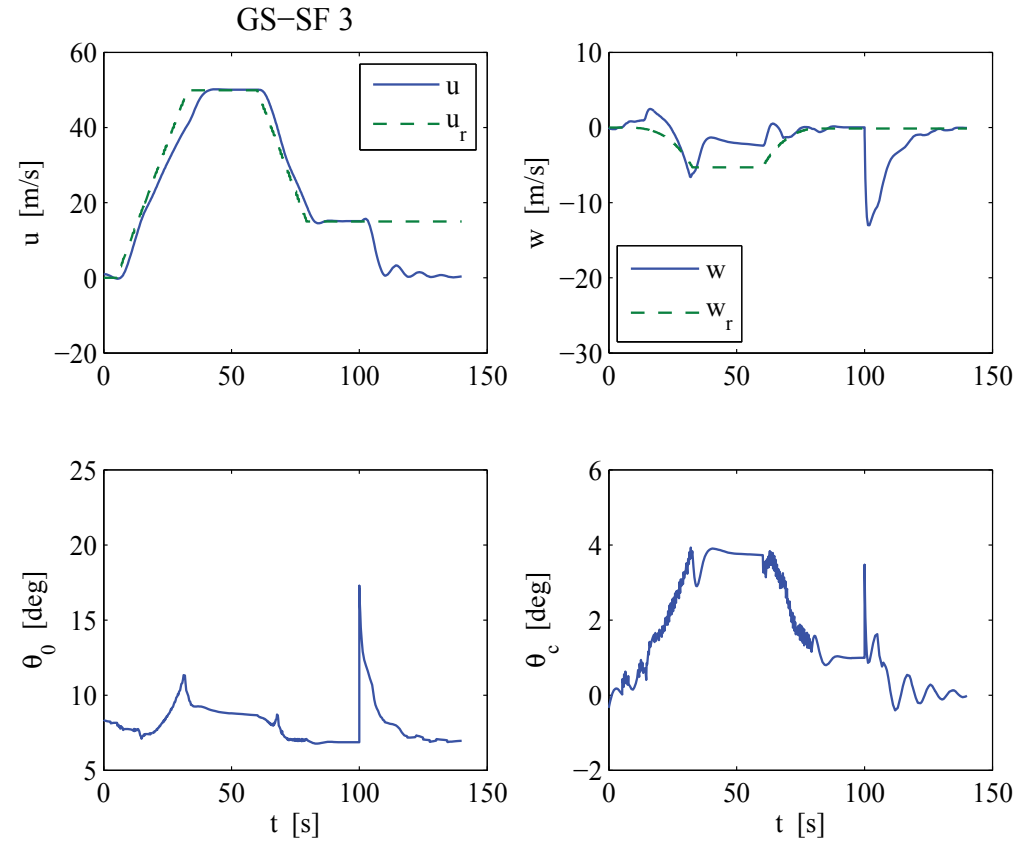

(a) Controlled variables and inputs

GS-SF 3
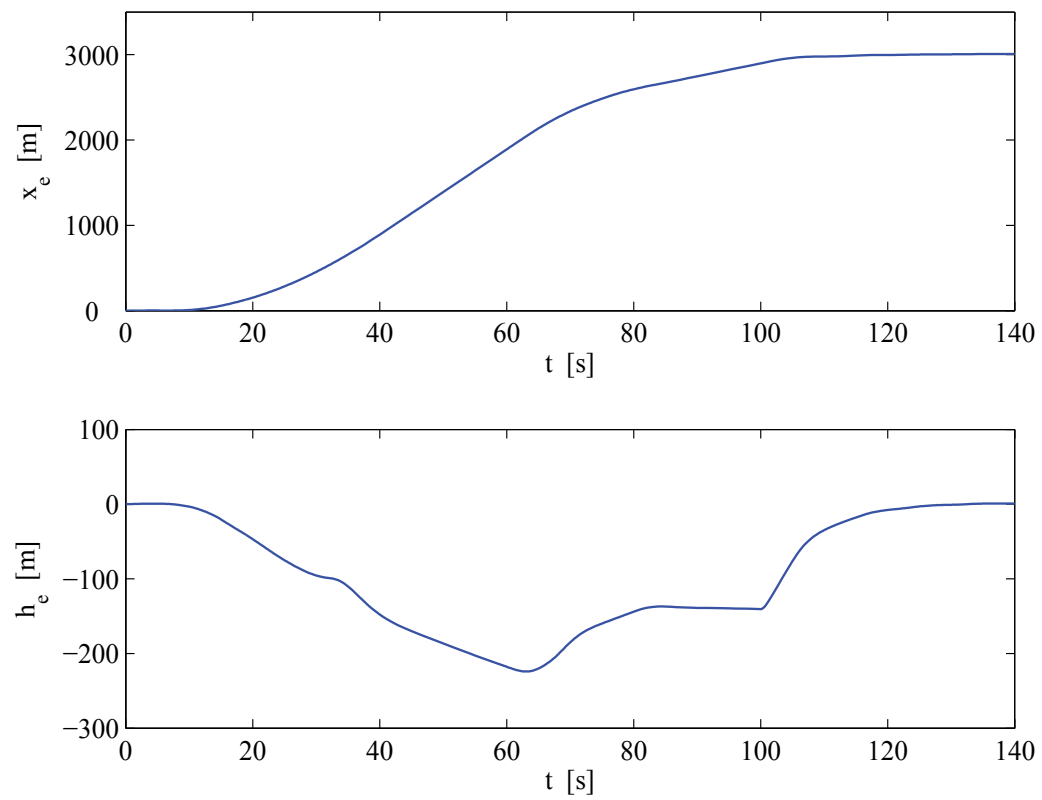

(b) Positions

Fig. 12. Time responses using $F_{g s-3}$ 
They were used for both of GS-SF and Fixed-SF. Three GS-SF gains denoted as $F_{g s-i}(i=$ $1,2,3)$ were designed according to Section 4.2, while three Fixed-SF gains denoted as $F_{f i x-i}$ $(i=1,2,3)$ were designed by LQR technique in which the weights of the quadratic index were given by $C_{1}^{T} C_{1}$ and $D_{1}^{T} D_{1}$.

Figure 6 shows the $\mathcal{H}_{2}$ cost of the closed-loop system which the designed $F$ is combined with Eq. (30). The $\mathcal{H}_{2}$ cost by $F_{f i x-3}$ was minimized at $V=40[\mathrm{~m} / \mathrm{s}]$ which was near the design point $V_{d}=50[\mathrm{~m} / \mathrm{s}]$, but was increased in the low flight velocity region. The $\mathcal{H}_{2}$ cost by $F_{\text {fix }-1}$ and $F_{f i x-2}$ showed the similar result. On the other hand, the $\mathcal{H}_{2}$ cost by $F_{g s-2}$ and $F_{g s-3}$ was kept small over the entire flight region. The $\mathcal{H}_{2}$ cost by $F_{g S-1}$ was small in the middle flight velocity region but was increased in the low and the high flight velocity regions.

\subsection{Tracking evaluation}

The flight mission given in Fig. 3 was performed in Simulink. Figures 7 - 12 show the time histories of the closed-loop system with the three GS-SF and three Fixed-SF gains. In the case of $F_{f i x-1}$ shown in Fig. 7, the controlled variables $u$ and $w$ tracked their references until the acceleration phase $(5 \leq t<30$ [s]) but they were diverged in the cruise phase (30 $\leq t<60$ [s]). In the deceleration phase $(60 \leq t<80$ [s]), the closed-loop system was stabilized again but it was de-stabilized in the approach phase $(t \geq 100$ [s]). Although the closed-loop system remained stable for the entire flight region in the case of $F_{f i x-2}$ shown in Fig. 8, oscillatory responses were observed in the cruise and approach phases. The responses using $F_{f i x-3}$ shown in Fig. 9 were better than those using $F_{f i x-2}$.

On the other hand, the three GS-SF gains provided stable responses as shown in Figs. 10 12 , In particular, The responses by $F_{g s-3}$ showed improved tracking and settling properties compared to other cases.

Summarizing the simulation in MATLAB/Simulink, polytopic model $P_{\text {poly-3 }}$ made the $v$-gap metric smaller than other models for the entire flight region. $F_{g s-3}$ designed by using $P_{p o l y-3}$ showed better control performance.

\section{Concluding remarks}

This paper has presented an autonomous flight control design for the longitudinal motion of helicopter to give insights for developing autopilot techniques of helicopter-type UAVs. The characteristics of the equation of helicopter was changed during a specified flight mission because the trim values of the equation were widely varied. In this paper, gain scheduling state feedback (GS-SF) was included in the double loop flight control system to keep the vehicle stable for the entire flight region. The effectiveness of the proposed flight control system was evaluated by computer simulation in MATLAB/Simulink. The model error of the polytopic model was smaller than that of LTI models which were obtained at specified flight velocity. Flight control systems with GS-SF showed better control performances than those with fixed-gain state feedback. The double loop flight control structure was useful for accomplishing flight mission considered in this paper.

\section{References}

[1] Boyd, S.; Ghaoui, L. E.; Feron, E. \& Balakrishnan, V. (1994). Linear Matrix Inequalities in System and Control Theory, SIAM, Vol. 15, Philadelphia.

[2] Bramwell, A. R. S. (1976). Helicopter Dynamics, Edward Arnold, London, 1976. 
[3] Cho, S.-J.; Jang, D.-S. \& Tahk, M.-L. (2005). Application of TCAS-II for Unmanned Aerial Vehicles, Proc. CD-ROM of JSASS 19th International Sessions in 43rd Aircraft Symposium, Nagoya, 2005

[4] Fujimori, A.; Kurozumi, M.; Nikiforuk, P. N. \& Gupta, M. M. (1999). A Flight Control Design of ALFLEX Using Double Loop Control System, AIAA Paper, 99-4057-CP, Guidance, Navigation and Control Conference, 1999, pp. 583-592.

[5] Fujimori, A.; Nikiforuk, P. N. \& Gupta, M. M. (2001). A Flight Control Design of a Reentry Vehicle Using Double Loop Control System with Fuzzy Gain-Scheduling, IMechE Journal of Aerospace Engineering, Vol. 215, No. G1, 2001, pp. 1-12.

[6] Fujimori, A.; Miura, K. \& Matsushita, H. (2007). Active Flutter Suppression of a High-Aspect-Ratio Aeroelastic Using Gain Scheduling, Transactions of The Japan Society for Aeronautical and Space Sciences, Vol. 55, No. 636, 2007, pp. 34-42.

[7] Johnson, E. N. \& Kannan, S. K. (2005). Adaptive Trajectory Control for Autonomous Helicopters, Journal of Guidance, Control and Dynamics, Vol. 28, No. 3, 2005, pp. 524-538.

[8] Langelaan, J. \& Rock, S. (2005). Navigation of Small UAVs Operating in Forests, Proc. CD-ROM of AIAA Guidance, Navigation, and Control Conference, San Francisco, 2005.

[9] Padfield, G. D. (1996). Helicopter Dynamics: The Theory and Application of Flying Qualities and Simulation Modeling, AIAA, Reston, 1996.

[10] Van Hoydonck, W. R. M. (2003). Report of the Helicopter Performance, Stability and Control Practical AE4-213, Faculty of Aerospace Engineering, Delft University of Technology, 2003.

[11] Vinnicombe, G. (2001). Uncertainty and Feedback ( $\mathcal{H}_{\infty}$ loop-shaping and the v-gap metric), Imperial College Press, Berlin.

[12] Wilson, J. R. (2007). UAV Worldwide Roundup 2007, Aerospace America, May, 2007, pp. 30-38. 


\title{
Autonomous Flight Control for RC Helicopter Using a Wireless Camera
}

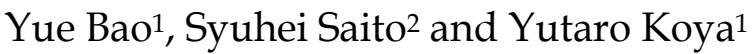 \\ ${ }^{1}$ Tokyo City University, \\ ${ }^{2}$ Canon Inc. \\ Japan
}

\section{Introduction}

In recent years, there are a lot of researches on the subject of autonomous flight control of a micro radio control helicopter. Some of them are about flight control of unmanned helicopter (Sugeno et al., 1996) (Nakamura et al., 2001). The approach using the fuzzy control system which consists of IF-Then control rules is satisfying the requirements for the flight control performance of an unmanned helicopter like hovering, takeoff, rotating, and landing. It is necessary to presume three dimensional position and posture of micro RC helicopter for the autonomous flight control.

A position and posture presumption method for the autonomous flight control of the RC helicopter using GPS (Global Positioning System), IMU (Inertial Measurement Unit), Laser Range Finder (Amida et al., 1998), and the image processing, etc. had been proposed. However, the method using GPS cannot be used at the place which cannot receive the electric waves from satellites. Therefore, it is a problem that it cannot be used for the flight control in a room. Although the method which uses various sensors, such as IMU and Laser Range Finder, can be used indoors, you have to arrange many expensive sensors or receivers in the room beforehand. So, these methods are not efficient. On the other hand, the method using an image inputted by a camera can be used in not only outdoors but also indoors, and is low price. However, this method needs to install many artificial markers in the surroundings, and it is a problem that the speed of the image inputting and image processing cannot catch up the speed of movement or vibration of a RC helicopter. A method presuming the three dimensional position and posture of a RC helicopter by the stereo measurement with two or more cameras installed in the ground was also proposed. In this case, the moving range of the $\mathrm{RC}$ helicopter is limited in the place where two or more cameras are installed. Moreover, there is a problem for which a high resolution camera must be used to cover a whole moving range. (Ohtake et al., 2009)

Authors are studying an autonomous flight of a RC helicopter with a small-wireless camera and a simple artificial marker which is set on the ground. This method doesn't need to set the expensive sensors, receivers, and cameras in the flight environment. And, we thought that a more wide-ranging flight is possible if the natural feature points are detected from the image obtained by the camera on the RC helicopter. This chapter contains the following contents. 
a. Input method of image from a small, wireless camera which is set on a RC helicopter.

b. Extraction method of feature points from an image of flight environment taken with a camera on RC helicopter.

c. Calculation method of three dimensional position and posture of RC helicopter by image processing.

d. Experiment of autonomous flight of a RC helicopter using fuzzy logic control.

\section{Composition of system}

The overview of a micro RC helicopter with coaxial counter-rotating blades used in our experiment is shown in Fig.1. Since this RC helicopter can negate a running torque of a body by a running torque between an up propeller and a down propeller, it has the feature that it can fly without being shakier than the usual RC helicopter. The composition of our experiment system for automatic guidance of $\mathrm{RC}$ helicopter is shown in Fig.2. A small wireless camera is attached on the RC helicopter as shown in Fig.3, and the image of the ground is acquired with this camera, and this image is sent to the receiver on ground, and then sent to the computer through a video capture. The position and posture of the RC

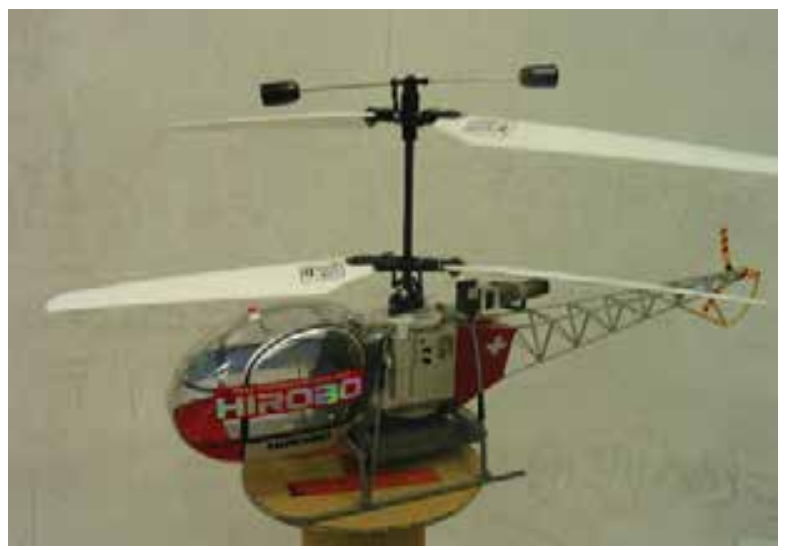

Fig. 1. Micro RC helicopter with the coaxial contra-rotating rotors

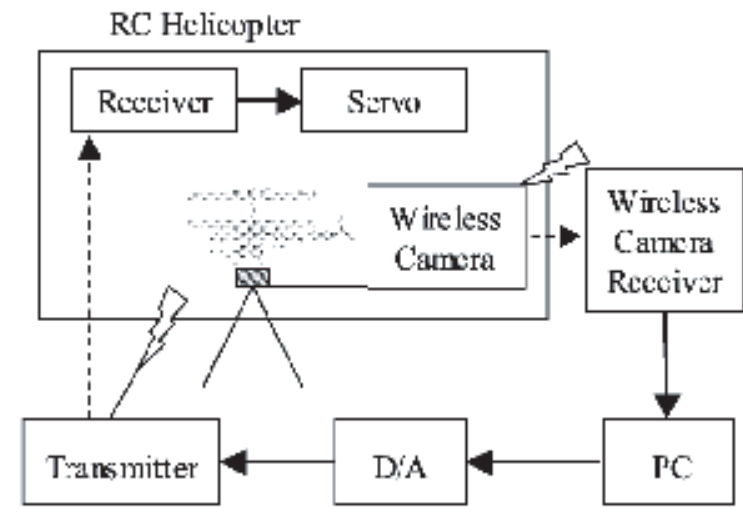

Fig. 2. Composition of automatic guidance system 


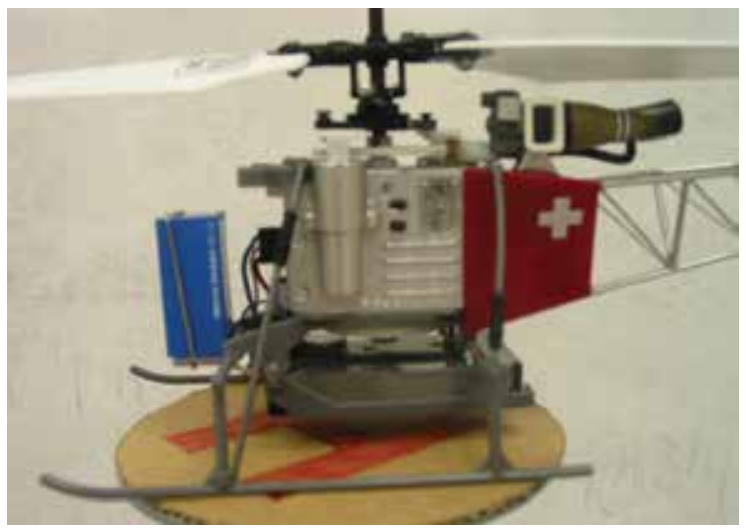

Fig. 3. RC helicopter equipped with a micro wireless camera

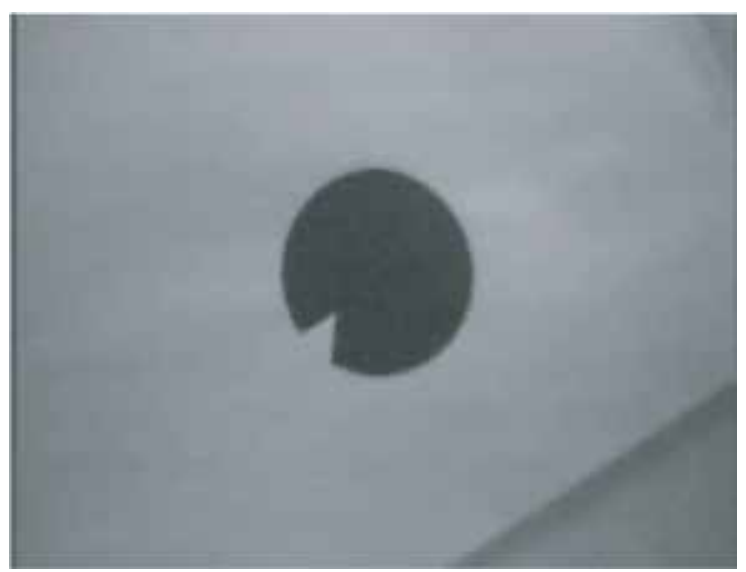

Fig. 4. An artificial marker

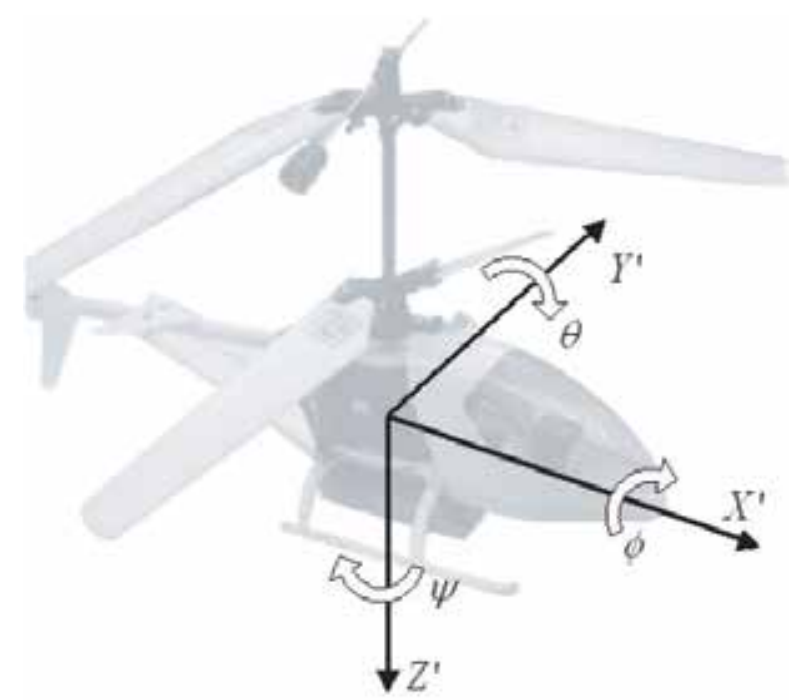

Fig. 5. Coordinate axes and attitude angles 
helicopter are computed with image processing by the computer set on the ground, and, this image processing used the position and shape of an artificial marker in the camera image like Fig.4. The three dimensional position of the RC helicopter $(x(t), y(t), z(t))$ and the changing speed of the position $(\dot{x}(t), \dot{y}(t), \dot{z}(t))$ and the attitude angles $\psi(t)$ and changing speed of attitude angles $\dot{\psi}(t)$ can be obtained by this calculation. Fig.5 shows the relation between these coordinate axes and attitude angles.

This micro RC helicopter is controlled by four control signals, such as Aileron, Elevator, Rudder, and Throttle, and, the control rule of fuzzy logic control is decided by using measurement data mentioned above. The control signals are sent to micro RC helicopter through the digital-analog converter.

\section{Image processing}

\subsection{Image input}

The micro wireless camera attached on the RC helicopter takes an image by interlaces scanning. If the camera takes an image during $\mathrm{RC}$ helicopter flying, since the vibration of the $\mathrm{RC}$ helicopter is quicker than the frame rate of the camera, the image taken by the camera will be a blurred image resulting from an interlace like Fig.6. We devised a method skipping the odd number line (or, even number line) of input image to acquire an clear input image while the RC helicopter is flying.

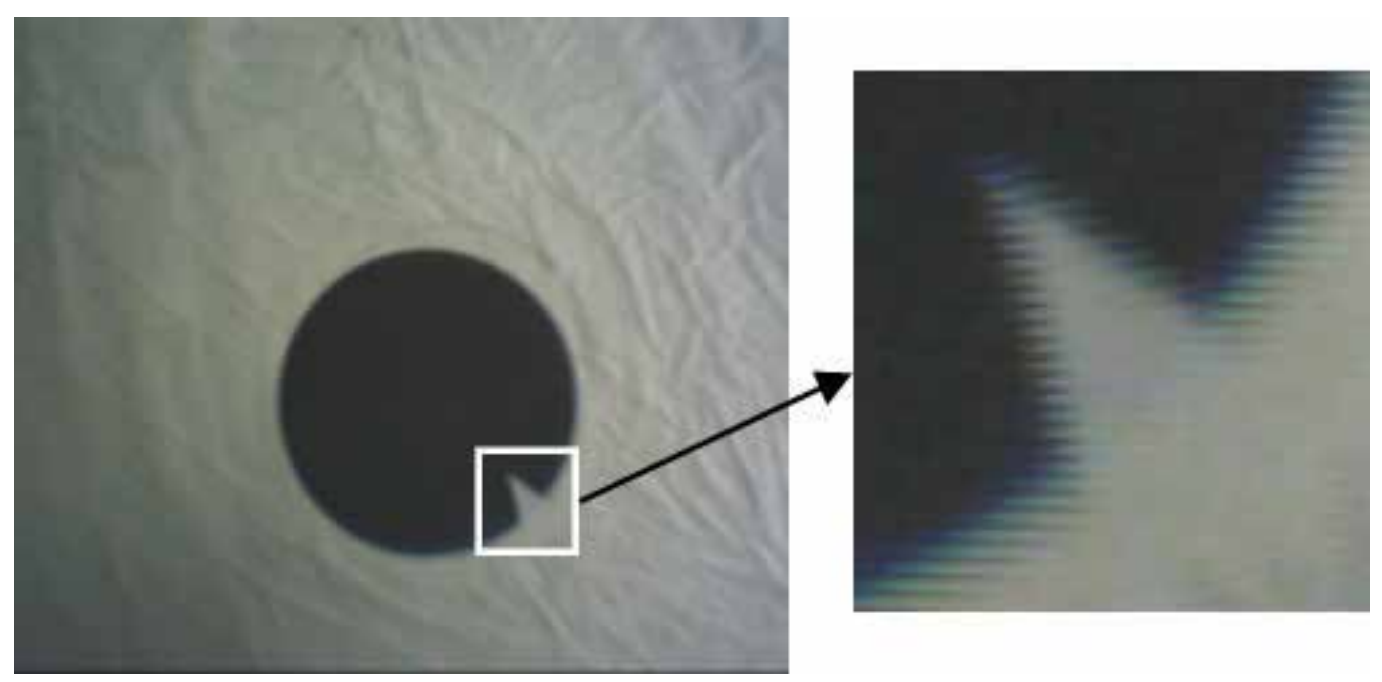

Fig. 6. A blurring image acquired by wireless camera

\subsection{Feature point extraction}

Feature point detection is defined in terms of local neighborhood operations applied to an image such as an edge and corner. Harris operator (Harris and Stephens, 1988) (Schmid et al., 1998) and SUSAN operator (Smith and Brady, 1997) are well known as common feature detectors. The methods (Neumann and You, 1999) (Bao and Komiya, 2008) to estimate 
position and attitude by using natural feature points or marker in the input image are proposed too. Our prototype experiment system used the Harris operator which can extract the same feature points in higher rate more than other feature point extraction methods (Schmid et al., 1998). First, we obtain a grayscale image from camera. Let us consider taking an image patch over an area $(u, v)$ from an image $I$ and shifting it by $(x, y)$. The Harris matrix $M$ can be found by taking the second derivative of the sum of squared differences between these two patches around $(x, y)=(0,0)$. $M$ is given by:

$$
M=\left(\begin{array}{cc}
G_{\sigma}\left(\frac{\partial^{2} I}{\partial^{2} x}\right) & G_{\sigma}\left(\frac{\partial I}{\partial x} \frac{\partial I}{\partial y}\right) \\
G_{\sigma}\left(\frac{\partial I}{\partial x} \frac{\partial I}{\partial y}\right) & G_{\sigma}\left(\frac{\partial^{2} I}{\partial^{2} y}\right)
\end{array}\right)
$$

Let the standard deviation of $G$ in the equation be $\sigma$ with Gaussian function for performing smoothing with Gaussian filter. The strength of a corner is decided by second derivative. Here, the eigenvalue of $M$ is $\left(\lambda_{1}, \lambda_{2}\right)$, and the value of eigenvalue can be got from the following inference.

- If $\lambda_{1} \approx 0$ and $\lambda_{2} \approx 0$, then there are no features at this pixel $(x, y)$.

- If either $\lambda_{1}$ or $\lambda_{2}$ is large positive value, then an edge is found.

- If $\lambda_{1}$ and $\lambda_{2}$ are both large positive values, then a corner is found.

Because the exact calculation of eigenvalue by the method of Harris will increase computational amount, the following functions $\mathrm{R}$ were proposed instead of those calculation methods.

$$
\begin{gathered}
R=\operatorname{det}(M)-k(\operatorname{tr}(M))^{2} \\
R=\lambda_{1} \lambda_{2}-k\left(\lambda_{1}+\lambda_{2}\right)^{2}
\end{gathered}
$$

The det expresses a determinant and $t r$ expresses the sum of the diagonal element of a matrix, and $k$ is a value decided experientially.

The kanade-Tomasi corner detector (Shi and Tomasi, 1994) uses min $\left(\lambda_{1} \lambda_{2}\right)$ as measure of feature point. For example, Fig.7 shows a feature point detection using Harris operator for photographed image. The Harris operator detects the corner point mainly from the image as a feature point.

The position of feature point is estimate able by related position information of an artificial marker to feature point from camera image after coordinate transformation. The flight control area of RC helicopter can be expanded(see Fig.8) by using the information of natural feature points around an artificial marker. Harris operator is suitable for detecting natural points. Our system saves the areas including the natural feature points as templates when the artificial marker is detected. In the range that can take the image of the artificial marker, the system uses the position information of the artificial marker. If the system can't take the image of an artificial marker, the position of the helicopter is estimated by template matching between the area of natural feature points and the template area. 

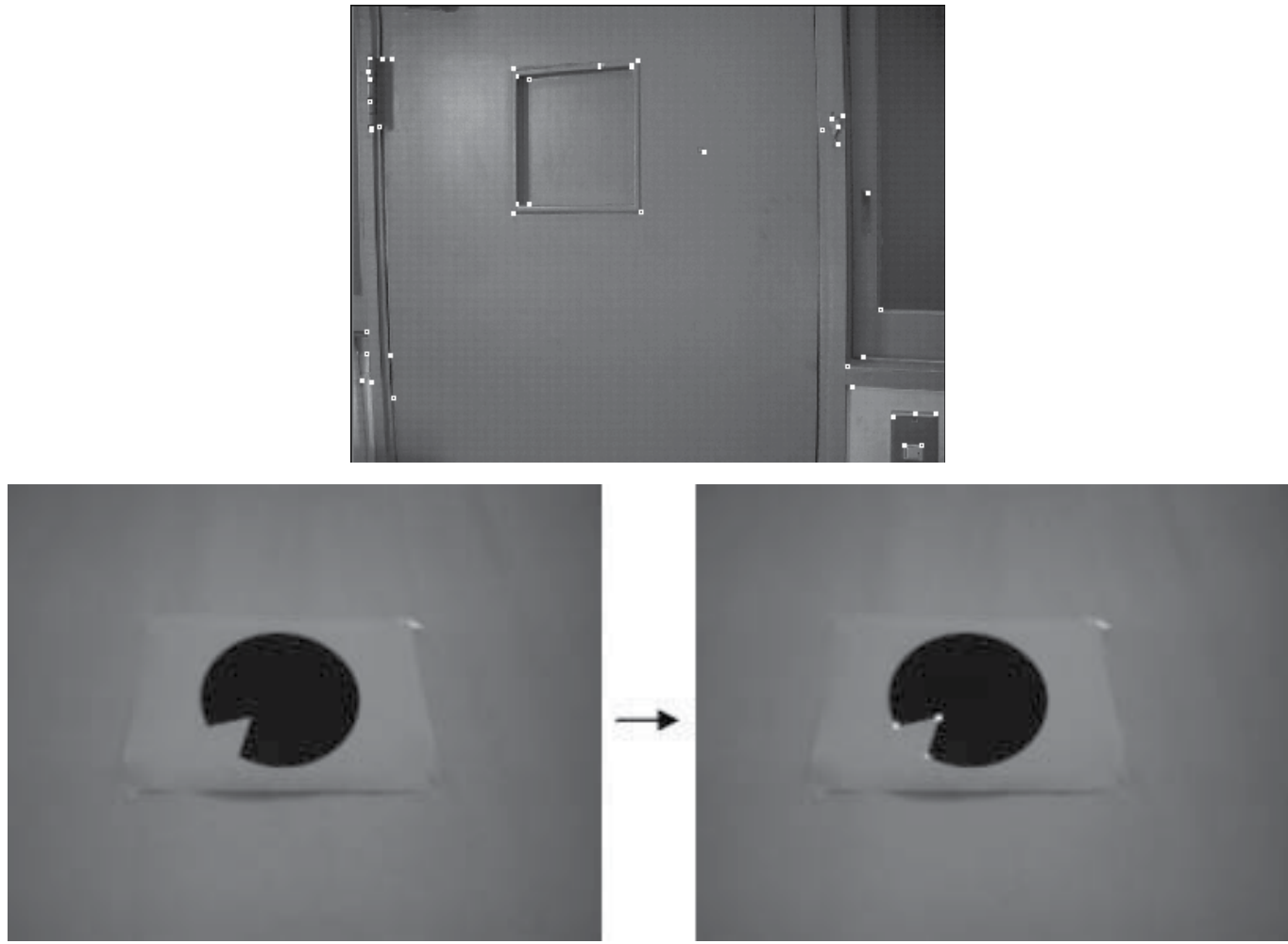

Fig. 7. Feature point extraction by a Harris operator

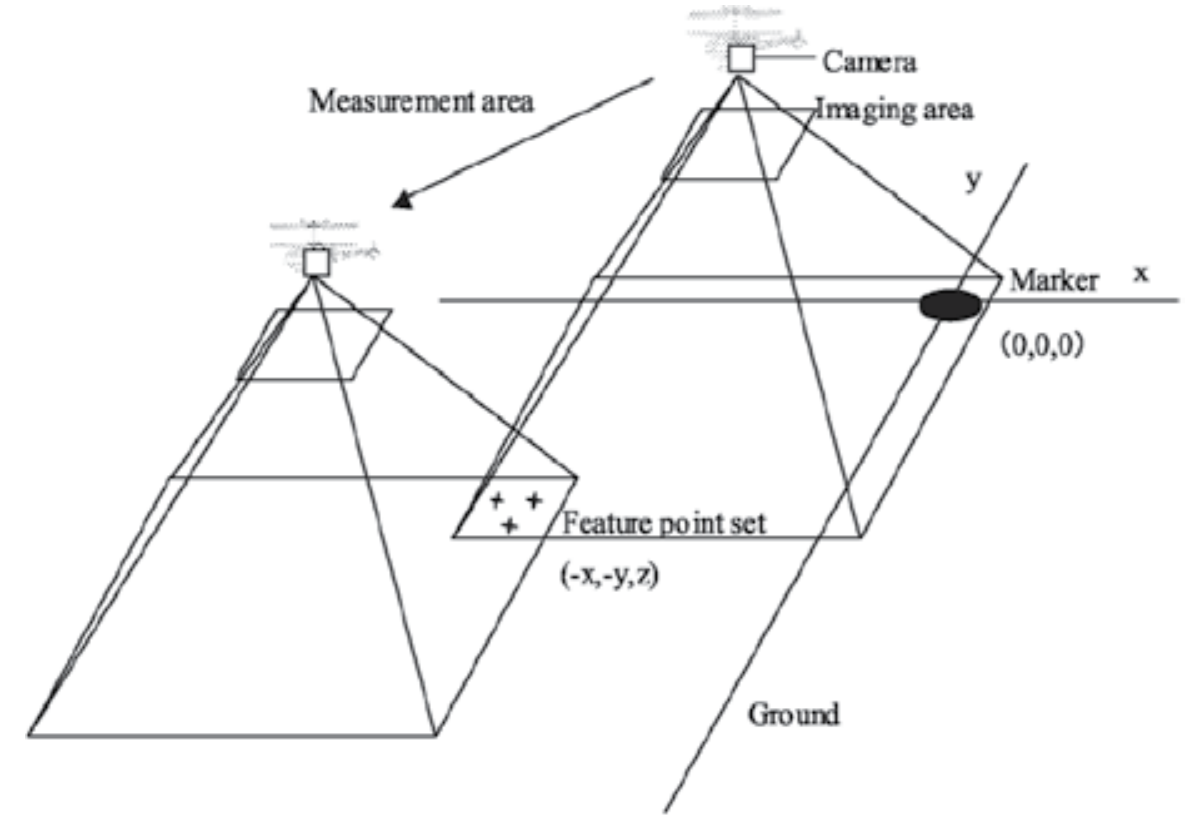

Fig. 8. The expansion of flight area 


\subsection{Detection of a marker}

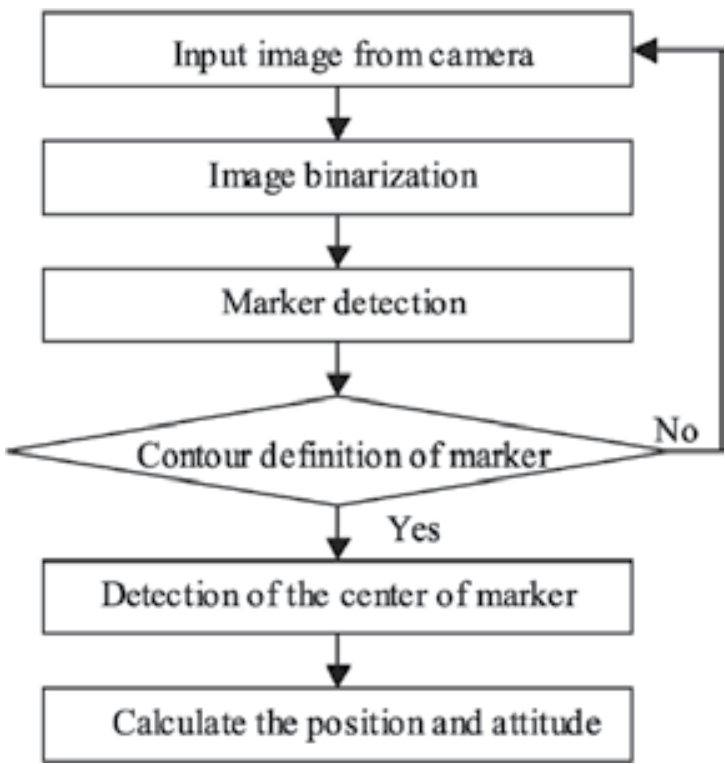

Fig. 9. The flow chart of marker detection

The image of the marker photographed with the wireless camera on micro RC helicopter is shown in Fig.4. The yaw angle can be calculated by the center of the marker and a position of a cut of triangle of the marker, and the angle of the pitch and the angle of roll can be acquired by coordinate transformation between a camera coordinate system and a world coordinate system. The flow chart about the image processing for the marker extraction and the calculation of position and posture are shown in Fig.9. First, a binarization processing is performed at the input image from the camera installed on the micro RC helicopter. Then, the marker in the image is searched, and the outline of the marker is extracted. If the outline cannot be extracted, an image is acquired from the camera again and a marker is searched again. A marker center is searched after the outline of a marker is extracted. The method of search is shown in Fig.10. The maximum values and the minimum values about the $x$-coordinate and $y$-coordinate are searched from of the extracted outline, and their middle values are given as the coordinates of the marker center. A length of major axis and a length of minor axis of the marker are calculated by the distance between the center coordinates of the marker and the pixel on the outline of the marker. The calculation method of the major axis and a minor axis is shown in Fig.11. When the center coordinate is defined as $P\left(x_{C}, y_{C}\right)$, and the coordinate of the pixel which is on the outline is defined as $I(x, y)$, the distance PI from the center to the pixel of the outline is calculated by equation (4).

$$
P I=\sqrt{\left(x-x_{c}\right)^{2}+\left(y-y_{c}\right)^{2}}
$$

For obtaining the maximum value $G_{1}$ of $P I$ and the minimum value $G_{2}$ of $P I$, all pixels on the outline are calculated. And the segment of $G_{1}$ is defined as the major axis $P O$, and the segment of $G_{2}$ is defined as a minor axis $P Q$. The position and posture of the micro RC helicopter are calculated by the method shown in Section 4 . 


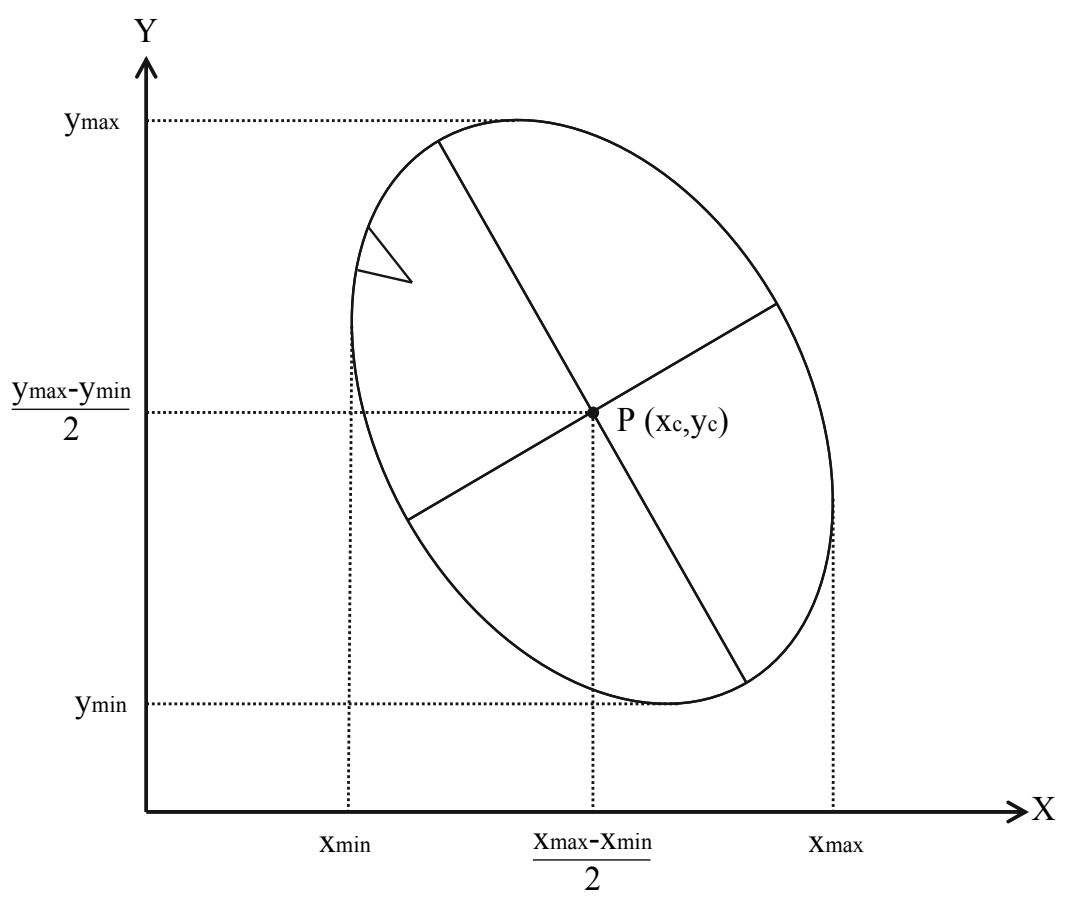

Fig. 10. The marker center

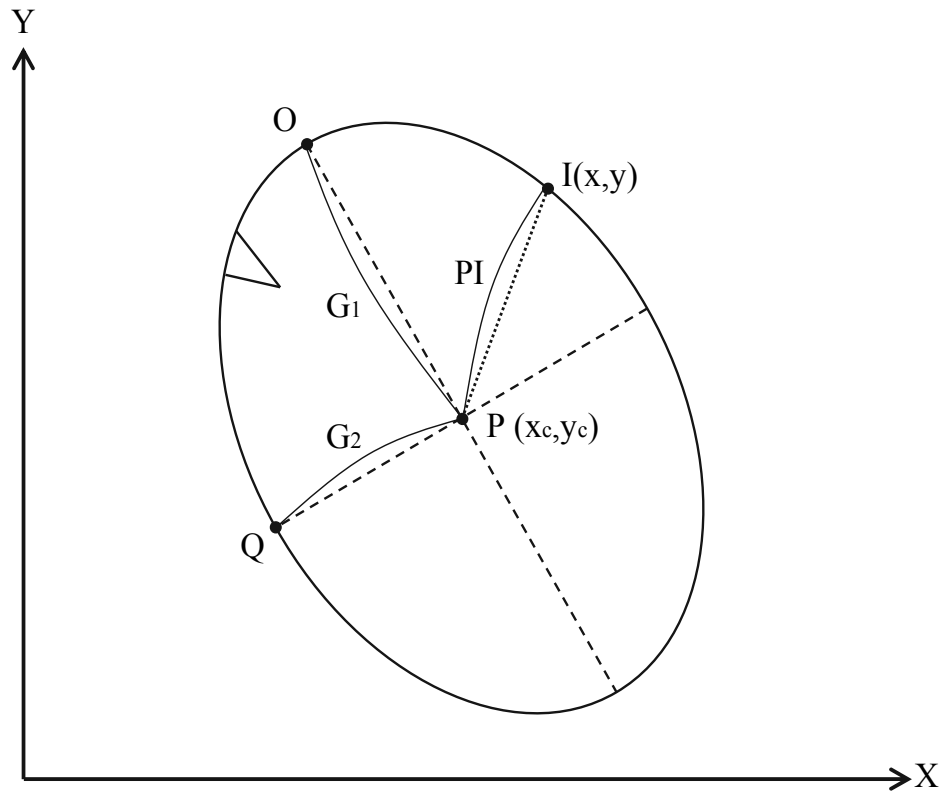

Fig. 11. The calculation method of the major axis and the minor axis of the marker 
Since the vibration of the RC helicopter is quicker than the frame rate of the camera, when the camera which attached on the micro RC helicopter takes the image, the taken image has become a blurred image resulting from the interlace like Fig.6. Therefore, an exact result cannot be obtained by a usual image processing. The image processing that we devised scans with skipping the odd (or even) number lines only about y axial direction at pixel scanning. The method of scanning is shown in Fig.12. If the pixels processed are on odd lines, the even number lines are skipped only about $y$ axial direction. About $x$ axial direction, the system scans 1 pixel at a time like the usual pixel scanning. By this method, a stable profile tracking can be performed without being blurred by interlace.

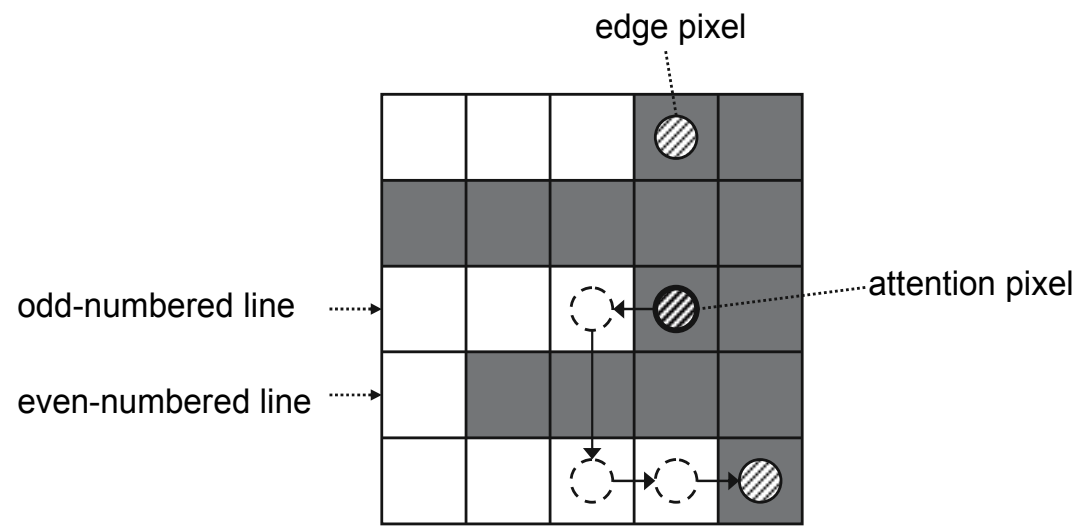

Fig. 12. The method of outline tracking

\section{Calculation of position and posture}

\subsection{Calculation of the position coordinates of RC helicopter}

In order to measure the position of the RC helicopter, it is necessary to calculate the focal distance of the wireless camera on the RC helicopter first. If the size of marker and the location of $\mathrm{z}$ axial direction of $\mathrm{RC}$ helicopter are got, the focal distance $\mathrm{f}$ of the wireless camera can be calculated easily. The three-dimensional coordinates of a moving RC helicopter are calculated by using the focal distance, a center of gravity and the value of radius of the marker. The image formation surface of the camera and the circular marker surface are parallel if RC helicopter is hovering above the circular marker like Fig.13.

Let's consider that a marker is in the center of the photographed image. The radius of circular marker in the camera image is defined as $D_{1}$, and the center coordinates of the circle marker are defined as $\left(x_{C 1}, y_{C 1}\right)$. The radius of the actual marker is defined as $d_{1}$, and the center coordinates of the actual marker in a world coordinate system are defined as $\left(x_{1}, y_{1}, z_{1}\right)$. Then, the focal distance $f$ of a camera can be calculated from the following two equations from parallel relation.

$$
\begin{gathered}
z_{1}: d_{1}=f: D_{1} \\
f=\frac{z_{1} \cdot D_{1}}{d_{1}}
\end{gathered}
$$


When the RC-helicopter is in moving, the radius of the marker in the image after moving is defined as $D_{2}$, the center coordinates of the marker after moving are defined as $\left(x_{\mathrm{C} 2}, y_{\mathrm{C} 2}\right)$, and the center coordinates of actual marker is defined as $\left(x_{2}, y_{2}, z_{2}\right)$. Then, the following equation is acquired.

$$
z_{2}: d_{1}=f: D_{2}
$$

Here, since the focal distance $\mathrm{f}$ and the radius $d_{1}$ of actual marker are not changing, the following relation is obtained from equation (5) and equation (7).

$$
D_{1}: D_{2}=z_{2}: z_{1}
$$

$z_{2}$ can be acquired by the following equation. Moreover, $x_{2}$ and $y_{2}$ can be calculated by the following equations from parallel relation. Therefore, the coordinate of the helicopter after moving is computable by using equation (9), equation (10), and equation (11), using the focal distance of the camera.

$$
\begin{aligned}
& z_{2}=\frac{D_{1} \cdot z_{1}}{D_{2}} \\
& x_{2}=\frac{X_{2} \cdot z_{2}}{f} \\
& y_{2}=\frac{Y_{2} \cdot z_{2}}{f}
\end{aligned}
$$
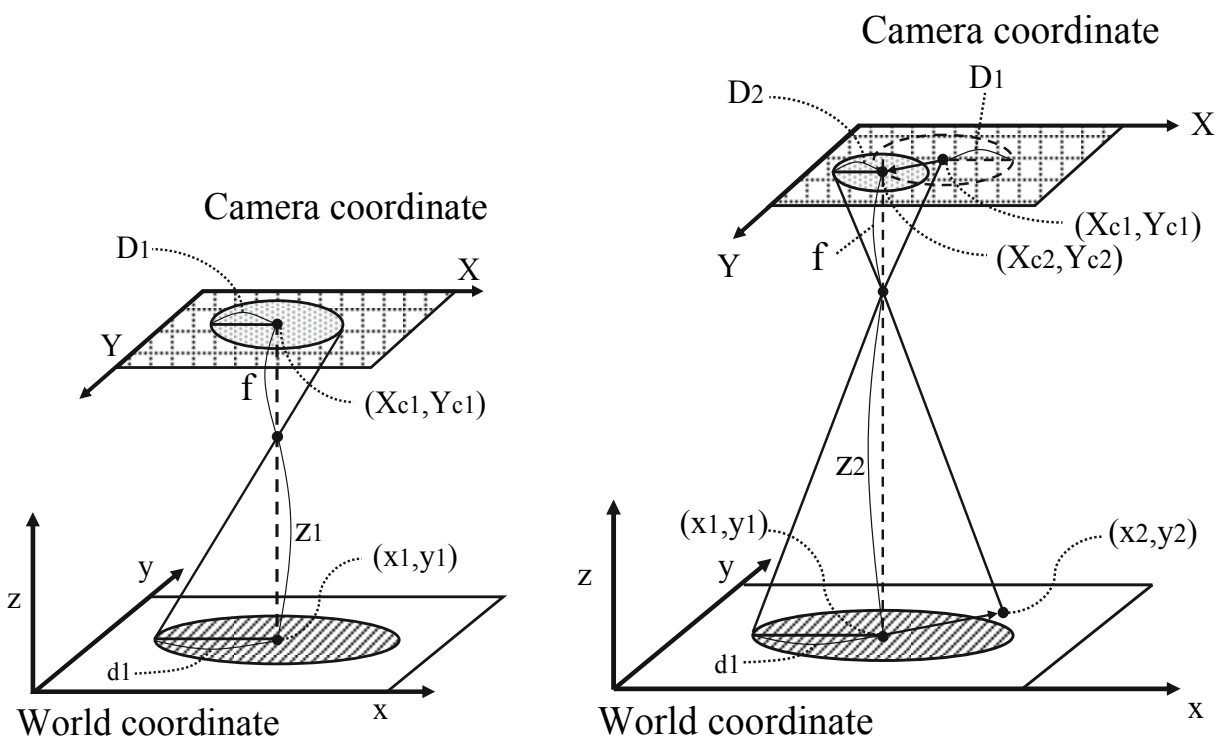

Fig. 13. The location calculation method of RC helicopter 


\subsection{Calculation of the attitude angle of RC helicopter}

The relation between an angle of RC helicopter and an image in the camera coordinate system is shown in Fig.14. When RC helicopter is hovering above a circular marker, the circular marker image in the camera coordinate system is a right circle like an actual marker. If RC helicopter leans, the marker in a camera coordinate system becomes an ellipse. To calculate the attitude angle, first, the triangular cut part of the circular marker is extracted as a direction feature point. Then the deformation of the marker image is corrected for calculating a yaw angle using the relation between the center of the circular marker and the location of the direction feature point of the circular marker. The pitch angle and the roll angle are calculated performing coordinate transformation from the camera coordinate system to the world coordinate by using the deformation rate of the marker in the image from the wireless camera.
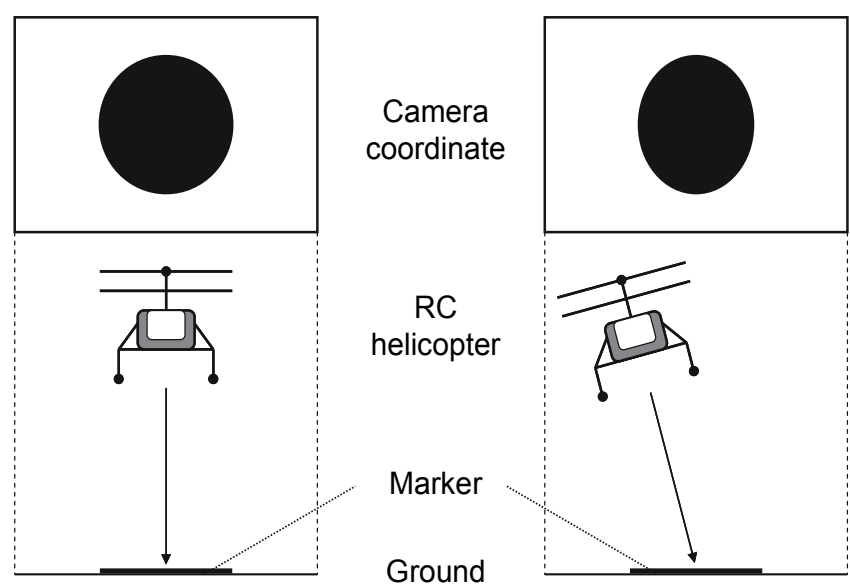

Fig. 14. Relation between attitude angle of RC helicopter and image in wireless camera.

\section{Calculation of a yaw angle}

The value of yaw angle can be calculated using the relation of positions between the center of circular marker image and the direction feature point of the circular marker image.

However, when the marker image is deforming into the ellipse, an exact value of the yaw angle cannot be got directly. The yaw angle has to be calculated after correcting the deformation of the circular marker. Since the length of the major axis of the ellipse does not change before and after the deformation of marker, the angle $\alpha$ between $x$ axis and the major axis can be correctly calculated even if the shape of the marker is not corrected.

As shown in Fig.15, the center of a marker is defined as point $P$, the major axis of a marker is defined as $P O$, and the intersection point of the perpendicular and $x$ axis which were taken down from Point $O$ to the $x$ axis is defined as $C$. The following equation is got if $\angle \mathrm{OPC}$ is defined as $\alpha^{\prime}$.

$$
\alpha^{\prime}=\arctan \left(\frac{O C}{P C}\right)
$$

Here, when the major axis exists in the 1st quadrant like Fig.15(a), $\alpha$ is equal to the value of $\alpha^{\prime}$, and when the major axis exists in the 2nd quadrant, $\alpha$ is calculated by subtracting $\alpha^{\prime}$ 
from 180 degrees like Fig.15(b). If the $x$-coordinate of Point $O$ is defined as $x O$, the value of $\alpha$ is calculated by the following equation.

$$
\alpha=\left\{\begin{array}{l}
\alpha^{\prime}\left(x_{o} \geq 0\right) \\
180-\alpha^{\prime}\left(x_{o}<0\right)
\end{array}\right.
$$

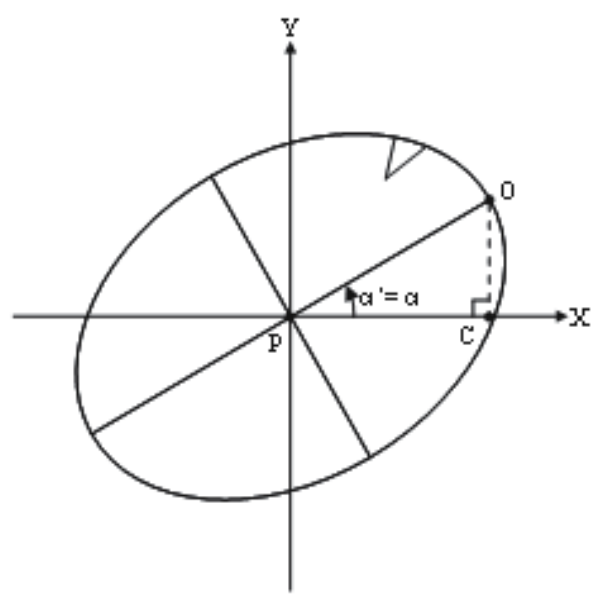

(a) When the major axis is in the 1st quadrant

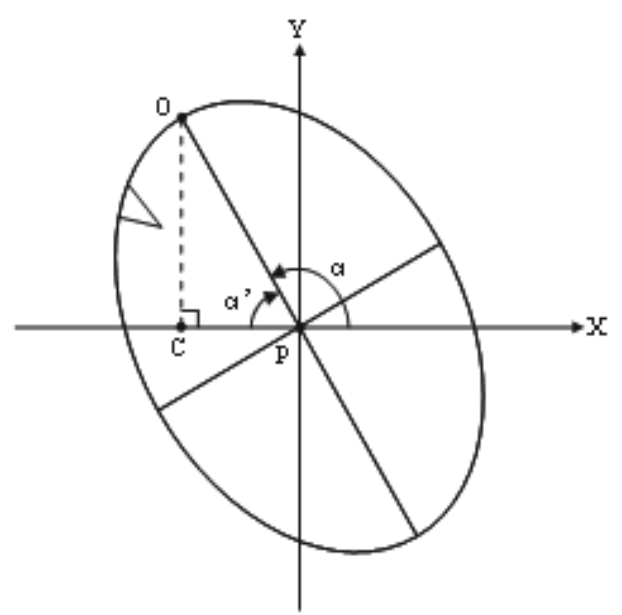

(b) When the major axis is in the 2nd quadrant

Fig. 15. An angle between the major axis and coordinate axes

Next, the angle $\gamma$ between the major axis and the direction of direction feature point is calculated. When taking a photograph from slant, a circular marker transforms and becomes an ellipse-like image, so the location of the cut part has shifted compared with the original location in the circular image. The marker is corrected to a right circle from an ellipse, and the angle is calculated after acquiring the location of original direction feature point. First, the value for deforming an ellipse into a right circle on the basis of the major axis of an ellipse is calculated. The major axis of an ellipse is defined as PO like Fig.16, and a minor axis is defined as $P Q$. The ratio $R$ of the major axis to a minor axis is calculated by the following equation.

$$
R=\frac{P O}{P Q}=\frac{G_{1}}{G_{2}}
$$

If this ratio multiplies along the direction of a minor axis, an ellipse can be transformed to a circle. The direction feature point of the marker in the ellipse is defined as $a$, and the point of intersection formed by taking down a perpendicular from Point $a$ to the major axis $P O$ is defined as $S$. If the location of the feature point on the circle is defined as $A$, point $A$ is on the point of intersection between the extended line of the segment $a S$ and a right circle.

Because $a S$ is a line segment parallel to a minor axis, the length of a line segment $a S$ is calculated by the following equations.

$$
A S=a S \times R
$$


When the line segment between Point $A$ and the center of the marker is defined as $P A$, the angle $r$ which the line segment $P A$ and the major axis $P O$ make is calculated by the following equations.

$$
\gamma=\arctan \frac{A S}{P S}
$$

Finally, a yaw angle is calculable by adding $\alpha$ to $\gamma$.

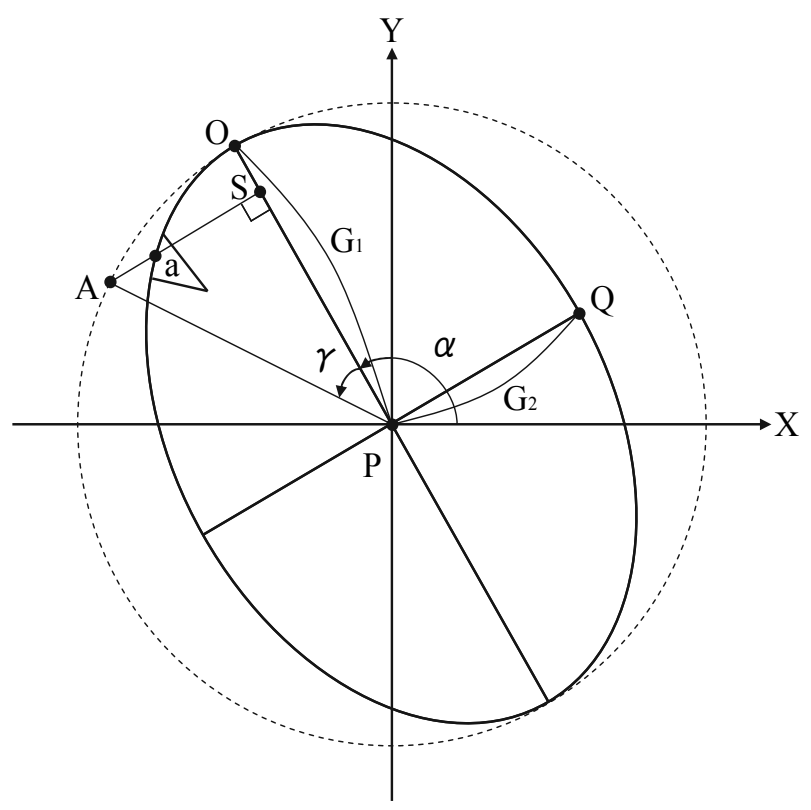

Fig. 16. An angle between the direction feature point and the major axis

\section{Calculation of pitch angle and roll angle}

By using the deformation rate of the marker in an image, a pitch angle and a roll angle can be calculated by performing coordinate transformation from a camera coordinate system to a world coordinate system. In order to get the pitch angle and rolling angle, we used a weak perspective projection for the coordinate transformation (Bao et al., 2003).

Fig.17 shows the principle of the weak perspective projection. The image of a plane figure which photographed the plane figure in a three-dimensional space by using a camera is defined as $I$, and the original configuration of the plane figure is defined as $T$. The relation between $I$ and $T$ is obtained using the weak perspective projection transformation by the following two steps projection.

a. $\quad T^{\prime}$ is acquired by a parallel projection of $T$ to $P$ paralleled to camera image surface $C$.

b. $\quad I$ is acquired by a central projection of $T^{\prime}$ to $C$.

The attitude angle $\beta^{\prime}$ is acquired using relation between $I$ and $T$. The angle $\beta^{\prime}$ shown in Fig.18 expresses the angle between original marker and the marker in the camera coordinate system. In that case, the major axis $G_{1}$ of the marker image and a minor axis $G_{2}$ of the marker image can show like Fig.19. 


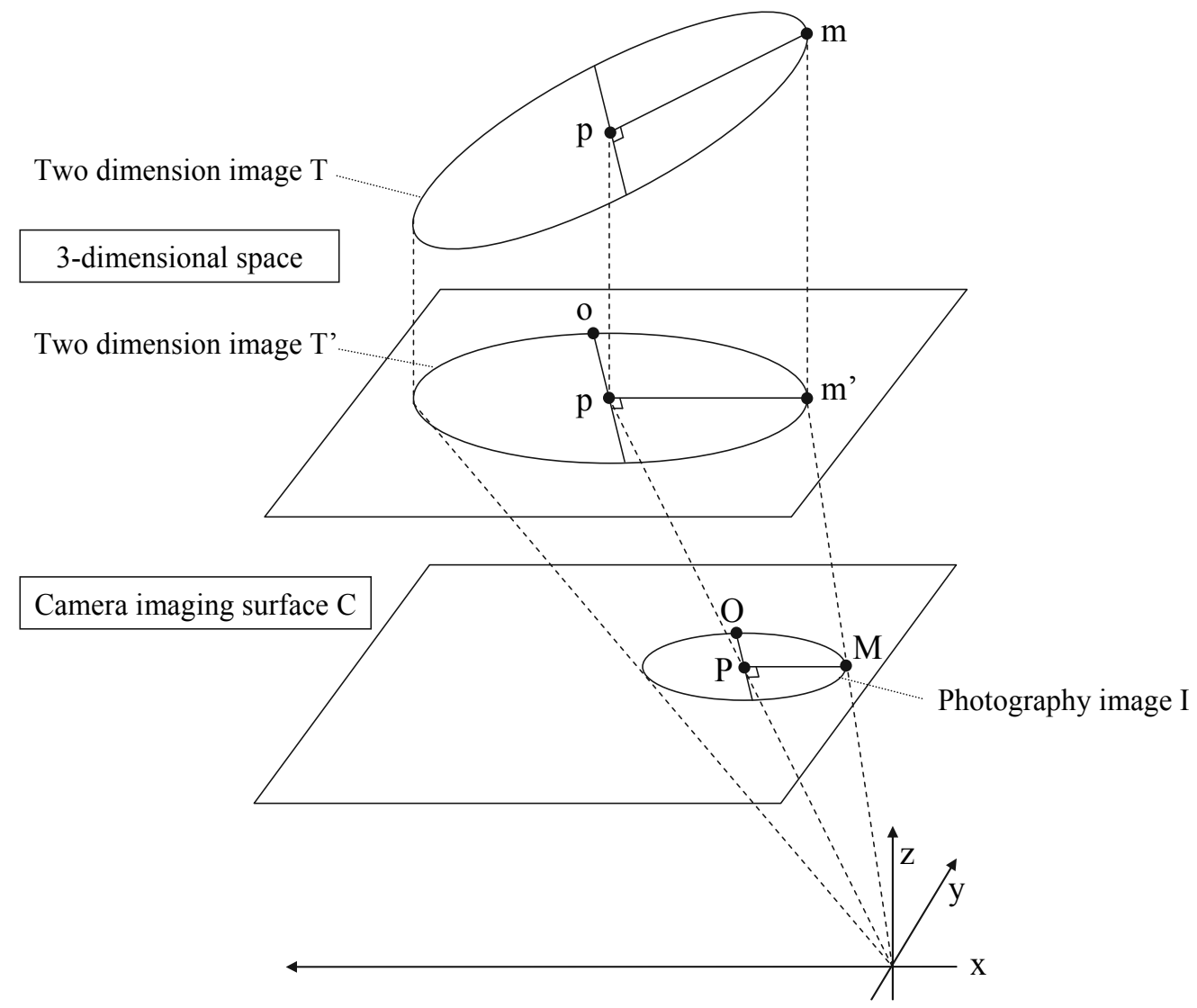

Fig. 17. The conceptual diagram of weak central projection

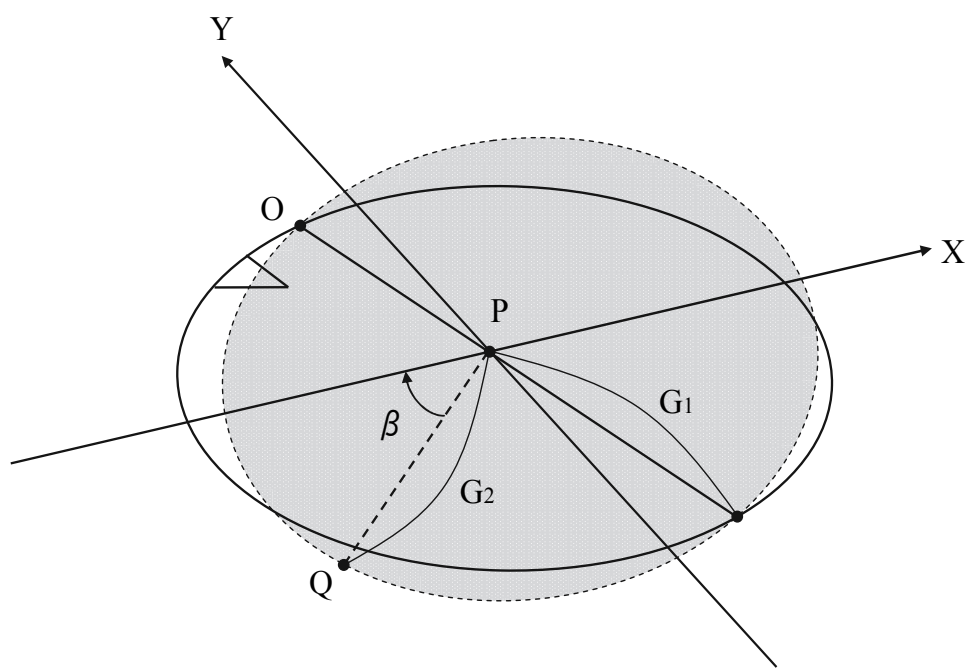

Fig. 18. The schematic diagram of the attitude angle $\beta^{\prime}$ 


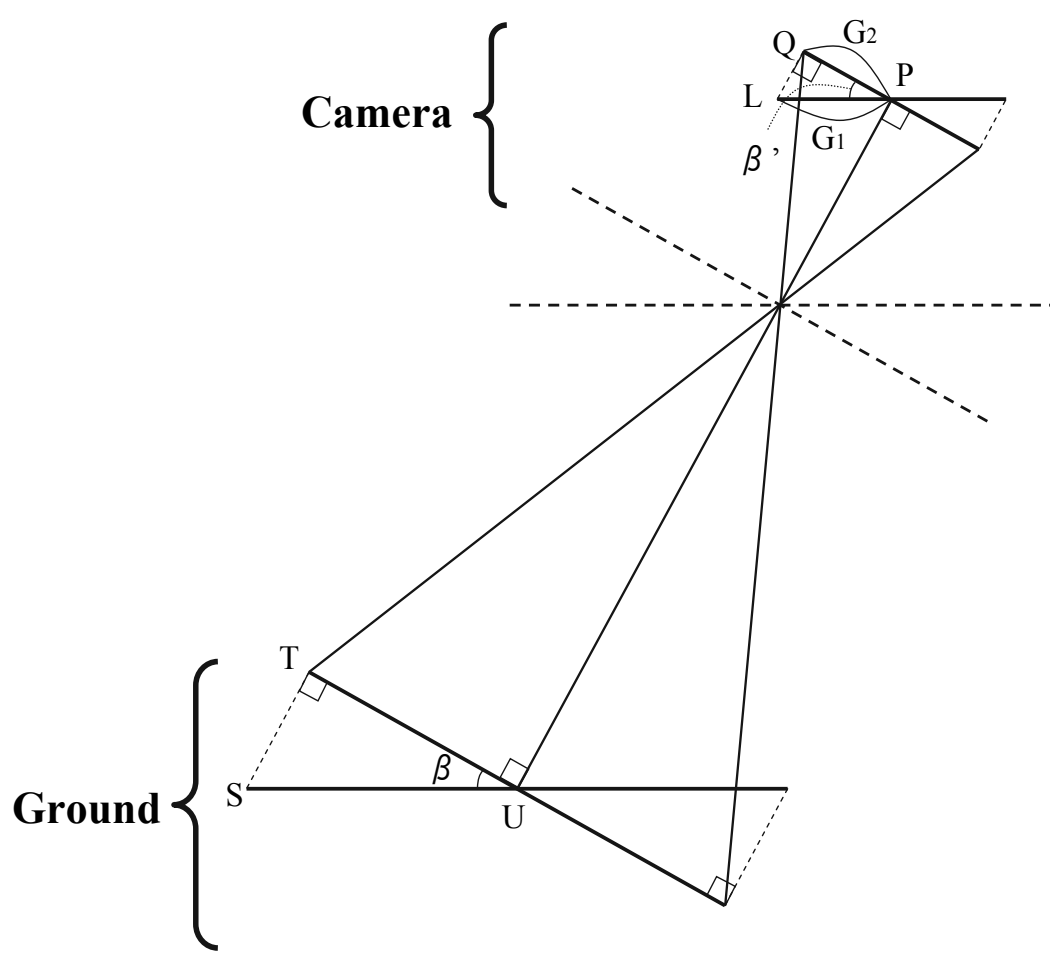

Fig. 19. Calculation of an attitude angle

Fig. 19 shows the calculation method of $\beta^{\prime}$. PQ is transformed into $L P$ if along optical axis of a camera an inverse parallel projection is performed to the minor axis $P Q$. Since the original configuration of a marker is a right circle, $L P$ becomes equal to the length $G_{1}$ of the major axis in a camera coordinate system. $\beta^{\prime}$ is calculated by the following equation.

$$
\beta^{\prime}=\arcsin \left(\frac{G_{2}}{G_{1}}\right)
$$

To get the segment $T U, S U$ is projected orthogonally on the flat surface parallel to $P Q$. $P Q$ and TU are in parallel relationship and $L P$ and $S U$ are also in parallel relationship.

Therefore, the relation between $\beta^{\prime}$ and $\beta$ can be shown by equation (18), and the inclination $\beta^{\prime}$ of the camera can be calculated by the equation (19).

$$
\begin{gathered}
\beta^{\prime}=\beta \\
\beta=\arcsin \left(\frac{G_{2}}{G_{1}}\right)
\end{gathered}
$$

\section{Control of RC helicopter}

Control of RC helicopter is performed based on the position and posture of the marker acquired by Section 4 . When RC helicopter is during autonomous hovering flight, the position 
data of RC helicopter are obtained by tracking the marker from definite height. The fuzzy rule of the Throttle control input signal during the autonomous flying is defined as follows.

- If $z(t)$ is $\mathrm{PB}$ and $\dot{z}(t)$ is $\mathrm{PB}$, Then Throttle is NB

- If $z(t)$ is $\mathrm{PB}$ and $\dot{z}(t)$ is $\mathrm{ZO}$, Then Throttle is NS

- If $z(t)$ is $\mathrm{PB}$ and $\dot{z}(t)$ is $\mathrm{NB}$, Then Throttle is $\mathrm{ZO}$

- If $z(t)$ is $Z O$ and $\dot{z}(t)$ is $\mathrm{PB}$, Then Throttle is NS

- If $z(t)$ is $\mathrm{ZO}$ and $\dot{z}(t)$ is $\mathrm{ZO}$, Then Throttle is $\mathrm{ZO}$

- If $z(t)$ is $Z O$ and $\dot{z}(t)$ is NB, Then Throttle is PS

- If $z(t)$ is NB and $\dot{z}(t)$ is $\mathrm{PB}$, Then Throttle is ZO

- If $z(t)$ is NB and $\dot{z}(t)$ is ZO, Then Throttle is PS

- If $z(t)$ is NB and $\dot{z}(t)$ is NB, Then Throttle is PB

The fuzzy rule design of Aileron, Elevator, and Rudder used the same method as Throttle. Each control input $u(t)$ is acquired from a membership function and a fuzzy rule. The adaptation value $\omega_{i}$ and control input $u(t)$ of a fuzzy rule are calculated from the following equations.

$$
\begin{gathered}
\omega_{i}=\prod_{k=1}^{n} \mu_{A k i}\left(x_{k}\right) \\
u(t)=\frac{\sum_{i=1}^{r} \omega_{i} c_{i}}{\sum_{i=1}^{r} \omega_{i}}
\end{gathered}
$$

Here, $i$ is the number of a fuzzy rule, $n$ is the number of input variables, $r$ is the quantity of a fuzzy rule, $\mu_{A k i}$ is the membership function, $x_{k}$ is the adaptation variable of a membership function, and $c_{i}$ is establishment of an output value (Tanaka, 1994) (Wang et al., 1997).

\section{Experiments}

In order to check whether parameter of a position and a posture can be calculated correctly, we compared actual measurement results with the calculation results by several experiments. The experiments were performed indoors. In the first experiment, a wireless camera shown in Fig.20 is set in a known three-dimensional position, and a marker is put on the ground like Fig.21.

The marker is photographed by this wireless camera. A personal computer calculated the position and the posture of this wireless camera and compared the calculated parameters with the actual parameters.

Table 1 shows the specification of the wireless camera and Table 2 shows the specification of the personal computer. A marker of $19 \mathrm{~cm}$ radius is used in experiments because it is considered that the marker of this size can be got easily when this type of wireless camera which has the resolution of 640x480 pixels photographs it at a height between $1 \mathrm{~m}$ and $2 \mathrm{~m}$.

Table 3 shows experimental results of $\mathrm{z}$ axis coordinates. Table 4 shows experimental results of moving distance. Table 5 shows experimental results of yaw angle $\left(\beta^{\prime}+\gamma\right)$. Table 6 shows experimental results of $\beta^{\prime}$ angle. According to the experimental results, although there are some errors in these computed results, these values are close to actual measurement. 


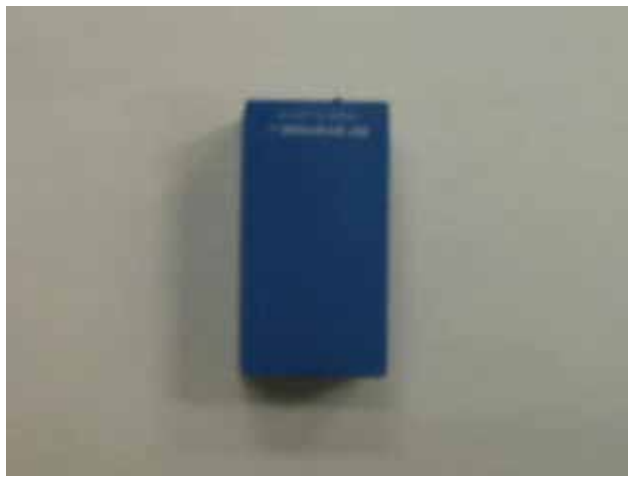

Fig. 20. The wireless camera

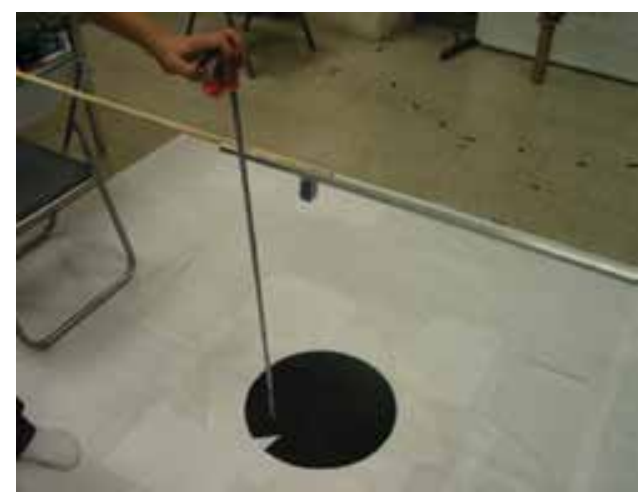

Fig. 21. The first experiment

\begin{tabular}{|l|l|}
\hline Maker & RF SYSTEM lab. \\
\hline Part number & Micro Scope RC-12 \\
\hline Image sensor & 270,000 pixel , $1 / 4$ inch, color CMOS \\
\hline Lens & $\varphi 0.8 \mathrm{~mm}$ Pin lens \\
\hline Scan mode & Interlace \\
\hline Effective distance & $30 \mathrm{~m}$ \\
\hline Time of charging battery & About 45 minutes \\
\hline Size & $15 \times 18 \times 35(\mathrm{~mm})$ \\
\hline Weight & $14.7 \mathrm{~g}$ \\
\hline
\end{tabular}

Table 1 . The specification of the wireless camera

\begin{tabular}{|l|l|}
\hline Maker & Hewlett Packard \\
\hline Model name & Compaq nx 9030 \\
\hline OS & Windows XP \\
\hline CPU & Intel Pentium M 1.60GHz \\
\hline Memory & $768 \mathrm{MB}$ \\
\hline
\end{tabular}

Table 2. The specification of PC 


\begin{tabular}{|l|l|l|l|l|}
\hline Actual distance $(\mathrm{mm})$ & 800 & 1000 & 1200 & 1400 \\
\hline Calculated value & 785 & 980 & 1225 & 1372 \\
\hline
\end{tabular}

Table 3. The experimental results of $\mathrm{z}$ axis coordinates

\begin{tabular}{|l|l|l|l|l|}
\hline Actual moving distance $(\mathrm{mm})$ & 50 & -50 & 100 & -100 \\
\hline Computed value of $x$ axis coordinates & 31 & -33 & 78 & -75 \\
\hline Computed value of y axis coordinates & 29 & -33 & 101 & -89 \\
\hline
\end{tabular}

Table 4. The experimental results of moving distance

\begin{tabular}{|l|l|l|l|l|}
\hline Actual degree (degree) & 45 & 135 & 225 & 315 \\
\hline Calculated value & 64 & 115 & 254 & 350 \\
\hline
\end{tabular}

Table 5. The experimental results of yaw angle ( $\alpha$ angle $+\gamma$ angle)

\begin{tabular}{|l|l|l|l|l|}
\hline Actual degree (degree) & 0 & 10 & 20 & 40 \\
\hline Calculated value & 12 & 28 & 36 & 44 \\
\hline
\end{tabular}

Table 6. The experimental results of $\beta$ angle

In next experiment, we attached the wireless camera on RC helicopter, and checked if parameters of a position and a posture would be calculated during the flight. Table 7 shows the specification of RC helicopter used for the experiment. A ground image like Fig.22 is photographed with the wireless camera attached at RC helicopter during the flight. The marker is detected by the procedures of Fig.9 using image processing program. A binarization was performed to the inputted image from the wireless camera and the outline on the marker was extracted like Fig. 23. The direction feature point was detected from the image of the ground photographed by the wireless camera like Fig.24.

Fig. 25 shows the measurement results on the display of a personal computer used for the calculation. The measurement values in Fig. 25 were $x$-coordinate $=319, y$-coordinate $=189$, z-coordinate $=837$, angle $\alpha=10.350105$, angle $\gamma=-2.065881$, and angle $\beta^{\prime}=37.685916$. Since our proposal image input method which can improve blurring was used, the position and the posture were acquirable during flight. However, since the absolute position and posture of the RC helicopter were not measureable by other instrument during the flight. We confirmed that by the visual observation the position and the posture were acquirable almost correctly.

\begin{tabular}{|l|l|}
\hline Length & $360 \mathrm{~mm}$ (Body), 62mm(Frame) \\
\hline Width & $90 \mathrm{~mm}$ \\
\hline Height & $160 \mathrm{~mm}$ \\
\hline Gross load & $195 \mathrm{~g}$ \\
\hline Diameter of a main rotor & $350 \mathrm{~mm}$ \\
\hline Gear ratio & $9.857: 1$ \\
\hline Motor & XRB Coreless Motor \\
\hline
\end{tabular}

Table 7. The specification of RC helicopter 


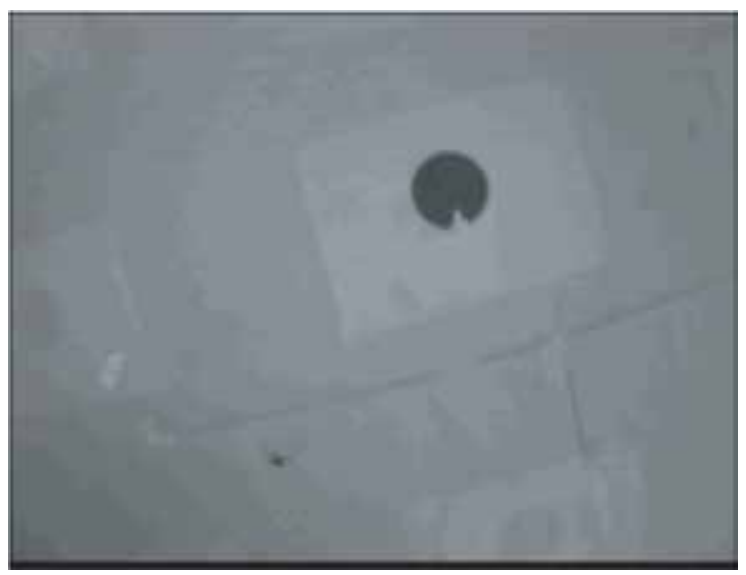

Fig. 22. An image photographed by the wireless camera

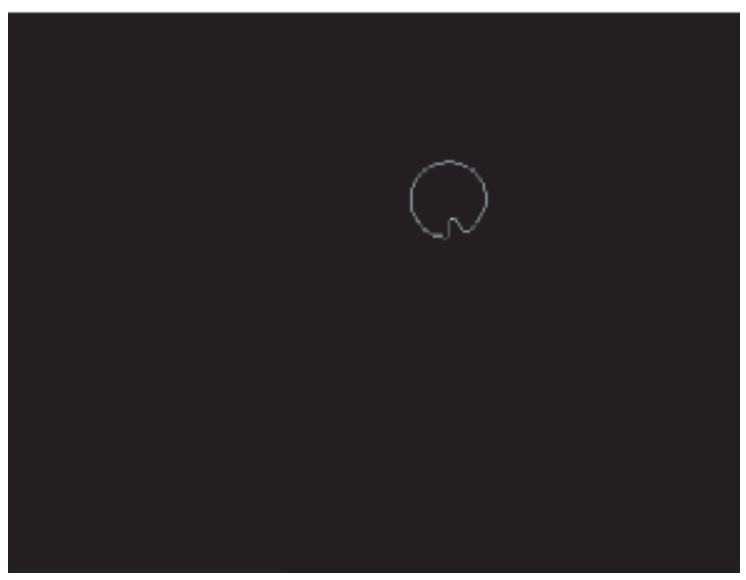

Fig. 23. The result of marker detection

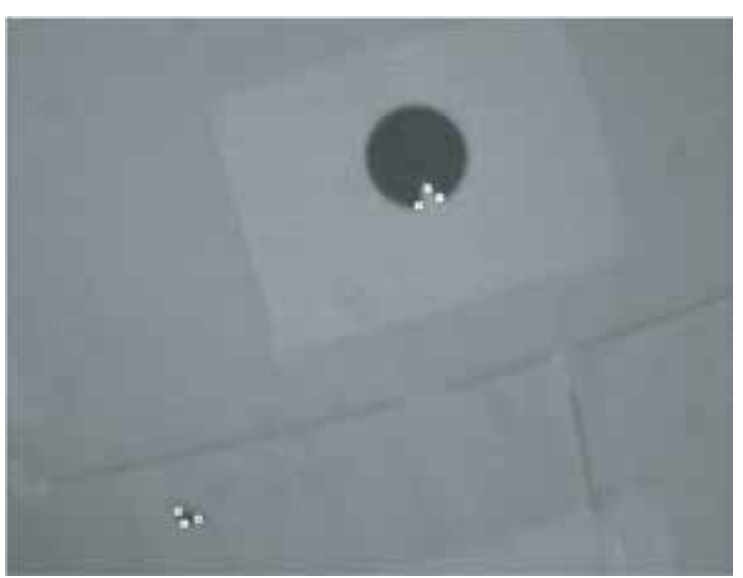

Fig. 24. The result of feature point extraction 


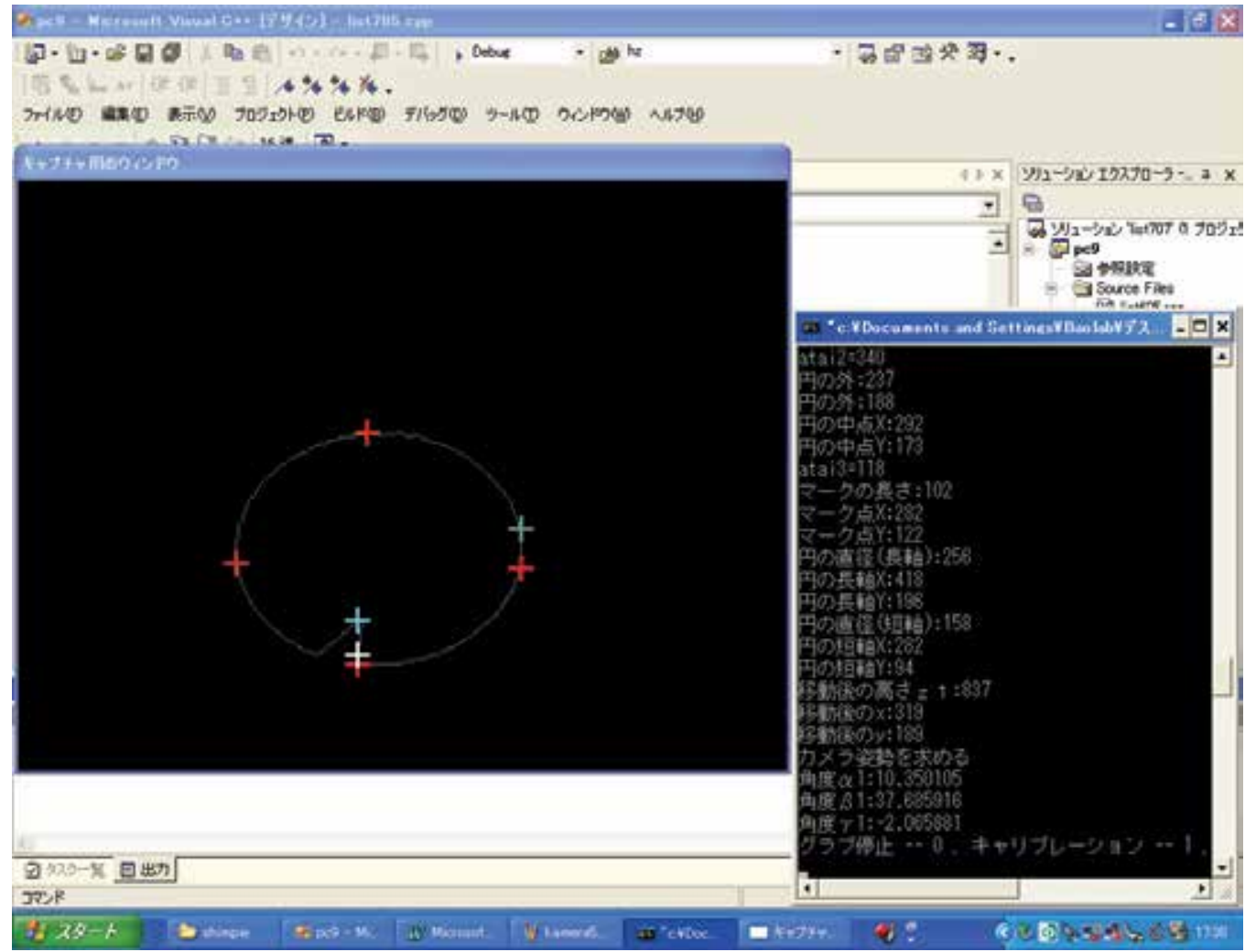

Fig. 25. The measurement results during flight

At the last, the autonomous flight control experiment of the RC helicopter was performed by detecting the marker, calculating the position and the posture,and fuzzy control. Fig. 26 shows a series of scenes of a hovering flight of the RC helicopter. The results of image processing can be checked on the display of the personal computer. From the experimental results, the marker was detected and the direction feature point was extracted correctly during the autonomous flight. However, when the spatial relation of the marker and the RC helicopter was unsuitable, the detection of position and posture became unstable, then the autonomous flight miscarried. We will improve the performance of the autonomous flight control for RC helicopter using stabilized feature point detection and stabilized position estimation.

\section{Conclusion}

This Chapter described an autonomous flight control for micro RC helicopter to fly indoors. It is based on three-dimensional measuring by a micro wireless camera attached on the micro RC helicopter and a circular marker put on the ground. First, a method of measuring the self position and posture of the micro RC helicopter simply was proposed. By this method, if the wireless camera attached on the RC helicopter takes an image of the circular marker, a major axis and a minor axis of the circular marker image is acquirable. Because this circular marker has a cut part, the direction of the circular marker image can be 


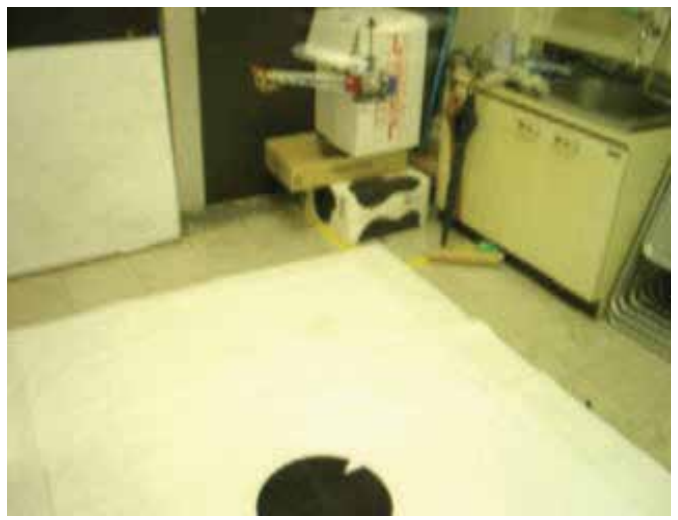

Time 1

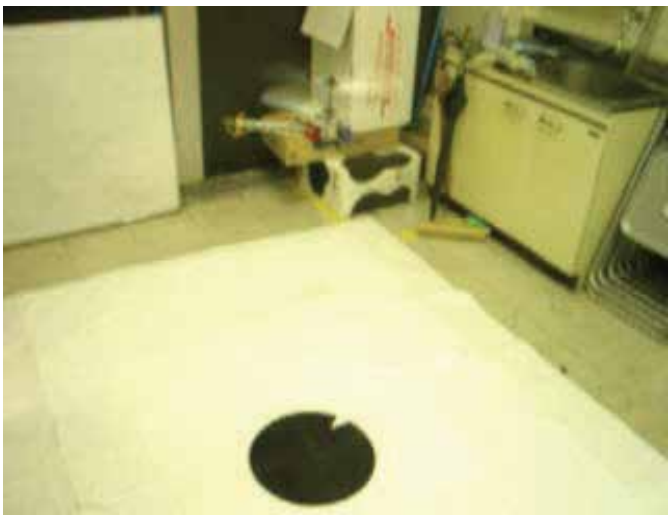

Time 3

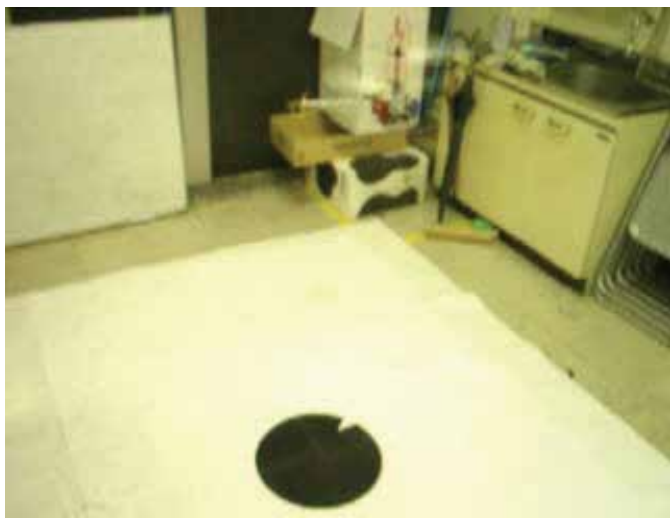

Time 2

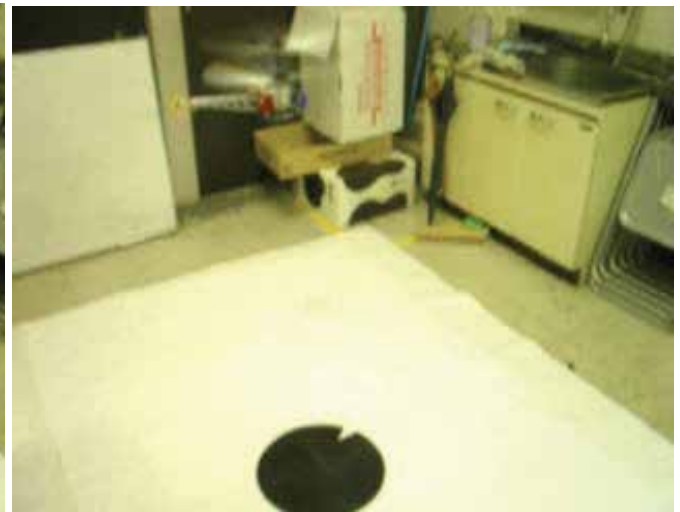

Time 4

Fig. 26. The experiment of autonomous flight

acquired by extracting the cut part as a direction feature point of the circular marker. Therefore, the relation between the circular marker image and the actual circular marker can be acquired by a coordinate transform using the above data. In this way, the threedimensional self position and posture of the micro RC helicopter can be acquired with image processing and weak perspective projection. Then, we designed a flight control system which can perform fuzzy control based on the three-dimensional position and posture of the micro RC helicopter. The micro RC helicopter is controlled by tracking the circle marker with a direction feature point during the flight.

In order to confirm the effectiveness of our proposal method, in the experiment, the position and the posture were calculated using an image photographed with a wireless camera fixed in a known three-dimensional position. By the experiment results, the calculated values near the actually measuring values were confirmed. An autonomous flight control experiment was performed to confirm that if our proposal image input method is effective when using a micro wireless camera attached on the micro RC Helicopter. By results of the autonomous flight control experiment of the RC helicopter, the marker was detected at real-time during the flight, and it was confirmed that the autonomous flight of the micro RC helicopter is possible. However, when the spatial relation of the marker and the RC helicopter was 
unsuitable, the detection of position and posture became unstable and then the autonomous flight miscarried. We will improve the performance of autonomous flight control of the RC helicopter to more stable. We will improve the system so that the performance of the autonomous flight control of the RC Helicopter may become stability more.

\section{Reference}

Amida, O.; Kanade, T. \& Miller, J.R. (1998). Vision-Based Autonomous Helicopter Research at Carnegie Mellon Robotics Institute 1991-1997, American Helicopter Society International Conf. Heli, Japan.

Harris, C. \& Stephens, M. (1988). A Combined Corner and Edge Detecter, Proc. 4th Alvey Vision Conf., pp.147-151.

Nakamura, S.; Kataoka, K. \& Sugeno, M. (2001). A Study on Autonomous Landing of an Unmanned Helicopter Using Active Vision and GPS, J.RSJ Vol.18, No.2, pp.252-260.

Neumann, U. \& You, S. (1999). Natural Feature Tracking for Augmented-reality, IEEE Transactions on Multimedia, Vo.1, No.1, pp.53-64.

Ohtake, H.; Iimura, K. \& Tanaka, K. (2009). Fuzzy Control of Micro RC Helicopter with Coaxial Counter-rotating Blades, journal of Japan Society for Fuzzy Theory and Intelligent Informatics, Vol.21, No.1, pp.100-106.

Schmid, C.; Mohr, R. \& Bauckhage, C. (1998). Comparing and Evaluating Interest Points, Proc. 6th Int. Conf. on Computer Vision, pp.230-235.

Shi, J. \& Tomasi, C. (1994). Good Features to Track, Proc. IEEE Conf. Comput. Vision Patt. Recogn., pp.593-600.

Smith, S. M.; \& Brady, J. M. (1997). SUSAN - A New Approach to Low Level Image Processing, Int. J. Comput. Vis., vol.23, no.1, pp.45-78.

Sugeno, M. et al. (1996). Inteligent Control of an Unmanned Helicopter based on Fuzzy Logic., Proc. of American Helicopter Society 51st Annual Forum., Texas.

Tanaka, K. (1994). Advanced Fuzzy Control, Kyoritsu Shuppan Co.,LTD, Japan.

Wang, G.; Fujiwara, N. \& Bao, Y. (1997). Automatic Guidance of Vehicle Using Fuzzy Control. (1st Report). Identification of General Fuzzy Steering Model and Automatic Guidance of Cars., Systems, Control and Information, Vol.10, No.9, pp.470479.

Bao, Y.; Takayuki, N. \& Akasaka, H. (2003). Weak Perspective Projection Invariant Pattern Recognition without Gravity Center Calculation, journal of IIEEJ, Vol.32, No.5, pp.659--666

Bao, Y. \& Komiya, M. (2008). An improvement Moravec Operator for rotated image, Proc. of the ADVANTY 2008 SYMPOSIUM , pp.133-138. 


\title{
Hierarchical Control Design of a UAV Helicopter
}

\author{
Ali Karimoddini, Guowei Cai, Ben M. Chen, Hai Lin, Tong H. Lee \\ Graduate School for Integrative Sciences and Engineering (NGS) and Department of \\ Electrical and Computer Engineering, National University of Singapore \\ Singapore
}

\section{Introduction}

Nowadays, control design of Unmanned Aerial Vehicles (UAVs) has emerged as an attractive research area, due to the wide range of UAV applications in various military and civilian areas such as terrain and utility inspections, coordinated surveillance, search and rescue missions, disaster monitoring, rapid emergency response, aerial mapping, traffic monitoring, and reconnaissance missions (see, e.g., (Metni et al., 2007), (Kuroki et al., 2010 ), (Campbell \& Campbell, 2010 )). They can also be used as complex test-bed dynamic systems for implementation and verification of the control schemes for different research purposes (Kim \& Sukkarieh, 2007), (Saripalli et al., 2003), (Bortoff, 1999). Several research groups are involved in the modeling and control of UAVs (Bortoff, 1999), (Gavrilets et al., 2000), (Cai et al., 2006). The control methods such as the neural network approach (Enns \& Si, 2003), the differential geometry method (Isidori et al., 2003), feedback control with decoupling approach (Peng et al., 2009), and the model predictive approach (Shim et al., 2003) have been applied for the flight control of the UAV helicopters. In this chapter, however, we have used an analytical approach to design and analyze the whole system including the inner-loop and the outer-loop controllers for a small-scale UAV helicopter. Here, in the proposed hierarchical structure, the inner-loop is responsible for the internal stabilization of the UAV in the hovering state and control of the linear velocities and heading angle angular velocity whereas the outer-loop is used to drive the system, which is already stabilized by the inner-loop, to follow a desired path while keeping the system close to the hovering state. This hierarchical strategy is an intuitive way of controlling such a complex system. However, there is another reason that compels us to employ such a control structure. Indeed, the UAV model cannot be fully linearized, since, in practice, we cannot expect the heading angle of the UAV to be restricted to a small range of variation as depending on the mission, the heading of the UAV could be in any direction. This will impose some kind of nonlinearity on the system, which can be modeled by a simple transformation. To handle this semi-linearized model of the UAV, we can separate the linear and nonlinear parts, and then control the linear part in the inner-loop and the nonlinear part in the outer-loop.

In this hierarchy, for the inner-loop, we have used an $H_{\infty}$ controller to both stabilize the system and suboptimally achieve the desired performance of the UAV attitude control. Assuming that the inner-loop has already been stabilized by an $H_{\infty}$ controller, a proportional feedback controller combined with a nonlinear compensator block have been used in the outer-loop to bring the UAV into the desired position with desired heading angle. 
Although designing a proportional feedback controller for SISO systems is straightforward, the situation for MIMO systems is different. This is because, in MIMO systems, it is not easy to use the popular tools, such as the Nyquist stability theorem or the root-locus approach, that are well-established for the SISO systems. The current approaches employed for MIMO systems are rather complicated and are mostly extensions of the existing results for SISO systems (Wang et al., 2007). In this chapter, we propose a design method of a decentralized P-controller for MIMO systems that, although conservative, can be effectively used in practical problems, particularly for the case that the system is close to a decoupled system. The approach is an extension of the Nyquist theorem to MIMO systems, and its application to the NUS UAV system provides a successful flight controlled system.

The test-bed is Helion (Fig. 1), the first developed UAV helicopter in our NUS UAV research group (Peng et al., 2007). In (Cai et al., 2008a), a systematic procedure for the construction of a small-scale UAV helicopter is described, and in (Cai et al., 2005), the hardware parts of the NUS UAV, including both the avionic system and the ground station, are illustrated in detail.

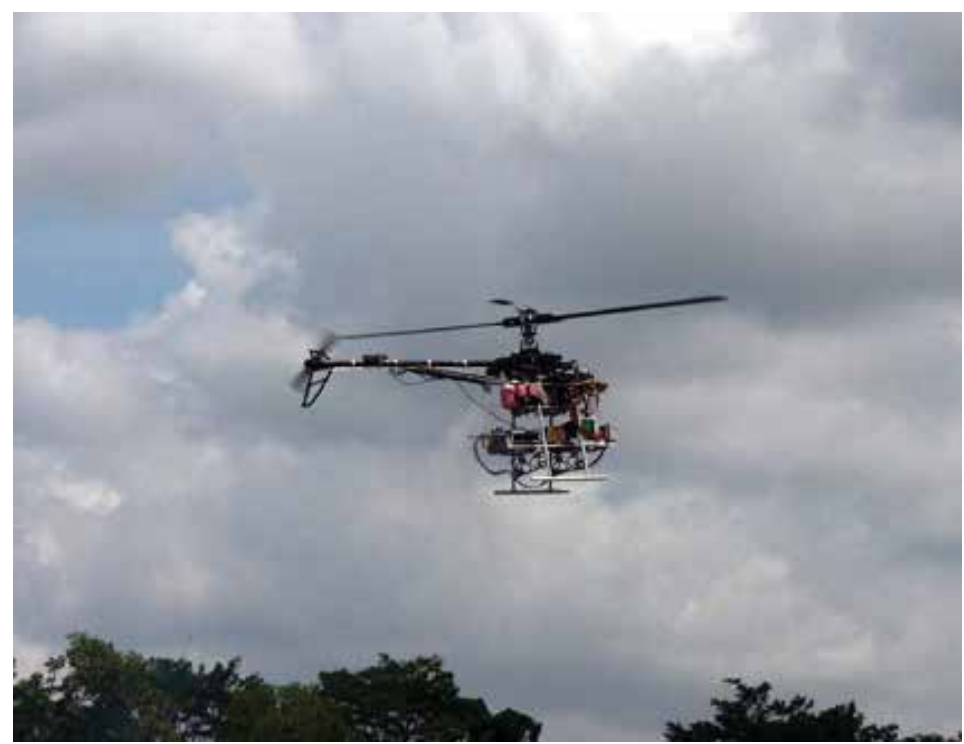

Fig. 1. The NUS UAV helicopter.

The remaining parts of this chapter are organized as follows. In Section II, the model and the structure of the NUS UAV is described. The UAV model consists of two decoupled subsystems. In Section III, a hierarchical controller, including an inner-loop and an outer-loop controller, is designed for both subsystems. Actual flight tests are presented in Section IV, and the chapter is concluded in Section V. For the convenience of the reader, a nomenclature part is provided at the end of this chapter.

\section{Modeling and structure of the UAV helicopter}

A typical UAV helicopter consists of several parts: physical parts such as engine and fuselage; ground station to monitor the flight situation and collect realtime flight data, and the avionic system to implement the control strategy to have an autonomous flight control. Among these elements, the avionic part is in the center of our interest in this chapter and we will focus on the control structure which is embedded in the airborne computer system. Here, the avionic 
system consists of an airborne computer system which can be extended modularly by some extension boards such as $\mathrm{A} \backslash \mathrm{D}$ card, DC-DC convertor card, and serial communication board. In addition, the avionic system has been equipped with some analog and digital sensors to collect the information of the current state of the UAV. The major sensor used in the avionic system, is the NAV-IMU sensor. The IMU sensor provides three axis velocities, acceleration and angular rates in the body frame, as well as longitude, latitude, relative height and heading, pitch and roll angles. Moreover, the avionic system has a fuel level sensor as well as a magnetic RPM sensor to measure the speed of the rotor. Furthermore, it comprises five servo actuators that could manipulate the helicopter to move forward and backward, up and down, to turn left and right, to regulate the nose angle and finally to control the spinning speed of the rotor. All of these servos are controlled by a servo board as a local controller. In addition, the servo board gives the ability of driving the servo system into either the manual mode or the automatic mode. In the manual mode, a pilot can drive the helicopter by a radio controller which is useful in the emergency situations; however, in the automatic mode the helicopter is under the control of the computer system and all control signals are generated by the avionic system and the computer board, autonomously.

Using some basic physical principles, we can obtain a general nonlinear UAV model. These principles will result in several equations that represent the effects of different factors such as gravity, the main rotor, and tail rotor forces and moments. The model equations will be obtained in two coordinate systems: the body frame and the ground frame. The body frame is centered at the center of gravity of the UAV, and the ground frame is an NED (North - East - Down) coordinate system (Stevens \& Lewis, 1992) with a fixed origin at the starting point of the UAV flight. Clearly, the UAV dynamic equations should be derived in the body frame, while the position of the UAV is considered in the ground frame.

Neglecting the gyroscopic effect of the engine-driven train, the equations of the helicopter motion in the body frame are obtained as follows:

$$
\begin{gathered}
\dot{\vec{V}}_{b}=-\vec{\omega}_{b} \times \vec{V}_{b}+B_{b} \vec{g}+m^{-1} \vec{F} \\
\dot{\vec{\omega}}_{b}=-J^{-1} \omega_{b} \times J \vec{\omega}_{b}+J^{-1} \vec{M}
\end{gathered}
$$

where in these equations, $\times$ denotes the cross product of the vectors, and the concatenation of two matrices or vectors represents the normal matrix multiplication. Moreover, $\vec{F}$ and $\vec{M}$ are the resultant force and moment in the body frame, including those generated from the main rotor, the tail rotor and the fuselage. Other symbols' definition can be found in the nomenclature part provided at the end of this chapter.

The Euler angles that show the orientation of the body frame relative to the ground frame are as follows:

$$
\left[\begin{array}{c}
\dot{\phi} \\
\dot{\theta} \\
\dot{\psi}
\end{array}\right]=\left[\begin{array}{ccc}
1 & \tan \theta \sin \phi & \tan \theta \cos \phi \\
0 & \cos \phi & -\sin \phi \\
0 & \frac{\sin \phi}{\cos \theta} & \frac{\cos \phi}{\cos \theta}
\end{array}\right] \dot{\vec{\omega}}_{b}
$$

where $(\phi \theta \psi)^{\prime}$ is a vector that contains the Euler angles to describe the attitude of the helicopter with respect to the NED frame.

The relation between the UAV position in the ground frame and the UAV velocity in the body frame is:

$$
\dot{\vec{P}}_{g}=\dot{B}_{b} \vec{V}_{b}
$$


where $B_{b}$ is the transformation matrix from the ground frame to the body frame, which has the following form:

$$
B_{b}=\left[\begin{array}{ccc}
\cos \theta \cos \psi & \cos \theta \sin \psi & -\sin \theta \\
-\cos \phi \sin \psi+\sin \phi \sin \theta \cos \psi & \cos \phi \cos \psi+\sin \phi \sin \theta \sin \psi & \sin \phi \cos \theta \\
\sin \phi \sin \psi+\cos \phi \sin \theta \cos \psi & -\sin \phi \cos \psi+\cos \phi \sin \theta \cos \psi & \cos \phi \cos \theta
\end{array}\right]
$$

The details of this UAV model are described in (Peng et al., 2007). From the above model description, it can be seen that the UAV model is nonlinear. Furthermore, the main problem encountered in the modeling of our UAV is that the process of buying a radio-control helicopter from the market and upgrading it to an autonomous flying vehicle leaves us with many unknown parameters and aerodynamic data. Therefore, for practical reasons, we need to linearize the UAV model and then, identify the resulting linearized model through the recorded in-flight data. The in-flight data can be collected through the manual mode and by injecting perturbed input signals to the flying helicopter. We have obtained a linearized model at the hovering state as it has been presented in (Cai et al., 2006). By hovering, we mean that $\vec{V}_{0}=0, \vec{\omega}_{0}=0, \theta_{0}=0, \phi_{0}=0$. The obtained linearized model is as follows:

$$
\dot{x}=A x+B u+E w
$$

where the state variable $x$ includes 11 variables as $x=\left[V_{z_{b}}(\mathrm{~m} / \mathrm{s}) \omega_{z_{b}}(\mathrm{rad} / \mathrm{s}) w_{z f}(\mathrm{rad} / \mathrm{s})\right.$ $\left.V_{x_{b}}(\mathrm{~m} / \mathrm{s}) V_{y_{b}}(\mathrm{~m} / \mathrm{s}) \omega_{x_{b}}(\mathrm{rad} / \mathrm{s}) \omega_{y_{b}}(\mathrm{rad} / \mathrm{s}) \phi(\mathrm{rad}) \theta(\mathrm{rad}) \tilde{a}_{s}(\mathrm{rad}) \tilde{b}_{s}(\mathrm{rad})\right]^{\prime}$. These parameters are shown in Fig. 2. $w_{z f}$ is the yaw rate feedback, which is related to $\delta_{\text {pedal }}$ by a first-order differential equation (Cai et al., 2008b).

Furthermore, the control input $u$ includes commands to the servos embedded for the control of the helicopter blades as $u=\left[\delta_{\text {col }}(\mathrm{rad}) \delta_{\text {pedal }}(\mathrm{rad}) \delta_{\text {roll }}(\mathrm{rad}) \delta_{\text {pitch }}(\mathrm{rad})\right]^{\prime}$. Matrices $A, B$, and $E$ are obtained as follows:

$$
A=\left[\begin{array}{cc}
A_{1} & 0_{3 \times 8} \\
0_{8 \times 3} & A_{2}
\end{array}\right], B=\left[\begin{array}{cc}
B_{1} & 0_{3 \times 2} \\
0_{8 \times 2} & B_{2}
\end{array}\right], E=\left[\begin{array}{cc}
E_{1} & 0_{3 \times 2} \\
0_{8 \times 1} & E_{2}
\end{array}\right]
$$

$$
\begin{aligned}
& A_{1}=\left[\begin{array}{ccc}
-0.6821 & -0.1070 & 0 \\
-0.1446 & -5.5561 & -36.6740 \\
0 & 2.7492 & -11.1120
\end{array}\right], B_{1}=\left[\begin{array}{cc}
15.6491 & 0 \\
1.6349 & -58.4053 \\
0 & 0
\end{array}\right] \text {, } \\
& E_{1}=\left[\begin{array}{c}
-0.5995 \\
-1.3832 \\
0
\end{array}\right], B_{2}=\left[\begin{array}{cc}
0 & 0 \\
0 & 0 \\
0 & 0 \\
0 & 0 \\
0 & 0 \\
0 & 0 \\
0.0496 & 2.6224 \\
2.4928 & 0.1740
\end{array}\right], E_{2}=\left[\begin{array}{cc}
-0.1778 & 0 \\
0 & -0.3104 \\
-0.3326 & -0.2051 \\
0.0802 & -0.2940 \\
0 & 0 \\
0 & 0 \\
0 & 0 \\
0 & 0
\end{array}\right] \text {, } \\
& A_{2}=\left[\begin{array}{cccccccc}
-0.1778 & 0 & 0 & 0 & 0 & -9.7807 & -9.7808 & 0 \\
0 & -0.3104 & 0 & 0 & 9.7807 & 0 & 0 & 9.7807 \\
-0.3326 & -0.5353 & 0 & 0 & 0 & 0 & 75.7640 & 343.86 \\
-0.1903 & -0.2940 & 0 & 0 & 0 & 0 & 172.620 & -59.958 \\
0 & 0 & 1 & 0 & 0 & 0 & 0 & 0 \\
0 & 0 & 0 & 1 & 0 & 0 & 0 & 0 \\
0 & 0 & 0 & -1 & 0 & 0 & -8.1222 & 4.6535 \\
0 & 0 & -1 & 0 & 0 & 0 & -0.0921 & -8.1222
\end{array}\right] .
\end{aligned}
$$

Remark 1. In the linearized model described by (6), the saturation level for the servos is $\left|\delta_{\star_{\max }}\right|=0.5$. We need to provide a control law such that the resulting control signals always remain within the linear unsaturated range. 


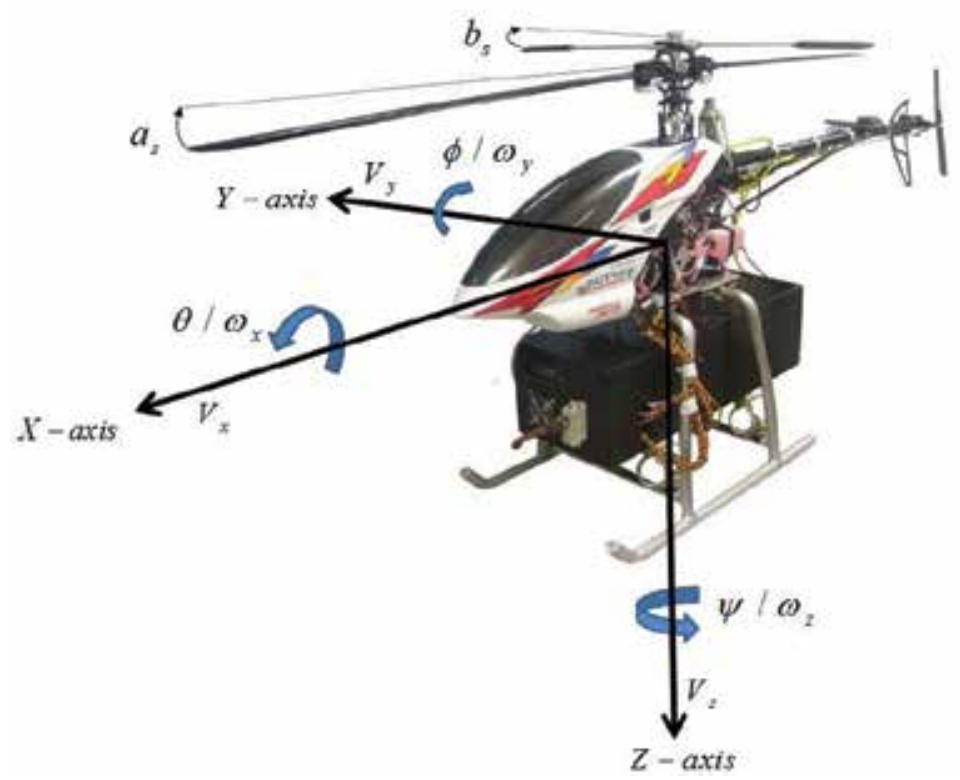

Fig. 2. Helicopter states in the body frame.

Although (6) describes the relation between the control input and the state variable $x$, it still does not describe the whole dynamics of the system, and particularly, $\vec{P}$ and $\varphi$ are not reflected in the model. Thus, considering (2) and (4), a more complete model containing $\vec{P}$ and $\varphi$ is as follows:

$$
\left\{\begin{array}{l}
\dot{x}=A x+B u+E w \\
\dot{\psi}=\omega_{z_{b}} \\
\dot{\vec{P}}_{g}=\dot{B}_{b} \vec{V}_{b}
\end{array}\right.
$$

Remark 2. Matrix $B_{b}$ includes some time-dependent terms. Therefore, matrix $B_{b}$ can not be considered as a constant term and it is not simple to integrate both sides of (7) in order to obtain the position in the ground frame. This is due to the fact that the body frame is a moving coordinate system. Hence, to obtain the displacement, it is necessary to first obtain the velocities in a fixed coordinate system such as the ground frame. Then, the displacement can be calculated by integrating of the velocity vector in the fixed coordinate system.

The presence of nonlinear terms of $B_{b}$, in the third equation of (7), makes it difficult to design a controller for the system; however, we can further simplify the model. Indeed, matrix $B_{b}$ in (5), which introduces some nonlinear terms to the model, can be linearized at the hovering state. In practice, the heading angle of the helicopter can take any arbitrary value; however, the roll and pitch angles are usually kept close to the hovering condition. Therefore, linearizing matrix $B_{b}$ at the hovering state will result in:

$$
B_{b}=\left[\begin{array}{ccc}
\cos \psi & \sin \psi & 0 \\
-\sin \psi & \cos \psi & 0 \\
0 & 0 & 1
\end{array}\right]=\left[\begin{array}{cc}
R & \mathbf{0}_{\mathbf{2} \times \mathbf{1}} \\
\mathbf{0}_{\mathbf{1} \times \mathbf{2}} & 1
\end{array}\right]
$$

The physical interpretation is that by keeping $\vec{\theta}$ and $\vec{\phi}$ close to zero, the Euler rotation in a three-dimensional space will be converted into a simple rotation in a two-dimensional space 
with respect to $\psi$. In this case, the rotation matrix is:

$$
R=\left[\begin{array}{cc}
\cos \psi & \sin \psi \\
-\sin \psi & \cos \psi
\end{array}\right]
$$

In the following section, as we design the outer-loop controller, it will be shown that this new formulation of $B_{b}$ helps us to keep the system decoupled, even after using the outer-loop controller.

The semi-linearized UAV model, presented in (7) can be controlled in separate parts: the linear part in the inner-loop and the nonlinear part in the outer-loop, as described in the following section.

\section{Controller design}

We use a hierarchical approach to design a controller for the UAV (Fig. 3). In this framework, the system is stabilized in the inner-loop, and then it is driven to track a desired trajectory in the outer-loop. Besides this rational strategy, there is another reason that compels us to select this particular architecture. As mentioned previously, we need to derive the model equations in two coordinate systems: the velocities and accelerations should be obtained in the body coordinate system as a moving frame, whereas the displacement must be derived by the integration of the velocities in a fixed frame. The velocity transformation from the body frame to the ground frame, modeled by matrix $B_{b}$, imposes some kind of nonlinearity as described in (7). This nonlinearity can be handled in the outer-loop. Using this control strategy, we have separated the nonlinear term from the linear part and put it in the outer-loop.

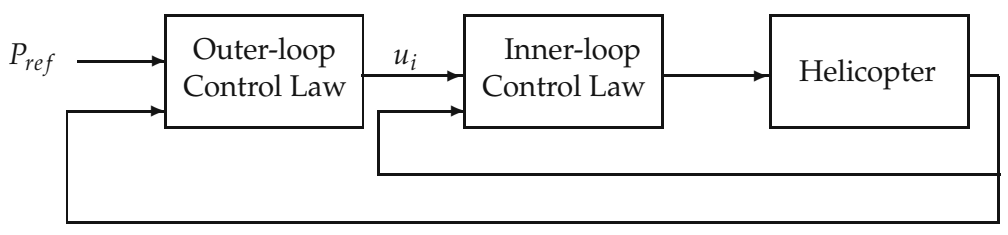

Fig. 3. Schematic diagram of the flight control system.

In this control architecture, the references for the inner-loop controller, $u_{i}$, are the linear velocities $\left(V_{x_{b}}, V_{y_{b}}\right.$, and $\left.V_{z_{b}}\right)$ and the yaw rate, $\omega_{z_{b}}$, which should all be provided by the outer-loop. The outer-loop, however, is responsible for the control of the position and heading angle of the UAV and will guide the UAV to follow a desired trajectory. Therefore, the references for the outer-loop are the position $\left(X_{r}, Y_{r}, Z_{r}\right)$ and the yaw angle $\psi_{r}$. In other words, the UAV will follow the generated path by the position and the yaw angle control in the outer-loop, and the linear velocity and the angular rate control in the inner-loop.

looking at matrices $A, B$, and $E$ in (6), we can see that, the system has been already decoupled into two independent parts. Therefore, (6) can be rewritten into two separate subsystems as follows:

$$
\begin{aligned}
& \dot{x}_{1}=A_{1} x_{1}+B_{1} u_{1}+E_{1} w \\
& \dot{x}_{2}=A_{2} x_{2}+B_{2} u_{2}+E_{2} w
\end{aligned}
$$

where $x_{1}=\left[V_{z_{b}}(\mathrm{~m} / \mathrm{s}) \omega_{z_{b}}(\mathrm{rad} / \mathrm{s}) w_{z f}(\mathrm{rad} / \mathrm{s})\right]^{\prime}, u_{1}=\left[\delta_{\text {col }} \delta_{\text {pedal }}\right]^{\prime}, x_{2}=\left[V_{x_{b}}(\mathrm{~m} / \mathrm{s}) V_{y_{b}}(\mathrm{~m} / \mathrm{s})\right.$ $\left.\omega_{x_{b}}(\mathrm{rad} / \mathrm{s}) \omega_{y_{b}}(\mathrm{rad} / \mathrm{s}) \phi(\mathrm{rad}) \theta(\mathrm{rad}) \tilde{a}_{s}(\mathrm{rad}) \tilde{b}_{s}(\mathrm{rad})\right]^{\prime}$, and $u_{2}=\left[\delta_{\text {roll }}(\mathrm{rad}) \delta_{\text {pitch }}(\mathrm{rad})\right]^{\prime}$. 
Considering (7), (8), (10), and (11), the above-mentioned hierarchical control strategy can be implemented for the decoupled model of our UAV, as shown in Fig. 4 and Fig. 5. In these figures, subscripts $g, b$, and $r$ stand for ground frame, body frame, and reference, respectively. Moreover, matrices $C_{1}$ and $C_{2}$ are:

$$
C_{1}=\left[\begin{array}{lll}
1 & 0 & 0 \\
0 & 1 & 0
\end{array}\right], C_{2}=\left[\begin{array}{llllllll}
1 & 0 & 0 & 0 & 0 & 0 & 0 & 0 \\
0 & 1 & 0 & 0 & 0 & 0 & 0 & 0
\end{array}\right]
$$

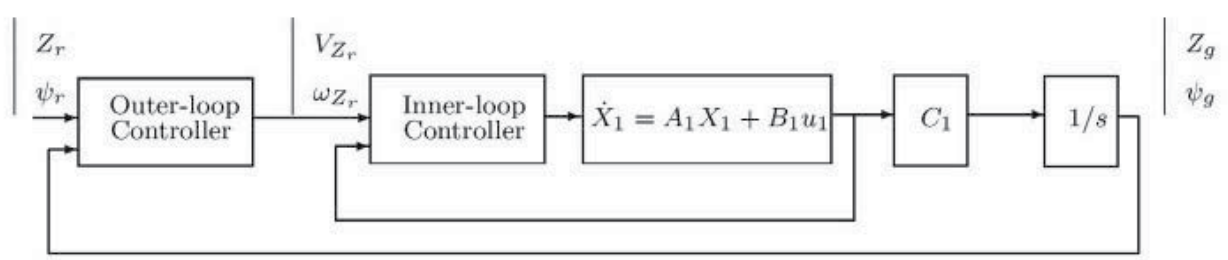

Fig. 4. Control schematic for Subsystem 1.

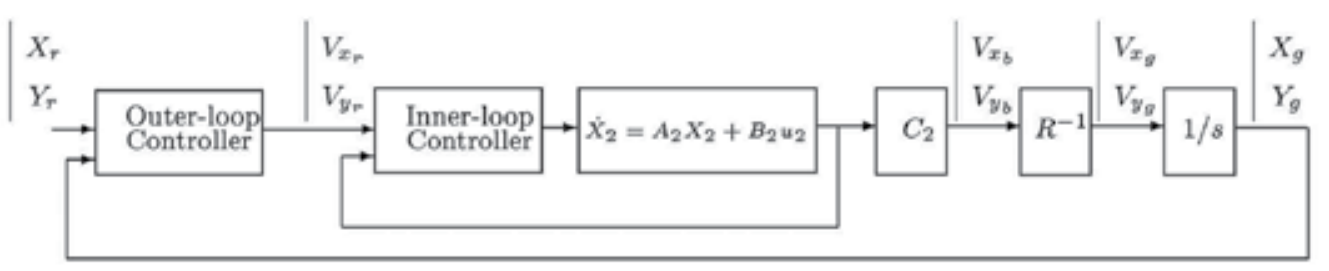

Fig. 5. Control schematic for Subsystem 2.

Due to the special structure of the linearized form of $B_{b}$, Subsystem 1 is a fully linearized model. However, in the outer-loop of Subsystem 2, the term $R^{-1}$ appears as a nonlinear element, and thus Subsystem 2 is more complicated than Subsystem 1. In the following parts, we will describe the control design for both subsystems.

\subsection{Controller for subsystem 1}

Subsystem 1 is a fully linearized model so that linear design tools can be applied to the system. We will use the $H_{\infty}$ control design technique for the inner-loop and a P-controller for the outer-loop.

\subsubsection{Inner-loop controller}

Using an $H_{\infty}$ controller for the inner-loop, both robust stability and proper performance of the system can be achieved simultaneously. To design the $H_{\infty}$ controller, using notation analogous with (Chen, 2000), we define the measurement output simply as the state feedback in the form of $y_{1}=C_{11} x$ with $C_{11}=I$. Since our primary task is to design a control law to internally stabilize the system, and to achieve a good response of the state variables that are directly related and linked to the outer-loop, while considering the constraints on the inputs and some state variables, we define the controlled output $h_{1}$ in the form of $h_{1}=C_{12} x+D_{12} u$, where

$$
C_{12}=\left[\begin{array}{ccc}
0_{2 \times 3} & \\
3.1623 & 0 & 0 \\
0 & 3.1623 & 0 \\
0 & 0 & 1.7321
\end{array}\right], D_{12}=\left[\begin{array}{cc}
44.7214 & 0 \\
0 & 28.2843 \\
0 & 0
\end{array}\right]
$$


The nonzero entries of $C_{12}$ and $D_{12}$ are used for tuning the controller, and here, are determined experimentally to achieve the desired performance. Meanwhile, the $H_{\infty}$ design guarantees internal stability and robustness of the system. Indeed, $H_{\infty}$ control design minimizes the effect of the wind gust disturbance, i.e., minimizes the $H_{\infty}$ norm of the closed-loop transfer function from the disturbance $w$ to the controlled output $h_{1}$, denoted by $T_{1}$. The $H_{\infty}$ norm of the transfer function $T_{1}$ is defined as follows:

$$
\left\|T_{1}\right\|_{\infty}=\sup _{0 \leq \omega<\infty} \sigma_{\max }\left[T_{1}(j \omega)\right]
$$

where $\sigma_{\max }[$.$] denotes the maximum singular value of the matrix.$

It should be highlighted that the $H_{\infty}$ norm is the worst case gain in $T_{1}(s)$. Therefore, minimization of the $H_{\infty}$ norm of $T_{1}$ is equivalent to the minimization of the disturbance effect from the disturbance $w$ to the controlled output $h_{1}$ in the worst case situation. Having the matrix $C_{12}$ and $D_{12}$, one can find the $\gamma_{\infty}^{*}$ which is the optimal $H_{\infty}$ performance for the closed-loop system from the disturbance input $w$ to the controlled output $h_{1}$ over all the possible controllers that internally stabilize the system. As practically, $\gamma_{\infty}^{*}$ is not achievable, we will try to reach $\gamma_{\infty}$ which is slightly larger than $\gamma_{\infty}^{*}$.

With this choice of the control parameters, $D_{11}$ and $D_{12}$ are full rank and the quadruples $\left(A_{1}, B_{1}, C_{12}, D_{12}\right)$ and $\left(A_{1}, E_{1}, C_{11}, D_{11}\right)$ are left invertible and are free of invariant zeros. Therefore, we have a so-called regular problem, for which we can use well-established $H_{\infty}$ control theory (Chen, 2000). The resulting closed loop system suboptimality minimizes the $H_{\infty}$ norm of the transfer function from the disturbance $w$ to the controlled output $h_{1}$. To design this controller we consider the control law in the following form:

$$
u_{1}=F_{1} x_{1}+G_{1} r_{1}
$$

where $r_{1}=\left(V_{z_{r}}, \omega_{z_{r}}\right)^{\prime}$ is the reference signal generated by the outer-loop controller, $G_{1}=$ $-\left(C_{1}\left(A_{1}+B_{1} F_{1}\right)^{-1} B_{1}\right)^{-1}$ is the feedforward gain, and $F_{1}$ is the $H_{\infty}$ control gain that can be achieved as follows:

$$
\left.F_{1}=-\left(D_{12}^{\prime} D_{12}\right)^{-1}\left(D_{12}^{\prime} C_{12}+B_{1}^{\prime} P_{1}\right)\right)
$$

where matrix $P_{1}$ is the positive semi-definite solution of the following $H_{\infty}$ algebraic Riccati equation:

$$
\begin{array}{r}
A_{1}^{\prime} P_{1}+P_{1} A_{1}+C_{12}^{\prime} C_{12}+P_{1} E_{1} E_{1}^{\prime} P_{1} / \gamma^{2}- \\
\left(P_{1} B_{1}+C_{12}^{\prime} D_{12}\right)\left(D_{12}^{\prime} D_{12}\right)^{-1}\left(D_{12}^{\prime} C_{12}+B_{1}^{\prime} P_{1}\right)=0
\end{array}
$$

For this system and these control parameters values, the value of $\gamma_{\infty}^{*}$ is 1.4516 . Hence, we select $\gamma_{\infty}=1.4616$. Therefore, matrices $F_{1}$ and $G_{1}$ are obtained as follows:

$$
F_{1}=\left[\begin{array}{ccc}
-0.0935 & -0.0005 & 0.0027 \\
0.0008 & 0.0364 & -0.0481
\end{array}\right], G_{1}=\left[\begin{array}{cc}
0.1371 & 0.0066 \\
-0.0020 & -0.2748
\end{array}\right]
$$

To evaluate the controller performance and its effect on the disturbance attenuation, we simulated the closed loop system with an initial state of $x_{1}\left(\begin{array}{l}0 \\ 0\end{array}=\left[\begin{array}{lll}1.5 & 0 & 0\end{array}\right]^{\prime}\right.$, and also we injected wind gust disturbance for $20 \mathrm{sec}$ (Fig. 6). The injected disturbance has a maximum amplitude of $3 \mathrm{~m} / \mathrm{s}$ along the $\mathrm{z}$ axis (the other directions do not affect the dynamics of Subsystem 1). The controlled system reaches the steady hovering state after $3.5 \mathrm{sec}$, and the disturbance effect is reduced to less than $0.25 \%$. The control inputs are within the unsaturated region. 

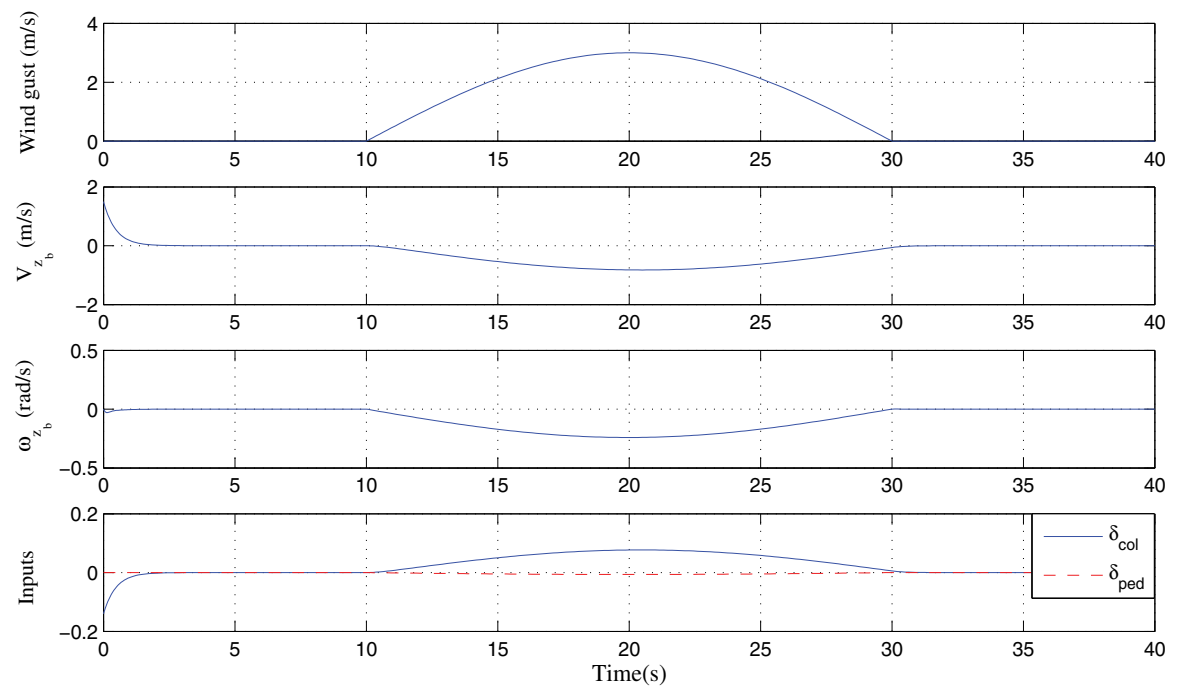

Fig. 6. Simulation of the inner-loop of Subsystem 1.

\subsubsection{Outer-loop controller}

In the outer-loop of Subsystem 1, we use a P-controller $K_{P_{1}}$ (Fig. 7). We can redraw this system as shown in Fig. 8, in which $G_{i n_{1}}=\frac{1}{S} C_{1}\left(S I-\left(A_{1}+B_{1} F_{1}\right)\right)^{-1} B_{1} G_{1}$. It can be shown that $G_{i n_{1}}$ is a $2 \times 2$ multi-variable system. Unfortunately, in general, designing a P-controller for an MIMO system is difficult. However, if we consider $K_{p_{1}}$ in diagonal form as $K_{p_{1}}=k_{p_{1}} I_{2 \times 2}$, we can apply the generalized Nyquist theorem (Postlethwaite \& MacFarlane, 1979) to design $k_{p_{1}}$ such that it stabilizes the system as described in the following part.

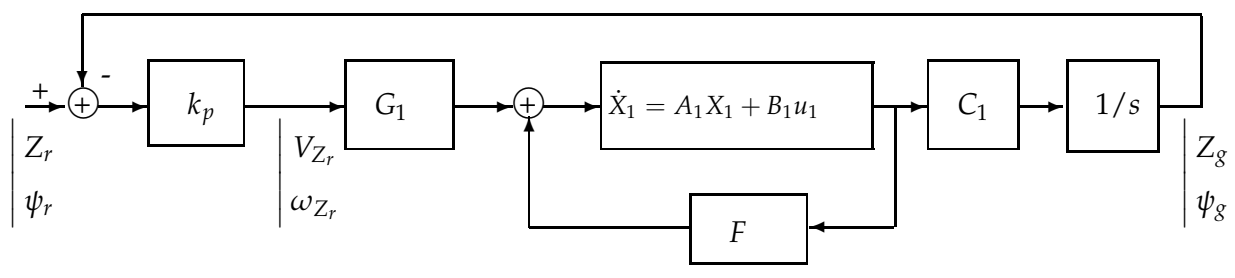

Fig. 7. Control structure of Subsystem 1.

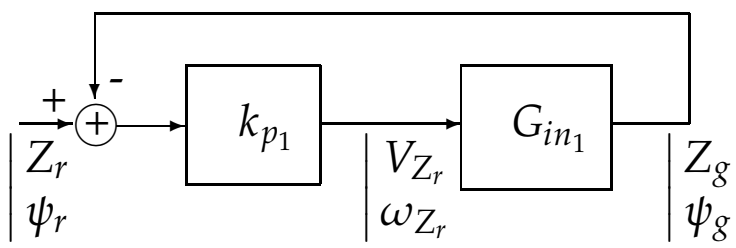

Fig. 8. Redrawing the control structure of Subsystem 1.

\subsubsection{Stability analysis}

The characteristic loci of $G_{i n_{1}}$ are shown in Fig. 9, where the dash-dot lines correspond to the infinite values. In Subsystem 1, Fig. 8, the inner-loop has already been stabilized, using an $H_{\infty}$ 
controller. Therefore, due to the presence of the integral term, $G_{i n_{1}}$ has two poles at the origin and the remaining poles are in the LHP plane. Hence, $G_{i n_{1}}$ has no pole in the Nyquist contour. It follows from the form of the characteristic loci of $G_{i n_{1}}$ in Fig. 9, that $k_{p_{1}} \in(0, \infty)$ will keep the entire system stable. However, in practice, we are subjected to the selection of small values of $k_{p_{1}}$ to avoid saturation of the actuators. $k_{p_{1}}=1.5$ is a typical value.

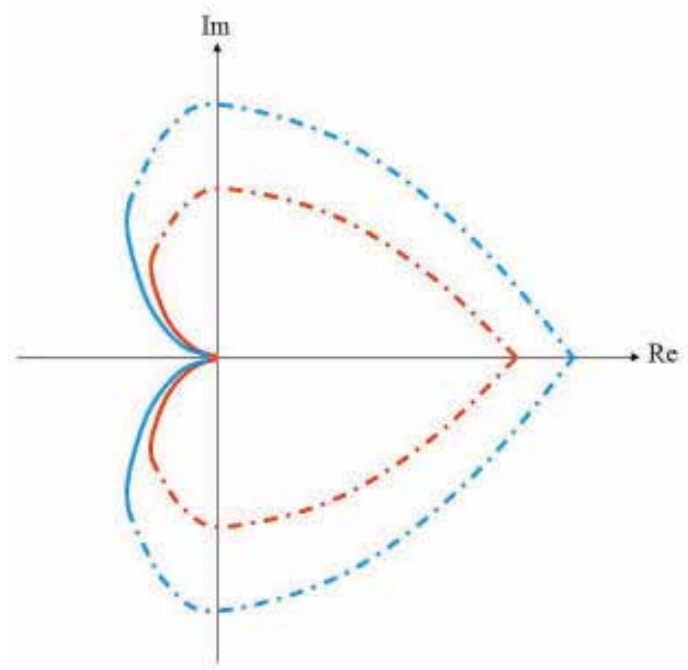

Fig. 9. Characteristic loci of $G_{i n_{1}}$.

\subsubsection{Tuning the controller}

With the above outer-loop controller, the stability of the whole system has been achieved; however, the controller in the form of $K_{p_{1}}=k_{p_{1}} \times I_{2 \times 2}$ with only one control parameter is not an appropriate choice. We need to have more degrees of freedom to tune the controller and achieve better performance. By considering the proportional feedback gain $K_{p_{1}}=$ $\operatorname{diag}\left\{K_{p_{11}}, K_{p_{12}}\right\}$ as a diagonal matrix, we have more degrees of freedom and can control each of the output channels in a decentralized manner, while keeping the system decoupled.

Uncertainty analysis usually is used to investigate the effect of the plant uncertainty. Here, we borrow this idea to analyze the effect of deviation of the diagonal entries of the matrix $K_{p_{1}}=k_{p_{1}} I_{2 \times 2}$ in the controller part. Alternatively, one can define $K_{p_{1}}$ as follows:

$$
K_{p_{1}}=\left[\begin{array}{cc}
K_{p_{11}} & 0 \\
0 & K_{P_{12}}
\end{array}\right]=k_{p_{1}} I_{2 \times 2}+\left[\begin{array}{cc}
\Delta_{K_{p_{11}}} & 0 \\
0 & \Delta_{K_{P_{12}}}
\end{array}\right]
$$

The objective is to design $\Delta=\operatorname{diag}\left\{\Delta_{K_{p_{11}}}, \Delta_{K_{p_{12}}}\right\}$ such that it does not affect the stability of the system. In fact, $\triangle$ is the tuning range (Fig. 10).

Following from Fig. 10, one can extract the internal model of the system as:

$$
\left\{\begin{array}{l}
y=G_{i n_{1}} k_{p_{1}}\left(I+G_{i n_{1}} k_{p_{1}}\right)^{-1} v+\left(I+G_{i n_{1}} k_{p_{1}}\right)^{-1} G_{i n_{1}} z \\
x=\left(I+G_{i n_{1}} k_{p_{1}}\right)^{-1} v-\left(I+G_{i n_{1}} k_{p_{1}}\right)^{-1} G_{i n_{1}} z
\end{array}\right.
$$

To simplify the notation, (20) can be rewritten as:

$$
\left\{\begin{array}{l}
y=G_{11} v+G_{12} z \\
x=G_{21} v+G_{22} z
\end{array}\right.
$$




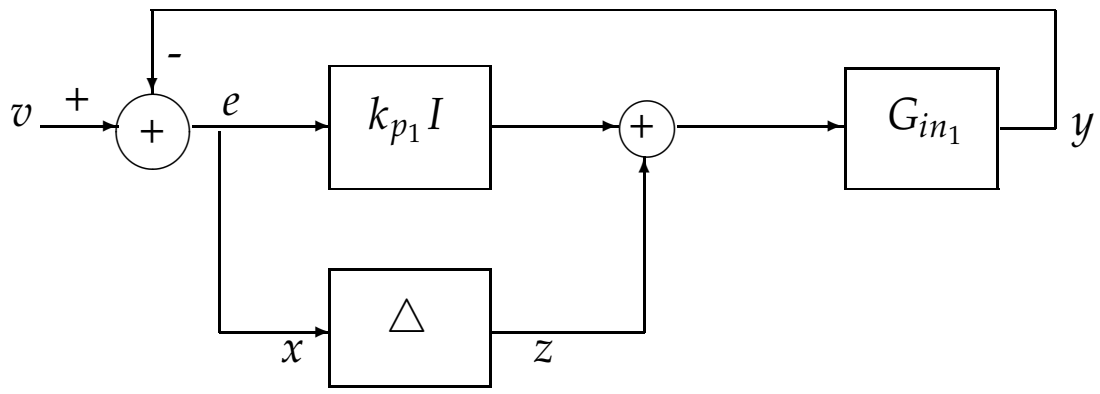

Fig. 10. Tuning the controller using uncertainty analysis.

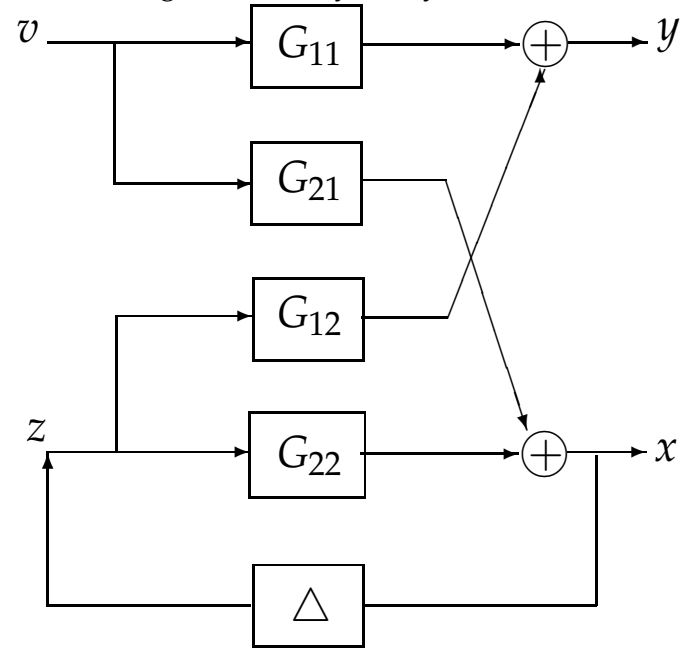

Fig. 11. Redrawing Subsystem 1 for uncertainty analysis.

Therefore, Fig. 10 can be redrawn as it is shown in Fig. 11. In the new diagram, since the nominal system with $\Delta=0$ is stable, all $G_{i j}$ are stable. $G_{11}, G_{12}$, and $G_{21}$ are outside the uncertain loop and cannot be affected by block $\triangle$; however, the loop includes $G_{22}$ and $\triangle$ may affect the internal stability of the system due to perturbations of the elements of $\triangle$. Since $G_{22}$ and $\triangle$ are stable, according to the generalized Nyquist theorem, the characteristic loci of the loop transfer function should not encircle the point $(-1+\mathrm{j} 0)$, or equivalently, $\left|\lambda_{i}\left(-G_{22} \Delta\right)\right|<1$. To satisfy this condition, since $\left|\lambda_{i}\left(G_{22} \Delta\right)\right| \leq \bar{\sigma}\left(G_{22} \Delta\right) \leq \sup \left(\bar{\sigma}\left(G_{22} \Delta\right)\right)=\left\|G_{22} \Delta\right\|_{\infty}$, it is sufficient that $\left\|G_{22} \Delta\right\|_{\infty}<1$. Using norm properties, we have:

$$
\left\|G_{22} \Delta\right\|_{\infty} \leq\left\|G_{22}\right\|_{\infty}\|\Delta\|_{\infty}
$$

Therefore, the sufficient condition for the stability of the system is:

$$
\left\|G_{22}\right\|_{\infty}\|\Delta\|_{\infty}<1
$$

For these values of the controller and plant and for a frequency range of $(0,10000)$, we obtain $\left\|G_{22}\right\|_{\infty}=0.6986$. Therefore, the perturbation of $K_{p_{1}}$ should be such that $\|\Delta\|_{\infty} \leq 1.4315$. Recall that $\triangle$ has a diagonal structure, and hence, all diagonal entries of $K_{p_{1}}$ should have less than a 1.4315-unit deviation from their nominal value. In fact, using this approach, we first 
obtained a nominal controller that provides the stability of the system, and then, we attempted to tune the controller to improve the performance, while keeping the system stable. After tuning the controller, the value of $K_{p_{1}}=\operatorname{diag}\{0.5,0.7\}$ was selected as an appropriate value that satisfies the above mentioned condition and gives a satisfactory performance. The method is conservative as $\Delta$ is structured and real, but applying to the UAV plant it has provided sufficient degree of freedom for tuning the controller and improving the performance.

To simulate the resulting system, let the outer-loop reference be $\left(Z_{r}, \psi_{r}\right)=(-2,0.5)$ and the current position and heading angle be $\left(Z_{g}, \psi_{g}\right)=(0,0)$. The system will reach its target after approximately $8 \mathrm{sec}$ as shown in Fig. 12.
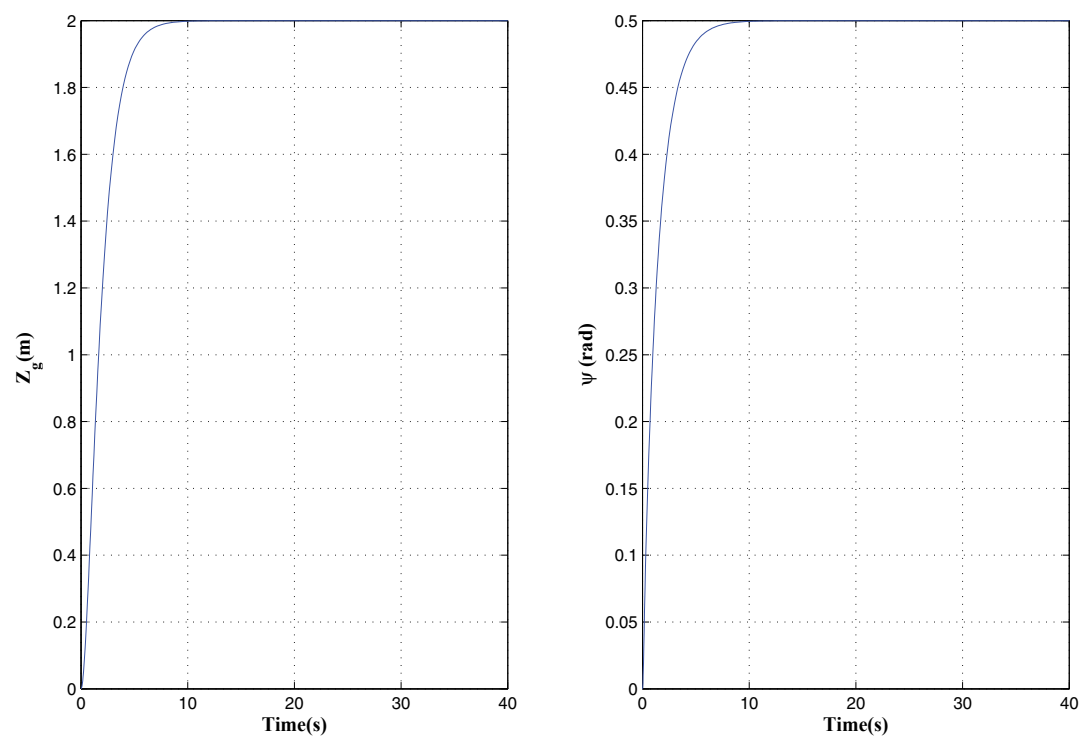

Fig. 12. Simulation of the outer-loop of Subsystem 1.

\subsection{Designing the controller for subsystem 2}

\subsubsection{Inner-loop controller}

For Subsystem 2, described by (11), we use an $H_{\infty}$ controller for the inner-loop controller design, similar to Subsystem 1. Analogous with Subsystem 1, we define $h_{2}$ as $h_{2}=C_{22} x_{2}+D_{22} u_{2}$, where

$$
C_{22}=\left[\begin{array}{cccccccc}
0.3162 & 0 & 0 & 0 & 0 & 0 & 0 & 0 \\
0 & 0.3162 & 0 & 0 & 0 & 0 & 0 & 0 \\
0 & 0 & 0.3162 & 0 & 0 & 0 & 0 & 0 \\
0 & 0 & 0 & 0.3162 & 0 & 0 & 0 & 0 \\
0 & 0 & 0 & 0 & 1 & 0 & 0 & 0 \\
0 & 0 & 0 & 0 & 0 & 1 & 0 & 0 \\
0 & 0 & 0 & 0 & 0 & 0 & 1 & 0 \\
0 & 0 & 0 & 0 & 0 & 0 & 0 & 1
\end{array}\right]
$$


$D_{22}=\left[\begin{array}{cc}5.4772 & 0 \\ 0 & 5.4772 \\ & 0 \\ 0 & \end{array}\right]$

With these parameters, we obtain $\gamma_{\infty}^{*}=0.0731$, and choosing $\gamma_{\infty}=0.0831$, we have:

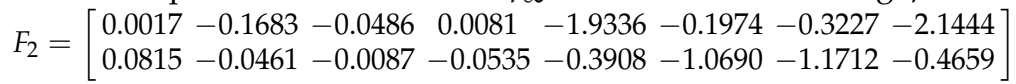

Moreover, $G_{2}=-\left(C_{2}\left(A_{2}+B_{2} F_{2}\right)^{-1} B_{2}\right)^{-1}$, is the feedforward gain for Subsystem 2 and can be calculated as:

$$
G_{2}=\left[\begin{array}{ll}
-0.0029 & 0.2335 \\
-0.0978 & 0.0632
\end{array}\right]
$$

The simulation of the system is shown in Fig. 13. In this figure, the initial state of the system is $x_{2}(0)=\left[\begin{array}{llllllll}1.5 & 0 & 0 & 0 & 0.17 & 0 & 0 & 0\end{array}\right]^{\prime}$. The injected disturbance has a maximum amplitude of 10 $\mathrm{m} / \mathrm{s}$ along the $x$ and $y$ axes (the $z$ direction does not affect the dynamics of Subsystem 2). The controlled system reaches the steady hovering state after $3.5 \mathrm{sec}$, and the disturbance effect is reduced to less than $0.25 \%$. In addition, the control inputs are within the unsaturated region.
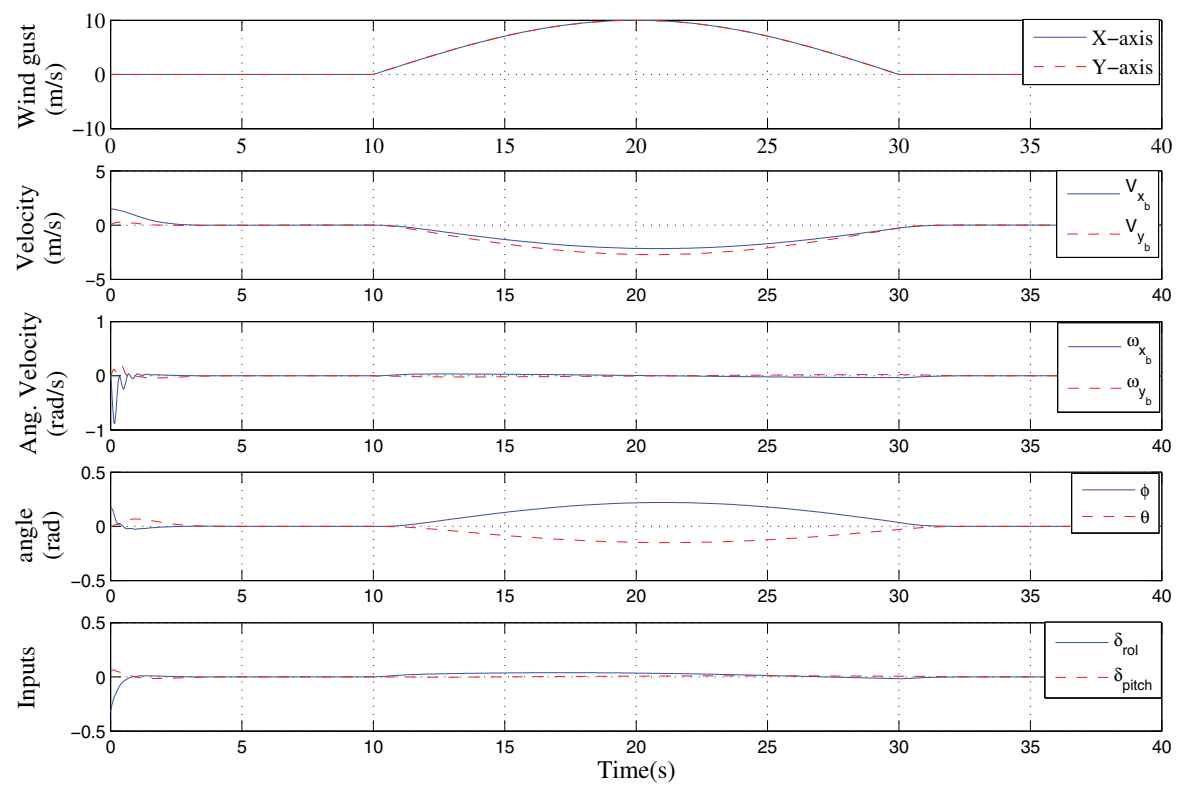

Fig. 13. Simulation of the inner-loop of Subsystem 2.

\subsubsection{Outer-loop controller}

Although the outer-loop of Subsystem 2 is similar to the outer-loop of Subsystem 1, the main difference lies in the presence of the nonlinear term, $R^{-1}$, in the outer-loop of Subsystem 2, as shown in Fig. 4. In this structure, it can be seen that the error signal is the difference between the actual position and the reference position, which both are in the ground frame. Therefore, the resulting control signal, which is the reference for the inner-loop, will be obtained in the ground frame; however, the inner-loop is in the body frame. Hence, it is reasonable that 
we transform the control signal to the body frame before delivering it to the inner-loop as the reference to be tracked. To implement this idea, we can use the transformation term, $R$, to obtain a control signal in the body frame. The new structure is shown in Fig. 14, in which $G_{i n_{2}}=C_{2}\left(S I-\left(A_{2}+B_{2} F_{2}\right)\right)^{-1} B_{2} G_{2}$ is a $2 \times 2$ multi-variable system. In Fig. 15, it is shown that the inner-loop block $G_{i n_{2}}$ is very close to a decoupled system with equal diagonal elements. Indeed, Subsystem 2 corresponds to the dynamics of the helicopter for the $x-y$ plane movement. In practice, we expect the dynamics of the UAV in the $x$ and $y$ directions to be similar and decoupled, since the pilot can easily drive the UAV in either of directions independently. Using this concept, we can take the block $G_{i n_{2}}$ out so that the two rotation matrices $R$ and $R^{-1}$ will cancel each other.

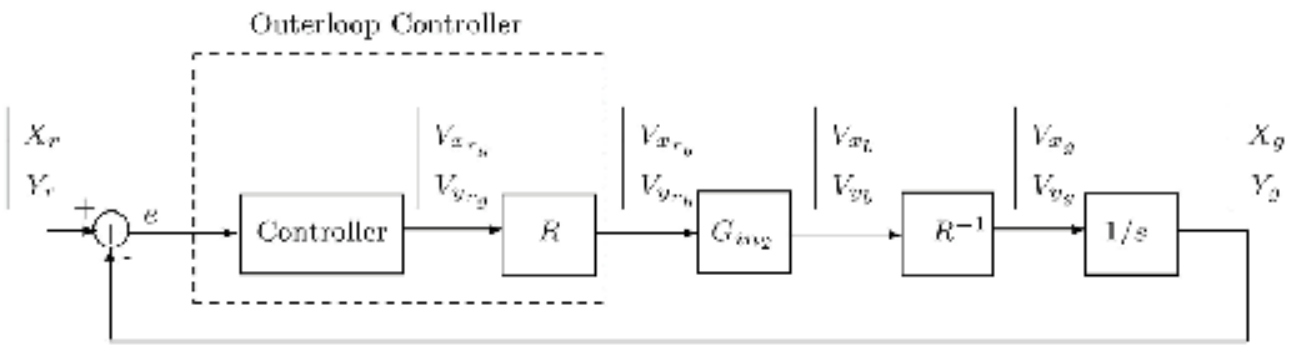

Fig. 14. Control diagram of Subsystem 2.
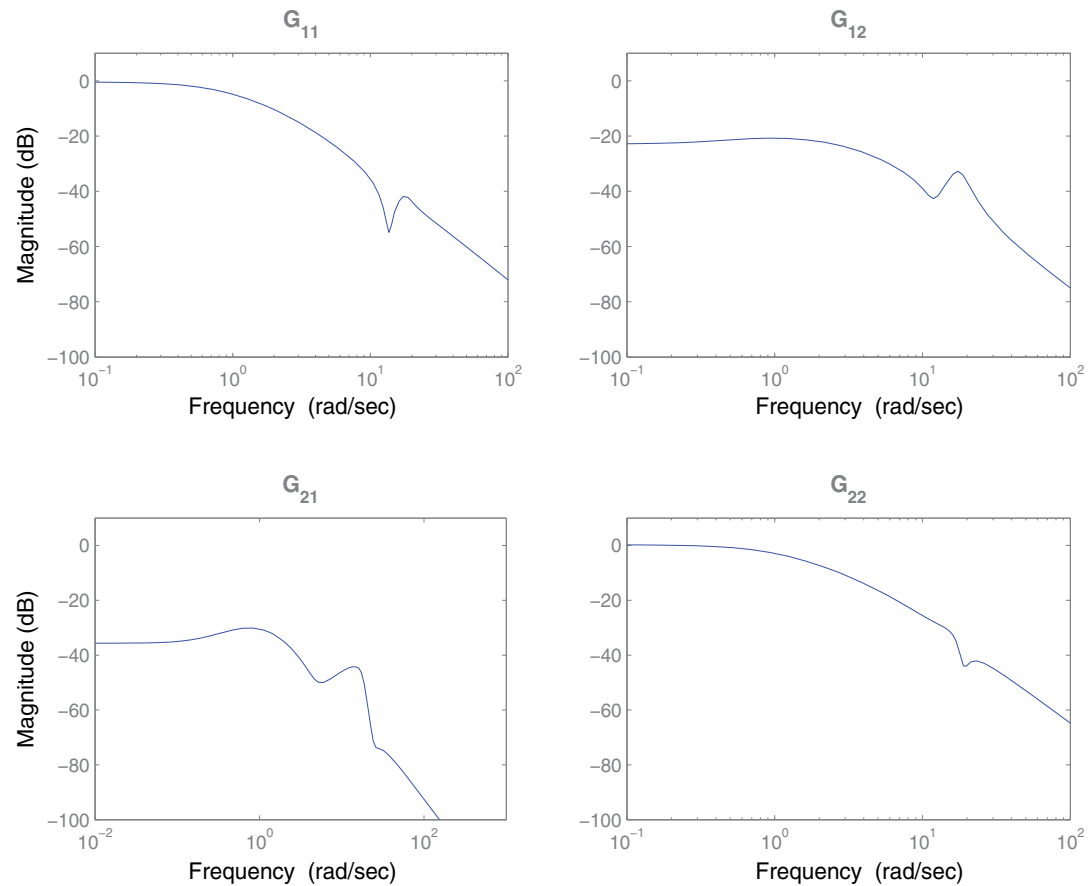

Fig. 15. Bode plot of entries of $G_{i n_{2}}$ 
The remaining job is simple, and we can repeat the procedure of designing the outer-loop controller for Subsystem 1 and design a P-controller in the form of $K_{p_{2}}=\operatorname{diag}\left\{K_{p_{21}}, K_{P_{22}}\right\}$ that stabilizes $G_{i n_{2}}$ (Fig. 16). As an appropriate choice of control parameters, we can select $K_{p_{2}}=\operatorname{diag}\{0.3,0.3\}$. Rationally, $K_{p_{21}}$ and $K_{P_{22}}$ should be the same, since we expect a similar behavior of the UAV system in the $x$ and $y$ directions.

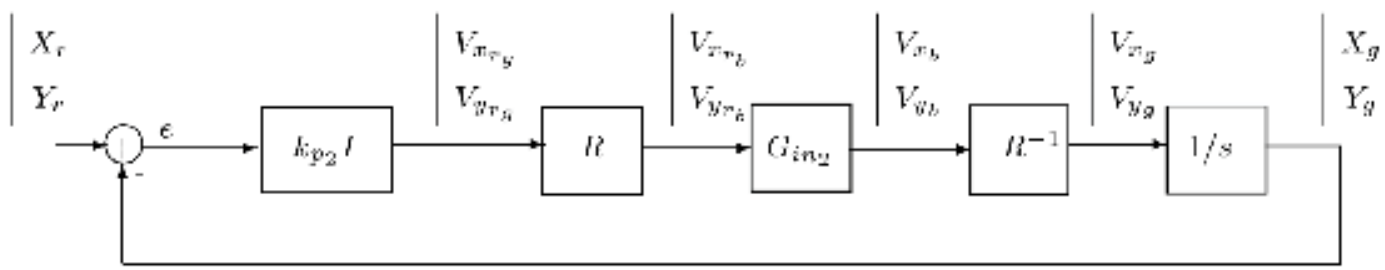

Fig. 16. Redrawing the control diagram of Subsystem 2.

For an outer-loop reference at $\left(x_{r}, y_{r}\right)=(2,2)$ and the current UAV position at $\left(x_{g}, y_{g}\right)=$ $(0,0)$, the simulation results are shown in Fig. 17, in which the UAV reaches to the desired position after approximately $10 \mathrm{sec}$, smoothly and without overshooting.
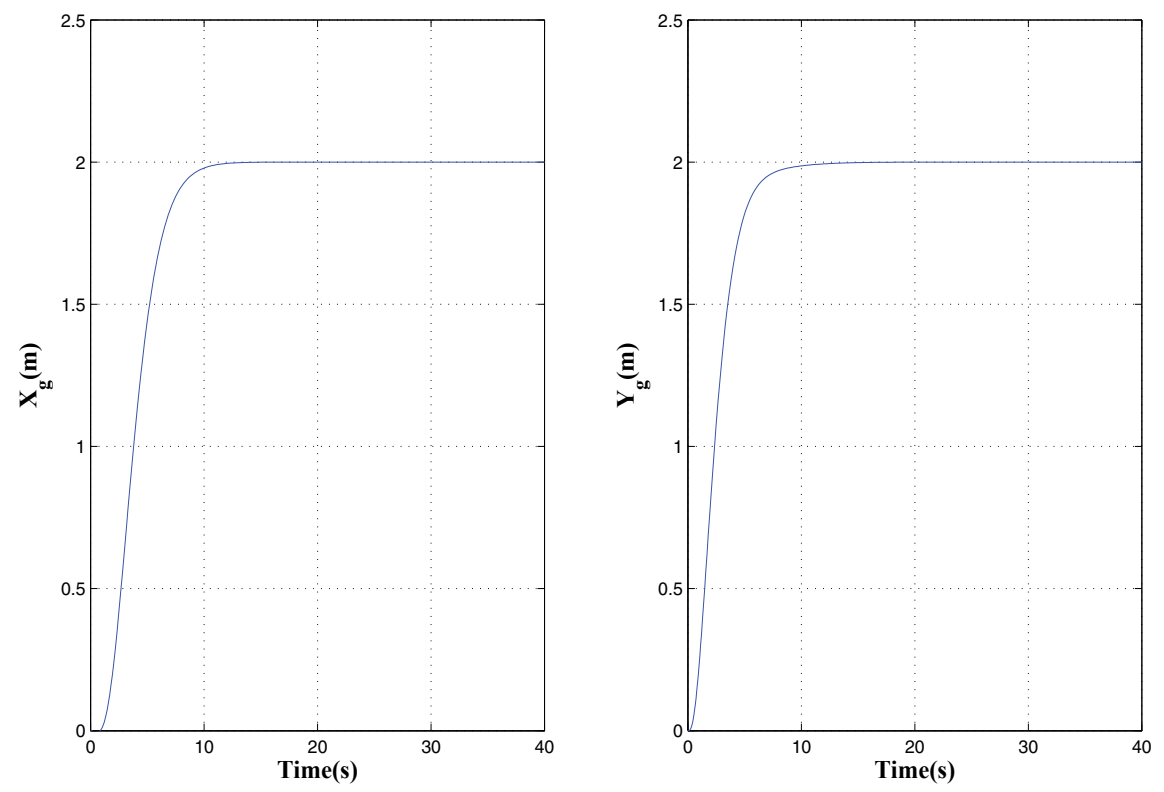

Fig. 17. Simulation of the outer-loop of Subsystem 2.

\section{Experimental results}

Before using the designed controller in an actual flight test, we first evaluate it through a hardware-in-the-loop simulation platform (Cai et al., 2009). In this platform, the nonlinear dynamics of the UAV has been replaced with its nonlinear model, and all software and hardware components that are involved in a real flight test remain active during the 
simulation. Using the hardware-in-the-loop simulation environment, the behavior of the system is very close to the real experiments.

Then, we conducted actual flight tests to observe the in-flight behavior of the helicopter. First, we used the UAV in the hovering state for $80 \mathrm{sec}$. Figure 18 shows the state variables in the hovering experiment at $(x, y, z, \psi)=(-16,-34,10,-1.5)$. To evaluate the hovering control performance, the position of the UAV is depicted in a 2D $x-y$ plane in Fig. 19. As can be seen, the position of the UAV has at most a 1-meter deviation from the desired hovering position, which is quite satisfactory. The control inputs are also shown in Fig. 20. All of the control inputs are within the unsaturated region.

Next, we used the UAV to follow a circle with a diameter of 20 meters as a given trajectory. This trajectory determines the reference $(x(t), y(t), z(t), \psi(t))$ for the system. With this trajectory, the UAV should complete the circle within $63 \mathrm{sec}$, while keeping a fixed altitude. Then, it will hover for $7 \mathrm{sec}$. In Fig. 21, it is shown that the UAV is able to follow this trajectory successfully. The UAV path tracking in the $x-y$ plane is shown in Fig. 22. Moreover, to have a better insight of the system behavior, all of the states of the UAV and the control inputs are represented in Fig. 23 and Fig. 24, respectively. These results show that the UAV is able to track the desired trajectory in situations close to the hovering state. The small deviations in the hovering mode or path tracking mode could be due to environmental effects such as wind disturbances or the GPS signal inaccuracy as with the installed sensors the measurable steady accuracy of the heading angle is $2.5^{\circ}$ and the positioning accuracy of the GPS is $3 m(1 \sigma)$. Videos of the hovering experiment and circle path tracking are available at http://uav.ece.nus.edu.sg/video/hover.mpg and http://uav.ece.nus.edu.sg/video/circle.mpg , respectively.
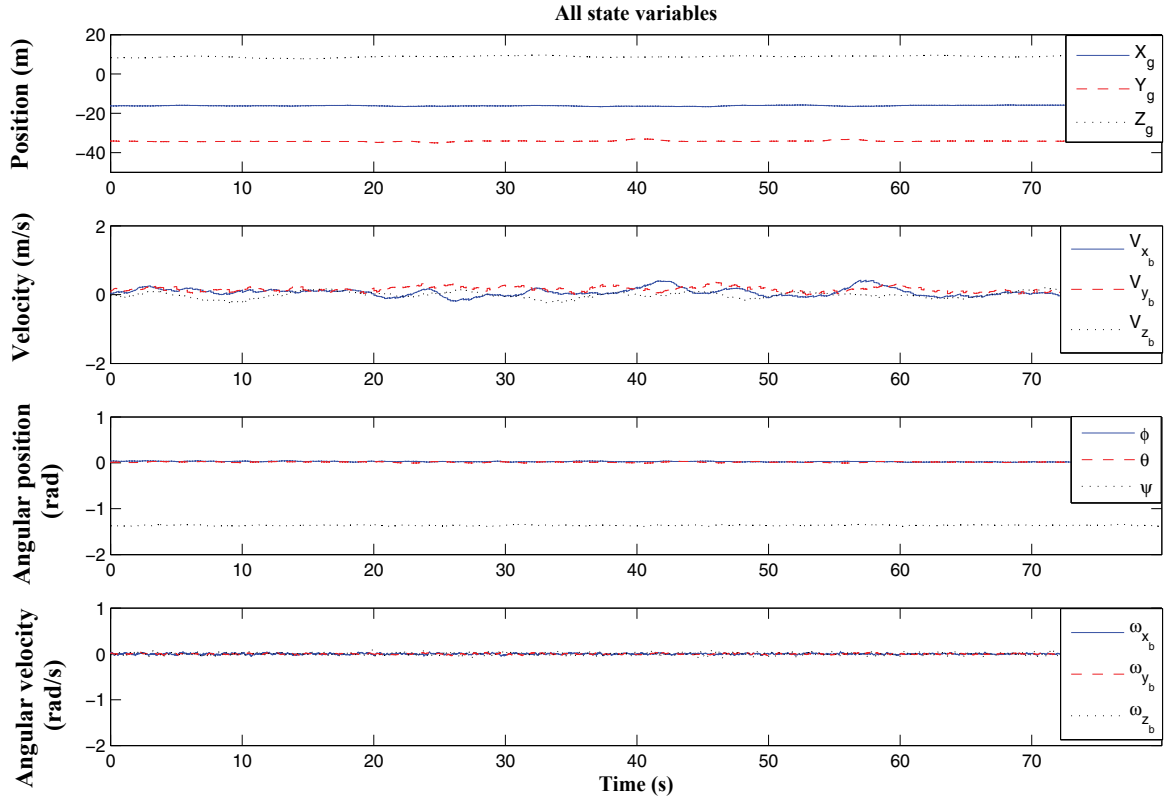

Fig. 18. State variables of the UAV for the hovering. 


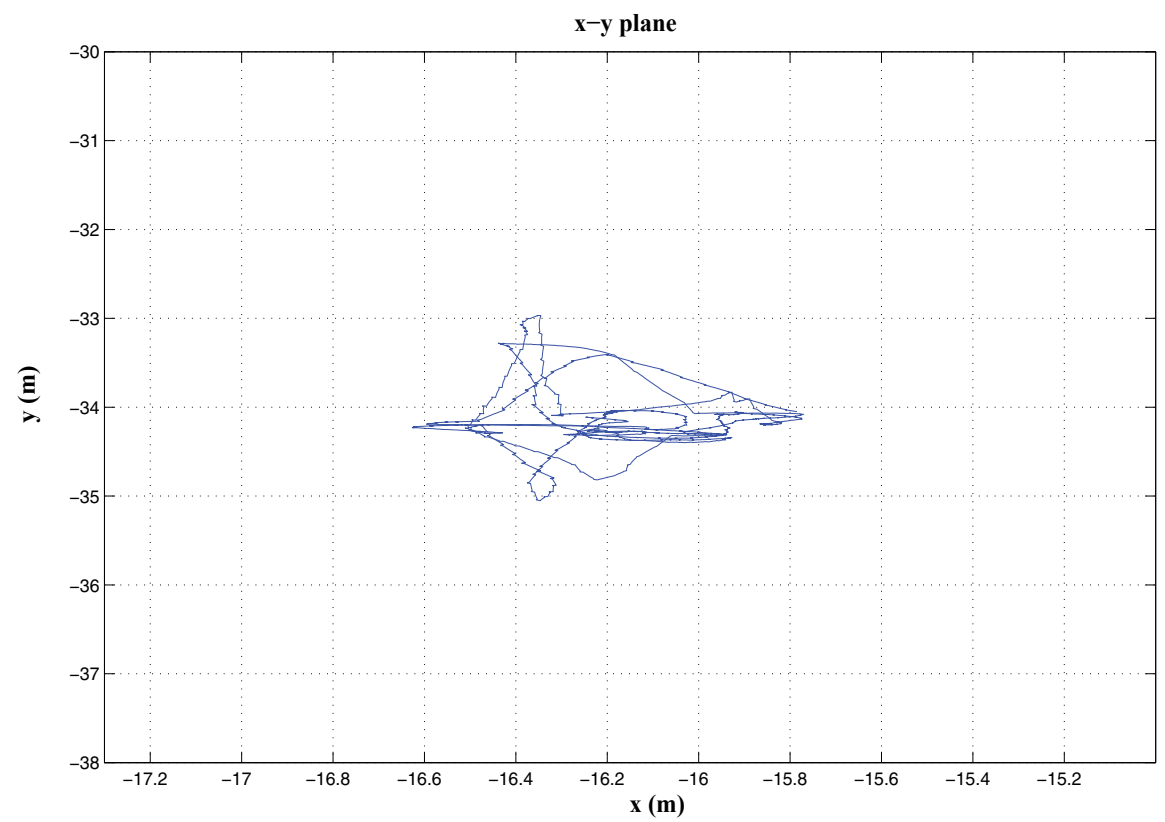

Fig. 19. UAV position in $x-y$ plane at hovering

\section{Conclusion}

In this chapter, we presented a systematic approach for the flight control design of a small-scale UAV helicopter in a hierarchical manner. In this structure, the lower level aims at stabilization of the system, and the upper level focuses on the reference tracking. For the disturbance attenuation and stabilization of the UAV, we used an $H_{\infty}$ controller in the inner-loop of the system. Due to the presence of some nonlinear terms in the outer-loop of the system, we first compensated for the nonlinearity by an inverse rotation; then, we used a decentralized P-controller to enable the UAV to follow a desired trajectory. We also proposed a new method of designing a P-controller for MIMO systems that was successfully applied to the UAV system. The simulations and actual flight tests show the efficacy of the control structure. In the future, we will use this structure to accomplish more complex missions such as formation control (Karimoddini et al., 2010). Such missions will require an embedded decision-making unit to support the tasks and to switch between the controllers. This concept will guide us in designing a hybrid supervisory controller in the path planner level of the UAV to comprehensively analyze the reactions between the continuous dynamics of the system and discrete switching between the controllers (Karimoddini et al., 2009).

\section{Acknowledgments}

The authors gratefully acknowledge the technical support of Mr. Dong Xiangxu and Mr. Lin Feng during the implementations and flight tests. 

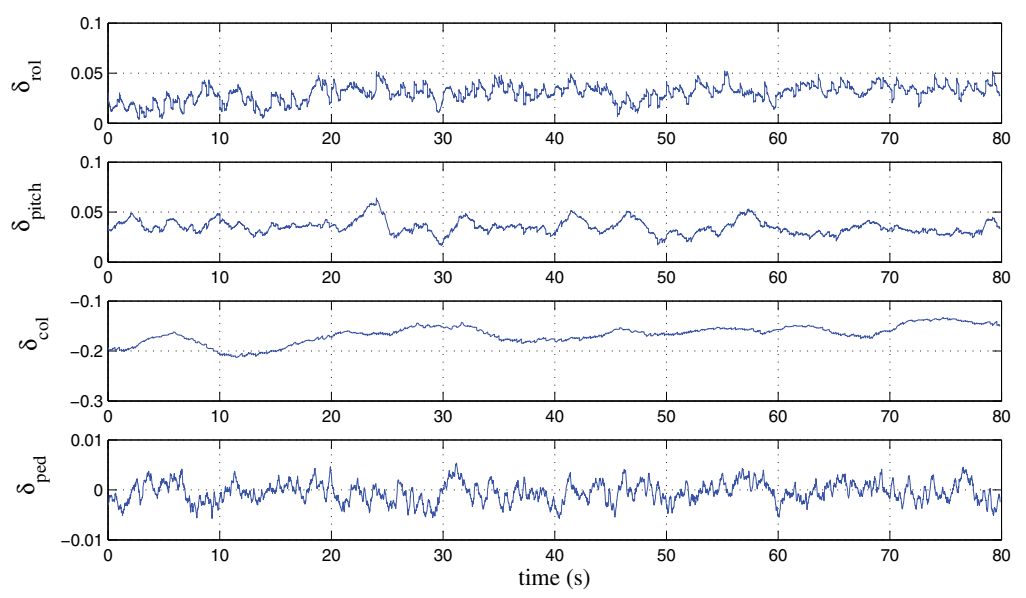

Fig. 20. Control signals at hovering
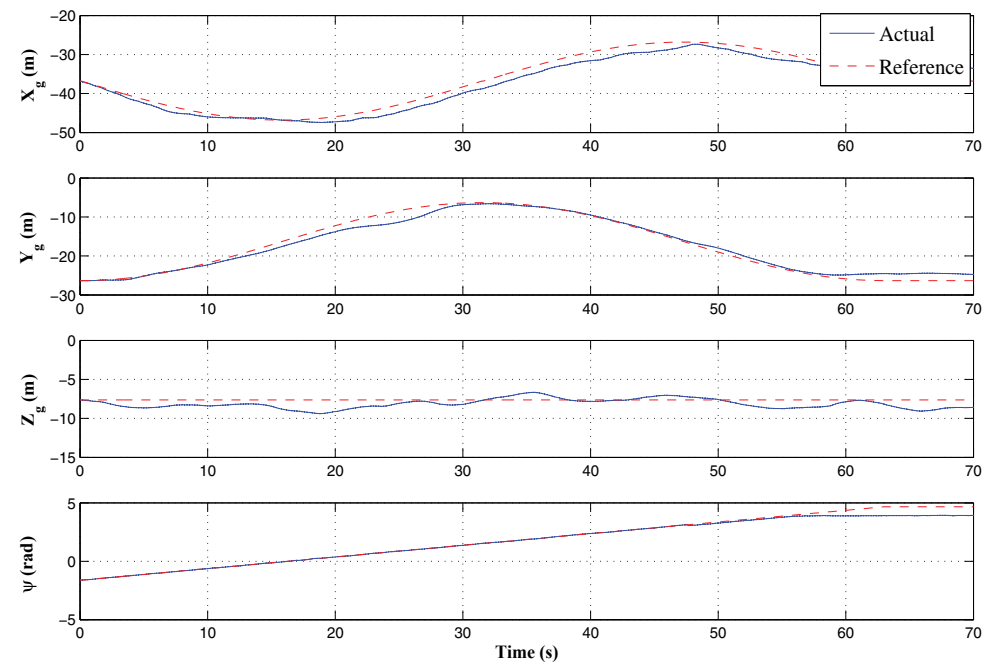

Fig. 21. Tracking a desired path. 


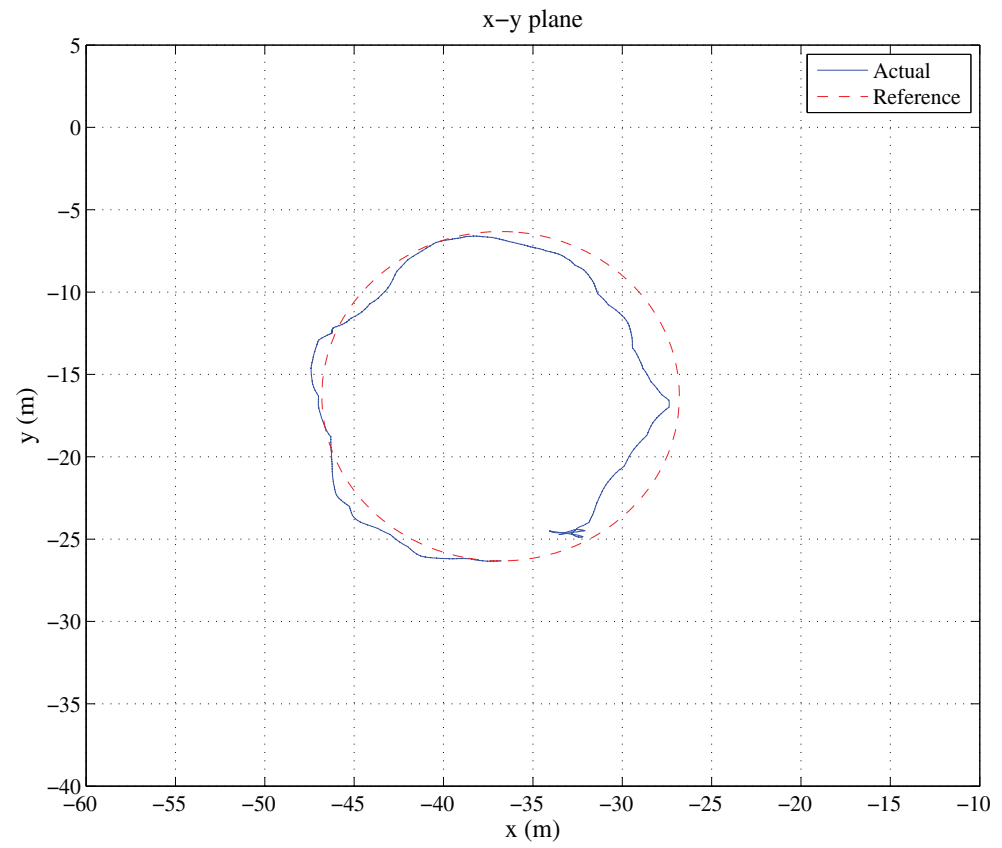

Fig. 22. Circle path tracking in $x-y$ plane.
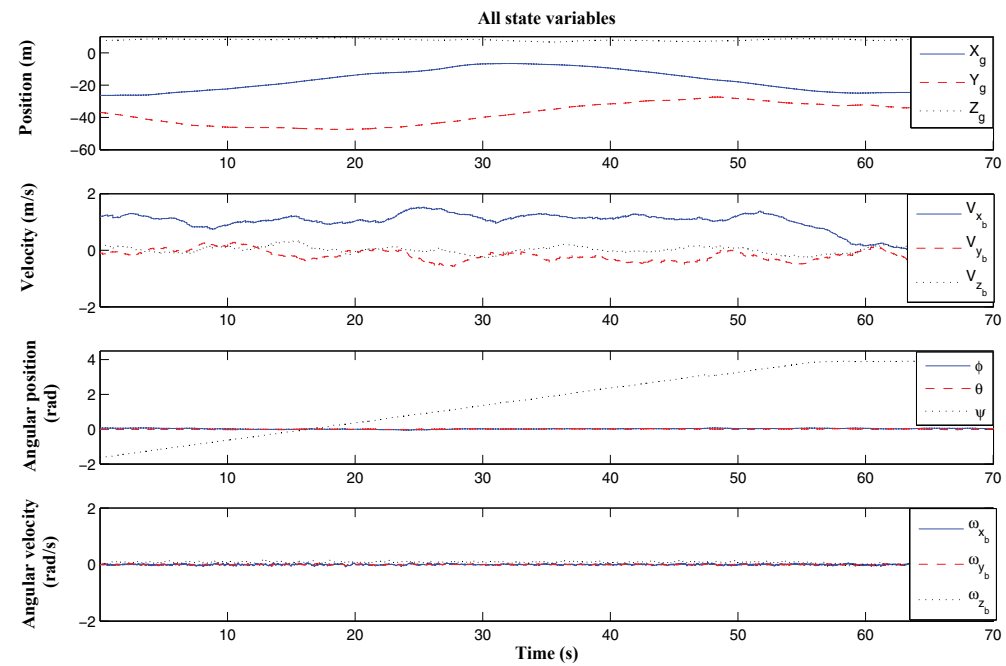

Fig. 23. States of the UAV in the circle path tracking behavior. 


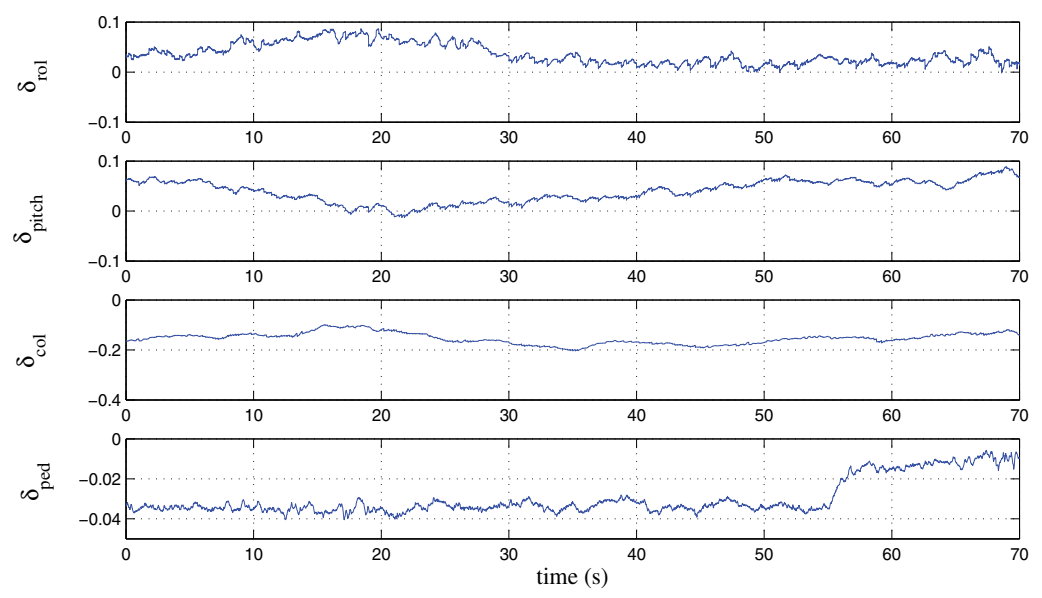

Fig. 24. Control inputs in the circle path tracking behavior.

\section{Nomenclature}

$A \quad=$ system matrix

$a_{S} \quad=$ longitudinal blade angle

$B=$ control input matrix

$B_{b} \quad=$ transformation matrix from the ground to the body frame

$b_{S} \quad=$ lateral blade angle

$E \quad=$ disturbance matrix

$\vec{F} \quad=$ resultant force in the body frame

$\vec{g} \quad=$ acceleration due to gravity, $\vec{g}=[0,0, g]^{\prime}$

$J \quad=$ inertia matrix

$\vec{M} \quad$ = resultant moment in the body frame

$\vec{P} \quad=$ position of the UAV, $\vec{P}=(X, Y, Z)^{\prime}$

$u=$ control input vector

$\vec{V} \quad=$ linear velocity vector of the $\mathrm{UAV}, \vec{V}=\left(V_{x}, V_{y}, V_{z}\right)^{\prime}$

$\vec{\omega} \quad=$ angular velocity vector of the $\mathrm{UAV}, \vec{\omega}=\left(\omega_{x}, \omega_{y}, \omega_{z}\right)^{\prime}$

$w \quad=$ wind gust disturbance in the body frame, $w=\left(w_{x}, w_{y}, w_{z}\right)^{\prime}$

$\theta \quad=$ pitch angle

$\phi \quad=$ roll angle

$\psi \quad=$ yaw angle

$\delta_{\text {roll }}=$ roll channel input

$\delta_{\text {pitch }}=$ pitch channel input

$\delta_{\text {pedal }}=$ pedal channel input

$\delta_{c o l}=$ collective channel input

$\star_{g}=$ the value of $\star$ in the ground frame

$\star_{b}=$ the value of $\star$ in the body frame

$\star_{r}=$ the value of $\star$ as control reference 


\section{References}

Bortoff, S. (1999). The university of toronto rc helicopter: a test bed for nonlinear control, Proceedings of the IEEE International Conference on Control Applications, IEEE, Vol. 1, pp. 333-338.

Cai, G., Peng, K., Chen, B. M. \& Lee, T. H. (2005). Design and assembling of a uav helicopter system, International Conference on Control and Automation, Vol. 2, pp. 697-702.

Cai, G., Chen, B. M., Peng, K., Dong, M. \& Lee, T. H. (2006). Modeling and control system design for a uav helicopter, 14th Mediterranean Conference on Control and Automation, pp. 1-6.

Cai, G., Feng, L., Chen, B. M. \& Lee, T. H. (2008a). Systematic design methodology and construction of uav helicopters, Mechatronics, 18(10): 545-558.

Cai, G., Chen, B. M., Peng, K., Dong, M. \& Lee, T. H.(2008b). Comprehensive modeling and control of the yaw channel of a UAV helicopter, IEEE Transactions on Industrial Electronics, 55(9): 3426-3434.

Cai, G., Chen, B. M., Lee, T. H. \& Dong, M. (2009). Design and implementation of a hardware-in-the-loop simulation system for small-scale UAV helicopters, Mechatronics, 19(7): 1057-1066.

Campbell, M. E. \& Wheeler, M. (2010). Vision-Based Geolocation Tracking System for Uninhabited Aerial Vehicles, AIAA Journal of Guidance, Control, and Dynamics, 33(2): 521-532.

Chen, B. M. (2000). Robust and $H_{\infty}$ Control, Springer, London.

Dong, M., Chen, B. M., Cai, G. \& Peng, K. (2007). Development of a real-time onboard and ground station software system for a UAV helicopter, AIAA Journal of Aerospace Computing, Information, and Communication, 4(8): 933-955.

Enns, R. \& Si, J. (2003). Helicopter trimming and tracking control using direct neural dynamic programming, IEEE Transactions on Neural Networks, IEEE, Vol. 14, pp. 929-939, 2003.

Gavrilets, V., Shterenberg, A., Dahleh, M. \& Feron, E. (2000). Avionics system for a small unmanned helicopter performing aggressive maneuvers, Proceedings of the 19th Digital Avionics Systems Conferences, Vol. 1, pp. 1E2/1-1E2/7.

Isidori, A., Marconi, L. \& Serrani, A.(2003) Robust nonlinear motion control of a helicopter, IEEE Transactions on Automatic Control, IEEE, Vol. 48, pp. 413-426, 2003.

Karimoddini, A., Lin, H., Chen, B.M. \& Lee, T.H. (2009) Developments in hybrid modeling and control of unmanned aerial vehicles, Proceedings of the 7th IEEE International Conference on Control and Automation, Christchurch, New Zealand, pp. 228-233.

Karimoddini, A., Lin, H., Chen, B.M. \& Lee, T.H. (2010) Hybrid formation control of the Unmanned Aerial Vehicles, Mechatronics, doi:10.1016/ j.mechatronics.2010.09.007.

Kim, J. \& Sukkarieh, S. (2007). Real-time implementation of airborne inertial-SLAM, Journal of Robotics and Autonomous Systems, 55(1): 62-71.

Kuroki, Y., Young, G.S. \& Haupt, S.E. (2010). UAV navigation by an expert system for contaminant mapping with a genetic algorithm, Journal of Expert Systems with Applications, 37(6): 4687-4697.

Metni, N. \& Hamel, T. (2007). A UAV for bridge inspection: Visual servoing control law with orientation limits, Journal of Automation in Construction, 17(1): 3-10.

Peng, K., Dong, M., Chen, B. M., Cai, G., Lum, K. Y. \& Lee, T. H. (2007). Design and implementation of a fully autonomous flight control system for a uav helicopter, Chinese Control Conference, pp. 662-667. 
Peng, K. , Cai, G., Chen, B. M., Dong, M., Lum, K. Y. \& Lee, T. H. (2009). Design and implementation of an autonomous flight control law for a UAV helicopter, Automatica, 45(10), pp. 2333-2338.

Postlethwaite, I. \& MacFarlane, A. G. I. (1979). A Complex Variable Approach to the Analysis of Linear Multivariable Feedback Systems, in Lecture Notes in Control and Information Sciences, Vol. 12/1979, Springer, Berlin, pp. 58-76.

Saripalli, S., Montgomery, J. \& Sukhatme, G. (2003). Visually guided landing of an unmanned aerial vehicle, IEEE Transactions on Robotics and Automation, 19(3): 371-380.

Shaferman, V. \& Shima, T. (2008). Unmanned Aerial Vehicles Cooperative Tracking of Moving Ground Target in Urban Environments, AIAA Journal of Guidance, Control, and Dynamics, 31(5): 1360-1371.

Shim, D. H., Kim, H. J. \& Sastry, S. (2003). Decentralized nonlinear model predictive control of multiple flying robots, Proceedings of the 42nd IEEE Conference on Decision and Control, IEEE, Hawaii, pp. 3621-3626.

Stevens, B. L. \& Lewis, F. L. (1992). Aircraft control and simulation, Wiley, New York.

Wang, Q.G., Lin, C., Ye, Z., Wen, G., He, Y. \& Hang, C. C. (2007). A quasi-lmi approach to computing stabilizing parameter ranges of multi-loop pid controllers, Journal of Process Control, 17(1): 59-72. 


\title{
Comparison of Flight Control System Design Methods in Landing
}

\author{
S.H. Sadati, M.Sabzeh Parvar and M.B. Menhaj \\ Aerospace Engineering Department, \\ Amir Kabir University, Hafez Ave., Tehran,
}

Iran

\section{Introduction}

The development and application of most present-day systems and control theory were spurred on by the need to resolve aerospace problems. This is roughly the problem of analyzing and designing flight control systems for tactical missiles or aircraft. The control laws used in current tactical missile or aircraft are mainly based on classical control design techniques. These control laws were developed in the 1950s and have evolved into fairly standard design procedures [1].

Current autopilot design processes contain time-and resource-consuming trial-and-error approaches. Especially late changes in the flight control laws contribute to high cost and delay of first delivery. The automatic landing mode development is a good example of a process with trial-and-error design phases, because of the many parameters the system has to be robust against. These parameters originate from different runway, terrain, ILS and weather characteristics, and from aircraft uncertainties like configuration and landing weight. Additional uncertainties arise from uncertain aerodynamic parameters, actuator model uncertainties, etc. The autonomous aircraft landing is an issue that implies three main aspects: the performance of equipment, the process models and the ethics. Generally, the landing is not a standard flight task as it could be thinking. We consider it a nonstandard flight stage because it has a very high sensitivity versus environment perturbation and to the psychological factors.

In the last three decades, optimality-based designs have been considered to be the most effective way for a guided missile engaging the target [2-4]. However, it is also known from the optimal control theory that a straightforward solution to the optimal trajectory shaping problem leads to a two point boundary-value problem [2], which is too complex for realtime onboard implementation. Based on the reasons given above, advanced control theory must be applied to a control system to improve its performance. One of the best ways to solve this problem is to approach the artificial intelligence modeling technology based on fuzzy logic and neural network [5].

Intelligent control is a control technology that replaces the human mind in making decisions, planning control strategies, and learning new functions whenever the environment does not allow or does not the presence of a human operator. Artificial neural networks and fuzzy logic are two potential tools for use in applications in intelligent control engineering. Artificial neural networks offer the advantage of performance improvement 
through learning by means of parallel and distributed processing. Many neural control schemes with back propagation training algorithms, which have been proposed to solve the problems of identification and control of complex nonlinear systems, exploit the nonlinear mapping abilities of neural networks [6,7]. Recently, adaptive neural network algorithms have also been used to solve highly nonlinear flight control problems. A fuzzy logic-based design that can resolve the weaknesses of conventional approaches has been cited above. The use of fuzzy logic control is motivated by the need to deal with highly nonlinear flight control and performance robustness problems. It is well known that fuzzy logic is much closer to human decision making than traditional logical systems. Fuzzy control based on fuzzy logic provides a new design paradigm such that a controller can be designed for complex, ill-defined processes without knowledge of quantitative data regarding the inputoutput relations, which are otherwise required by conventional approaches [8-11]. An overview of neural and fuzzy control designs for dynamic systems was presented by Dash et al.[12].

\section{Aircraft model}

Aircraft landing process enhanced several phases that define the so-called standard landing trajectory. The landing operation concerning two controlled maneuvers: first for guiding the aircraft in the horizontal plane, in order to align it onto the axe of the runway and the second, for aircraft guiding in the vertical plane in order to do the approaching of runway surface. Basically, the automatic landing systems provide the information for instrument navigation along the standard trajectory. In this paper only control about the longitudinal axis is considered. With this restriction, the equations of motion describing the aircraft take the form [13]:

$$
\begin{gathered}
\dot{V}=\frac{g}{W}\left(T_{b a l}-D\right)-g \sin \gamma \\
\dot{\gamma}=\frac{g}{V}\left(\frac{L}{W}-\cos \gamma\right) \\
\dot{q}=\frac{\bar{q} S C}{I_{y}}\left(C_{m}\right) \\
\dot{h}=V \sin \gamma \\
\dot{x}=V \cos \gamma \\
\dot{\theta}=q
\end{gathered}
$$

The state variables are $V=$ airspeed, $\gamma=$ flight path angle, $\theta=$ pitch angle, and $q=$ pitch rate, $h$ is the altitude, $T$ is the engine thrust force. The aerodynamic and propulsive forces are assumed to have the following form:

$$
C_{m}=C_{m_{0}}+C_{m_{q}} q+C_{m_{\alpha}} \alpha+C_{m_{\delta_{e}}} \delta_{e}+C_{m_{\delta_{f}}} \delta_{f}
$$




$$
\begin{aligned}
& C_{L_{b a l}}=\frac{2 m g \cos \gamma_{g l}}{\rho S V_{l}^{2}} \\
& \alpha_{b a l}=\frac{C_{L_{b}}-C_{L_{0}}}{C_{L_{\alpha}}} \\
& T_{b a l}=C_{D\left(C_{L_{b a l}}\right)} \frac{\rho V_{l}^{2} S}{2}+m g \sin \gamma_{g l} \\
& \delta_{b a l}=-\left(C_{m_{0}}+C_{m_{\alpha}} \alpha_{b a l}+C_{m_{\delta_{f}}} \delta_{f}\right) / C_{m_{\delta_{e}}} \\
& C_{D}(\alpha)=B_{0}+B_{1} \alpha+B_{2} \alpha^{2} \\
& C_{L}(\alpha)=\left\{\begin{array}{l}
C_{0}+C_{1} \alpha, \\
C_{0}+C_{1} \alpha+C_{2} \alpha^{2},
\end{array}\right\},\left\{\begin{array}{l}
\alpha \leq \alpha_{*} \\
\alpha^{*} \leq \alpha \leq \alpha_{\max }
\end{array}\right\}
\end{aligned}
$$

The model parameter data given in Table 1 refer to a Boeing B727 aircraft powered by three JT8D-17 turbofan engines [13].

\section{Conventional design methods}

Generally, the controller is in a form of proportional-integral-derivative (PID) parameters, and the control gains are determined by using classical control theory, such as the root locus method, Bode method or Nyquist stability criterion [14-16]. Modern control theory has been used extensively to design the flight control system, such as in the linear quadratic techniques [17,18], generalized singular linear quadratic technique [19], $H_{\infty}$ design technique and $\mu$ synthesis technique [20].

\section{A. Optimal Control}

The optimization of dynamic processes frequently requires the solution of an optimal control problem. That is, given a process model in terms of differential equations for the state variables, find the vector of control functions that minimizes some performance index, subject to boundary conditions and possibly additional equality or inequality constraints on the state and controls. Application of the necessary condition for optimal control of systems defined by ordinary differential equations results in a two-point boundary value problem. A suitable Method to solve such problems is the well-known Multiple Shooting Method [21]. The method reduces the boundary value problem to the solution of a set of algebraic equations and initial value problems with partially unknown initial conditions that are solve by Newton iteration. Based upon this method, this boundary value problem is solved numerically by the Multiple Shooting code BNDSCO [22]. The optimal trajectory problem consists of minimizing [2]

$$
J(u)=\int\left[\left(\delta_{e}-\delta_{e_{b a l}}\right)^{2}+\left(H^{*}-H\right)^{2}\right] d t
$$

with boundary conditions are, 


$$
\begin{aligned}
& V(0)=V_{0}, \\
& \gamma(0)=\gamma_{0}, \\
& q(0)=q_{0} \\
& h(0)=h_{0}, h\left(t_{f}\right)=h_{f} \\
& X(0)=X_{0} \\
& \theta(0)=\theta_{0,}, \theta\left(t_{f}\right)=\theta_{f}
\end{aligned}
$$

and the final time $t_{f}$ is free

The variational Hamiltonian is formed by adjoining the right-hand sides of the system of state differential Eqs. (1-6) with the costate variables

$$
\begin{aligned}
& H=\left(\delta_{e}-\delta_{e_{b}}\right)^{2}+\left(H^{*}-H\right)^{2}+ \\
& \lambda_{V}\left(\frac{g}{W}\left(T_{b}-D\right)-g \sin \gamma\right)+\lambda_{\gamma}\left(\frac{g}{V}\left(\frac{L}{W}-\cos \gamma\right)\right) \\
& +\lambda_{q}\left(\frac{\bar{q} S C}{I_{y}} C_{m}\right)+\lambda_{h}(V \sin \gamma)+\lambda_{x}(V \cos \gamma)+\lambda_{\theta}(q)
\end{aligned}
$$

The costate variables are defined by differential equation

$$
\begin{gathered}
\frac{d \lambda_{v}}{d t}=-\frac{\partial H}{\partial v}, v=V, \gamma, q, h, x, \theta \\
\dot{\lambda}_{V}=\lambda_{V}\left[\frac{g}{W}\left(D_{v}\right)\right]+\lambda_{\gamma} \frac{g}{V^{2}}\left(\frac{L}{W}-\cos \gamma\right)-\lambda_{q} \frac{\bar{q}_{v} C S C_{m}}{I_{y}}-\lambda_{h} \sin \gamma-\lambda_{x} \cos \gamma \\
\dot{\lambda}_{\gamma}=\lambda_{V} g \cos \gamma-\lambda_{\gamma} \frac{g \sin \gamma}{V}-\lambda_{h} V \cos \gamma+\lambda_{x} V \sin \gamma \\
\dot{\lambda}_{q}=-\lambda_{q} \frac{\bar{q} C S C_{m_{q}}}{I_{y}}-\lambda_{\theta} \\
\dot{\lambda}_{h}=2\left(H^{*}-H\right) \\
\dot{\lambda}_{x}=0 \\
\dot{\lambda}_{\theta}=0
\end{gathered}
$$

Solving these equations with the appropriate boundary conditions is a two-point boundaryvalue problem (TPBVP). Generally, at the initial point, all six states are specified and the costates are free; whereas, at the final time, the $H, \theta$ is fix and the remaining four states are free. Thus, at the final time all costates are free. The free final time $t_{f}$ can be considered as an additional variable of the problem and, by the standard transformation

$$
t=t_{f} \xi
$$




$$
d / d \xi=t_{f} d / d t
$$

The equations are transformed into a system with the independent variable $\xi$ ranging in the interval $0 \leq \xi \leq 1$. Further, the additional trivial differential equation

$$
\frac{d}{d \xi} t_{f}=0
$$

is added to the system. At the final time, the transversality condition requires that

$$
H\left(t_{f}\right)=0
$$

The optimal control function is obtained by means of the minimum principle. With respect to the elevator, we find the relation

$$
\delta_{e}=\delta_{e_{b}}-\lambda_{q} \frac{\bar{q} \operatorname{CSC}{m_{\delta_{e}}}}{2 I_{y}}
$$

Result of optimal trajectory for the selected B-727 is presented in Fig (3) through (8). The costate variable $\lambda_{q}$, which is responsible for the determination of the extermal controller, is shown in Fig.8.

\section{B. Pole Placement Method}

An alternative and very powerful method for designing feedback gains for autostabilization systems is the pole placement method. The method is based on the manipulation of the equations of motion in state space form and makes full use of the appropriate computational tools in the analytical process. Practical application of the method to airplanes is limited since it assumes that all state variables are available for use in an augmentation system, which is not usually the case. The open loop state equation may then be written

$$
\dot{\mathrm{X}}=\mathrm{AX}+\mathrm{BU} \& \mathrm{Y}=\mathrm{CX}
$$

$$
\mathrm{A}=\left[\begin{array}{ccccc}
-0.04436 & 29.988 & 0 & -32.1629 & 0 \\
-0.001458 & -0.53075 & 1 & 0.007357 & 0 \\
0 & -0.4914 & -0.431 & 0 & 0 \\
0 & 0 & 1 & 0 & 0 \\
0 & -210 & 0 & 210 & 0
\end{array}\right] \& \mathrm{~B}=\left[\begin{array}{c}
0 \\
0 \\
-0.5849 \\
0 \\
0
\end{array}\right]
$$

To track the target successfully, the closed-loop tracking system must be fast enough. To improve the flying qualities of this airplane more short-period damping is needed. A short period mode damping ratio of 0.7 was chosen to have gives a good margin of stability. Therefore, the pole placement method is employed to give the augmented aircraft the following closed loop characteristic polynomial $\Delta_{\text {aug }}(s)=(s+0.00019)\left(s^{2}+11.2 s+64\right)\left(s^{2}+0.12 s+0.0684\right)$. The feedback gain matrix required to give the augmented aircraft the characteristic polynomial was determined $\mathrm{K}=\left[\begin{array}{lllll}0.1411 & -85.6292 & -17.6339 & -14.6896 & -0.0004\end{array}\right]$ 


\section{Intelligent control techniques}

Intelligent control achieves automation via the emulation of biological intelligence. It either seeks to replace a human who performs a control task or it borrows ideas from how biological systems solve problems and applies them to the solution of control problems. In this section we will provide an overview of several techniques used for intelligent control and discuss challenging industrial application domains where these methods may provide particularly useful solutions. The objective here is not to provide a comprehensive treatment. We only seek to present the basic ideas to give a favor of the approaches.

\section{A. Neural Net-based Control Design}

Artificial neural networks are circuits, computer algorithms, or mathematical representations loosely inspired by the massively connected set of neurons that form biological neural networks. The success of neural networks is mainly attributed to their unique features:

1. Parallel structures with distributed storage and processing of massive amounts of information.

2. Learning ability made possible by adjusting the network interconnection weights and biases based on certain learning algorithms.

The neural flight control architecture is based upon the augmented model inversion controller, developed by Rysdyk and Calise [23]. The NN adaptation rule results from nonlinear stability analysis, which ensures that the error signals and network weights are bounded. One of the common methods for controlling nonlinear dynamical systems is based on approximate feedback linearization [24]. The form that is employed in each control channel depends on the relative degree of the controlled variable. To simplify our discussion, we assume that the system has full relative degree, where each controlled variable (element of the state vector $x$ ) has a relative degree of two

$$
\ddot{x}=f(x, \dot{x}, \delta)
$$

In the case of aircraft, typically $x, \delta \in R$, where the element of $x$ correspond to the pitch attitude angle. A variant of this form arises in which angular rate is controlled. Here, the equation of motion for that degree of freedom is expressed in first order form [25]. A pseudo-control $\mathrm{v}$ is defined such that the dynamic relation between it and the system state is linear

$$
v=f(x, \dot{x}, \delta)
$$

Ideally, the actual controls $\delta$ are obtained by inverting Eq. (30). The total pseudo control signal is constructed of three components

$$
v=v_{r m}+v_{p d}-v_{a d}
$$

where $v_{r m}$ the pseudo-control component is generated by the reference model, $v_{p d}$ is the output of the linear compensator, and $v_{a d}$ is generated by the adaptive element introduced to compensate for the model inversion error. The linear compensator is designed so that the error dynamics are stabilized. This is most often achieved using standard proportionalderivative (PD) controllers, although additional integral action can be incorporated to improve steady state performance. For the second order system, PD compensation is expressed by 


$$
v_{p d}=\left[\begin{array}{ll}
K_{P} & K_{D}
\end{array}\right] e
$$

The compensator gain matrices $K_{P}, K_{D} \in R$ are chosen so that the tracking error dynamics given by

$$
\begin{gathered}
\dot{e}=A e+B\left[v_{a d}-\Delta\right] \\
A=\left[\begin{array}{cc}
0 & I \\
-K_{P} & -K_{D}
\end{array}\right], B=\left[\begin{array}{l}
0 \\
I
\end{array}\right]
\end{gathered}
$$

are stable, i.e., the eigenvalues of $A$ are prescribed. It is evident from Eq. (33) that the role of the adaptive component $v_{a d}$ is to cancel $\Delta$.The adaptive signal is chosen to be the output of a single hidden layer [26].

$$
v_{a d}=W^{T} \sigma\left(V^{T} \bar{x}\right)
$$

where $V$ and $W$ are the input and output weighting matrices, respectively, and $\sigma$ is a sigmoid activation function. Although ideal weighting matrices are unknown and usually cannot be computed, they can be adapted in real time using the following NN weights training rules [27]:

$$
\begin{gathered}
\dot{W}=-\left[\left(\sigma-\sigma^{\prime} V^{T} \bar{x}\right) e^{T} P B+\kappa\|e\| W\right] \Gamma_{W} \\
\dot{V}=-\Gamma_{V}\left[\bar{x} e^{T} P B W^{T}+\kappa\|e\| V\right]
\end{gathered}
$$

where $\Gamma_{W}, \Gamma_{V}$ and $\Gamma_{W}, \Gamma_{V}$ are the positive definite learning rate matrices, and $\mathrm{\kappa}$ is the emodification parameter. Here, $P$ is a positive definite solution of the Lyapunov equation $A^{T} P+P A+Q=0$ for any positive definite $Q$.

\section{B. Fuzzy Logic-Based Control Design}

The existing applications of fuzzy control range from micro-controller based systems in home applications to advanced flight control systems. The main advantages of using fuzzy are as follows:

1. It is implemented based on human operator's expertise which does not lend itself to being easily expressed in conventional proportional integral-derivative parameters of differential equations, but rather in action rules.

2. For an ill-conditioned or complex plant model, fuzzy control offers ways to implement simple but robust solutions that cover a wide range of system parameters and, to some extent, can cope with major disturbances.

The aircraft landing procedures admit a linguistic describing. This is practiced, for example, in case of guiding for landing in non-visibility conditions or in piloting learning. This approach permits to build a model for landing control based on the reasoning rules using the fuzzy logic. The process requires the control of the following parameters: the current altitude to runway surface $(H)$, the aircraft's vertical speed and aircraft flight speed. The goal of the control is formulated as follow: the aircraft should touch the runway ( $H$ becomes 0$)$ at the conventional point of landing with admitted vertical touch speed and the recommended 
landing speed. The input of FLC normally includes the error between the state variable and its set point, $\left(e=x_{d}-x\right)$ and the first derivative of the error, $\dot{e}$. A typical form of the linguistic rules is represented as

Rule $i$ Th: If e is $A_{i}$ and $\dot{e}$ is $B_{i}$ then $u^{*}$ is $C_{i}$

Where $A_{i}, B_{i}$, and $C_{i}$ are the fuzzy sets for the error, the error rate, and the controller output at rule $\mathrm{i}$, respectively, and $\mathrm{u}^{*}$ is the controller output.

The resulting rule base of FLC is shown in Table 2. The abbreviations representing the fuzzy sets $\mathbf{N}, \mathbf{Z}, \mathbf{P}, \mathbf{S}$, and $\mathbf{B}$ in linguistic form stand for negative, zero, positive, small, and big, respectively, for example negative big (NB). Five fuzzy sets in triangular membership functions are used for FLC input variables, e and è, and FLC output, u* . For the fuzzy inference or rule firing, Mamdani-type min-max composition is employed. In the defuzzification stage, by adopting the method of center of gravity, the deterministic control $u$ is obtained. The membership functions have been designed for input and output diagram using the trapezoidal shapes, as shown in Figures 1, 2. The fuzzy control system design with a simple longitudinal aircraft model given by Eqs. (1-6).

\section{Advantages over Conventional Designs}

1. Fuzzy guidance and control provides a new design paradigm such that a control mechanism based on expertise can be designed for complex, ill-defined flight dynamics without knowledge of quantitative data regarding the input-output relations, which are required by conventional approaches. A fuzzy logic control scheme can produce a higher degree of automation and offers ways to implement simple but robust solutions that cover a wide range of aerodynamic parameters and can cope with major external disturbances.

2. Artificial Neural networks constitute a promising new generation of information processing systems that demonstrate the ability to learn, recall, and generalize from training patterns or data. This specific feature offers the advantage of performance improvement for ill-defined flight dynamics through learning by means of parallel and distributed processing. Rapid adaptation to environment change makes them appropriate for guidance and control systems because they can cope with aerodynamic changes during flight.

\section{Simulation results and discussion}

Simulations are performed at sea level, airspeed of $210 \mathrm{ft} / \mathrm{s}$, corresponding to the flare maneuver configuration of the Boeing 727. The simulation results are presented in Figs 3 to 8. Figure 3, which depicts the flight speed variation, demonstrates that the engines can regulate slight speed until that is compromised for attitude rate control. Time histories of the controls are shown in Fig. 4. The time response of pitch angle is shown in fig.5. A comparison between the commanded altitude profile and the actual aircraft response is presented in Fig. 6, it shows that the difference between the actual and desired trajectory (the fuzzy logic, neural net-based adaptive and optimal controls) is kept less than about $6 \mathrm{ft}$. This figure, so shows that the sink rate (the rate of descent) is reduced to less than $1.0 \mathrm{ft} / \mathrm{sec}$, which is small enough to achieve a smooth landing. The fuzzy logic, neural net-based adaptive and optimal control approaches do the flare maneuver well, while the Pole Placement Method has substantially large error. Neural network adaptation signal $v_{a d}$ for compensate inversion error is presented in Fig. 7. Summarizing the results presented so far, the nonlinear controller performance for this maneuver has been found very good. 


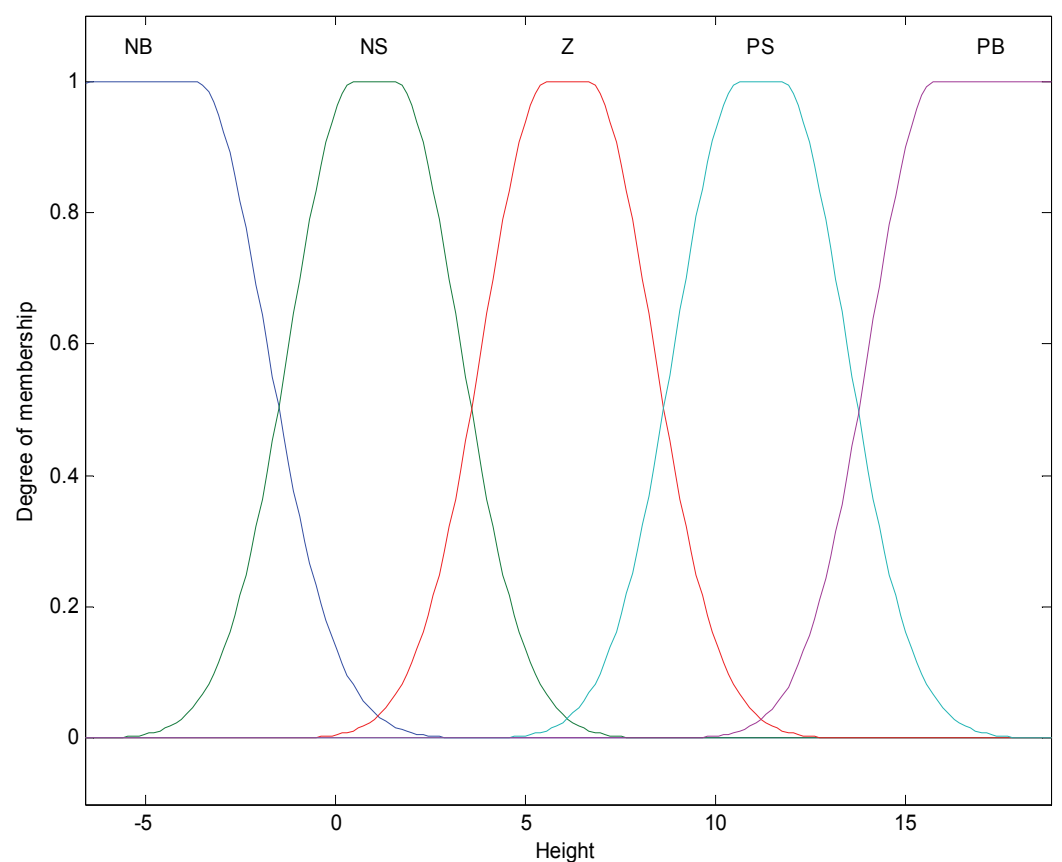

Fig. 1. Altitude Membership Functions

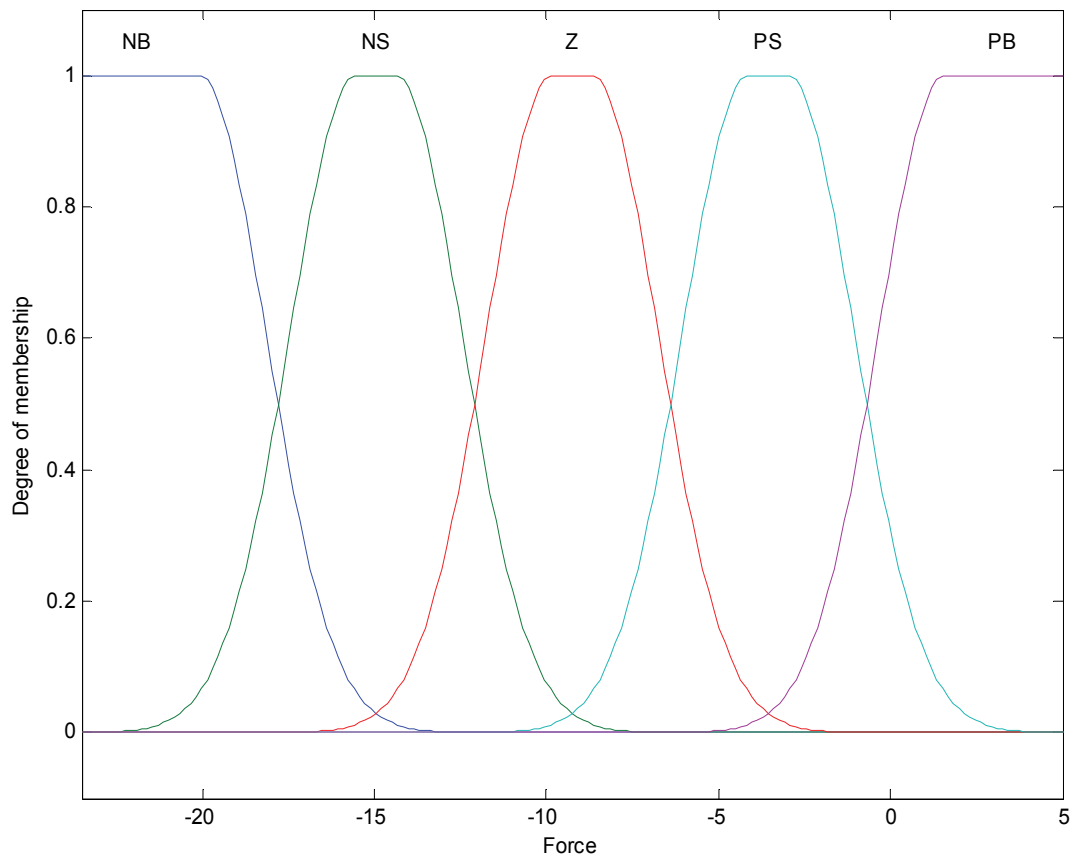

Fig. 2. Force Membership Functions 


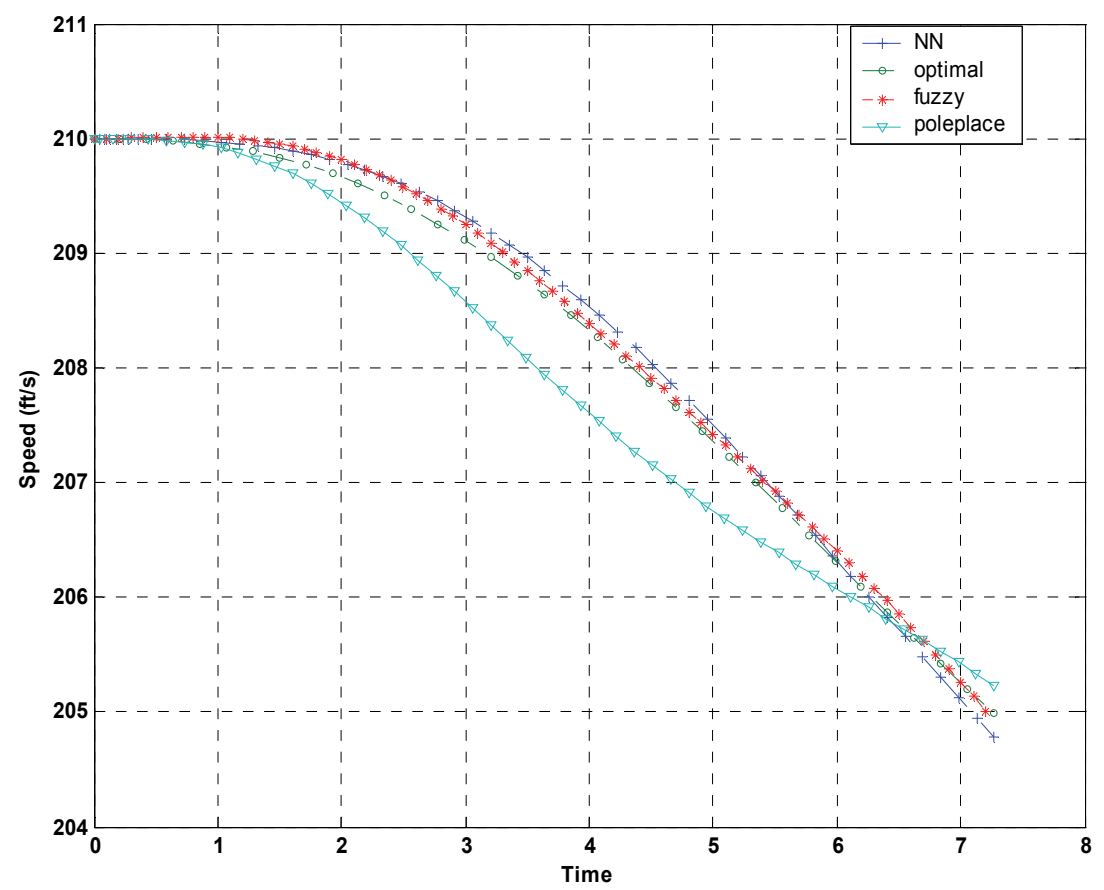

Fig. 3. Time response of the airspeed

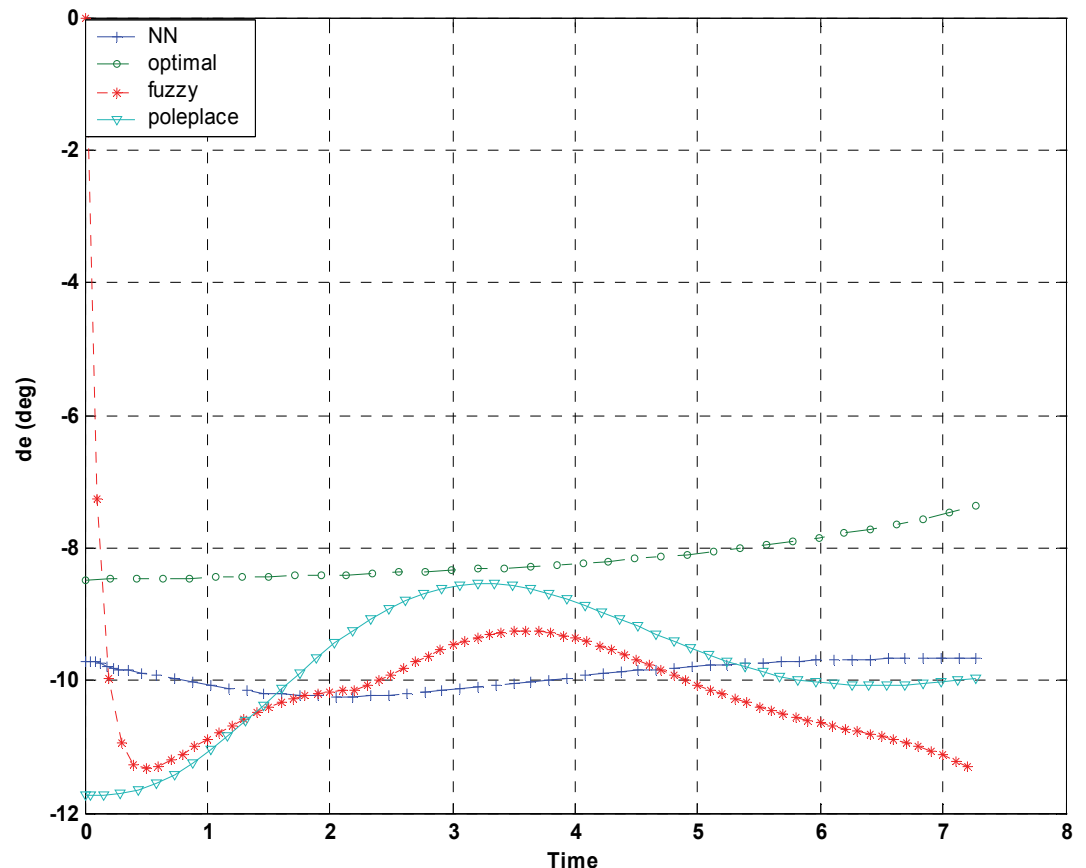

Fig. 4. Time response of the elevator 


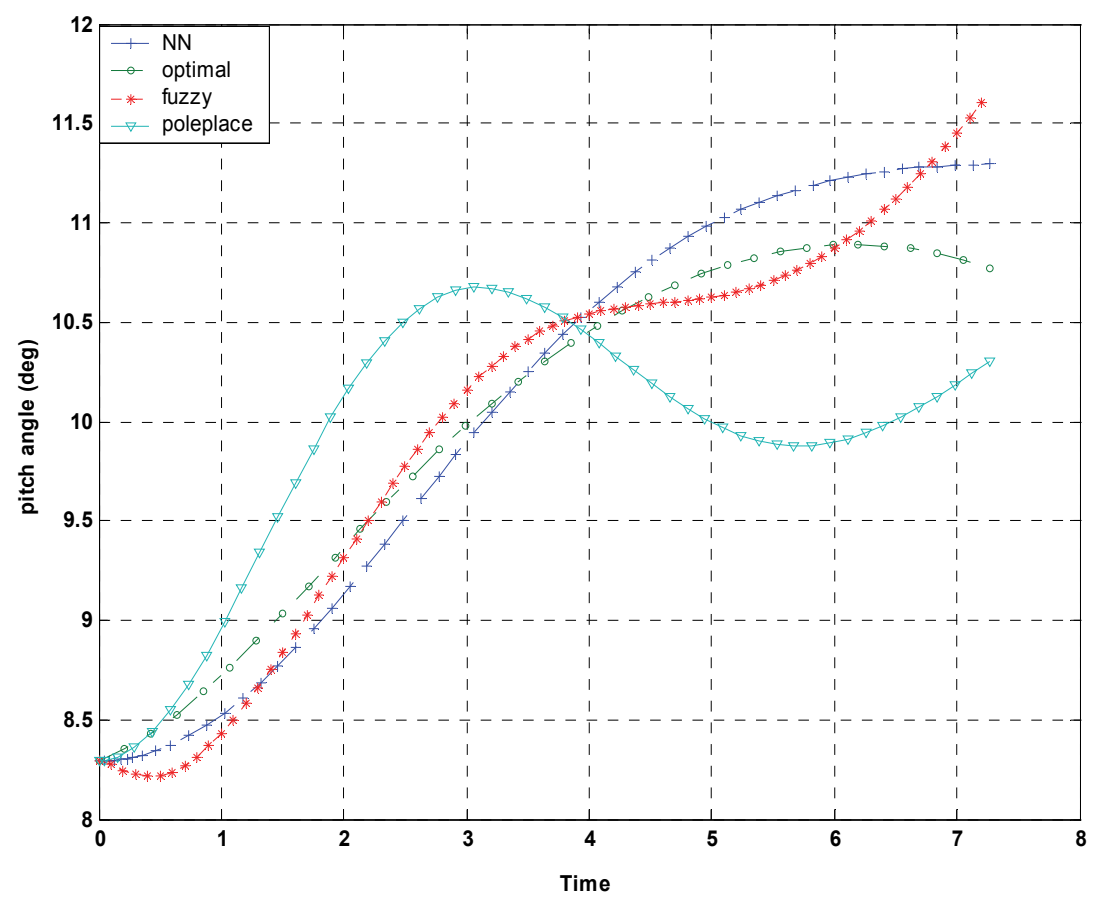

Fig. 5. Time response of the pitch angle

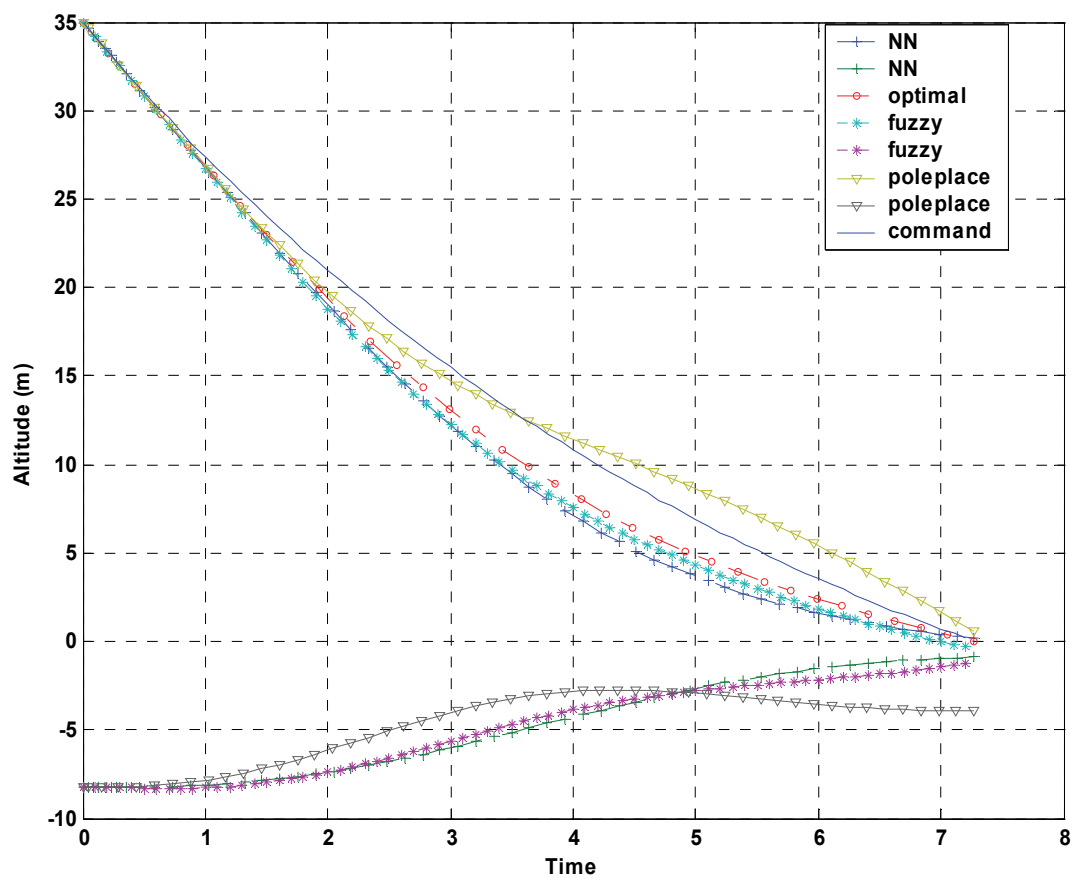

Fig. 6. Desired and actual flare trajectories 


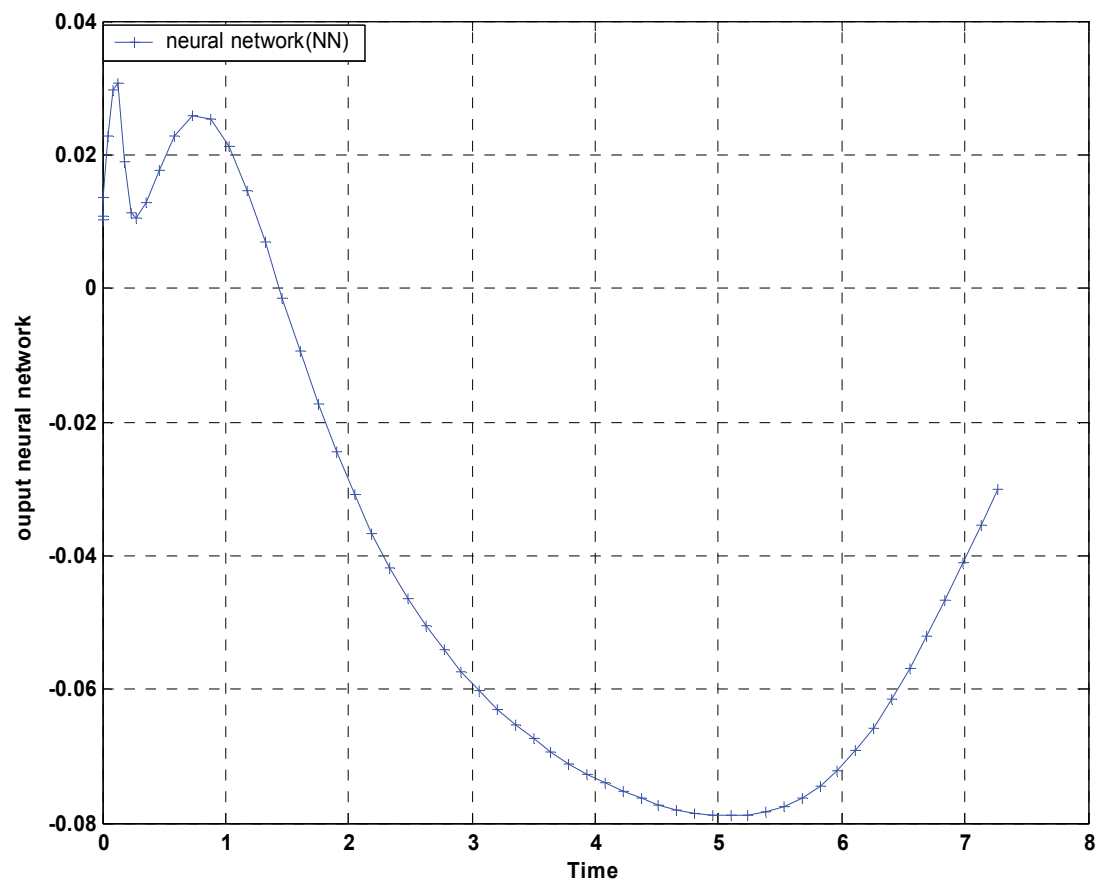

Fig. 7. NN adaptation signal $v_{a d}$

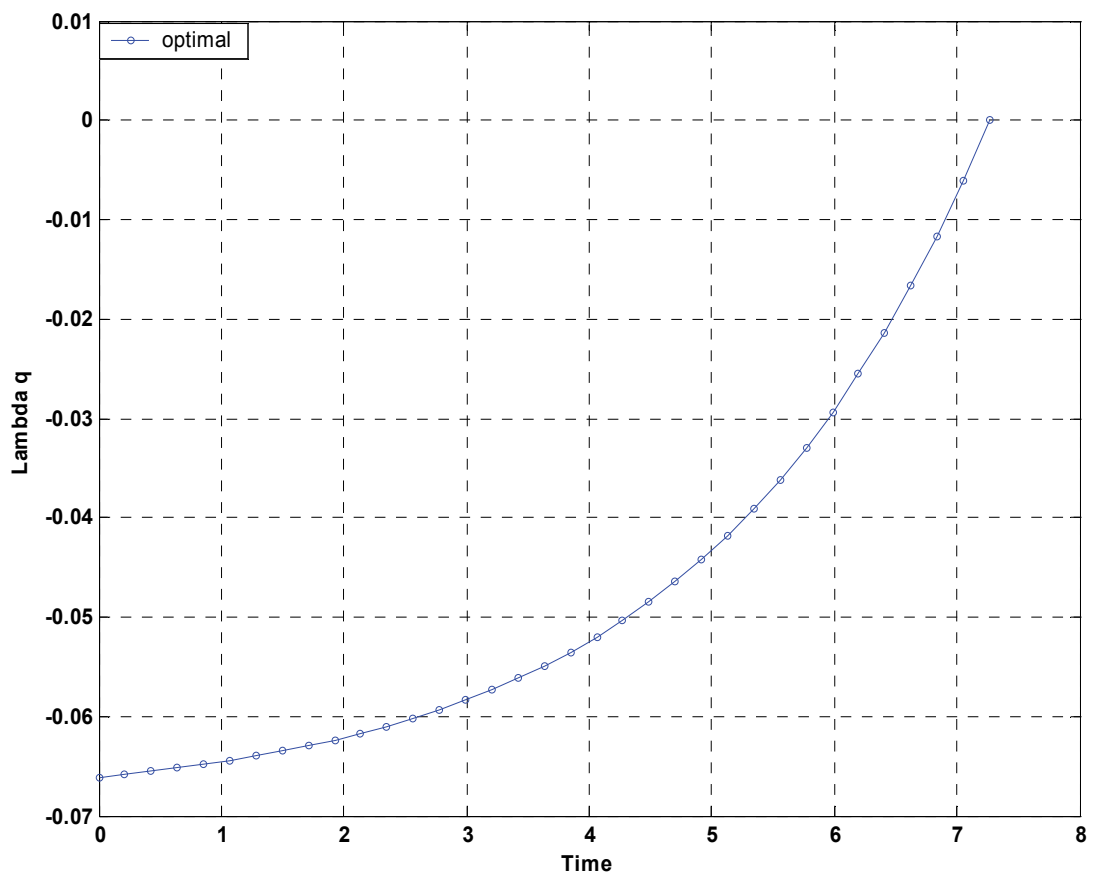

Fig. 8. Time response of the $\lambda_{q}$ 


\section{Conclusions}

It has been the general focus of this paper to summarize the basic knowledge about intelligent control structures for the development of control systems. For completeness, conventional, adaptive neural net-based, fuzzy logic-based, control techniques have been briefly summarized. Our particular goal was to demonstrate the potential intelligent control systems for high precision maneuvers required by aircraft landing. The proposed model reveals the functional aspect for realistic simulation data. The method does not require the existing controller to be designed based on a linear model.

\begin{tabular}{|c|c|}
\hline $\mathrm{a}_{\max }=17.2$ & $\mathrm{a}_{*}=12 \mathrm{deg}$ \\
\hline $\mathrm{g}=32.2 \mathrm{ft}^{2}$ & $\mathrm{I}_{\mathrm{y}}=3 \times 10^{6}$ \\
\hline $\mathrm{C}_{0}=0.7125$ & $\mathrm{~B}_{0}=0.1552$ \\
\hline $\mathrm{B}_{1}=0.12369 \mathrm{rad}^{-1}$ & $\mathrm{~S}=0.156 \times 10^{4} \mathrm{ft}^{2}$ \\
\hline $\mathrm{B}_{2}=2.4203 \mathrm{rad}^{-2}$ & $\mathrm{~W}=150000 \mathrm{Ib}$ \\
\hline $\mathrm{C}_{2}=-9.0277 \mathrm{rad}^{-2}$ & $\mathrm{C}_{1}=6.0877 \mathrm{rad}^{-1}$ \\
\hline
\end{tabular}

Table 1. model parameter data B-727

\begin{tabular}{|c|c|c|c|c|c|}
\hline \multicolumn{7}{|c|}{ Fuzzy set, $\dot{e}$} \\
\hline Fuzzy set, $\boldsymbol{e}$ & NB & NS & Z & PS & PB \\
\hline NB & PB & PB & PS & PS & NS \\
\hline NS & PB & PS & PS & NS & NB \\
\hline Z & PB & PS & Z & NS & NB \\
\hline PS & PB & PS & NS & NS & NB \\
\hline PB & PS & NS & NS & NB & NB \\
\hline
\end{tabular}

Table 2. Rule base for FLC

\section{References}

[1] Chicago, IL, U.S.A. Locke, A. S., Guidance, D. Van Nostrand Co., Princeton, NJ, U.S.A (1955).

[2] Bryson, A. E., Jr. and Y. C. Ho, Applied Optimal Control., Blaisdell, Waltham, MA, U.S.A (1969).

[3] Lin, C. F., Modern Navigation, Guidance, and Control Processing, Prentice-Hall, Englewood Cliffs, NJ, U.S.A (1991).

[4] Zarchan, P., Tactical and Strategic Missile Guidance, 2nd Ed., AIAA, Inc., Washington, D.C., U.S.A (1994).

[5] Bezdek, J., "Fuzzy Models: What are they and Why," IEEE Trans. Fuzzy Syst., Vol.1 No.1, pp, 1-6 (1993).

[6] Miller, W. T., R. S. Sutton, and P. J. Werbos., Neural Networks for Control., MIT Press, Cambridge, MA, U.S.A. Mishra (1991).

[7] Narendra, K. S. and K. Parthasarthy., Identification and control of dynamical systems using neural networks. IEEE Trans. Neural Networks, 1(1), 4-27 (1990). 
[8] Mamdani, E. H. and S. Assilian., An experiment in linguistic synthesis with a fuzzy logic controller. Int. J. Man Machine Studies, 7(1), 1-13 (1975).

[9] Lee, C. C., Fuzzy logic in control systems: fuzzy logic controller part I. IEEE Trans. Syst. Man and Cyb., 20(2), 404-418 (1990).

[10] Lee, C. C., Fuzzy logic in control systems: fuzzy logic controller part II. IEEE Trans. Syst. Man and Cyb., 20(2), 419-435 (1990).

[11] Driankov, D., H. Hellendoorn, and M. Reinfrank., "An Introduction to Fuzzy Control". Springer, Berlin, Germany. Driankov (1993).

[12] Dash, P. K., S. K. Panda, T. H. Lee and J. X. Xu., Fuzzy and neural controllers for dynamic systems: an overview. Proc. Int. Conf. Power Electronics, Drives and Energy Systems, Singapore (1997).

[13] BULIRSCH,R., F. Montone, and H. Pesch, "Abort Landing in the Presence of Windshear as a minimax optimal Control Problem, Part 1: Necessary Conditions", J. Opt. Theory Appl., Vol. 70,pp. 1-23 (1991).

[14] Price, C. F. and R. S. Warren, "Performance Evaluation of Homing Guidance Laws for Tactical Missiles," TASC Tech (1973).

[15] Nesline, F. W., B. H. Wells, and P. Zarchan, "Combined optimal/classical approach to robust missile autopilot design," AIAA J. Guid. Contr., Vol.4,No.3, pp.316-322 (1981).

[16] Nesline, F.W. and M.L. Nesline, "How Autopilot Requirements Constrain the Aerodynamic Design of Homing Missiles," Proc. Amer. Contr. Conf., San Diego, CA, USA, pp.176-730 (1984).

[17] Stallard, D. V., "An Approach to Autopilot Design for Homing Interceptor Missiles," AIAA Paper 91-2612, AIAA, Washington, D.C., U.S.A, pp. 99-113 (1991).

[18] Lin, C.F., J. Cloutier, and J. Evers, "Missile Autopilot Design Using a Generalized Hamiltonian Formulation," Proc. IEEE 1st Conf. Aero. Contr. Syst., Westlake Village, CA, USA, pp. 715-723 (1993).

[19] Lin, C. F. and S. P. Lee, "Robust missile autopilot design using a generalized singular optimal control technique," J. Guid., Contr., Dyna., Vol. 8, No. 4, pp. $498-507$ (1985).

[20] Lin, C. F. Advanced Control System Design. Prentice- Hall, Englewood Cliffs, NJ, U.S.A (1994).

[21] Stoer J. and R. Burlisch, Introduction to Numerical Analysis, Springer Verlag, New York, (1980).

[22] Oberle, H.J, "BNDSCO-A Program for the Numerical Solution of Optimal Control Problems," Internal Report No.515-89/22, Institute for Flight Systems Dynamics, DLR, Oberpfaffenhofen, Germany (1989).

[23] Rysdyk, R., B. Leonhardt, and A.J. Calise, "Development of an Intelligent Flight Propulsion Control System: Nonlinear Adaptive Control," AIAA- 2000-3943, Proc. Guid. Navig. Contr. Conf., Denver, CO, USA (2000).

[24] Isodori, A., Nonlinear Control Systems, Springer Verlag, Berlin (1989).

[25] Calise, A. J., S. Lee, and M. Sharma, "Development of a reconfigurable flight control law for the X-36 tailless fighter aircraft," Proc. AIAA Guid. Navig. Contr. Conf., Denver, CO, USA., AIAA-2000-3940 (2000).

[26] Hornik, K., M. Stinchcombe, M. and H. White, “Multilayer Feedforward Networks are Universal Approximators," Neural Networks, Vol. 2, pp. 359-366 (1989).

[27] Johnson, E. and A.J. Calise, "Neural Network Adaptive Control of Systems with Input Saturation," Proc. Amer. Contr. Conf., pp. 3527-3532. (2001). 


\title{
Oscillation Susceptibility of an Unmanned Aircraft whose Automatic Flight Control System Fails
}

\author{
Balint Maria-Agneta and Balint Stefan \\ West University of Timisoara \\ Romania
}

\section{Introduction}

Interest in oscillation susceptibility of an aircraft was generated by crashes of high performance fighter airplanes such as the YF-22A and B-2, due to oscillations that were not predicted during the aircraft development. Flying qualities and oscillation prediction, based on linear analysis, cannot predict the presence or the absence of oscillations, because of the large variety of nonlinear interactions that have been identified as factors contributing to oscillations. Pilot induced oscillations have been analyzed extensively in many papers by numerical means.

Interest in oscillation susceptibility analysis of an unmanned aircraft, whose flight control system fails, was generated by the need to elaborate an alternative automatic flight control system for the Automatic Landing Flight Experiment (ALFLEX) reentry vehicle for the case when the existing automatic flight control system of the vehicle fails.

The purpose of this chapter is the analysis of the oscillation susceptibility of an unmaned aircraft whose automatic flight control system fails. The analysis is focused on the research of oscillatory movement around the center of mass in a longitudinal flight with constant forward velocity (mainly in the final approach and landing phase). The analysis is made in a mathematical model defined by a system of three nonlinear ordinary differential equations, which govern the aircraft movement around its center of mass, in such a flight. This model is deduced in the second paragraph, starting with the set of 9 nonlinear ordinary differential equations governing the movement of the aircraft around its center of mass.In the third paragraph it is shown that in a longitudinal flight with constant forward velocity, if the elevator deflection outruns some limits, oscillatory movement appears. This is proved by means of coincidence degree theory and Mawhin's continuation theorem. As far as we know, this result was proved and published very recently by the authors of this chapter (research supported by CNCSIS--UEFISCSU, project number PNII - IDEI 354 No. 7/2007) and never been included in a book concerning the topic of flight control.The fourth paragraph of this chapter presents mainly numerical results. These results concern an Aero Data Model in Research Environment (ADMIRE) and consists in: the identification of the range of the elevator deflection for which steady state exists; the computation of the manifold of steady states; the identification of stable and unstable steady states; the simulation of successful and unsuccessful maneuvers; simulation of oscillatory movements. 


\section{The mathematical model}

Frequently, we describe the evolution of real phenomena by systems of ordinary differential equations. These systems express physical laws, geometrical connections, and often they are obtained by neglecting some influences and quantities, which are assumed insignificant with respect to the others. If the obtained simplified system correctly describes the real phenomenon, then it has to be topologically equivalent to the system in which the small influences and quantities (which have been neglected) are also included. Furthermore, the simplified system has to be structurally stable. Therefore, when a simplified model of a real phenomenon is build up, it is desirable to verify the structural stability of the system. This task is not easy at all. What happens in general is that the results obtained in simplified model are tested against experimental results and in case of agreement the simplified model is considered to be authentic. This philosophy is also adapted in the description of the motion around the center of gravity of a rigid aircraft. According to Etkin \& Reid, 1996; Cook, 1997, the system of differential equations, which describes the motion around the center of gravity of a rigid aircraft, with respect to an $x y z$ body-axis system, where $x z$ is the plane of symmetry, is:

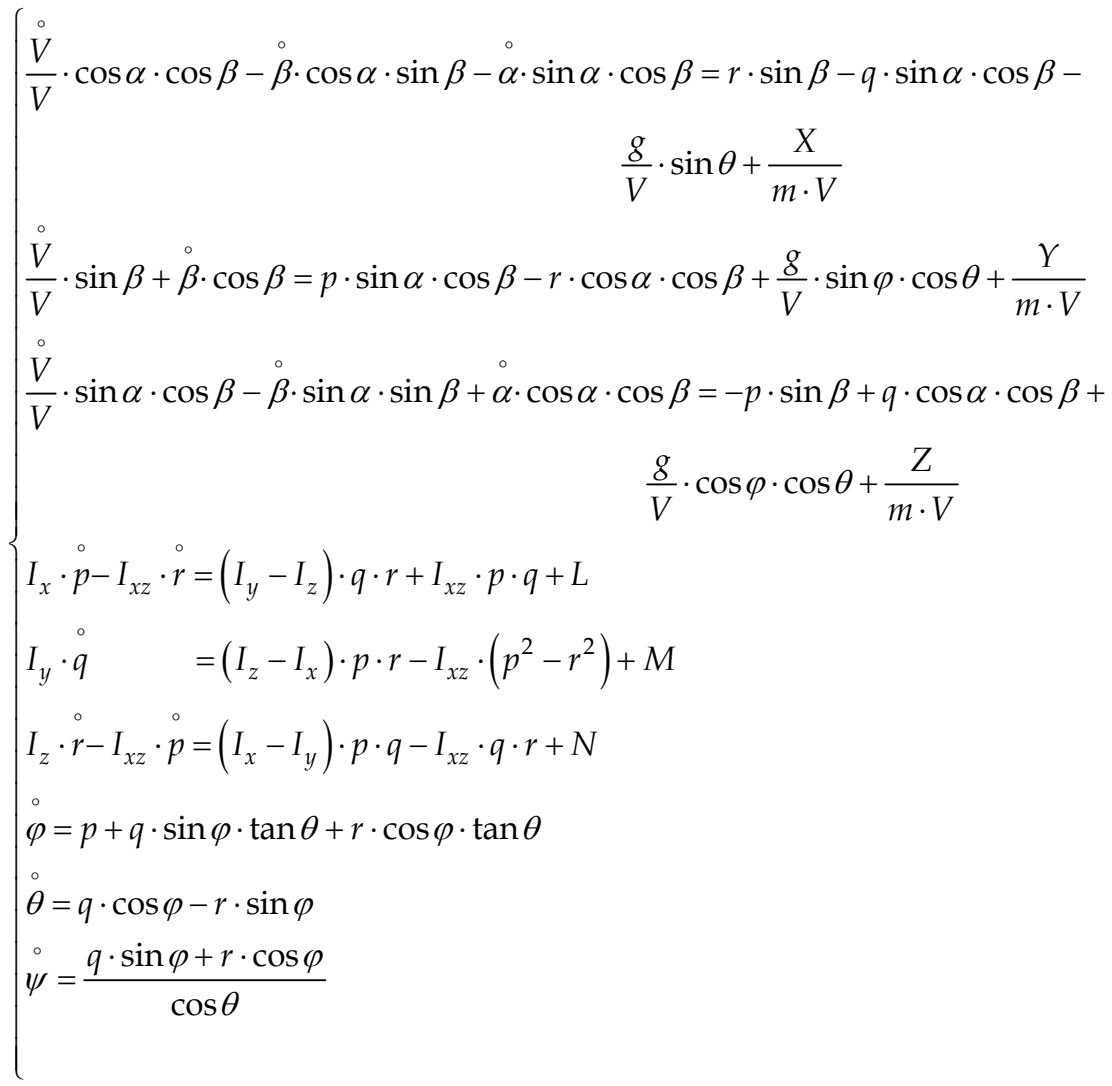

The state parameters of this system are: forward velocity $V$, angle of attack $\alpha$, sideslip angle $\beta$, roll rate $p$, pitch rate $q$, yaw rate $r$, Euler roll angle $\varphi$, Euler pitch angle $\theta$ and Euler yaw angle $\psi$. The constants $I_{x}, I_{y}$ and $I_{z}$-moments of inertia about the $x, y$ and $z$-axis, respectively; 
$I_{x z}$ - product of inertia, $g$-gravitational acceleration; and $m$ - mass of the vehicle. The aero dynamical forces $X, Y, Z$ and moments $L, M, N$ are functions of the state parameters and the control parameters: $\delta_{a}$-aileron deflection; $\delta_{e}$ - elevator deflection; and $\delta_{r}$-rudder deflection (the body flap, speed break, $\delta_{c}, \delta_{\mathrm{ca}}$ are available as additional controls but, for simplicity, they are set to 0 in the analysis to follow). When the automatic flight control system is in function, then the control parameters are functions of the state parameters, describing how the flight control system works. When the automatic flight control system fails, then the control parameters are constant. This last situation will be analyzed in this chapter.

A flight with constant forward velocity $V$ is defined as a flight for which $V=$ const (i.e. $\stackrel{\circ}{V}=0)$.

In a flight with constant forward velocity $V$ the following equalities hold:

$$
\left\{\begin{array}{l}
-\stackrel{\circ}{\beta} \cdot \cos \alpha \cdot \sin \beta-\alpha \cdot \sin \alpha \cdot \cos \beta=r \cdot \sin \beta-q \cdot \sin \alpha \cdot \cos \beta-\frac{g}{V} \cdot \sin \theta+\frac{X}{m \cdot V} \\
\stackrel{\circ}{\beta} \cdot \cos \beta=p \cdot \sin \alpha \cdot \cos \beta-r \cdot \cos \alpha \cdot \cos \beta+\frac{g}{V} \cdot \sin \varphi \cdot \cos \theta+\frac{Y}{m \cdot V} \\
\stackrel{\circ}{\beta} \cdot \sin \alpha \cdot \sin \beta+\alpha \cdot \cos \alpha \cdot \cos \beta=-p \cdot \sin \beta+q \cdot \cos \alpha \cdot \cos \beta+\frac{g}{V} \cdot \cos \varphi \cdot \cos \theta+\frac{Z}{m \cdot V}
\end{array}\right.
$$

Replacing $\stackrel{\circ}{V}$ by 0 in the system (1), the equalities(2) are obtained.

If in a flight with constant forward velocity $V$ one has $\beta \equiv(2 n+1) \cdot \pi / 2$, then the following equalities hold:

$$
\left\{\begin{array}{l}
(-1)^{n} \cdot r-\frac{g}{V} \cdot \sin \theta+\frac{X}{m \cdot V} \equiv 0 \\
\frac{g}{V} \cdot \sin \varphi \cdot \cos \theta+\frac{Y}{m \cdot V} \equiv 0 \\
(-1)^{n+1} \cdot p+\frac{g}{V} \cdot \cos \varphi \cdot \cos \theta+\frac{Z}{m \cdot V} \equiv 0
\end{array}\right.
$$

Replacing $\stackrel{\circ}{\beta}=0$ and $\beta \equiv(2 n+1) \cdot \pi / 2$ in (2), the equalities (3) are obtained.

If in a flight with constant forward velocity $V$ one has $\beta \neq(2 n+1) \cdot \pi / 2$, then the following equality holds:

$$
\begin{gathered}
g[\sin \beta \cdot \sin \varphi \cdot \cos \theta-\cos \alpha \cdot \cos \beta \cdot \sin \theta+\sin \alpha \cdot \cos \beta \cdot \cos \varphi \cdot \cos \theta]+\frac{Y}{m} \cdot \sin \beta+ \\
+\frac{X}{m} \cdot \cos \alpha \cdot \cos \beta+\frac{Z}{m} \cdot \sin \alpha \cdot \cos \beta \equiv 0
\end{gathered}
$$

Equation (4) is the solvability (compatibility) condition, with respect to $\stackrel{\circ}{\alpha}, \stackrel{\circ}{\beta}$, of the system (2) when $\beta \neq(2 n+1) \cdot \pi / 2$. 
If $\beta \neq(2 n+1) \cdot \pi / 2$ and equality (4) holds, then the system (2) can be solved with respect to $\dot{\alpha}, \dot{\circ}$, obtaining in this way the explicit system of differential equations, which describes the motion around the center of mass of the aircraft in a flight, with constant forward velocityV:

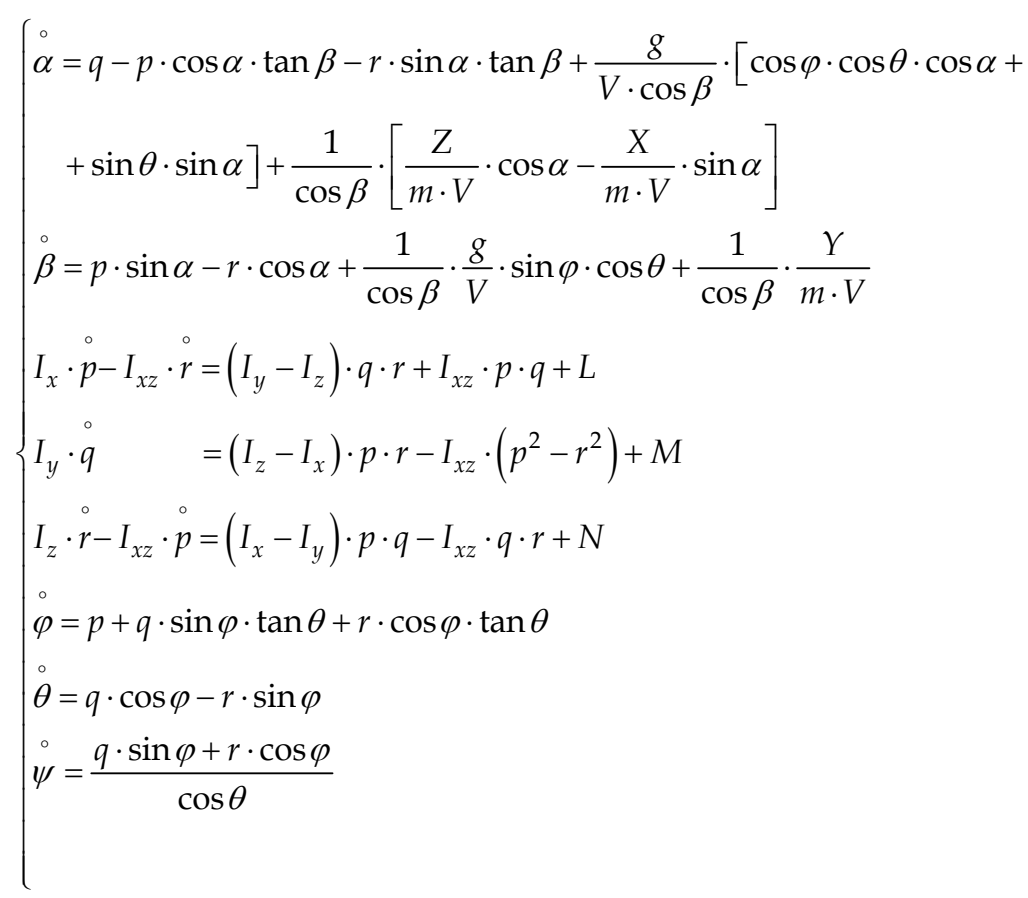

System (5) is obtained solving system (2) with respect to $\stackrel{\circ}{\alpha}, \stackrel{\circ}{\beta}$ and replacing in system (1) the equations $(1)_{1},(1)_{2},(1)_{3}$ with the obtained $\alpha$ and $\beta$.

A longitudinal flight is defined as a flight for which the following equalities hold:

$$
\beta \equiv p \equiv r \equiv \varphi \equiv \psi \equiv 0 \text { and } \delta_{a}=\delta_{r}=0 .
$$

A longitudinal flight is possible if and only if $Y=L=N=0$ for $\beta=p=r=\varphi=\psi=0$ and $\delta_{a}=\delta_{r}=0$.

This result is obtained from (1) taking into account the definition of a longitudinal flight. The explicit system of differential equations which describes the motion of the aircraft in a longitudinal flight is:

$$
\left\{\begin{array}{l}
\stackrel{\circ}{V}=g \cdot \sin (\alpha-\theta)+\frac{X}{m} \cdot \cos \alpha+\frac{Z}{m} \cdot \sin \alpha \\
\stackrel{\circ}{\alpha}=q+\frac{g}{V} \cdot \cos (\theta-\alpha)-\frac{X}{m \cdot V} \cdot \sin \alpha+\frac{Z}{m \cdot V} \cdot \cos \alpha \\
\stackrel{\circ}{q}=\frac{M}{I_{y}} \\
\stackrel{\circ}{\theta}=q
\end{array}\right.
$$


This result is obtained from (1) taking into account the definition of a longitudinal flight. In system (7) $X, Z, M$ depend only on $\alpha, q, \theta$ and $\delta_{e}$. These dependences are obtained replacing in the general expression of the aerodynamic forces and moments: $\beta=p=r=\varphi=\psi=0$ and $\delta_{a}=\delta_{r}=0$.

The explicit system of differential equations which describes the motion around the center of gravity of the aircraft in a longitudinal flight with constant forward velocity $V$ is:

$$
\left\{\begin{array}{l}
\stackrel{\circ}{\alpha}=q+\frac{g}{V} \cdot \cos (\theta-\alpha)-\frac{X}{m \cdot V} \cdot \sin \alpha+\frac{Z}{m \cdot V} \cdot \cos \alpha \\
\circ=\frac{M}{I_{y}} \\
\dot{\theta}=q
\end{array}\right.
$$

This system is obtained from (7) taking into account $\stackrel{\circ}{V}=0$.

A longitudinal flight with constant forward velocity is possible if the following equalities hold:

$$
\begin{gathered}
Y=L=N=0 \text { for } \beta=p=r=\varphi=\psi=0 \text { and } \delta_{a}=\delta_{r}=0 \\
g \cdot \sin (\alpha-\theta)+\frac{X}{m} \cdot \cos \alpha+\frac{Z}{m} \cdot \sin \alpha=0
\end{gathered}
$$

This result is obtained from system (7), taking into account the fact that $\stackrel{\circ}{V}$ is equal to zero. In (8) $X, Z, M$ depend on $\alpha, q, \theta, \delta_{e}$ and $V$. Taking into account (10), the system (8) can be written as:

$$
\left\{\begin{array}{l}
\dot{\alpha}=q+\frac{g}{V} \cdot \cos (\theta-\alpha)-\frac{g}{V} \cdot \sin (\theta-\alpha) \cdot \tan \alpha+\frac{Z}{m \cdot V} \cdot \frac{1}{\cos \alpha} \\
\dot{q}=\frac{M}{I_{y}} \\
\dot{\theta}=q
\end{array}\right.
$$

The system (11) describes the motion around the center of gravity of an aircraft in a longitudinal flight with constant forward velocity $V$ and defines the general nonlinear model.

In system (11) the functions $Z=Z\left(\alpha, q, \theta ; \delta_{e}, V\right)$ and $M=M\left(\alpha, q, \theta ; \delta_{e}, V\right)$ are considered known. When the automated flight control system fails, $\delta_{e}$ and $V$ are parameters.

Frequently, in a research environment for the description of the movement around the center of the gravity of some types of aircrafts in a flight with constant forward velocity $V$, the explicit system of differential equations (12) is employed by Balint et al., 2009a,b,c; 2010a,b; Kaslik \& Balint, 2007; Goto \& Matsumoto, 2000.

The model defined by equations (12) is called Aero Data Model In a Research Environment (ADMIRE). 
System (12) can be obtained from (5) substituting the general aero dynamical forces and moments (see for example section 4), assuming that $a$ and $\beta$ are small and making the approximations (13).

Due to the approximations (13), the ADMIRE model defined by (12) is also called the simplified ADMIRE model.

The simplified system which governs the longitudinal flight with constant forward velocity $V$ of the ADMIRE aircraft is (14).

System (14) is obtained from (12) for $\beta=p=r=\varphi=0, \delta_{a}=\delta_{r}=\delta_{c}=\delta_{c a}=0$ and defines the simplified nonlinear model of the motion around the center of gravity of the aircraft in a longitudinal flight with constant forward velocity $V$.

In system (14) $g, V, z_{\alpha}, z_{\delta_{e}}, m_{\alpha}, m_{q}, \overline{m_{\alpha}}, c_{2}, a_{2}, a, m_{\delta_{e}}$ are considered constants (see Section 4).

$$
\begin{aligned}
& \stackrel{\circ}{\alpha}=q-p \cdot \beta+\frac{g}{V} \cdot \cos \theta \cdot \cos \varphi+z_{\alpha} \cdot \alpha+y_{\beta} \cdot \beta^{2}+y_{p}(\alpha, \beta) \cdot p \cdot \beta+y_{r}(\beta) \cdot r \cdot \beta+ \\
& +y_{\delta_{a}} \cdot \beta \cdot \delta_{a}+z_{\delta_{e}} \cdot \delta_{e}+y_{\delta_{r}} \cdot \beta \cdot \delta_{r} \\
& \stackrel{\circ}{\beta}=p \cdot \alpha-r+\frac{g}{V} \cdot \sin \varphi \cdot \cos \theta-z_{\alpha} \cdot \alpha \cdot \beta+y_{\beta} \cdot \beta-y_{p}(\alpha, \beta) \cdot p-y_{r}(\beta) \cdot r+ \\
& +y_{\delta_{a}} \cdot \delta_{a}-z_{\delta_{e}} \cdot \beta \cdot \delta_{e}+y_{\delta_{r}} \cdot \delta_{r} \\
& \dot{p}=-i_{1} \cdot q \cdot r+l_{\beta}(\alpha) \cdot \beta+l_{p} \cdot p+l_{r}(\alpha) \cdot r+l_{\delta_{a}} \cdot \delta_{a}+l_{\delta_{r}} \cdot \delta_{r} \\
& \dot{q}=i_{2} \cdot p \cdot r+m_{\alpha} \cdot \alpha+m_{q} \cdot q-\overline{m_{\circ}} \cdot p \cdot \beta+\overline{y_{p}} \cdot p \cdot \beta+\overline{y_{\beta}} \cdot \beta^{2}+\overline{y_{r}}(\beta) \cdot r \cdot \beta+ \\
& +\frac{g}{V} \cdot\left(\overline{m_{\alpha}} \cdot \cos \theta \cdot \cos \varphi-\frac{c_{2}}{a} \cdot a_{2} \cdot \sin \theta\right)+\overline{y_{\delta_{a}}} \cdot \beta \cdot \delta_{a}+m_{\delta_{c}} \cdot \delta_{c}+ \\
& +m_{\delta_{e}} \cdot \delta_{e}+\overline{y_{\delta_{r}}} \cdot \beta \cdot \delta_{r} \\
& r=-i_{3} \cdot p \cdot q+n_{\beta} \cdot \beta+n_{p}(\alpha, \beta) \cdot p+n_{r}(\alpha, \beta) \cdot r+n_{\delta_{a}} \cdot \delta_{a}+n_{\delta_{c a}}(\alpha) \cdot \delta_{c a}+n_{\delta_{r}} \cdot \delta_{r} \\
& \dot{\phi}=p+(q \cdot \sin \varphi+r \cdot \cos \varphi) \cdot \tan \theta \\
& \text { 。 } \\
& \theta=q \cdot \cos \varphi-r \cdot \sin \varphi \\
& \dot{\psi}=\frac{q \cdot \sin \varphi+r \cdot \cos \varphi}{\cos \theta}
\end{aligned}
$$

$$
\begin{aligned}
& \text { in }(5)_{1} \quad \cos \beta \approx 1 ;-p \cdot \cos \alpha \cdot \tan \beta \approx-p \cdot \beta ;-r \cdot \sin \alpha \cdot \tan \beta \approx 0 ; \cos \alpha \approx 1 ; \sin \alpha \approx 0 \\
& \text { in }(5)_{2} \quad \cos \beta \approx 1 \\
& \left\{\frac{I_{x z} \cdot\left(I_{x}+I_{z}-I_{y}\right)}{I_{x} \cdot I_{z}-I_{x z}^{2}} \approx 0 ; \quad \frac{\left(I_{y}-I_{z}\right) \cdot I_{z}-I_{x z}^{2}}{I_{x} \cdot I_{z}-I_{x z}^{2}} \approx-i_{1} ; \quad \frac{I_{x z}}{I_{y}} \approx 0 ;\right. \\
& \frac{I_{x z}^{2}+\left(I_{x}-I_{y}\right) \cdot I_{x}}{I_{x} \cdot I_{z}-I_{x z}^{2}} \approx-i_{3} ; \frac{I_{x z} \cdot\left(I_{y}-I_{z}-I_{x z}\right)}{I_{x} \cdot I_{z}-I_{x z}^{2}} \approx 0 ;
\end{aligned}
$$




$$
\left\{\begin{array}{l}
\stackrel{\circ}{\alpha}=q+\frac{g}{V} \cdot \cos \theta+z_{\alpha} \cdot \alpha+z_{\delta_{e}} \cdot \delta_{e} \\
\dot{\circ}=m_{\alpha} \cdot \alpha+m_{q} \cdot q+\frac{g}{V} \cdot\left(\overline{m_{\alpha}} \cdot \cos \theta-\frac{c_{2}}{a} \cdot a_{2} \cdot \sin \theta\right)+m_{\delta_{e}} \cdot \delta_{e} \\
\stackrel{\circ}{\theta}=q
\end{array}\right.
$$

\section{Theoretical proof of the existence of oscillatory movements}

Interest in oscillation susceptibility of an aircraft is generated by crashes of high performance fighter airplanes such as the YF-22A and B-2, due to oscillations that were not predicted during the aircraft development (Mehra \& Prasanth, 1998).Flying qualities and oscillation prediction are based on linear analysis and their quasi-linear extensions. These analyses cannot, in general, predict the presence or the absence of oscillations, because of the large variety of nonlinear interactions that have been identified as factors contributing to oscillations. The effects of some of these factors have been reported by Mehra et al., 1977; Shamma \& Athans, 1991; Kish et al., 1997; Klyde et al., 1997. The oscillation susceptibility analysis in a nonlinear model involves the computation of nonlinear phenomena including bifurcations, which lead sometimes to large changes in the stability of the aircraft.

Interest in oscillation susceptibility analysis of an unmanned aircraft, whose flight control system fails, was generated by the elaboration of an alternative automatic flight control for the case when the existing automatic flight control system of the aircraft fails Goto \& Matsumoto, 2000. Numerical results concerning this problem for the ALFLEX unmanned reentry vehicle, considered by Goto \& Matsumoto, 2000, for the final approach and landing phase are reported by Kaslik et al., 2002; 2004 a; 2004 b; 2005 a; 2005 b; 2005 c; Caruntu et al., 2005.

A theoretical proof of the existence of oscillatory solutions of the ALFLEX unmanned reentry vehicle in a longitudinal flight with constant forward velocity and decoupled flight control system is reported Kaslik \& Balint, 2010. Numerical results related to oscillation susceptibility analysis along the path of the longitudinal flight equilibriums of a simplified ADMIRE-model, when the automated flight control system fails are reported by Balint et al., 2009 a; 2009 b; 2009 c; 2010 a.

In this section we give a theoretical proof of the existence of oscillatory solutions in longitudinal flight in the simplified ADMIRE model of an unmanned aircraft, when the flight control system fails. This result was established by Balint et al., 2010b.

The simplified system of differential equations which governs the motion around the center of mass in a longitudinal flight with constant forward velocity of a rigid aircraft, when the automatic flight control system fails, is given by (see Balint et al., 2010b):

$$
\left\{\begin{array}{l}
\stackrel{\circ}{\alpha}=z_{\alpha} \cdot \alpha+q+\frac{g}{V} \cdot \cos \theta+z_{\delta_{e}} \cdot \delta_{e} \\
\dot{\circ}=m_{\alpha} \cdot \alpha+m_{q} \cdot q+\frac{g}{V} \cdot\left(\overline{m_{\dot{\alpha}}} \cdot \cos \theta-\frac{c_{2}}{a} \cdot a_{2} \cdot \sin \theta\right)+m_{\delta_{e}} \cdot \delta_{e} \\
\dot{\circ}=q
\end{array}\right.
$$


In this system, the state parameters are: angle of attack $\alpha$, pitch rate $q$ and Euler pitch angle $\theta$. The control parameter is the elevator angle $\delta_{e} . V$ is the forward velocity of the aircraft, considered constant and $g$ is the gravitational acceleration.

The aero dynamical data appearing in (15) are given in section 4.

The following proposition (Balint et al., 2010b) addresses the existence of equilibrium states for the system (15).

Proposition 1. $(\alpha, q \theta)^{T}$ is an equilibrium state of the system (15) corresponding to $\delta_{e}$ if and only if $\alpha$ is a solution of the equation:

$$
A \cdot \alpha^{2}+B \cdot \delta_{e} \cdot \alpha+C \cdot \delta_{e}^{2}+D=0
$$

$q$ is equal to zero and $\theta$ is a solution of the equation:

$$
\cos \theta=-\frac{V}{g}\left[z_{\alpha} \cdot \alpha+z_{\delta_{e}} \cdot \delta_{e}\right]
$$

where $A, B, C, D$ are given by:

$$
\begin{aligned}
& A=\left(m_{\alpha}-\overline{m_{\dot{\alpha}}} \cdot z_{\alpha}\right)^{2}+\frac{c_{2}{ }^{2}}{a^{2}} \cdot a_{2}{ }^{2} \cdot z_{\alpha}{ }^{2} \\
& B=2 \cdot\left(m_{\alpha}-\overline{m_{\dot{\alpha}}} \cdot z_{\alpha}\right) \cdot\left(m_{\delta_{e}}-\overline{m_{\dot{\alpha}}} \cdot z_{\delta_{e}}\right)+2 \cdot \frac{c_{2}{ }^{2}}{a^{2}} \cdot a_{2}{ }^{2} \cdot z_{\alpha} \cdot z_{\delta_{e}} \\
& C=\left(m_{\delta_{e}}-\overline{m_{\dot{\alpha}}} \cdot z_{\delta_{e}}\right)^{2}+\frac{c_{2}{ }^{2}}{a^{2}} \cdot a_{2}{ }^{2} \cdot z_{\delta_{e}}{ }^{2} \\
& D=-\frac{g^{2}}{V^{2}} \cdot \frac{c_{2}{ }^{2}}{a^{2}} \cdot a_{2}{ }^{2}
\end{aligned}
$$

Proof. By computation.

Proposition 2. Equation (16) has real solutions if and only if $\delta_{e}$ satisfies:

$$
\left|\delta_{e}\right| \leq \sqrt{\frac{4 \cdot A \cdot D}{B^{2}-4 \cdot A \cdot C}}
$$

Proof. By computation.

Proposition 3. If $z_{\alpha}<0$ and $\alpha$ is a real solution of Eq.(16), then Eq.(17) has a solution if and only if for $\alpha$ the following inequality holds:

$$
\frac{1}{z_{\alpha}} \cdot\left[\frac{g}{V}-z_{\delta_{e}} \cdot \delta_{e}\right] \leq \alpha \leq-\frac{1}{z_{\alpha}} \cdot\left[\frac{g}{V}+z_{\delta_{e}} \cdot \delta_{e}\right]
$$

Proof. By computation.

Remark. For $\delta_{e}=0$ the solutions of Eq.(16) are:

$$
\alpha= \pm \sqrt{\frac{\frac{g^{2}}{V^{2}} \cdot \frac{c_{2}{ }^{2}}{a^{2}} \cdot a_{2}{ }^{2}}{\frac{c_{2}{ }^{2}}{a^{2}} \cdot a_{2}{ }^{2} \cdot z_{\alpha}{ }^{2}+\left(m_{\alpha}-\overline{m_{\dot{\alpha}}} \cdot z_{\alpha}\right)^{2}}}
$$


and both verify (20) for $z_{\alpha}<0$.

In the following assume that $z_{\alpha}<0$ and consider $\underline{\delta_{e}}, \overline{\delta_{e}}$ defined as follows:

$$
\begin{aligned}
& \underline{\delta_{e}}=\inf \left\{\delta_{e} \mid \delta_{e}<0, \exists \text { a real solution of eq.(16) for which (20) holds }\right\} \\
& \overline{\delta_{e}}=\sup \left\{\delta_{e} \mid \delta_{e}>0, \exists \text { a real solution of eq.(16) for which (20) holds }\right\}
\end{aligned}
$$

Let $I$ be the closed interval $I=\left[\underline{\delta_{e}}, \overline{\delta_{e}}\right]$.

\section{Proposition 4.}

a. If $\delta_{e} \in I$, then for the system (15) there exists a countable infinity of equilibriums corresponding to $\delta_{e}$, namely for any $n \in Z$

$$
\begin{aligned}
& \left(\alpha_{1,2}=-\frac{B}{2 \cdot A} \cdot \delta_{e} \pm \frac{1}{2 \cdot A} \cdot \sqrt{B^{2} \cdot \delta_{e}^{2}-4 \cdot A \cdot\left(C \cdot \delta_{e}^{2}+D\right)} ; \quad q=0 ;\right. \\
& \left.\theta_{1,2}=2 \cdot \pi \cdot n \pm \arccos \left[\frac{V}{g} \cdot\left(-z_{\alpha} \cdot \alpha_{n}{ }^{ \pm}-z_{\delta_{e}} \cdot \delta_{e}\right)\right]\right)
\end{aligned}
$$

b. If $\delta_{e} \in \partial I=\left\{\delta_{e}, \overline{\delta_{e}}\right\}$, then the equilibriums corresponding to $\delta_{e}$ are saddle-node bifurcation points.

c. If $\delta_{e} \in I$, then for the system (15) there are no equilibriums corresponding to $\delta_{e}$.

Proof. By computation.

Proposition 4 translates into the following necessary and sufficient condition for the existence of equilibrium states for (15): $\delta_{e} \in I=\left[\underline{\delta_{e}}, \overline{\delta_{e}}\right]$. At $\delta=\underline{\delta_{e}}, \overline{\delta_{e}}$ saddle-node bifurcation occurs.

It can be easily verified that the following proposition is valid.

\section{Proposition 5.}

a. If $(\alpha(t), q(t), \theta(t))^{T}$ is a solution of the system (15), then $\theta(t)$ is a solution of the third order differential equation:

$$
\begin{aligned}
& \dddot{\theta}-\left(z_{\alpha}+m_{q}\right) \cdot \ddot{\theta}+\left[z_{\alpha} \cdot m_{q}-m_{\alpha}+\frac{g}{V} \cdot\left(\overline{m_{\dot{\alpha}}} \cdot \sin \theta+\frac{c_{2}}{a} \cdot a_{2} \cdot \cos \theta\right)\right] \cdot \dot{\theta}= \\
& =\frac{g}{V} \cdot\left(m_{\alpha}-z_{\alpha} \cdot \overline{m_{\circ}}\right) \cdot \cos \theta+\frac{g}{V} \cdot z_{\alpha} \cdot \frac{c_{2}}{a} \cdot a_{2} \cdot \sin \theta+\left(m_{\alpha} \cdot z_{\delta_{e}}-z_{\alpha} \cdot m_{\delta_{e}}\right) \cdot \delta_{e}
\end{aligned}
$$

b. If $\theta(t)$ is a solution of (22), then

$$
\begin{aligned}
& \alpha(t)=\frac{1}{m_{\alpha}} \cdot\left[\ddot{\theta}-m_{q} \cdot \dot{\theta}-\frac{g}{V} \cdot\left(\overline{m_{\dot{\alpha}}} \cdot \cos \theta-\frac{c_{2}}{a} \cdot a_{2} \cdot \sin \theta\right)-m_{\delta_{e}} \cdot \delta_{e}\right] \\
& q(t)=\dot{\theta}(t) \\
& \theta(t)=\theta(t)
\end{aligned}
$$

is a solution of the system (15).

Proof. By computation. 
A solution $\theta(t)$ of Eq.(22) is called monotonic oscillatory solution if the derivative $\dot{\theta}(t)$ is a strictly positive or a strictly negative periodic function.

Proposition 6. a. If there exists an increasing oscillatory solution $\theta(t)$ of Eq.(22)and $T>0$ is the period of $\dot{\theta}(t)$, then there exists $n \in N^{*}$ such that $\theta(t+T)=\theta(t)+2 n \pi$ and there exists a $2 n \pi$ periodic solution $x(s)$ of the equation:

$$
\begin{aligned}
& x^{\prime \prime}=2\left(x^{\prime}\right)^{2}+\left(z_{\alpha}+m_{q}\right) \cdot x^{\prime} \cdot e^{x}+\left[z_{\alpha} \cdot m_{q}-m_{\alpha}+\frac{g}{V} \cdot\left(\overline{m_{\dot{\alpha}}} \cdot \sin s+\frac{c_{2}}{a} \cdot a_{2} \cdot \cos s\right)\right] \cdot e^{2 x}- \\
& -\left[\frac{g}{V} \cdot\left(m_{\alpha}-z_{\alpha} \cdot \overline{m_{\alpha}}\right) \cdot \cos s+\frac{g}{V} \cdot z_{\alpha} \cdot \frac{c_{2}}{a} \cdot a_{2} \cdot \sin s+\left(m_{\alpha} \cdot z_{\delta_{e}}-z_{\alpha} \cdot m_{\delta_{e}}\right) \cdot \delta_{e}\right] \cdot e^{3 x}
\end{aligned}
$$

satisfying

$$
\int_{0}^{2 n \pi} e^{x(s)} \cdot d s=T
$$

b. If for $n \in N$ * there exists a $2 n \pi$-periodic solution $x(s)$ of Eq.(23), then there exists an increasing oscillatory solution $\theta(t)$ of Eq.(22) satisfying $\theta(t+T)=\theta(t)+2 n \pi$, where $T$ is given by (24).

Proof. See Balint et al., 2010b.

In order to prove that Eq.(22) has an increasing oscillatory solution, it is sufficient to prove that there exists a $2 n \pi$ - periodic solution of the Eq.(23).

Denoting $x_{1}=x$ and $x_{2}=-\left(z_{\alpha}+m_{q}\right) \cdot e^{-x}-x^{\prime} \cdot e^{-2 x}$, eq.(23) is replaced by the system:

$$
\begin{aligned}
& x_{1}{ }^{\prime}=-\left(z_{\alpha}+m_{q}\right) \cdot e^{x_{1}}-x_{2} \cdot e^{2 x_{1}} \\
& x_{2}{ }^{\prime}=\left[\left(-z_{\alpha} \cdot m_{\delta_{e}}+m_{\alpha} \cdot z_{\delta_{e}}\right) \cdot \delta_{e}+\frac{g}{V} \cdot\left(m_{\alpha}-z_{\alpha} \cdot \overline{m_{\dot{\alpha}}}\right) \cdot \cos s+\frac{g}{V} \cdot z_{\alpha} \cdot \frac{c_{2}}{a} \cdot a_{2} \cdot \sin s\right] \cdot e^{x_{1}}- \\
& \quad-\left[z_{\alpha} \cdot m_{q}-m_{\alpha}+\frac{g}{V} \cdot\left(\overline{m_{\dot{\alpha}}} \cdot \sin s+\frac{c_{2}}{a} \cdot a_{2} \cdot \cos s\right)\right]
\end{aligned}
$$

Proposition 7. a. If there exists a decreasing oscillatory solution $\theta(t)$ of (22) and $T>0$ is the period of $\dot{\theta}(t)$, then there exists $n \in N^{*}$ such that $\theta(t+T)=\theta(t)-2 n \pi$ and there exists a $2 n \pi$-periodic solution $x(s)$ of the equation:

$$
\begin{aligned}
x^{\prime \prime} & =2\left(x^{\prime}\right)^{2}-\left(z_{\alpha}+m_{q}\right) \cdot x^{\prime} \cdot e^{x}+\left[z_{\alpha} \cdot m_{q}-m_{\alpha}+\frac{g}{V} \cdot\left(\overline{m_{\dot{\alpha}}} \cdot \sin s+\frac{c_{2}}{a} \cdot a_{2} \cdot \cos s\right)\right] \cdot e^{2 x}+ \\
& +\left[\frac{g}{V} \cdot\left(m_{\alpha}-z_{\alpha} \cdot \overline{m_{\dot{\alpha}}}\right) \cdot \cos s+\frac{g}{V} \cdot z_{\alpha} \cdot \frac{c_{2}}{a} \cdot a_{2} \cdot \sin s+\left(m_{\alpha} \cdot \delta_{e}-z_{\alpha} \cdot m_{\delta_{e}}\right) \cdot \delta_{e}\right] \cdot e^{3 x}
\end{aligned}
$$

satisfying

$$
T=\int_{0}^{2 n \pi} e^{x(s)} \cdot d s
$$


b. If for $n \in N^{*}$, there exists a $2 n \pi$-periodic solution $x(s)$ of(26), then there exists a decreasing oscillatory solution $\theta(t)$ of (22), satisfying $\theta(t+T)=\theta(t)-2 n \pi$ with $T$ given by (27).

Proof. See Balint et al., 2010b.

It follows that in order to prove that Eq.(22) has a decreasing oscillatory solution, it is sufficient to prove that there exists a $2 n \pi$ - periodic solution of the Eq.(26).

Denoting by $x_{1}=x$ and $x_{2}=\left(z_{\alpha}+m_{q}\right) \cdot e^{-x}+x^{\prime} \cdot e^{-2 x}$ Eq.(26) is replaced by the system:

$$
\begin{aligned}
x_{1}{ }^{\prime}= & \left(z_{\alpha}+m_{q}\right) \cdot e^{x_{1}}+x_{2} \cdot e^{2 x_{1}} \\
x_{2}{ }^{\prime}= & {\left[\left(-z_{\alpha} \cdot m_{\delta_{e}}+m_{\alpha} \cdot z_{\delta_{e}}\right) \cdot \delta_{e}+\frac{g}{V} \cdot\left(m_{\alpha}-z_{\alpha} \cdot \overline{m_{\dot{\alpha}}}\right) \cdot \cos s+\frac{g}{V} \cdot z_{\alpha} \cdot \frac{c_{2}}{a} \cdot a_{2} \cdot \sin s\right] \cdot e^{x_{1}} } \\
& \quad\left[z_{\alpha} \cdot m_{q}-m_{\alpha}+\frac{g}{V} \cdot\left(\overline{m_{\dot{\alpha}}} \cdot \sin s+\frac{c_{2}}{a} \cdot a_{2} \cdot \cos s\right)\right]
\end{aligned}
$$

Hence, in order to show the existence of a decreasing oscillatory solution of Eq.(22) it is sufficient to show the existence of a $2 n \pi$ - periodic solution of (28).

For a continuous $2 n \pi$ periodic function we define:

$$
|f|_{M}=\max _{s \in[0,2 n \pi]} f(s) \quad|f|_{L}=\min _{s \in[0,2 n \pi]} f(s)
$$

Proposition 8. If $f$ is a smooth $2 n \pi$ periodic function, then

$$
|f|_{M} \leq|f|_{L}+\frac{1}{2} \cdot \int_{0}^{2 n \pi}|\dot{f}(s)| \cdot d s .
$$

Proof. See Balint et al., 2010b.

Let $X, Y$ be two infinite dimensional Banach spaces. A linear operator $L: D o m L \subset X \rightarrow Y$ is called a Fredholm operator if $\operatorname{Ker} L$ has finite dimension and $\operatorname{Im} L$ is closed and has finite codimension. The index of a Fredholm operator $L$ is the integer $i(L)=\operatorname{dim} \operatorname{Ker} L-\operatorname{codim} \operatorname{Im} L$.

In the following, consider $L: D o m L \subset X \rightarrow Y$ a Fredholm operator of index zero, which is not injective. Let $P: X \rightarrow X$ and $Q: Y \rightarrow Y$ be continuous projectors, such that $\operatorname{Ker} Q=\operatorname{Im} L, \operatorname{Im} P=\operatorname{Ker} L, X=\operatorname{Ker} L \oplus \operatorname{Ker} P \quad$ and $\quad Y=\operatorname{Im} L \oplus \operatorname{Im} Q . \quad$ The operator $L_{P}=\left.L\right|_{\text {DomL } \cap \text { KerP }}: \operatorname{Dom} L \cap \operatorname{KerP} \rightarrow \operatorname{Im} L$ is an isomorphism. Consider the operator $K_{P Q}: Y \rightarrow X$ defined by $K_{P Q}=K_{P}{ }^{-1}(I-Q)$. Let $\Omega \subset X$ be an open bounded set and $N: \Omega \rightarrow Y$ be a continuous nonlinear operator. We say that $N$ is $L$ compact if $K_{P Q} N$ is compact, $Q N$ is continuous and $Q N(\bar{\Omega})$ is a bounded set in $Y$. Since $\operatorname{Im} Q$ is isomorphic to $\operatorname{KerL}$, there exists an isomorphism $I: \operatorname{Im} Q \rightarrow \operatorname{KerL}$.

Mawhin, 1972; Gaines \& Mawhin, 1977 established the Mawhin's continuation theorem : Let $\Omega \subset X$ be an open bounded set, let $L$ be a Fredholm operator of index zero and let $N$ be L-compact on $\bar{\Omega}$. Assume:

a. $L x \neq \lambda N x$ for any $\lambda \in(0,1)$ and $x \in \partial \Omega \cap D o m L$.

b. $Q N x \neq 0$ for any $x \in \operatorname{KerL} \cap \partial \Omega$. 
c. Brouwer degree $\operatorname{deg}_{B}(I Q N, \Omega \cap \operatorname{KerL}, 0) \neq 0$.

Then $L x=N x$ has at least one solution in Dom $L \cap \bar{\Omega}$.

Consider the Eq.(22) and denote by:

$$
\begin{aligned}
& \tau=-\left(z_{\alpha}+m_{q}\right) \\
& \delta=z_{\alpha} \cdot m_{q}-m_{\alpha}+\frac{g}{V} \cdot\left(\overline{m_{\dot{\alpha}}} \cdot \sin \theta+\frac{c_{2}}{a} \cdot a_{2} \cdot \cos \theta\right) \\
& \gamma=\left(m_{\alpha} \cdot z_{\delta_{e}}-z_{\alpha} \cdot m_{\delta_{e}}\right) \cdot \delta_{e}+\frac{g}{V} \cdot z_{\alpha} \cdot \frac{c_{2}}{a} \cdot a_{2} \cdot \sin \theta \\
& \varepsilon=-\frac{g}{V} \cdot\left(m_{\alpha}-z_{\alpha} \cdot \overline{m_{\dot{\alpha}}}\right)
\end{aligned}
$$

With these notations Eq.(22) can be written as:

$$
\dddot{\theta}+\tau \cdot \ddot{\theta}+\delta \cdot \dot{\theta}=\gamma-\varepsilon \cdot \cos \theta
$$

As concerns the quantities $\tau, \delta, \gamma$, we make the following assumptions:

$$
\tau>0, \quad \delta>0 \text { and } \tau^{2}>4 \delta
$$

Remark that the above inequalities can be assured as follows:

$$
\begin{aligned}
& z_{\alpha}+m_{q}<0 \quad(\Leftrightarrow \tau>0) \\
& z_{\alpha} \cdot m_{q}-m_{\alpha}>\frac{g}{V} \cdot \sqrt{{\overline{m_{\dot{\alpha}}}}^{2}+\frac{c_{2}^{2}}{a^{2}} \cdot a_{2}^{2}} \quad(\Rightarrow \delta>0) \\
& \left(z_{\alpha}+m_{q}\right)^{2}>4 \cdot\left(z_{\alpha} \cdot m_{q}-m_{\alpha}\right)+\frac{4 g}{V} \cdot \sqrt{{\overline{m_{\dot{\alpha}}}}^{2}+\frac{c_{2}{ }^{2}}{a^{2}} \cdot a_{2}{ }^{2}} \quad\left(\Rightarrow \tau^{2}>4 \delta\right)
\end{aligned}
$$

Remark also that since $z_{\alpha}<0$ for $\gamma$ the following inequality holds:

$$
\left(m_{\alpha} \cdot z_{\delta_{e}}-z_{\alpha} \cdot m_{\delta_{e}}\right) \cdot \delta_{e}-\frac{g}{V} \cdot z_{\alpha} \cdot\left|\frac{c_{2}}{a} \cdot a_{2}\right| \geq \gamma \geq\left(m_{\alpha} \cdot z_{\delta_{e}}-z_{\alpha} \cdot m_{\delta_{e}}\right) \cdot \delta_{e}+\frac{g}{V} \cdot z_{\alpha} \cdot\left|\frac{c_{2}}{a} \cdot a_{2}\right|
$$

In terms of $\tau, \delta, \gamma, \varepsilon$ the systems (25) and (28) can be written in the forms:

$$
\left\{\begin{array}{l}
x_{1}{ }^{\prime}=\tau \cdot e^{x_{1}}-x_{2} \cdot e^{2 x_{1}} \\
x_{2}{ }^{\prime}=[\gamma(s)-\varepsilon \cdot \cos s] \cdot e^{x_{1}}-\delta(s)
\end{array}\right.
$$

and

$$
\left\{\begin{array}{l}
x_{1}{ }^{\prime}=-\tau \cdot e^{x_{1}}+x_{2} \cdot e^{2 x_{1}} \\
x_{2}{ }^{\prime}=[\gamma(s)-\varepsilon \cdot \cos s] \cdot e^{x_{1}}+\delta(s)
\end{array}\right.
$$

respectively. 
Theorem 1. If the inequalities (32) hold and

$$
\left(m_{\alpha} \cdot z_{\delta_{e}}-z_{\alpha} \cdot m_{\delta_{e}}\right) \cdot \delta_{e}+\frac{g}{V} \cdot z_{\alpha} \cdot\left|\frac{c_{2}}{a} \cdot a_{2}\right|>|\varepsilon|
$$

then for any $n \in N^{*}$ the system (2.20) has at least one $2 n \pi$-periodic solution.

Proof. See Balint et al., 2010b.

Theorem 2. If inequalities (32) hold and

$$
\left(m_{\alpha} \cdot z_{\delta_{e}}-z_{\alpha} \cdot m_{\delta_{e}}\right) \cdot \delta_{e}-\frac{g}{V} \cdot z_{\alpha} \cdot\left|\frac{c_{2}}{a} \cdot a_{2}\right|<-|\varepsilon|
$$

then for any $n \in N$ * the system (2.21) has at least one $2 n \pi$-periodic solution.

Proof. See Balint et al., 2010b.

The conclusion of this section can be summarized as:

Theorem 3. If inequalities (32) and (36) hold, then for any $n \in N^{*}$ equation (22) has at least one solution $\theta(t)$, such that its derivative $\dot{\theta}(t)$ is a positive $2 n \pi$-periodic function (i.e. $\theta(t)$ is an increasing oscillatory solution).

If inequalities (32) and (37) hold, then for any $n \in N^{*}$ equation (22) has at least one solution $\theta(t)$, such that its derivative $\dot{\theta}(t)$ is a negative periodic function (i.e. $\theta(t)$ is a decreasing oscillatory solution).

\section{Numerical examples}

To describe the flight of ADMIRE (Aero Data Model in a Research Environment) aircraft with constant forward velocity $V$, the system of differential equations (12) is employed: where:

$$
\begin{aligned}
& z_{\alpha}=a \cdot C_{N}^{\alpha} \quad z_{\delta_{e}}=a \cdot C_{N}^{\delta_{e}} \quad y_{\beta}=a \cdot C_{y}^{\beta} \quad y_{r}(\beta)=a \cdot C_{y}^{r}(\beta) \quad y_{\delta_{r}}=a \cdot C_{y}^{\delta_{r}} \\
& y_{\delta_{a}}=a \cdot C_{y}^{\delta_{a}} \quad y_{p}(\alpha, \beta)=a \cdot C_{y}^{p}(\alpha, \beta) m_{\alpha}=a_{2} \cdot\left(C_{m}^{\alpha}-c_{1} \cdot C_{N}^{\alpha}+c_{2} \cdot a \cdot C_{T}^{\alpha}+C_{m}^{\alpha} \cdot a \cdot C_{N}^{\alpha}\right) \\
& m_{\delta_{e}}=a_{2} \cdot\left(C_{m}^{\delta_{e}}-c_{1} \cdot C_{N}^{\delta_{e}}+C_{m}^{\alpha} \cdot a \cdot C_{N}^{\delta_{e}}\right) \quad m_{\delta_{c}}=a_{2} \cdot C_{m}^{\delta_{c}} \quad m_{q}=a_{2} \cdot\left(C_{m}^{q}+C_{m}^{\alpha}\right) \quad m_{\alpha}=a_{2} \cdot C_{m}^{\alpha} \\
& l_{\beta}(\alpha)=a_{1} \cdot C_{l}^{\beta}(\alpha) \quad l_{p}=a_{1} \cdot C_{l}^{p} \quad l_{r}(\alpha)=a_{1} \cdot C_{l}^{r}(\alpha) \quad l_{\delta_{r}}=a_{1} \cdot C_{l}^{\delta_{r}} \quad l_{\delta_{a}}=a_{1} \cdot C_{l}^{\delta_{a}} \\
& n_{\beta}=a_{3} \cdot\left(C_{n}^{\beta}+c_{3} \cdot C_{y}^{\beta}\right) n_{p}(\alpha, \beta)=a_{3} \cdot\left(C_{n}^{p}(\alpha, \beta)+c_{3} \cdot C_{y}^{p}(\alpha, \beta)\right) n_{\delta_{c a}}(\alpha)=a_{3} \cdot c_{3} \cdot C_{n}^{\delta_{c a}}(\alpha) \\
& n_{r}(\alpha, \beta)=a_{3} \cdot\left(C_{n}^{r}(\alpha, \beta)+c_{3} \cdot C_{y}^{r}(\beta)\right) \quad n_{\delta_{r}}=a_{3} \cdot\left(C_{n}^{\delta_{r}}+c_{3} \cdot C_{y}^{\delta_{r}}\right) \quad n_{\delta_{a}}=a_{3} \cdot\left(C_{n}^{\delta_{a}}+c_{3} \cdot C_{y}^{\delta_{a}}\right) \\
& \overline{y_{\beta}}=a_{2} \cdot c_{2} \cdot a \cdot C_{y}^{\beta} \quad \overline{y_{r}}=a_{2} \cdot c_{2} \cdot a \cdot C_{y}^{r}(\beta) \quad \overline{y_{p}}=a_{2} \cdot c_{2} \cdot a \cdot C_{y}^{p}(\alpha, \beta) \\
& \overline{y_{\delta_{r}}}=a_{2} \cdot c_{2} \cdot a \cdot C_{y}^{\delta_{r}} \quad \overline{y_{\delta_{a}}}=a_{2} \cdot c_{2} \cdot a \cdot C_{y}^{\delta_{a}} \quad C_{T}^{\alpha}=-0.157\left[\mathrm{rad}^{-1}\right] \quad C_{l}^{p}=-0.28\left[\mathrm{rad}^{-1}\right] \\
& C_{N}^{\alpha}=3.295\left[\mathrm{rad}^{-1}\right] \quad C_{l}^{r}(\alpha)=(0.344 \cdot \alpha+0.02)\left[\mathrm{rad}^{-1}\right] \quad C_{N}^{\delta_{e}}=1.074\left[\mathrm{rad}^{-1}\right] \\
& C_{n}^{\beta}=0.0907\left[\mathrm{rad}^{-1}\right] \quad C_{m}^{\alpha}=0.267\left[\mathrm{rad}^{-1}\right] \quad C_{n}^{\delta_{r}}=-0.0846\left[\mathrm{rad}^{-1}\right] \quad C_{m}^{\delta_{e}}=-0.426\left[\mathrm{rad}^{-1}\right] \\
& C_{n}^{\delta_{a}}=0.051\left[\mathrm{rad}^{-1}\right] \quad C_{m}^{\delta_{c}}=0.2\left[\mathrm{rad}^{-1}\right] \quad C_{n}^{\delta_{c a}}(\alpha)=-0.49 \cdot \alpha+0.0145[\mathrm{rad}]
\end{aligned}
$$


$C_{m}^{\alpha}=-0.44\left[\mathrm{rad}^{-1}\right] \quad C_{m}^{q}=-1.45\left[\mathrm{rad}^{-1}\right] \quad C_{1}^{\beta}(\alpha)=0.896 \cdot \alpha^{2}-0.47 \cdot \alpha-0.04[\mathrm{rad}]$

$C_{y}^{\beta}=0.804\left[\mathrm{rad}^{-1}\right] \quad C_{y}^{\delta_{r}}=-0.185\left[\mathrm{rad}^{-1}\right] \quad C_{y}^{\delta_{a}}=0.122\left[\mathrm{rad}^{-1}\right] \quad C_{y}^{r}(\beta)=2.725 \cdot \beta^{2}[\mathrm{rad}]$

$C_{y}^{p}(\alpha, \beta)=(6.796 \cdot \alpha+0.315) \cdot \beta^{2}+(0.237 \cdot \alpha-0.498) \cdot 10^{-3}[\mathrm{rad}]$

$C_{n}^{r}(\alpha, \beta)=\left(1.572 \cdot \alpha^{2}-0.368 \cdot \alpha-1.07\right) \cdot \beta^{2}-0.005[\mathrm{rad}]$

$C_{l}^{\delta_{r}}=0.024[\mathrm{rad}] ; \quad C_{l}^{\delta_{a}}=0.192[\mathrm{rad}] ; \quad C_{n}^{p}(\alpha, \beta)=(2.865 \cdot \alpha+0.3) \cdot \beta^{2}[\mathrm{rad}]$

$H=500[\mathrm{~m}] \quad M=0.25 \quad a_{s}=338\left[\mathrm{~m} \cdot \mathrm{s}^{-1}\right] \quad \rho=1.16\left[\mathrm{~kg} \cdot \mathrm{m}^{-3}\right]$

$g=9.81\left[\mathrm{~m} \cdot \mathrm{s}^{-2}\right] \quad V=M \cdot a_{\mathrm{s}}=84.5\left[\mathrm{~m} \cdot \mathrm{s}^{-1}\right] \quad g / V=0.116\left[\mathrm{~s}^{-1}\right]$

$S=45\left[\mathrm{~m}^{2}\right] \quad \bar{c}=5.2[\mathrm{~m}] \quad b=10[\mathrm{~m}] \quad \bar{m}=9100[\mathrm{~kg}] \quad x_{G}=1.3[\mathrm{~m}] \quad z_{e}=-0.15$

$I_{x}=21000\left[\mathrm{~kg} \cdot \mathrm{m}^{2}\right] \quad I_{y}=81000\left[\mathrm{~kg} \cdot \mathrm{m}^{2}\right] \quad I_{z}=101000\left[\mathrm{~kg} \cdot \mathrm{m}^{2}\right]$

$a=-0.485\left[\mathrm{~s}^{-1}\right] \quad a_{1}=88.743\left[\mathrm{~s}^{-1}\right] \quad a_{2}=11.964\left[\mathrm{~s}^{-1}\right] \quad a_{3}=18.45\left[\mathrm{~s}^{-1}\right]$

$c_{1}=0.25 \quad c_{2}=-0.029 \quad c_{3}=0.13 \quad i_{1}=0.952 \quad i_{2}=0.987 \quad i_{3}=0.594$

All the other derivatives are equal to zero.

The system which governs the longitudinal flight with constant forward velocity $V$ of the ADMIRE aircraft, when the automatic flight control fails, is:

$$
\left\{\begin{array}{l}
\stackrel{\circ}{\alpha}=q+\frac{g}{V} \cdot \cos \theta+z_{\alpha} \cdot \alpha+z_{\delta_{e}} \cdot \delta_{e} \\
\dot{\circ}=m_{\alpha} \cdot \alpha+m_{q} \cdot q+\frac{g}{V} \cdot\left(\overline{m_{\alpha}} \cdot \cos \theta-\frac{c_{2}}{a} \cdot a_{2} \cdot \sin \theta\right)+m_{\delta_{e}} \cdot \delta_{e} \\
\dot{\theta}=q
\end{array}\right.
$$

When the automatic flight control system is in function, then $\delta_{e}$ in (38) is given by:

$$
\delta_{e}=k_{\alpha} \cdot \alpha+k_{q} \cdot q+k_{p} \cdot \theta
$$

with $k_{\alpha}=-0.401 ; k_{q}=-1.284$ and $k_{p}=1 \div 8$.

System (38) is obtained from the system (12) for $\beta=p=r=\varphi=0 \quad \delta_{a}=\delta_{r}=\delta_{c}=\delta_{c a}=0$. The equilibriums of (38) are the solutions of the nonlinear system of equations:

$$
\left\{\begin{array}{l}
q+\frac{g}{V} \cdot \cos \theta+z_{\alpha} \cdot \alpha+z_{\delta_{e}} \cdot \delta_{e}=0 \\
m_{\alpha} \cdot \alpha+m_{q} \cdot q+\frac{g}{V} \cdot\left(\overline{m_{\alpha}} \cdot \cos \theta-\frac{c_{2}}{a} \cdot a_{2} \cdot \sin \theta\right)+m_{\delta_{e}} \cdot \delta_{e}=0 \\
q=0
\end{array}\right.
$$

System (40) defines the equilibriums manifold of the longitudinal flight with constant forward velocity $V$ of the ADMIRE aircraft. 
It is easy to see that (40) implies:

$$
A \cdot \alpha^{2}+B \cdot \delta_{e} \cdot \alpha+C \cdot \delta_{e}{ }^{2}+D=0
$$

where $A, B, C, D$ are given by:

$$
\begin{aligned}
& A=\left(m_{\alpha}-\bar{m}_{\alpha} \cdot z_{\alpha}\right)^{2}+\frac{c_{2}{ }^{2}}{a^{2}} \cdot a_{2}{ }^{2} \cdot z_{\alpha}{ }^{2} \\
& B=2 \cdot\left(m_{\alpha}-\bar{m}_{\dot{\alpha}} \cdot z_{\alpha}\right) \cdot\left(m_{\delta_{e}}-\bar{m}_{\alpha} \cdot z_{\delta_{e}}\right)+2 \cdot \frac{c_{2}{ }^{2}}{a^{2}} \cdot a_{2}{ }^{2} \cdot z_{\alpha} \cdot z_{\delta_{e}} \\
& C=\left(m_{\delta_{e}}-\bar{m}_{\alpha} \cdot z_{\delta_{e}}\right)^{2}+\frac{c_{2}{ }^{2}}{a^{2}} \cdot a_{2}{ }^{2} \cdot z_{\delta_{e}}{ }^{2} \\
& D=-\frac{g^{2}}{V^{2}} \cdot \frac{c_{2}{ }^{2}}{a^{2}} \cdot a_{2}{ }^{2}
\end{aligned}
$$

Solving Eq.(41) two solutions $a_{1}=a_{1}\left(\delta_{e}\right)$ and $a_{2}=a_{2}\left(\delta_{e}\right)$ are obtained. Replacing in (17) $a_{1}=$ $a_{1}\left(\delta_{e}\right)$ and $a_{2}=a_{2}\left(\delta_{e}\right)$ the corresponding $\theta_{1}=\theta_{1}\left(\delta_{e}\right)+2 k \pi$ and $\theta_{2}=\theta_{2}\left(\delta_{e}\right)+2 k \pi$ are obtained $(k \in Z)$. Hence a part of the equilibrium manifold $\mathcal{M}_{V}(k=0)$ is the union of the following two pieces:

$$
\mathscr{P}_{1}=\left\{\left(\alpha_{1}\left(\delta_{e}\right), 0, \theta_{1}\left(\delta_{e}\right)\right) \vdots \delta_{e} \in I\right\} ; \quad \mathscr{P}_{2}=\left\{\left(\alpha_{2}\left(\delta_{e}\right), 0, \theta_{2}\left(\delta_{e}\right)\right) \vdots \delta_{e} \in I\right\} .
$$

The interval $I$ where $\delta_{e}$ varies follows from the condition that the angles $\alpha_{1}\left(\delta_{e}\right)$ and $\alpha_{2}\left(\delta_{e}\right)$ have to be real.

Using the numerical values of the parameters for the ADMIRE model aircraft and the software MatCAD Professional it was found that:

$\underline{\delta_{e}}=-0.04678233231992[\mathrm{rad}]$ and $\overline{\delta_{e}}=0.04678233231992[\mathrm{rad}]$.

The computed $\alpha_{1}\left(\delta_{e}\right), \theta_{1}\left(\delta_{e}\right), \alpha_{2}\left(\delta_{e}\right), \theta_{2}\left(\delta_{e}\right)$ are represented on Fig.1, 2.

Fig.1 shows that $\alpha_{1}\left(\underline{\delta_{e}}\right)=\alpha_{2}\left(\underline{\delta_{e}}\right), \alpha_{1}\left(\overline{\delta_{e}}\right)=\alpha_{2}\left(\overline{\delta_{e}}\right)$ and $\alpha_{1}\left(\delta_{e}\right)>\alpha_{2}\left(\delta_{e}\right)$ for $\delta_{e} \in\left(\underline{\delta_{e}}, \overline{\delta_{e}}\right)$.

Fig.2 shows that $\theta_{1}\left(\underline{\delta_{e}}\right)=\theta_{2}\left(\underline{\delta_{e}}\right), \theta_{1}\left(\overline{\delta_{e}}\right)=\theta_{2}\left(\overline{\delta_{e}}\right)$ and $\theta_{1}\left(\delta_{e}\right)<\theta_{2}\left(\delta_{e}\right)$ for $\delta_{e} \in\left(\underline{\delta_{e}}, \overline{\delta_{e}}\right)$.

The eigenvalues of the matrix $A\left(\underline{\delta_{e}}\right)$ are: $\lambda_{1}=-22.6334 ; \lambda_{2}=-1.5765 ; \lambda_{3}=1.0703 \times 10^{-8} \approx 0$.

For $\delta_{e}>\underline{\delta_{e}}$ the equilibriums of $\mathcal{P}_{1}$ are exponentially stable and those of $\mathscr{P}_{2}$ are unstable. These facts were deduced computing the eigenvalues of $A\left(\delta_{e}\right)$.

More precisely, it was obtained that the eigenvalues of $A\left(\delta_{e}\right)$ are negative at the equilibriums of $\mathscr{P}_{1}$ and two of the eigenvalues are negative and the third is positive at the equilibriums of $\mathscr{P}_{2}$. Consequently, $\delta_{e}$ is a turning point. Maneuvers on $\mathscr{P}_{1}$ are successful and on $\mathscr{P}_{2}$ are not successful, Fig.3, 4 .

Moreover, numerical tests show that when $\delta_{e}{ }^{\prime}, \delta_{e}{ }^{\prime \prime} \in\left(\underline{\delta_{e}}, \overline{\delta_{e}}\right)$, the maneuver $\delta_{e}{ }^{\prime} \rightarrow \delta_{e}{ }^{\prime \prime}$ transfers the ADMIRE aircraft from the state in which it is at the moment of the maneuver in the asymptotically stable equilibrium $\left(\alpha_{1}\left(\delta_{e}{ }^{\prime \prime}\right), 0, \theta_{1}\left(\delta_{e}{ }^{\prime \prime}\right)\right)$. 


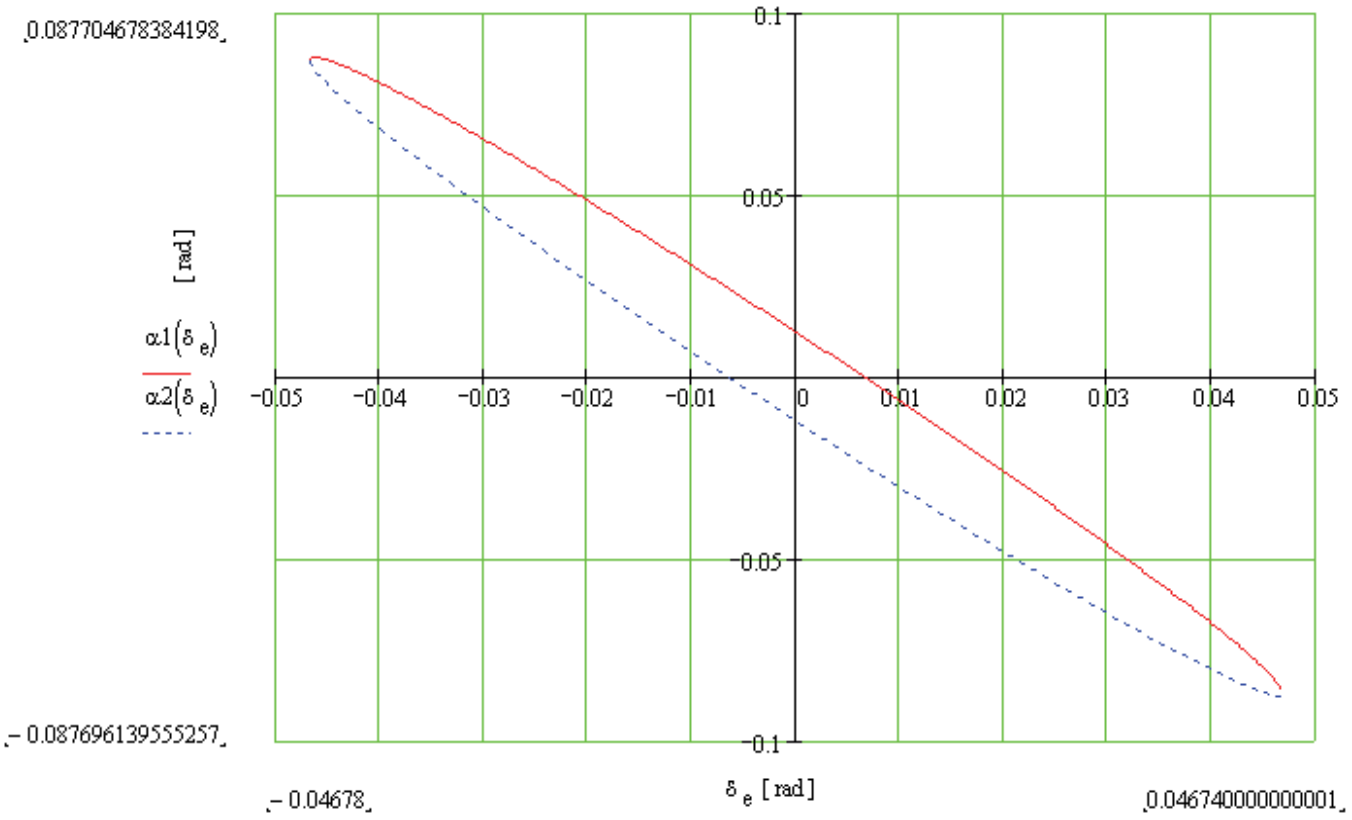

Fig. 1. The $a_{1}\left(\delta_{e}\right)$ and $a_{2}\left(\delta_{e}\right)$ coordinates of the equilibriums on the manifold $\mathrm{M}_{V}$.

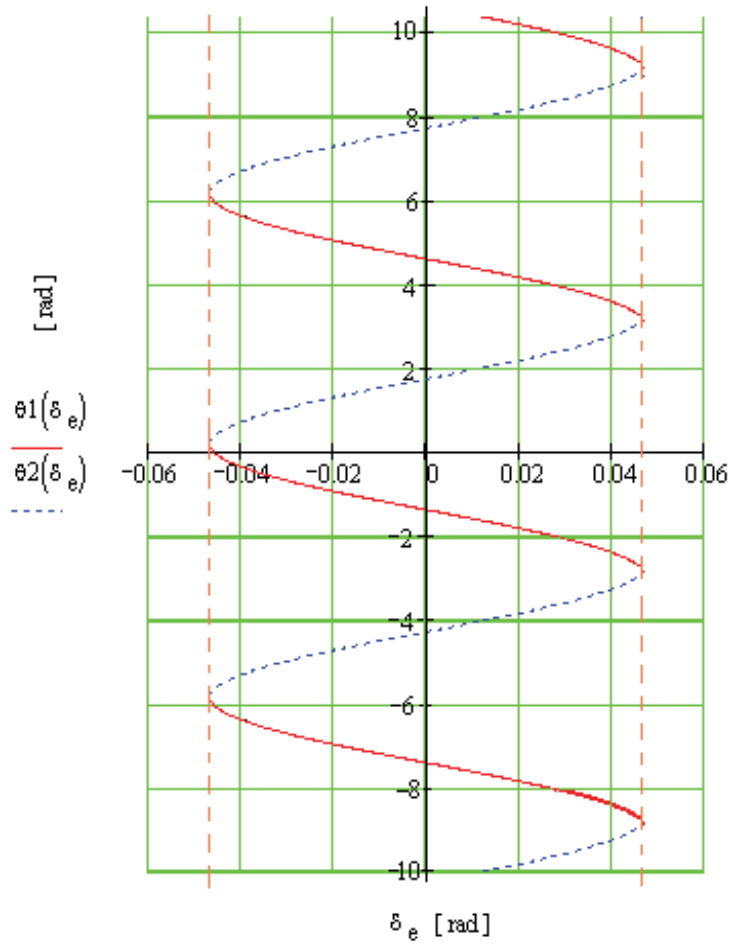

Fig. 2. The $\theta_{1}\left(\delta_{e}\right)+2 k \pi$ and $\theta_{2}\left(\delta_{e}\right)+2 k \pi$ coordinates of the equilibriums on the manifold $\mathcal{M}_{V}$. 

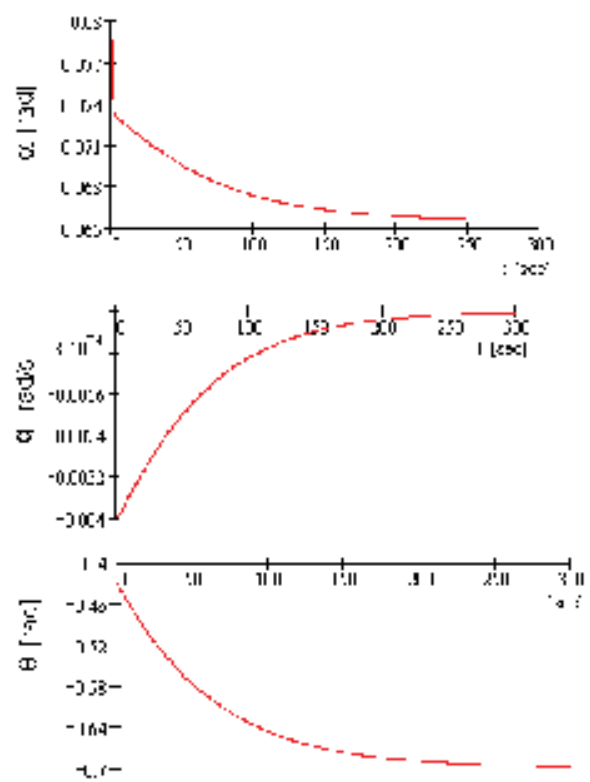

Fig. 3. A successful maneuver on $\mathscr{P}_{1}$ :

$a_{1}{ }^{1}=0.078669740237840[\mathrm{rad}] ; q_{1}{ }^{1=} 0[\mathrm{rad} / \mathrm{s}] ; \theta_{1}{ }^{1}=0.428832005303479[\mathrm{rad}] \rightarrow$ $a_{1}^{2}=0.065516737567037[\mathrm{rad}] ; q_{1}^{2}=0[\mathrm{rad} / \mathrm{s}] ; \theta_{1}^{2}=-0.698066723826469[\mathrm{rad}]$
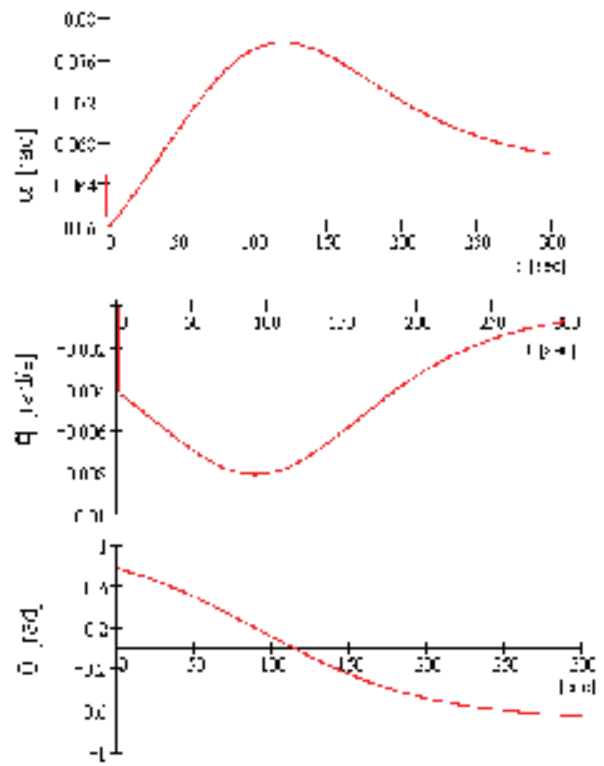

Fig. 4. An unsuccessful maneuver on $\mathscr{P}_{2}$ :

$a_{2}{ }^{1}=0.064883075974905[\mathrm{rad}] ; q_{2}{ }^{1=} 0[\mathrm{rad} / \mathrm{s}] ; \theta_{2} 1=0.767462467841413[\mathrm{rad}] \rightarrow$ $a_{1}{ }^{2}=0.065516737567037[\mathrm{rad}] ; q_{1}{ }^{2}=0[\mathrm{rad} / \mathrm{s}] ; \theta_{1}{ }^{2}=0.698066723826469$ [rad] instead of $a_{2}{ }^{1}=0.064883075974905[\mathrm{rad}] ; q_{2}{ }^{1}=0[\mathrm{rad} / \mathrm{s}] ; \theta_{2}{ }^{1}=0.767462467841413[\mathrm{rad}] \rightarrow$ $a_{2^{2}}=0.046845089090947[\mathrm{rad}] ; q_{2}{ }^{2}=0$ [rad/s] ; $\theta_{2}{ }^{2}=1.036697186364400$ [rad]. 

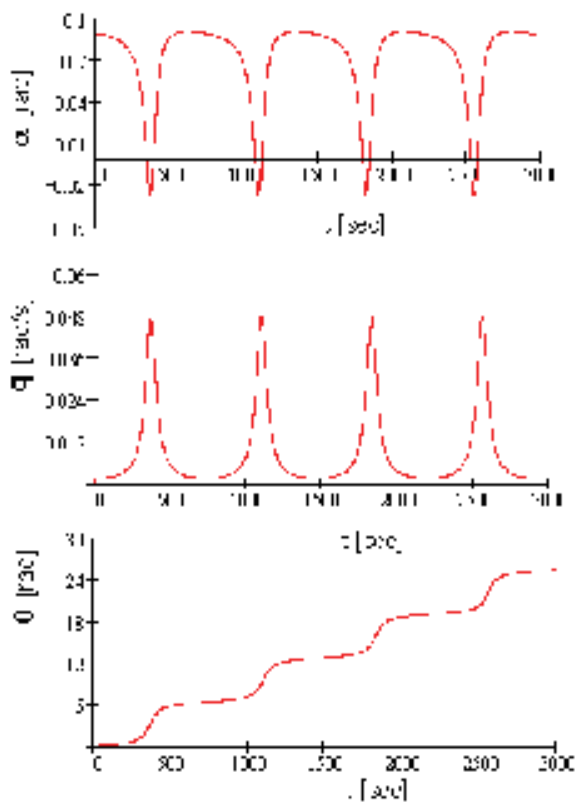

Fig. 5. Oscillation when $\delta_{e}=-0.05[\mathrm{rad}]$ and the starting point is : $a_{1}=0.086974288419088$ [rad]; $q_{1}=0$ [rad/sec]; $\theta 1=0.159329728679884[\mathrm{rad}]$.

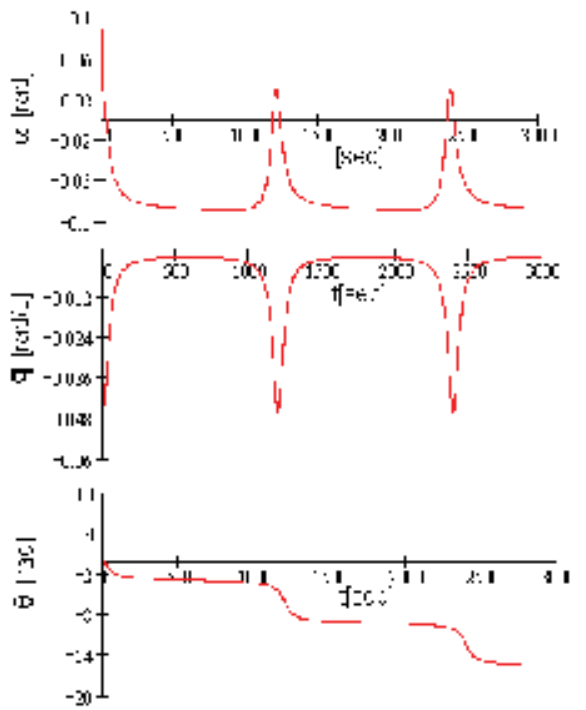

Fig. 6. Oscillation when $\delta_{e}=0.048[\mathrm{rad}]$ and the starting point is : $\alpha_{1}=0.086974288419088$ [rad]; $q_{1}=0$ [rad/sec]; $\theta 1=0.159329728679884[\mathrm{rad}]$. 
The behavior of the ADMIRE aircraft changes when the maneuver $\delta_{e}{ }^{\prime} \rightarrow \delta_{e}{ }^{\prime \prime}$ is so that $\delta_{e}{ }^{\prime} \in\left(\underline{\delta_{e}}, \overline{\delta_{e}}\right)$ and $\delta_{e}{ }^{\prime \prime} \notin\left(\underline{\delta_{e}}, \overline{\delta_{e}}\right)$. Computation shows that after such a maneuver $\alpha$ and $q$ oscillate with the same period and $\theta$ tends to $+\infty$ or $-\infty$. (Figs.5, 6)

The oscillation presented in Figs. 5,6 is a non catastrophic bifurcation, because if $\delta_{e}$ is reset, then equilibrium is recovered, as it is illustrated in Fig.7.
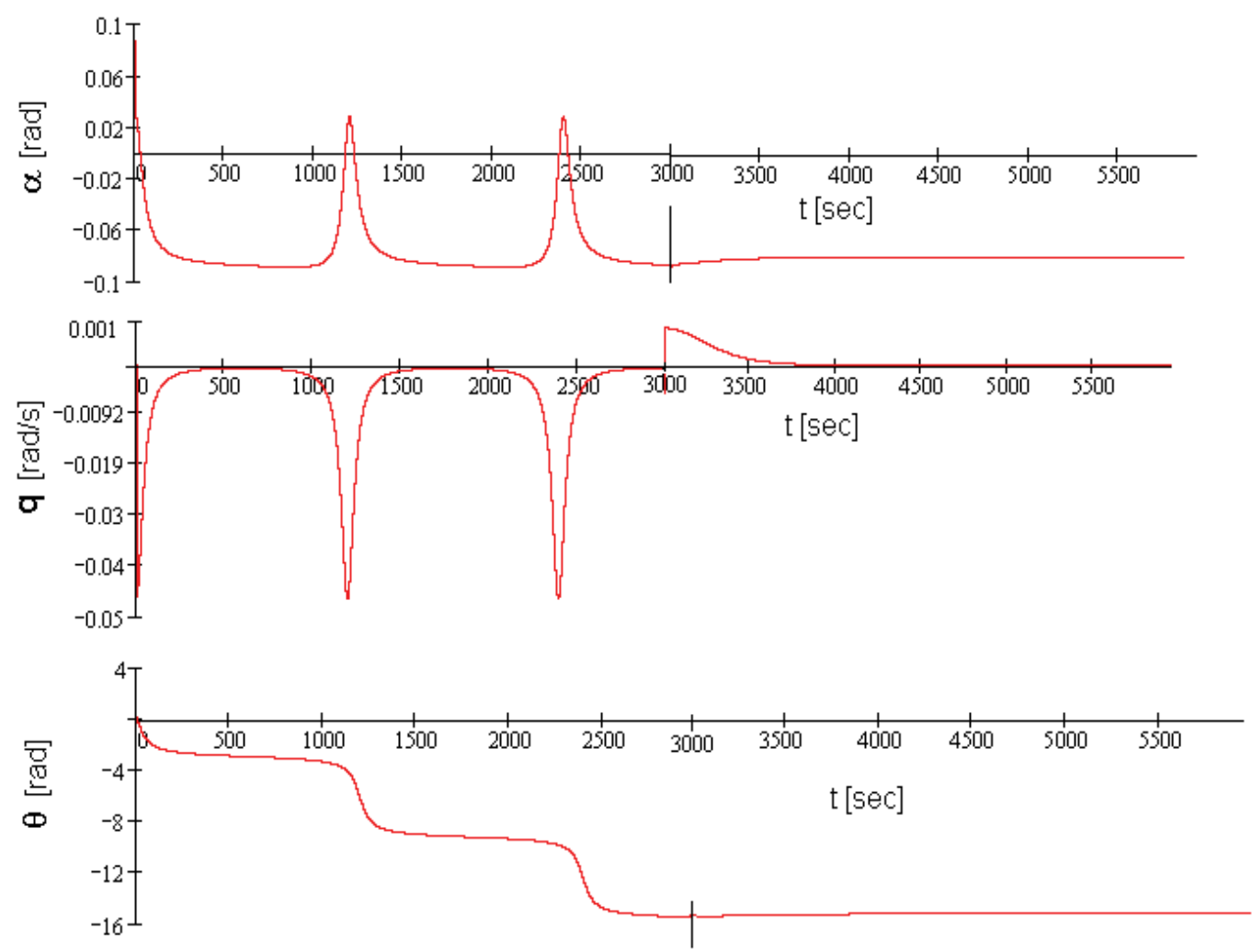

Fig. 7. Resetting $\delta_{e}=0.048[\mathrm{rad}]<\delta_{e 0}$ after 3000 [s] of oscillations to $\delta_{e}=\delta_{e 0}$, equilibrium is recovered.

\section{Conclusion}

For an unmanned aircraft whose automatic flight control system during a longitudinal flight with constant forward velocity fails, the following statements hold:

1. If the elevator deflection is in the range given by formula (19), then the movement around the center of mass is stationary or tends to a stationary state.

2. If the elevator deflection exceeds the value given by formula (36), then the movement around the center of mass becomes oscillatory decreasing and when the elevator 
deflection is less than the value given by formula (37), then the movement around the center of mass becomes oscillatory increasing.

3. This oscillatory movement is not catastrophic, because if the elevator deflection is reset in the range given by (19), then the movement around the center of mass becomes stationary.

4. Numerical investigation of the oscillation susceptibility (when the automatic flight control system fails) in the general non linear model of the longitudinal flight with constant forward velocity reveals similar behaviour as that which has been proved theoretically and numerically in the framework of the simplified model. As far as we know, in the general non linear model of the longitudinal flight with constant forward velocity the existence of the oscillatory solution never has been proved theoretically.

5. A task for a new research could be the proof of the existence of the oscillatory solutions in the general model.

\section{References}

Balint, St.; Balint, A.M. \& Ionita, A. (2009a). Oscillation susceptibility along the path of the longitudinal flight equilibriums in ADMIRE model. J. Aerospace Eng. 22, 4 (October 2009) 423-433 ISSN 0893-1321.

Balint, St.; Balint, A.M. \& Ionita, A. (2009b). Oscillation susceptibility analysis of the ADMIRE aircraft along the path of longitudinal flight equilibrium. Differential Equations and Nonlinear Mechanics 2009. Article ID 842656 (June 2009) 1-26. ISSN: 1687-4099

Balint, St.; Balint, A.M. \& Kaslik, E. (2010b) Existence of oscillatory solutions along the path of longitudinal flight equilibriums of an unmanned aircraft, when the automatic flight control system fails J. Math. Analysis and Applic., 363, 2(March 2010) 366-382. ISSN 0022-247X.

Balint, St.; Kaslik E.; Balint A.M. \& Ionita A. (2009c). Numerical analysis of the oscillation susceptibility along the path of the longitudinal flight equilibria of a reentry vehicle. Nonlinear Analysis:Theory, Methods and applic., 71, 12 (Dec.2009) e35-e54. ISSN: 0362-546X

Balint, St.; Kaslik, E. \& Balint, A.M. (2010a). Numerical analysis of the oscillation susceptibility along the path of the longitudinal flight equilibria of a reentry vehicle. Nonlinear Analysis: Real World Applic., 11, 3 (June 2010)1953-1962.ISSN: 1468-1218

Caruntu, B.; Balint, St. \& Balint, A.M. (2005). Improved estimation of an asymptotically stable equilibrium-state of the ALFLEX reentry vehicle Proceedings of the $5^{\text {th }}$ International Conference on Nonlinear Problems in Aviation and Aerospace Science, pp.129-136, ISBN: 1-904868-48-7, Timisoara, June 2-4, 2004, Cambridge Scientific Publishers, Cambridge.

Cook, M. (1997). Flight dynamics principles, John Wiley \& Sons, ISBN-10: 047023590X, ISBN13: 978-0470235904 , New York.

Etkin, B. \& Reid, L. (1996). Dynamics of flight: Stability and Control, John Wiley \& Sons, ISBN: 0-471-03418-5, New York. 
Gaines, R.\& Mawhin J. (1977). Coincidence Degree and Nonlinear Differential Equations, Springer, ISBN: 3-540-08067-8, Berlin - New York.

Goto, N. \& Matsumoto, K.(2000). Bifurcation analysis for the control of a reentry vehicle. Proceedings of the $3^{\text {rd }}$ International Conference on Nonlinear Problems in Aviation and Aerospace Science,pp.167-175 ISBN 0952664321 Daytona Beach, May 10-12, 2000 Cambridge Scientific Publishers, Cambridge

Kaslik, E. \& Balint, St.. (2007). Structural stability of simplified dynamical system governing motion of ALFLEX reentry vehicle, J. Aerospace Engineering, 20, 4 (Oct. 2007) 215219. ISSN 0893-1321.

Kaslik, E.; Balint, A.M.; Chilarescu, C. \& Balint, St. (2002). The control of rolling maneuver Nonlinear Studies, 9,4, (Dec.2002) 331-360. ISSN: 1359-8678 (print) 2153-4373 (online)

Kaslik, E.; Balint, A.M.; Grigis, A. \& Balint, St. (2004a). The controllability of the "path capture" and "steady descent flight of ALFLEX. Nonlinear Studies, 11,4, (Dec.2004) 674-690. ISSN: 1359-8678 (print) 2153-4373 (online)

Kaslik, E.; Balint, A.M.; Birauas, S. \& Balint, St. (2004 b). On the controllability of the roll rate of the ALFLEX reentry vehicle, Nonlinear Studies 11, 4 (Dec.2004) 543-564. ISSN: 1359-8678 (print) 2153-4373 (online)

Kaslik, E.; Balint, A.M.; Grigis, A. \& Balint, St. (2005a). On the set of equilibrium states defined by a simplified model of the ALFLEX reentry vehicle Proceedings of the $5^{\text {th }}$ International Conference on Nonlinear problems in aviation and aerospace science, pp.359372. ISBN: 1-904868-48-7, Timisoara, June 2-4 , 2004, Cambridge Scientific Publishers, Cambridge

Kaslik, E.; Balint, A.M.; Grigis, A. \& Balint, St. (2005b) Considerations concerning the controllability of a hyperbolic equilibrium state. Proceedings of the $5^{\text {th }}$ International Conference on Nonlinear problems in aviation and aerospace science, pp.383389 ISBN: 1-904868-48-7, Timisoara, June 2-4 , 2004, Cambridge Scientific Publishers, Cambridge

Kaslik, E.; Balint, A.M.; Grigis, A. \& Balint, St. (2005c) Control procedures using domains of attraction, Nonlinear Analysis, 63, 5-7, (Nov.-Dec.2005) e2397-e2407 ISSN: 0362$546 X$

Kaslik, E.\& Balint, St. (2010). Existence of oscillatory solutions in longitudinal flight dynamics. Int. J. Nonlinear Mechanics, 45(2) (March 2010) 159-168. ISSN: 00207462

Kish, B.A. ; Mosle, W.B. \& Remaly, A.S. (1997) A limited flight test investigation of pilotinduced oscillation due to rate limiting. Proceedings of the AIAA Guidance, Navigation, and Control Conference AIAA-97-3703 New Orleans (August 1997) 1332-1341

Klyde, D.H.; McRuer, D.T.; \& Myers, T.T. (1997). Pilot-induced oscillation analysis and prediction with actuator rate limiting. J. Guidance, Control and Dynamics, 20,1, (Jan.1997) 81-89 ISSN 0731-5090

Mawhin, J. (1972). Equivalence theorems for nonlinear operator equations and coincidence degree theory, Journal of Differential Equations 12, 3, 610-636 (Nov. 1972). ISSN: 00220396 
Mehra, R.K. \& Prasanth, R.K. (1998). Bifurcation and limit cycle analysis of nonlinear pilot induced oscillations, (1998) AIAA Paper98-4249 AIIA Atmosphere Flight Mechanics Conf., 10-12, August 1998, Boston, MA.

Mehra, R.K. ; Kessel, W.C. \& Carroll, J.V. (1977). Global stability and control analysis of aircraft at high angles of attack. ONR-CR215-248, vol.1-4 (June 1977) 81-153.

Shamma,I. \& Athans, M. (1991). Guaranted properties of gain scheduled control for linear parameter varying plants Automatica, 27,3, 559-564 (May 1991), ISSN: 0005-1098. 

